\title{
PLUTONIUM METALLURGY NOTEBOOK
}

\author{
Compiled by M.E. Hasbrouck \\ Edited by M.P. Burns
}

September, 1965

MESEARCH and

DEVELOPMENT REPORT 


\section{LEGAL NOTICE}

This report was prepared as an occount of Government sponsored work. Neither the United States; nor the Cammission, nor any person acting on behalf of the Commissian:

A. Makes any warranty ar representation, expresied or implind, with respect to the accuracy, completeness, or vaefuiness of the information comtained in this report, or that the use of any information, apparatus, method, or process ditclosed in this repoet moy not infringe privately owned rightss of

B. Assumes any liabilities with respect to the use of, or for damages cesulting from the use of any information, opparatus, mothod, of process disclosed in this report.

As used in the above, "persan acting on behalf of the Commission? includes any employece or contractor of the Commission, or employes of such contractor, to the extent that such emplayee or contractor of the Commission, or emplayee of sueh controctor prepores, disseminates, of provides accerss to, any information pursuant to his employment or contract with the Commission, or his employment with such contractor.

\section{PACIFIC NORTHWEST LABORATORY RICHLAND, WASHINGTON \\ operated by BATTELLE MEMORIAL INSTITUTE}

for the

UNITED STATES ATOMIC ENERGY COMMISSION UNDER CONTRACT AT(45-1)-1830 
BNWL-37

UC-25, Metals, Ceramics, and Materials

\section{PLUTONIUM METALLURGY NOTEBOOK}

Compiled by M. E. Hasbrouck*

Edited by M. P. Burns

Technical Information Section

Technical and Public Services Department

September, 1965

\section{PACIFIC NORTHWEST LABORATORY \\ RICHLAND, WASHINGTON}

\footnotetext{
*Present address: Lawrence Radiation Laboratory; University of
} California; Livermore, California 
:

• 


\section{TABLE OF CONTENTS}

$\underline{\text { Page }}$

INTRODUCTION ................ . . . v v

OUTLINE . . . . . . . . . . . . . . vi vi

PART I: PLUTONIUM . . . . . . . . . . 1

PART II: BINARY ALLOYS . . . . . . . . . . . 61

PART III: TERNARY ALLOYS . . . . . . . . 175

BIBLIOGRAPHY . . . . . . . . . . 205

ACKNOWLEDGEMENTS . . . . . . . . . . 209 



\section{INTRODUCTION}

This report is a compilation of data on the structure and properties of plutonium and its alloys. Included are phase diagrams, crystal structure data, transformation kinetics, physical and mechanical properties, corrosion and corrosive properties, and radiation effects information.

The data were obtained from reports, books, and journals. When results reported by two or more laboratories differed, the most recent data from each laboratory were included. Tentative or uncertain results were included only if later, more positive information was not available. The information is current to September, 1964.

The report is divided into three major sections. The first section presents data for unalloyed plutonium. In the second section, the binary alloys of plutonium are arranged alphabetically by the symbol for the alloying element. The third section consists of information on ternary alloys.

The properties for each material are arranged in a standard order with a number for each property, as shown in the outline on the following pages. In the sections on alloys, the number is preceded by the symbol of the alloying element. For example, the marginal heading 202.1 represents thermal expansion of plutonium, A1 202.1 represents thermal expansion of aluminum-plutonium alloys. The absence of a particular outline topic for a material indicates that no information was found on the subject.

Supplements to this report will be issued periodically. Within two years, a new edition will be issued, together with a volume on the properties of plutonium ceramics. The editor will welcome any suggestions of additions or changes. 
PART I. PLUTONIUM

PART II. BINARY ALLOYS

$\mathrm{Ag}$
$\mathrm{Al}$
$\mathrm{Au}$
$\mathrm{Be}$
ete.

PART III. TERNARY ALLOYS

General subject outline for use under PLUTONIUM and each alloy:

\author{
100 Structural Properties \\ 200 Physical Properties \\ 300 Mechanical Properties \\ 400 Corrosion \\ 500 Corrosive Properties \\ 600 Radiation Effects
}

The subdivisions under each of these main headings are given in the detailed outline which follows. This detailed outline will also be used under each alloy, with the chemical symbol of the alloying element and the outline number appearing in the margin. For example, information on the density of aluminum-plutonium alloys will be found under the marginal heading Al 201. 


\section{DETAILED OUTLINE}

100

\section{Structural Properties}

101 Phase diagrams

102 Transformation kinetics

103 Crystal structure

104 Stability of the various phases

105 Recrystallization and grain growth

106 Diffusion

200 Physical Properties

201 Density

202 Thermal properties (See also Transformation Kinetics)

202.1 Thermal expansion

202.2 Thermal conductivity

202.3 Specific heat and heat capacity

202.4 Entropy

202.5 Temperature of and volume change during transition

202.6 Heats of transition

203 Electrical properties

203.1 Electrical conductivity

203.2 Superconductivity

203.3 Thermoelectric power

204 Magnetic properties

204.1 Magnetic susceptibility

204.2 Hall effect

205 Optical, thermionic and electronic properties

205.1 Emissivity

206 Viscosity

300 Mechanical Properties

301 Tensile properties

302 Compressive properties

303 Torsional and shear properties

304 Impact strength

305 Creep

306 Fatigue

307 Hardness 
308.1 Young's modulus

308.2 Shear modulus

308.3 Bulk modulus

30). 4 Poisson's ratio

308.5 (ompressibility (reciprocal of hulk modulus)

400

Corrosion

401 Reactions with gases

$$
\begin{array}{ll}
401.1 & \text { Air } \\
401.2 & \text { Oxygen } \\
401.3 & \text { Iyydrogen } \\
\text { 101.4 } & \text { Inert gases }
\end{array}
$$

402 Aqueous corrosion

$$
\begin{aligned}
& 402.1 \text { Water and steam } \\
& 402.2 \text { Acids } \\
& \text { 102.3 Alkaline solutions }
\end{aligned}
$$

403 Organic compounds

40.4 Reactions with liquid metals

tos properties of colrosion films

500 Corrosive Propertics

600 Radiation Elfects

601 Structure

602 Physical properties

603 Mechanical properties 


\title{
PLUTONIUM METALLURGY NOTEBOOK
}

\author{
PART I \\ PLUTONIUM
}




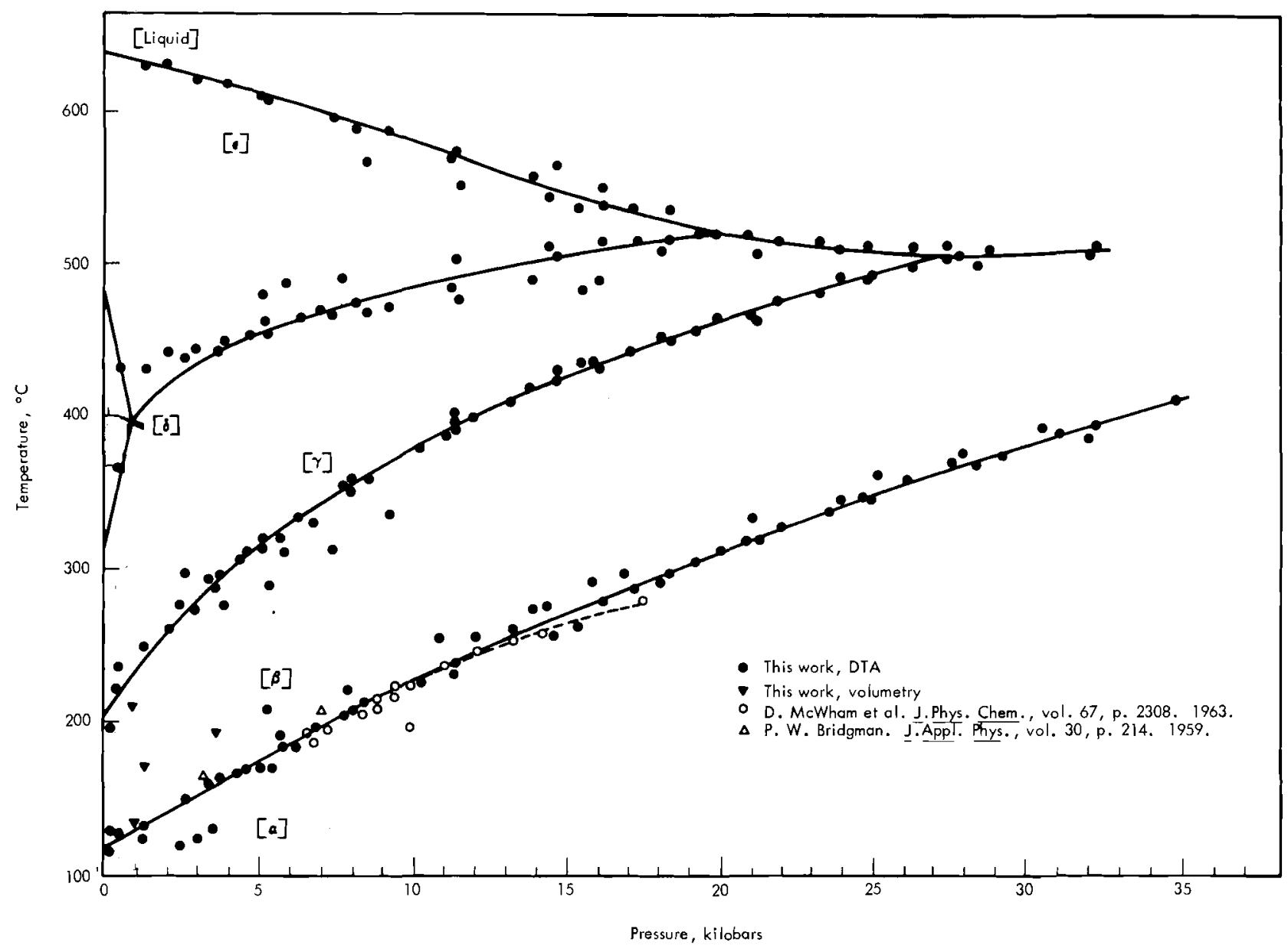

The Phase Diagram of Plutonium. From D. R. Stephens,

J. Phys. Chem. Solids, vol. 24, p. 1200.1963.

TRIPLE POINTS AND INITIAL TRANSITION SLOPES

From D. R. Stephens, J. Phys. Chem. Solids, vol. 24, p. 1201.1963.

$$
\begin{aligned}
& 398 \pm 10^{\circ} \mathrm{C} \\
& 0.9 \pm 0.2 \mathrm{kbar} \\
& 518+10^{\circ} \mathrm{C} \\
& 19.5+1 \mathrm{kbar} \\
& 500+10^{\circ} \mathrm{C}
\end{aligned}
$$

Experimental Values

Predicted by R. G. Loasby

Transition Initial Slope of Transition Lines, ${ }^{\circ} \mathrm{C} / \mathrm{kbar}$

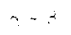

$-i$

$x-s$

$x-8$

f- 1iquid
Experimental Slope Predicted by R. G. Loasby

$\begin{array}{lr}11.2 & 13.1 \\ 27.3 & 38 \\ 88 & 95 \\ -87 & -190 \\ -4.4 & -26\end{array}$

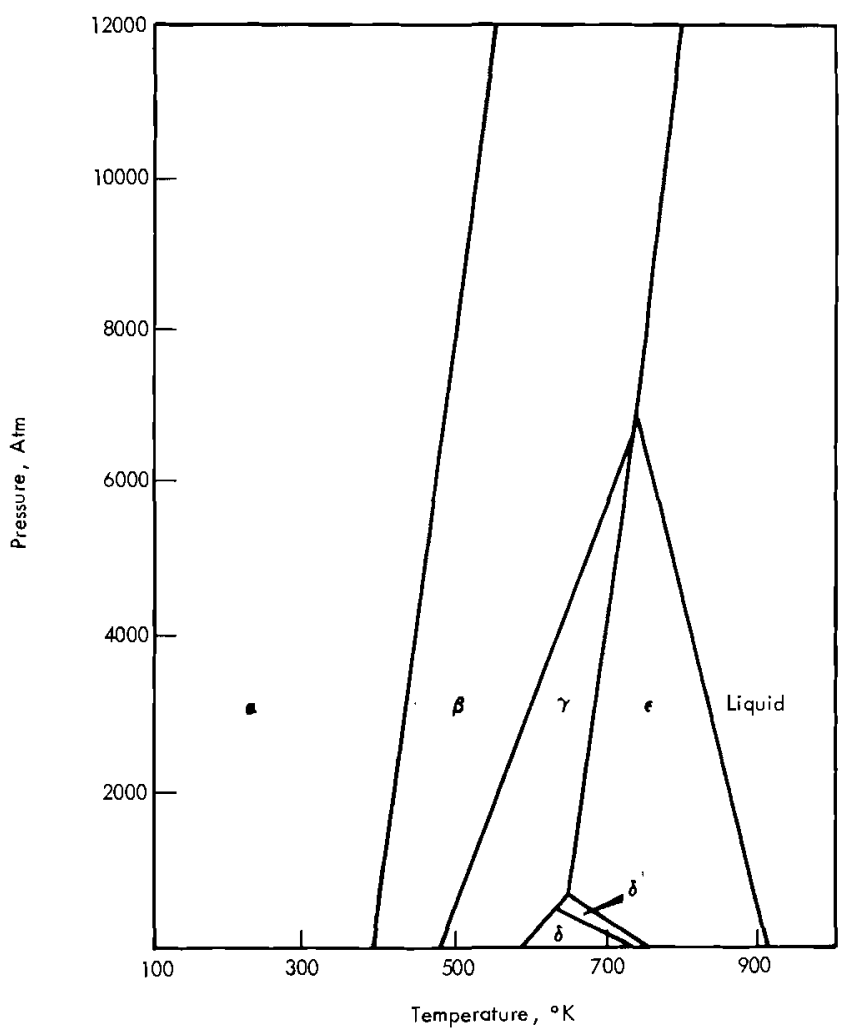

Derived Phase Diagram for Plutonium. From R G. Loasby Plutonium 1960, p. 102. 3 
PHASE DIAGRAM DATA

From R. G. Loasby, Plutonium 1960, p. 101.

\begin{tabular}{|c|c|c|}
\hline Phase Change & $\mathrm{dH} / \mathrm{dt}$, atm $/{ }^{\circ} \mathrm{C}$ & $\mathrm{dT} / \mathrm{dP},{ }^{\circ} \mathrm{C} /$ bar $10^{2}$ \\
\hline$n-3$ & 77.5 & 1.31 \\
\hline $2-\gamma$ & 27.0 & 3.8 \\
\hline$y-\delta$ & 10.7 & 9.5 \\
\hline$\delta-\delta^{\prime}$ & -5.3 & -19.0 \\
\hline $8^{1}-\varepsilon$ & -5.8 & -17.0 \\
\hline$\epsilon-1$ iquid & 39.6 & -2.6 \\
\hline$\gamma-\delta^{2}$ & 11.1 & 9.1 \\
\hline$y-\epsilon$ & 70.5 & 1.4 \\
\hline $3-\epsilon$ & 44.7 & 2.3 \\
\hline s-liquid & 91.6 & 1.1 \\
\hline
\end{tabular}

TRIPLE POINTS

From R. G. Loasby, Plutonium 1960, p. 101.

Bounding Phases

$v-8-\delta^{\prime}$

$v-s-\epsilon$

$B-\vee-\varepsilon$

$B-\epsilon-$ liquid
Pressure, atm

500

690

6750

6850

Temperature, ${ }^{\circ} \mathrm{K}$

635

647

735

737

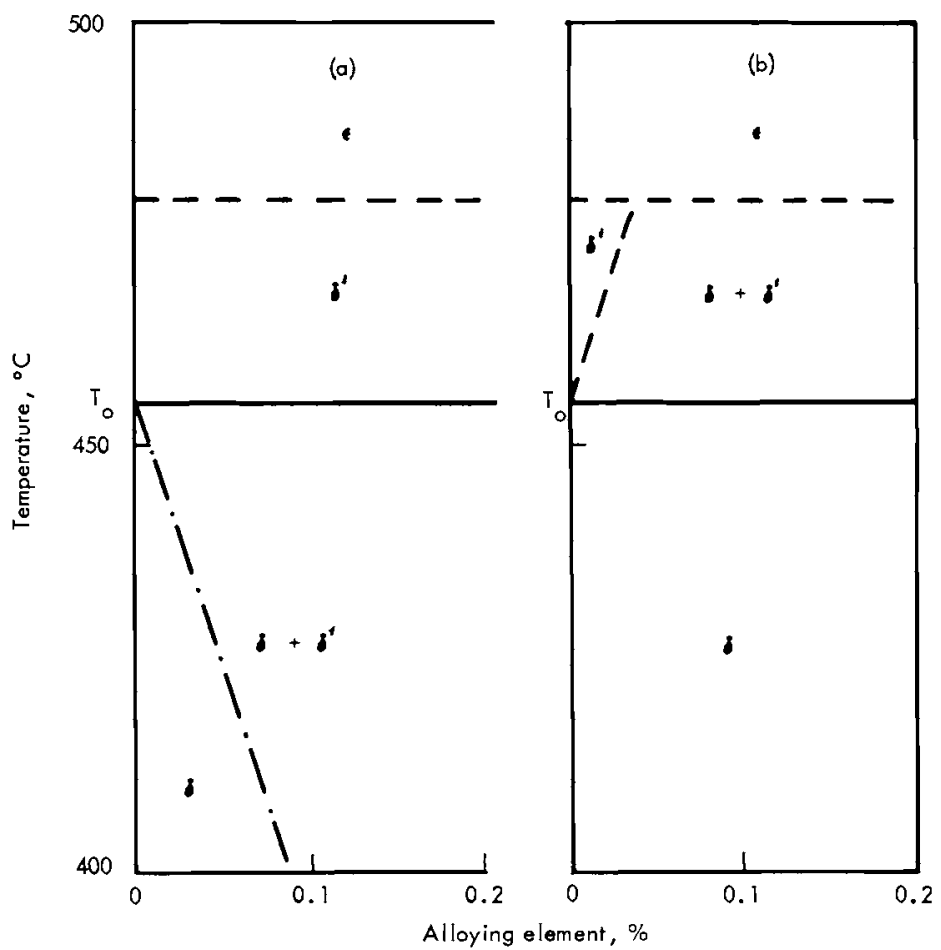

Limiting Cases for the Effect of Alloying Elements on the $\delta \Rightarrow \delta^{\prime}$ Transition in Plutonium.

In (a) above the impurity, addition does not affect the lower limit of the pure $s$ region, whereas in (b) the pure 8 region is effectively eliminated by the addition.

From R. G. Cope, Nature, vol. 186, p. 540. 1960. 


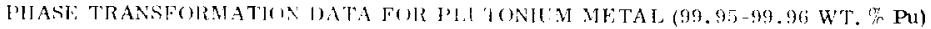

\begin{tabular}{|c|c|c|c|c|c|c|c|}
\hline \multirow[b]{3}{*}{$a \rightarrow B$} & \multirow[t]{2}{*}{ Diliatsometerer 11} & \multirow[t]{2}{*}{ Thermal dualygis (2) } & \multicolumn{2}{|c|}{ Retsistanee Measurements } & \multicolumn{2}{|c|}{ Inch/Inch of Ifngth At (2) } & \multirow[t]{2}{*}{$\varnothing(4)$} \\
\hline & & & Spocine:n 1 & Sperimen $2(i)$ & Tomperature $\mathrm{x}$ & & \\
\hline & $122(+1,-2)$ & 122 & $12: 3.9$ & $12: 3,0 \div 0,1$ & $248(i 2 ; j,-.3 i j)$ & 8,9 & $8,9-11,3$ \\
\hline$\beta \rightarrow \gamma$ & $200(-3,-2)$ & $20: 3$ & $20 \pi .5-22 i .1$ & $201.11+0.2$ & $78(16,-11)$ & 2.4 & $2.3-2.77$ \\
\hline$\gamma \rightarrow \delta$ & $339(+4,-3)$ & 317 & $3] 9.1 ;: 0.5$ & $317.0: 0.5$ & $205(+22,-11)$ & (i. 8 & 6. $7-6.8$ \\
\hline$\delta \rightarrow \delta^{\prime}$ & $451(-4,-2)$ & 453 & $4.1 .4-158.6 \mathrm{i}$ & $156.1+1.0$ & $-12(+7,-5)$ & -11.1 & $-0.1-0.4$ \\
\hline$\delta^{\prime} \rightarrow$ & $476(+3,-6)$ & $17 x$ & $4 \times(1) .(i-4861.8$ & $480.1-4 \times 7.5$ & $-126(+10,-12)$ & -3.0 & -3.0 \\
\hline$\rightarrow$ liquid & & $(j ; 29.6 \cdot 2$ & & & & & $\sim 0.1$ \\
\hline
\end{tabular}

(1) From F. M. Cramer et al. in The Metal 1'lutonjum, p. 119.

(2) From E. R. Jette in NucTear Metal lurgy, P. 35.

(3) From T. A. Sindenaw and R. B. Gibney. I. Phys. Chem. Solids, vol. 6, p. 86. 1958

(4) From W. D. Wilkinson in Extractive and rhysical Metallurgy of Plutonium and its Alloys, r. 20

JHASE-TRANSFORMATION TFMPFIRATURES FOR ZONE-MELTED PLUTONTUM (ABOIT 99, $\times$ AT. $\%$ PU, 250 PPM TOTAI, IM IIIRITTES) From M. B. Waldron el al. W/71, Proe. second Geneva Internat. Conf. vol. $6, \mathrm{p}, \mathrm{1} 62.195 \%$.

Trinsformation

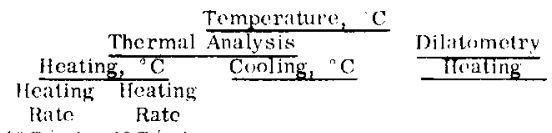

Alphis to betia Beta to gamm Gamma to delta Del ta to delta-prime Delta-prime to

epsilon

Fusilon to liquid

$$
\underline{1 .} \mathrm{C} / \mathrm{min} 44^{\circ} \mathrm{C} / \mathrm{min}
$$

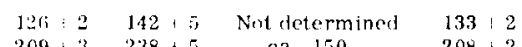

$$
\begin{aligned}
& 209: 3 \quad 238+5 \quad \text { ca. } 150 \quad 200+2 \\
& 316: 2 \quad 325: 5 \quad \text { ca. } 230 \quad 3014: 2 \\
& 451 ; 5 \quad 451 \div 5 \quad \text { Not certain } \quad 460 \div 2 \\
& 484: 2 \quad 481 \div 5 \quad 487: 2 \quad 460: 2
\end{aligned}
$$

TRANSITTON THMUPERATIRES IPOSSIRIA FRROR:

DILATOMFTRY, 99. (!) Wt.? PU.

From R. Abramson et al. pi327, Proc. Second Genova Internat. Conf. vol. 6, p. 175.1958 .

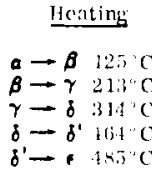

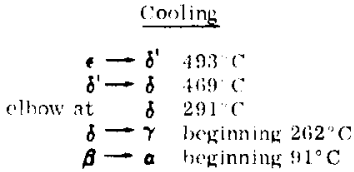

TEMPFRATIRFS OF PHASF, TRANSITTONS

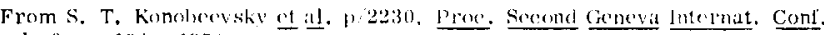
vol. $6, p .194 .19) \mathrm{s}$.

\section{Transition}
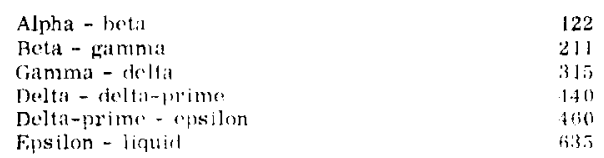

211

\begin{tabular}{|c|c|c|c|c|c|c|c|c|}
\hline \multirow{4}{*}{$\begin{array}{c}\text { Total lmpuritices } \\
\left.\text { (l.ess } \mathrm{O}_{2}\right) \\
\text { InWt. pym } \\
\text { Transformation }\end{array}$} & \multicolumn{6}{|c|}{ Ordinary Parity } & \multicolumn{2}{|c|}{ High-Purity } \\
\hline & \multirow{2}{*}{\multicolumn{2}{|c|}{$\begin{array}{l}1-1332-5,3 \\
\leq 3 \times 3 \\
\end{array}$}} & \multirow{2}{*}{\multicolumn{2}{|c|}{$\begin{array}{l}1-3(1): 5 \\
<: 3 \pi 11 \\
\end{array}$}} & \multirow{2}{*}{\multicolumn{2}{|c|}{$\begin{array}{r}1-30.37 \\
<-719 \\
\end{array}$}} & \multirow{2}{*}{\multicolumn{2}{|c|}{$\begin{array}{l}\text { KJ } J 329 \\
\leq 221 \\
\end{array}$}} \\
\hline & & & & & & & & \\
\hline & Start & End & $\underline{\text { Start }}$ & Endi & Start & End & Start & Find \\
\hline$\delta \rightarrow$ & 137 & & 437 & & $12 i$ & & & \\
\hline$\delta \rightarrow \delta^{\prime}$ & 4515 & 460 & 454 & $?$ & 155 & $45 !$ & $42=$ & \\
\hline$\delta \rightarrow$ & & $<482$ & & 176 & & $4 \pi$ & $1 \div 1$ & +400 \\
\hline
\end{tabular}

315

160

DFITA/DELTA-TRIME ANT DELTA-PRIME/FISSILON TIANSITION TFMTPRATIRES OF PLITTONI!M ON HEATING (IN * ()

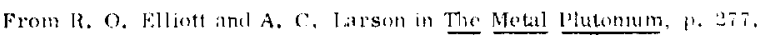

A small proportion of epsilon phase formed in the ordinary-purity metals at

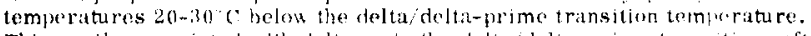
This epsilon coexisted with deltat up to the delta/delta-prime transition, aftes which it coexisted with delti-prime. The amount of epsilen appested to increase slightly as the temperature was ralsed, hut the main. ripitit iteltitfrime, cpsilon transition did not aecur mueh below $480 . \mathrm{C}$.

In high-purity plutonium, dol'a transformed isothermally to deltil-prime at $452: 20 \mathrm{C}$. With continued hoiting, a small amount of delta-prime was next observed to change to epsilon near $474^{\circ} \mathrm{C}$, and this phase nuxtule remained unchanged to $480^{\circ}: 2 \cdot C$, where the delta-prime epsilon trinsition tork place ripindly.

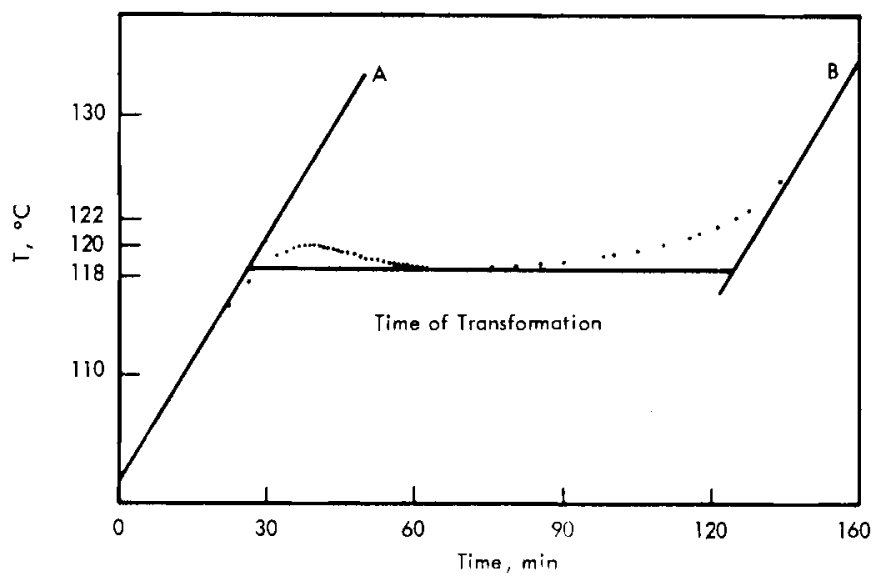

Detail of the a-s Transformation in Plutonium. From R. (i. Loashy, Plutontum 19t0, p. 100

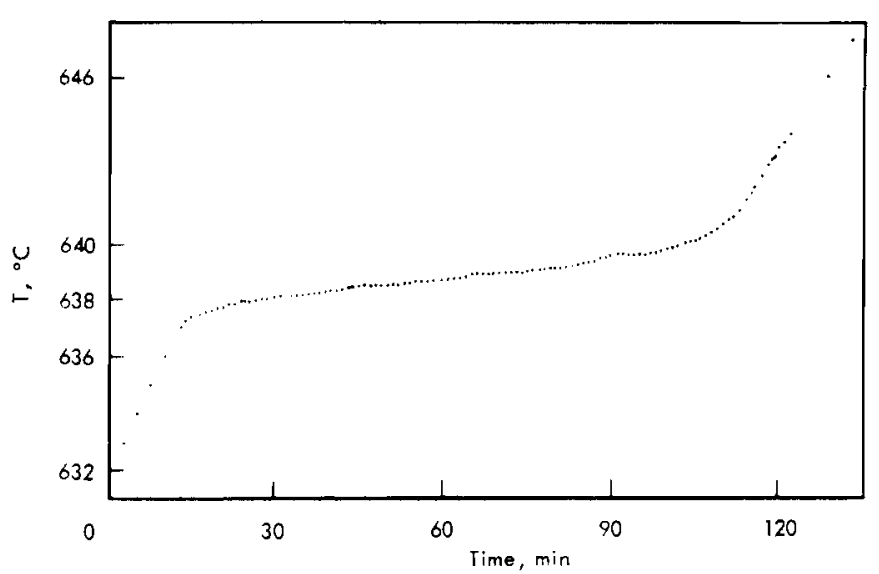

Detail of the -Liquid Transiormation in Plutonium. From R, G. loashy, Plutonium $1960, \mathrm{p} .101$. 


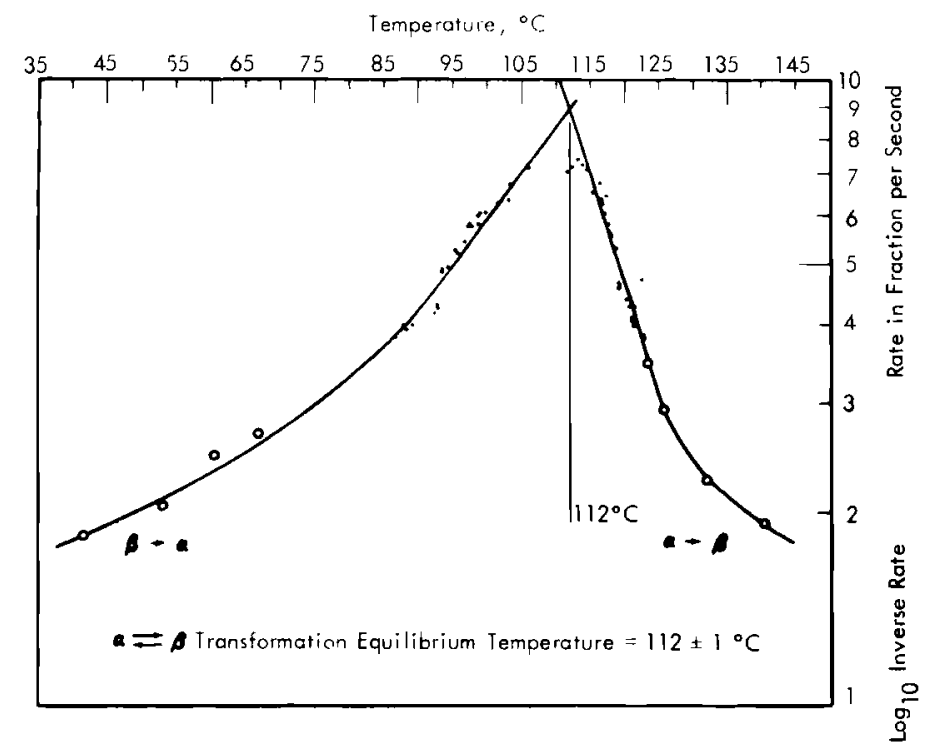

$a=\boldsymbol{\beta}$ "Maximum Rate" Study. From J. i). Hill, Nature, vol. 186, p. 305. 1960.

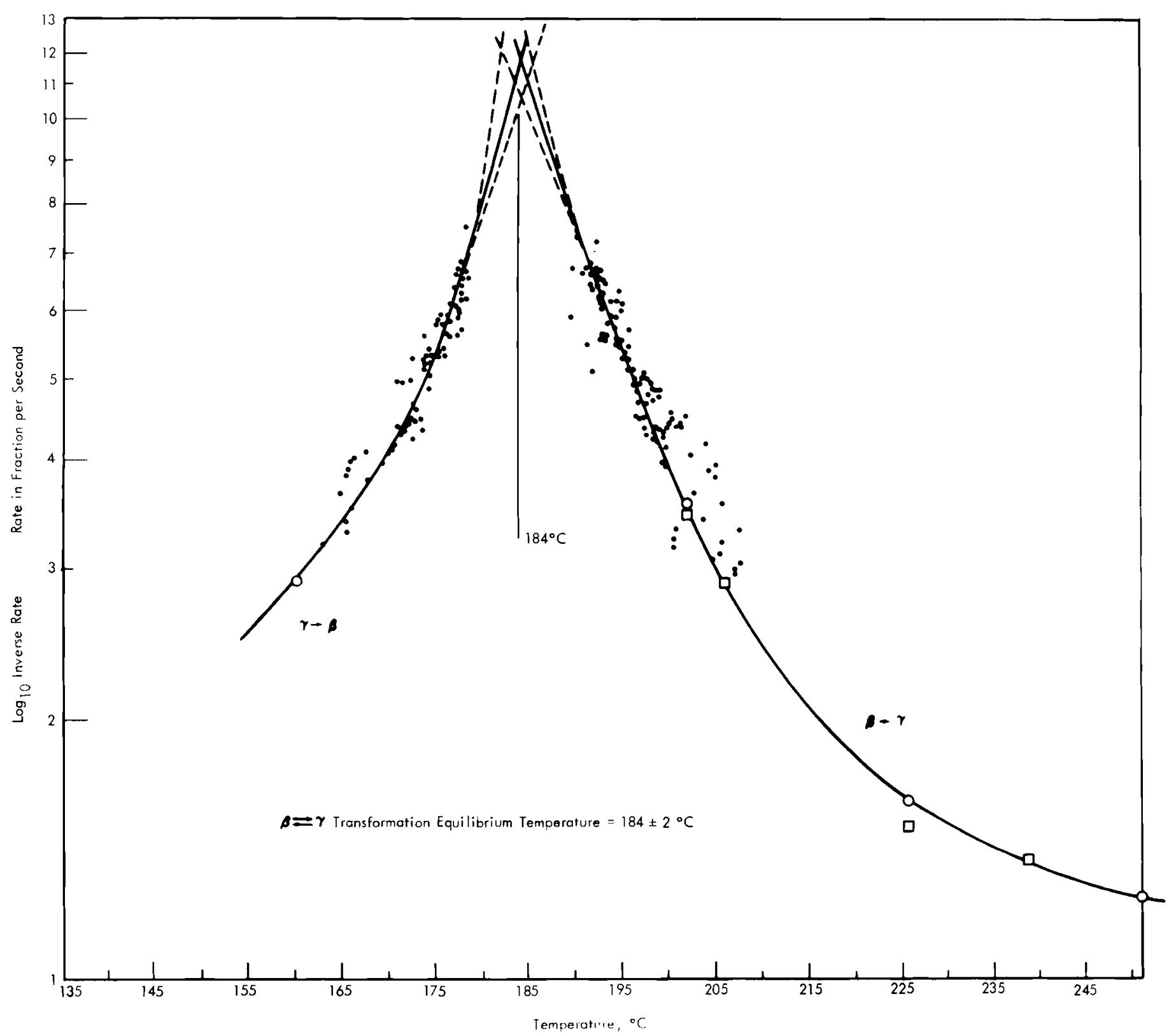




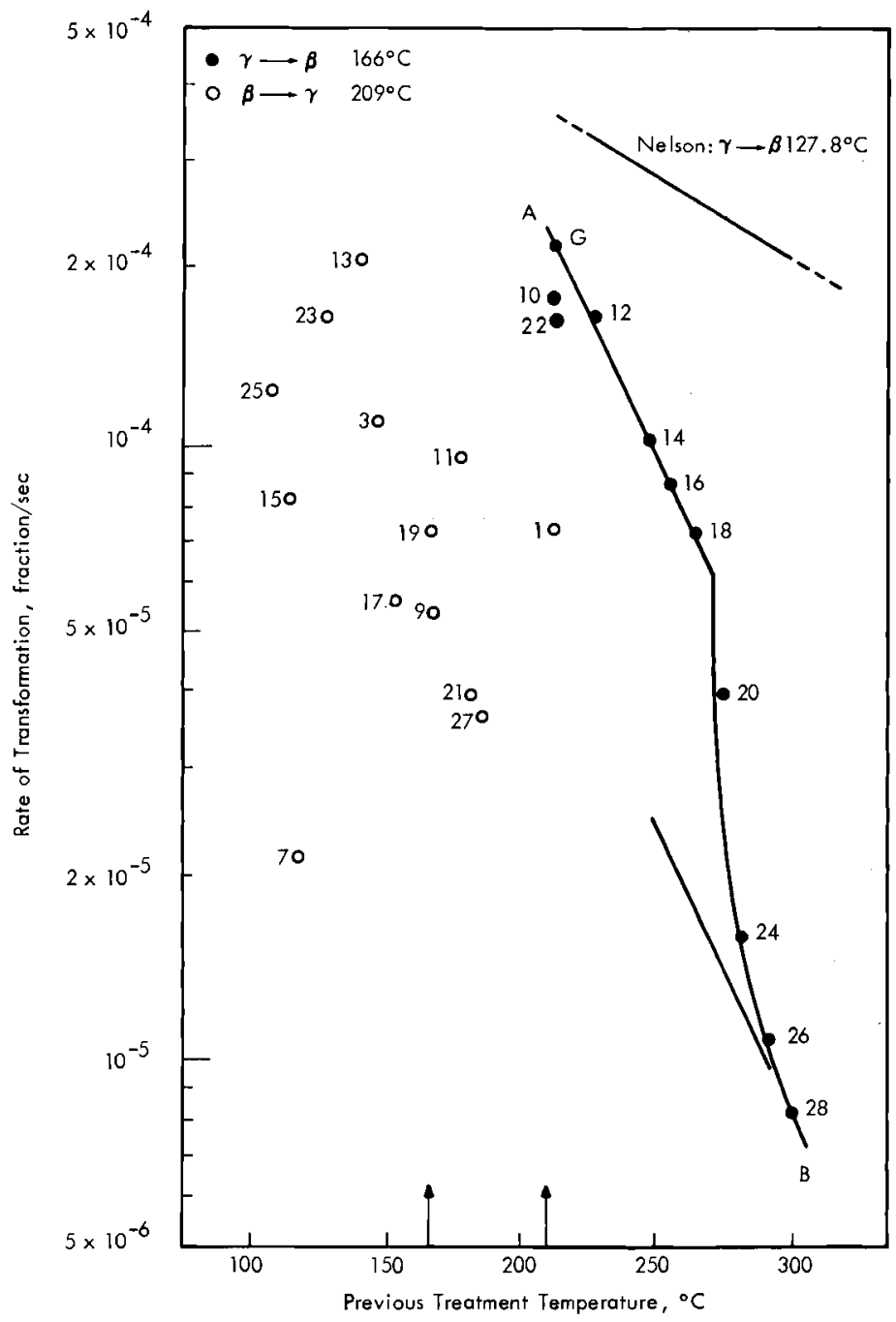

From J. D. Hill, Less-Common Metals, vol. 4, p. 381. 1962. R. D. Nelson, as shown in J. D Hill, AERE-R-3568.

THE $-\delta$ TRANSFORMATION IN PLUTONIUM. From $w$. M. Lomer, Solid State Commun., vol. 1, p. 96. 1963.

The $a-\delta$ phase transformation in I $5 \%$ Ce seems to be martensitic, A correspondence matrix is proposed which relates the lattices and accounts for most of the atomic positions.

It has been suggested that the a phase of Pu is in some way particularly closely associated with the $\delta$ phase. It is pointed out that the general form of the resistivity-temperature curve is similar in the two phases, and that in the $5 \% \mathrm{Ce} \mathrm{Pu}$ alloy a reversible transformation takes place at temperatures well below room temperature, where presumably atomic mobilities are so small that only a shear (martensitic) transformation seems admissible. There is, however, no direct microscopic evidence for this mechanism.

The details of such a transformation must be discussed in two stages; first a "correspondence" relation between the two lattices must be found. This relationship identifies that block of primitive unit cells of the simpler lattice which is to be deformed slightly to make a single unit cell of the complex lattice. Then secondly the macroscopic deformation required to bring this block to the correct dimensions must be evaluated, and microscopically inhomogeneous deformations discovered to accommodate these strains in a constrained crystal. This stage of the argument is very difficult indeed to carry through even when good metallographic studies are available for guidance. This is not yet the case for Pu.

An apparently satisfactory correspondence matrix has, however, been discovered. The $\delta$ lattice is referred to the cube axes, the cube edge $\left(a_{0}=4.637 \mathrm{~A}\right)$ being defined as unity. The monoclinic a lattice is taken with conventional parameters

$$
a-6.182 \mathrm{~A} ; \mathrm{b}=4.826 \mathrm{~A} ; \mathrm{c}=10.956 \mathrm{~A} ; \boldsymbol{B}-101.7^{\circ}
$$

the unit cell contains 16 atoms, and is therefore constructed from four unit cells of the face-centered cubic lattice. The correspondence proposed is

$$
M=\left(\begin{array}{rrr}
-1 & -1 & 0 \\
0 & 0 & 1 \\
3 / 2 & -5 / 2 & 0
\end{array}\right) \quad M^{-1}=\left(\begin{array}{rrr}
-5 / 8 & 0 & 1 / 4 \\
-3 / 8 & 0 & -1 / 4 \\
0 & 1 & 0
\end{array}\right)
$$

The rows of $M$ give the vectors in the lattice from which the $a, b$, and $c$ vectors are derived. The determinant of $\mathrm{M}$ is four, as required by the number of atoms per unit cell.

The parameters of the pseudo a cell defined by $\mathrm{M}$ in an undistorted $\delta$ lattice are

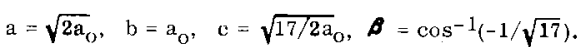

Numerically these values are

$$
\mathrm{a}=6.557, \mathrm{~b}=4.637, \mathrm{c}=13.52, \boldsymbol{B}=104.1^{\circ}
$$

Conversely the a cell is transformed by the correspondence matrix into a pseudo-cubic cell of dimensions

$$
\mathrm{x}=4.259, \quad \mathrm{y}=3.931, \quad \mathrm{z}=4 . k 26, \quad \gamma=82.06
$$

Such a cell would have been produced by a strain matrix $\epsilon$ acting on the $\delta$ lattice where $e$ is the matrix

$$
=\left(\begin{array}{ccc}
-0.084 & 0.061 & 0 \\
0.061 & -0.154 & 0 \\
0 & 0 & 0.041
\end{array}\right)
$$

The detailed atomic positions in Pu of course differ from the ideal ones which would be derived from the homogeneously-distorted f.c.c. lattice. We list below the ideal values and observed values for the 16 atoms in the strueture, referred to the center of symmetry as origin. This center is midway between two atoms of the f,c.c. lattice at $(0,3 / 4,-1 / 4) \delta=(-9 / 32,-1 / 4$, $-3 / 16)_{a}=-(0.281,0.25,0.187)_{a}$.

The remaining atoms are at the positions given by reflection in the origin. It

\begin{tabular}{|c|c|c|c|c|c|c|c|c|}
\hline \multicolumn{3}{|c|}{ Ideal } & \multicolumn{3}{|c|}{ Ideal Referred to C. o S } & \multicolumn{3}{|c|}{ Actual } \\
\hline 0 & 0 & u & 0.281 & 0.25 & 0.187 & 0.332 & 0.25 & 0.152 \\
\hline $7 / 8$ & 0 & $1 / 4$ & 0.156 & 0.25 & 0.437 & 0.138 & 0.25 & 0.337 \\
\hline $3 / 4$ & 0 & $1 / 2$ & 0.031 & 0.25 & 0.687 & 0.013 & 0.25 & 0.617 \\
\hline $5 / 8$ & 0 & $3 / 4$ & 0.906 & 0.25 & 0.937 & 0.885 & 0.15 & 0.897 \\
\hline $1 / 2$ & 0 & 0 & 0.781 & 0.25 & 0.187 & 0.767 & 0.25 & 0.167 \\
\hline $3 / 8$ & 0 & $1 / 4$ & 0.656 & 0.25 & 0.437 & 0.651 & 0.25 & 0.456 \\
\hline $1 / 4$ & 0 & $1 / 2$ & 0.531 & 0.25 & 0.617 & 0.459 & 0.25 & 0.642 \\
\hline $1 / 8$ & 0 & $3 / 4$ & 0.406 & 0.25 & 0.937 & 0.335 & 0.25 & 0.924 \\
\hline
\end{tabular}
will be seen that all the atom movements are small - only the second atom listed is displaced by as much as $1 \mathrm{~A}(=0.1 \mathrm{c}$ or 0.25 a).

This listing sequence has been chosen to emphasize the well-marked rows of atoms along the $(\overline{102})$ direction in the a structure, which are derived from the $(1 \overline{1} 0) \delta$ rows.

HIGH TEMPERA TURE OXIDATION OF PLUTONIUM. From J. T. Waber, Metallurgy and Fuels, series V, vol. 4, p. 890 .

Plutonium is not very reactive towards nitrogen. For example, plutoniun after being melted in a nitrogen atmosphere and held for several hours at $100^{\circ} \mathrm{C}$, contained only a few nitride dendrites near the surface. Thus the high temperature properties in air can be reduced to the behaviour towards pure oxygen at equivalent temperatures.

The oxidation of plutonjum in pure oxygen in the range between 315 and $400^{\circ} \mathrm{C}$ has been studied manometrically. Specimens made from $0.020 \mathrm{in}$. ribbon were oxidized to completion in about $20 \mathrm{~min}$. at $343^{\circ} \mathrm{C}$ and in about 6 min. at $400^{\circ} \mathrm{C}$. Time dependences greater than one were observed; thus the reaction may be regarded as accelerating in this temperature range. The acceleration is undoulstedly due to the spallation of the oxide during the run. In contrast, the reaction rate decreases with time at $315^{\circ}$ to $325^{\circ} \mathrm{C}$ and possibly at lower temperitures. The reactions went to completion on 0.020 in. sheet in a little over an hour at these temperatures.

A $10 \mathrm{wt}$. p plutonium alloy in aluminum which consists of the intermetallic compound $\mathrm{PuAl}_{4}$ dispersed in a matrix of aluminum, was held one week at $400^{\circ} \mathrm{C}$ in pure oxygen. The pick-up of oxygen was harely detectable by the manometric method employed for the oxidation of pure plutonium. An upper limit on the amount of oxide formed is $8 \mu$. An X-ray diffraction study of the oxidized surface showed only a weak pattern of $\mathrm{Al}_{2} \mathrm{O}_{3}$ and the patterns of the underlying alloy phises but no $\mathrm{PuO}_{2}$. Conprarison with the rate of oxidation of pure aluminum suggests that plutonium niay slightly reduce the protection afforded aluminum by its oxide. 


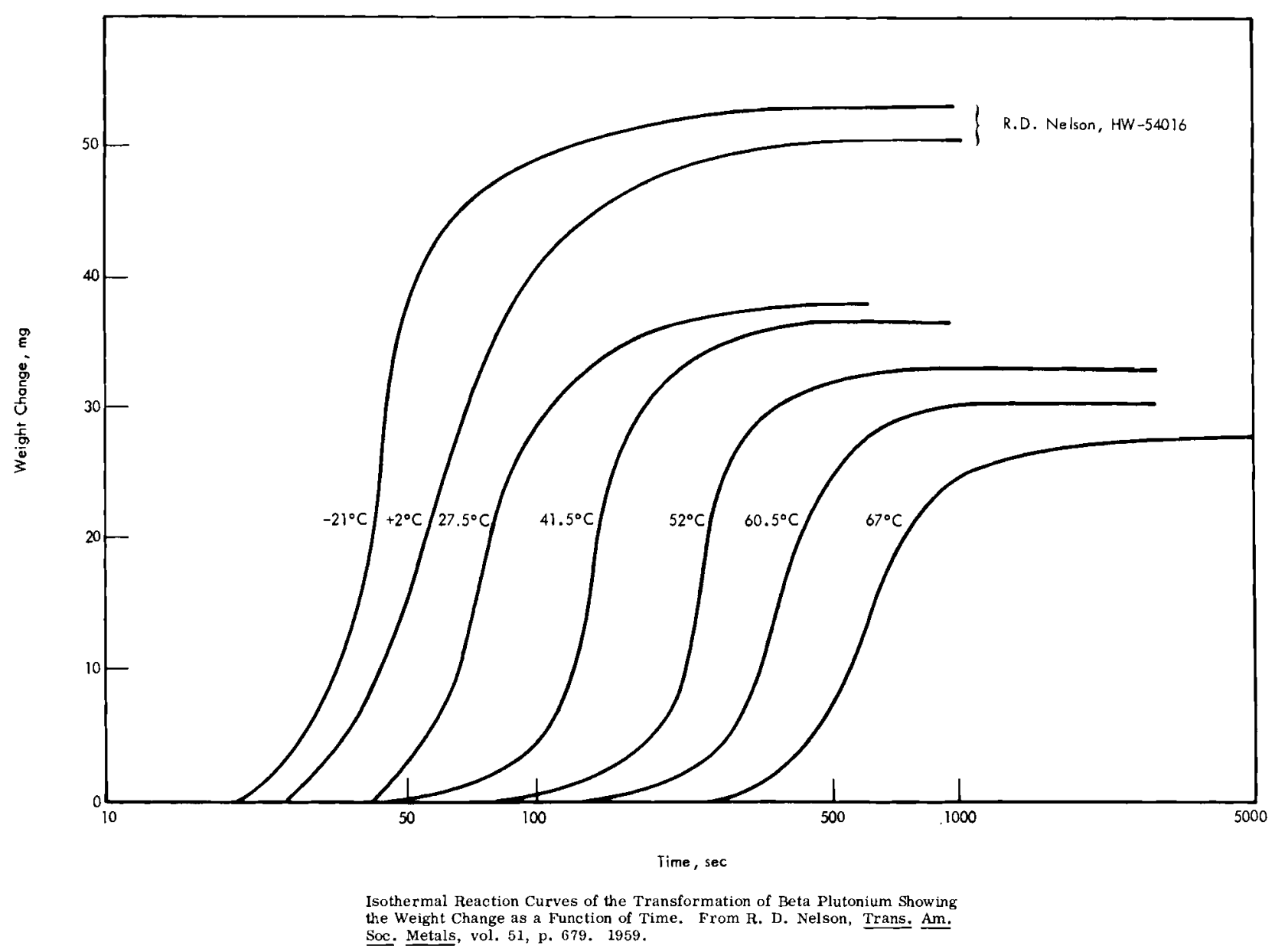

103

Crystal Structure

CELl dimensions (A) AND CalCulated DENSities, $\left(\mathrm{gcm}^{-3}\right)$ 99. 9\% Pure Plutonium of Isotopic Composition
Corresponding to Atomic Weight 239.05 .

From W. H. Zachariagen and F. H. Ellinger, Acta Cryst. . vol. 16, p. $780,1963$.

\begin{tabular}{|c|c|c|c|c|}
\hline & $21^{\circ} \mathrm{C}$ & $51^{\circ} \mathrm{C}$ & $96{ }^{\circ} \mathrm{C}$ & $104^{\circ} \mathrm{C}$ \\
\hline & $6.183 \pm 0.001$ & 6.194 & 6.211 & 6.214 \\
\hline b & $4.822 \pm 0.001$ & 4. 831 & 4.847 & 4.852 \\
\hline & $10.963 \pm 0.001$ & 10.973 & 10.987 & 10.989 \\
\hline & $101.79 \pm 0.01^{\circ}$ & $101.78^{\circ}$ & $101.77^{\circ}$ & $101.75^{\circ}$ \\
\hline & $19.86 \pm 0.01$ & 19.77 & 19.62 & 19.59 \\
\hline
\end{tabular}

The space group is $\mathrm{P} 2 / \mathrm{m}$. All atoms are in the reflection planes with coordinates $\pm(x, 1 / 4, z)$. There are 16 atoms per unit cell.
POSITION PARAMETERS FROM LEAST -SQUARE REFINEMENT From W. H. Zachariasen and F. H. Ellinger, Acta Cryst., vol. 16, p. $782,1963$.

\begin{tabular}{l} 
Atom Typ \\
\hline I \\
II \\
III \\
IV \\
V \\
VI \\
VII \\
VIII
\end{tabular}

\begin{tabular}{c}
$z$ \\
\hline $0.162 \pm 0.002$ \\
$0.168 \pm 0.002$ \\
$0.340 \pm 0.003$ \\
$0.457 \pm 0.003$ \\
$0.618 \pm 0.003$ \\
$0.653 \pm 0.002$ \\
$0.926 \pm 0.002$ \\
$0.894 \pm 0.002$
\end{tabular}


"SIIORT" AND "LONG" BONDS

From W. H. Zarharisson and F. H. Ellinger, Acta Cryst., vol.16, p. 783, 1963.

Ators '?

T

II

III

IV

$\mathrm{r}$

II

VII

IIII

\begin{tabular}{|c|c|}
\hline vinming. & ianse.A \\
\hline$\overline{1}$ & $2.77 \cdot 276$ \\
\hline 4 & $2.601-2.64$ \\
\hline 4 & $2.58-2.66$ \\
\hline 4 & $2.58-2.7+$ \\
\hline+ & $\therefore 58-2.72$ \\
\hline 4 & $2.64 \quad 2.74$ \\
\hline 4 & $2.57-2.78$ \\
\hline 3 & $2.76-3.78$ \\
\hline
\end{tabular}

$\begin{array}{cc}\text { Number } & \frac{\text { larig Ponds }}{\text { Rangt, A }} \\ 71 & 3.21-3.71 \\ 11 & 3.14-3.62 \\ 10 & 3.24-3.65 \\ 10 & 3.26-3.12 \\ 11 & 3.24-3.51 \\ 10 & 3.21-3.65 \\ 10 & 3.30-3.51 \\ 13 & 3.14-3.71\end{array}$

\begin{tabular}{cc} 
Number & All Bonds \\
\hline 12 & Mean Length, A \\
14 & 3.10 \\
14 & 3.18 \\
14 & 3.13 \\
14 & 3.19 \\
14 & 3.22 \\
14 & 3.15 \\
16 & 3.32
\end{tabular}

The structure an viewed along the b-axis is shown below. The atoms are arranged in peruto-jexagonal lavers nornial to the b-axis and 2.411 .1 apart. Each atom forms six bonds with atoms in the same reflection plane and six to ten bonds with atoms of adjacent lavers. The distortion from hexaconal configuration in the reflection plane is so grest that the bond lengths in this plane range from 2.57 to $3.71 \mathrm{~A}$. From $\mathrm{W}$. II, \%achariasen and $\mathrm{F}$. II. Ellinger, Acta Cryst., rol. 16, 1, 782, 1969.

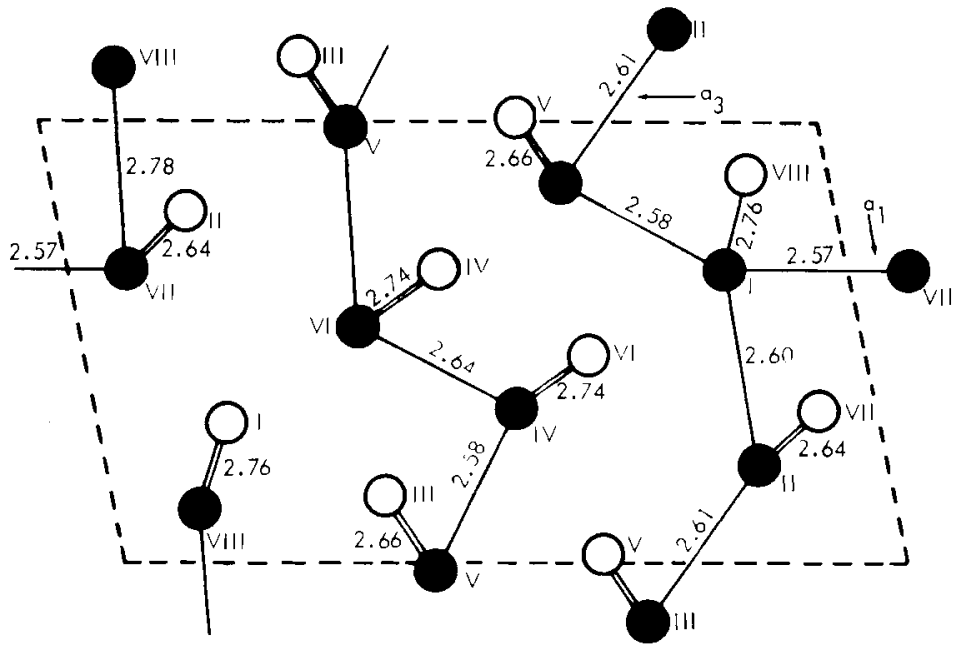

Projection of the Structure on the (010) Plane. From W. H. Zachariasen and F. H. Elliner, Actia Cryst., vol. lii, p. i-2, 1963.

Filled circles represent atoms at 1 it omen circles ztome ilf $y-1 / 4$

Only the short bonds are indicated in the projection. When corrections are

made for the effect of coordination number, these values give $1.58 \mathrm{~A}$ for the metallic radius of plutonium in the -phase. From W. H. Zachariasen and

F. II. Ellinger, Acta Cryst., vol. 16, p. 782, 1963.

COMPARISON OF CRYSTALLOCIRAPHIC GONSTANTSOF - NIITONILM AT 23,77, A VI) $29.4^{\prime \prime} \mathrm{k}$

Trom R. B. Finf I! I A-2 2

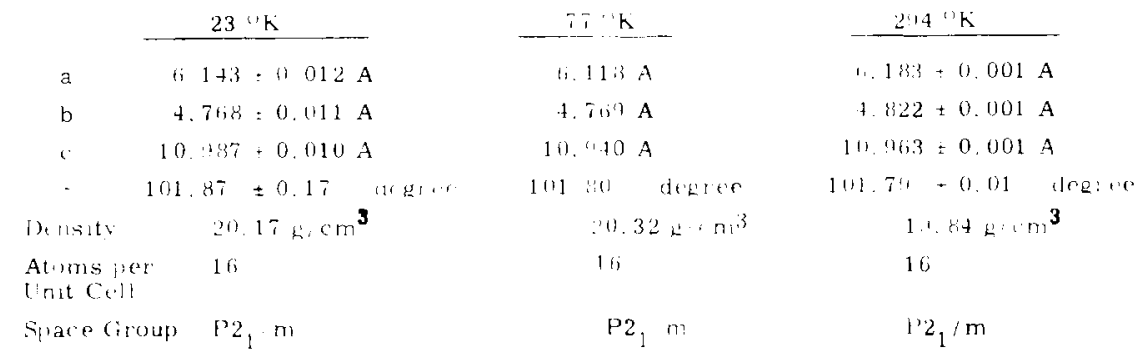




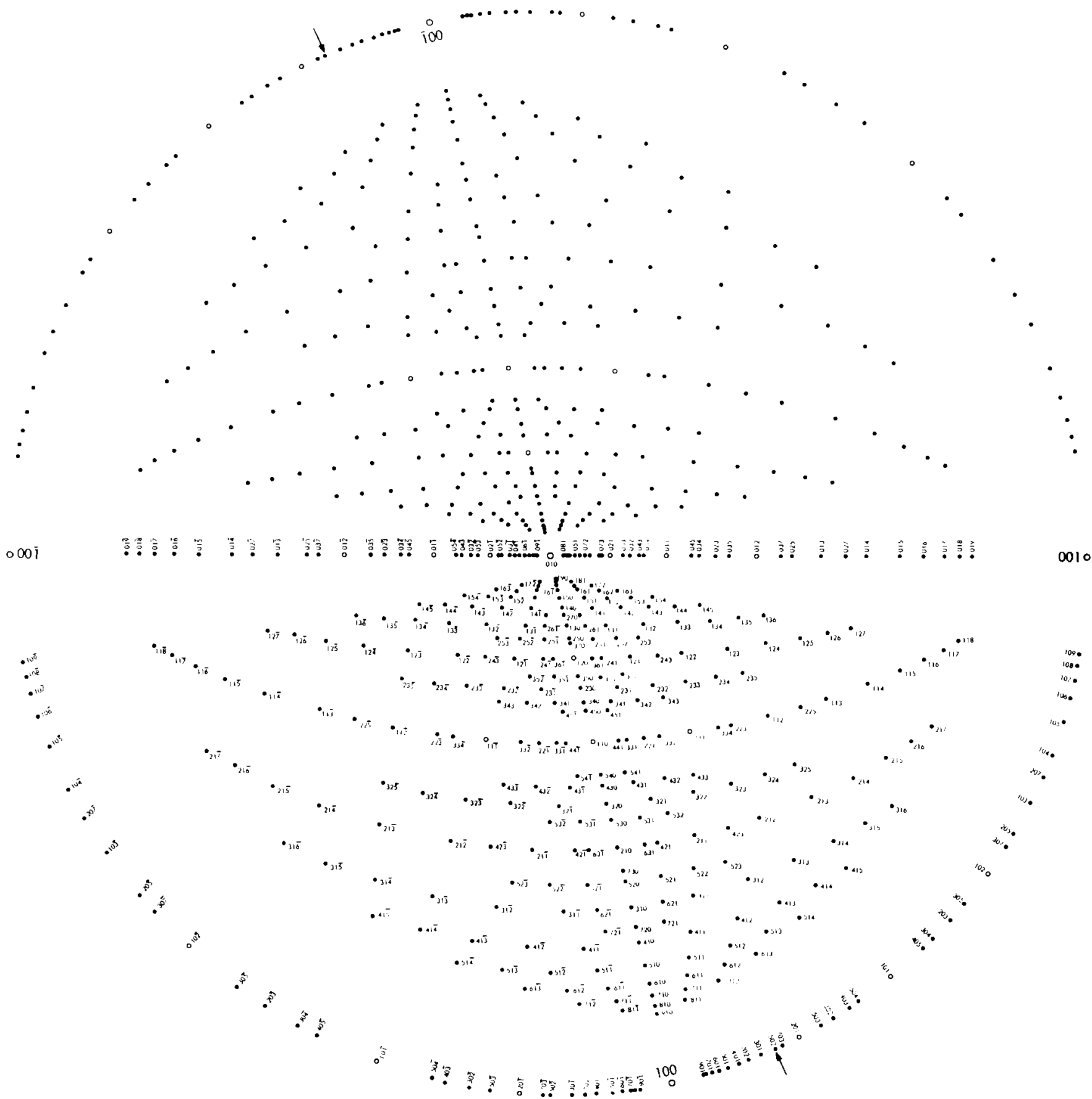

Alpha Plutonium, (010) Standard Projection of Plane Poles. From L. T. Lloyd and L. J. Nowicki, J. Nucl Mater., vol. 10, p. 254.1963. 


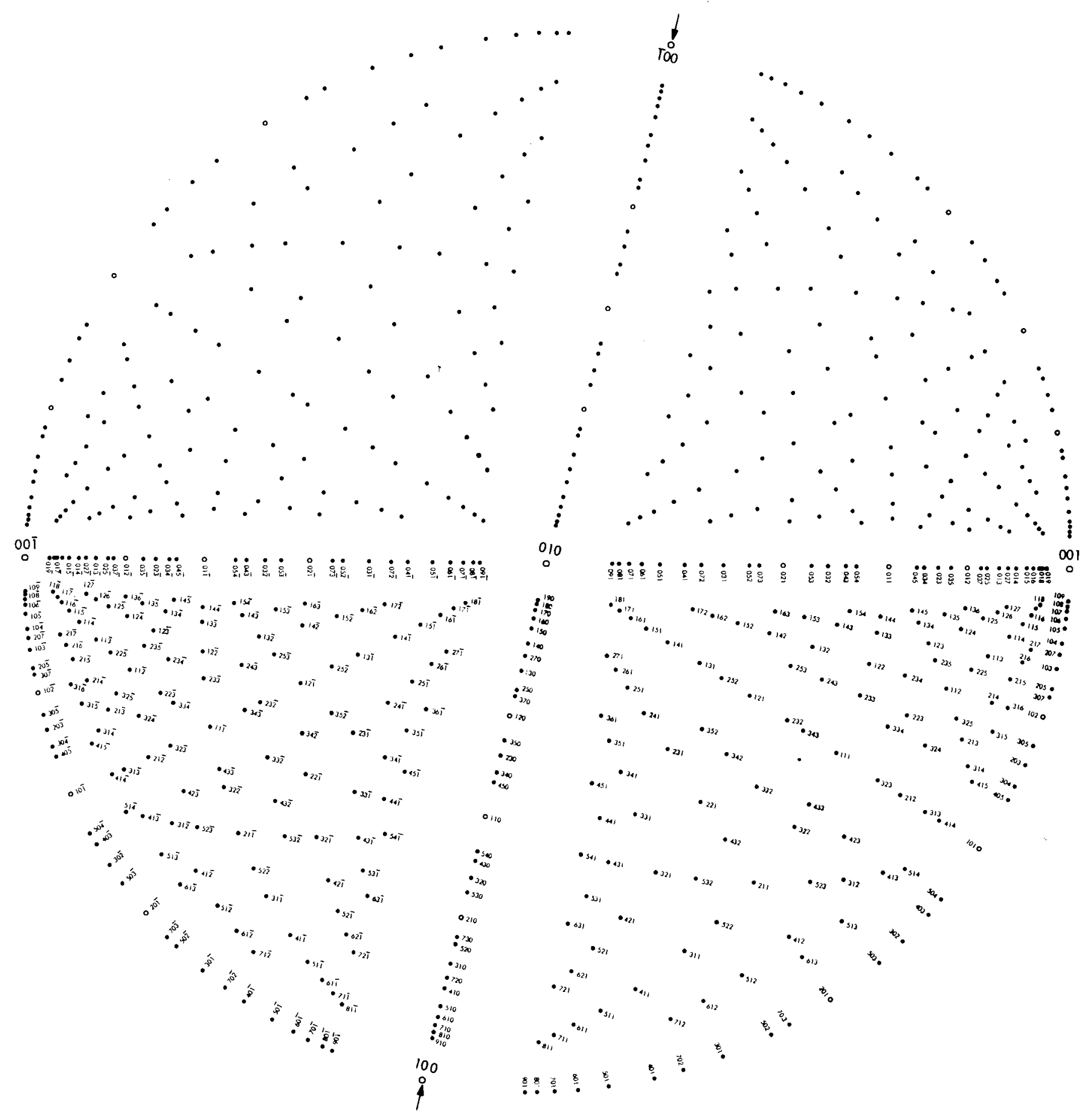

Alpha Plutonium, (010) Standard Projection of Directions. From. L. T. Lloyd and L. J. Nowicki, J. Nucl Mater., vol. 10, p. 256.1963. 


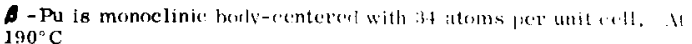

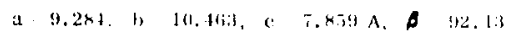

and the deneity is $17.710 \mathrm{gem}^{-3}$.

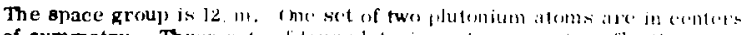

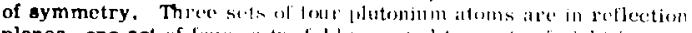
planes, one set of four an twolold axes and two sets of eight in genema positions. The thirteren pinsitmeters in the structure have been determined to an accuricy of ithout 10 . olt $\mathrm{A}$.

The seven different kinfls of plutonium atoms in the structure have coordination number of twelve, thirteen or fourteen. The mean atemic radius is $1,60 \mathrm{~A}$ tor coorrimation number twelve. From $\mathrm{W}$. II.

Zachariasen and F. H. Fllinger, Acta Cryst, vol, 16, 1, 369. I!6i3.

INIT CELL DIMENSIONS *

From W. H. Zachariagen and F. H. Ellinger Acta (ryst., vol. 16, p. 371. 1963.

\begin{tabular}{|c|c|c|c|c|c|}
\hline Sample & Temperature, ${ }^{\circ} \mathrm{C}$ & $a_{1} A$ & b) $A$ & c, A & A. degree \\
\hline Pure Pu & 193 & 9.227 & 10.449 & 7.824 & 92.54 \\
\hline Pure Pu & $1 ! ! 1 !$ & 9.284 & 10.463 & 7.859 & 92.13 \\
\hline $99 \mathrm{Pu}-1 \mathrm{Ce}$ & 133 & 9.251 & 10.436 & 7.834 & 92.3 .5 \\
\hline $99 \mathrm{Pu}-1 \mathrm{Ce}$ & 172 & 9.260 & 10.449 & 7.846 & 92.24 \\
\hline $99 \mathrm{Pu}-\mathrm{L} \mathrm{Ce}$ & 202 & 9.286 & 10.451 & 7.852 & 92.06 \\
\hline $98 \mathrm{Pu}-2 \mathrm{U}$ & $8: 3$ & 9.215 & 10.418 & 7. 815 & 92.52 \\
\hline $98 \mathrm{Pu}-2 \mathrm{U}$ & $13 !$ & 9.242 & 10.433 & 7.824 & 92.36 \\
\hline $98 \mathrm{Pu}-2$ U & 186 & 9.264 & 10.439 & 7.841 & 92.22 \\
\hline $98 \mathrm{Pu}-2 \mathrm{U}$ & 252 & 9.302 & 10.444 & 7.859 & 91.97 \\
\hline
\end{tabular}

${ }^{*}$ Calculated density for pure plutonium is $17.70 \mathrm{gcm}^{-3}$ at $190^{\circ} \mathrm{C}$. Precision is $+0.002 \mathrm{~A}$ for $\mathrm{a}, \mathrm{b}, \mathrm{c}$, and $\mathrm{t} 0.02^{\mathrm{O}}$ for $\mathrm{A}$.

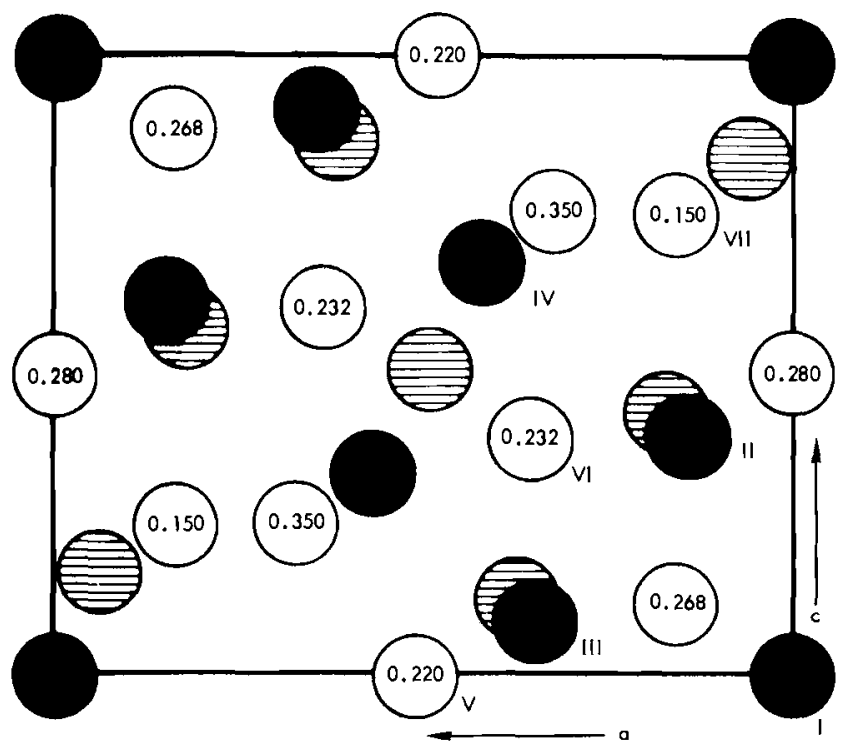
The structure projected on the Y-plane. Filled circles represent atoms with
$y$, 0 ; shaded circles, atoms with $y=0.50$. The numbers within the open circles give the $y$-coordinates for atoms at heights t above the projection plane. From \begin{tabular}{l} 
W. H. Zachariasen and F. H. Fllinger, Acta Cryst., vol, 16, p. 375. 1963. \\
\hline
\end{tabular}
HIS

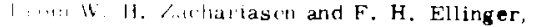

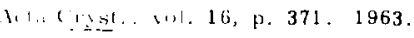

$\frac{1}{44}$
84
794

Her. In $(9) P_{11-1 C_{0}}$

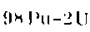

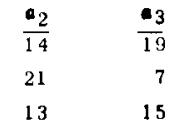

POSITION PARAMETERS

From W. H, Zachariasen and F. H. Ellinger, Acta Cryst., vol. 16, p. 3721963.

Tyle
I
II
III
IV
V
VI
VII
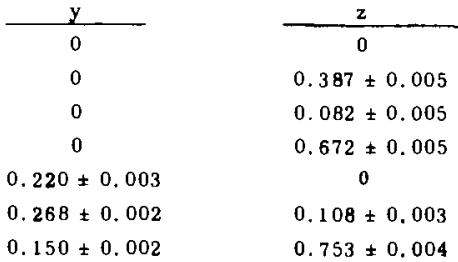

$0.387 \pm 0.005$

$0.082 \pm 0.005$

$0.672 \pm 0.005$ 0

$0,108 \pm 0,003$

$0.753 \pm 0.004$
MET ALLIC RADIUS ( $(=12)$

From W. H. Zachariagen and F. H. Ellinger, Acta Cryst., vol. 16, p. 374. 1963.

\begin{tabular}{|c|c|c|c|}
\hline Phase & Temperature, ${ }^{\circ} \mathrm{C}$ & Radius, $\mathbf{A}$ & $\begin{array}{l}\text { Radius at Room } \\
\text { Temperature, A }\end{array}$ \\
\hline a & 25 & 1.58 & 1. 58 \\
\hline $\boldsymbol{A}$ & 93 & 1.60 & 1.59 \\
\hline$\gamma$ & 235 & 1.601 & 1. 589 \\
\hline $\boldsymbol{t}$ & 320 & 1.640 & 1.644 \\
\hline $\boldsymbol{b}^{\prime}$ & 46.5 & 1.638 & 1.644 \\
\hline - & 490 & 1.622 & 1.594 \\
\hline
\end{tabular}

BONI) LENGTHS IN OTHER PLUTONIUM PHASES

From W. H. Zachariasen and F. H. Ellinger, Acta Cryst., vol. 16, p. 375.1963

\begin{tabular}{cccc} 
Phase Temperature. ${ }^{\circ} \mathrm{C}$ & Bond Number & Bond Length, A \\
\cline { 3 - 4 }$r$ & 235 & 4 & 3.026 \\
& & 4 & 3.159 \\
6 & 320 & 12 & 3.288 \\
$\gamma^{\prime}$ & 465 & 8 & 3.279 \\
& & 4 & 3.249 \\
1 & 490 & 8 & 3.327
\end{tabular}

SHORT ANI LONG BONDS IN a - AND - PLLTONIUM

from W. H, Zachariasen and F. H. Ellinger, Acta Cryst, vol, 16, p. 375, 1963.

\begin{tabular}{|c|c|c|c|c|}
\hline \multirow[b]{2}{*}{ Type } & \multicolumn{2}{|c|}{ Shoit } & \multicolumn{2}{|c|}{ Long } \\
\hline & Number & Hange $\mathrm{A}$ & Number & Range, A \\
\hline I & 5 & $2.50-2.76$ & 7 & $3.38-3.51$ \\
\hline II & 4 & $2.62-2.66$ & 10 & $3.36-3.64$ \\
\hline III & 4 & $2.56-2.67$ & 10 & $3.17-3.53$ \\
\hline IV & 4 & $2.55-2.67$ & 10 & $3.17-3.52$ \\
\hline$v$ & 4 & $2.55-2.72$ & 10 & $3.32-3.54$ \\
\hline VI & $t$ & $2.56-2.72$ & 10 & $3.34-3.64$ \\
\hline VII & + & $2.50-2.73$ & 10 & $3.34-3.55$ \\
\hline VIII & 3 & $2.73-2.76$ & 13 & $3.21-3.55$ \\
\hline
\end{tabular}

A - Ficutonium

\begin{tabular}{|c|c|c|c|c|}
\hline \multirow[b]{2}{*}{ Typ: } & \multicolumn{2}{|c|}{ Short } & \multicolumn{2}{|c|}{ Long } \\
\hline & Number & Range, A & Number & Range, $A$ \\
\hline I & 4 & 2. 197 & 8 & $3.15-3.26$ \\
\hline II & 3 & $3.03-3.10$ & 11 & $3.26-3.55$ \\
\hline III & 4 & $2.79-3.03$ & 9 & $3.15-3.43$ \\
\hline IV & 4 & $2.79-3.01$ & 8 & $3.16-3.48$ \\
\hline v & 4 & $2.80-2.34$ & 10 & $3.36-3.63$ \\
\hline VI & 4 & $2.11-3.10$ & 10 & $3.16-3.55$ \\
\hline VIl & 5 & $2 . i^{4}-2.05$ & 7 & $3.14-3.63$ \\
\hline
\end{tabular}


CRYSTAL STRUCTURE DATA FOR THE PLUTONIUM PHASES

From A. S. Coffinberry and M. B. Waldron in Metallurgy and Fuels, series V, vol. 1, p.373.

\begin{tabular}{|c|c|c|c|c|}
\hline Phase & Crystal Lattice & Unit Cell Dimensions, A & $\begin{array}{l}\text { Number of Atoms in } \\
\text { Unit Cell }\end{array}$ & $\begin{array}{c}\text { Calculated } \\
\text { Density, } \mathrm{g} / \mathrm{cm}^{3}\end{array}$ \\
\hline Alpha & Monoelinie(1) & $\begin{array}{l}\text { At } 21^{\circ} \mathrm{C} \\
\mathrm{a}=6.1835 \pm 0.0005 \\
\mathrm{~b}=4.8244 \pm 0.0005 \\
\mathrm{c}=10.973 \pm 0.001 \\
\boldsymbol{\beta}=101.81^{\circ} \pm 0.02^{\circ}\end{array}$ & $\frac{16}{16}$ & $19.816 \pm 0.006$ \\
\hline Beta & $\begin{array}{l}\text { Crystal structure } \\
\text { unknown }\end{array}$ & & & \\
\hline Gamma & $\begin{array}{l}\text { Face-centered } \\
\text { orthorhombic }(2)\end{array}$ & $\begin{array}{l}\text { At } 235^{\circ} \mathrm{C}: \\
\mathrm{a}=3.1587 \pm 0.0004 \\
\mathrm{~b}=5.7682 \pm 0.0004 \\
\mathrm{c}=10.162 \pm 0.002\end{array}$ & 8 & $17.14 \pm 0.01$ \\
\hline Delta & $\begin{array}{l}\text { Face-centered } \\
\text { cubic }(3)(4)(5)\end{array}$ & $\begin{array}{l}\text { At } 320^{\circ} \mathrm{C} \\
\mathrm{a}=4.6371 \pm 0.0004\end{array}$ & 4 & 15.92 \\
\hline Delta-prime & $\begin{array}{l}\text { Body-centered } \\
\text { tetragonal (3) (4) (5) }\end{array}$ & $\begin{array}{l}\text { At } 465^{\circ} \mathrm{C}: \\
\mathrm{a}=3.327 \pm 0.003 \\
\mathrm{c}=4.482 \pm 0.007\end{array}$ & 2 & 16.00 \\
\hline Epsilon & $\begin{array}{l}\text { Body-centered } \\
\text { cubic }(3)(4)(5)\end{array}$ & $\begin{array}{l}\text { At } 490^{\circ} \mathrm{C} \text { : } \\
\mathrm{a}=3.6361 \pm 0.0004\end{array}$ & 2 & 16.51 \\
\hline
\end{tabular}

(1) From W. H. Zachariasen. Private communication as reported by A. S. Coffinberry and M. B. Waldron.

(2) From W. H. Zachariasen and F. H. Ellinger, Acta Cryst., vol. 8, p. 431.1955.

(3) E. R. Jette, J. Chem. Phys., vol. 23, p. 367. 1955 .

(4) E. R. Jette. Nuclear Metallurgy, Inst. of Metals Div. Report Series No, 1, p. 29. 1955.

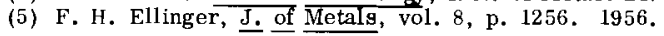

Pu body centered tetragonal unit cell dimensions:

at $465^{\circ} \mathrm{C} \mathrm{a}=3.327 \pm 0.003 \mathrm{~A}, \mathrm{c}=4.482 \pm 0.07 \mathrm{~A}$, calculated density $16.0 \mathrm{~g} / \mathrm{cm}^{3}$

UNIT-CELL DIMENSIONS AND VOLUMES OF DELTA-PRIME PLUTONIUM AT $450^{\circ} \mathrm{C}$

From F. H. Ellinger, J. Metals, vol. 8, p. 1256. 1956.

and from R. O. Elliott and A. C. Larson, The Metal Plutonium, pp. 278

Elliott and Larson

(X-Ray Diff ractometer)

\begin{tabular}{|c|c|c|c|}
\hline Units & $\begin{array}{c}\text { High-Purity; } \\
\text { Total Impurities } \\
\left.\quad \text { less } \mathrm{O}_{2}\right) \\
<221 \mathrm{ppm}^{(w t)} \\
\end{array}$ & $\begin{array}{l}\text { Ordinary Purity; } \\
\text { Total Impurities } \\
\quad\left(1 \mathrm{ess} \mathrm{O}_{2}\right) \\
<386 \mathrm{ppm}(\mathrm{wt}) \\
\end{array}$ & $\begin{array}{c}\text { Ellinger } \\
\text { (X-Ray Powder Method) } \\
\text { 99.97\% } \\
\text { Purity } \\
\end{array}$ \\
\hline $\mathrm{BCT}\left(\mathrm{a}_{0}\right), \mathrm{A}$ & $3.3261 \pm 0.0008$ & $3.3283 \pm 0.0010$ & $3.3119 \pm 0.0021$ \\
\hline $\mathrm{BCT}\left(\mathrm{c}_{0}\right), \mathrm{A}$ & $4.4630 \pm 0.0014$ & $4.4512 \pm 0.0016$ & $4.5265 \pm 0.0056$ \\
\hline Unit-cell volume, $\mathbf{A}^{3}$ & $49.374 \pm 0.023$ & $49.308 \quad \pm 0.027$ & $49.650 \pm 0.076$ \\
\hline
\end{tabular}

Measured lattice constant values were fitted to straight lines giving the following equations, from which the unit cell dimensions above were calculated:

$$
\begin{aligned}
& \mathrm{a}=3.3220 \pm 0.0018+(10.14 \pm 1.16) 10^{-4}(\mathrm{t}-460) \\
& \mathrm{c}=4.4969 \pm 0.0047-(29.56 \pm 3.01) 10^{-4}(\mathrm{t}-460) \\
& a_{t}=3.3261 \pm 0.0008+(14.80 \pm 0.40) \times 10^{-4}(t-450) \text { for high } \\
& \left.c_{t}=4.4630 \pm 0.0014-(47.46 \pm 0.81) \times 10^{-4}(t-450)\right\} \text { purity Pu }
\end{aligned}
$$

where $t$ is in ${ }^{\circ} \mathrm{C}$ and the uncertainties are standard deviations. Two atoms / unit cell at 000 and $1 / 2,1 / 2,1 / 2$. Structure Type $14 / \mathrm{mmm}$. Each Pu atom has eight neighbors at $3.249 \mathrm{~A}$ and four at $3.327 \mathrm{~A}$. From F. H. Ellinger, J. Metals, vol. 8, p. 1256. 1956, and from R. O. Elliott and A. C. Larson, The Metal Plutonium, pp. 278-279. 


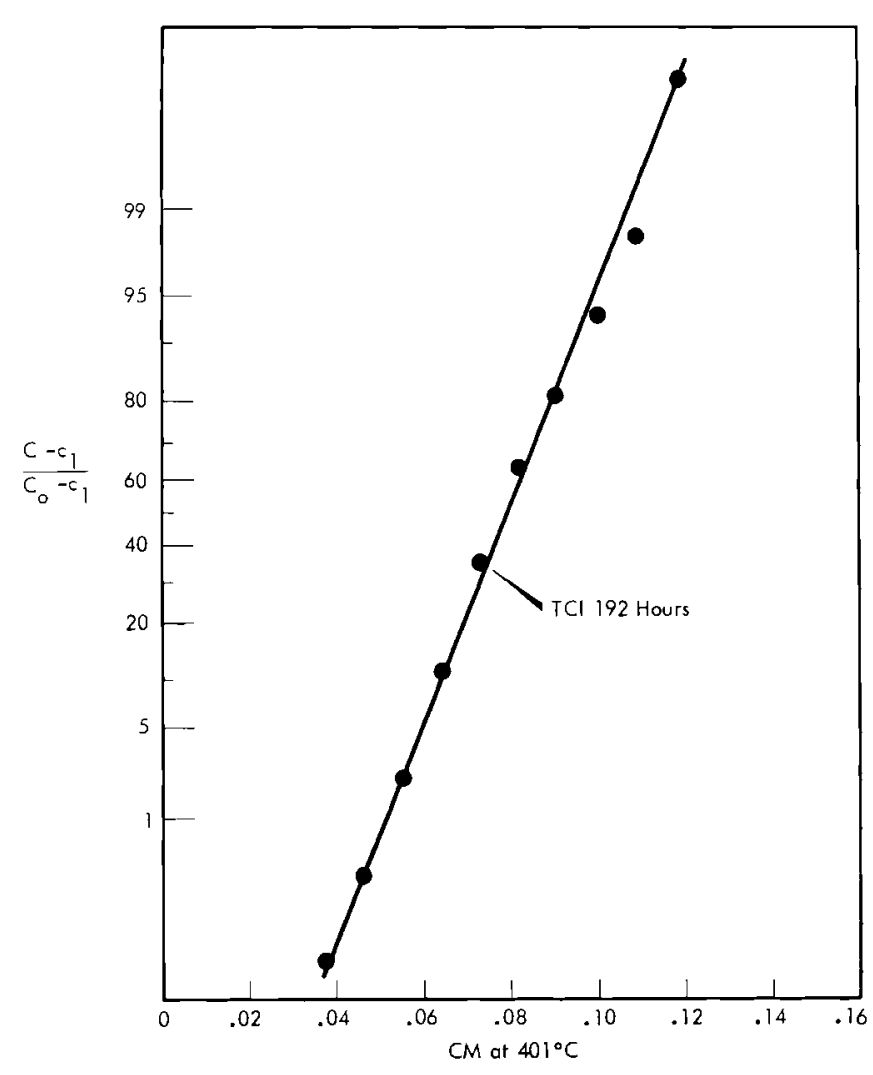

P'robulitity Plot of Data for Couple TCl Annealed for $192 \mathrm{Hr}$ at $401^{\circ} \mathrm{C}$. l'rom R. F. T:te and F. M. Cramer, Trans. Met. Soc. AIME, vol, 230 1.. 1341 . 1!t6it.

I) $\frac{c-c_{\perp}}{c_{0}-c_{1}} \frac{1}{2}\left[1+\frac{2}{\sqrt{\pi}} \int_{0}^{\frac{x}{2 \sqrt{D_{t}}}} e^{-\lambda^{2}} d \lambda\right]$

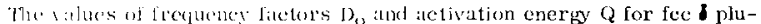
The tontan ice rivith bec $r$ urance surprising whes the very low heat of fusion, $L_{m}=693 \mathrm{cal}$ per $\mathrm{g}$-ittom, is taken int constle thion. The lnensured actiontion is hisher than that fredicteri by Nachtrieb's correlation; i.e., $Q=16.5 \mathrm{~L}_{\mathrm{m}}$ If the heats of transformation alsove the $\delta$ range are added together $(\delta$ to f' 24 cal pere gratom, of to 444 cal per g-atom, and to liquid $693 \mathrm{cal}$ per g-atom, a totil of 1157 call jer g-atom), Nachtrieb's correlation predie:1s 19, 190 cal per g-atom for the activation energy of $\delta$ plutonium comfared with the measured value of $23,800 \mathrm{cal}$ jer gr-atom.

Low $D_{0}$, and Q values are usually attributed to short-eircuiting diffusion patths. The autoradiographic experiments indicate that grain boundary cliffusion is unlikely. Further experiments which might shed additional light on the diffusion behavior of plutonium are being studied. From $\mathrm{B}, \mathrm{E}$. Tilte and F. M. Cramer, Trans. Met. Soc. AIME, vol. 230, p. 642, 1964 DISCUSSION - p. 642, 1st columin (first 2 paragraphs)

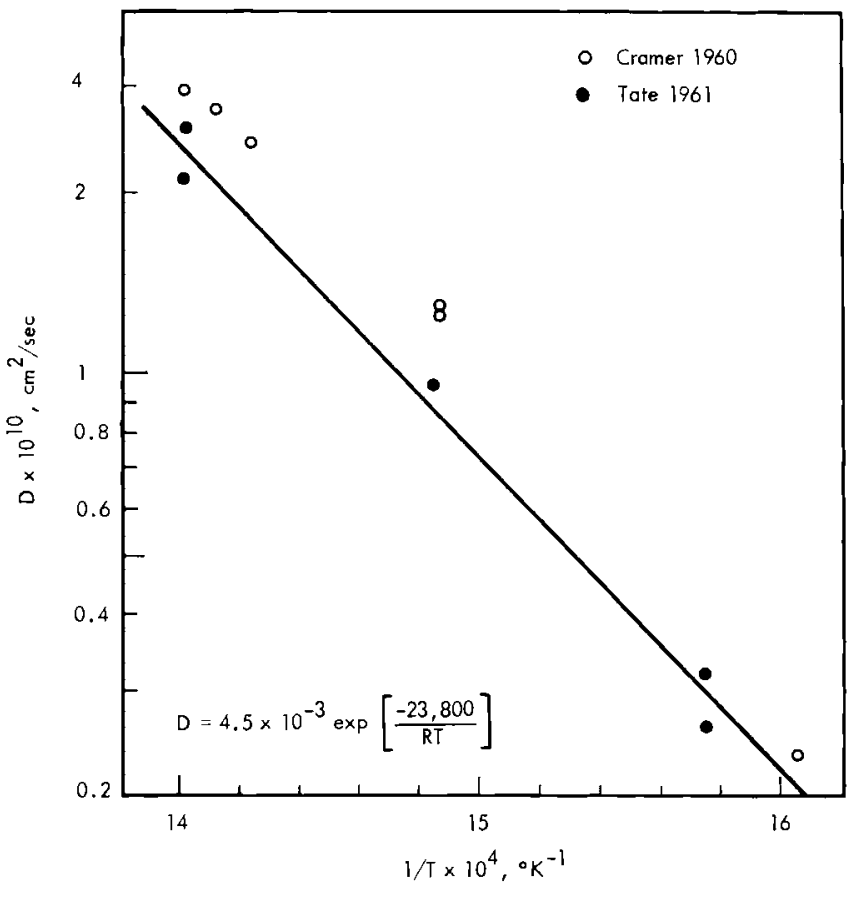

Arrhenius Plot for Self-Diffusion in $\delta$ Plutonium. From R. E. Tate and E. M. Cramer, Trans. Mel. Soc: AIME, vol. 230 , p. 642. 1964.

\section{$200 \quad$ Physical Properties}

PHASE DATA

(Probably 99.96 wt\% Plutonium)

From E. R. Jette, in Nuclear Metallurgy, p. 36. 1955.

\begin{tabular}{|c|c|c|}
\hline Phase & $\underline{\text { Resistivity, } \rho \times 10^{6}}$ & $1 / 0$ d dt $\times 10^{5}$ \\
\hline a & $145\left(25^{\circ}\right)$ & -21 \\
\hline $\boldsymbol{\rho}$ & $108\left(150^{\circ}\right)$ & -6 \\
\hline $\boldsymbol{\gamma}$ & $107.5\left(235^{\circ}\right)$ & -5 \\
\hline$\delta$ & $100\left(370^{\circ}\right)$ & +7 \\
\hline$b^{\prime}$ & $103\left(475^{\circ}\right)$ & +45 \\
\hline 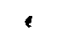 & $111\left(510^{\circ}\right)$ & 0 \\
\hline
\end{tabular}

At same temperatures as the resistance values

Note: $\mathrm{t}={ }^{\circ} \mathrm{C}$ 
CALCULATED DENSITY DETERMINED

FROM SELF-HEATING DILATOMETER EXPERIMENTS WITH 99.95 wt\% PLUTONIUM

E. M. Cramer et al. in The Metal Plutonium, pp. 112-122. (Table Compiled by M. E. Hasbrouck)

$$
\begin{array}{r}
\text { Pha } \\
\boldsymbol{r} \\
\boldsymbol{\gamma} \\
\boldsymbol{y}
\end{array}
$$

Density, $\mathrm{g} / \mathrm{cm}^{3}$

19.8

17.8

17. 2

16.0

16.2

17. 0
Temperature, ${ }^{\circ} \mathrm{C}$

25

122

206

319

451

476

\section{DENSITY OF PLUTONIUM}

From W. D. Wilkinson, in Extractive and Physical

Metallurgy of Piutonium and Its Alloys, p. 21.

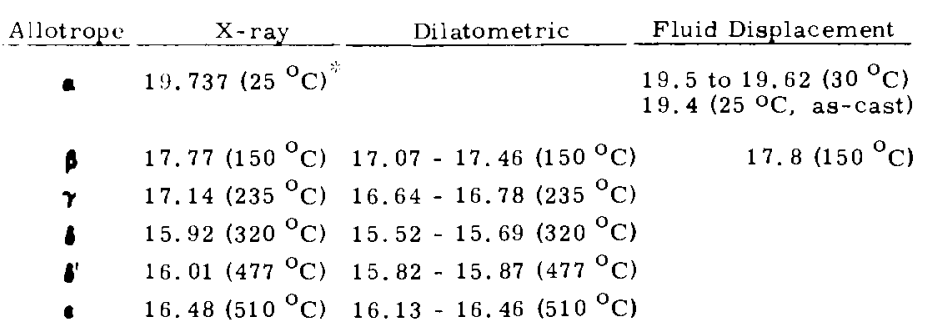

Cooled from $300^{\circ} \mathrm{C}$ under high pressure (Pressed at 50,000 psi at $300^{\circ} \mathrm{C}$ )

\section{DENSITY OF PLUTONIUM}

From C. Z. Serpan, Jr., and L. J. Wittenberg,

Trans. Met. Soc. AIME, vol. 221, p. 1019.1961.

\begin{tabular}{ccccc}
$\begin{array}{c}\text { Temperature, } \\
{ }_{\text {OC }}\end{array}$ & $\begin{array}{c}\text { Experimental } \\
\text { Density, } / \mathrm{cm}^{3}\end{array}$ & $\begin{array}{c}\text { Density from } \\
\text { Curve, g/ } / \mathrm{cm}^{3}\end{array}$ & $\begin{array}{c}\text { Deviation, } \\
\%\end{array}$ \\
\cline { 2 - 2 } 664 & 16.617 & & 16.624 & 0.04 \\
791 & 16.602 & & 16.584 & 0.11 \\
748 & 16.522 & 16.527 & 0.03 \\
752 & 16.439 & 16.498 & 0.36 \\
771 & 16.499 & 16.483 & 0.07 \\
788 & 16.533 & 16.460 & 0.44 \\
& 16.402 & 16.433 & 0.19
\end{tabular}

p- $\left[17.63_{2}-1.52_{2} \times 10^{-3} \mathrm{t}\right] \pm 0.03_{8} \mathrm{~g} / \mathrm{cc}$ where $\mathrm{t}={ }^{\circ} \mathrm{C}$

The volume expansion coefficient is

$$
93 \times 10^{-6} \text { per }{ }^{\circ} \mathrm{C}
$$

The density of the liquid at the melting point, $640^{\circ} \mathrm{C}$, is calculated to be 16.66 $\mathrm{g}$ per cc while an extrapolation of two different measurements of the solid phase give 16.24 (calculated from X-ray data) and $16.26 \mathrm{~g}$ per cc as the density of the phase at $640^{\circ} \mathrm{C}$. Since the liquid is, therefore, of 2.4 pct greater density than the solid at the melting point, plutonium metal joins that group of anomalous materials in which the solid floats on the liquid. From C. $z$.

Serpan, Jr, and L. J. Wittenberg, Trans. Met. Soc. AIME, vol, 221, p. 1020 , last paragraph. 1961 .

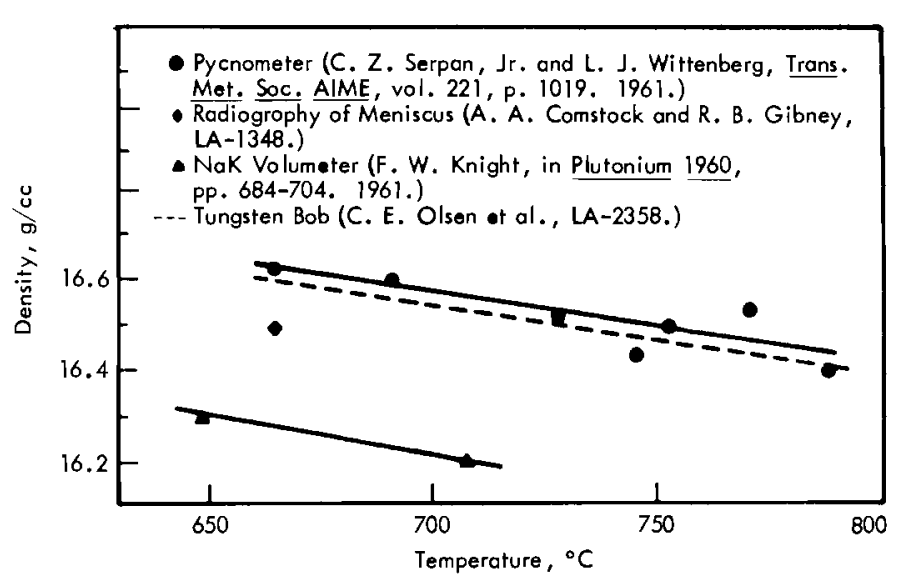

Density of Plutonium Vs Temperature for Several Techniques of Measurenent. From C. Z. Serpan, Jr. and L. J. Wittenberg, Trans. Met. Soc. AIME, vol. 221 , p. 1019. 1961 .

\section{SPECTROGRAPHIC ANALYSIS OF PLUTONIUM}

From C. Z. Serpan, Jr., and L. J. Wittenberg,

Trans. Met. Soc. AIME, vol. 221, p. 1019.1961.

$\begin{array}{lcc}\frac{\text { Element }}{\mathrm{Al}{ }^{*}} & & \text { PPY } \\ \mathrm{B} & & 90 \\ \mathrm{Be} & <0.5 \\ \mathrm{C} & <0.2 \\ \mathrm{Ca} & 95 \\ \mathrm{Cr} & 50 \\ \mathrm{Cu} & 10 \\ \mathrm{~F} & <2 \\ \mathrm{Fe} & 340\end{array}$

* Chemical analyais

* Spectrophotometric analyeis 


\section{LINEAR EXPANSION PER ${ }^{\circ} \mathrm{C}$}

From W. D. Wilkinson in Extractive and Physical Metallurgy of Plutonium and Its Alloys, pls. 15-20.

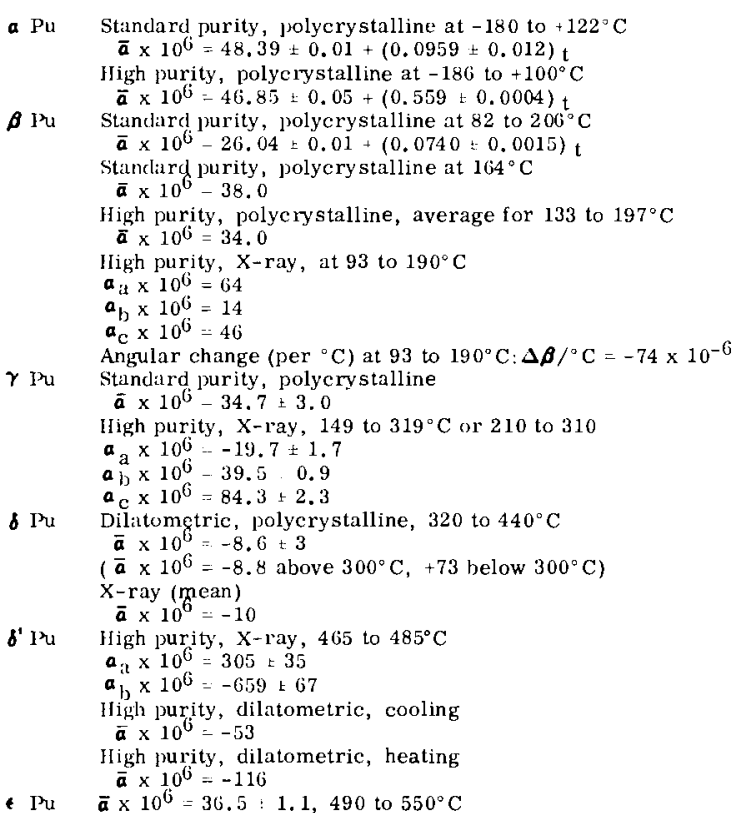

RESIITSS OF SELF-HEATING DILATOMETER EXPERIMENTS WITH 99.95 wt\% PLUTONIUM

E. M. Cramer et al. in The Metal Plutonium, pp. 112-122

\begin{tabular}{|c|c|c|}
\hline Phase & $\begin{array}{l}\text { Temperature } \\
\text { Range, } \\
{ }^{\circ} \mathrm{C} \\
\end{array}$ & $\begin{array}{c}\text { Linear Expansion Coefficient, } \\
{ }^{\circ} \mathrm{C}^{-1} \times 10^{-6}, \\
\text { Averages }\end{array}$ \\
\hline$a$ & $58-106$ & $58(+3,-3)$ \\
\hline$\beta$ & $144-201$ & $35(+2,-3)$ \\
\hline$\gamma$ & $224-300$ & $33(+2,-2)$ \\
\hline 8 & $336-447$ & $-16(+2,-2)$ \\
\hline $\boldsymbol{b}^{\prime}$ & $459-474$ & $-118(+53,-21)$ \\
\hline e & $490-527$ & $\begin{array}{c}27 \text { (X-ray diffraction } \\
=36.5 \pm 1.1)\end{array}$ \\
\hline
\end{tabular}

The limits given are actual maximum variations in values given by seven measurements involving three specimens. Plastic deformation was observed during six of the measurements on $\lesssim-p l u t o n i u m$.

Tube with $0.017 \mathrm{in.}$ Wall Thickness

$\begin{array}{lrl}\text { a } & -180-100 & 46.847+0.055881 \mathrm{~T} \\ & 133-197 & 33.86\end{array}$

THERMAL EXPANSION COEFFICIENTS

From M. B. Waldron et al., p/71, Proc. Second Geneva Internat, Conf., vol. $6, \mathrm{p}$ 162. 1958 .

\begin{tabular}{lc} 
Phase & $\begin{array}{c}\text { Linear Coefficient of Thermal Expansion, } \\
10^{\circ} /{ }^{\circ} \mathrm{C}\end{array}$ \\
\cline { 2 - 2 } Alpha & $67\left(80-120^{\circ} \mathrm{C}\right)$ \\
Beta & $41\left(160-200^{\circ} \mathrm{C}\right)$ \\
Gamma & $35\left(220-280^{\circ} \mathrm{C}\right)$ \\
Delta & $-8.6\left(340-440^{\circ} \mathrm{C}\right)$ \\
Delta-prime & $-596^{*, k}\left(470^{\circ} \mathrm{C}\right)$ \\
Epsilon & $15\left(490-550^{\circ} \mathrm{C}\right)$
\end{tabular}

COEFIICIEN'TS OF EXPANSION, $10^{-6} /{ }^{\circ} \mathrm{C}$

R. Abramson et ill, p/327, Proc. Second Geneva Internat. Conf., vol. $6, \frac{1}{\text { p. } 177} .1958$.

$\begin{array}{lc}\text { a phase } & 59 \\ \text { A phase } & 30 \\ \boldsymbol{\gamma} \text { phase } & 33 \\ \delta \text { phase during } \\ \text { heating } \\ \boldsymbol{\delta}^{\prime} \text { phase during } \\ \text { heating } \\ \begin{array}{l}\text { phase during } \\ \text { cooling }\end{array} \\ \delta \text { ' phase during } \\ \text { cooling } \\ \delta \text { phase during } \\ \text { cooling } \\ \text { (1) above the elbow } \\ \text { (2) below the elbow }\end{array}$

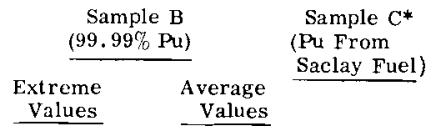
$\begin{aligned} & \text { Sample A } \\ &\left(\sim 99.9^{\prime} \mathrm{Pu}\right)\end{aligned}$

Values $\quad$ Values
37

* Average of two tests

EXPANSION DURING PHASE TRANSFORMATIONS

R. Abramson et al., p/327,

Proc. Second Geneva Internat. Conf., vol. 6, p. 177. 1958.

\begin{tabular}{|c|c|c|c|}
\hline & \multicolumn{3}{|c|}{ Expansion, \% } \\
\hline & Sample A & Sample B & Sample C \\
\hline Total & 5.6 & 7.25 & 7.6 \\
\hline$a \rightarrow \beta$ & 2 & 3.2 & 3.2 \\
\hline$\beta \rightarrow \gamma$ & 0.8 & 0.85 & 0.96 \\
\hline $\boldsymbol{\gamma} \rightarrow \boldsymbol{\delta}$ & 1.8 & 2.05 & 2.1 \\
\hline$e \rightarrow \delta^{\prime}$ & -0.72 & -0.77 & -0.66 \\
\hline
\end{tabular}

EXPANSION COEFFICIENTS FOR ALPHA-PLUTONIUM $99.9 \%$ PURE, $10^{-6} /{ }^{\circ} \mathrm{C}$

W. H. Zachariasen and F. H. Ellinger, Act a Cryst., vol. 16, p. 780. 1963.

\begin{tabular}{|c|c|c|c|c|}
\hline & $21-51{ }^{\circ} \mathrm{C}$ & $21-96{ }^{\circ} \mathrm{C}$ & $21-104^{\circ} \mathrm{C}$ & $\begin{array}{c}\text { Mean, } \\
21^{\circ}-100^{\circ} \mathrm{C} \\
\end{array}$ \\
\hline$a_{a}$ & 59 & 60 & 60 & $60 \pm 1$ \\
\hline$a_{b}$ & 57 & 69 & 75 & $72 \pm 3$ \\
\hline$a_{\mathrm{c}}$ & 30 & 29 & 29 & $29 \pm 1$ \\
\hline$\Delta$ & -6 & -5 & -8 & $-7 \pm 2$ \\
\hline$a_{1}$ & - & - & - & $62 \pm 1$ \\
\hline$a_{2}$ & - & - & - & $72 \pm 3$ \\
\hline$a_{3}$ & - & - & - & $29 \pm 1$ \\
\hline$\phi$ & - & - & - & $13 \pm 2$ \\
\hline
\end{tabular}

$a_{a}, a_{b}, a_{c}=$ linear coefficients in the directions of the crystallographic axes. $\Delta=$ change in angle (in radians) per degree rise in temperature. These quantities are related to the principle coefficients $a_{1}, a_{2}, a_{3}$ and the angle $\phi$ describing the orientation of the tensor elipsoid by the equations: (see W. H. Zachariasen and F. H. Ellinger, Acta Cryst., vol. 12, p. 175. 1959.)

$$
\begin{aligned}
a_{1}-a_{3} & =-\Delta / \sin \beta \cos (2 \phi-\beta) . \\
\tan (2 \phi-\beta) & =\left(a_{a}-a_{c}\right) / \Delta . \\
a_{2} & =a_{b} .
\end{aligned}
$$

$a_{1}+a_{3}=a_{2}+a_{c}+\Delta \operatorname{cotan} \beta$ 


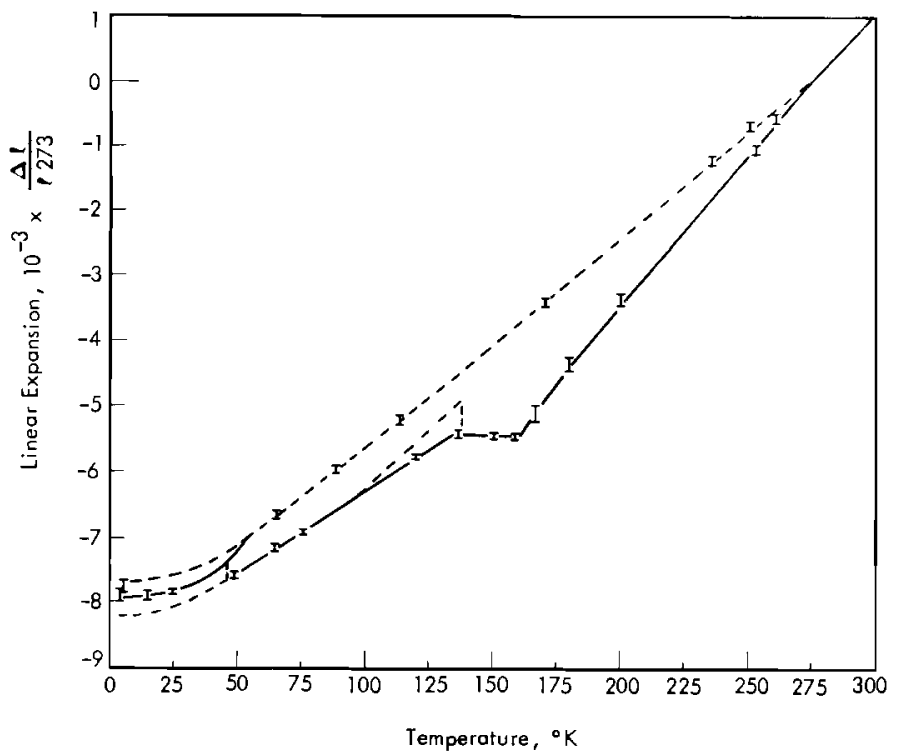

Thermal Expansion of Normal Purity Plutonium Metal Relative to Fused Quart\%. (a) Top dashed line represents curve for relatively rapid cooling and wal iming. (b) solid line between $\sim 48^{\circ} \mathrm{K}$ and $300^{\circ} \mathrm{K}$ represents results found in $27-1 / 2 \mathrm{~h}$ cooling period. (c) Solid line between $2^{\circ} \mathrm{K}$ and $60^{\circ} \mathrm{K}$ represents results from warming trend. [99.9\% Pu (combined iron and nickel eontent $150 \mathrm{ppm}$ ).] From T. A. Sandenaw in Plutonium 1960, p. 85

LEAST-SQUARES EQUATION FOR LINEAR EXPANSION OF PLUTONIUM METAL BELOW $273^{\circ} \mathrm{K}$

T. A. Sandenaw in Plutonium $\underline{1960}$, last paragraph on $p .87$.

Because of the offset observed at $\sim 50^{\circ} \mathrm{K}$ and the region of zero coefficient of lincar exlsansion (between $135^{\circ}$ and $160^{\circ} \mathrm{K}$ ), the data were divided into threc temperature regions. The equations, uncorrected for expansion ixdaviour of lused quartz, for those three regions were found to be as follows:

$$
\begin{aligned}
& \text { Tomperature range: } 2^{\circ} \text { to } 55^{\circ} \mathrm{K} \\
& \Delta 1_{\mathrm{T}} \cdot \mathrm{l}_{273}\left(\mathrm{~A}+\mathrm{BT}+\mathrm{CT}^{2}\right) \\
& \begin{array}{l}
A=-7.9421680 \times 10^{-3} \\
B=-6.4305347 \times 10^{-6}
\end{array} \\
& \mathrm{C}=4.1447317 \times 10^{-7} \\
& \sigma=1.3 \times 10^{-4} \\
& \text { Tomperature range: } 50^{\circ} \text { to } 135^{\circ} \mathrm{K} \\
& \Delta 1_{\mathrm{T}} \cdot 1_{273}(\mathrm{~A}-\mathrm{BT}) \\
& \mathrm{A}=-8.8183670 \times 10^{-3} \\
& \begin{array}{l}
A=-8.8183670 \times 10^{-5} \\
B=2.5303415 \times 10^{-5}
\end{array} \\
& \begin{array}{l}
B=6.4 \times 10^{-5} \\
\sigma=6.5
\end{array} \\
& \text { Tomperature range: } 160^{\circ} \text { to } 273^{\circ} \mathrm{K} \\
& \Delta 1_{\mathrm{T}}=1_{273}(\mathrm{~A}+\mathrm{BT}) \\
& A=-1.3240679 \times 10^{-2} \\
& \begin{array}{l}
B-4.8509769 \\
\sigma=1.6 \times 10^{-4}
\end{array}
\end{aligned}
$$

THE'RMAL EXPANSION COEFFICIENTS FOR $=-$ PLUTONIUM, $\times 10^{-6} /{ }^{\circ} \mathrm{C}$

From W. H. Zachariasen and $\mathbf{F}$. H. Ellinger, Acta Cryst., vol. 12, p. 176. 1959.

$\begin{array}{cc} & 93-190^{\circ} \mathrm{C} \\ r_{a} & 64 \\ \gamma_{b} & 14 \\ \vdots c & 46 \\ \therefore & -74 \\ 1 & 94 \\ 2 & 14 \\ -3 & 19 \\ - & 42 \\ & 40\end{array}$

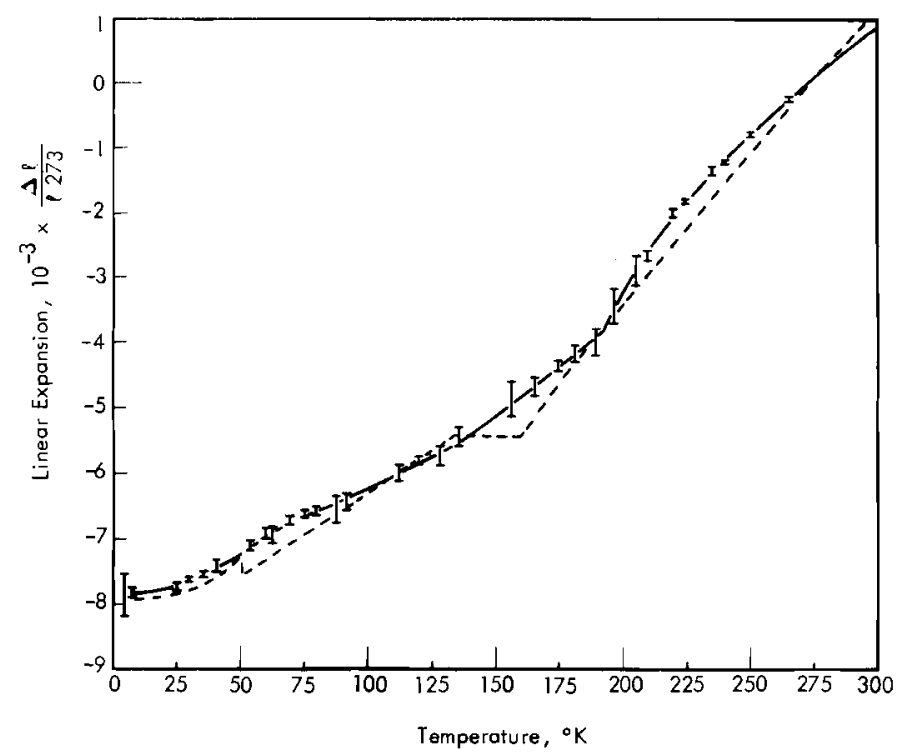

Thermal Expansion of a Plutonium Specimen With 600 PPM Iron Content Relative to Fused Quartz (Solid Line). Dashed curve represents data for the normal purity specimen (combined iron and nickel content $150 \mathrm{ppm}$ ). From T. A. Sandenaw in Plutonium 1960, p. 86.

\section{THERMAL EXPANSION COEFFICIENTS} FOR GAMMA PLUTONIUM

From W. H. Zacharlasen and F. H. Ellinger, Acta Cryst., vol. B, p. 433. 1955.
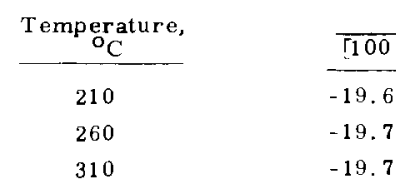

$\begin{array}{cc}\text { True } \approx \times 10^{-6} /{ }^{\circ} \mathrm{C} & \\ \frac{[010]}{39.48} & 5001] \\ 39.40 & 84.21 \\ 39.32 & 83.86 \\ 1.4 \% & 83.51 \\ & 1.9 \%\end{array}$

Standard Deviation

$5.1 \%$

1. $4 \%$

$1.9 \%$

Mean linear coefficients for the range $210-310^{\circ} \mathrm{C}-19.7 \pm 1.0 \quad 39.5 \pm 0.6 \quad 84.3 \pm 1.6$

Mean linear coefficient for polycrystalline gamma plutonium $=(34.7 \pm 0.7) \times 10^{-6} /{ }^{\circ} \mathrm{C}$ Mean volume coefficient $\left(\bar{a}_{v}=3 \bar{a}\right)=(104 \pm 2) \times 10^{-6} /{ }^{\circ} \mathrm{C}$

LINEAR THERMAL E XPANSION OF DELTA-PRIME PLUTONIUM, PER ${ }^{\circ} \mathrm{C}$

From R. O. Elliott and A. C. Larson in The Metal Plutonium, p. 279

Elliot and Larson (X-Ray Diffractometer)

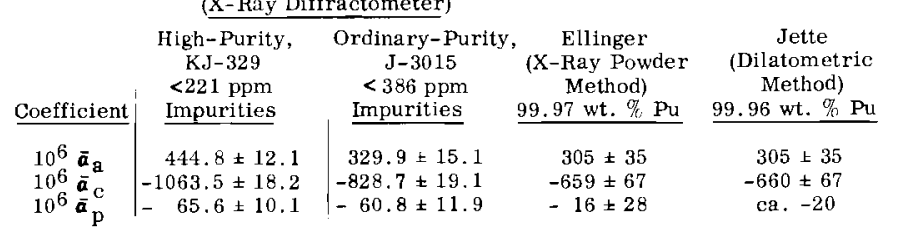

The mean linear coefficient of thermal expansion, $\mathbf{a}_{n}$, for the bulk, randomly oriented polycrystalline material was calculated from the single-crystal coefficients, using the relation $\left(1+\bar{a}{ }_{p} \Delta\right)^{3}=\left(1+\bar{a}_{a} \Delta t\right)^{2}\left(1+\bar{a} c_{C} \Delta t\right)$. 


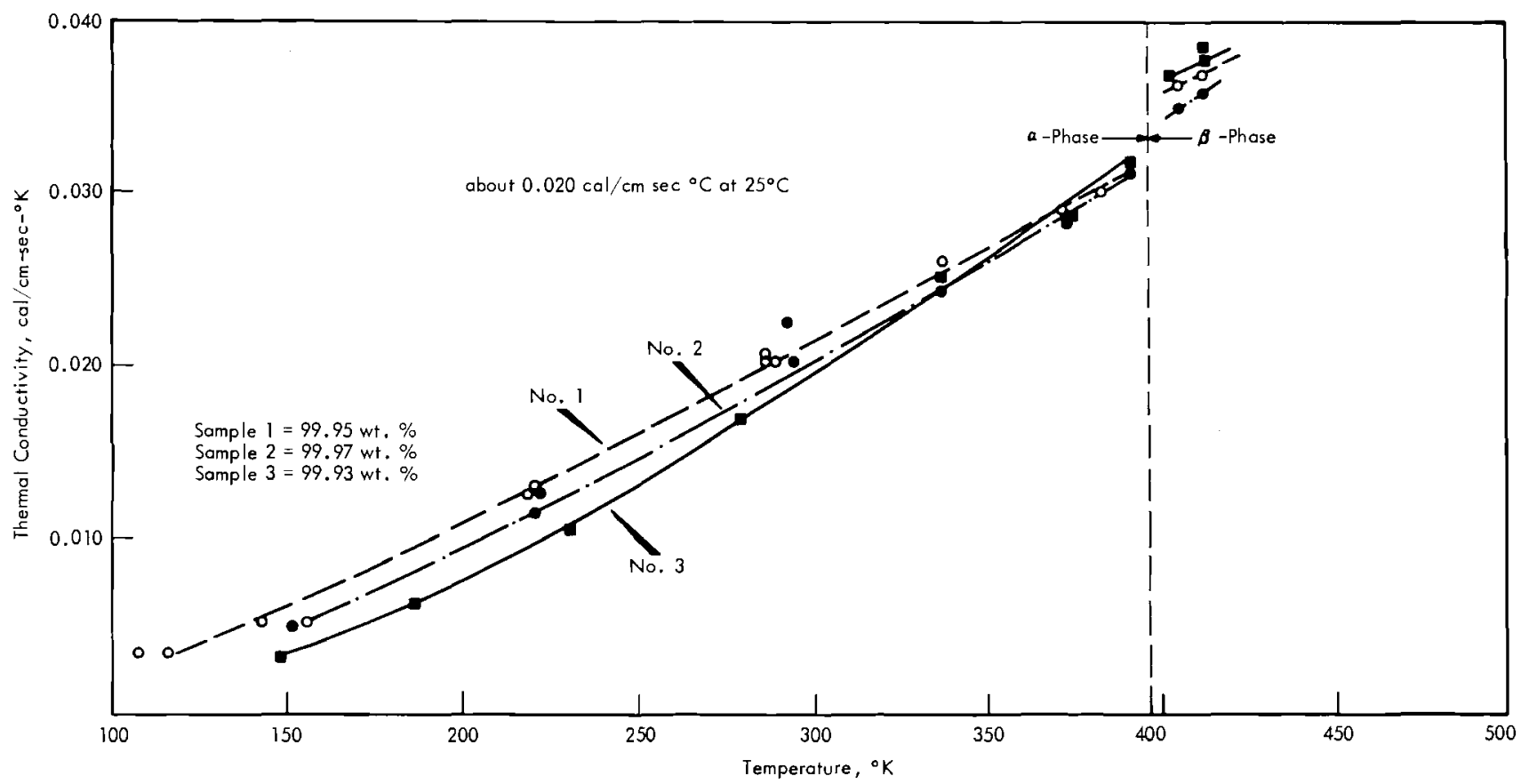

Thermal Conductivity as a Function of Absolute Temperature. From

T. A. Sandenaw and R. B. Gibney, J. Phys. Chem. Solids, vol. 6,

p. 87. 1958 .

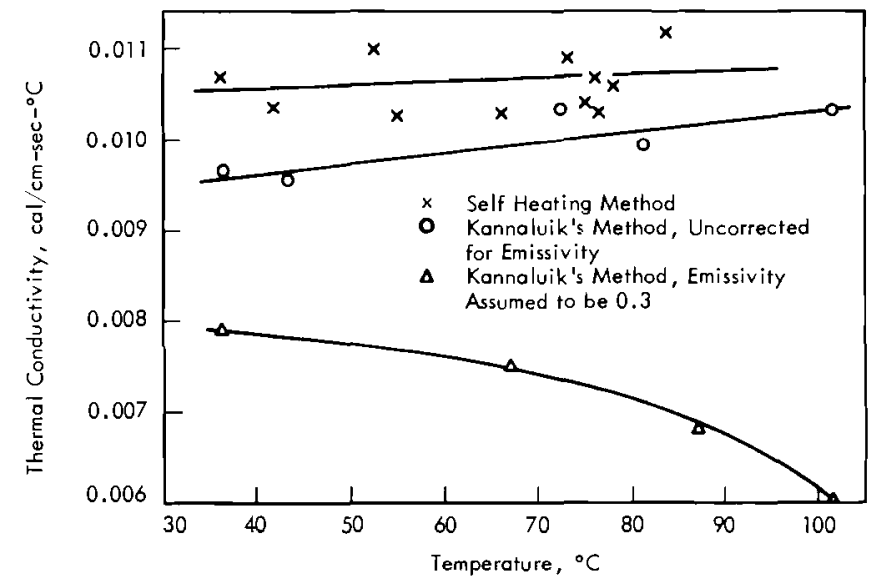

Thermal Conductivity of Plutonium. From M. B. Waldron et al. p/71, Proc. Second Geneva Internat. Conf., vol. 6, p. 164. 1958

\section{3 specific Heat and Heat Capacity}

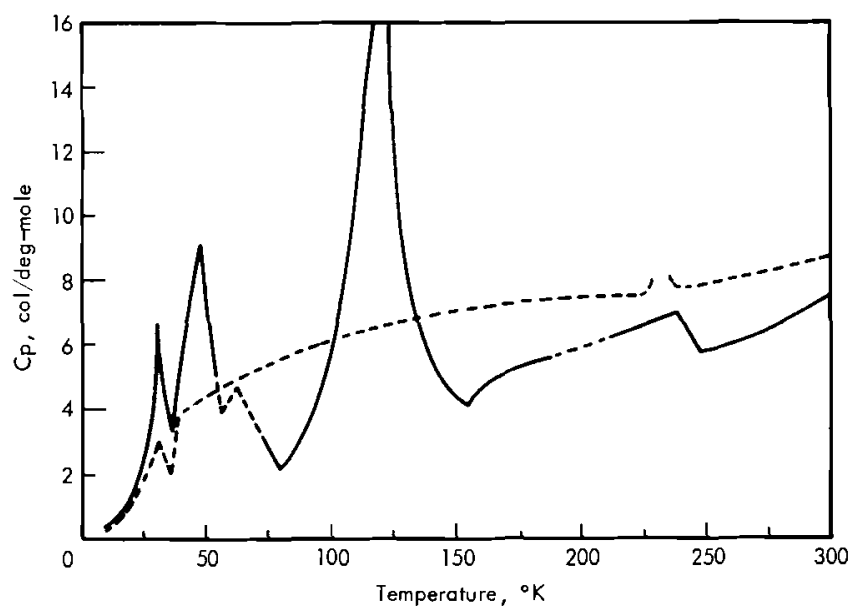

Heat Capacity as a Funetion of Temperature for High-Purity (Predominantly Solid Line) and Normal-Purity (Dashed Line) Plutonium Specimens. From T. A. Sandenaw et al in plutonium 1960, p. 72 . 


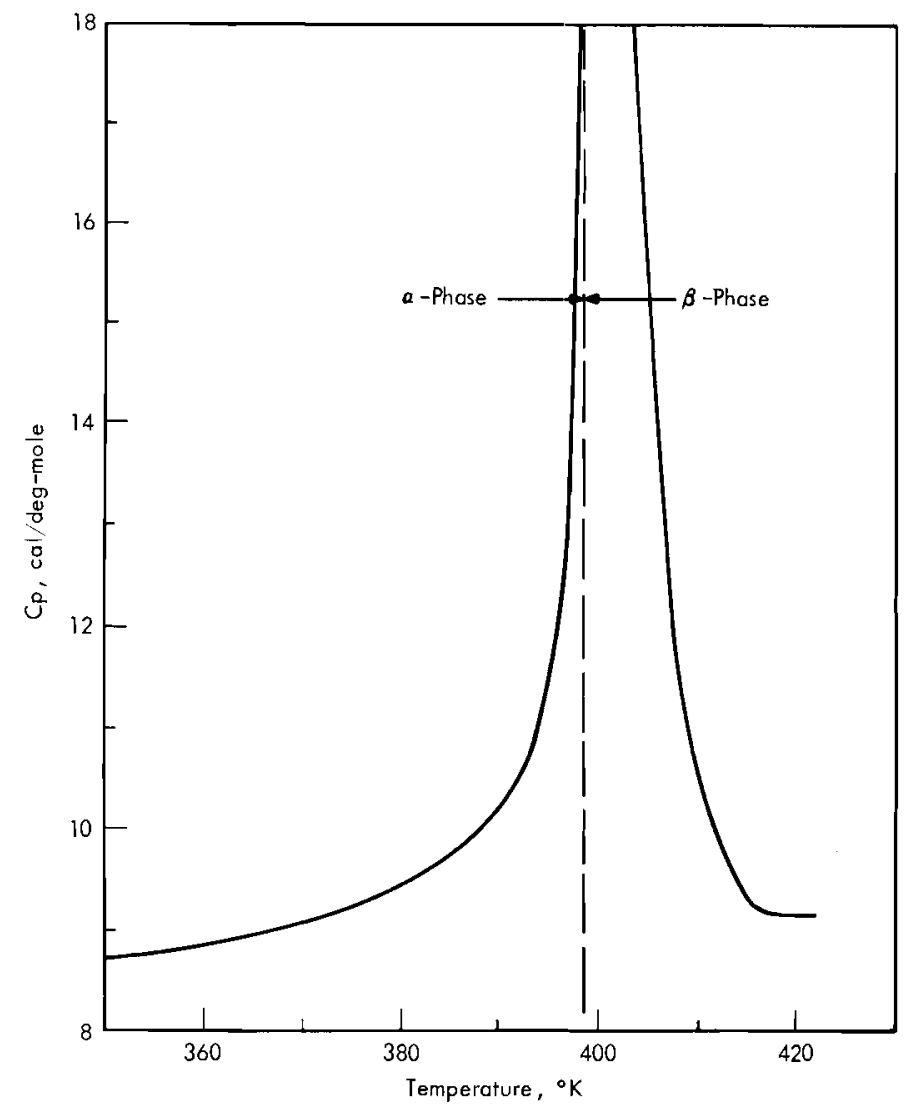

Shape of Heat Capacity Peak at the $\boldsymbol{a}-\boldsymbol{\beta}$ Phase Transition. From 'T. A. Sandenaw et al in Plutonium 1960, p. 72 .

Plutonium containing $160 \mathrm{ppm} \mathrm{Ni}, 200 \mathrm{ppm} \mathbf{F c}, 205 \mathrm{ppm} \mathrm{C}$ (4 runs)

Temperature range below $21.25^{\circ} \mathrm{K}$

$$
\begin{aligned}
& \mathrm{C}_{\mathrm{p}}=\gamma \mathrm{T}+\mathrm{B} \mathrm{\Gamma}^{3} \\
& \gamma \quad 1.167 \times \times 10^{-2} \\
& \mathrm{~J} \quad 9.27\left(\mathrm{i} \times 10^{-5}\right. \\
& \sigma \text { (standarid deviation) } \quad 0.066
\end{aligned}
$$

Temperature range $21.25^{\circ}$ to $31.81^{\circ} \mathrm{K}$

$$
\begin{array}{ll}
C_{\mathrm{p}} & \gamma \mathrm{T}: \mathrm{B}^{\mathrm{T}} \mathrm{C}^{3} \\
\gamma & 2.2306 \times 10^{-2} \\
B & 7.1010 \times 10^{-5} \\
\sigma & 0.226
\end{array}
$$

Temperature range 40 to $298.15^{\circ} \mathrm{K}$

(Peali-free portion of curve for nornal-purity speeimen No. 1)

$$
\begin{array}{ll}
C_{p} & A=13 \Gamma+\mathrm{C}^{2} \Gamma \Gamma^{3} \\
A & 1.12 \times 2876 \\
13 & 8.2517711 \times 10^{-2} \\
C & -3.8530037 \times 10^{-4} \\
D & 10.4728553 \times 10^{-7} \\
\sigma & 0.226
\end{array}
$$

The very low temperature data from seven runs with 99.96 and 99.89 wh. If Pu specimens gave the following equation below $21.66^{\circ} \mathrm{K}$ (7 runs):

Temperiture range lielow $21.166^{\circ} \mathrm{K}$

$$
\begin{array}{ll}
\mathrm{C}_{\mathrm{P}} & \gamma \mathrm{T} \cdot \mathrm{RT}^{3} \\
\gamma & 2.05 \mathrm{~T} 5 \times 10^{-2} \\
\mathrm{~B} & 8.4-167 \times 10^{-5} \\
\sigma & 0.04 .4
\end{array}
$$

\begin{tabular}{|c|c|c|c|c|c|}
\hline & $\begin{array}{c}\text { Tempera- } \\
\text { ture, } \\
{ }^{\circ} \mathrm{C}\end{array}$ & $\begin{array}{l}\text { Experi- } \\
\text { mental } \\
\text { Molar } \\
\text { Specific } \\
\text { Heat } \mathrm{C}_{\mathrm{p}} \text {, } \\
\text { call/ } /{ }^{\circ} \\
\text { molc- } \mathrm{C} \\
\end{array}$ & $\begin{array}{l}\text { Calculated } \\
\text { Lattice } \\
\text { Specific } \\
\text { Heat } \\
\mathrm{C}_{\mathrm{v}}, \\
\text { cal/mole- }{ }^{\circ} \mathrm{C} \\
\end{array}$ & $\begin{array}{l}{\left[\mathrm{C}_{\mathrm{p}}-\mathrm{C}_{\mathrm{v}}\right]_{\mathrm{d}}} \\
\mathrm{cal} / \mathrm{mole}-{ }^{\circ} \mathrm{C}\end{array}$ & $\begin{array}{c}\text { Excess } \\
\text { Specific } \\
\text { Heat } \\
\mathrm{C}_{p} \cdots \\
\mathrm{C}_{\mathrm{p}_{\mathrm{exp}}-\mathrm{C}_{\mathrm{p}}} \mathrm{p}_{\mathrm{ealc}} \\
\end{array}$ \\
\hline$a$ & $\begin{array}{r}-13 \\
27 \\
47 \\
67\end{array}$ & $\begin{array}{l}8.0 \\
8.48 \\
8.66 \\
8.84\end{array}$ & $\begin{array}{l}5.79 \\
5.83 \\
5.85 \\
5.86\end{array}$ & $\begin{array}{l}0.89 \\
1.09 \\
1.22 \\
1.31\end{array}$ & $\begin{array}{l}1.32 \\
1.56 \\
1.59 \\
1.67\end{array}$ \\
\hline $\boldsymbol{\beta}$ & $\begin{array}{l}140 \\
150 \\
160 \\
170 \\
180 \\
190\end{array}$ & $\begin{array}{l}8.20 \\
8.24 \\
8.28 \\
8.34 \\
8.41 \\
8.46\end{array}$ & $\begin{array}{l}5.93 \\
5.93 \\
5.93 \\
5.93 \\
5.93 \\
5.93\end{array}$ & $\begin{array}{l}0.62 \\
0.63 \\
0.65 \\
0.66 \\
0.68 \\
0.69\end{array}$ & $\begin{array}{l}1.65 \\
1.68 \\
1.70 \\
1.75 \\
1.80 \\
1.84\end{array}$ \\
\hline$\gamma$ & $\begin{array}{l}230 \\
240 \\
250 \\
260 \\
270\end{array}$ & $\begin{array}{l}8.58 \\
8.62 \\
8.67 \\
8.76 \\
8.85\end{array}$ & $\begin{array}{l}5.93 \\
5.93 \\
5.93 \\
5.93 \\
5.93\end{array}$ & $\begin{array}{l}0.73 \\
0.74 \\
0.76 \\
0.77 \\
0.78\end{array}$ & $\begin{array}{l}1.92 \\
1.95 \\
1.98 \\
2.06 \\
2.14\end{array}$ \\
\hline$\delta$ & $\begin{array}{l}327 \\
377 \\
427\end{array}$ & $\begin{array}{l}9.0 \\
9.0 \\
9.0\end{array}$ & $\begin{array}{l}5.94 \\
5.94 \\
5.94\end{array}$ & $\begin{array}{l}0.026 \\
0.024 \\
0.022\end{array}$ & $\begin{array}{l}3.0 \\
3.0 \\
3.0\end{array}$ \\
\hline$\epsilon$ & $\begin{array}{l}500 \\
600\end{array}$ & $\begin{array}{l}8.4 \\
8.4\end{array}$ & $\begin{array}{l}5.94 \\
5.94\end{array}$ & $\begin{array}{l}(0.90) \\
(1.00)\end{array}$ & $\begin{array}{l}(1.56) \\
(1.46)\end{array}$ \\
\hline
\end{tabular}

['rom ']' A. Siandenaw et al. in Plutonium 1960., pp. 73, 75.

Specific Heat

$$
\begin{array}{ll}
\text { a Plutonium } & 8.0 \mathrm{cal} / \mathrm{gran} \text {-atom } /{ }^{\circ} \mathrm{C} \\
\beta \text { Plutonium } & \text { 8.2 cal } / \text { gram-atom } /{ }^{\circ} \mathrm{C}
\end{array}
$$

W. 1). Wilkinsom in Extractive and Physical Metallurgy of Plutonium and Its Alloys, (1). $15-20$
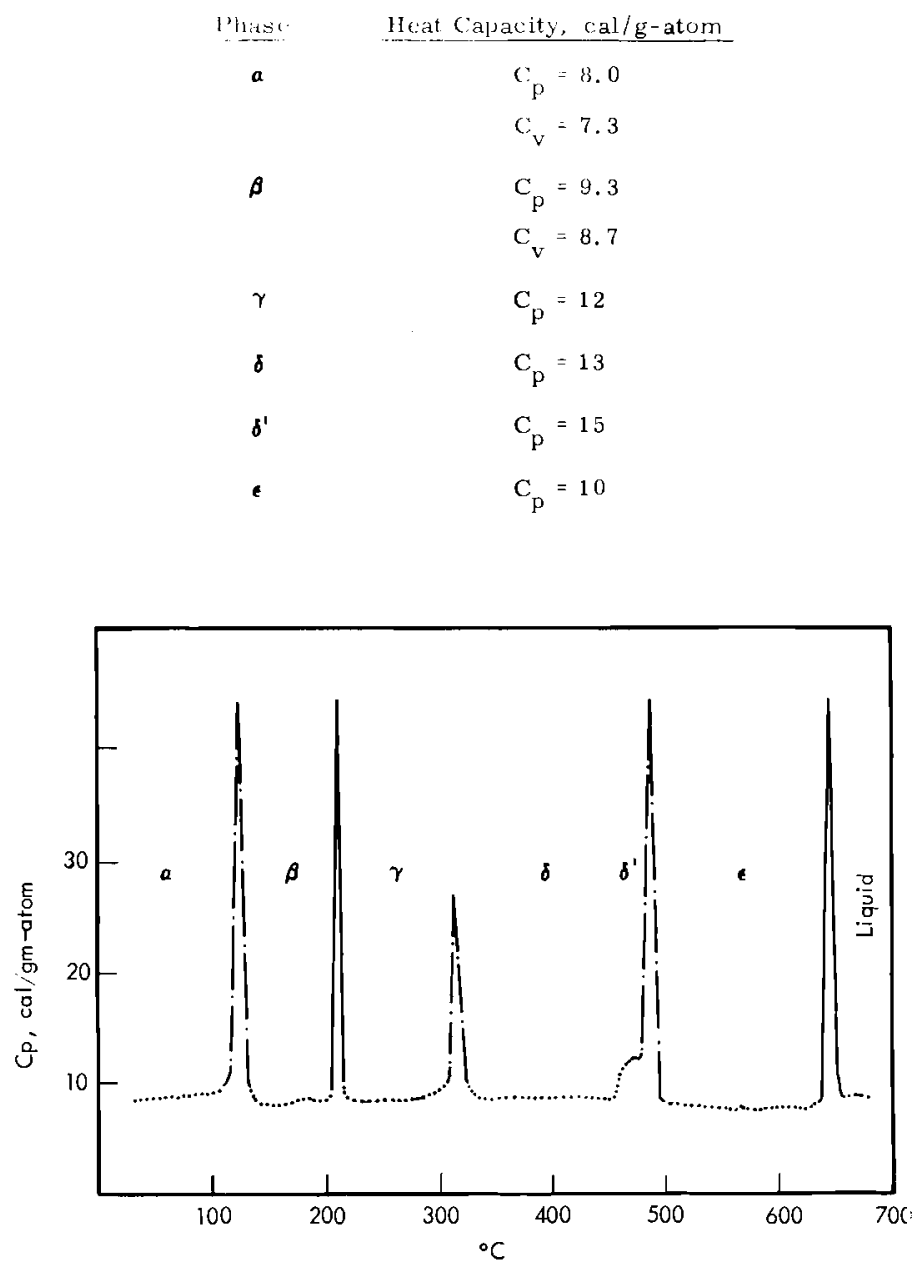

Variation of Specific Ileat with Temperature. From A. F. Kay and R. G. Loashy. Phil. Mag., eighth series, vol. 9, no. 97, p. 42.1964.

SPECIFIC HEA'TS

From A. F. Kay and R. G. Loasby, Phil. Mag., eighth series, vol. 9, no. 97, p. 13. 1964 


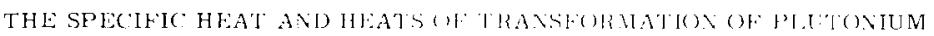

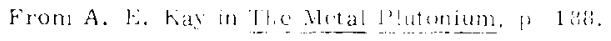

\begin{tabular}{|c|c|c|c|c|c|}
\hline $\begin{array}{c}\text { Temperature, } \\
{ }_{\mathrm{O}}^{\mathrm{C}}\end{array}$ & Specific Heat & 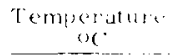 & Specifie that & Temperature, & Specific Heat \\
\hline-62.0 & 33.4 & 184 & +1.6 & 374 & 46.4 \\
\hline-58.5 & 33.4 & 190 & 41.11 & 376 & 46.9 \\
\hline-50 & 34.2 & 192 & 43.3 & 379 & 47.1 \\
\hline-44 & 35.2 & 197 & 43.4 & 383 & 46.4 \\
\hline-32 & 37.5 & 200 & 42.3 & 389 & 48.3 \\
\hline-25 & 38.9 & 204 & 42.8 & 397 & 45.7 \\
\hline-21 & 38.7 & 208 & $43 . t$ & 407 & 45.6 \\
\hline-13 & 38.8 & 210 & $4 \$ .7$ & 412 & 47.8 \\
\hline-1.5 & 35.7 & 211 & 10.3 & 420 & 48.6 \\
\hline-1.0 & 35.5 & $8-$ tra & ition; & 426 & 48.6 \\
\hline 5.0 & 32.4 & latent $h$ & $L=140$ & 433 & 45.0 \\
\hline 13 & 35.5 & $\pm 15 \mathrm{ca}$ & -atom & 442 & 47.0 \\
\hline 15 & 33.5 & 212 & $6,6.5$ & 449 & 49.7 \\
\hline 20 & 33.8 & 212 & 59.9 & 450 & 50.2 \\
\hline 29 & 34.1 & 213 & 42.6 & 452 & 51.0 \\
\hline 38 & 34.5 & 218 & 40.8 & 454 & 53.9 \\
\hline 44 & 34.9 & 226 & 42.3 & 456 & 56.6 \\
\hline 52 & 36.6 & 230 & 44.0 & 457 & 64.1 \\
\hline 62 & 36.6 & 236 & 43.0 & 459 & 86.8 \\
\hline 65 & 35.0 & 242 & 44.8 & \multicolumn{2}{|c|}{$\delta-\delta^{\prime}$ transition; } \\
\hline 70 & 38.7 & 243 & 44.4 & \multicolumn{2}{|c|}{ lat ent heat $=17$} \\
\hline 74 & 38.6 & 250 & 45.0 & \multicolumn{2}{|c|}{ $\pm 10 \mathrm{cal} / \mathrm{g}$-atom } \\
\hline 81 & 41.3 & 254 & 46.3 & 461 & 62.9 \\
\hline 85 & 41.5 & 260 & 45.2 & 464 & 58.6 \\
\hline 90 & 41.3 & 267 & 46.9 & 468 & 65.8 \\
\hline 96 & 42.5 & 272 & 45.9 & 470 & 75.0 \\
\hline 104 & 44.9 & 279 & 46.1 & 473 & 79.4 \\
\hline 111 & 44.9 & 284 & 46.8 & 473 & 100.6 \\
\hline 113 & 47.8 & 288 & 45.3 & 477 & 141.6 \\
\hline 118 & 52.8 & 291 & 47.8 & 480 & 155.2 \\
\hline 121 & 56.0 & 297 & 47.9 & 481 & 157.8 \\
\hline 122 & 60.9 & 303 & 49.9 & 483 & 268.1 \\
\hline 124 & 76.7 & 305 & 50.5 & \multicolumn{2}{|c|}{$\delta^{\prime} \rightarrow \in$ transition; } \\
\hline 126 & 101.7 & 307 & 60.7 & \multicolumn{2}{|c|}{ lat ent heat $=470$} \\
\hline 126 & 129.2 & 312 & 89.6 & \multicolumn{2}{|c|}{ $\pm 10 \mathrm{cal} / \mathrm{g}$-atom } \\
\hline \multicolumn{2}{|c|}{$127^{\circ}$ : superheat; the true } & 315 & 128.1 & 486 & 55.6 \\
\hline \multirow{2}{*}{\multicolumn{2}{|c|}{$\begin{array}{l}\text { transformation temperature } \\
-121{ }^{\circ} \mathrm{C} \text {; the latent heat of }\end{array}$}} & \multicolumn{2}{|c|}{ 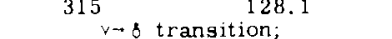 } & 488 & 43.8 \\
\hline & & \multicolumn{2}{|c|}{$\begin{array}{l}\forall \rightarrow \& \text { transition; } \\
\text { latent heat }=156\end{array}$} & 491 & 43.4 \\
\hline \multicolumn{2}{|c|}{ the $:-8$ transition $=958$} & \multicolumn{2}{|c|}{$\begin{array}{l}\text { latent heat }=156 \\
\pm 5 \mathrm{cal} / \mathrm{g} \text {-atom }\end{array}$} & 498 & 43.1 \\
\hline$\div 10 \mathrm{cal} / \mathrm{g}$ & tom & 318 & 87.3 & 505 & 44.0 \\
\hline & & 320 & 82.0 & 513 & 42.2 \\
\hline 127 & 161.9 & 322 & 73.9 & 518 & 39.4 \\
\hline 130 & 99.0 & 324 & 62.3 & 527 & 41.7 \\
\hline 132 & 72.2 & 326 & 56.8 & 533 & 35.4 \\
\hline 133 & 57.9 & 327 & 52.8 & 538 & 36.9 \\
\hline 135 & 50.9 & 332 & 48.5 & 546 & 37.6 \\
\hline 140 & 43.1 & 334 & 47.1 & \multicolumn{2}{|c|}{$\varepsilon \rightarrow$ liquid transition at } \\
\hline 145 & 40.5 & 343 & 44.6 & \multicolumn{2}{|c|}{$639^{\circ} \mathrm{C}$; latent heat $=$} \\
\hline 152 & 39.9 & 346 & 45.5 & \multicolumn{2}{|c|}{$940 \pm 150 \mathrm{cal} / \mathrm{g}$-atom; } \\
\hline 158 & 41.1 & 350 & 45.5 & \multicolumn{2}{|c|}{ total heat of plutonium } \\
\hline 167 & 41.9 & 356 & 48.1 & \multicolumn{2}{|c|}{ in solid state $=11.7$} \\
\hline 171 & 42.0 & 361 & 46.5 & \multicolumn{2}{|c|}{ $\pm 0.5 \mathrm{Kcal} / \mathrm{g}$-atom } \\
\hline 176 & 42.5 & 368 & 46.9 & & \\
\hline
\end{tabular}

Rate of heat generation, $Q=2.2125 \mathrm{~mW} / \mathrm{g}$ (normal deviation almost $0.1-0.2 \%$ )

L. H. Treeman reported a value of $Q=2.2157 \mathrm{~mW} / \mathrm{g}$ (private communication to Sandenaw)

From T. A. Sandenaw, J. Phys. Chem. Solids, vol. 23, p. 825. 1962.

Some $C_{\nu}$ values may be obtained using the data of Linford given in Paper 14 (Plutonium 1960, p. $51-8$ ), and these are given below.

$\begin{array}{cccc}\text { Phase } & \begin{array}{c}\text { Temperature, } \\ { }^{\circ} \mathrm{C}\end{array} & \begin{array}{c}\mathrm{C}_{\mathrm{P}}, \\ \mathrm{cal}_{\mathrm{g}}\end{array} & \begin{array}{c}\mathrm{C}_{\mathrm{V}}, \\ \mathrm{cal} / \mathrm{g}\end{array} \\ \boldsymbol{\beta} & 5 & 0.036 & 0.030 \\ \gamma & 150 & 0.034 & 0.030 \\ \gamma & 230 & 0.035 & 0.032 \\ & 320 & 0.043 & 0.042\end{array}$

From R. G. Loasby in Plutonium 1960, p. 102.

From T. A. Sandenaw et al. in plutonium $1960, p, 74$.

\begin{tabular}{|c|c|c|c|c|c|c|c|c|}
\hline \multirow[b]{2}{*}{ Specimen No. } & \multicolumn{2}{|c|}{$36.0^{\circ}$ to $36.5^{\circ} \mathrm{K}$} & \multicolumn{2}{|c|}{$88^{\circ}$ to $90^{\circ} \mathrm{K}$} & \multicolumn{2}{|c|}{$154^{\circ}$ to $156^{\circ} \mathrm{K}$} & \multicolumn{2}{|c|}{$298.15^{\circ} \mathrm{K}$} \\
\hline & $\mathrm{S}_{\mathrm{T}}$ & $\mathrm{H}_{\mathrm{T}} \mathrm{T}_{0}^{-\mathrm{H}_{0}}$ & $\mathrm{~s}_{\mathrm{T}}$ & $\mathrm{H}_{\mathrm{T}}-\mathrm{H}_{0}$ & $\mathrm{~S}_{T}$ & $\mathrm{H}_{\mathrm{T}^{-\mathrm{H}_{0}}}$ & $\underline{\mathrm{s}_{\mathrm{T}}}$ & ${ }^{\mathrm{H}_{\mathrm{T}}}{ }^{-\mathrm{I}}{ }_{0}$ \\
\hline$\overline{\mathrm{H}}$ & $2 . \overline{75(5)}$ & $65.0(5)$ & $7 . \overline{07(5)}$ & $328(5)$ & $11.37(5)$ & $834(5)$ & $15.17(3)$ & $1707(3)$ \\
\hline NP & $1.94(6)$ & 4 & $5.91(6)$ & $283(6)$ & $9.58(5)$ & $728(5)$ & $14.55(5)$ & $1826(5)$ \\
\hline P2 and NP2 $(99.96,99$ & $2.33(7)$ & $55.3(7)$ & $7.15(6)$ & $338(6)$ & $10.28(1)$ & $764(1)$ & $15.09(1)$ & $1812(1)$ \\
\hline Av. of all values & $2.32(18)$ & $54.1(18)$ & $6.69(17)$ & $316(17)$ & $10.46(11)$ & $779(11)$ & $14.82(9)$ & $1785(9)$ \\
\hline
\end{tabular}

CALCULATED EXTRA ENTROPY IN HFAT CAPACTTY PEAKS

From T. A. Sandenaw et al. in Plutonium 1960, p. 74

\begin{tabular}{cc} 
Temp. at Crest, ${ }^{\circ} \mathrm{K}$ & Extra Entropy, e.u. \\
\hline 31 & $1.15=\sim \mathrm{R} \ln 2$ \\
47 & $2.00=\sim \mathrm{R} \ln 3$ \\
123 & $2.95=\sim \mathrm{R} \ln 4$
\end{tabular}

The heat capacity curve at the $\boldsymbol{\alpha}-\boldsymbol{\beta}$ phase transition for a rather impure plutonium specimen was shown in section 202.3. Assuming that the entropy and enthalpy at $298^{\circ} \mathrm{K}$ were equivalent to the average found for the three specimens, it has been calculated that the entropy and enthalpy at $420^{\circ} \mathrm{K}$ would be $20.05 \pm 0.40 \mathrm{e} . \mathrm{u}$. and $3900 \pm 100 \mathrm{cal} / \mathrm{mole}$, respectively.

From T. A. Sandenaw et al. in Plutonium 1960, p. 74 . 
See the following tables in section for entilled

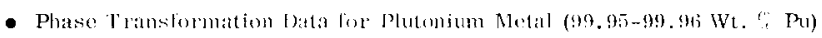

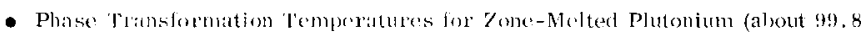

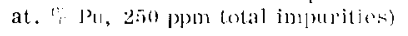

- Transiliom Temperatures (jossible error

- Temperatures ol Phalse Transitians

- Delta/Delta-Prime and Delta-Prime/Fpsilon Transition Temperatures of Plutonium on Heating (in $\left.{ }^{\circ} \mathrm{C}\right)$

Also see section 602 .

\section{Heats of Transition}

W. L. Wilkinson in Extractive and Physical Metallurgy of Plutonium and Its Aloys, P. 20.

\begin{tabular}{|c|c|}
\hline 'I'ransition & $\Delta \mathrm{H}, \mathrm{cal} / \mathrm{g}$-atom \\
\hline$\alpha-\beta$ & $925 *$ \\
\hline & $958 \pm 10\left(\right.$ at $\left.121^{\circ}{ }^{\circ}\right)+$ \\
\hline$\beta-\gamma$ & $140 \pm 15$ (at $208^{\circ} \mathrm{C}$ ) \\
\hline$\gamma-\delta$ & $156 \pm 5\left(\right.$ at $\left.316^{\circ} \mathrm{C}\right)$ \\
\hline$\delta-\delta$ & $17 \pm 10$ (at $455^{\circ} \mathrm{C}$ ) \\
\hline$\delta^{\prime}-\epsilon$ & $470 \pm 10\left(\right.$ at $\left.479^{\circ} \mathrm{C}\right)$ \\
\hline$\epsilon-$ liquid & $790 \pm 190$ \\
\hline
\end{tabular}

* Normal purity plutonium (99.8\% plutonium)

+ The rest of the values are for high-purity plutonium $(99.96 \%$ plutonium $)$

1. K. kngel et al. USAEC Report TID-7641, pp. 266-272.

\begin{tabular}{ccc} 
Transformation & $\begin{array}{c}\text { Temperature, } \\
\text { (on Heating) }\end{array}$ & $\begin{array}{r}\text { Latent Heat, } \\
\text { cal/g-atom }\end{array}$ \\
\cline { 2 - 2 }$\cdot \boldsymbol{\beta}$ & 127 & $810+5$ \\
$\boldsymbol{\beta} \cdot \boldsymbol{\gamma}$ & 207 & $124+12$ \\
$\boldsymbol{\gamma} \cdot \delta$ & 320 & $136+7$ \\
$\delta \cdot \delta$ & 460 & $25+8$ \\
$\delta \cdot \boldsymbol{C}$ & 481 & $441+8$ \\
$\boldsymbol{\epsilon}-$ liquid & 640 & $655+6$
\end{tabular}

\section{L.ATENT HEATS}

From A. E. Kay and R. G. Loasby, 1'ril. Mag , eighth series, vol, 9, no. 97, p. 43, 1964.

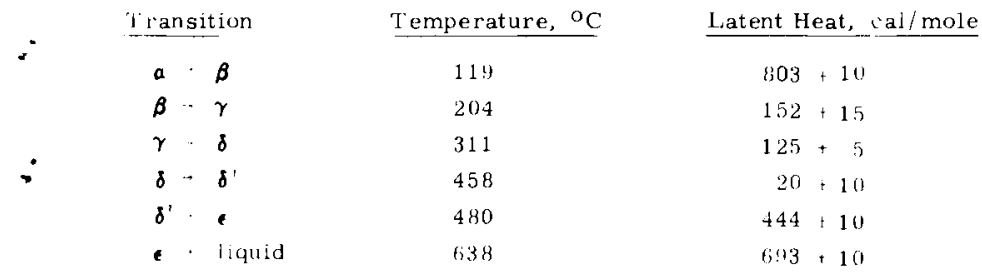

\section{Electrical Conductivity}

From W. D. Wilkinson, in Extractive and l'hysical Metallurgy of Plutonium and Its Alloys, pp. 15-20.

\begin{tabular}{|c|c|c|}
\hline Phase & 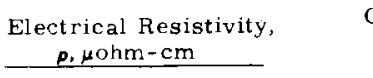 & $\begin{array}{l}\text { Coefficient of Electrical Resistivity, } \\
\qquad \frac{1}{\rho}(\mathrm{d} \rho / \mathrm{dT}) \times 10^{-5}\end{array}$ \\
\hline a & 145 at $28^{\circ} \mathrm{C}$ & -21 to -40 \\
\hline$\beta$ & 110.5 at $132{ }^{\circ} \mathrm{C}, 117$ average & -5 to -11 (average $=-6)$ \\
\hline$\gamma$ & 110 at $230^{\circ} \mathrm{C}, 115$ average & $-4 t o-8(a$ prage $=-5)$ \\
\hline$\delta$ & 103 at $353^{\circ} \mathrm{C}$ & 7 to 18 \\
\hline$\delta^{\prime}$ & 105 at $462^{\circ} \mathrm{C}$ & 45 \\
\hline
\end{tabular}

The: unusual behaviour of the $\delta$-phase in having a negative coefficient of expansion and the contraction in volume which occurs on passing from the f.c.c. $\delta$-phase to the b.c.c. $c$-phase are two other features which might be explained in terms of the thermodynamic data. Varley (1956) has considered the main fictors controlling the volume expansion of solids and concluded that a negative coefficient might arise if the density of states at the $\mathrm{F}$ crmi surface varies rapidly with energy and if the electronic specific heat is also high. Measurements of the specific heat and a number of other properties from high temperalures down to liquid helium temperatures are being undertaken in an attempt to understand the metallic behaviour of plutonium.

Two problems arise in attempting to measure the low temperature specific heat of plutonium. These are the extremely rapid rate of rise in temperature $(1 \% / \mathrm{sec}$ helow $10^{\circ} \mathrm{K}$ ) which oceurs at low temperatures due to the immutalle self-heating capacity of the material and the self-irradiation energy (Sandenaw 1962) which is stored in the lattice if the samples are held at these temperatures for any appreciable time. Low temperature measurements are restricted to the $a$ and $\boldsymbol{\delta}$-phase with possibly the inclusion of the $\boldsymbol{\beta}$-phase. The a-phase can he examined in the pure state, whereas the $\boldsymbol{\delta}$ and $\boldsymbol{\beta}$-phases nust be statilized by the addition of an alloying element. Data on pure metal phases can the rofore only be olstnined at temperatures where they a re normally stable.

From A. E. Kily and R. G. Loashy. Phil Mag., eighth series, vol. 9, no, 97, 1). 37 (leginning with th line through the 2 nd paragraph). 1966 .

S. T. Konobeevsky et al. p/2230, Proc. Second Geneva Internat. Conf., vol. 6, p. 194. 1958.

\begin{tabular}{cc} 
Transition & Latent Heat of Transformation, cal $/ \mathrm{g}^{-1}$ \\
${$\cline { 2 - 2 }$-\boldsymbol{\beta}} }$ & $3.2 \pm 0.5$ \\
$\boldsymbol{\beta}-\boldsymbol{\gamma}$ & $0.7 \pm 0.1$ \\
$\boldsymbol{\gamma}-\boldsymbol{\delta}$ & $0.8 \pm 0.1$ \\
$\boldsymbol{\delta}-\boldsymbol{\delta}$ & 0 \\
$\boldsymbol{\delta}^{\prime}-\boldsymbol{\epsilon}$ & $1.6+0.3$ \\
$\boldsymbol{\epsilon}-$ liquid & $3.3+0.5$
\end{tabular}

Results obtained using a differential vacuum calorimeter.

The thermal arrest due to the $\boldsymbol{a} \rightarrow \boldsymbol{\beta}$ transformation indicated a heat of transition of $938+10 \mathrm{cal} / \mathrm{mole}$

From T. A. Simdenaw and R. B. (dibney. J, Phys, (hem, Solids, vol, d, p. 86 1958.

The latent heat of the $\delta \rightarrow \delta$ transformation appen $r$ s to he about 100 cal/g-aton The latent heat of the $\delta \rightarrow$ transformation is larger, the ratio being $3.5 \pm 1$.

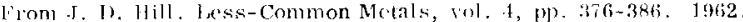

Heat of Vaporization. B., $16 ; 0.34 \mathrm{k}: \mathrm{al} / \mathrm{mole}$.

Frem F. R. Jette and A. S. cofinbery. LSAFC laport AE(C)-3647, p. 237.
J. G. Ball and W. B. H. Lord, J. Inst. Metals, vol, 86, p. 376. 1957-58.

\begin{tabular}{lcc} 
Phase & $\begin{array}{c}\text { Resistivity, } \\
\mu o h m-\mathrm{cm}\end{array}$ & $\begin{array}{c}\text { Temperature Coefficient of Resistance, } \\
10^{-5 / 0 \mathrm{C}}\end{array}$ \\
\cline { 2 - 3 } Alpha & 150 & -22 to -40 \\
Beta & 117 & -5 to -11 \\
Gamma & 115 & -4 to 8 \\
Jelta & 108 & +8 to +18
\end{tabular}




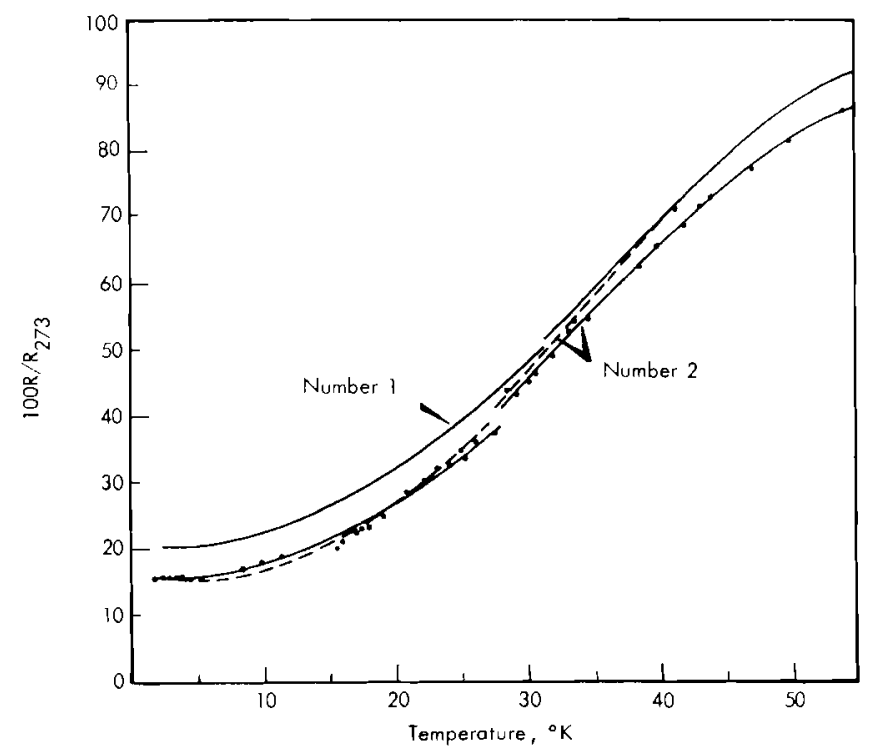

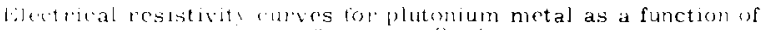

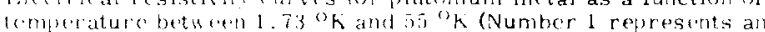
arrage of three suns uith specinen Number 1, - maximum

Weviation : 1" Number 2 shows branihing observed with specimen

vinmbere?

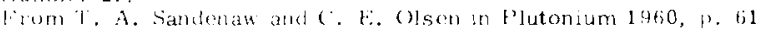

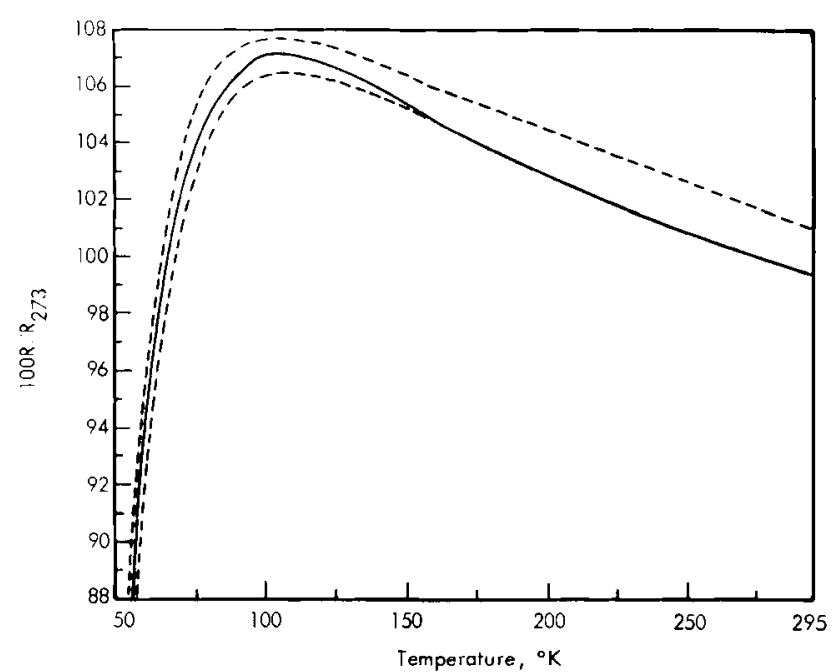

Filectrical Resistivity of Plutonium Metal Between $50^{\circ} \mathrm{K}$ and 23.5 " $\mathrm{K}$. (Dashed line repregents obgerved limits; solid line represents normal curve.)

From I. A. Sandenaw and C. E. Olsen in Plutonium 1960, p. 63.

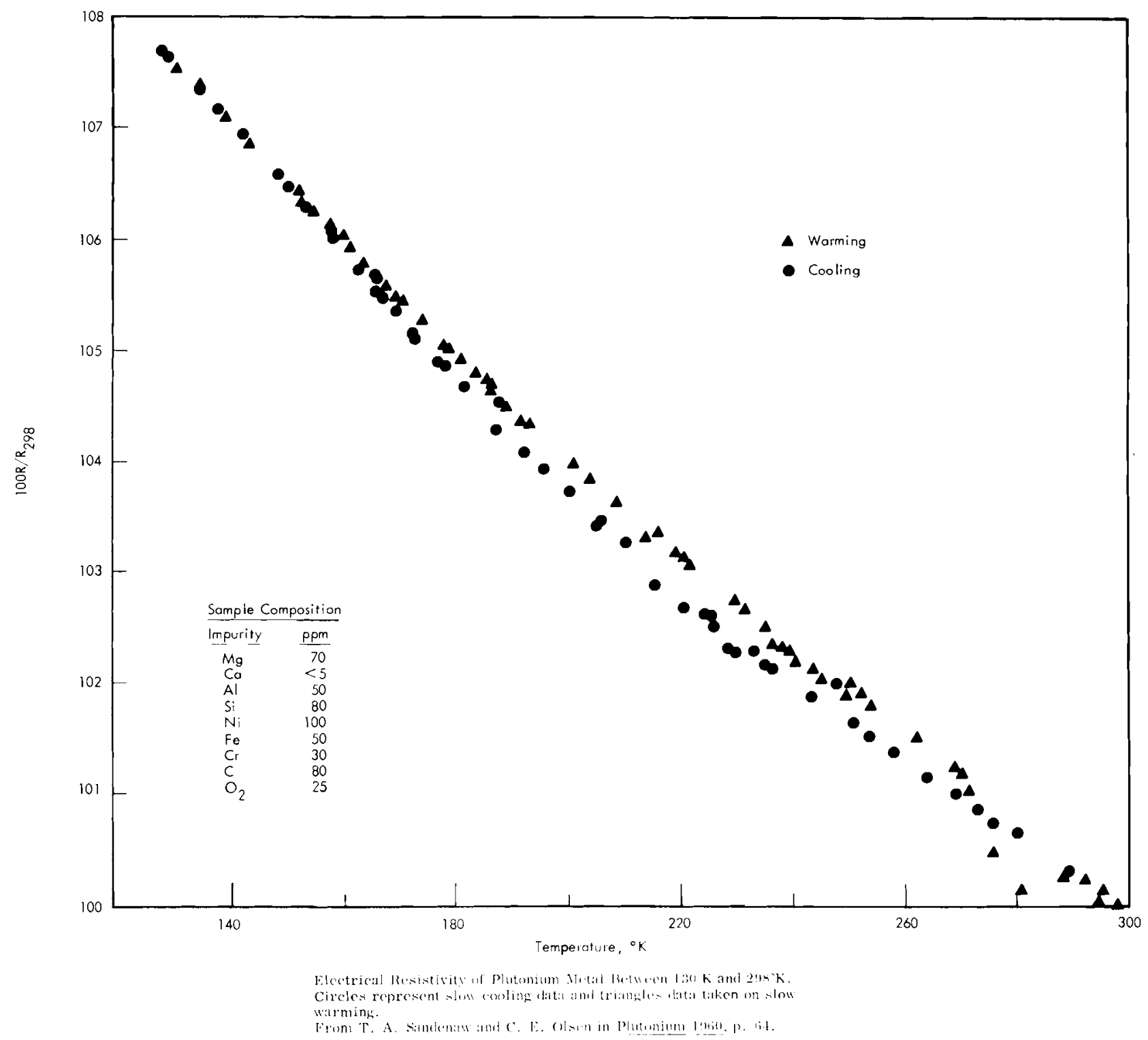




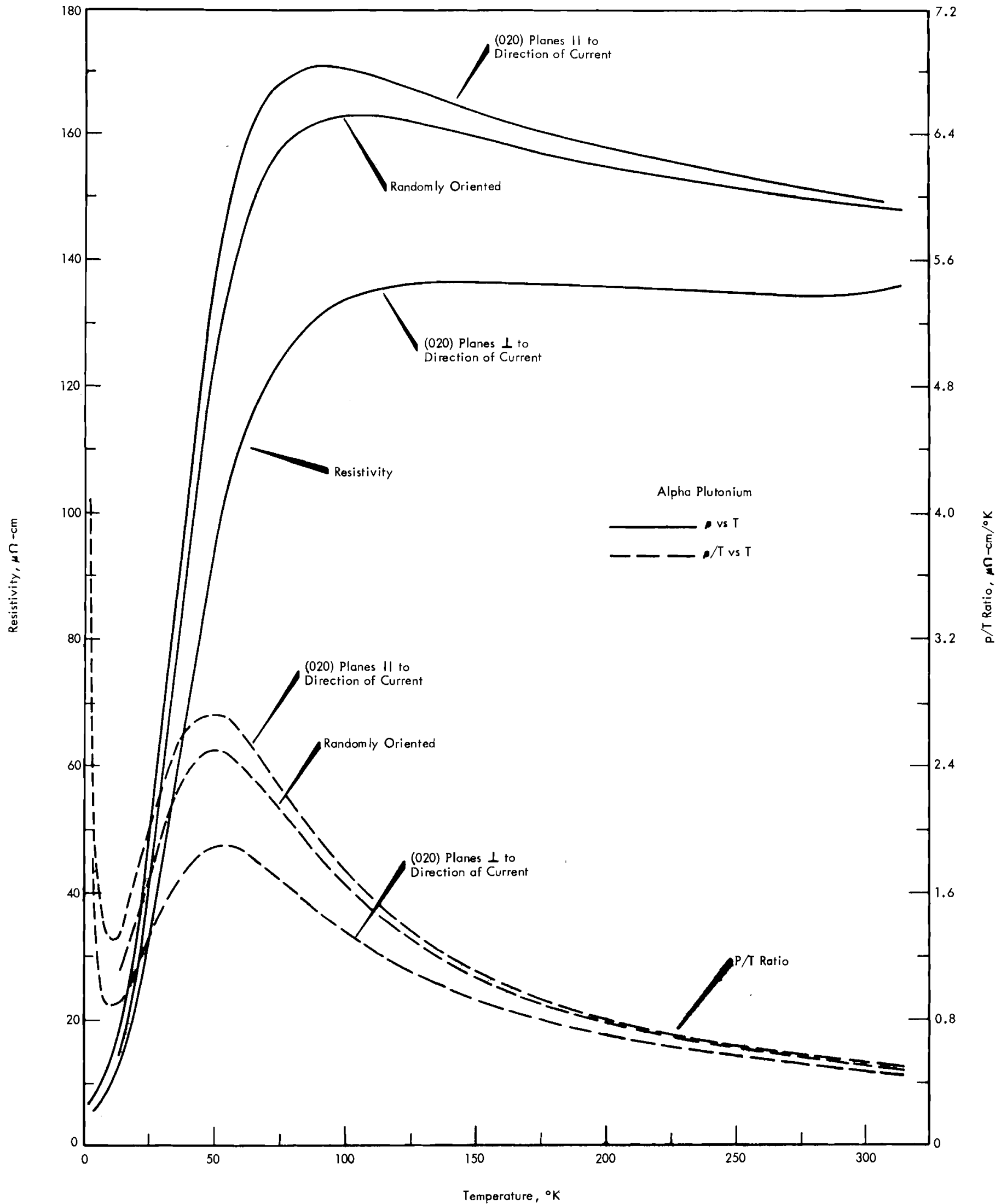

Resistivity Data vs Temperature for Highly Oriented and Randomly Oriented Alpha Plutonium, From R. O. Elliott et al. Phys. Rev. Letters, vol. 12,

p. 277. 1964. 
COMPARISON OF RESISTIVITY DATA FOR HIGHLY ORIENTED AND RANDOMLY ORIENTED ALPHA PLUTONIUM

From R. O. Elliott et al. Phys. Rev. Letters, vol. 12, p. 278.1964.

\begin{tabular}{|c|c|c|c|c|}
\hline $\begin{array}{l}\text { Orientation of } \\
\text { Specimen }\end{array}$ & $\begin{array}{c}\text { Residual } \\
\text { Resistivity, } \\
\mu \|-\mathrm{cm}\end{array}$ & $\begin{array}{l}\text { Temp at } \\
\boldsymbol{P}_{\max },{ }^{\circ} \mathbf{K}\end{array}$ & $\begin{array}{l}\rho \max , \\
\mu \curvearrowleft-\mathrm{cm}\end{array}$ & 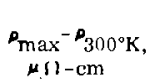 \\
\hline $\begin{array}{l}\text { (020) planes } \perp \text { to } \\
\text { direction of current }\end{array}$ & 6.2 & $125-170^{*}$ & 137 & 1 \\
\hline Randomly oriented & . & 105 & 163.5 & 14 \\
\hline $\begin{array}{l}\text { (020) planes II to } \\
\text { direction of current }\end{array}$ & 7.5 & 92 & 171.3 & 20.7 \\
\hline
\end{tabular}

*Resistivity maximum is flat in this region.

COM PARISON OF THE ELECTRICAL RESISTIVITIES OF TWO PLUTONIUM SPECIMENS

From G. T. Meaden. Proc. Roy, Soc. (London), Ser. A, vol 276, p. 564.1963

\begin{tabular}{|c|c|c|c|c|}
\hline & $\begin{array}{r}\text { About } 200 \mathrm{ppm}, \\
\text { Density }=19.625\end{array}$ & $\begin{array}{l}\text { Iachined, } \\
0.02 \mathrm{~g} / \mathrm{cm}^{3}\end{array}$ & $\begin{array}{l}\text { About } 275 \mathrm{ppm}, \\
\text { Density }=19.195\end{array}$ & $\begin{array}{l}\text { st to Shape } \\
0.02 \mathrm{~g} / \mathrm{cm}\end{array}$ \\
\hline & $\stackrel{P T}{\mu(1 \mathrm{cn}}$ & $\mathrm{d} \rho / \mathrm{d} T$ & $\begin{array}{r}P_{\mathrm{T}} \\
\mu\{\mathrm{Cm}\end{array}$ & $\mathrm{d} \rho / \mathrm{d} T$ \\
\hline $1,4^{\circ} \mathrm{K}$ & 20.75 & 0 & 49.25 & 0 \\
\hline 10 & 22.6 & +0.75 & 55.1 & +1.21 \\
\hline 20 & 39.3 & 2.36 & 72.4 & 2.21 \\
\hline 40 & 102.6 & 3.12 & 117.8 & 1.81 \\
\hline 60 & 140.1 & 1.10 & 142.0 & 0.72 \\
\hline 80 & 152.5 & 0.37 & 152.0 & 0.28 \\
\hline 100 & 155.5 & ${ }^{-0.005}$ & 155.6 & +0.005 \\
\hline 150 & 153.6 & -0.061 & 154.8 & -0.040 \\
\hline 200 & 150.9 & -0.041 & 153.0 & -0.034 \\
\hline 250 & 148.9 & -0.031 & 151.4 & -0.031 \\
\hline 273 & 148.1 & -0.026 & 150.8 & -0.026 \\
\hline 300 & $147.5=0.2$ & & $150.5 \pm 0.2$ & \\
\hline
\end{tabular}

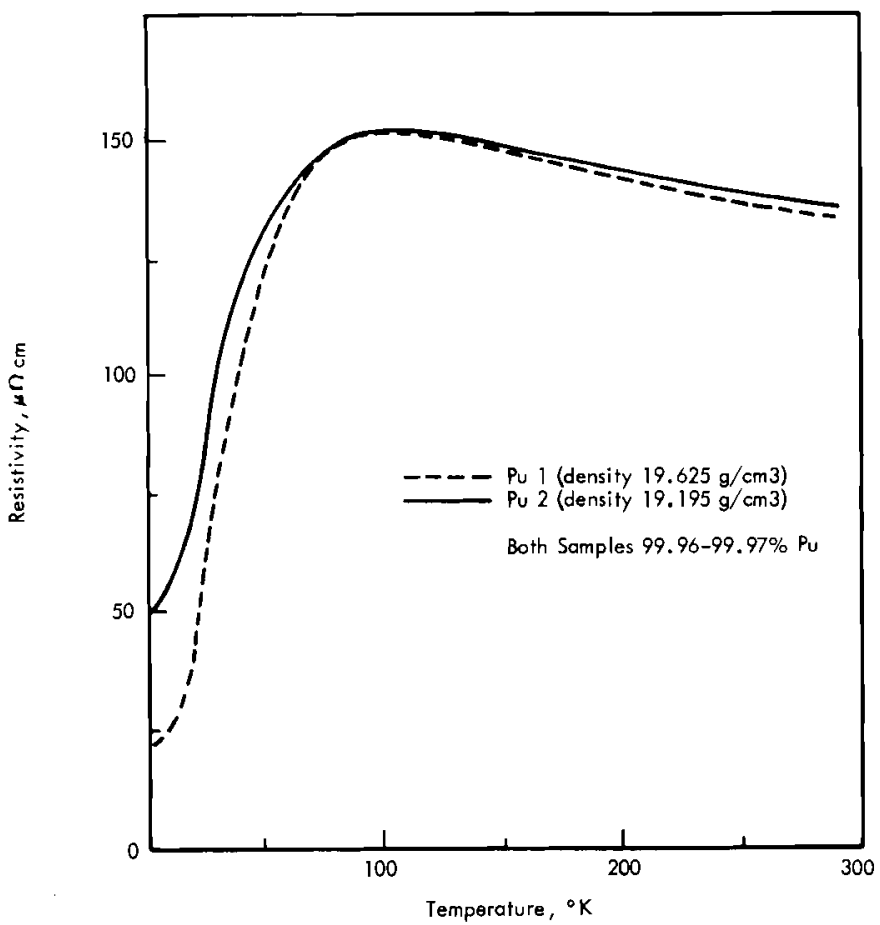

Resistivity vs Temperature for Two Specimens of Plutonium in the a - Phase. Experimental points were too numerous to be drawn on a - Phase. Experimental points were too numerous to be drawn on
the graph. The scatter was such that all the points lay within the thicknesses of the lines. From J. A. Lee et al. Cryogenics, vol. 1, p. 52. 1960 .

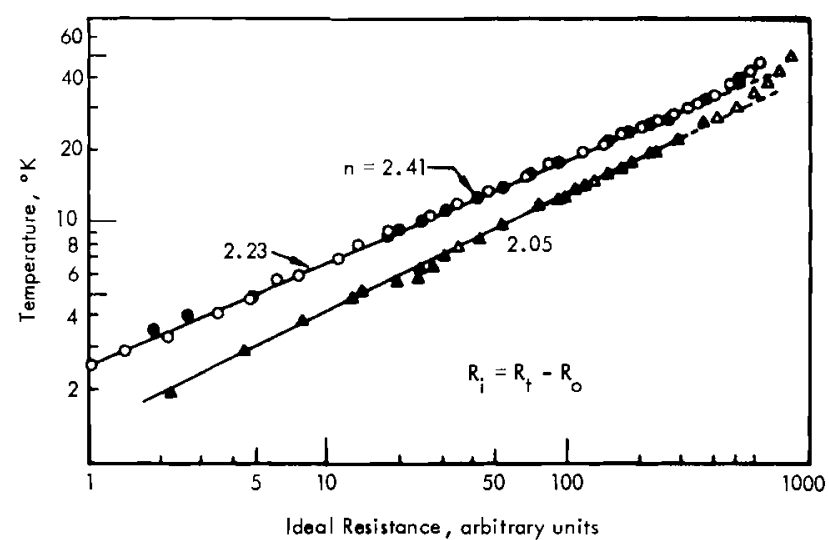

Ideal Resistances Plotted Iogarithmically. Specimens: O, Pu 3 run $5 ; \bullet, P u$ run $7 ; \Delta$, Pu 4 run $1 a ; \Delta$, Pu 4 run $1 b$. From G. T. Meaden. Proc. Roy. Soc. (London), Ser. A, vol. 276, p. 565. 1963

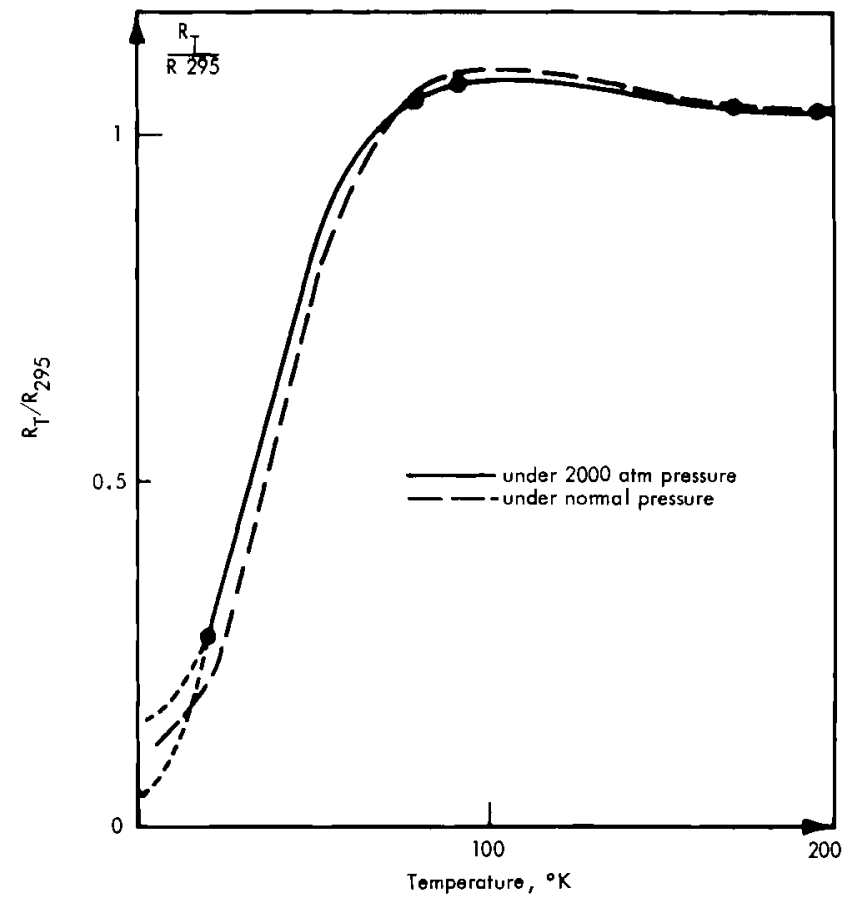

Resistivity of a-Pu. From R, Lallement. Physics Letters, vol. 8, p. 166.1964.

See section 602 . 


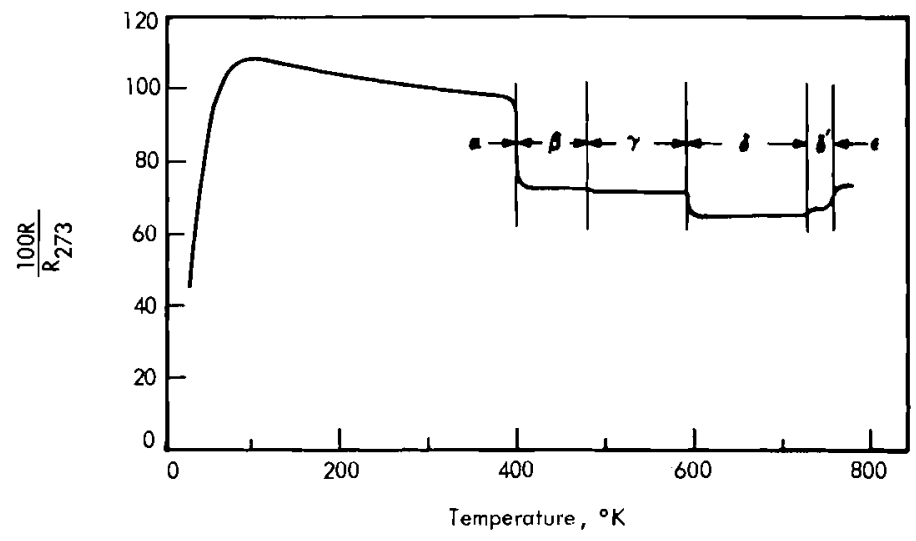

Electrical resistance, plotted as $100 \mathrm{R} / \mathrm{R}_{273}$ versus absolute temperature. From T. A. Sandenaw in The Metal Plutonium, p. 153.

RESISTIVITY VALUES OF PLUTONIUM SPECIMENS

(from amoothed curves)

From T. A. Sandenaw and R. B. Gibney,

J. Phys. Chem. Solids, vol. 6, p. 85, 1958.

Temperature, ${ }^{\circ} \mathrm{K}$

Resistivity, $\mu \Omega-\mathrm{cm}$

Specimen 1

99.95 wt\% Plutonium $\quad 99.96$ wt $\%$ Plutonium

25.8

27.2

50

100

150

273.15

380

420

475

505

590

625

725

735

774

787

61.1
128.0
$157.0 \pm 0.10$
153.5
$146.3 \pm 0.25$
141.0
107.5
107.0
106.2
105.0
97.7
98.5
99.4
107.1
-

68.5

128. 0

$156.8 \pm 0.06$

153.5

$146.6 \pm 0.04$

141.8

109.5

110.0

109.5

109.3

103.0

104.0

104.8

114.0

114.1

TEMPERATURE COEFFICIENT OF RESISTIVITY OF PLUTONIUM SPECIMENS

From T. A. Sandenaw and R. B. Gibney, J. Phys. Chem. Solids, vol. 6, p. 86. 1958.

Temperature Range, ${ }^{o} \mathrm{~K}$

$27-50$

$126-293$

$302-381$

$420-470$

$503-590$

$625-725$

$736-747$

$761-775$
Phase

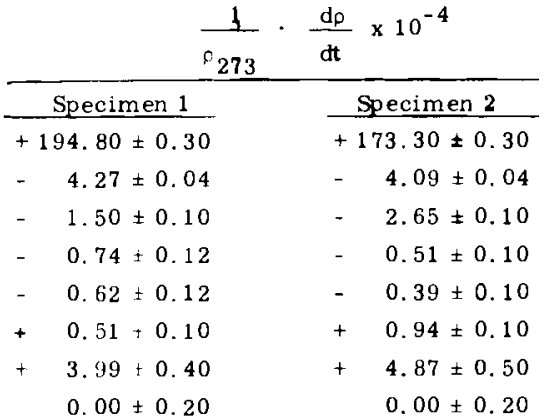




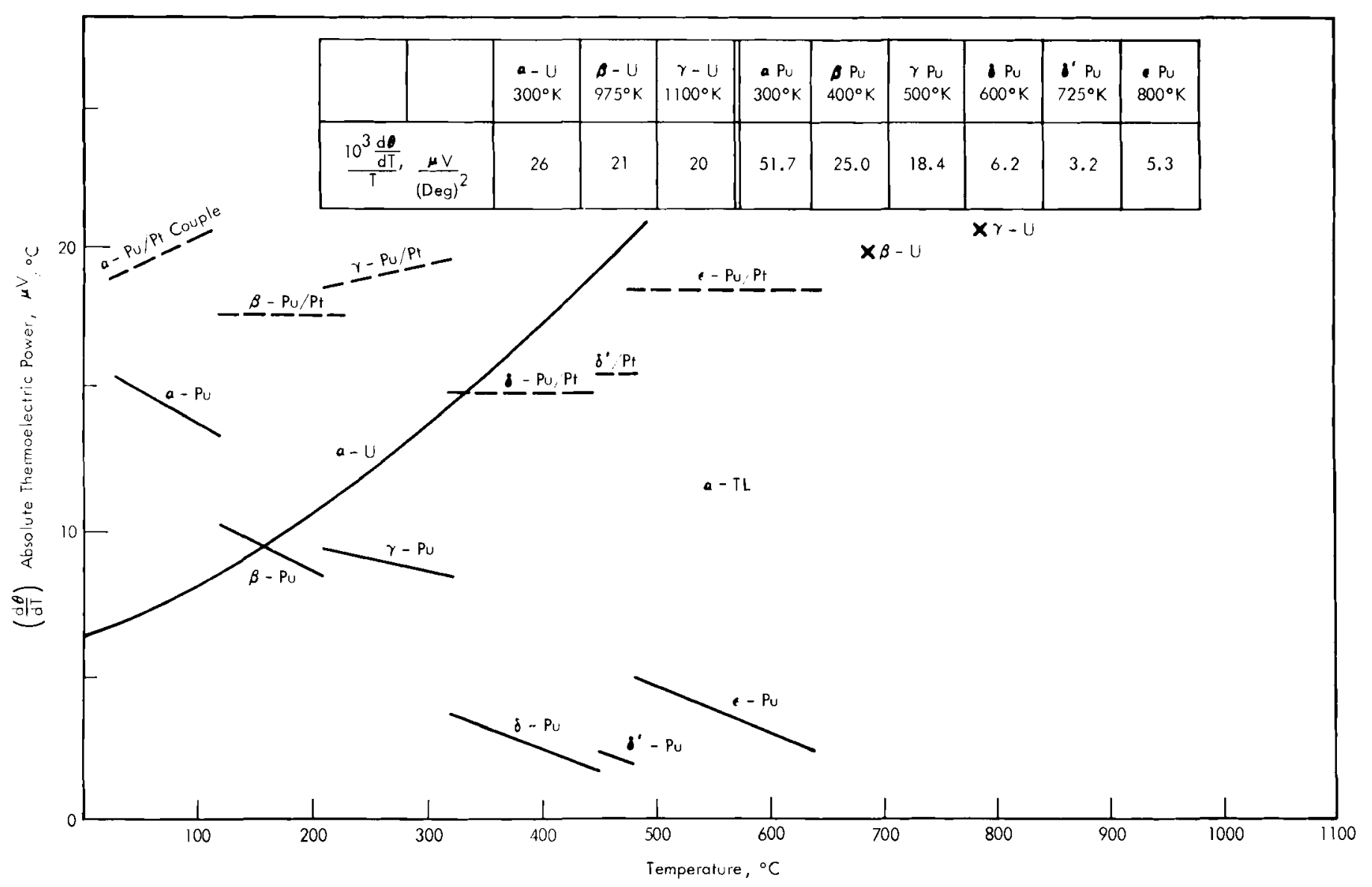

Absolute Thermuclectric Purer. From $\mathrm{J}$ A. He and $\mathrm{R}$ O. A. Hall, Less-Common Nickals, vol. 1, 1. 35x. 1959.

The thermal (.,m.f. al a plutonium-platimun thermorenple was measured between $20^{\circ}$ and $630^{\circ} \mathrm{C}$. The alose ligure shows the thermoelectric power of the plutoniumplatinum eouple caloulated from the stepe il the e.m. if temperature curve and the absolute thermoselectic powers derival fom llis curve. The scatter in the results for the relative the rmoelectric powe was within $\pm 5 \%$ from run to run, and sample to sample. Values for urimium a de included for comparison. The inplicatinns of these results in rolation to the form of the density of states/energy curve at the Fermi surfice and thus in relation lo Varley's eriterion for a negative volume coefficient of cxpansion are cliscussed. 


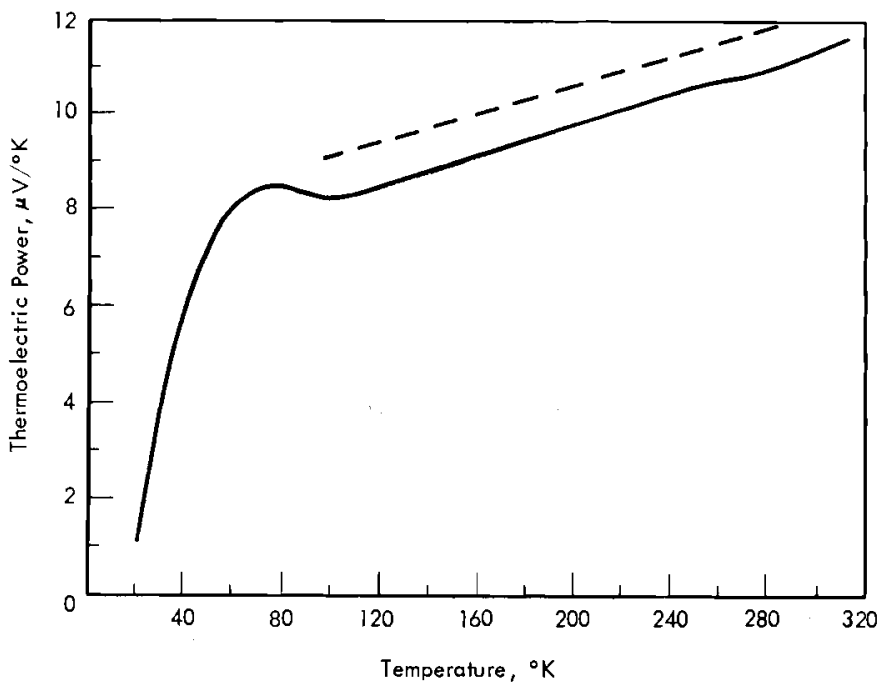

The Absolute Thermoelectric Power of High Purity Pu. From G. T. Meaden. Proc. Roy. Soc. (London), Ser. A, vol. 276, p. 565. 1963.

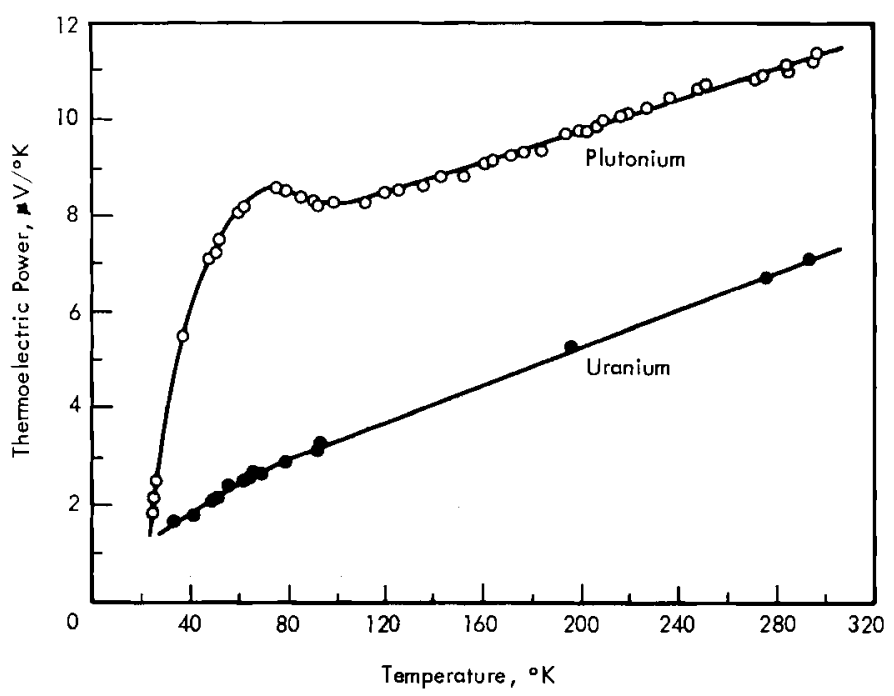

The Thermoelectric Powers of Uranium and Plutonium as Functions of the Temperature. From G. 'T. Meaden and J. A. Lee. Cryogenics, vol. 2 p. $182,1962$.

\section{Magnetic Susceptibility}

From E. R. Jette, J. Chem. Phys., vol. 23, p. 368, 1955. W. D. Wilkinson in Extractive and Physical Metallurgy of Plutonium and Its Alloys, pp. $15-20$.

\begin{tabular}{lc} 
Phase & $\begin{array}{c}\text { Typical Magnetic Sugceptibility, } \\
10^{6} x_{\mathrm{m}}\end{array}$ \\
\cline { 2 - 2 } O (at $25 \circ \mathrm{C})$ & 532 \\
B (middle of stability range) & 553 \\
Y (middle of stability range) & 531 \\
3 (middle of stability range) & 515 \\
S'(middle of stability range) & 515 \\
(middle of stability range) & 522
\end{tabular}

For the a-phase, susceptibility is practically independent of temperature for each of the other phases it decreases with increasing temperature, particularly in the b-phase.

THE MAGNETIC SUSCEPTIBILITY OF PLUTONIUM ${ }^{*}$

From J. K. Dawson, J. Chem. Soc. (London), Part III, p. 3394. 1954

Sample A

Weight, 6. $74 \mathrm{mg}$. Length, $5 \mathrm{~mm}$. Purity, 99.66\% excluding oxygen (this sample had a rather thick yellow oxide coating).

\begin{tabular}{ccc} 
Temperature, ${ }^{\circ} \mathrm{C}$ & $\mathrm{t}$ & $10^{6} \mathrm{x}_{\mathrm{g}}$ \\
\hline-183 & - & 4.20 \\
-72 & - & 2.75 \\
+27 & 1 & 2.68 \\
62 & 1 & 2.62 \\
84 & 1 & 2.63 \\
86 & 2 & 2.62 \\
110 & 1 & 2.62 \\
114 & 1 & 2.62 \\
116 & 1 & 2.65 \\
118 & 1.5 & 2.68 \\
118 & 2.5 & 2.70 \\
125 & 1 & 2.69 \\
127 & 1 & 2.69 \\
133 & 0.5 & 2.70 \\
135 & 1 & 2.70 \\
156 & 1 & 2.68 \\
175 & 1 & 2.70 \\
190 & 0.5 & 2.69 \\
195 & 0.5 & 2.69 \\
201 & 1 & 2.71 \\
208 & 1. & 2.66 \\
234 & 0.5 & 2.57 \\
283 & 0.5 & 2.56 \\
294 & 0.3 & 2.51 \\
304 & 0.3 & 2.51 \\
327 & 0.3 & 2.52 \\
& &
\end{tabular}

Sample B

Weight, $19.84 \mathrm{mg}$. Length, $2.8 \mathrm{~mm}$ Purity, 99. 8\% excluding oxygen

but no appreciable oxidation visible.

\begin{tabular}{ccc} 
Temperature, ${ }^{\circ} \mathrm{C}$ & $\mathbf{t}$ & $10^{6} \times \mathbf{B}$ \\
\hline 19 & - & 5.23 \\
50.5 & 0.5 & 4.90 \\
79 & 0.5 & 4.55 \\
103 & 0.5 & 4.18 \\
110.5 & 0.5 & 4.07 \\
118 & 1 & 4.01 \\
120 & 0.5 & 4.02 \\
120 & 1 & 4.06 \\
120 & 2 & 4.08 \\
129 & 1 & 3.88 \\
143 & 0.5 & 3.52 \\
155 & 0.5 & 3.28 \\
166 & 0.5 & 3.02 \\
176 & 0.5 & 2.80 \\
185 & 0.5 & 2.67 \\
195 & 0.5 & 2.52 \\
196 & 0.5 & 2.51 \\
200 & 0.5 & 2.40 \\
200 & 1 & 2.40 \\
210 & 0.5 & 2.33 \\
220 & 1 & 2.30 \\
248 & 0.3 & 2.26 \\
263 & 0.3 & 2.25 \\
280 & 0.3 & 2.24 \\
295 & 0.3 & 2.23 \\
304 & 0.3 & 2.19 \\
318 & 0.3 & 2.19 \\
341 & 0.3 & 2.19 \\
& & \\
\hline & &
\end{tabular}

Sample C

Weight, $20.57 \mathrm{mg}$. Length, $1.6 \mathrm{~mm}$. Purity, 99.87\% excluding oxygen

(no appreciable oxidation visible).

\begin{tabular}{ccc} 
Temperature, ${ }^{\circ} \mathrm{C}$ & $\mathrm{t}$ & $10^{6} \times \mathbf{g}$ \\
\hline 20 & - & 2.52 \\
48 & 0.5 & 2.51 \\
76.5 & 0.7 & 2.51 \\
97 & 1.5 & 2.50 \\
101 & 0.7 & 2.50 \\
115.5 & 1.5 & 2.49 \\
117.5 & 0.5 & 2.49 \\
120 & 2 & 2.58 \\
140 & 1 & 2.61 \\
140 & 17 & 2.61 \\
167 & 1 & 2.61 \\
186 & 1 & 2.59 \\
196 & 0.5 & 2.59 \\
203 & 0.7 & 2.59 \\
216.5 & 0.7 & 2.52 \\
239 & 0.5 & 2.53 \\
282 & 0.5 & 2.52
\end{tabular}




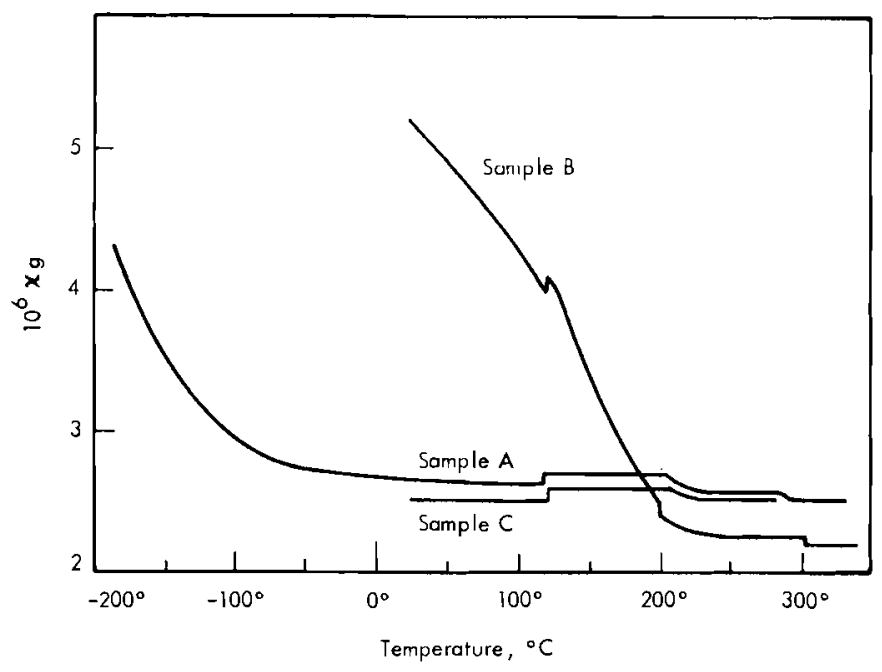

The Thermomagnetic Behaviour of Plutonium. From J, K. Dawson, J. Chem. Soc. (London), Part חI, p. 3395. 1954.

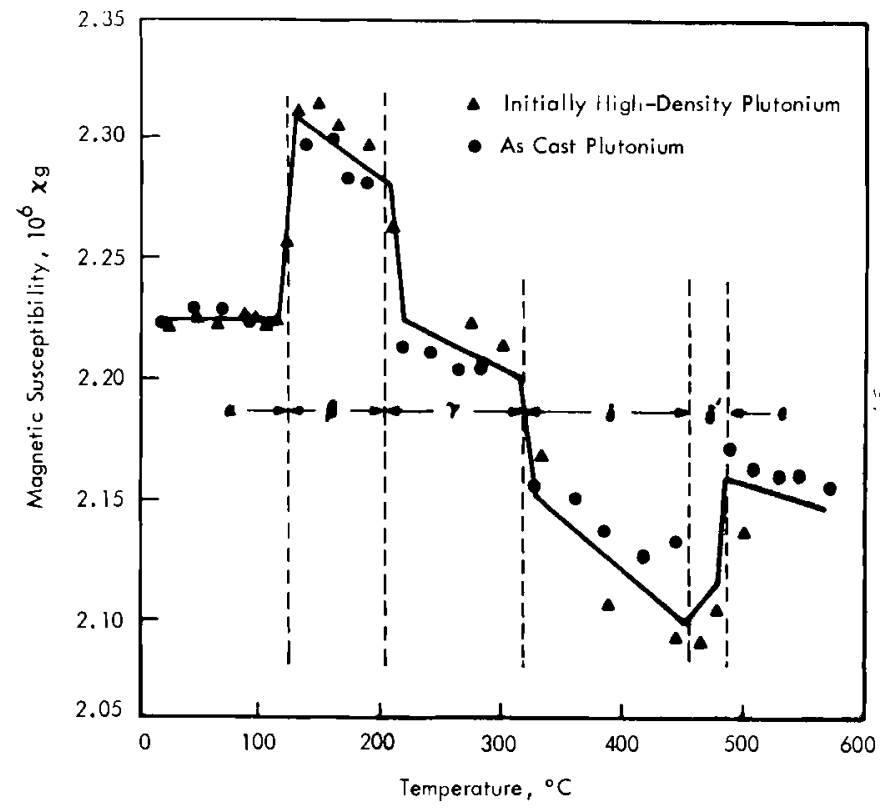

Magnetic Susceptibility as a Funetion of Temperature for Samples of 99. $9^{\text {\% }}$ or Purer Pu. From T. A. Sandenaw in The Metal Plutonium, p. 154 .

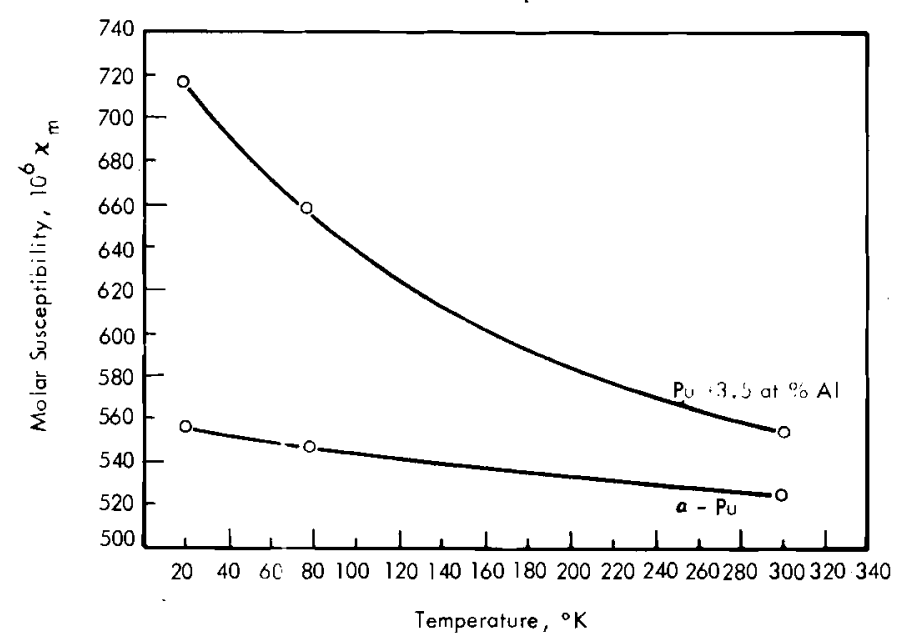

Paramagnetic Susceptibilities. From J. A. Lee et al. in Plutonium 1960, p. 43 .

\subsection{Hall Eftert}

I'la lliall cocflietent of al lohil plutunium was found to be $+2.8 \times 10^{-13}$ at $388^{\circ} \mathrm{K}$

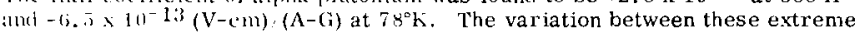
comperatures wis found to change wrictually with temperature and the value changes sign at $1900^{\prime} \mathrm{k}$. No lijeld dependence of Hall voltage was found, nor did

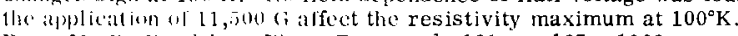

From M. 13. Brodski. Plys. Rev, vol. 131, p. 137. 1963.

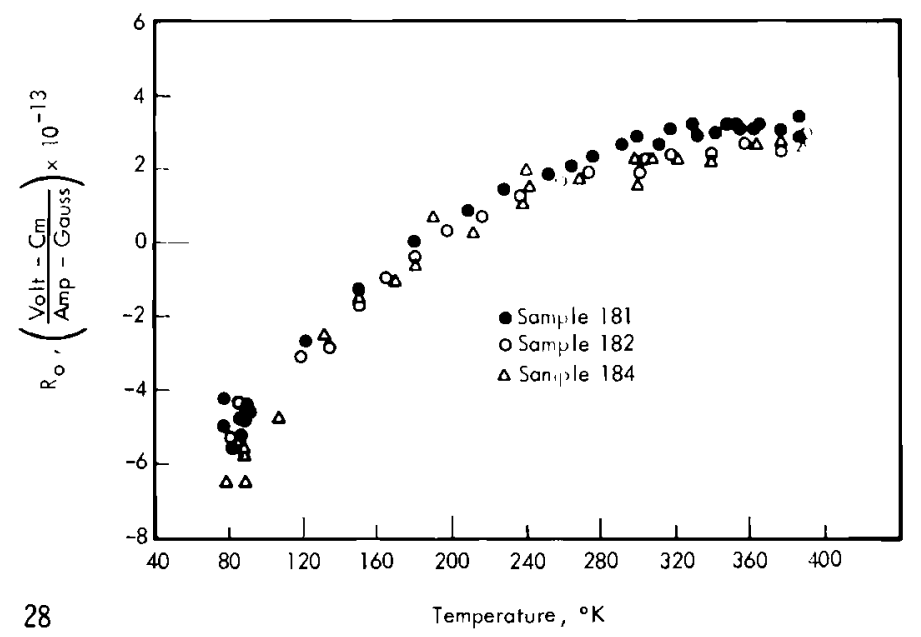

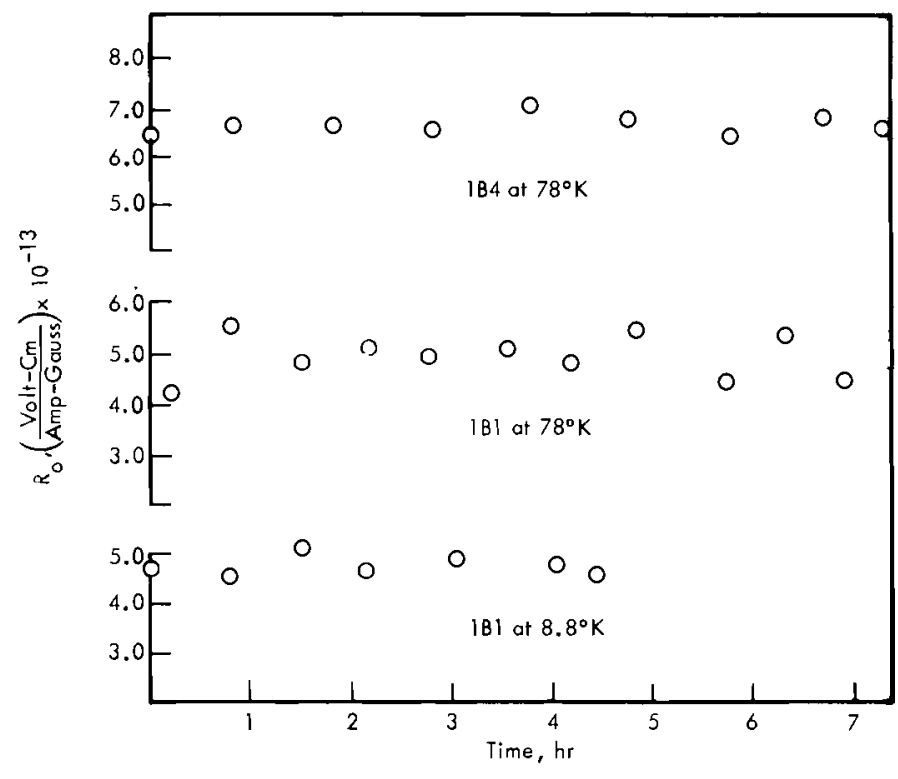


R. G. Loasby and J. C. Taylor, Proc. Phys. Soc. (London), vol. 78, pp. $776-777.1961$.

\begin{tabular}{|c|c|c|}
\hline Temperature, ${ }^{\circ} \mathrm{k}$ & $\mathrm{R}_{\mathrm{H}}, \mathrm{mi}^{3} \mathrm{Co}$ & $\times 10^{-11}$ \\
\hline & $x-P^{3} u$ & $\mathrm{i}-\mathrm{Pu}$ \\
\hline 293 & $+11.1+1.0$ & $+6.9 \pm 0.5$ \\
\hline 77 & $+16.2 \pm 1.5$ & $-9.5 \pm 0.6$ \\
\hline
\end{tabular}

Note: for comparison with previous data,

$$
\frac{\text { volt }-\mathrm{cm}}{\text { ampere-gauss }}=10^{2} \frac{\mathrm{m}^{3}}{\text { coulomb }}
$$

Viscosity

CALCULATED VISCOSITIES OF PLUTONIUM

From L. V. Jones et al., Am. Soc. Metals,

Trans. Quart., vol. 55, p. 822. 1962.

\begin{tabular}{r} 
Tempe \\
${ }^{\circ} \mathrm{C}$ \\
\hline \\
91 \\
82 \\
739 \\
699 \\
650 \\
64 \\
\\
\\
950 \\
902 \\
859 \\
809 \\
765 \\
712 \\
670 \\
645
\end{tabular}

$$
\begin{gathered}
\begin{array}{c}
\text { Period, } \\
\text { sec }
\end{array} \quad \begin{array}{c}
\text { Logarithmic } \\
\text { Decrement }
\end{array} \\
\hline
\end{gathered}
$$
First Series

$\begin{array}{ll}917 & 11.45 \\ 824 & 11.46 \\ 739 & 11.48 \\ 699 & 11.487 \\ 650 & 11.49 \\ 648 & 11.49\end{array}$

$\begin{array}{ll}950 & 11.374 \\ 902 & 11.376 \\ 859 & 11.386 \\ 809 & 11.395 \\ 765 & 11.405 \\ 712 & 11.414 \\ 670 & 11.422 \\ 645 & 11.423\end{array}$

0.0711022 0.0733421 0.075873

0.0758731

0.0812432

0.0808763

Second Series

0.0689083

0.0697351

0.0706259

0.0719241

0.0727018

0.0749486

0.0766835

0.0792345

16.235
16.370
16.495
16.548
16.620
16.623

16.185

16. 255

16.320

16.320

16.392

16.440

16.530

16.592

16.628

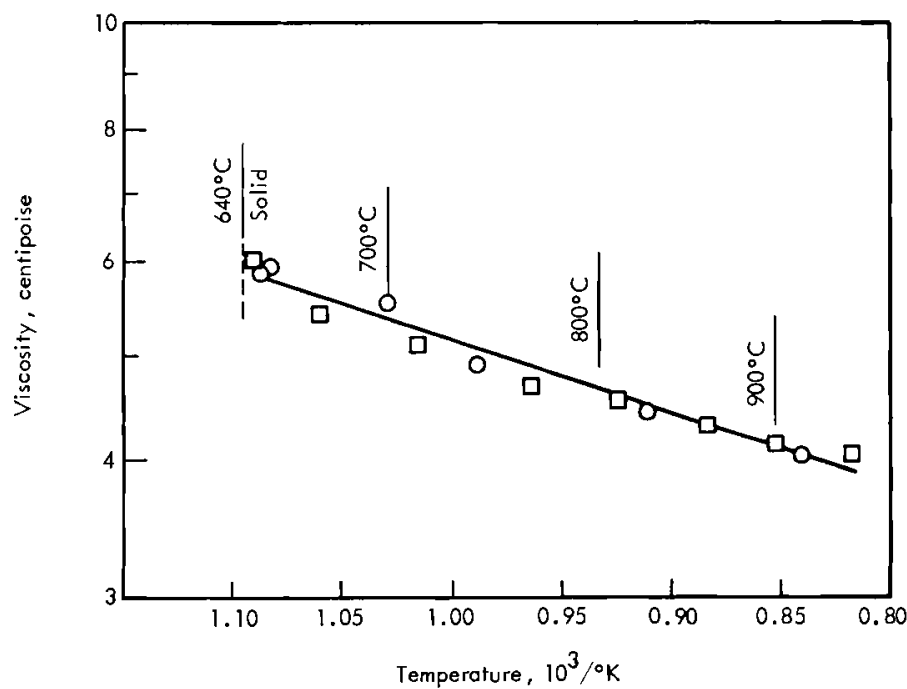

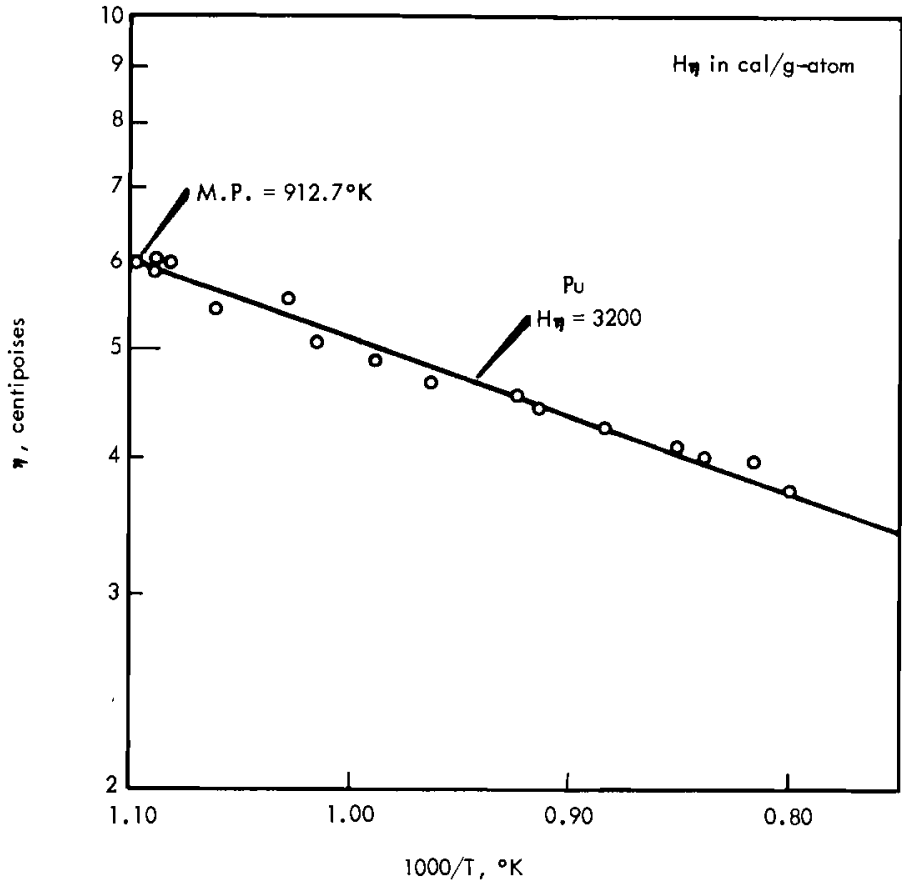

In the above figure, the straight line has a slope of $3200 \mathrm{cal} / \mathrm{g}$-atom, the predicted value for $\mathrm{H}_{\eta}$ (A. V. Grosse, J. Inorg. Nucl. Chem., Vol. 23, p. 333. 1961) and the points are experimental results obtained at Mound Laboratory. From A. V. Grosse. J. Inorg. Nucl. Chem., vol, 25,

p. 138. 1963. The viscosity of plutonium, in poises, at a temperature $\mathrm{T}$, in ${ }^{\circ} \mathbf{K}$, is expressed by the equation:

Viscosity of Plutonium (99.95\% pure) as a Function of Absolute

Temperature. From L. V. Jones et al. Am. Soc. Metals, Trans.

Quart., vol. 55, p. 821 . 1962 .

The equation of the line in the above Figure is:

$$
\log _{10}=0.672 \frac{1}{\mathrm{~T}}+0.037
$$

where:

$7=$ centipoise

$\mathrm{T}={ }^{\circ} \mathrm{K}+10^{-3}$

Tensile Properties

H. R. Gardner et al., p/1081, Proc. Second Geneva Internat. Conf., vol. 6, p. 688 CHEMICAI ANALYSIS OF PLUTONIUM STUDIED 


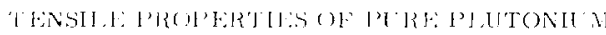

From H. R. Gardner et it. p./104l, bocond Genevil Internat. Conf, vol. 6,

p. $688.19 \%$.

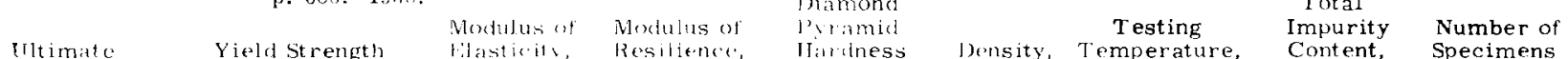

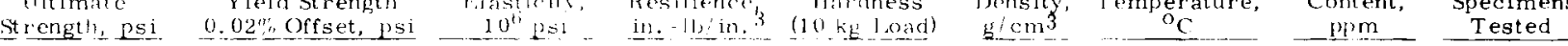

\begin{tabular}{|c|c|c|c|c|c|c|}
\hline Ninmum & 43,800 & 30,600 & 11.5 & $\because .1$ & 250 & 14.29 \\
\hline Naximam & 77,200 & 45,800 & 14,6 & $+1,2$ & $283: 3$ & 19.58 \\
\hline Astoratse & 62,800 & 35.300 & 13.6 & 23.5 & $21 ; 2$ & $1 \div .51$ \\
\hline \multicolumn{7}{|c|}{$0.250 \mathrm{in}$. Dram tor - As Cust, Dolta Annealed and cold Treatrd } \\
\hline Minimum & 43,700 & 33,800 & 11.4 & 18.8 & 250 & 19.41 \\
\hline Maximum & 74,300 & 42,000 & 14.5 & 45.0 & 277 & 19.58 \\
\hline Average: & 61,000 & 38,700 & 12.8 & 28.6 & 259 & 19.51 \\
\hline \multicolumn{7}{|c|}{$0.500 \mathrm{in}$. Diameter - As Cast and Cold Treated } \\
\hline Minimum & 47,500 & 32,700 & $13.1 i$ & 11.6 & 260 & 19.41 \\
\hline Naximum & 63,700 & 40,300 & $1+.11$ & 21.8 & 276 & 19.56 \\
\hline Averate & 59,100 & 35,300 & 14.1 & 16.3 & 289 & 19.49 \\
\hline \multicolumn{7}{|c|}{$0.500 \mathrm{in.}$ Diamor - As Cast, Delta Annealed and Cold Treated } \\
\hline Minimum & 60,500 & 36,200 & 12.9 & 11.7 & 266 & 19.05 \\
\hline Maximum & 65,300 & 43,200 & 14.2 & 22.9 & 275 & 19.47 \\
\hline Average & 63,100 & 38,900 & 13,4 & 18.2 & 270 & 19.33 \\
\hline
\end{tabular}

Average

63,100

38,900

13.4

18.2

19.33

$\begin{array}{rr}29 & 420 \\ 35 & 1129\end{array}$

129
1375

$-$

$28 \quad 500-$

1091

$31-525$

$37 \quad 2510$

1514

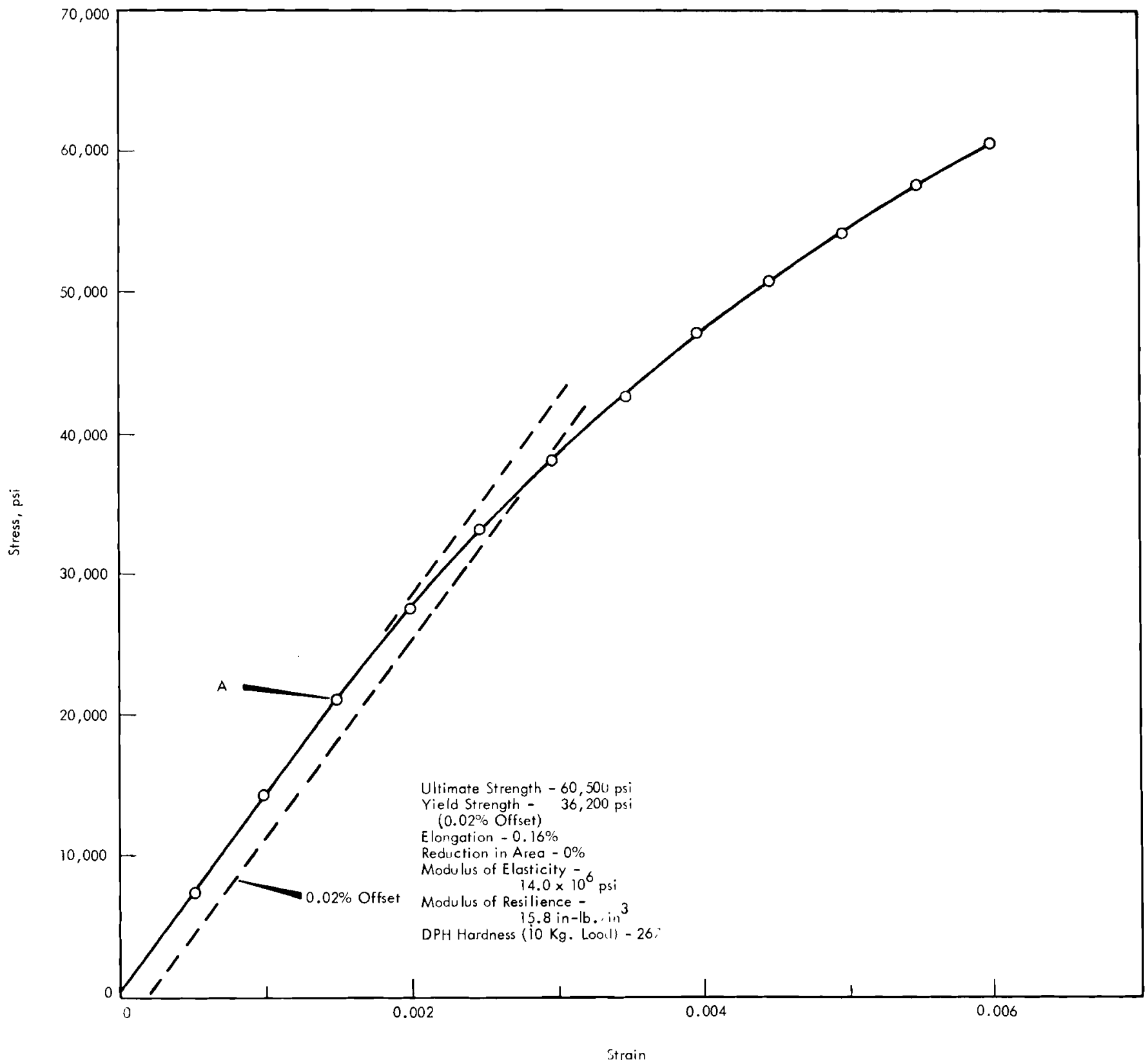

Stress Strain Relationship for Plutonitun (59. 1' Pu) at $32 \mathrm{C}$ Cold Treated at $-23 \mathrm{C}$ after Casting. 
Ultimate tensile streneti: 50,000 to 51,800 psi (as-cast, unmachined, a Pu)

Yield strength: 45,000 psi (a Pu)

From W. D. Wilkinson in Extractive and Plyysical Metallurgy of Plutonium and Its Alloys, p. 15

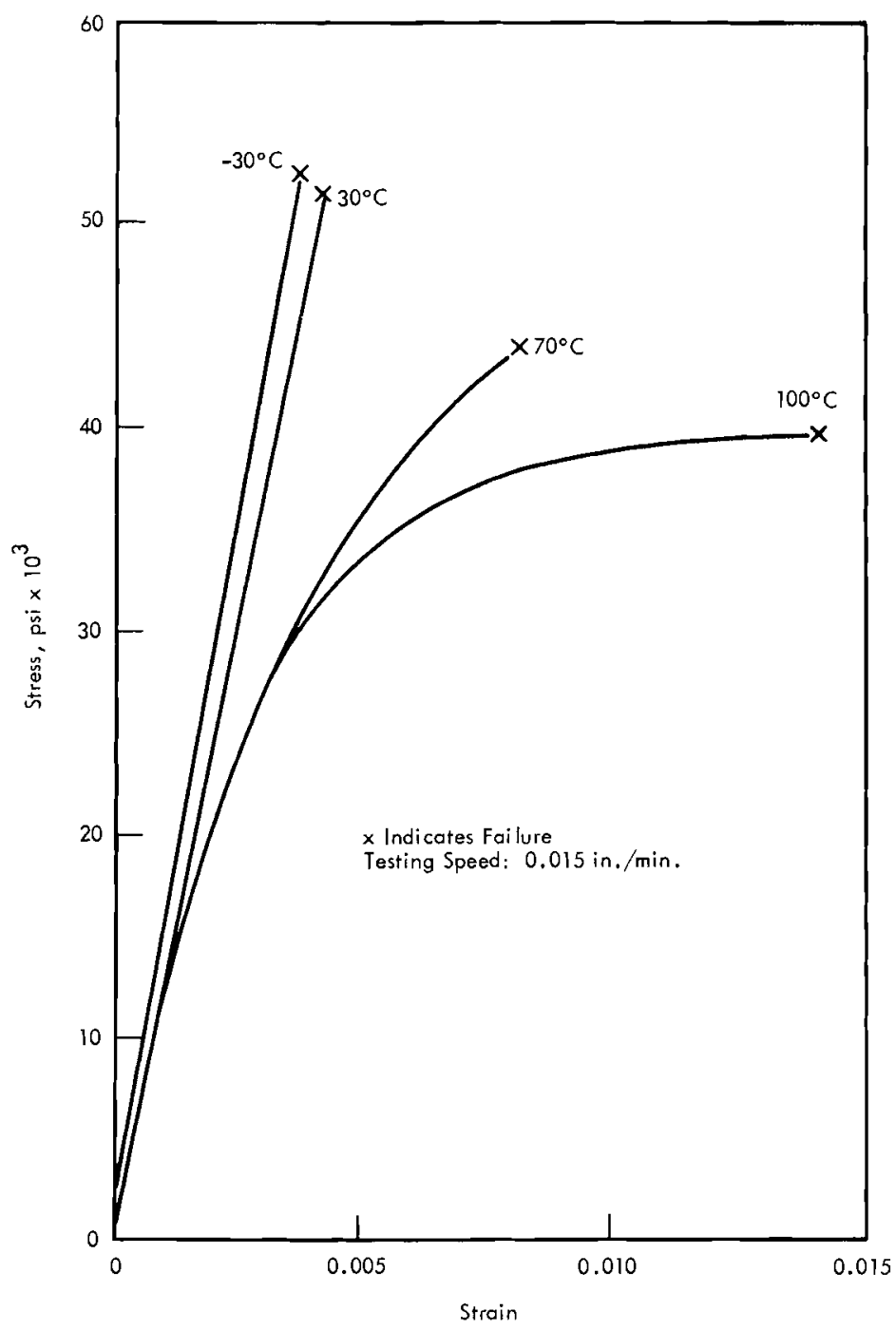

Effect of Temperature on Stress-Strain Curves for Alpha-Plutonium in Tension. From H. R. Gardner. Unpublisherl Data. 
DOREC"I HLATJNG (T:RVHS

From C. T. Horton and 13. T. Ward in Plutonium 1960, p. 507.

\begin{tabular}{|c|c|c|c|}
\hline $\begin{array}{l}\text { lesting } \\
\text { Tenperature, }\end{array}$ & $\begin{array}{l}\text { Atrut } \\
\text { ferestment }\end{array}$ & $\begin{array}{l}\text { 1). T. S. } \\
\text { ton/in. } 2\end{array}$ & Flongation, \\
\hline 25 & Neme & 21.6 & 1 \\
\hline 25 & None. & $26 y+$ & $<1$ \\
\hline 25 & Nont. & 14.7 & 1 \\
\hline 100 & Held $60 \mathrm{r}^{\circ} 20 \mathrm{mu}$ & 14.98 & -1 \\
\hline 130 & Held for 2 h & 11.68 & 3 \\
\hline 130 & Held lor 30 min & 13.54 & 4 \\
\hline 140 & Held for $20 \mathrm{~min}$ & 8.9 & 3 \\
\hline 160 & Held for $25 \mathrm{~min}$ & 4.94 & 150 \\
\hline $\begin{array}{l}175 \\
175 \\
175 \\
175 \\
175 \\
175\end{array}$ & $\begin{array}{l}\text { Held for } 1 \mathrm{~h}, 30 \mathrm{~min} \\
\text { Held for } 25 \mathrm{~min} \\
\text { Held for } 1 \mathrm{~h}, 30 \mathrm{~min} \\
\text { Held for } 25 \mathrm{~min} \\
\text { Held for } 30 \mathrm{~min} \\
\text { Held for } 30 \mathrm{~min}\end{array}$ & $\begin{array}{l}2.05 \\
2.2 \\
2.62 \\
2.61 \\
2.64 \\
2.17\end{array}$ & $\begin{array}{l}212 \\
256 \\
224 \\
240 \\
(55) \ddagger \\
350\end{array}$ \\
\hline 200 & Held for 30 min & 1. 49 & 344 \\
\hline \multicolumn{4}{|c|}{$\begin{array}{c}\text { TESTS AFTER } \boldsymbol{\beta} \text { - PHASF ANNEAL } \\
\left.\text { (held at } 175^{\circ} \mathrm{C} \text { for } 45 \mathrm{~min}\right)\end{array}$} \\
\hline 175 & Tested & 2.62 & 224 \\
\hline 150 & Cooled to $150^{\circ} \mathrm{C}$. Tested & 4.32 & 168 \\
\hline 140 & Cooled to $140^{\circ} \mathrm{C}$. Testred & 6.12 & 154 \\
\hline 130 & Cooled to $130^{\circ} \mathrm{C}$. Tested & 6.83 & 180 \\
\hline 120 & Cooled to $120^{\circ} \mathrm{C}$. Testud & 7.08 & 78 \\
\hline 100 & Cooled to $100{ }^{\circ} \mathrm{C}$. Tested & 9.53 & 80 \\
\hline 90 & Conled to $90^{\circ} \mathrm{C}$. Tested & 13.95 & 60 \\
\hline \multicolumn{4}{|c|}{ TESTS IN $\because$-AND $*$-HASES } \\
\hline 260 & Held for $30 \mathrm{~min}$ & 3.5 & 36 \\
\hline 400 & $\mathrm{Held}$ [or $30 \mathrm{~min}$ & 0.25 & 30 \\
\hline
\end{tabular}

EFFHCT OF TEMPEIRATERE ON TENSILL: PROPERTIES OF a-PHASE PLUTONIUA*

From H. R. Garderer :nd I. H. Mann in Plutonium 1960, p. 522.

\begin{tabular}{|c|c|c|c|c|c|c|c|}
\hline & $\begin{array}{c}\text { Test } \\
\text { Tenyperature, } \\
{ }^{\circ} \mathrm{C} \\
\end{array}$ & $\begin{array}{l}\text { Ullimate } \\
\text { Strengtli, } \\
\text { lb/in.2 } \\
\end{array}$ & $\begin{array}{r}0.01^{c} \\
\text { Yiclel } \\
\text { Strength, } \\
\text { lb/in. } 2 \\
\end{array}$ & $\begin{array}{l}\text { Modulus of } \\
\text { Flasticity, } \\
10^{6} \mathrm{ll} / \mathrm{in} .^{2} \\
\end{array}$ & Elongation, ${ }^{+}$ & $\begin{array}{l}\text { Reduction } \\
\text { in Area, } \\
y \\
\end{array}$ & $\begin{array}{c}\text { Nuniber of } \\
\text { Samples } \\
\text { Tested } \\
\end{array}$ \\
\hline $\mathrm{Min}$ & & 52500 & 16700 & 14.0 & 0.016 & 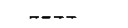 & \\
\hline Max & -30 & 63300 & $\lceil\leftrightarrow 800$ & 15.2 & 0.020 & --- & 4 \\
\hline Avr & & 57300 & 50304 & $14.7(12.7)^{++}$ & 0.018 & --- & \\
\hline Win & & 17800 & 28200 & $13 . \overline{5}$ & 0.054 & $\ldots$ & \\
\hline Max & (10) & 56800 & 35600 & 14.8 & 0.077 & -- & 5 \\
\hline Aver & & 50900 & 32100 & 14.3 & 0.068 & ---- & \\
\hline Min & & 42900 & 1.1900 & 12.6 & 0.41 & --- & \\
\hline $\operatorname{Max}$ & 70 & 44600 & 15600 & 12.7 & 0.45 & $\ldots$ & 2 \\
\hline Avg & & 43800 & 15200 & 12.6 & 0.43 & ---- & \\
\hline $\operatorname{Min}$ & & 29100 & 11800 & 8.5 & 0.91 & --- & \\
\hline Nax & 100 & 38700 & $1+100$ & 9.4 & 1.08 & --- & 3 \\
\hline Aig & & 85100 & 13300 & $9.0(9.2)^{++}$ & 1.00 & --- & \\
\hline Min & & $-\infty--$ & 11600 & 9.0 & --- & --- & \\
\hline M:Ix & 110 & - & 13000 & 30.9 & ---- & ---- & 2 \\
\hline Avg & & 33100 & 12300 & 10.0 & $-\infty-$ & ---- & \\
\hline
\end{tabular}


From H. R. Gardner and I. B. Mann in Plutonium 1960, p. 523.

\begin{tabular}{|c|c|c|c|c|c|c|c|c|}
\hline & $\begin{array}{c}\text { Test } \\
\text { Temperature, } \\
{ }^{\circ} \mathrm{C} \\
\end{array}$ & $\begin{array}{l}\text { Ultimate } \\
\text { Strength, } \\
1 \mathrm{~b} / \text { in. }^{2} \\
\end{array}$ & $\begin{array}{l}\text { Yield } \\
\text { Strength, } \\
\text { lb/in.2 } \\
\end{array}$ & $\begin{array}{l}\text { Modulus of } \\
\text { Elasticity, } \\
10^{6} \text { lb/in. } \\
\end{array}$ & $\begin{array}{c}\text { Elongation, + } \\
\% \\
\end{array}$ & $\begin{array}{c}\text { Reduction } \\
\text { in Area, } \\
\% \\
\end{array}$ & $\begin{array}{c}\text { Number of } \\
\text { Samples } \\
\text { Tested } \\
\end{array}$ & Phase \\
\hline $\begin{array}{l}\text { Min } \\
\text { Max } \\
\text { Avg }\end{array}$ & 130 & $\begin{array}{l}11700 \\
12500 \\
12100\end{array}$ & $\begin{array}{l}10240 \\
10570 \\
10400\end{array}$ & $\begin{array}{l}2.0 \\
3.9 \\
3.0\end{array}$ & $\begin{array}{l}272.0 \\
316.0 \\
294.0\end{array}$ & $\begin{array}{l}100.0 \\
100.0 \\
100.0\end{array}$ & 2 & $\boldsymbol{B}$ \\
\hline $\begin{array}{l}\text { Min } \\
\text { Max } \\
\text { Avg }\end{array}$ & 160 & $\begin{array}{l}5500 \\
5830 \\
5620\end{array}$ & $\begin{array}{l}3720 \\
4380 \\
3950\end{array}$ & $\begin{array}{l}--- \\
--- \\
---\end{array}$ & $\begin{array}{l}519.0 \\
621.8 \\
570.4\end{array}$ & $\begin{array}{l}100.0 \\
100.0 \\
100.0\end{array}$ & 3 & $\boldsymbol{\beta}$ \\
\hline $\begin{array}{l}\text { Min } \\
\text { Max } \\
\text { Avg }\end{array}$ & 180 & $\begin{array}{l}3550 \\
3600 \\
3580\end{array}$ & $\begin{array}{l}2540 \\
2600 \\
2620\end{array}$ & $\begin{array}{l}--- \\
---\end{array}$ & $\begin{array}{l}481.3 \\
525.0 \\
503.2\end{array}$ & $\begin{array}{l}100.0 \\
100.0 \\
100.0\end{array}$ & 2 & $\boldsymbol{\rho}$ \\
\hline $\begin{array}{l}\text { Min } \\
\text { Max } \\
\text { Avg }\end{array}$ & 190 & $\begin{array}{l}2950 \\
3590 \\
3280\end{array}$ & $\begin{array}{l}1730 \\
2630 \\
2210\end{array}$ & $\begin{array}{l}1.4 \\
1.7 \\
1.6\end{array}$ & $\begin{array}{l}271.5 \\
406.2 \\
325.9\end{array}$ & $\begin{array}{r}93.5 \\
100.0 \\
97.8\end{array}$ & 6 & $\boldsymbol{\rho}$ \\
\hline $\begin{array}{l}\text { Min } \\
\text { Max } \\
\text { Avg }\end{array}$ & 200 & $\begin{array}{l}2730 \\
3390 \\
2940\end{array}$ & $\begin{array}{l}1950 \\
2630 \\
2210\end{array}$ & $\begin{array}{l}--- \\
---\end{array}$ & $\begin{array}{r}93.8 \\
151.0 \\
121.0\end{array}$ & $\begin{array}{l}60.2 \\
97.1 \\
82.1\end{array}$ & 6 & $\boldsymbol{\beta}$ \\
\hline $\begin{array}{l}\text { Min } \\
\text { Max } \\
\text { Avg }\end{array}$ & 230 & $\begin{array}{l}3330 \\
4990 \\
4420\end{array}$ & $\begin{array}{l}3250 \\
4210 \\
3860\end{array}$ & $\begin{array}{l}1.8 \\
1.8 \\
1.8\end{array}$ & $\begin{array}{l}37.5 \\
65.8 \\
50.0\end{array}$ & $\begin{array}{l}\theta 1.0 \\
92.5 \\
75.0\end{array}$ & 7 & $\gamma$ \\
\hline $\begin{array}{l}\text { Min } \\
\text { Max } \\
\text { Avg }\end{array}$ & 265 & $\begin{array}{l}2500 \\
4750 \\
3470\end{array}$ & $\begin{array}{l}2340 \\
3660 \\
2900\end{array}$ & $\begin{array}{l}--- \\
-\cdots \\
---\end{array}$ & $\begin{array}{l}33.0 \\
84.4 \\
57.2\end{array}$ & $\begin{array}{l}64.0 \\
84.7 \\
77.0\end{array}$ & 7 & $\gamma$ \\
\hline $\begin{array}{l}\text { Min } \\
\text { Max } \\
\text { Avg }\end{array}$ & 300 & $\begin{array}{l}1540 \\
2630 \\
2020\end{array}$ & $\begin{array}{l}1360 \\
2100 \\
1650\end{array}$ & $\begin{array}{l}0.9 \\
1.2 \\
1.0\end{array}$ & $\begin{array}{l}37.5 \\
68.8 \\
50.1\end{array}$ & $\begin{array}{l}57.9 \\
98.6 \\
82.8\end{array}$ & 8 & $\gamma$ \\
\hline $\begin{array}{l}\text { Min } \\
\text { Max } \\
\text { Avg }\end{array}$ & 325 & $\begin{array}{l}837 \\
953 \\
895\end{array}$ & $\begin{array}{l}731 \\
848 \\
790\end{array}$ & $\begin{array}{l}0.35 \\
0.40 \\
0.38\end{array}$ & $\begin{array}{l}65.8 \\
68.8 \\
67.3\end{array}$ & $\begin{array}{l}97.5 \\
98.4 \\
98.0\end{array}$ & 2 & $\boldsymbol{J}$ \\
\hline
\end{tabular}

* Testing speed: $0.015 \mathrm{in} . / \mathrm{min}$.

+ Based on $1 \mathrm{ln}$. gauge length.

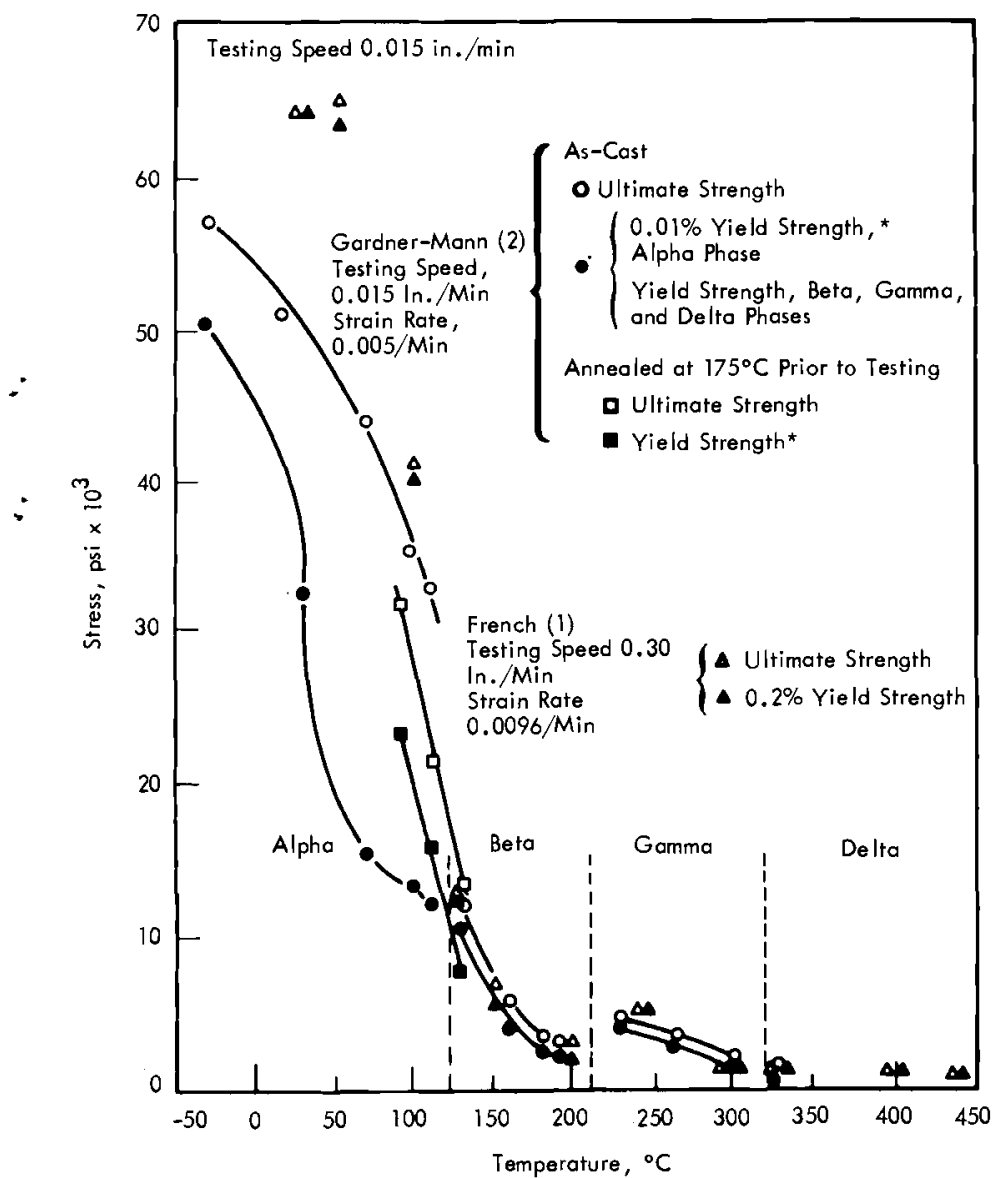

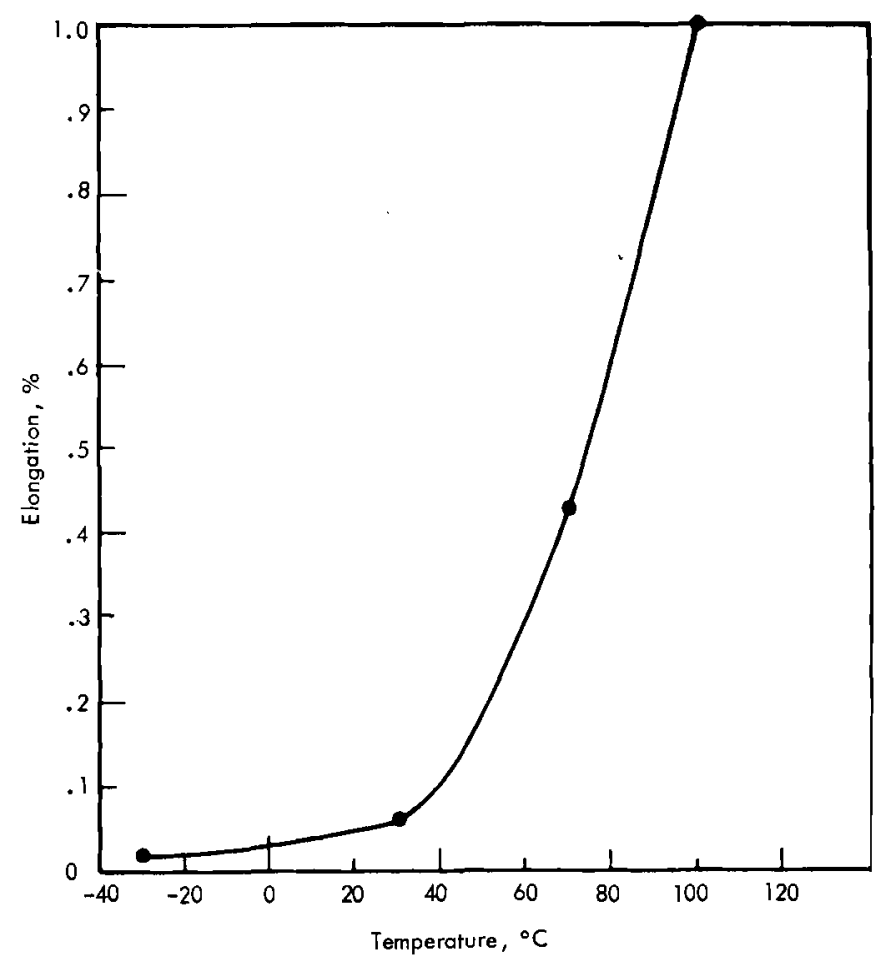

Effect of Test Temperature on Alpha Phase Elongation (Test speed $0.015 \mathrm{in.} / \mathrm{miri}$ ). From H. R. Gardner and I. B. Mann in Plutonium 1960, p. 522. 
From H. R. Gardner and I. B. Mann, in I'lutonium 1960, p. 533.

\begin{tabular}{|c|c|c|c|c|c|c|c|}
\hline $\begin{array}{l}\text { Specimen } \\
\text { Number } \\
\end{array}$ & $\begin{array}{c}\text { Test Speed, } \\
\text { in. / min } \\
\end{array}$ & $\begin{array}{l}\text { Density, } \\
\mathrm{g}^{2} \mathrm{~cm}^{3} \\
\end{array}$ & $\begin{array}{l}\text { Total Impurity } \\
\text { Content, ppm }\end{array}$ & $\begin{array}{l}\text { Uitimate Strength, } \\
\text { lb/in. } 2 \\
\end{array}$ & $\begin{array}{r}\text { Yield Strength, } \\
\mathrm{lb} / \mathrm{in} .{ }^{2} \\
\end{array}$ & $\underset{\%}{\text { Elongation, }}$ & $\begin{array}{c}\text { Reduction in Area, } \\
\%\end{array}$ \\
\hline $19-9-5 \mathrm{~L}$ & 0.002 & 19.54 & 326 & 3400 & 2550 & - & - \\
\hline \multicolumn{8}{|c|}{$\boldsymbol{\beta}$-phase $-160^{\circ} \mathrm{C}$} \\
\hline $\begin{array}{l}19-9-3 \mathrm{H} \\
19-9-7 \mathrm{C} \\
19-5-7 . J\end{array}$ & $\begin{array}{l}0.015 \\
0.015 \\
0.015\end{array}$ & $\begin{array}{l}19.56 \\
19.56 \\
19.52\end{array}$ & $\begin{array}{l}295 \\
347 \\
321\end{array}$ & $\begin{array}{l}5500 \\
5520 \\
5830\end{array}$ & $\begin{array}{l}3760 \\
4380 \\
3720\end{array}$ & $\begin{array}{l}- \\
621.8 \\
519.0\end{array}$ & $\begin{array}{l}100 \\
100\end{array}$ \\
\hline $\begin{array}{l}19-10-2 \mathrm{C} \\
19-10-5 \mathrm{M}\end{array}$ & $\begin{array}{l}0.060 \\
0.060\end{array}$ & $\begin{array}{l}19.52 \\
19.54\end{array}$ & $\begin{array}{l}570 \\
326\end{array}$ & $\begin{array}{l}8840 \\
8170\end{array}$ & $\begin{array}{l}6480 \\
5920\end{array}$ & $\begin{array}{l}406.2 \\
315.6\end{array}$ & $\begin{array}{l}100 \\
100\end{array}$ \\
\hline $\begin{array}{l}19-10-2 D \\
19-10-1 A\end{array}$ & $\begin{array}{l}0.250 \\
0.250\end{array}$ & $\begin{array}{l}19.52 \\
19.54\end{array}$ & $\begin{array}{l}570 \\
438\end{array}$ & $\begin{array}{l}12900 \\
11900\end{array}$ & $\begin{array}{r}10200 \\
9600\end{array}$ & $\begin{array}{l}306.2 \\
196.9\end{array}$ & $\begin{array}{l}98.9 \\
98.3\end{array}$ \\
\hline $\begin{array}{l}19-10-2 E \\
19-10-1 \mathrm{~B}\end{array}$ & $\begin{array}{l}1.0 \\
1.0\end{array}$ & $\begin{array}{l}19.52 \\
19.54\end{array}$ & $\begin{array}{l}570 \\
438\end{array}$ & $\begin{array}{l}18800 \\
18600\end{array}$ & $\begin{array}{l}14800 \\
15700\end{array}$ & $\begin{array}{r}78.1 \\
143.7\end{array}$ & $\begin{array}{l}61.1 \\
79.0\end{array}$ \\
\hline \multicolumn{8}{|c|}{$\gamma$-phase $-265^{\circ} \mathrm{C}$} \\
\hline $\begin{array}{l}19-11-1 \mathrm{C} \\
18-10-1 \mathrm{~S} \\
18-10-3 \mathrm{~B} \\
18-10-4 \mathrm{G} \\
19-5-5 \mathrm{~F} \\
19-9-8 \mathrm{E} \\
19-9-8 \mathrm{H}\end{array}$ & $\begin{array}{l}0.002 \\
0.015 \\
0.015 \\
0.015 \\
0.015 \\
0.015 \\
0.015\end{array}$ & $\begin{array}{l}19.60 \\
19.46 \\
19.53 \\
19.56 \\
19.60 \\
19.56 \\
19.56\end{array}$ & $\begin{array}{l}412 \\
219 \\
935 \\
388 \\
295 \\
553 \\
553\end{array}$ & $\begin{array}{l}3250 \\
3610 \\
4750 \\
4160 \\
2800 \\
2580 \\
2500\end{array}$ & $\begin{array}{c}2280 \\
2960 \\
- \\
3650 \\
2340 \\
2440 \\
2340\end{array}$ & $\begin{array}{l}75.0 \\
37.5 \\
37.5 \\
33.0 \\
82.8 \\
84.4 \\
84.4\end{array}$ & $\begin{array}{l}90.9 \\
= \\
82.7 \\
83.2 \\
84.7 \\
71.2 \\
76.0\end{array}$ \\
\hline $\begin{array}{l}19-9-4 M \\
19-10-5 N \\
19-11-3 J\end{array}$ & $\begin{array}{l}0.060 \\
0.060 \\
0.060\end{array}$ & $\begin{array}{l}19.53 \\
19.54 \\
19.55\end{array}$ & $\begin{array}{r}468 \\
326 \\
1277\end{array}$ & $\begin{array}{l}3280 \\
4240 \\
4200\end{array}$ & $\begin{array}{l}3100 \\
3540 \\
3270\end{array}$ & $\begin{array}{l}44.0 \\
40.7 \\
64.1\end{array}$ & $\begin{array}{l}49.4 \\
57.4 \\
99.0\end{array}$ \\
\hline $\begin{array}{l}19-9-4 N \\
19-10-5 D \\
19-11-3 H\end{array}$ & $\begin{array}{l}0.250 \\
0.250 \\
0.250\end{array}$ & $\begin{array}{l}19.53 \\
19.54 \\
19.55\end{array}$ & $\begin{array}{r}468 \\
326 \\
1277\end{array}$ & $\begin{array}{l}3710 \\
4590 \\
5200\end{array}$ & $\begin{array}{l}3520 \\
3980 \\
4600\end{array}$ & $\begin{array}{l}50.0 \\
46.8 \\
31.9\end{array}$ & $\begin{array}{l}52.3 \\
69.6 \\
85.6\end{array}$ \\
\hline $\begin{array}{l}19-9-40 \\
19-11-1 B \\
19-11-3 K\end{array}$ & $\begin{array}{l}1.0 \\
1.0 \\
1.0\end{array}$ & $\begin{array}{l}19.53 \\
19.60 \\
19.55\end{array}$ & $\begin{array}{r}468 \\
412 \\
1277\end{array}$ & $\begin{array}{l}4160 \\
5300 \\
5350\end{array}$ & $\begin{array}{l}4050 \\
5070 \\
5030\end{array}$ & $\begin{array}{l}37.8 \\
53.2 \\
43.7\end{array}$ & $\begin{array}{l}38.0 \\
76.7 \\
93.2\end{array}$ \\
\hline
\end{tabular}
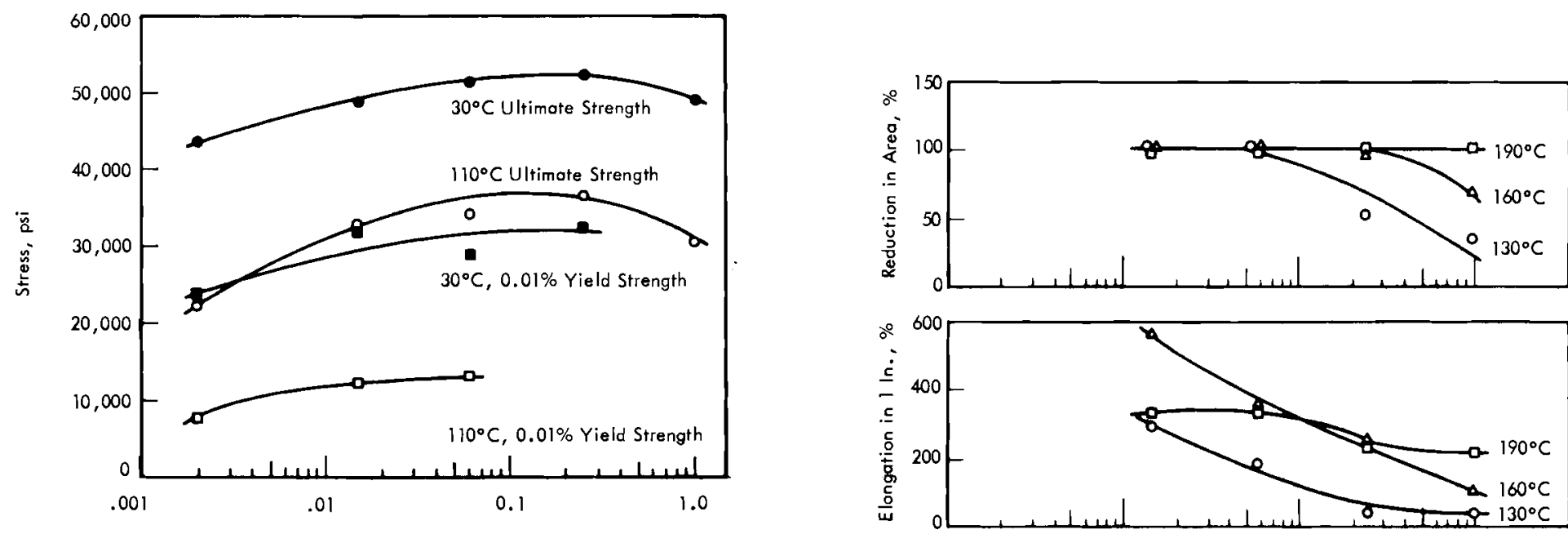

Effect of Testing Speed on a-Phase Tensile Strength. From H. R. Gardner and I. B. Mann in Plutonium 1960 p. 531.
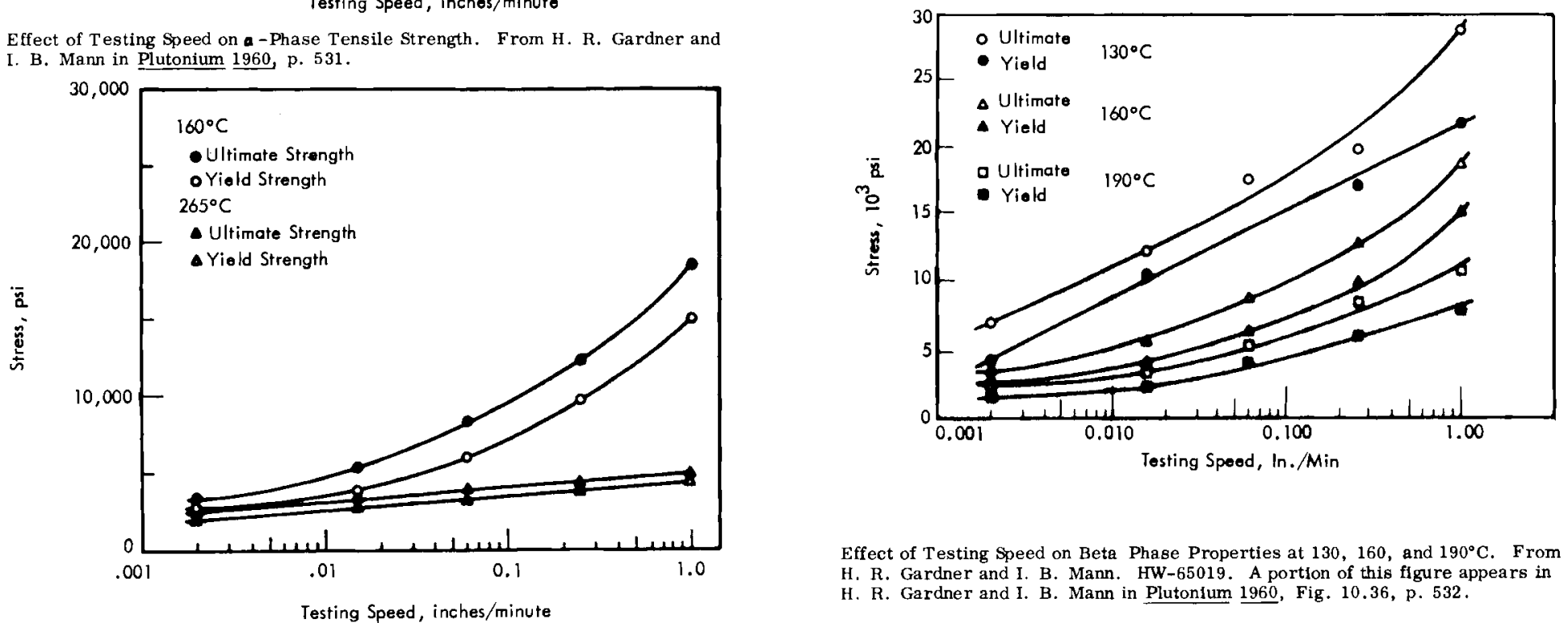

Effect of Testing Speed on Beta Phase Properties at 130,160 , and $190^{\circ} \mathrm{C}$. From H. R. Gardner and I. B. Mann. HW-65019. A portion of this figure appears in H. R. Gardner and 1. B. Mann in Plutontum 1960, Fig. 10.36, p. 532 . 


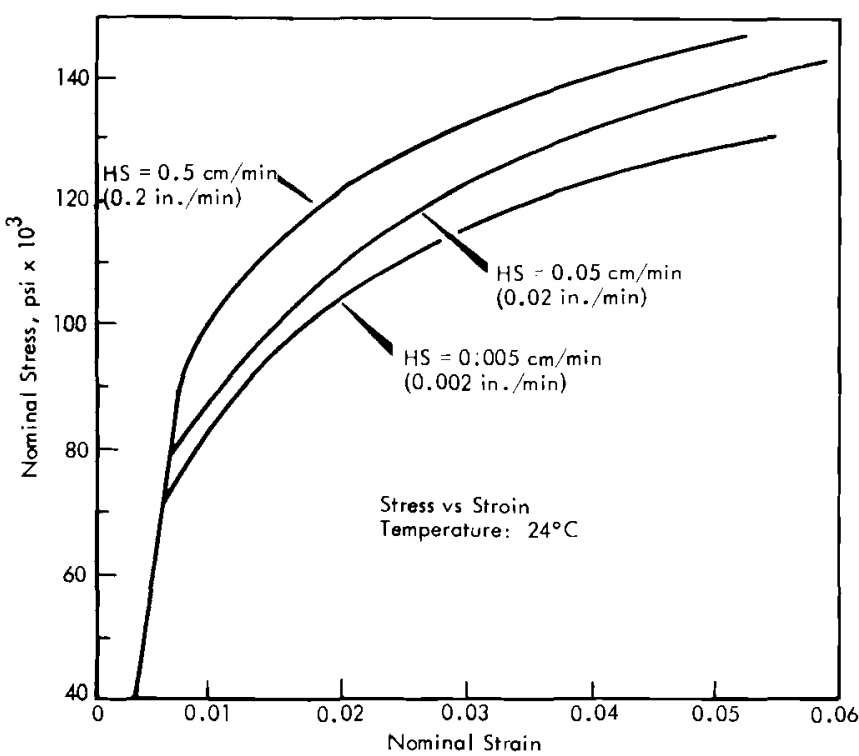

The Effect of Strain Rate on the Compression stress-Strain Curves for Alpha Plutonium at Room Temperature. (Fine grained polyerystalline eylinders. 500 ppm total impurities.)

Stress-strain compression tests were run in an oil bath at temperatures from $24^{\circ} \mathrm{C}$ to $100^{\circ} \mathrm{C}$ and at head speeds from 0.005 to $0.5 \mathrm{~cm} / \mathrm{min}$. Yicld stress varies in-
versely with temperature. Total deformation in compression ranged from $20^{\circ}$ at $24^{\circ} \mathrm{C}$ to $80 \%$ at $100^{\circ} \mathrm{C}$.

From S. E. Bronisz and A. F. Gorum, J. Nuel. Mater., vol. 2, p. 193.1960

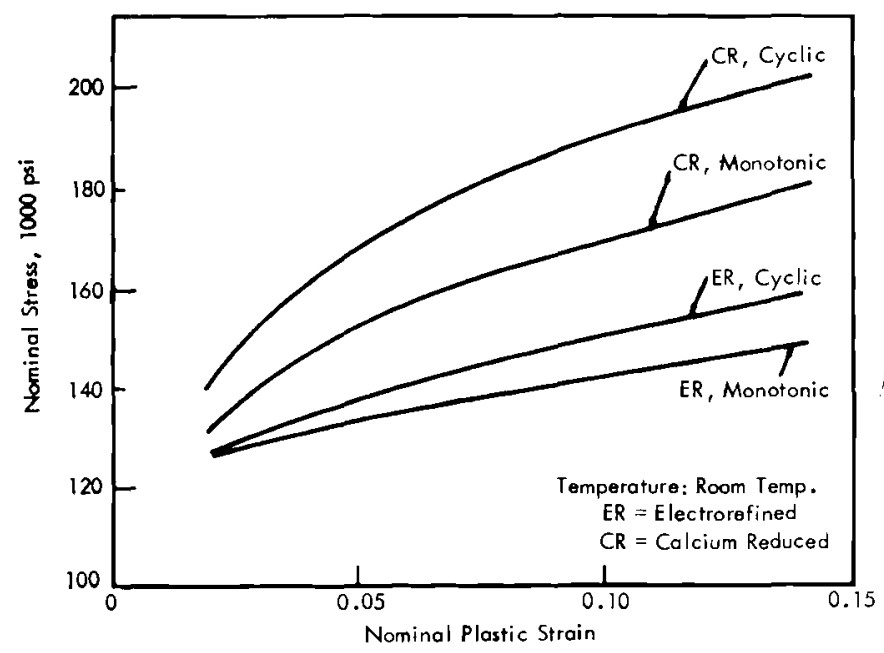

36

The Effect of Strain Aging on the Nominal Stress-Strain Curves of Alpha Plutonium Tegted in Compression at $24^{\circ} \mathrm{C}$ at a Hegd Speed of $0.02 \mathrm{in} / \mathrm{min}$. From

S. E. Bronisz. J. Nucl. Mater., vol. 9, p. 104.1963.

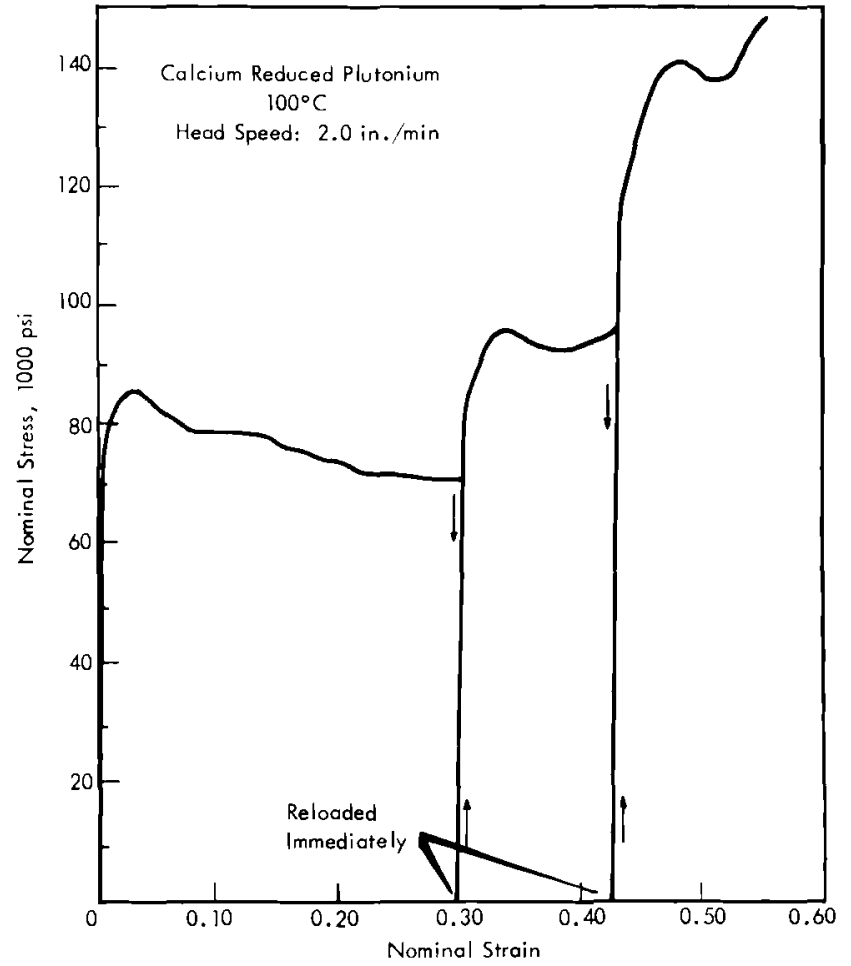

Strain Afing Field Points Olsserved in Alpha Plutonium Tested in Compression at $100^{\circ} \mathrm{C}$ at a llead speed of $2.0 \mathrm{in} / \mathrm{min}$. From S. E. Bronisz. J. Nucl. Mater., vol. 9, p. 1nt. 1963.

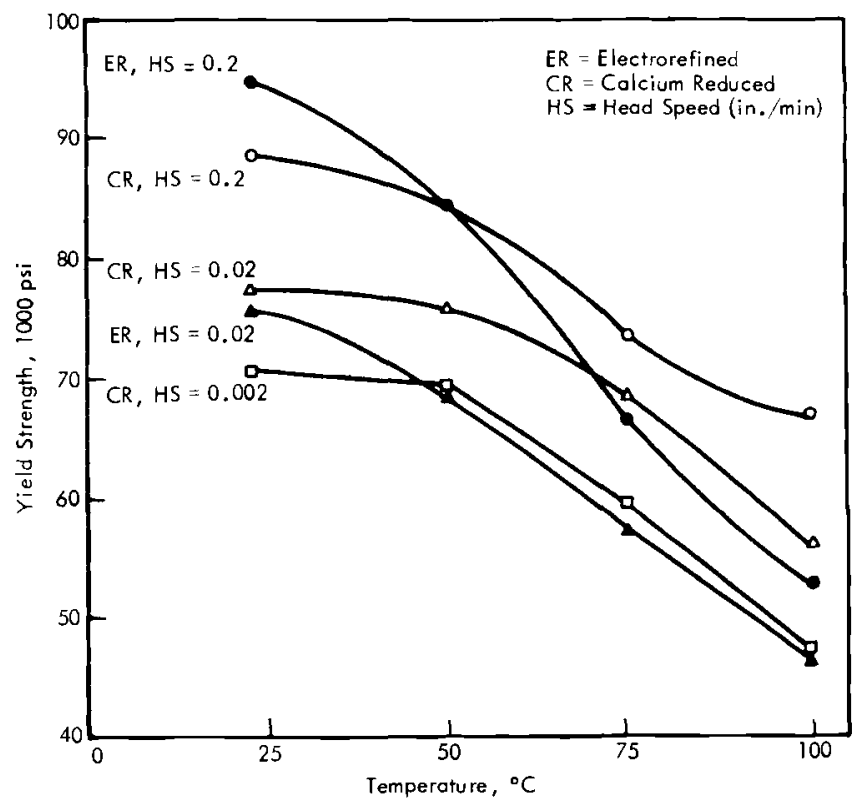

The Effect of Testing Temperature on the Compression Yield Strength of Alpha Plutonium at Various Testing Speeds. From S. E. Bronisz. J. Nucl. Mater. 


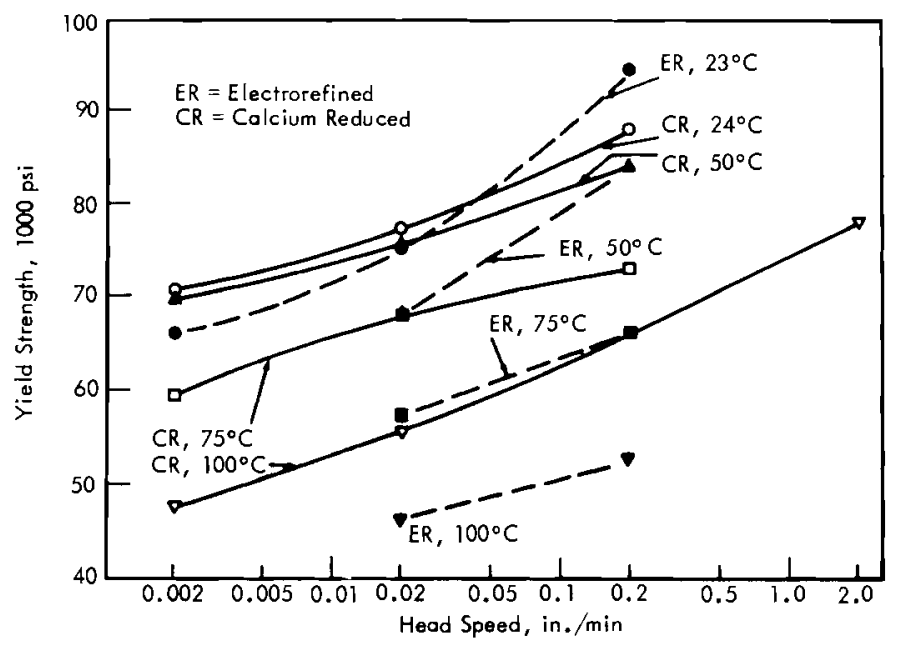

The Effect of Testing Speed on the Yield Strength of Alpha Plutonium at Various Temperatures. From S. E. Bronisz. I. Nucl. Mater., vol. 9, p. 104. 1963.
CHEMICAL ANALYSES OF PLUTONIUM USED FOR COM PRESSION TESTING

From S. E. Bronisz, J. Nucl. Mater., vol. 9, p. 102. 1963.

\begin{tabular}{|c|c|c|c|}
\hline & Electrorefined & Electrorefined & Electrorefi \\
\hline & $\begin{array}{l}\text { Plutonium, } \\
\text { As }\end{array}$ & $\begin{array}{l}\text { Plutonium, } \\
\text { Once }\end{array}$ & $\begin{array}{l}\text { Plutonium, } \\
\text { Thrice }\end{array}$ \\
\hline & Deposited & Remelted & Remelted \\
\hline
\end{tabular}

Total

480

115

185

227

YIELD STRENGTH AND MODULUS OF ELASTICITY IN COMPRESSION* AT $30^{\circ} \mathrm{C}$

From H. R. Gardner and I. B. Mann in Plutonium 1960, p. 538.

\begin{tabular}{|c|c|c|c|c|}
\hline $\begin{array}{l}\text { Specimen } \\
\text { Nu mber } \\
\end{array}$ & $\begin{array}{c}0.01 \% \\
\text { Yield } \\
\text { Strength, } \\
\text { lb/in. } \\
\end{array}$ & $\begin{array}{c}\text { Modulus } \\
\text { of Elasticity, } \\
10^{6} \mathrm{lb} / \text { in. } 2 \\
\end{array}$ & $\begin{array}{l}\text { Density, } \\
\mathrm{g} / \mathrm{cm}^{3}\end{array}$ & $\begin{array}{l}\text { Diamond } \\
\text { Pyramid } \\
\text { Hardness, } \\
10 \mathrm{~kg} \text { Load }\end{array}$ \\
\hline $\begin{array}{l}18-8-1 C \\
18-8-1 D \\
18-8-4 O \\
18-8-4 P \\
18-8-5 R\end{array}$ & $\begin{array}{l}47300 \\
39600 \\
34200 \\
32100 \\
37500\end{array}$ & $\begin{array}{l}13.9 \\
14.5 \\
14.7 \\
14.2 \\
14.0\end{array}$ & $\begin{array}{l}19.49 \\
19.55 \\
19.56 \\
19.56 \\
19.55\end{array}$ & $\begin{array}{c}261 \\
- \\
- \\
259 \\
258\end{array}$ \\
\hline Average & 38200 & 14.3 & 19.54 & 259 \\
\hline
\end{tabular}

* Testing speed: $0.015 \mathrm{in}, / \mathrm{min} ; \mathrm{h} / \mathrm{d}: 5 \cdot 5$.

COMPRESSION PROPERTIES OF PLUTONIUM ${ }^{* *}$ AT $30^{\circ} \mathrm{C}$

From H. R. Gardner and I. B. Mann in Plutonium 1960, pp. 537-538.

\begin{tabular}{|c|c|c|c|c|c|c|}
\hline $\begin{array}{l}\text { Specimen } \\
\text { Number }\end{array}$ & $\begin{array}{l}\text { Density } \\
\mathrm{g} / \mathrm{cm}^{3}\end{array}$ & $\begin{array}{l}\text { Ultimate } \\
\text { Strength, } \\
\text { lb/in. } \\
\end{array}$ & $\begin{array}{l}\text { Yield } \\
\text { Strengtb, } \\
\text { lb/in.2 } \\
\end{array}$ & $\begin{array}{c}\text { Diamond } \\
\text { Pyramid } \\
\text { Hardness, } 10 \mathrm{~kg} \text { Load } \\
\end{array}$ & $\begin{array}{l}\text { Plastic Decrease } \\
\text { in Length Prior } \\
\text { to Failure } \dagger, \% \\
\end{array}$ & $\begin{array}{l}\text { Metal } \\
\text { Group }\end{array}$ \\
\hline \multicolumn{7}{|c|}{ Density Less Than $19.50 \mathrm{~g} / \mathrm{cm}^{3}$} \\
\hline $\begin{array}{l}17-12-1 \mathrm{G} 1 \\
17-12-1 \mathrm{G} 2\end{array}$ & $\begin{array}{l}19.42 \\
19.42\end{array}$ & $\begin{array}{l}178600 \\
175200\end{array}$ & $\begin{array}{l}- \\
-\end{array}$ & $\begin{array}{l}272 \\
272\end{array}$ & - & 1 \\
\hline $\begin{array}{l}18-1-1 A \\
18-1-1 B\end{array}$ & $\begin{array}{l}19.34 \\
19.47\end{array}$ & $\begin{array}{l}163900 \\
175600\end{array}$ & - & $\begin{array}{l}255 \\
255\end{array}$ & - & $\begin{array}{l}2 \\
3\end{array}$ \\
\hline $\begin{array}{l}18-1-4 G 1 \\
18-1-4 G 2\end{array}$ & $\begin{array}{l}19.43 \\
19.43\end{array}$ & $\begin{array}{l}177000 \\
177800\end{array}$ & $\begin{array}{l}- \\
-\end{array}$ & $\begin{array}{l}267 \\
267\end{array}$ & - & 4 \\
\hline $\begin{array}{l}18-1-6 \mathrm{~L} 1 \\
18-1-6 \mathrm{~L} 2\end{array}$ & $\begin{array}{l}19.47 \\
19.47\end{array}$ & $\begin{array}{l}156600 \\
149000\end{array}$ & - & $\begin{array}{l}265 \\
265\end{array}$ & - & 5 \\
\hline $\begin{array}{l}18-1-7 \mathrm{Ml} \\
18-1-7 \mathrm{M} 2\end{array}$ & $\begin{array}{l}19.43 \\
19.43\end{array}$ & $\begin{array}{l}163200 \\
172000\end{array}$ & $\begin{array}{l}- \\
-\end{array}$ & $\begin{array}{l}256 \\
256\end{array}$ & - & 6 \\
\hline $\begin{array}{l}18-1-7 N 1 \\
18-1-7 N 2\end{array}$ & $\begin{array}{l}19.46 \\
19.46\end{array}$ & $\begin{array}{l}165600 \\
164400\end{array}$ & - & $\begin{array}{l}275 \\
275\end{array}$ & - & 7 \\
\hline Average ${ }^{\dagger+}$ & 19.43 & 168500 & - & 260 & - & - \\
\hline \multicolumn{7}{|c|}{ Density Equal to or Greater than $19.50 \mathrm{~g} / \mathrm{cm}^{3}$} \\
\hline $\begin{array}{l}18-1-3 F 1 \\
18-1-3 F 2\end{array}$ & $\begin{array}{l}19.55 \\
19.55\end{array}$ & $\begin{array}{l}177600 \\
175600\end{array}$ & - & $\begin{array}{l}265 \\
265\end{array}$ & - & 8 \\
\hline $\begin{array}{l}18-1-6 \mathrm{~K} 1 \\
18-1-6 \mathrm{~K} 2\end{array}$ & $\begin{array}{l}19.56 \\
19.56\end{array}$ & $\begin{array}{l}166800 \\
169000\end{array}$ & - & $\begin{array}{l}256 \\
256\end{array}$ & - & 9 \\
\hline $\begin{array}{l}18-8-1 \mathrm{D} 1 \\
18-8-1 \mathrm{D} 2\end{array}$ & $\begin{array}{l}19.55 \\
19.55\end{array}$ & $\begin{array}{l}168200 \\
164000\end{array}$ & $\begin{array}{l}102400 \\
109100\end{array}$ & - & $\begin{array}{l}21.3 \\
18.6\end{array}$ & 10 \\
\hline $\begin{array}{l}18-8-4 P 1 \\
18-8-4 P 2\end{array}$ & $\begin{array}{l}19.56 \\
19.56\end{array}$ & $\begin{array}{l}184000 \\
178000\end{array}$ & $\begin{array}{l}103200 \\
109800\end{array}$ & $\begin{array}{l}259 \\
259\end{array}$ & $\begin{array}{l}24.0 \\
22.6\end{array}$ & 11 \\
\hline $\begin{array}{l}18-8-5 Q 1 \\
18-8-5 Q 2 \\
18-8-5 Q^{3}\end{array}$ & $\begin{array}{l}19.55 \\
19.55 \\
19.55\end{array}$ & $\begin{array}{l}183000 \\
181000 \\
189000\end{array}$ & $\begin{array}{r}99800 \\
112700 \\
107600\end{array}$ & $\begin{array}{l}- \\
-\end{array}$ & $\begin{array}{l}23.4 \\
23.4 \\
25.0\end{array}$ & 12 \\
\hline $\begin{array}{l}18-8-5 R 1 \\
18-8-5 R 2\end{array}$ & $\begin{array}{l}19.55 \\
19.55\end{array}$ & $\begin{array}{l}169200 \\
189100\end{array}$ & $\begin{array}{l}109900 \\
101700\end{array}$ & $\begin{array}{l}258 \\
258\end{array}$ & $\begin{array}{l}20.6 \\
25.6\end{array}$ & 13 \\
\hline $19-7-3-G 2$ & 19.53 & 167500 & 113200 & - & 26.4 & 14 \\
\hline $19-8-2-\mathrm{C} 5$ & 19.55 & 178000 & 106300 & - & 20.3 & 15 \\
\hline Average ${ }^{\dagger+}$ & 19.55 & 175000 & 107400 & 263 & 22.8 & - \\
\hline
\end{tabular}

* Testing speed: $0.015 \mathrm{in} . / \mathrm{min}$

$\uparrow$ Determined from load-piaten dispiacement curve at point of failure.

+ All average values were obtained by first finding the average within each metal group and then averaging these values for an overall average. This was done because the material 
From H. R. Gardner and I. B. Nann in Plutonium 1960, p. 540 .

\begin{tabular}{|c|c|c|c|c|c|c|c|}
\hline $\begin{array}{l}\text { Specimen } \\
\text { Number } \\
\end{array}$ & $\begin{array}{c}\text { Test } \\
\text { Temperature, } \\
{ }^{\circ} \mathrm{C} \\
\end{array}$ & $\begin{array}{l}\text { [lltimate } \\
\text { Strength, } \\
\text { lb/in. } 2\end{array}$ & $\begin{array}{c}\text { Yield } \\
\text { Strength, } \\
\text { lb/in. } 2 \\
\end{array}$ & 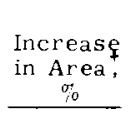 & $\begin{array}{l}\text { Plastic } \\
\text { Decrease } \\
\text { in Length, } \\
\% \\
\end{array}$ & $\begin{array}{l}\text { Density, } \\
\mathrm{g} / \mathrm{cm}^{3} \\
\end{array}$ & $\begin{array}{c}\text { Total } \\
\text { Impurity } \\
\text { Content, } \\
\text { PPm }\end{array}$ \\
\hline $\begin{array}{l}19-7-3-E 4 \\
19-7-3-E 5\end{array}$ & $\begin{array}{l}-30 \\
-30\end{array}$ & $\begin{array}{l}194200 \\
190300\end{array}$ & $\begin{array}{l}161600 \\
160600\end{array}$ & - & 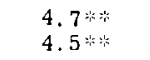 & $\begin{array}{l}19.53 \\
19.53\end{array}$ & $\begin{array}{l}745 \\
745\end{array}$ \\
\hline++ & 30 & 175000 & 107400 & - & $22.7 \div$ & 19.55 & - \\
\hline $\begin{array}{l}19-7-2-\mathrm{D} 5 \\
19-7-3-\mathrm{E} 1\end{array}$ & $\begin{array}{l}73 \\
70\end{array}$ & $11 \overline{8400}$ & $\begin{array}{l}74200 \\
80200\end{array}$ & - & $26.5 \div$ & $\begin{array}{l}19.54 \\
19.53\end{array}$ & $\begin{array}{l}324 \\
745\end{array}$ \\
\hline $\begin{array}{l}19-7-3-E 2 \\
19-7-3-E 3\end{array}$ & $\begin{array}{lll}1 & 1 & 0 \\
1 & 1 & 0\end{array}$ & $\begin{array}{l}78300 \\
77200\end{array}$ & $\begin{array}{l}65400 \\
64800\end{array}$ & - & $\begin{array}{c}18.3^{* * * ;} \\
-\end{array}$ & $\begin{array}{l}19.53 \\
19.53\end{array}$ & $\begin{array}{l}745 \\
745\end{array}$ \\
\hline $\begin{array}{l}19-5-4-B 4 \\
19-5-4-A 3 \\
19-5-4-A 4 \\
19-6-4-\mathrm{H} 4 \\
19-6-4-\mathrm{G} 1\end{array}$ & $\begin{array}{l}128 \\
130 \\
130 \\
131 \\
130\end{array}$ & $\begin{array}{l}- \\
- \\
- \\
-\end{array}$ & $\begin{array}{l}- \\
20900 \\
24100 \\
24600 \\
17700\end{array}$ & $\begin{array}{l}392 \\
409 \\
498 \\
497 \\
436\end{array}$ & $\begin{array}{l}79.8^{\dagger} \\
79.9^{\dagger} \\
82.2^{\dagger} \\
83.3^{\dagger} \\
81.6^{\dagger}\end{array}$ & $\begin{array}{l}19.59 \\
19.59 \\
19.59 \\
19.56 \\
19.56\end{array}$ & $\begin{array}{l}266 \\
266 \\
266 \\
412 \\
412\end{array}$ \\
\hline $\begin{array}{l}19-5-4-\mathrm{B} 3 \\
19-5-4-\mathrm{A} 2 \\
19-5-4-\mathrm{C} 3\end{array}$ & $\begin{array}{l}149 \\
149 \\
151\end{array}$ & $\begin{array}{l}- \\
-\end{array}$ & $\begin{array}{l}11650 \\
12000 \\
11970\end{array}$ & $\begin{array}{l}557 \\
557 \\
592\end{array}$ & $\begin{array}{l}84.8 \dagger \\
84.5^{\dagger} \\
85.2^{\dagger}\end{array}$ & $\begin{array}{l}19.59 \\
19.59 \\
19.59\end{array}$ & $\begin{array}{l}266 \\
266 \\
266\end{array}$ \\
\hline $\begin{array}{l}19-5-4-\mathrm{C} 1 \\
19-5-4-\mathrm{C} 2\end{array}$ & $\begin{array}{l}176 \\
176\end{array}$ & - & $\begin{array}{l}8170 \\
8770\end{array}$ & $\begin{array}{l}625 \\
625\end{array}$ & $\begin{array}{l}86.0^{+} \\
85.5^{+}\end{array}$ & $\begin{array}{l}19.59 \\
19.59\end{array}$ & $\begin{array}{l}266 \\
266\end{array}$ \\
\hline $\begin{array}{l}19-5-4-A 1 \\
19-5-4-D 4 \\
19-6-4-\mathrm{H} 3\end{array}$ & $\begin{array}{l}189 \\
190 \\
191\end{array}$ & $\begin{array}{l}- \\
-\end{array}$ & $\begin{array}{l}6820 \\
7130 \\
6910\end{array}$ & $\begin{array}{l}658 \\
658 \\
661\end{array}$ & $\begin{array}{l}86.6+ \\
86.3^{+} \\
86.5^{+}\end{array}$ & $\begin{array}{l}19.59 \\
19.59 \\
19.56\end{array}$ & $\begin{array}{l}266 \\
266 \\
412\end{array}$ \\
\hline $\begin{array}{l}19-4-3-\mathrm{C} 1 \\
19-4-3-\mathrm{B} 1 \\
19-5-4-\mathrm{B} 5\end{array}$ & $\begin{array}{l}230 \\
231 \\
232\end{array}$ & $\begin{array}{l}- \\
- \\
-\end{array}$ & $\begin{array}{l}4930 \\
5110 \\
4530\end{array}$ & $\begin{array}{l}945 \\
841 \\
800\end{array}$ & $\begin{array}{l}89.1^{+} \\
87.7^{+} \\
88.5^{+}\end{array}$ & $\begin{array}{l}19.55 \\
19.55 \\
19.59\end{array}$ & $\begin{array}{l}310 \\
310 \\
266\end{array}$ \\
\hline $\begin{array}{l}19-5-4-\mathrm{B} 2 \\
19-6-4-\mathrm{H} 2\end{array}$ & $\begin{array}{l}266 \\
266\end{array}$ & - & $\begin{array}{l}3970 \\
4340\end{array}$ & $\begin{array}{l}957 \\
942\end{array}$ & $\begin{array}{l}90.3^{+} \\
90.3^{+}\end{array}$ & $\begin{array}{l}19.59 \\
19.56\end{array}$ & $\begin{array}{l}266 \\
412\end{array}$ \\
\hline $\begin{array}{l}19-7-1-A 3 \\
19-5-4 B \\
19-4-3-B 2\end{array}$ & $\begin{array}{l}300 \\
300 \\
300\end{array}$ & $\begin{array}{l}- \\
- \\
-\end{array}$ & $\begin{array}{l}3430 \\
3380 \\
3500\end{array}$ & $\begin{array}{l}1145 \\
1353 \\
1196\end{array}$ & $\begin{array}{l}91.4^{\dagger} \\
92.6^{+} \\
92.2^{+}\end{array}$ & $\begin{array}{l}19.56 \\
19.59 \\
19.55\end{array}$ & $\begin{array}{l}343 \\
266 \\
310\end{array}$ \\
\hline $\begin{array}{l}19-4-3 A \\
19-7-1-A 1\end{array}$ & $\begin{array}{l}330 \\
331\end{array}$ & $\begin{array}{l}- \\
-\end{array}$ & $\begin{array}{r}816 \\
1265\end{array}$ & $\begin{array}{l}1593 \\
1610\end{array}$ & $\begin{array}{l}93.8^{+} \\
92.8^{+}\end{array}$ & $\begin{array}{l}19.55 \\
19.56\end{array}$ & $\begin{array}{l}310 \\
343\end{array}$ \\
\hline \multicolumn{8}{|c|}{$*$ Testing speed: $0.015 \mathrm{in} . / \mathrm{min}$. } \\
\hline
\end{tabular}

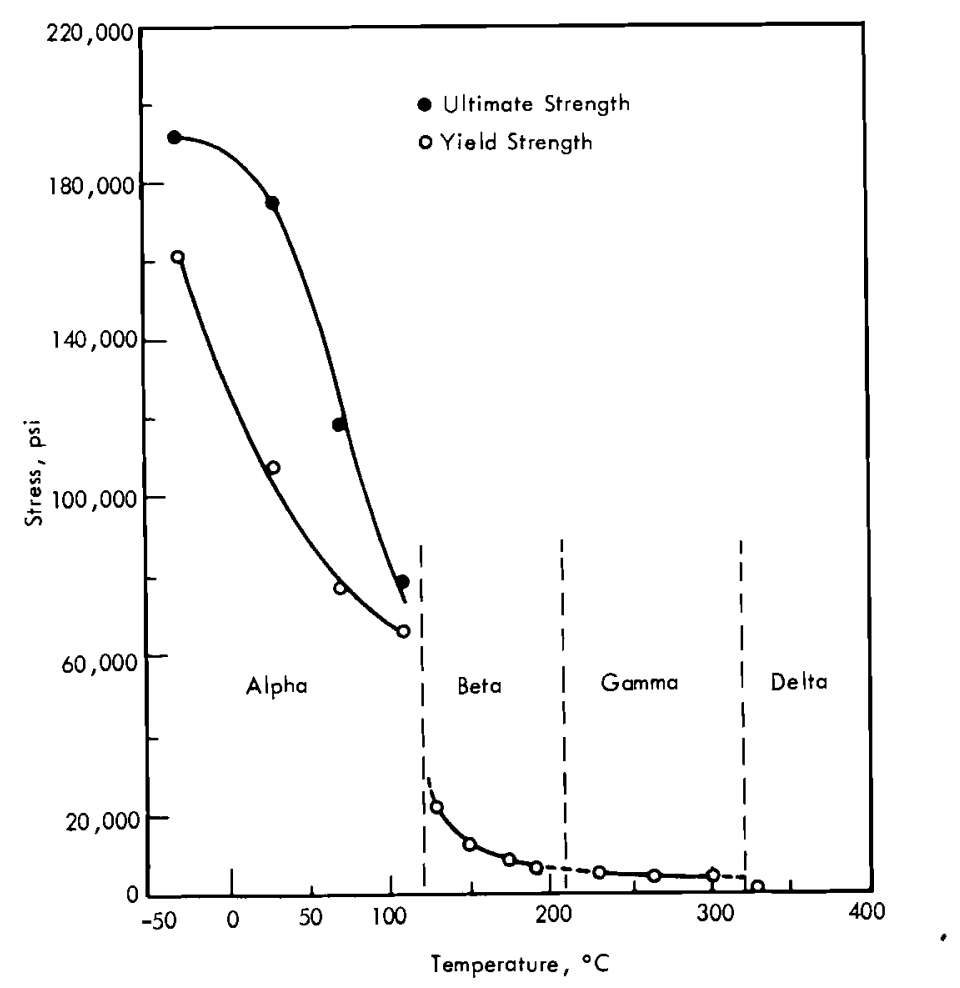

Effect of Temperature on Average Compressive Strength for High Density Plutonium. Testing speed: $0.015 \mathrm{in} / \mathrm{min}$. From H. R. Gardner and I. B. Mann in Plutonium 1960, p. 539 . 


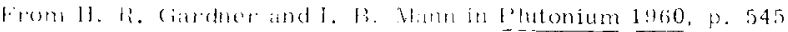

\begin{tabular}{|c|c|c|c|c|c|c|c|}
\hline $\begin{array}{l}\text { Spereinsen } \\
\text { Number }\end{array}$ & 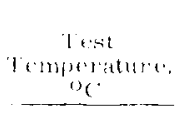 & 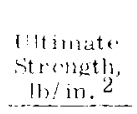 & $\begin{array}{r}\text { Yirid } \\
\text { Strerigth, } \\
\text { lb/in. } \\
\end{array}$ & $\begin{array}{l}\text { Increase } \\
\text { in Areat, }\end{array}$ & $\begin{array}{c}\text { Plastic } \\
\text { Decrease } \\
\text { in Iongth, } \\
\end{array}$ & $\begin{array}{r}\text { Density, } \\
\mathrm{g} / \mathrm{cm}^{3} \\
\end{array}$ & $\begin{array}{c}\text { Total } \\
\text { Impurity } \\
\text { Content } \\
\text { ppm } \\
\end{array}$ \\
\hline$+t$ & 30 & 1685500 & - & - & - & 19.43 & - \\
\hline $\begin{array}{l}19-2-2-7 \\
19-2-2-10\end{array}$ & $\begin{array}{l}100 \\
100\end{array}$ & $\begin{array}{l}97500 \\
90000\end{array}$ & $\begin{array}{l}68000 \\
67000\end{array}$ & - & - & $\begin{array}{l}19.41 \\
19.41\end{array}$ & $\begin{array}{l}1467 \\
1467\end{array}$ \\
\hline $\begin{array}{l}19-2-2-3 \\
19-2-2-9 \\
19-2-2-11 \\
19-2-2-12 \\
19-2-2-19\end{array}$ & $\begin{array}{l}150 \\
150 \\
150 \\
150 \\
150\end{array}$ & $\begin{array}{l}- \\
- \\
-\end{array}$ & $\begin{array}{l}10880 \\
14120 \\
12500 \\
15300 \\
15300\end{array}$ & $\begin{array}{c}- \\
- \\
- \\
599 \\
613\end{array}$ & $\begin{array}{l}- \\
- \\
- \\
- \\
86.1\end{array}$ & $\begin{array}{l}19.41 \\
19.41 \\
19.41 \\
19.41 \\
19.41\end{array}$ & $\begin{array}{l}1467 \\
1467 \\
1467 \\
1467 \\
1467\end{array}$ \\
\hline $\begin{array}{l}19-2-2-1 \\
19-2-2-4\end{array}$ & $\begin{array}{l}175 \\
175\end{array}$ & - & $\begin{array}{l}8750 \\
8160\end{array}$ & $6 \overline{83}$ & $\overline{86.8}$ & $\begin{array}{l}19.41 \\
19.41\end{array}$ & $\begin{array}{l}1467 \\
1467\end{array}$ \\
\hline $\begin{array}{l}19-2-2-2 \\
19-2-2-17\end{array}$ & $\begin{array}{l}190 \\
190\end{array}$ & - & $\begin{array}{l}5870 \\
5900\end{array}$ & $\begin{array}{c}- \\
725\end{array}$ & $\begin{array}{c}- \\
87.7\end{array}$ & $\begin{array}{l}19.41 \\
19.41\end{array}$ & $\begin{array}{l}1467 \\
1467\end{array}$ \\
\hline $\begin{array}{l}19-2-2-5 \\
19-2-2-14 \\
19-2-2-16\end{array}$ & $\begin{array}{l}230 \\
230 \\
230\end{array}$ & $\begin{array}{l}- \\
-\end{array}$ & $\begin{array}{l}6120 \\
6830 \\
7490\end{array}$ & $\begin{array}{l}958 \\
307 \\
853\end{array}$ & $\begin{array}{l}89.3 \\
89.2 \\
89.2\end{array}$ & $\begin{array}{l}19.41 \\
19.41 \\
19.41\end{array}$ & $\begin{array}{l}1467 \\
1467 \\
1467\end{array}$ \\
\hline $\begin{array}{l}19-2-2-15 \\
19-2-2-18\end{array}$ & $\begin{array}{l}265 \\
265\end{array}$ & - & $\begin{array}{l}5400 \\
4700\end{array}$ & $\begin{array}{l}1069 \\
1110\end{array}$ & $\begin{array}{l}91.7 \\
91.7\end{array}$ & $\begin{array}{l}19.41 \\
19.41\end{array}$ & $\begin{array}{l}1467 \\
1467\end{array}$ \\
\hline $\begin{array}{l}19-2-1-4 A \\
19-2-1-3 A\end{array}$ & $\begin{array}{l}300 \\
300\end{array}$ & $\begin{array}{l}- \\
-\end{array}$ & $\begin{array}{c}2600 \\
-\end{array}$ & $10 \overline{-}$ & $9 \overline{2.7}$ & $\begin{array}{l}19.45 \\
19.45\end{array}$ & $\begin{array}{l}2300 \\
2300\end{array}$ \\
\hline $\begin{array}{l}19-2-2-25 \\
19-2-1-1 A\end{array}$ & $\begin{array}{l}330 \\
330\end{array}$ & - & $\begin{array}{l}2350 \\
2575\end{array}$ & $\begin{array}{c}1473 \\
-\end{array}$ & 94.2 & $\begin{array}{l}19.41 \\
19.45\end{array}$ & $\begin{array}{l}1467 \\
2300\end{array}$ \\
\hline $\begin{array}{l}* \text { Testing } \\
+ \text { After ex } \\
\text { increase } \\
\text { tempera }\end{array}$ & $\begin{array}{l}\text { eed: } 0.015 \mathrm{in} . \\
\text { eding the yield } \\
\text { area and decr } \\
\text { e. }\end{array}$ & $\begin{array}{l}\text { nin. } \\
\text { rength the } \\
\text { ise in lengt }\end{array}$ & $\begin{array}{l}\text { Id was incre } \\
\text { lata were ta }\end{array}$ & $\begin{array}{l}\text { ed at a rap } \\
\text { after unl }\end{array}$ & $\begin{array}{l}\text { d rate to } 100 \\
\text { ading and co }\end{array}$ & $\begin{array}{l}0 \mathrm{lb} \text {. The } \\
\text { g to room }\end{array}$ & \\
\hline
\end{tabular}

IHLCO OF MUTAI, QUATITY ON YIET,D STRENGTH

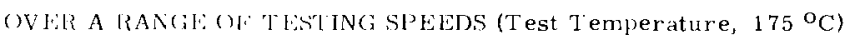

from II. R. Crardher and I. F. Maton in P'lutonium 1960, p. 554. L,ow-Granity Metal

\begin{tabular}{|c|c|c|c|c|}
\hline $\begin{array}{l}\text { lesting } \\
\text { Shrect } \\
\text { in. Inin }\end{array}$ & $\begin{array}{l}\text { Specimen } \\
\text { Number. }\end{array}$ & $\begin{array}{l}\text { Yieid } \\
\text { Strength } \\
\text { lb/in. }\end{array}$ & $\begin{array}{l}\text { Density, } \\
\mathrm{g} / \mathrm{cm}^{3}\end{array}$ & $\begin{array}{c}\text { Total } \\
\text { Impurity } \\
\text { Content, } \\
\text { ppm }\end{array}$ \\
\hline 0.003 & $19-2-2-22$ & 6650 & 19.41 & 1467 \\
\hline 0.007 & $19-2-2-24$ & 7650 & 19.41 & 1467 \\
\hline $0 .(11)$ & $1 ! 1-2-2-4$ & 8700 & 19.41 & 1467 \\
\hline 0.015 & $19-2-2-1$ & 8960 & 19.41 & 1467 \\
\hline 0.060 & $19-2-2-20$ & 14480 & 19.41 & 1467 \\
\hline 0.125 & $19-2-2-21$ & 17190 & 19.41 & 1467 \\
\hline 0.250 & $19-2-2-23$ & 20400 & 19.41 & 1467 \\
\hline
\end{tabular}

High-Quality Metal

\begin{tabular}{|c|c|c|c|c|}
\hline 0.0002 & $1: 1-7-1-A-4$ & 3900 & 19.56 & 343 \\
\hline 0.002 & $1 ! 1-7-1-A-5$ & 3870 & 19.56 & 343 \\
\hline 0.015 & $1: 5-4-C-1$ & 8170 & 19.59 & 266 \\
\hline 0.015 & 1.9 $=-4-C-2$ & 8770 & 19.59 & 266 \\
\hline 0.0600 & $194-7-1-A-6$ & 8930 & 19.56 & 343 \\
\hline $1.0(i 0$ & $1-7-1-B-1$ & 8910 & 19.56 & 343 \\
\hline (1. 250 & $1 A-7-1-B-3$ & 15600 & 19.56 & 343 \\
\hline 0.250 & $19-7-1-B-4$ & 14700 & 19.56 & 343 \\
\hline 1. 00 & $1 ! 1-7-1-13-5$ & 16900 & 19.56 & 343 \\
\hline 1.00 & $19-7-1-73-6$ & 20200 & 19.56 & 343 \\
\hline
\end{tabular}

TC'TAI. I'LASTIC DEFORMATION IN $\boldsymbol{a}$ - PHASE *

From II. R. Gardner and I. B. Mann

in Plutonium 1960, p. 554.

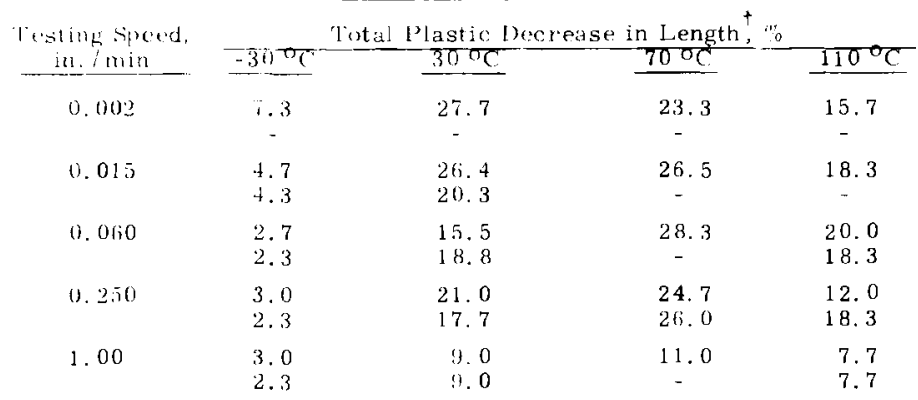

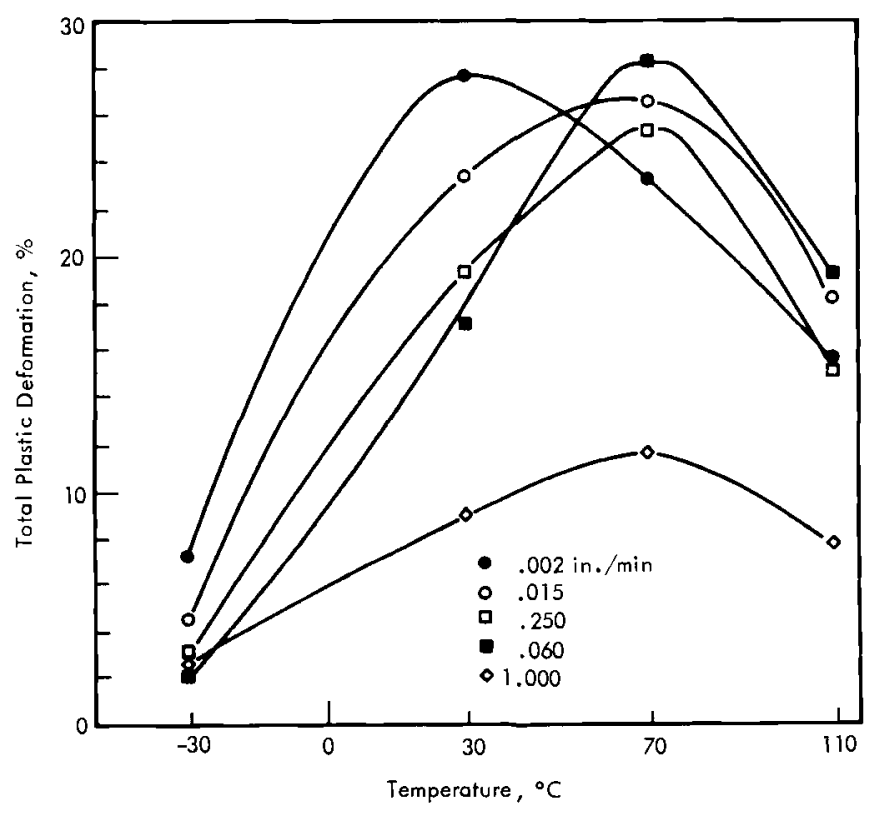

Effect of Temperature on Total Plastic Deformation Prior to Fracture. From H. R. Gardner and I. B. Mann in Plutonium 1960, p. 553. 


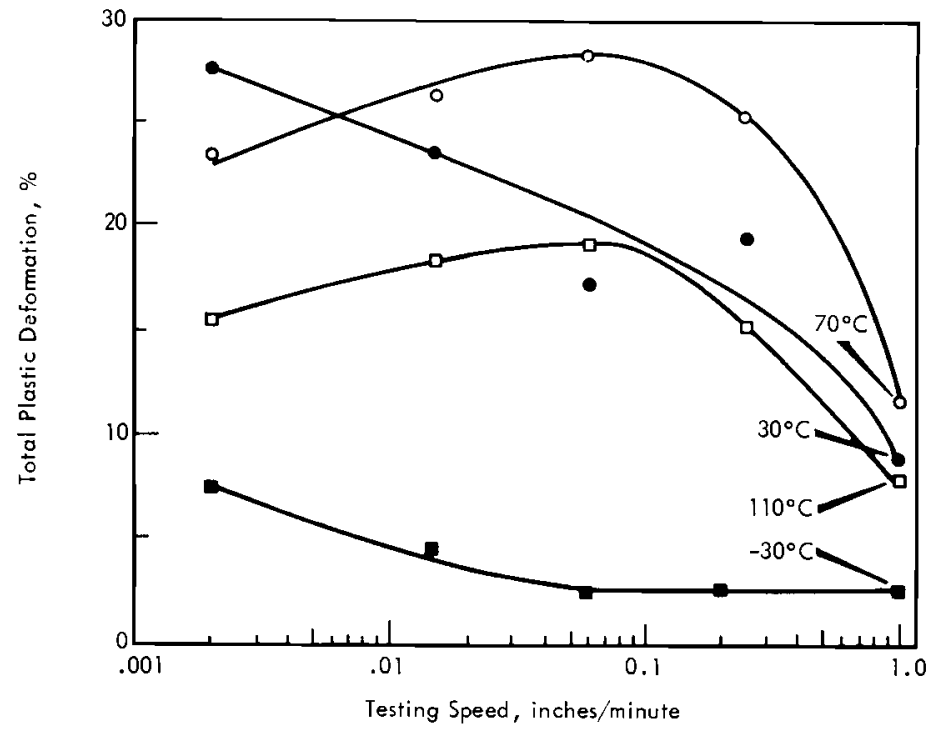

Effect of Testing Speed on Total Plastic Deformation Prior to Fracture. From H. R. Gardner and I. B. Mann in Plutonium 1960, p. 553.

EFFECT OF TESTING SPEED IN $\boldsymbol{a}-\boldsymbol{\beta} \boldsymbol{\beta}$ - AND $\boldsymbol{\gamma}$-PHASES ON COMPRESSIVE STRENGTH

From H. R. Gardner and I. B. Mann in Plutonium 1960, p. 551,

\begin{tabular}{|c|c|c|c|c|c|c|c|c|c|c|}
\hline \multirow{4}{*}{$\begin{array}{l}\text { Testing } \\
\text { Speed, } \\
\text { in. } / \mathrm{min} .\end{array}$} & \multicolumn{8}{|c|}{$a$-Phase } & \multirow{2}{*}{$\frac{\beta \text {-Phase }}{175^{\circ} \mathrm{C}}$} & \multirow{2}{*}{$\frac{\gamma \text {-Phase }}{265^{\circ} \mathrm{C}}$} \\
\hline & \multicolumn{2}{|c|}{$-30^{\circ} \mathrm{C}$} & \multicolumn{2}{|c|}{$29^{\circ} \mathrm{C}$} & \multicolumn{2}{|c|}{$70^{\circ} \mathrm{C}$} & \multicolumn{2}{|c|}{$110^{\circ} \mathrm{C}$} & & \\
\hline & Ultimate & Yield & Ultimate & Yield & Ultimate & Yield & Ultimate & Yield & Yield & Yield \\
\hline & $\begin{array}{l}\text { Strength, } \\
\text { lb/in. }{ }^{2}\end{array}$ & $\begin{array}{l}\text { Strength, } \\
1 \mathrm{~b} / \text { in. }^{2}\end{array}$ & $\begin{array}{l}\text { Strength, } \\
\text { lb/in. }\end{array}$ & $\begin{array}{l}\text { Strength, } \\
\text { lb/in. } 2\end{array}$ & $\begin{array}{l}\text { Strength, } \\
\text { lb/in. } 2\end{array}$ & $\begin{array}{l}\text { Strength, } \\
\text { lb/in.2 }\end{array}$ & $\begin{array}{l}\text { Strength, } \\
\text { lb/in. }{ }^{2}\end{array}$ & $\begin{array}{l}\text { Strength, } \\
1 \mathrm{~b} / \text { in.2 }\end{array}$ & $\begin{array}{l}\text { Strength, } \\
1 \mathrm{~b} / \text { in. }^{2}\end{array}$ & $\begin{array}{l}\text { Strength, } \\
1 \mathrm{~b} / \mathrm{in} .^{2}\end{array}$ \\
\hline \multirow[t]{2}{*}{0.002} & 190700 & 145200 & 142000 & 95300 & 95700 & 69000 & 63500 & 48100 & 3900 & 2620 \\
\hline & - & - & 149000 & 95900 & 96000 & 69000 & 68600 & 46900 & 3870 & 6000 \\
\hline \multirow[t]{2}{*}{0.015} & 190300 & 161600 & 178000 & 106300 & 118400 & 80200 & 77200 & 64800 & 8170 & 3970 \\
\hline & 194200 & 160600 & 167500 & 113200 & - & - & 78300 & 65400 & 8770 & 4340 \\
\hline \multirow[t]{2}{*}{0.060} & 179800 & 158600 & 156 & 123000 & 123200 & 85400 & 85000 & 64800 & 8930 & 3630 \\
\hline & 186400 & 161000 & 165500 & 117200 & 125800 & 79000 & 85000 & 69500 & 8910 & - \\
\hline \multirow[t]{2}{*}{0.250} & 183200 & 158500 & 136500 & 112100 & 117800 & 83700 & 75400 & 68300 & 14700 & 4040 \\
\hline & 184000 & 166000 & 147600 & 112800 & 114100 & 86000 & 75900 & 70400 & 15600 & - \\
\hline \multirow[t]{2}{*}{1.00} & 176800 & 140000 & 140100 & 111500 & 107000 & - & 77500 & 63500 & 16900 & 4420 \\
\hline & 175200 & 139000 & 141300 & 107300 & 103400 & 76300 & 75250 & 61200 & 20200 & 4500 \\
\hline
\end{tabular}

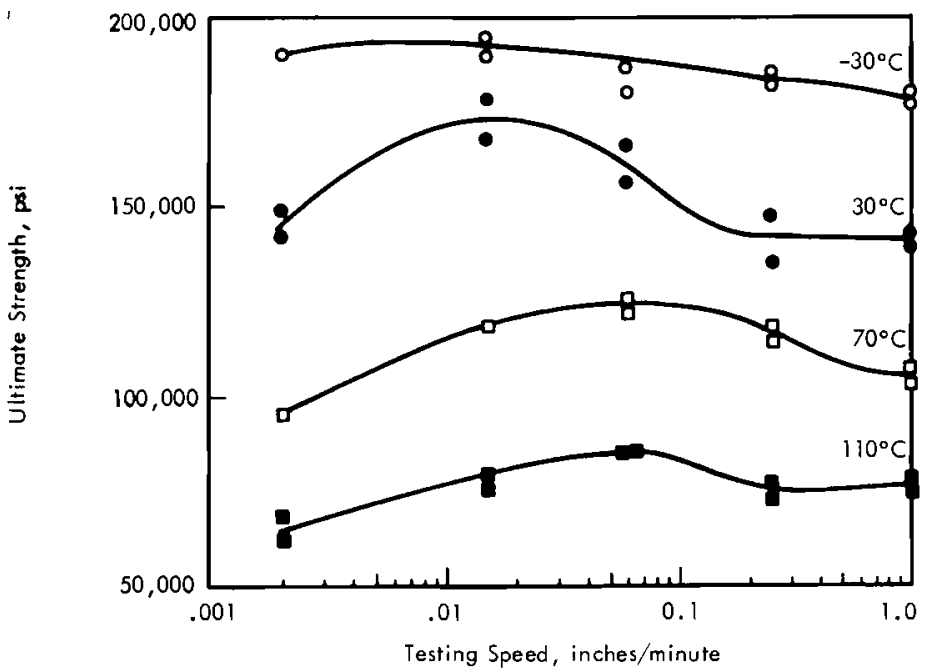

Effect of Testing Speed on Ultimate Strength in Compression. From H. R. Gardner and I. B. Mann in Plutonium 1960 , p. 550.

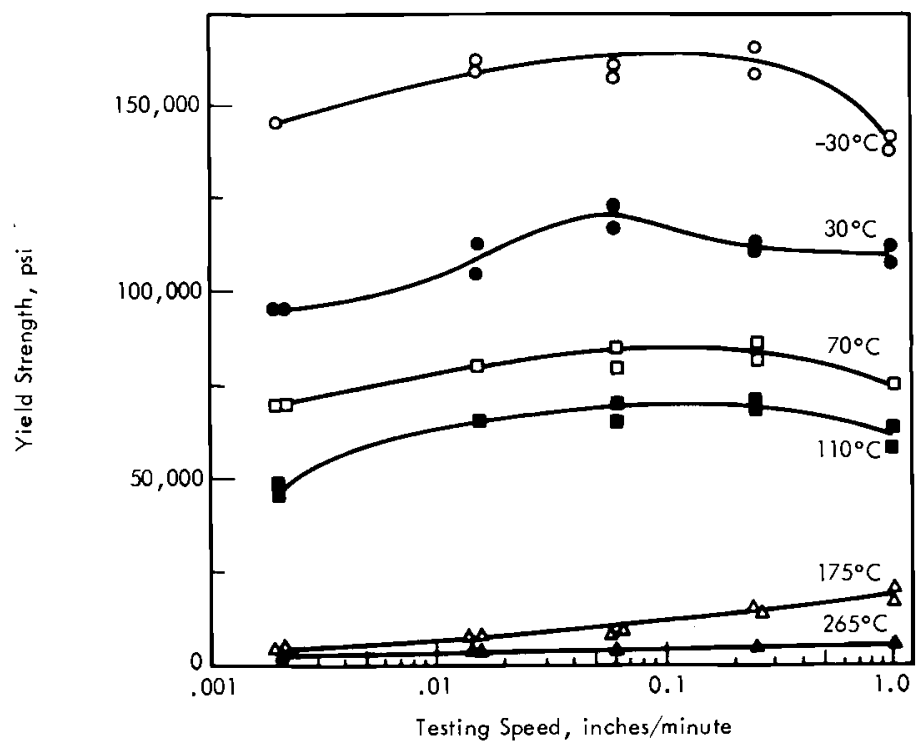

Effect of Testing Speed on Yield Strength in Compression. From H. R. Gardner and I. B. Mann in Plutonium 1960 , p. 552 

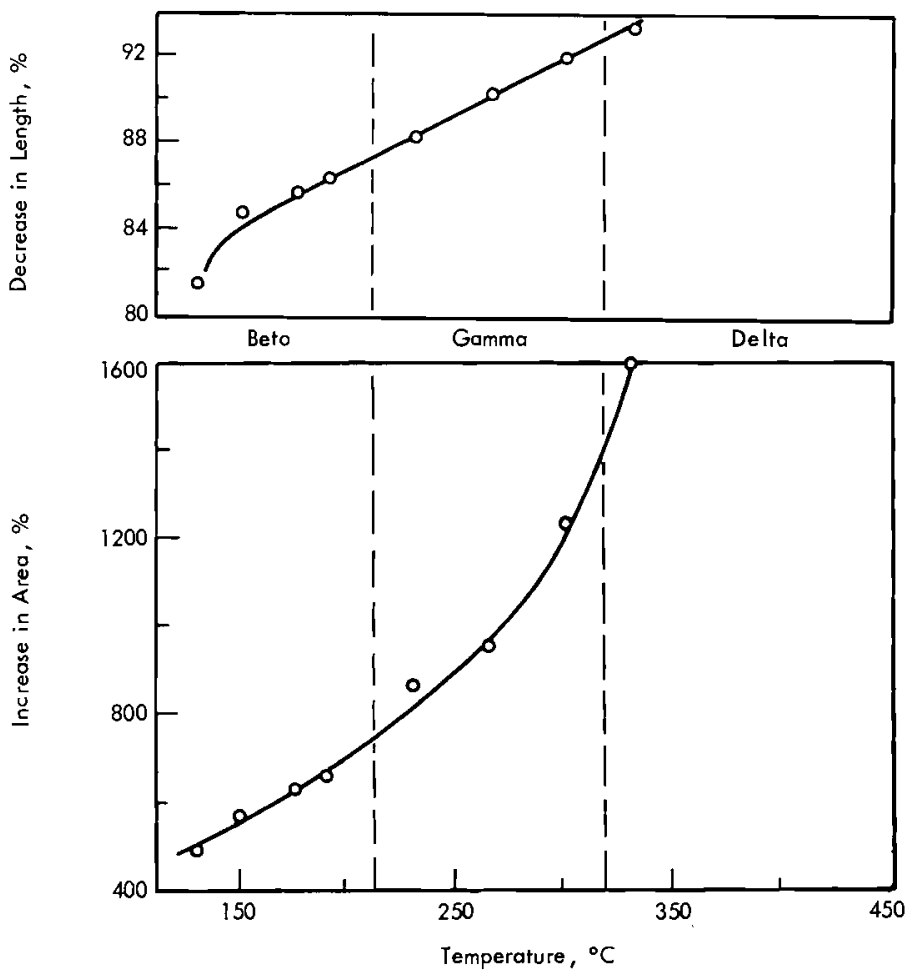

Effect of Temperature on Formability in Compression. From H. R. Gardner and I. B. Mann in Plutonium 1960 , p. 546.

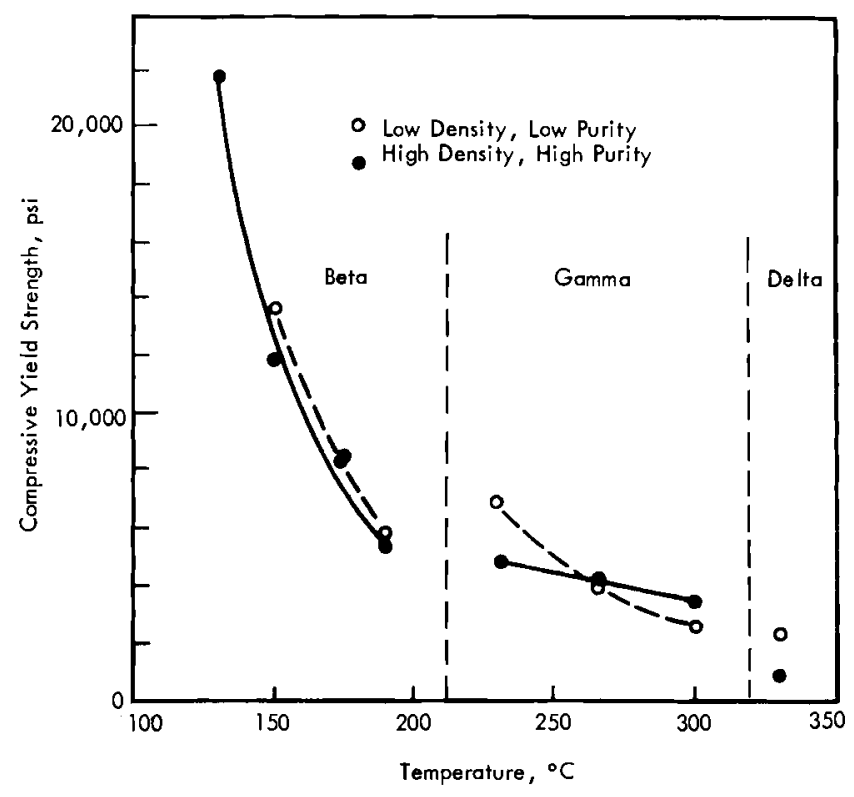

Effect of Metal Quality on Yield Strength in the,$- \boldsymbol{\gamma}$ - and - Phases. Testing speed: $0.015 \mathrm{in} / \mathrm{min}$. From H. R. Gardner and I. B. Mann in Plutonium 1960, p. 544 .

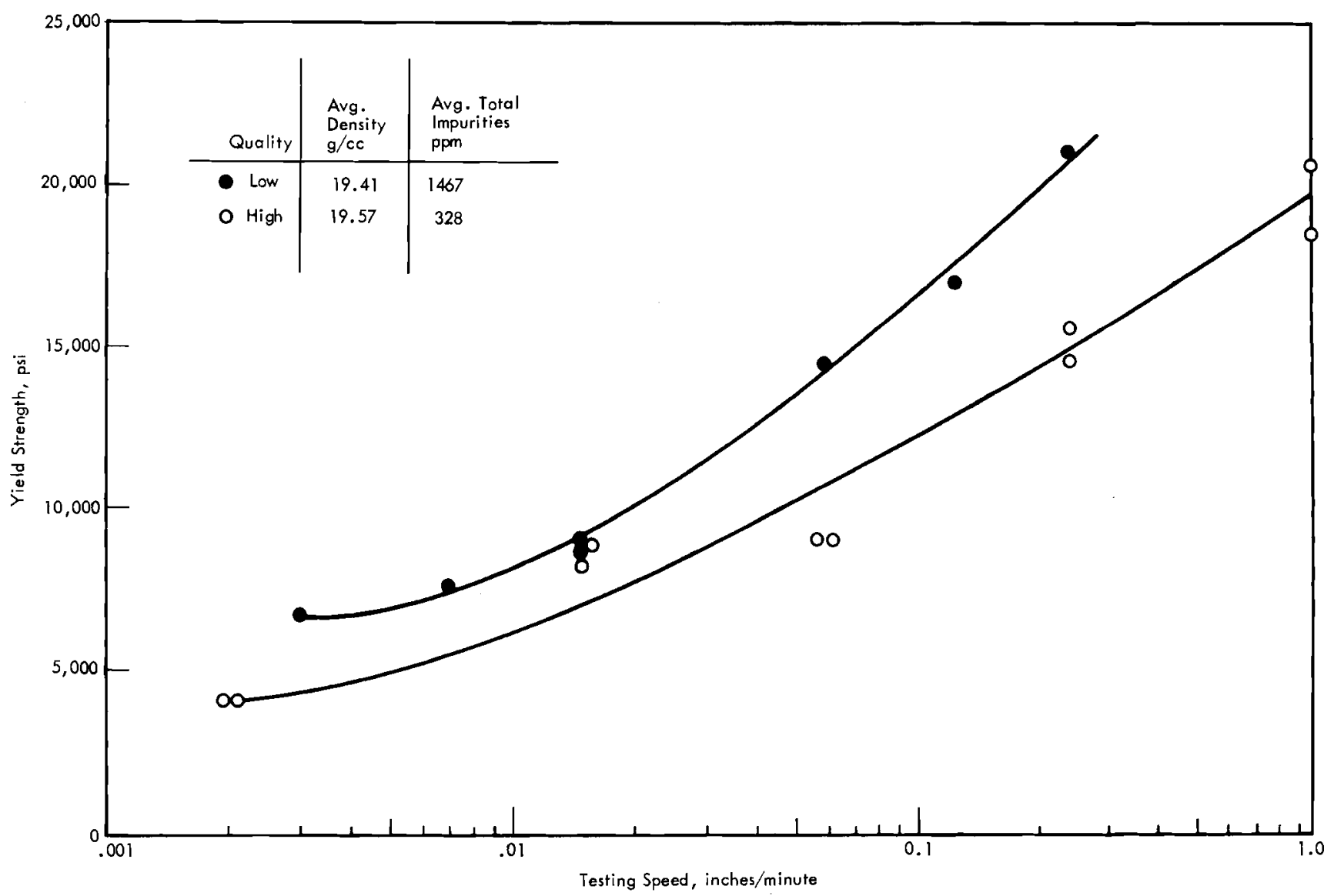

Effect of Metal Quality on Yield Strength Test Temperature, $175^{\circ} \mathrm{C}$. From H. R. Gardner and I. B. Mann in Plutonium 1960, p. 552. 


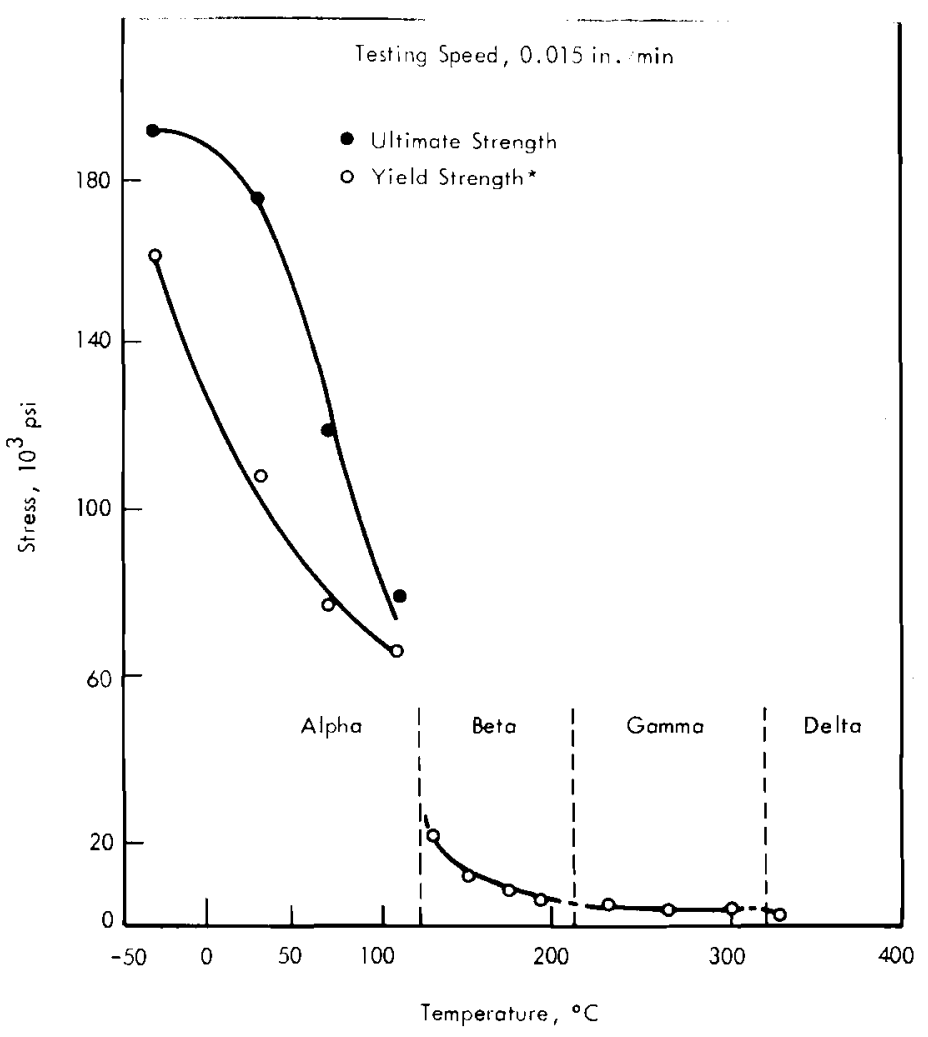

Effect of Temperature on Average Compressive Strength for High Density Plutonium. *Yield Strougth Determined as in Previous Figure. From H. R, Gardner and I. B. Nann in Plutonium 1960, p. 539.

\section{3}

\section{Torsional and Shear Properties}

Room temperature shear strength of a $\mathrm{Pl}$ :

from compression tests: $80,000 \mathrm{psi}$

from tensile tests of threaded joints: $35,000-45,000 \mathrm{psi}$

From A. S. Coffinberry et al. Reactor Handbook, 2nd ed., vol. 1, p. 266 .

Tubular torsion specimens were cast, cold trcated, and machined, then tested in a torsion testing machine at a speed of $0.01 \mathrm{RPM}$. A typical helicoidal fracture was obtained in each test bar.

Itimate Shear

Specimen Number Strength", psi Density, gice Content, l'lm

$19-6-4 B$

$19-10-8 \mathrm{~A}$

$19-10-8 B$

$19-10-8 C$

Average

Average Illtimate

Tensile Strength

46,400

54,700

53,700

57,800

53,200

50,900
The ultimate shear strength data and helicoidal fracture obtained indicate that Pu's ultimate tensile strength is less than its ultimate shear strength. Thus, failure under torsional loading occurs by tensile separation along a plane making a 45 degree angle with the plane of maximum shear.

It has been reported that the ratio of the true shear strength to true tensile strengh is in the range of 1.1 to 1.3 for brittle metals such as cast iron. Using a ratio of 1.2 and a value of 50,000 psi for the true ultimate tensile strength of Pu, an estimate of 61,100 psi can be ohtained for the true ultimate shear strength of Pu.

From H. R. Gardner and I. B. Mann in Plutonium 1960, p. 558.

\begin{tabular}{cr} 
Tension Impact & \\
Vulue, ft-lb & Density \\
\cline { 2 - 2 } 1.4 & 19.48 \\
1.6 & 19.48 \\
3.5 & $1 ! 1.41$ \\
4.2 & 19.41 \\
2.3 & 19.51 \\
4.8 & 19.51 \\
2.1 & 19.53 \\
1.3 & 19.53 \\
Average: & \\
2.6 & 19.48
\end{tabular}

Tintal Impurity content.

$+4 i$

$+7$

175.5

16.3

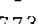




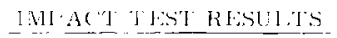

From IH. R. Ciarderer, HW-70607.

\begin{tabular}{|c|c|c|c|c|c|c|}
\hline \multirow{2}{*}{$\begin{array}{c}\text { Test } \\
\text { Temperature, } \\
{ }^{\circ} \mathrm{C} \\
\end{array}$} & \multicolumn{3}{|c|}{$\begin{array}{l}\text { Unnotched Charpy Specimens } \\
\text { Impact Energy, ft-1b }\end{array}$} & \multicolumn{3}{|c|}{$\begin{array}{l}\text { V-Notched Charpy Specimens } \\
\text { Impact Energy, ft-lb }\end{array}$} \\
\hline & Average & range & $\begin{array}{l}\text { Number } \\
\text { Jested }\end{array}$ & Average & Range & $\begin{array}{l}\text { Number } \\
\text { Tested }\end{array}$ \\
\hline-43 & 4.6 & $4.2-5.0$ & 5 & 1.8 & $1.7-1.8$ & 2 \\
\hline 30 & 6.5 & $4.5-8.8$ & 9 & 1.9 & - & 2 \\
\hline 70 & 5.6 & $5.5-5.7$ & 2 & - & - & - \\
\hline 100 & 6.9 & $5.1-10.0$ & 10 & 3.1 & $2.7-3.5$ & 3 \\
\hline 130 & 9.9 & $8.2-12.9$ & 3 & 1.9 & $1.8-2.0$ & 2 \\
\hline 175 & 7.7 & $6.5-9.0$ & 3 & 1.8 & $1.7-2.0$ & 4 \\
\hline 230 & $*$ & - & 1 & 11.1 & $9.9-13.5$ & 5 \\
\hline 300 & - & - & - & 11.0 & $9.0-13.8$ & 4 \\
\hline 330 & - & - & - & " & - & 1 \\
\hline
\end{tabular}

: Specimen bent in U-shape and did not break

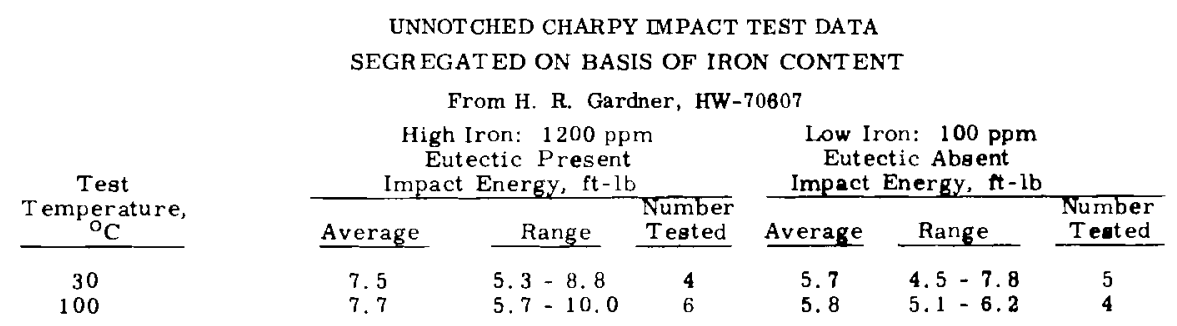

From H. R. Gardner, Trans. Met. Soc. AIME, vol. 224, p. 201.1962.

\begin{tabular}{|c|c|c|c|}
\hline Iron & Impurities Total & Density, $\mathrm{g} / \mathrm{cc}$ & Iron Class. \\
\hline 268 & 431 & 19.67 & Low \\
\hline L50 & 288 & 19.64 & Low \\
\hline 1142 & 1377 & 19.54 & High \\
\hline 80 & 303 & - & Low \\
\hline 1200 & 1560 & 19.52 & High \\
\hline
\end{tabular}

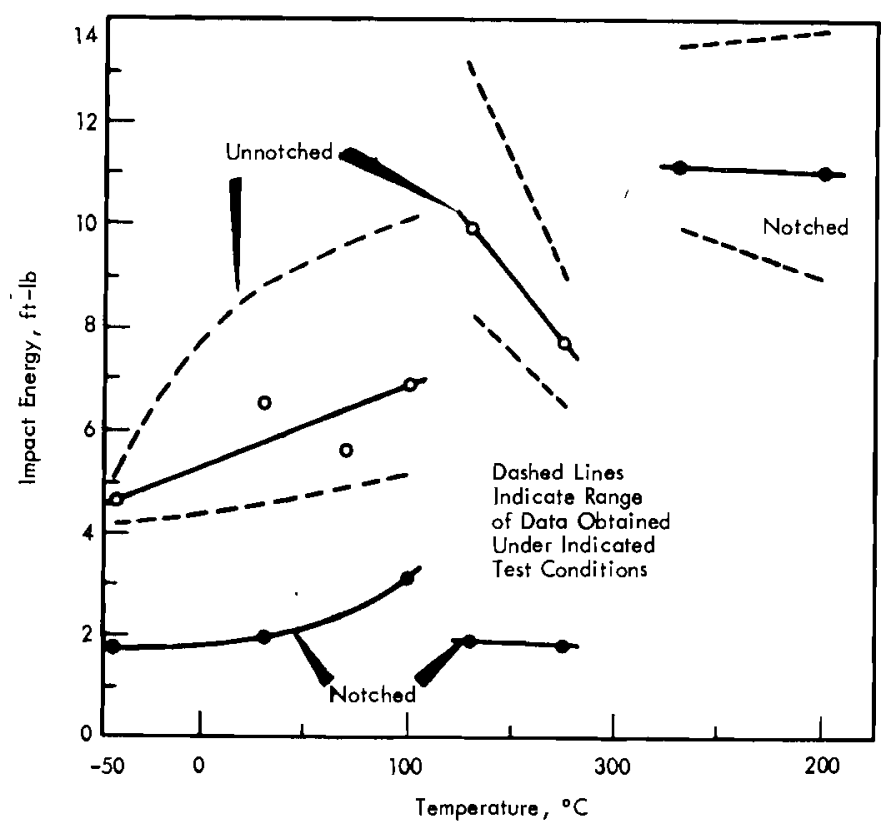

Effect of Temperature on Unnotched and Notched Impact Properties of Plutonium. From H. R. Gardner. Trans. Met. Soc. ALME, vol. 224 p. 201. 1962 .

From H. R. Gardner and I. B. Mann in Plutonium 1960, p. 557.

\begin{tabular}{|c|c|c|c|c|c|c|c|}
\hline $\begin{array}{l}\text { Specimen } \\
\text { Number }\end{array}$ & $\begin{array}{c}\text { Test } \\
\begin{array}{c}\text { Temperature, } \\
{ }^{\circ} \mathrm{C}\end{array} \\
\end{array}$ & $\begin{array}{l}\text { Applied } \\
\text { Stress. } \\
\text { lb/in. } \\
\end{array}$ & $\begin{array}{c}\text { Secondary } \\
\text { Creep } \\
\text { Rate } * \\
\end{array}$ & $\begin{array}{l}\text { Total } \\
\text { Strain } \\
\left(10^{-4}\right) \\
\end{array}$ & $\begin{array}{c}\text { Duration } \\
\text { of Test, } \\
\mathrm{hr} \\
\end{array}$ & $\begin{array}{l}\text { Density } \\
\mathrm{g} / \mathrm{cm}^{3} \\
\end{array}$ & $\begin{array}{l}\text { Total } \\
\text { Impurity } \\
\text { Content, } \\
\text { ppm }\end{array}$ \\
\hline $\begin{array}{l}18-10-12 A \\
19-3-2 A \\
19-3-2 A \\
18-10-12 B\end{array}$ & $\begin{array}{r}31.0 \\
26.0 \\
42.3 \\
100.0\end{array}$ & $\begin{array}{r}10000 \\
8000 \\
8000 \\
10000\end{array}$ & $\begin{array}{l}15.0 \\
15.2 \\
28.2 \\
330.0\end{array}$ & $\begin{array}{c}4.2 \\
4.3 \\
- \\
41.0\end{array}$ & $\begin{array}{r}1370 \\
1850 \\
870 \\
570\end{array}$ & $\begin{array}{l}19.43 \\
19.49 \\
19.49 \\
19.43\end{array}$ & $\begin{array}{l}520 \\
486 \\
486 \\
425\end{array}$ \\
\hline
\end{tabular}




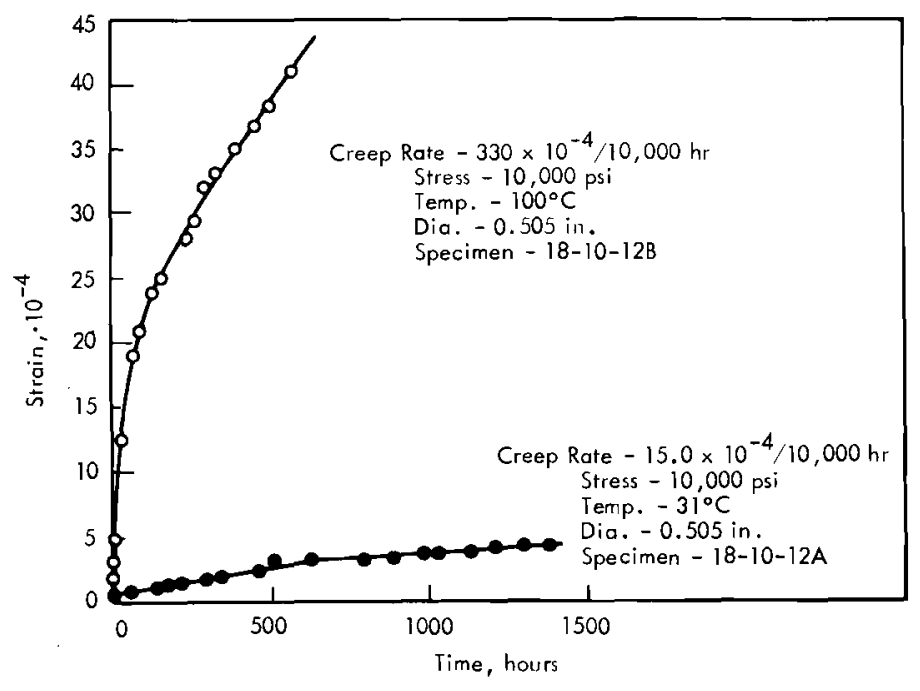

a -Plutonium Creep curves. I'mm H. R Fardncr and I. B. Mann in Plutonium $\underline{1960}$, p. 557 .

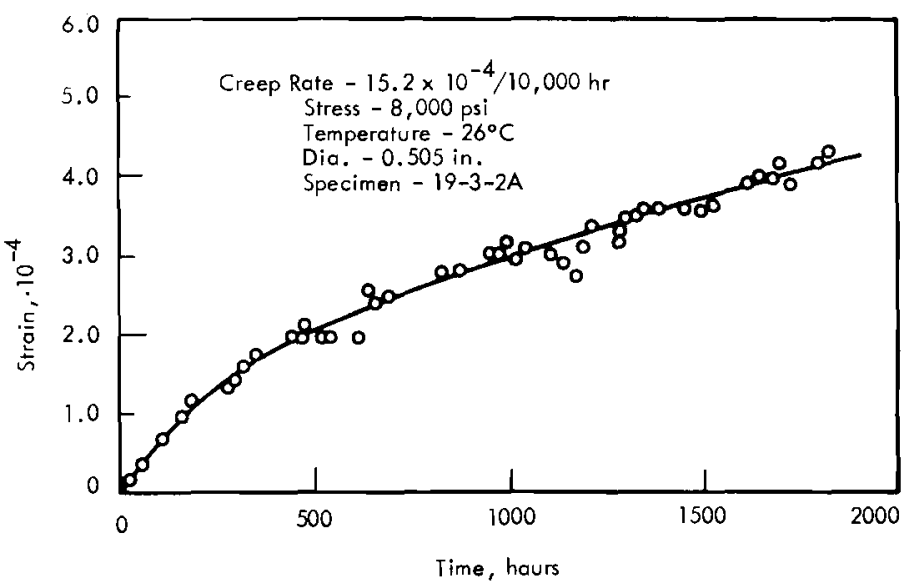

a-Plutonium Creep Curve. From H. R. Gardner and I. B. Mann in Plutonium 1960, p. 557.

('iripl rate (ander a 6 pil load)

Delta Pha (450) (d) No (reep occurs in $2 \mathrm{hr}$

l:psilon ma (inon: :-"C) $1.3 \times 10^{-3} / \mathrm{hr}$

From W. 11. Wilkinsen in lixtractive and Physical Metallurgy of Plutonium and

It Allngs, pl. $1-24$.

\section{Fatigue}

\section{rIIGMU"11. AVALYSES" AND DENSITY DATA}

From H. R. Hotratit, ]. Nurl. Mater, vol. 10, p. 259.1963.

\begin{tabular}{|c|c|c|c|c|c|c|c|c|c|c|}
\hline & $\therefore$ & $C^{\prime} \mathrm{I}$ & (iti & Fe & $\lg$ & $\mathrm{Mn}$ & $\mathrm{Ni}$ & $\mathrm{Si}$ & C & Density, $\mathrm{g} / \mathrm{cm}^{3}$ \\
\hline Mirtiaimoli & $<2$ & 15 & $\therefore i$ & 133 & 2 & 10 & 5 & $<2$ & 120 & 19.40 \\
\hline Naxjomon & 20 & 40 & 20 & $10+0$ & 25 & 40 & 200 & $<5$ & 500 & 19.64 \\
\hline litet age & 10 & 30 & 11 & ti2: & 11 & 24 & 83 & $<5$ & 310 & 19.53 \\
\hline
\end{tabular}

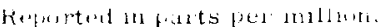

Whe fatipue results which fall on ol near the curve were obtained with good cuality specimens in which microcracking had been eliminated by the addilioul of (ivi-1060 pum the specimens failing below $20,000 \mathrm{psi}$ and from $10^{4}$ to $3.5 \times 10^{-1}$ eycles contained moderate microeracking. All speeifrom $10^{4}$ to $3.5 \times 10^{4}$ eycles contained moderate microeracking. All spect
nons fating below $26,000 \mathrm{nsi}$ and $10^{4}$ cyeles contained extensive nicromons failing below 2 i,, 000 psi and $10^{4}$ cyeles contained extensive nicro-
cracking, particularly those failing on the first cycle shown at the left hand sides of the figure.

The fatigue Iimit a usears to be approximately $14,000 \mathrm{psi}$ which is of the oriler of $20^{\prime \prime}$. of the ultimate tensile strength.

From H. R. Gardner, J Nucl. Mater., vol. 10, p. 259. 1963. 


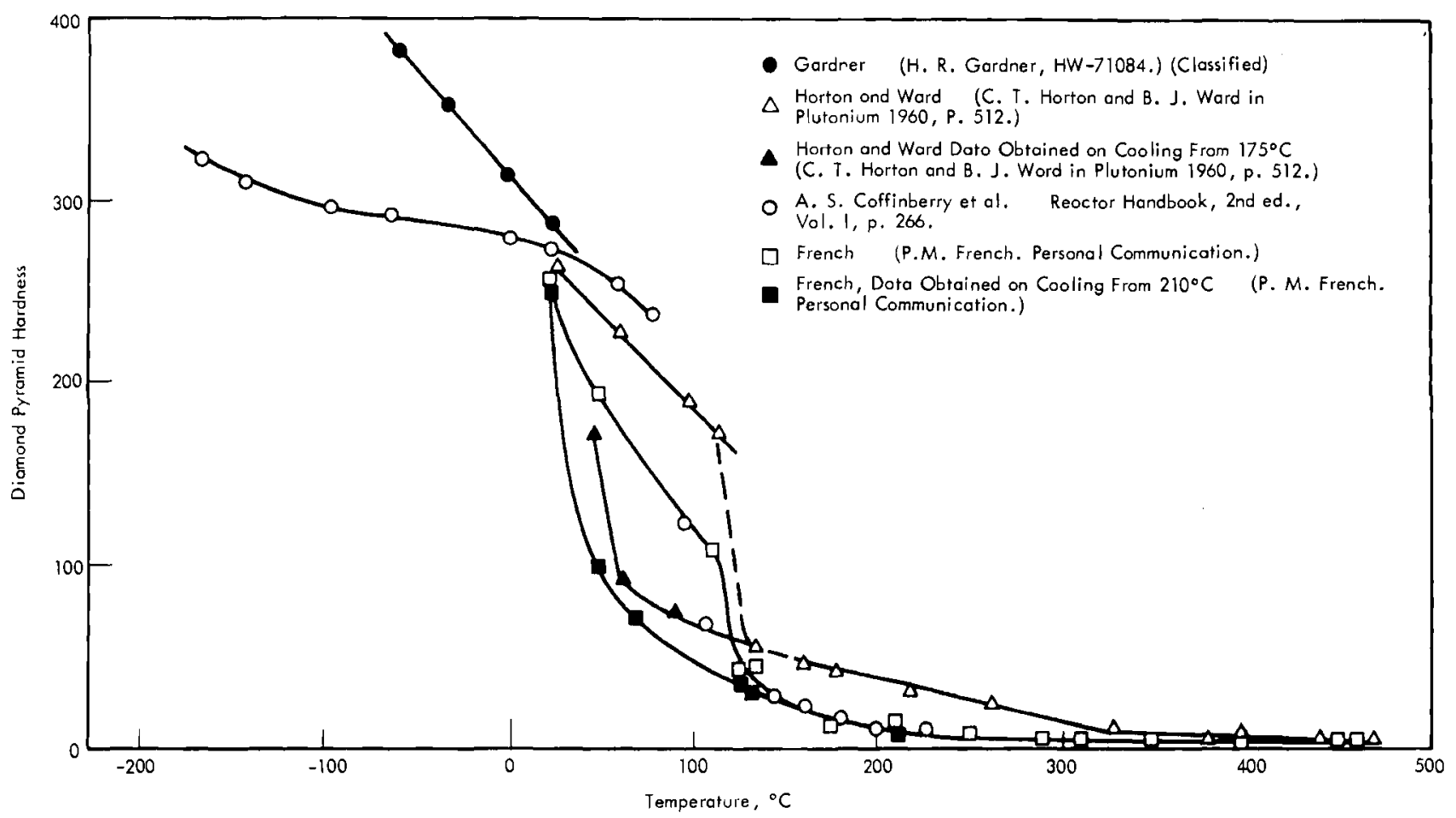

Compilod by ll, R. Garther, Ifanford Atomic products Operation, General

Filertile (cin. latit.

Microhardness of $\bullet \mathrm{Pu}_{\mathrm{u}}: 223-250 \mathrm{~kg} / \mathrm{mm}^{2}$

From W. D. Wilkinson in Fxtractive and Physical Metallurgy of Plutonium and

Its Alloys, p. 15

\section{Elastic Properties}

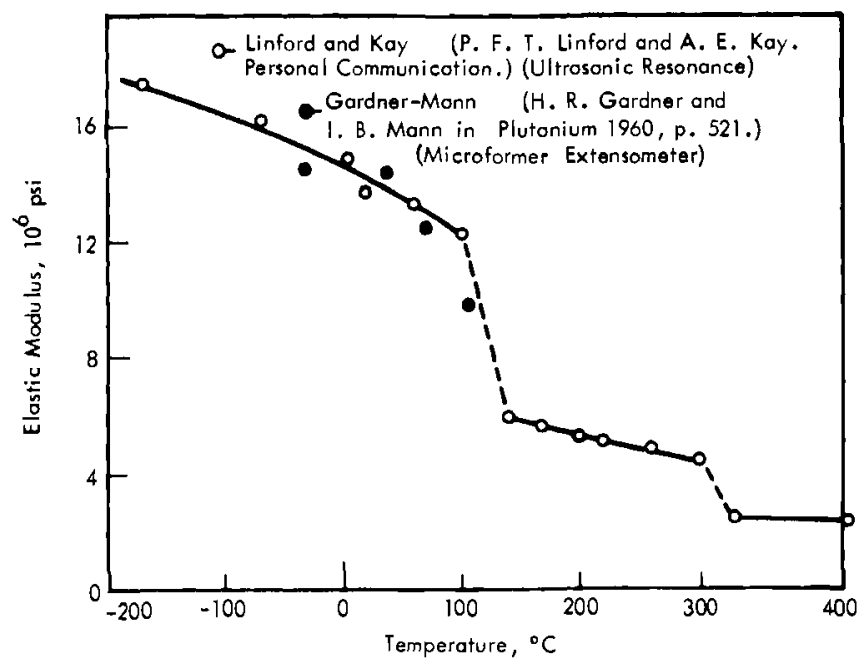




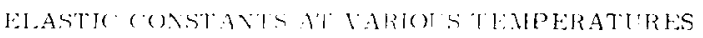

P. F. T. L inford and A, L. Ka, lersonit communication.

\begin{tabular}{|c|c|c|c|c|c|}
\hline $\begin{array}{c}\text { Temperature. } \\
{ }^{\circ} \mathrm{C} \\
\end{array}$ & Phase & $\begin{array}{l}\text { Nodulus of } \\
\text { litisticity } \\
10^{6} \text { psi }\end{array}$ & $\begin{array}{l}\text { Shear } \\
\text { Mordulus. } \\
10^{6} \text { psi } \\
\end{array}$ & $\begin{array}{c}\text { Poisson's } \\
\text { Ratio } \\
\end{array}$ & $\begin{array}{l}\text { Bulk Modulus, } \\
10^{6} \text { psi }\end{array}$ \\
\hline-170 & Alpha & 17.35 & 7.26 & 0,20 & 9.63 \\
\hline-80 & Alpha & 16.00 & 6. 63 & 0.20 & 8.90 \\
\hline 0 & Alpha & 14.65 & 6.17 & 0.20 & 8.18 \\
\hline 20 & Alpha & $1+.00$ & 6.08 & 0.16 & 6.88 \\
\hline 60 & Alpha & 13.30 & 5.80 & 0.15 & 6.33 \\
\hline 100 & Alpha & 12.40 & 5.45 & 0.14 & 5.76 \\
\hline 140 & Beta & 5.90 & 2.29 & 0.29 & 4.68 \\
\hline 170 & Beta & 5.58 & 2.20 & 0.27 & 4.05 \\
\hline 200 & Beta & 5.37 & 2. 12 & 0.27 & 3.80 \\
\hline 220 & Gamma & 5.28 & 2.13 & 0.24 & 3.38 \\
\hline 260 & Gamma & 4.94 & 1.97 & 0.25 & 3.29 \\
\hline 300 & Gamma & 4.58 & 1.52 & 0.25 & 3.06 \\
\hline 330 & Delta & 2.49 & 0.87 & 0.43 & 5.9 \\
\hline 380 & Delta & 2.45 & 0.84 & 0.45 & 8.1 \\
\hline 430 & Delta & 2.39 & 0.81 & 0.42 & 4.9 \\
\hline
\end{tabular}

ROOM TEMPERATURE $\left(20^{\circ} \mathrm{C}\right)$ ELASTIC CONSTANTS

P. F. T. Linford and A. E. Kay, Personal Communication.

\begin{tabular}{|c|c|c|c|c|c|c|}
\hline Specimen & Remarks & $\begin{array}{l}\text { Density } \\
\mathrm{g} / \mathrm{cm}^{3}\end{array}$ & $\begin{array}{r}\text { Modulus of } \\
\text { Elasticity, } \\
10^{6} \mathrm{psi} \\
\end{array}$ & $\begin{array}{c}\text { Shear } \\
\text { Modulus, } \\
10^{6} \mathrm{psi} \\
\end{array}$ & $\begin{array}{c}\text { Poisson's } \\
\text { Patio }\end{array}$ & $\begin{array}{c}\text { Bulk } \\
\text { Modulus, } \\
10^{6} \text { psi }\end{array}$ \\
\hline E1 & As-Cast & 19.50 & 14.15 & 6.02 & 0.175 & 7.26 \\
\hline E3 & As-Cast & 19.50 & 14.17 & 6.02 & 0.177 & 7.32 \\
\hline $\mathrm{E} 4$ & As-Cast & 19.48 & 14.26 & 6.03 & 0.182 & 7.47 \\
\hline F 2 & As-Cast & 19.45 & 14,00 & 6.00 & 0.171 & 7.08 \\
\hline F3 & As-Cast & 19,50 & 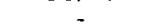 & 6.08 & - & - \\
\hline E5 & High Density & 19.71 & 14.60 & 6.12 & 0.186 & 7.74 \\
\hline $\mathrm{E} 6$ & High Density & 19.74 & 14.73 & - & - & - \\
\hline
\end{tabular}

Pu $99.5 \%$ pure or better, density $19.35 \mathrm{~g} / \mathrm{cm}^{3}$

\begin{tabular}{|c|c|}
\hline \multicolumn{2}{|c|}{ Velocity of longitudinal waves: $2370 \pm 7 \mathrm{~m} / \mathrm{sec}$} \\
\hline \multicolumn{2}{|c|}{ Velocity of transverse waves $=1453 \pm 4 \mathrm{~m} / \mathrm{sec}$} \\
\hline Young's moduius & $=(9.825 \pm 0.075) \times 10^{11}$ dynes $/ \mathrm{cm}^{2}$ \\
\hline $\begin{array}{l}\text { Modulus of transverse } \\
\text { rigidity }\end{array}$ & $=(4.080 \pm 0.040) \times 10^{11}$ dynes $/ \mathrm{cm}^{2}$ \\
\hline Poisson's ratio & $=0.199 \pm 0.002$ \\
\hline Compressive strength & $=(5.44 \pm 0.06) \times 10^{11}$ dynes \\
\hline & - from J. J. DeCadenet in Plutonium \\
\hline
\end{tabular}

$\underline{1960,}$, p. 107.

SUMMARY OF RESULTS AND EXTRAPOLATION TO X-RAY DENSITY AT $30^{\circ} \mathrm{C}$

From H. L. Laquer in The Metal Plutonium. p. 174.

\begin{tabular}{|c|c|c|c|c|c|}
\hline Quantity & Symbol & Range of Obse & erved Values & $\begin{array}{l}\text { Ext rapolation to } \\
\mathrm{X} \text {-Ray Density }\end{array}$ & Units \\
\hline Density & 8 & $(18.8) 19.0$ & -19.72 & 19.790 & $\mathrm{gm} / \mathrm{cm}_{3}$ \\
\hline Rod velocity & ${ }^{{ }} \mathrm{O}$ & 2.17 & -2.26 & $2.25_{5} \pm 0.01_{5}$ & $\times 10^{5} \mathrm{~cm} / \mathrm{sec}$ \\
\hline Inf. med, velocity & $\mathrm{v}_{\mathrm{L}}$ & 2.27 & -2.30 & $2.31_{3}+0.01$ & $\times 10^{5} \mathrm{~cm} / \mathrm{sec}$ \\
\hline Shear velocity & $v_{T}$ & $(1.37) 1.40$ & -1.49 & 1.4900 .007 & $\times 10^{5} \mathrm{~cm} / \mathrm{sec}$ \\
\hline Poisson's ratio & $\checkmark$ & $\begin{array}{l}0.14 \\
8.9\end{array}$ & $\begin{array}{l}-\quad 0.20 \\
-\quad 10.1\end{array}$ & $\begin{aligned} 0.14_{5} & +0.01 \\
10.0_{2} & \div 0.1_{3}\end{aligned}$ & $\times 10^{11}$ dynes/cm ${ }^{2}$ \\
\hline Young's modulus & $\mathrm{E}$ & 12.9 & -14.6 & $14.5_{4}^{2}+0.2^{5}$ & $\times 10^{6} \mathrm{psi}$ \\
\hline Shear modulus & is & $\begin{cases}(3.5) & 3.8 \\
(5.1) & 5.5\end{cases}$ & $\begin{array}{l}-4.3 \\
-\quad 6.2\end{array}$ & $\begin{array}{l}4.37 \\
6.34_{6}^{4} \pm 0.04 \\
\pm 0.06\end{array}$ & $\begin{array}{l}\times 10^{11} \text { dynes } / \mathrm{cm}^{2} \\
\times 10^{6} \text { psi }\end{array}$ \\
\hline Adiabatic compressibility & ${ }^{x} \mathrm{~s}$ & $\begin{array}{l}1.8 \\
1.8\end{array}$ & $\begin{array}{l}-2.2 \\
-\quad 2.2\end{array}$ & $\begin{array}{l}2.1_{2}^{2} \pm 0.1_{4}^{4} \\
2.1_{5} \pm 0.1_{4}\end{array}$ & $\begin{array}{l}\times 10^{-12} \mathrm{~cm}^{2} / \text { dyne } \\
\times 10^{-6}(\mathrm{dtm})^{-1}\end{array}$ \\
\hline Isothermal compressibility & ${ }^{x_{T}}$ & 2.1 & -2.5 & $2.4_{1} \pm 0.2$ & $\times 10^{-12} \mathrm{~cm}^{2} /$ dyne \\
\hline
\end{tabular}


APPROXIMATE TEMPERATURE COEFH ICINTHSAT:30 ${ }^{\circ}$ Reciprocal ${ }^{C} \times 10^{5}$

From H. L. Laquer in The Metal Plutonium, p. 178.

\begin{tabular}{|c|c|c|c|}
\hline Quantity & Low Purity & High Purity & Source \\
\hline $\begin{array}{l}\mathrm{L} \\
\mathrm{C}\end{array}$ & $\begin{array}{r}+5.127 \\
-15.381\end{array}$ & $\begin{array}{r}+5.127 \\
-15.381\end{array}$ & $\begin{array}{l}\text { Measured (10) } \\
\text { Derived from above }\end{array}$ \\
\hline $\mathrm{v}_{\mathrm{O}}$ & $-56 \pm 4$ & $-93 \pm 7$ & Measured \\
\hline $\mathrm{v}_{\mathrm{T}}$ & $-65 \pm 5$ & $-99 \pm 5$ & Measured \\
\hline$v_{L}$ & $-48 \pm 6$ & $-88+1 r$ & Derived \\
\hline 0 & $+138 \pm 70$ & $+90 \pm 130$ & Derived \\
\hline $\begin{array}{l}E \\
\mu \\
x_{S}\end{array}$ & $\begin{array}{r}-127 \pm 8 \\
-145 \pm 10 \\
+68 \pm 30\end{array}$ & $\begin{array}{l}-201+14 \\
-213+10 \\
+166+70\end{array}$ & $\begin{array}{l}\text { Derived } \\
\text { Derived } \\
\text { Derived }\end{array}$ \\
\hline
\end{tabular}

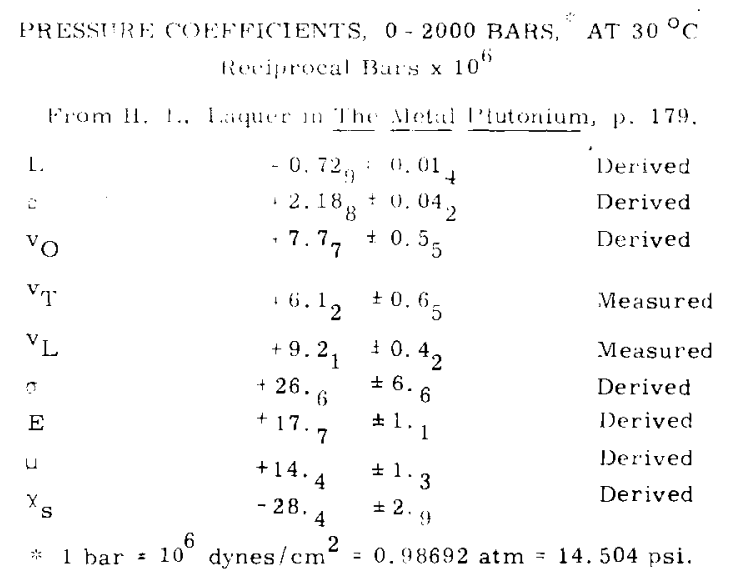

ELASTIC PROPERTIES AND SOUND VELOCITIES OF ALPHA PLUTONIUM AT $30^{\circ} \mathrm{C}$,

From H. L. Laquer in Metallurgy and Fuels, Progress in Nuclear Energy,

series $\mathrm{V}$, vol. 1, p. 383 .

\section{Property}

1. Young's modulus

2. Shear modulus

3. Poisson's ratio

4. Compressibility

5. Rod velocity ( $\mathrm{v}_{\mathrm{O}}$ )

6. Shear velocity $\left(v_{T}\right)$

7. Infinite medium velocity ( $v_{L}$ )

* Measurements by pulse technique on sample having $\Omega=19.722 \pm 0.005$ at $30^{\circ} \mathrm{C}$.

+ Measurements by pulse technique on sample having $\theta=19.62$ at $30^{\circ} \mathrm{C}$.

++ Measurements by resonance technique on two different samples ( $0=19.5$ and 19.6).

ROOM TEMPERATURE EI ASTIC CONSTANT DATA FROM VARIOUS SOURCES

P. F. T. J.inford and A. E. Kiay, Personal Communication

\begin{tabular}{|c|c|c|c|c|c|c|c|c|}
\hline Source & $\begin{array}{l}\text { Density } \\
\text { g/ } \mathrm{cm}^{3} \\
\end{array}$ & $\begin{array}{c}\text { Modulus ol } \\
\text { Elasticity } \\
10^{6} \text { psi } \\
\end{array}$ & $\begin{array}{l}\text { Shorl } \\
\text { Mndishs } \\
10^{f i} p s i \\
\end{array}$ & $\begin{array}{l}\text { Poisson's } \\
\text { Ratio } \\
\end{array}$ & $\begin{array}{l}\text { IBulk } \\
\text { Nodulss. } \\
10^{\mathrm{f}} \mathrm{psi} \\
\end{array}$ & $\begin{array}{c}\text { Adiahatic } \\
\text { Compressibilif } \\
10^{-7} \text { in. } 2: 1 \mathrm{~b}\end{array}$ & $\begin{array}{l}\text { Iwothermal } \\
\text { Compressibility, } \\
10-7 \text { in }\end{array}$ & Method \\
\hline $\begin{array}{l}\text { Linford }(1) \\
\text { (mean as-cast) }\end{array}$ & 19.49 & 14.15 & (i) 11 & $0.17 \mathrm{it}$ & 7.29 & 1.36 & 151 & Resomance \\
\hline $\begin{array}{l}\text { Linford } \\
\text { (high density) }\end{array}$ & 19.71 & 14.60 & 6.13 & 0.186 & 775 & 1.30 & 144 & Resonitues \\
\hline $\begin{array}{l}\text { Laquer (2) } \\
\text { (high density) }\end{array}$ & 19.72 & 14.40 & fi. 30 & 0.15 & fi. 77 & 1.48 & 1.62 & Pulse \\
\hline $\begin{array}{l}\text { DeCadenet } \\
\text { (as-cast) }\end{array}$ & 19.35 & 14.25 & 5.43 & 0.159 & $7: 10$ & 1.27 & $1 .+1$ & l'u!so \\
\hline Bridgman (4) & - & - & - & - & - & - & 1. 38 & cimpressibitits \\
\hline Robinson (5) & 19.6 & - & - & - & - & - & 1. 32 & (i) rupressibilla \\
\hline Gardner $(6)$ & 19.53 & 14.32 & - & - & - & - & - & Tensile Trest \\
\hline
\end{tabular}

(1) P. F. T. Linford, Personal Communication.

(2) H. I.. L.aquer, quoted in Metallurgy and Fuels, Progress in Nuclear Energy, series V, vol. 1, p. 383.1956

(3) I. I. Decadenet, in Plutonium 1960, p. 107.

(4) P'. W. Bridgman, J. Appl. Phys., vol. 30, p. 214.1959.

(5) A. C. Robinson, Personal Communication.

(6) H. R. Gardner, in Plutonium 1960, pp. 513-570. 


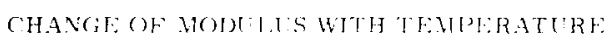

P. F. T. Linford and A. li. Kay. Persomis: Communisation

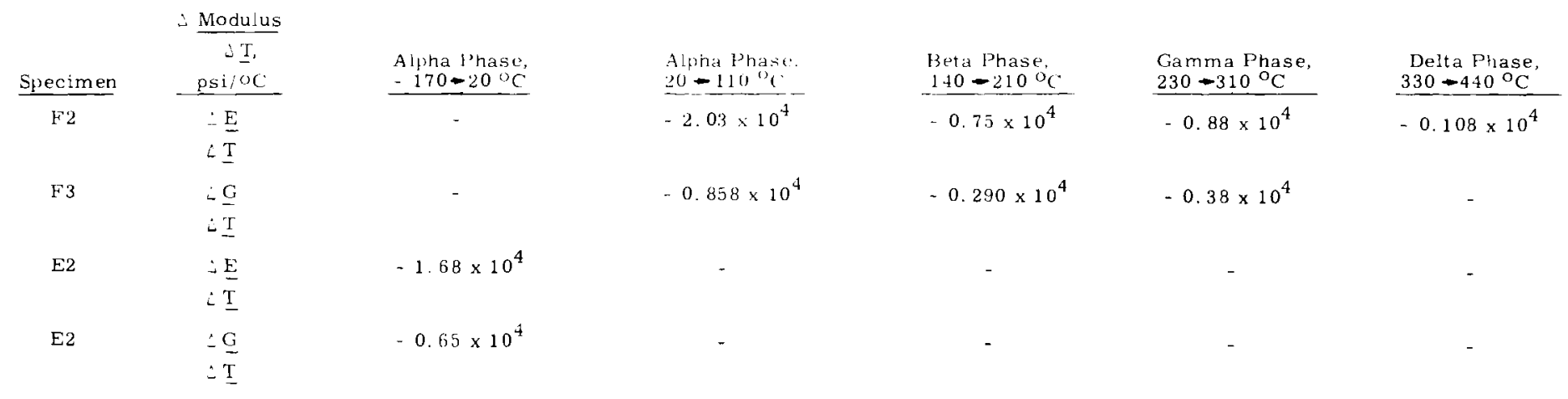

ELASTIC CONSTANT DATA CORRECTED FOR POROSITY

P. F. T. Linford and A. E. Kay. Personal Communication

\begin{tabular}{|c|c|c|c|c|c|}
\hline Specimen & $\begin{array}{l}\text { Density, } \\
\mathrm{g} / \mathrm{cm}^{3}\end{array}$ & $\begin{array}{l}\text { Modulus of } \\
\text { Elasticity. } \\
10^{6} \text { psi }\end{array}$ & $\begin{array}{l}\text { Shear } \\
\text { Modulus, } \\
10^{6} \text { psi } \\
\end{array}$ & $\begin{array}{c}\text { Bulk } \\
\text { Modulus, } \\
10^{6} \text { psi } \\
\end{array}$ & $\begin{array}{c}\text { Isothermal } \\
\text { Compressibility } \\
10^{-7} \text { in. } 2 / 1 \mathrm{~b}\end{array}$ \\
\hline $\mathrm{E} 3$ & 19.50 & 14.63 & 6.22 & 7.62 & 1.45 \\
\hline E5 & 19.71 & 14.73 & 6.22 & 7.80 & 1.42 \\
\hline
\end{tabular}

ROOM TEMPERATURE $\left(20^{\circ} \mathrm{C}\right)$ DATA

From A. E. Kay and P. F. T. Linford in Plutonium 1960, p. 56.

\begin{tabular}{|c|c|c|c|c|c|c|c|c|}
\hline Specimen & Remarks & Density, $\mathrm{g} / \mathrm{cm}^{3}$ & Length, $\mathrm{cm}$ & Diameter, $\mathrm{cm}$ & $\begin{array}{l}\text { Young's Modulus } \\
\text { E, dyn } / \mathrm{cm}^{2} \times 10^{-11}\end{array}$ & $\begin{array}{l}\text { Rigidity Modulus } \\
\mathrm{G}, \mathrm{dyn} / \mathrm{cm}^{2} \times 10^{-11} \\
\end{array}$ & $\begin{array}{c}\text { Poisson's Ratio } \\
\sigma \\
\end{array}$ & $\begin{array}{l}\text { Bulk Modulus } \\
\mathrm{K}, \text { dyn } / \mathrm{cm}^{2} \times 10^{-11} \\
\end{array}$ \\
\hline E1 & As-cast & $19.50 \pm 0.02$ & 2.541 & 0.30 & $9.76 \pm 0.01$ & $4.15 \pm 0.005$ & $0.175 \pm 0.005$ & $5.00 \pm 0.03$ \\
\hline E3 & As-cast & 19.50 & 2.542 & 0.30 & 9.77 & 4.15 & 0.177 & 5.04 \\
\hline $\begin{array}{l}\text { E4 } \\
\text { E5 }\end{array}$ & $\begin{array}{l}\text { As-cast } \\
\text { High density }\end{array}$ & $\begin{array}{l}19.48 \\
19.71\end{array}$ & $\begin{array}{l}2.250 \\
2.021\end{array}$ & $\begin{array}{l}0.30 \\
0.31\end{array}$ & $\begin{array}{r}9.84 \\
10.04\end{array}$ & $\begin{array}{l}4.17 \\
4.23\end{array}$ & $\begin{array}{l}0.181 \\
0.186\end{array}$ & $\begin{array}{l}5.14 \\
5.33\end{array}$ \\
\hline E6 & High density: & 19.74 & 1.502 & 0.30 & 10.17 & - & - & - \\
\hline $\begin{array}{l}\mathrm{E} 6 \\
\mathrm{E} 6\end{array}$ & $\begin{array}{l}\text { After } 1 \text { cycle } \\
\text { After } 2 \text { cycles }\end{array}$ & $\begin{array}{l}19.5 * \\
19.3 *\end{array}$ & $\begin{array}{l}1.53 \\
1.57\end{array}$ & - & $\begin{array}{l}9.55 \\
8.75\end{array}$ & - & - & - \\
\hline E6 & After 3 cycles & $19.1 \%$ & 1.57 & - & - & 3.45 & - & - \\
\hline
\end{tabular}

\footnotetext{
Estimated
} 


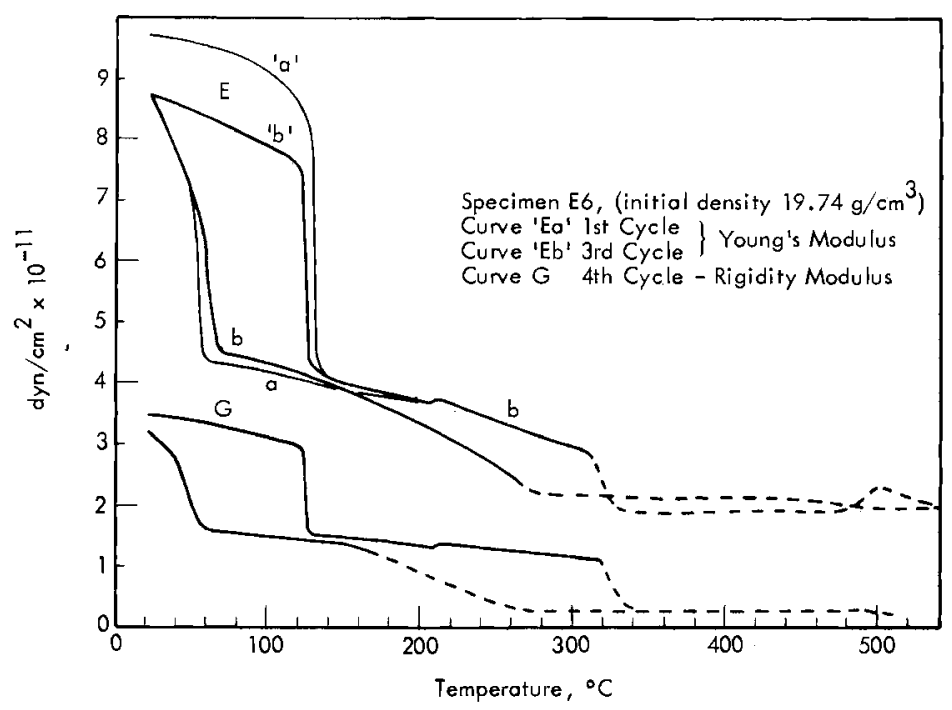

Variation of $\mathrm{E}$ and $\mathrm{G}$ with Temperature, From A. E. Kay and P. F. T. Linford in Plutonium 1960, p. 57.

ELASTIC CONST ANTS AT VARIOUS TEMPERATURES

From A. E. Kay and P. F. T. Linford in Plutonium 1960, p. 58.

\begin{tabular}{|c|c|c|c|}
\hline$\underset{{ }^{\circ} \mathrm{C}}{\text { Temperature, }}$ & Phase & 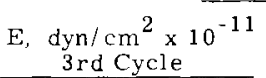 & $\begin{array}{l}\mathrm{G}, \mathrm{dyn} / \mathrm{cm}^{2} \times 10^{-11} \\
\text { 4th Cycle }\end{array}$ \\
\hline 21.5 & $a$ & 8.75 & 3.45 \\
\hline 60 & $a$ & 8.34 & 3.32 \\
\hline 100 & a & 7.91 & 3.14 \\
\hline 150 & $\beta$ & 3.97 & 1.42 \\
\hline 190 & $\beta$ & $3: 76$ & 1.35 \\
\hline 230 & $\gamma$ & 3.57 & 1.33 \\
\hline 270 & $\boldsymbol{\gamma}$ & 3.18 & 1.24 \\
\hline 310 & $\gamma$ & 2.86 & 1.21 \\
\hline
\end{tabular}

TEMPERATURE COEFFICIENTS

From A. E. Kay and P. F. T. Linford in Plutonium 1960, p. 58.

$\begin{array}{cccccc}\frac{\text { Specimen }}{\mathrm{E} 6} & \frac{\text { Remarks }}{3 \mathrm{rd} \mathrm{Cycle}} & \frac{\text { Modulus }}{\text { Young's }} & -126 \times 10^{-5} /{ }^{\circ} \mathrm{C} & -165 \times 10^{-5} /{ }^{\circ} \mathrm{C} & -251 \times 10^{-5} /{ }^{\circ} \mathrm{C} \\ \mathrm{E} 6 & 4 \text { th Cycle } & \text { Rigidity } & -150 \times 10^{-5} /{ }^{\circ} \mathrm{C} & -167 \times 10^{-5} /{ }^{\circ} \mathrm{C} & -183 \times 10^{-5} /{ }^{\circ} \mathrm{C}\end{array}$

ELASTIC CONSTANTS AND DEBYE TEMPERATURES

From A. E. Kay and R. G. Loasby,

Phil. Mag., eighth series, vol. 9, no. 97, p. 44. 1964.

\begin{tabular}{|c|c|c|c|c|}
\hline Phase & $\begin{array}{l}\text { Young's Modulus, } \\
\times 10^{-11} \text { dyne } / \mathrm{cm}^{2}\end{array}$ & $\begin{array}{c}\text { Poisson's } \\
\text { Ratio } \\
\end{array}$ & $\begin{array}{l}\text { Bulk Modulus } \\
\times 10^{-11} \text { dyne } / \mathrm{cm}^{2} \\
\end{array}$ & $\begin{array}{c}\text { Debye } \\
\text { Temperature, }{ }^{O} \mathrm{~K} \\
\end{array}$ \\
\hline a & 12.6 & 0.20 & 7.0 & 192 \\
\hline$\beta$ & 6.1 & 0.28 & 4.62 & 133 \\
\hline$\gamma$ & 6.1 & 0.25 & 4.06 & 136 \\
\hline$\delta$ & 5.1 & 0.33 & 5.1 & 120 \\
\hline
\end{tabular}




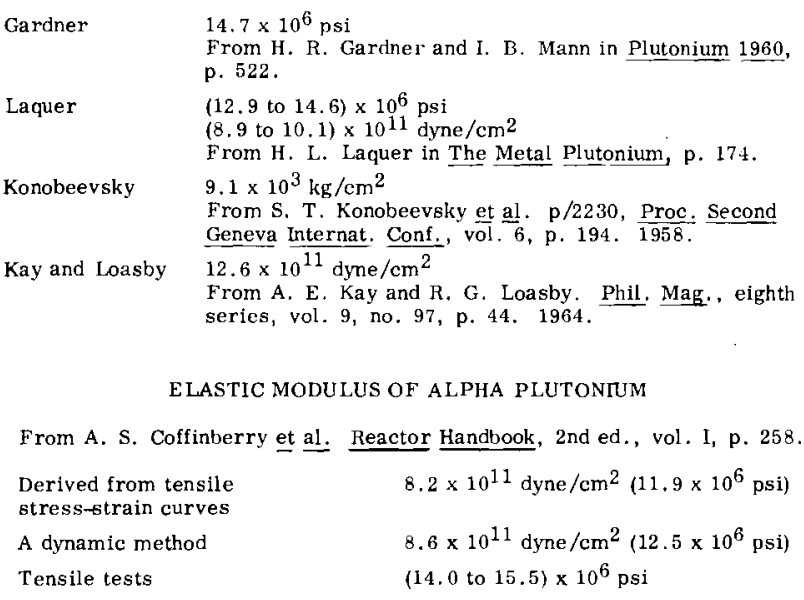

From A. S. Coffinberry et al. Reactor Handbook, 2nd ed., vol. 1, p. 258.

$$
\begin{array}{ll}
\begin{array}{l}
\text { Derived from tensile } \\
\text { stress-atrain curves }
\end{array} & 8.2 \times 10^{11} \mathrm{dyne} / \mathrm{cm}^{2}\left(11.9 \times 10^{6} \mathrm{psi}\right) \\
\text { A dynamic method } & 8.6 \times 10^{11} \mathrm{dyne} / \mathrm{cm}^{2}\left(12.5 \times 10^{6} \mathrm{psi}\right) \\
\text { Tensile tests } & (14.0 \text { to } 15.5) \times 10^{6} \mathrm{psi}
\end{array}
$$

Young's modulus of elạsticity for $\bullet \mathrm{Pu}$ :

tensile (from stress strain curve): $E=11.9 \times 10^{6} \mathrm{psi}$

dynamic: $E=12.5 \times 10^{6} \mathrm{psi}$

sonic: $E=(14.40 \pm 0.04) \times 10^{6} \mathrm{psi}$

compressive: $E=(14.0$ to 15.5$) \times 10^{6} \mathrm{psi}$

From W. D. Wilkinson in Extractive and Physical Metallurgy of Plutonium and Its Alloys, p. 15 .

A value of $E=9.1 \times 10^{3} \mathrm{~kg} / \mathrm{cm}^{2}$ was obtained by the resonance method at room temperature for alpha Pu extruded at $230^{\circ} \mathrm{C}$.

From S. T. Konobeevsky et al. p/2230, Proc. Second Geneva Internat. Conf. vol. 6 , p. 194.1958.

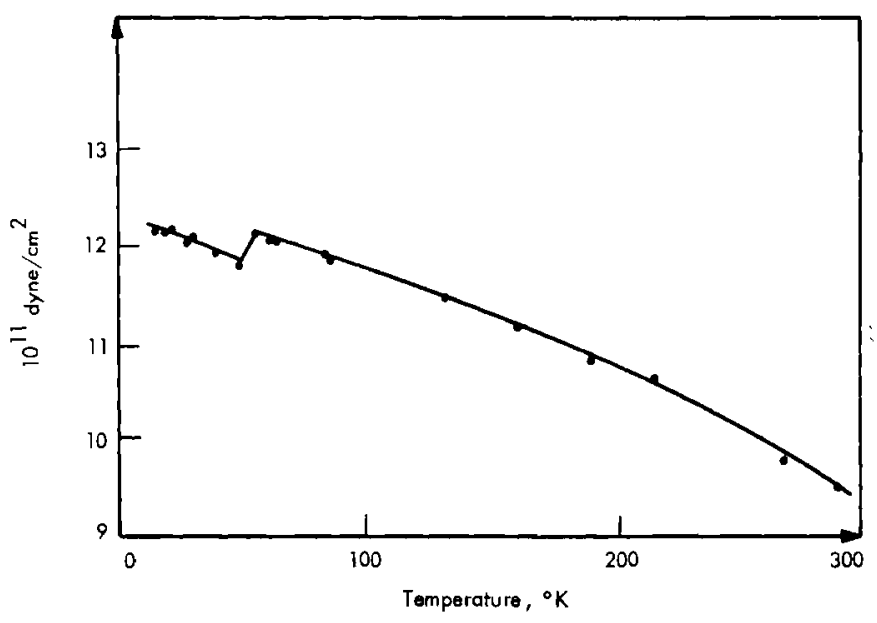

Young's Modulus for Alpha Plutonium. From R. Lallement. Physics Letters, vol. 5, p. 182.1963.

Young's modulus - room temperature, cast $\mathrm{Pu}$

$$
\begin{array}{ll}
\text { Tensile measurements } & 8.2 \times 10^{11} \mathrm{dyne} / \mathrm{cm}^{2} \\
\text { Compression measurements } & 9.9 \times 10^{11} \mathrm{dyne} / \mathrm{cm}^{2}
\end{array}
$$

Poisson's ratio 0.13

From A. E. Kay in The Metal Plutonium, p. 189.

\section{2}

Shear Modulus

Shear modulus of elasticity: $G=5.4 \times 10^{6} \mathrm{psi}$

$$
\text { (a Pu) }
$$

From W. D. Wilkinson in Extractive and Physical Metallurgy of Plutonium and Its Alloys, p. 15 .
A value for the shear modulus $\mathrm{G}$ of $3.8 \times 10^{3} \mathrm{~kg} / \mathrm{mm}^{2}$ was obtained by the resonance method at room temperature for alpha Pu extruded at $230^{\circ} \mathrm{C}$. From S. T. Konobeevsky et al. p/2230, Proc. Second Geneva Internat. Conf., vol. 6 , p. 194. 1958 .

\section{4}

\section{Poisson's Ratio}

\footnotetext{
for $* \mathrm{Pu} ; \boldsymbol{\nu}=0.15 \pm 0.01$

for * Pu extruded at $230^{\circ} \mathrm{C}: *=0.21$
}

From W. D. Wilkinson in Extractive and Physical Metallurgy of Plutonium and lts Alloys, p. 15
$=0.21$ (calculated from Young's modulus and shear modulus obtained by the resonance method at room temperature for alpha Pu extruded at $230^{\circ} \mathrm{C}$ ).

From S. T. Konobeevsky et al. p/2230, Proc. Second Geneva Internat. Conf. vol, 6, p. 194, 1958.

See end of section 308,1 .

Compressibility of $\propto \mathrm{Pu}$ at atmospheric pressure: $2.0 \times 10^{-6}$ /bar

From W. D. Wilkinson in Extractive and Physical Metalturgy of Plutonium and

Its Alloys, p. 15 .

\subsection{Reactions with Gases - Air}

EFFECT OF TEMPERATURE AND HUMIDITY

ON THE OXIDATION OF ALPHA PLUTONIUM

From A. S. Coffinberry et al. Reactor Handbook, 2nd ed., vol. I, p. 263.

\begin{tabular}{cccc}
$\begin{array}{c}\text { Temperature, } \\
{ }_{\mathrm{C}}\end{array}$ & $\begin{array}{c}\text { Relative } \\
\text { Humidity, \% }\end{array}$ & $\begin{array}{c}\text { Duration, } \\
\mathbf{h r}\end{array}$ & $\begin{array}{c}\text { Weight Gajn, } \\
\text { mg/cm }\end{array}$ \\
\cline { 2 - 3 } 25 & 40 & 900 & $0.01-0.07$ \\
50 & 100 & 900 & 6.5 \\
& 7 & 900 & 4.3 \\
& 7 & 200 & 0.6
\end{tabular}


EFFECT OF MOISTTRE ANT THATHRTTRH

(IN THE: ALR CORHOSION

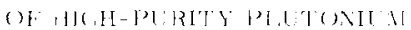

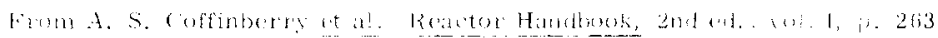

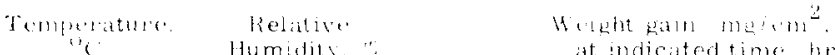

$$
\begin{aligned}
& \text { at indicated time hr }
\end{aligned}
$$

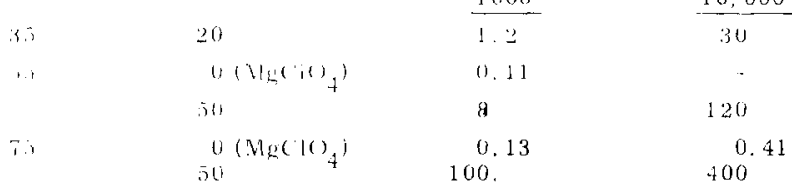

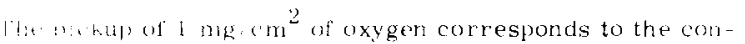
sulblifun prenetration of 3.74 s of alpha plutonium. The corrsponding figure for the delta phase is 4.86 . One-mil penetration of alpha corresponds to $6.74 \mathrm{mg} / \mathrm{cm}^{2}$ oxygen gained per square intimeter.

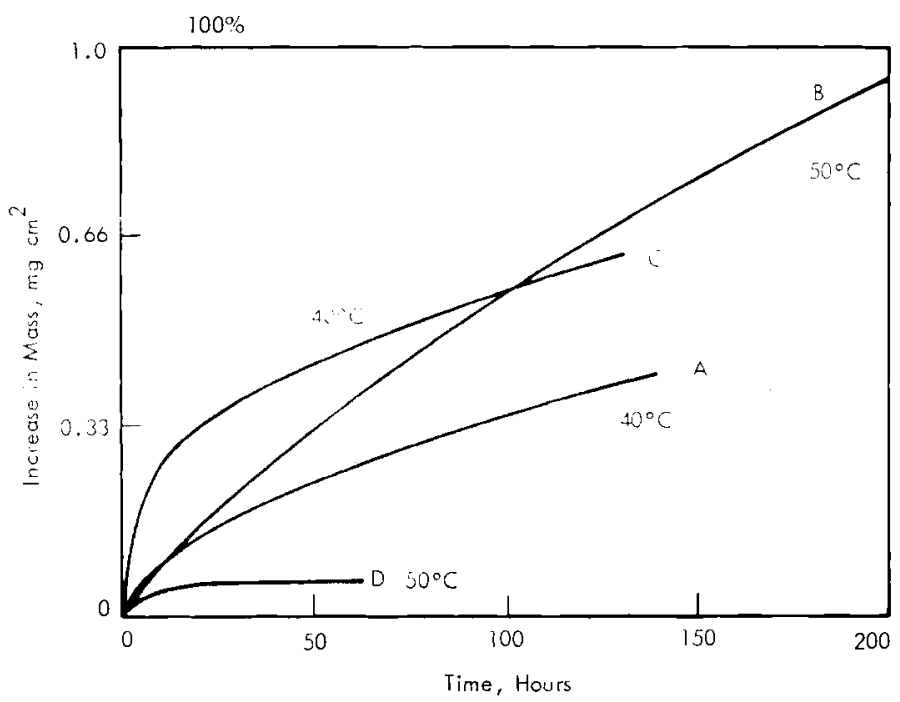

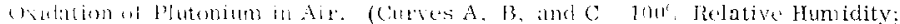

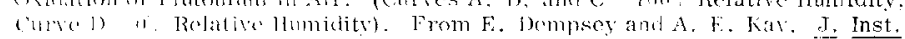

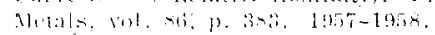

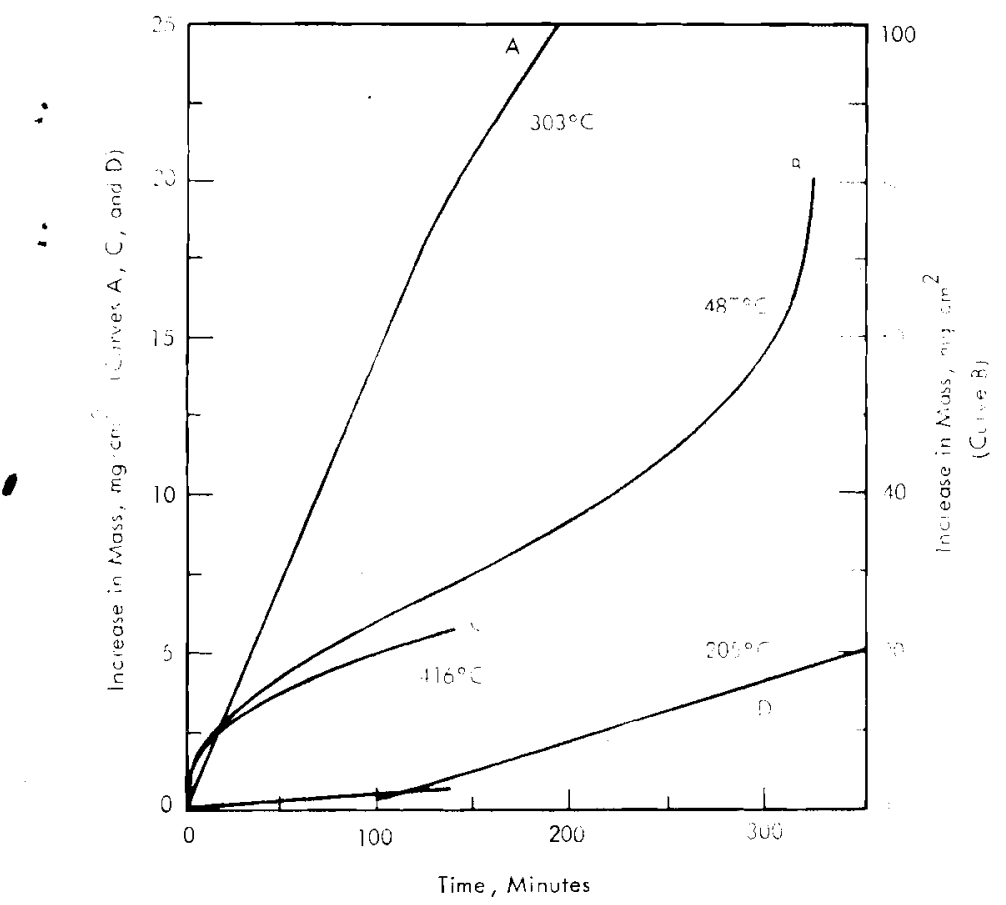

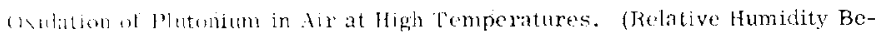

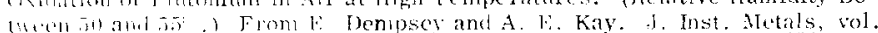

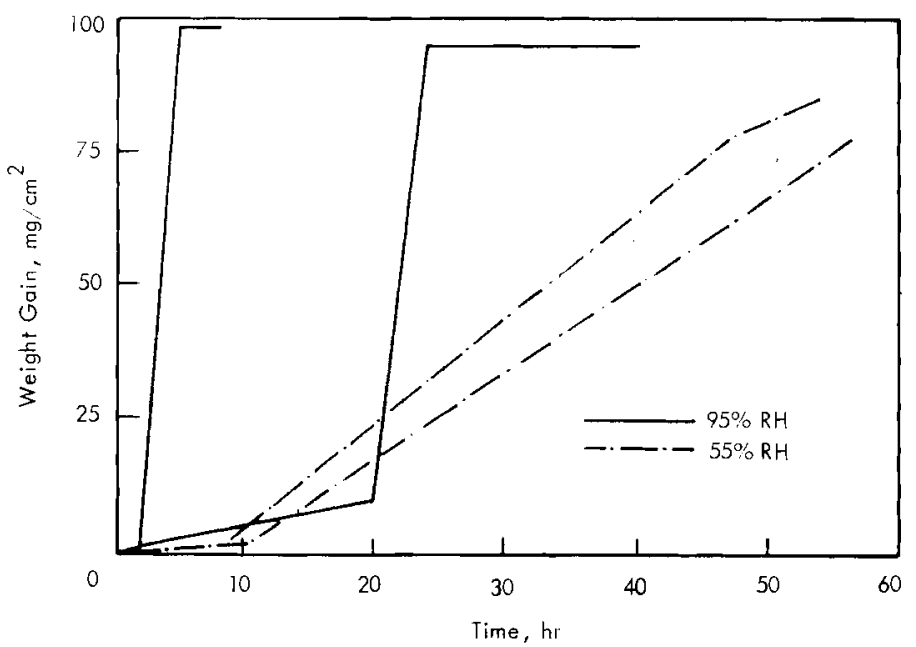

The Fffect of Inumidity on the Oxidation of a $-P u a t g 0^{\circ} \mathrm{C}$. From $J$. F. Sackman in Plutonium 1960, P. 224.

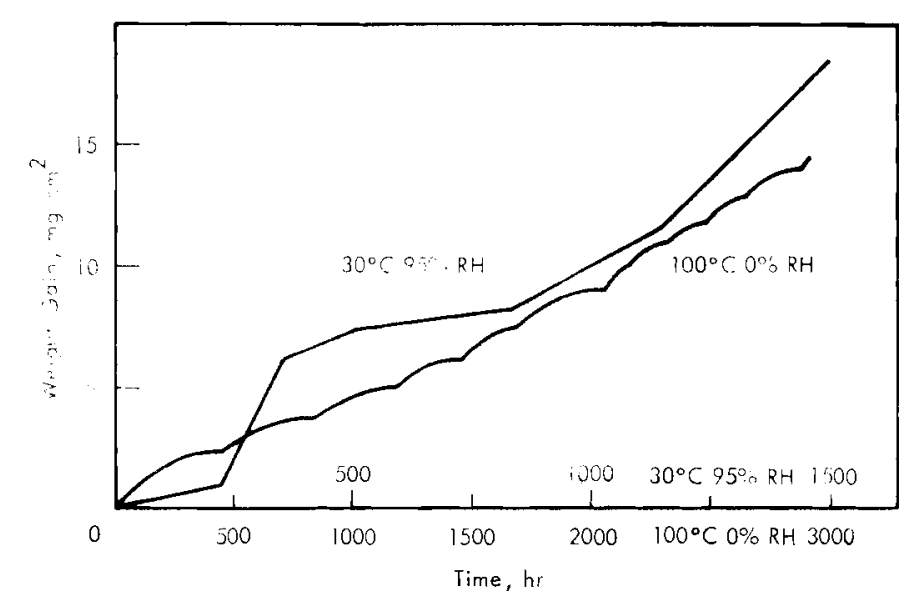

The widation of a-Pu Lndor. Mild comditions Showing the Linusual Behavios at $30{ }^{\circ} \mathrm{C}$ and $90^{\circ}$ pereent RH. From J. F. Sirliman in Plutonium 1960, p. 225.

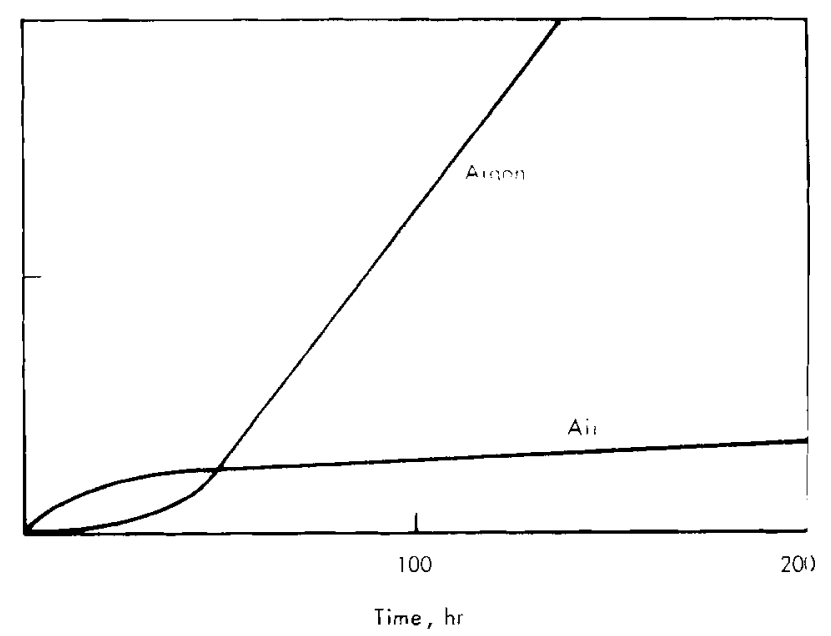

Lptake of Oxygen by Plutonium in Moist Air and in Moist Argon (95\% Relative Humidity at $95^{\circ} \mathrm{C}$. From J. B. Ravnor and J. F. Sackman. Nature, vol. 197, p. jor. 1963.3 
OXIDATION-RATE OF PLUTONIUM AT $25^{\circ} \mathrm{C}$

From J. B. Raynor and J. F, Sackman. Nature, vol. 197, p. 588 . 1963.

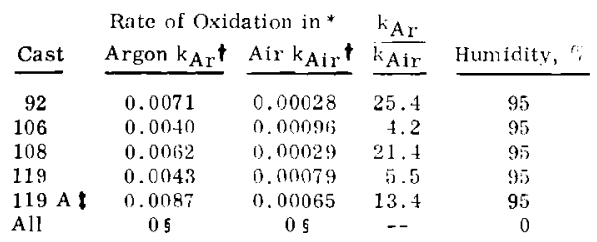

* Derived from slope of line after initial deviations from linea rity.

$\mathrm{Mg} / \mathrm{cm}^{2} / \mathrm{h}$.

Is 119, but electropolished surface.

5 Not detectable.

Abraded specimens of $\mathrm{Pu}$, both cast disks and rolled sheet, were oxidized for 1,800 hours in air and in argon. Results suggest that the reaction is anodically controlled.

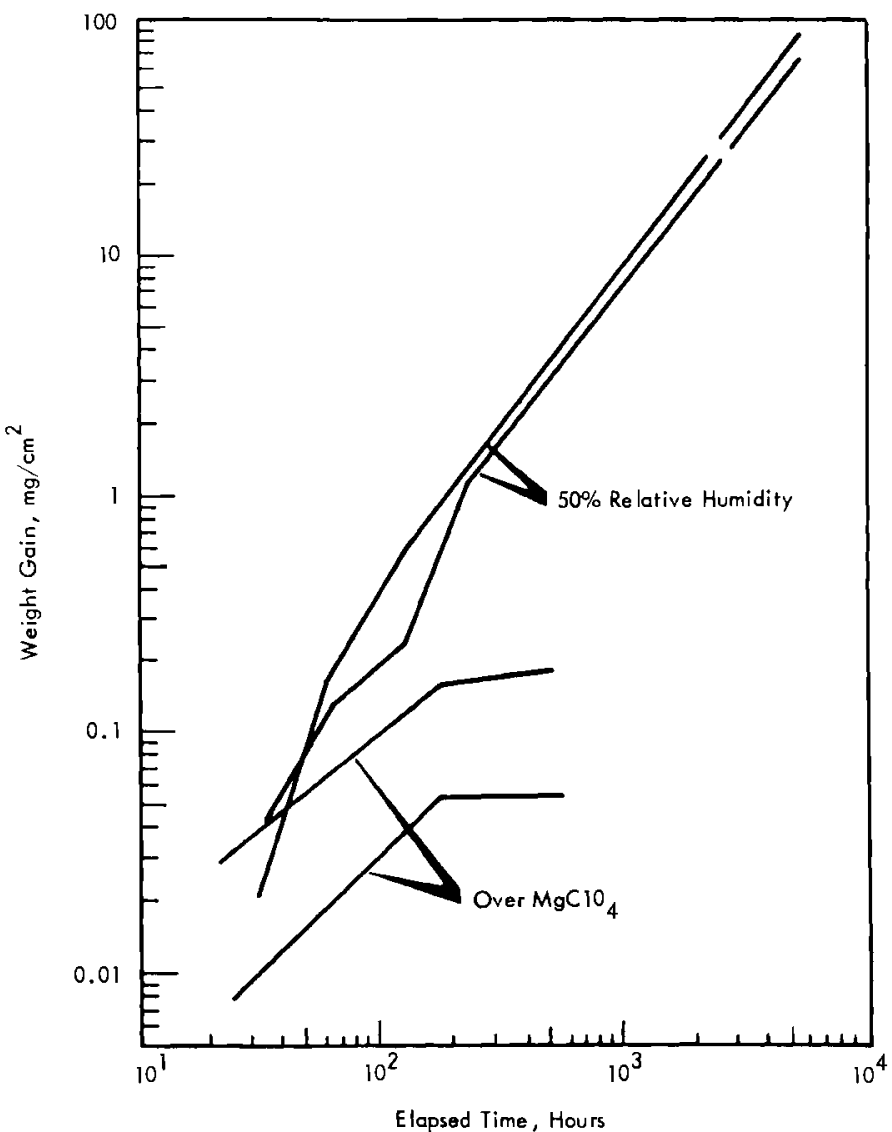

Comparison of the Corrosion Weight Gains in Dry and Moist Air at $55^{\circ} \mathrm{C}$. The approximately $50 \%$ relative humidity was maintained with a saturated solution of $\mathrm{NaBr}$. The dried air was continuously in contact with $\mathrm{MgClO}_{4}$. From J. T. Waher and E. S. Wright in The Metal Plutonium, p. 199

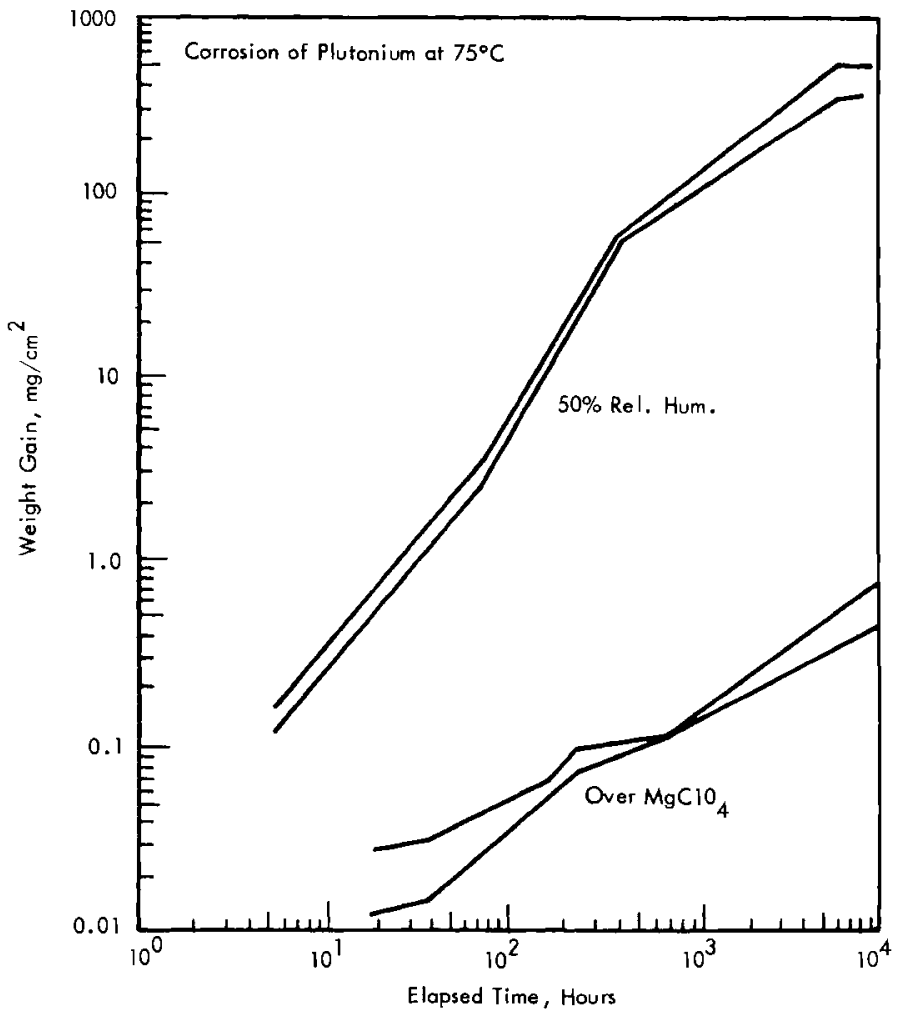

Comparison of the Corrosion Weight Gains of Pure Plutonium in Dry and Moist Air at $75^{\circ} \mathrm{C}$. The Approximately $50 \%$ Relative Humidity was Maintained with a Saturated Solution of $\mathrm{NaBr}$. The Dried Air was Continuously in Contact with

$\mathrm{MgClO}_{4}$. From J. T. Waber and E. S. Wright in The Metal Plutonium, p. 200. 


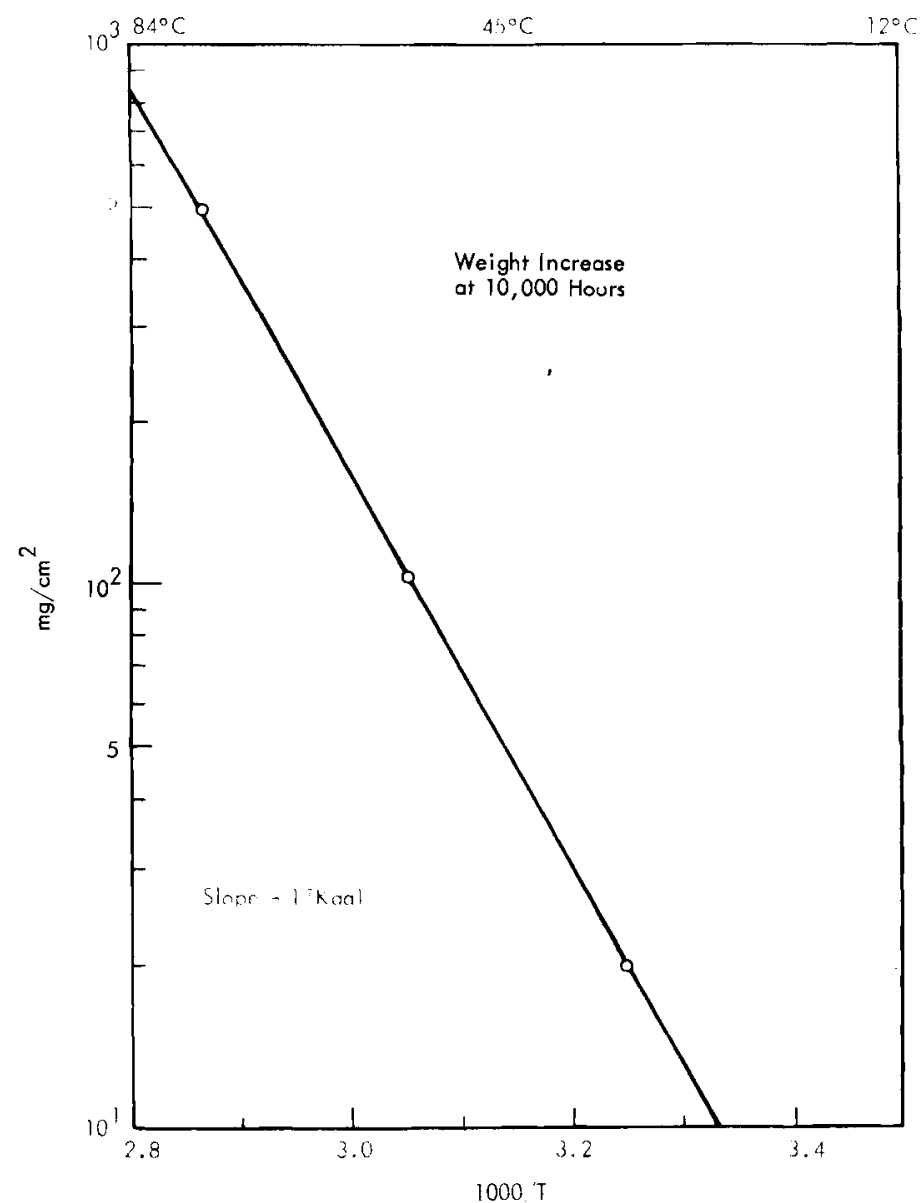

Appoximate Deternination of the Tenperature lependence of the Reaction Rate Constant for Plutonium Exposed to Moist Air. From J. T. Waber and E. S. Wright in The Metal Plutonium, p. 201.

COM PARISON OF OBSERVED CORROSION WEIGHT GAINS FOR ALPHA PLUTONIUM WITH CALCULATED WEIGHT GAINS FOR TWO COMPOSITIONS OF THE PRODUCTS $\left(75^{\circ} \mathrm{C}, 50 \% \mathrm{RH}\right)$

From J. T. Waber et al. J. Nucl. Mater., vol. 3, p. 210. 1961.

Initial WL

Meta], g

$$
\begin{gathered}
\begin{array}{c}
\text { Exposure } \\
\text { Time, hr }
\end{array} \\
\hline 3,360 \\
2,832 \\
2,832 \\
2,712 \\
2,352
\end{gathered}
$$

Final

Wt. $\mathrm{PuO}_{2}$

Calc., $\mathrm{g}$

Weight,

1.779133

1.969484

1.220691

2.541928

1.756114

1.945929

1.205912

1.00954

2.509454
2.293955

ion $\mathrm{PuO}_{2} \cdot \mathrm{xH}_{2} \mathrm{O}$

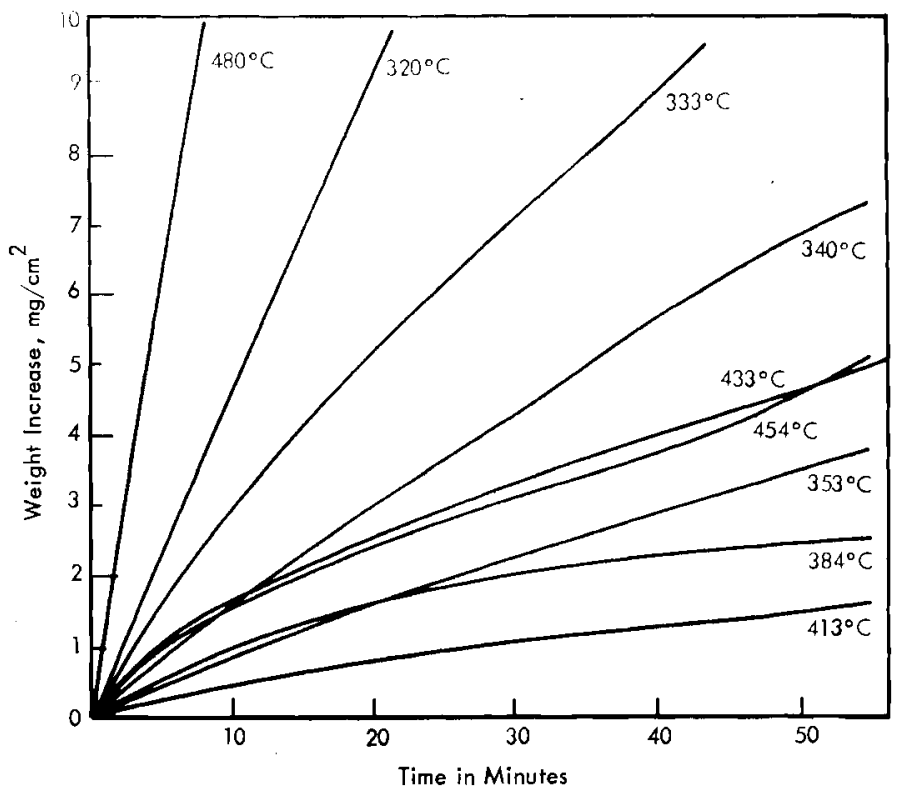

Oxidation of Electropolished Plutonium in Dry Air. From D. M. Poole et al. ir. Plutonium 1960 , p. 631

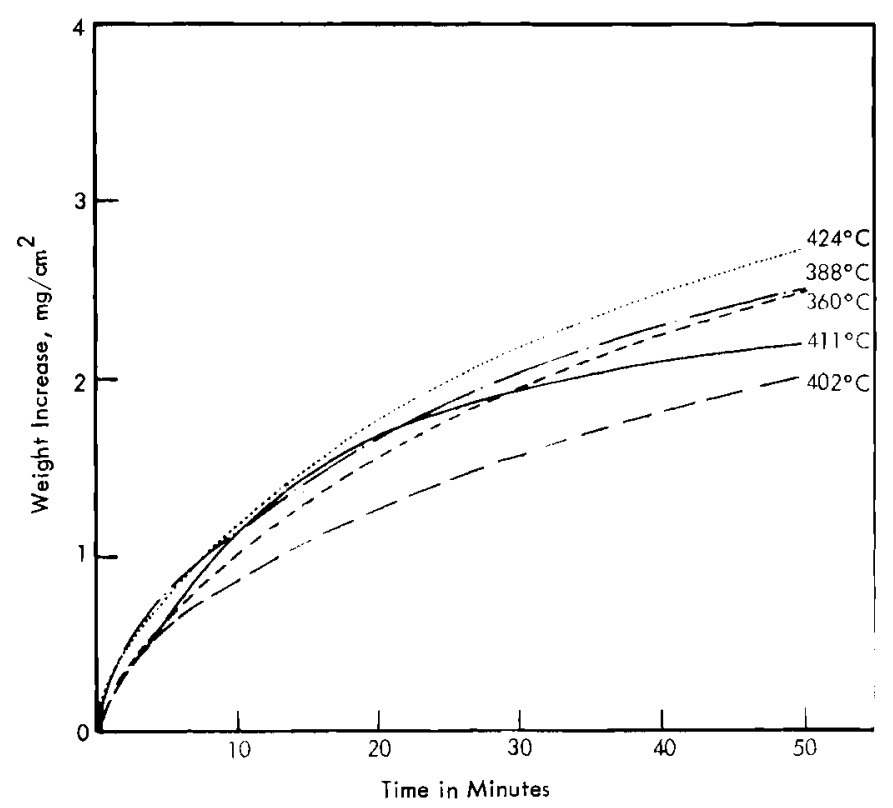

Oxidation of Mechanically Polished Plutonium in Air Dried Over Magnesium Perchlorate. The Temperature Measured is that of the Gas Stream. From D. M. Poole et al. in Plutonium 1960, p. 632 . 


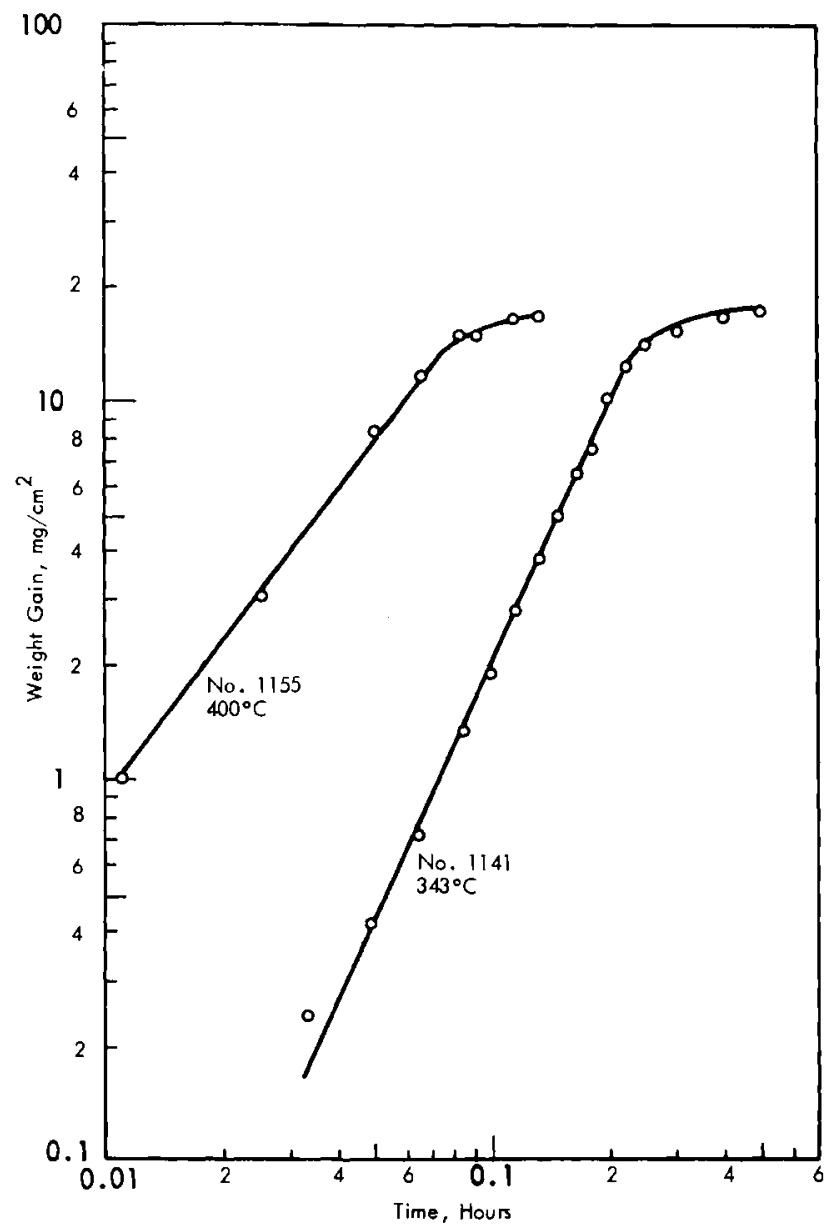

High-Temperature Oxidation of High-Purity Plutonium in Purified Oxygen. The Slopes in Excess of Unity Characterize the Reaction as Accelerating with Time.

The rate data are represented quite well by the following equation:

$$
w=k_{n} t^{n} \text {, }
$$

with the rate constant $k_{n}=320$ and exponent of time $n=2.2$ for the test at $343^{\circ} \mathrm{C}$, and $\mathrm{k}_{\mathrm{n}}=480, \mathrm{n}=1.4$ for the test at $400^{\circ} \mathrm{C}$.

From J. T. Waber and E. S. Wright in The Metal Plutonium, p. 201.

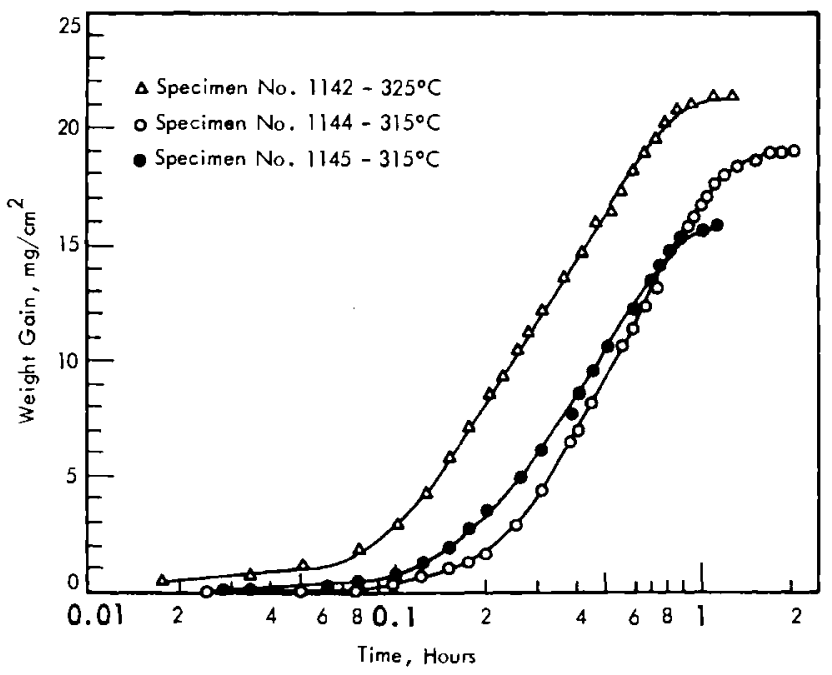

For the three runs shown to the left, the rate data can be represented by the logarithmic growth law:

$$
W=k_{L} \log _{10}(b t+c) .
$$

\begin{tabular}{|c|c|c|c|}
\hline $\begin{array}{c}\text { Specimen } \\
\text { No. } \\
\end{array}$ & $\begin{array}{c}\text { Test } \\
\text { Temp., }{ }^{\circ} \mathbf{C} \\
\end{array}$ & $\begin{array}{c}{ }^{\mathrm{k}} \mathrm{L} \\
\mathrm{mg} / \mathrm{cm}^{2}\end{array}$ & $\begin{array}{r}\mathrm{b}_{\mathrm{o}} \\
\mathrm{hr}^{-1} \\
\end{array}$ \\
\hline 1144 & 315 & 24.6 & 4.8 \\
\hline 1145 & 315 & 20.2 & 6.4 \\
\hline 1142 & 325 & 20.6 & 12.96 \\
\hline
\end{tabular}

with $k_{L}$ and $b$ appropriate kinetic constants:

It was found that $\mathrm{c}$ could be neglected.

From J. T. Waber and E. S. Wright in The Metal Plutonium, p. 202.

Semilogarithmic Plots of the Gas-Consumption Data for Pure Plutonium and Oxygen. The gas and metal were preheated in a molten salt bath before they were brought into contact. The sigmoid shape of the curves results from an increase in surface temperature at the beginning of the reaction, followed by depletion of metal at its end. From $J$. T. Waber and E. S. Wright in The 
Pltoniun metal at Joom temperature will take up hydrogen or deuterium wilh the evolution of lie:at. The lump of medit disinterrates during reaction and particles of hydride are thrown off the metal piece. At room temperature the formation of the hyclide or cleuteride may show an induction pertiod of some

minutes or mav be almost instantancous.

From $\mathrm{MUC}-\mathrm{JCW}-22 \mathrm{2}$ (

REACTIVITY OF PLUTONIUM METAL WITH VARIOUS SOLUTIONS

From A. S. Colfinberry et al. Reactor llandbook, 2nd ed., vol. I, p. 262.

Reagent Solution

Ammonium fluoride (conc. aqueous) Ammonium fluorid (sat. alcoholic) Nitric acid

Hydrochloric acid

Sulfuric acid

Acetic acid

Tricbloroacetic acid

Perchloric acid Phosphoric acid
Remarks

Slow attack with darkening of the surface

No attack at room temperature or on heating

No visible attack in any concentration range ove a period of several hours; some preferential dissolution expected from the experience with uranium.

Vigorous attack in all concentrations, becoming violent at high concentrations

Slow initial evolution of hydrogen in concentrated acid which eventually stops; moderate attack in dilute acid

No visible attack by glacial acetic acid even at elevated temperatures; very slow dissolution in dilute acid

Vigorous attack in concentrated acid; rate of solution dec reases upon dilution

Fairly rapid gas evolution in $72 \%$ acid

Fairly rapid attack in $85 \%$ acid

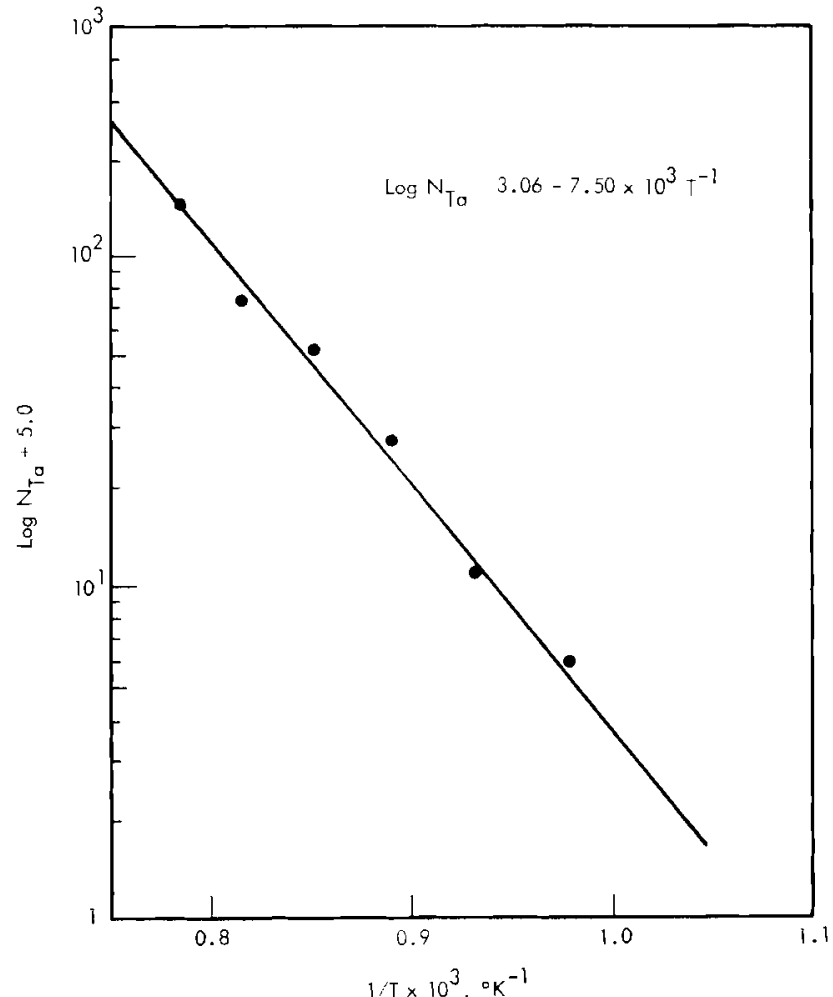

Plot of Log $\mathrm{N}_{\mathrm{T}}$ us 1 \% ( $\mathrm{N}_{\mathrm{Ta}}$ simlulility of Ta expressed as the molr

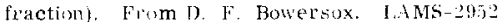


THE SOLLBILJTY OF Ta IN PU AS A FLNCTION OF TEM PERATURE

THE SOLUBILITY OF Ta in Pu AS A FUNCTION OF TIME AT $850^{\circ} \mathrm{C}$

From D. F. Bowersox. LAMS-2952

Total Time at $850^{\circ}, \quad$ Solubility,

\begin{tabular}{|c|c|}
\hline $\begin{array}{l}\text { Total Time at } 850^{\circ} \text {, } \\
\text { hours }\end{array}$ & $\begin{array}{l}\text { Solubility, } \\
\text { ppm Ta }\end{array}$ \\
\hline 1.0 & $<50$ \\
\hline 3.0 & $<35$ \\
\hline 6.0 & $<35$ \\
\hline 16.0 & $<35$ \\
\hline 22.0 & $<50$ \\
\hline 44.0 & 220 \\
\hline 66.0 & 210 \\
\hline 88.0 & 215 \\
\hline 112.0 & 195 \\
\hline 158.0 & 205 \\
\hline 187.0 & 215 \\
\hline
\end{tabular}

Physical Properties

VALUES OF S AND AH AT DIFFERENT TEMPERATURES

From T. A. Sandenaw. J. Phys. Chem. Solids, vol. 23, p. 1245. 1962.

$\underline{\text { Run }}$

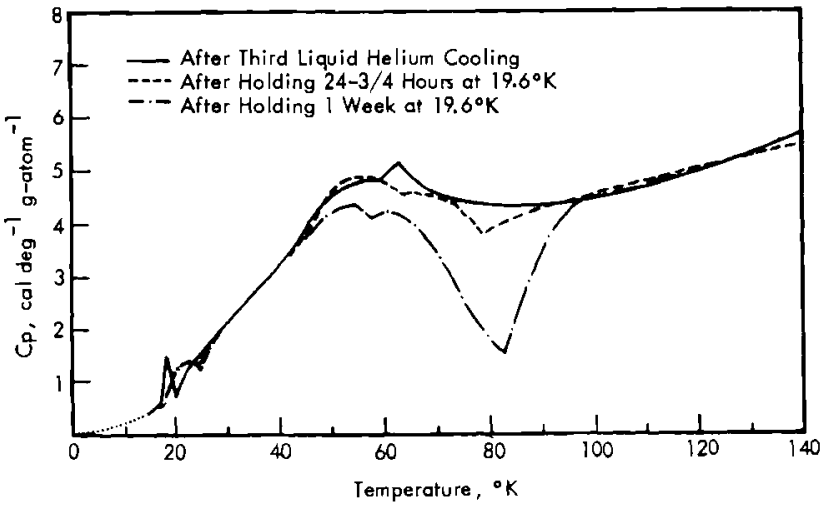

Heat Capacity Curves for an Electro-Refined Plutonium Specimen Showing Effects of Various Holding Times in Boiling Liquid Helium and Boiling Liquid Hydrogen. From T. A. Sandenaw. I. Phys. Chem. Solids, vol. 23, p. 1244. 1962.
From D, F, Bowersox. LAMS-2952.

\begin{tabular}{|c|c|c|c|}
\hline $\begin{array}{c}\text { Temperature, } \\
{ }^{\circ} \mathrm{C} \\
\end{array}$ & $\begin{array}{c}\begin{array}{c}\text { Hours at } \\
\text { Temperature }\end{array} \\
\end{array}$ & $\begin{array}{l}\text { Solubility, } \\
\text { ppm Ta* }\end{array}$ & $\begin{array}{l}\text { Mole Fraction Ta, } \\
\mathrm{N} \mathrm{Ta} \times 10^{4} \\
\end{array}$ \\
\hline $\begin{array}{l}700 \\
750 \\
800\end{array}$ & $\begin{array}{l}97.0 \\
24.0 \\
15.5 \\
18.0 \\
65.5\end{array}$ & $\begin{array}{r}<35 \\
45 \\
85 \\
85 \\
80 \\
\text { Avg. } \quad 83\end{array}$ & $\begin{array}{l}-. .- \\
0.59 \\
1.12 \\
1.12 \\
1.06 \\
1.10\end{array}$ \\
\hline 850 & $\begin{array}{r}44.0 \\
66.0 \\
88.0 \\
112.0 \\
158.0 \\
215.0\end{array}$ & $\begin{array}{r}220 \\
210 \\
215 \\
195 \\
205 \\
215 \\
\text { Avg. } 210\end{array}$ & $\begin{array}{l}2.90 \\
2.77 \\
2.84 \\
2.57 \\
2.70 \\
2.84 \\
2.77\end{array}$ \\
\hline 900 & $\begin{array}{l}16.0 \\
40.0 \\
45.0 \\
88.0\end{array}$ & $\begin{array}{r}\mathbf{3 7 5} \\
\mathbf{3 8 5} \\
\mathbf{3 8 5} \\
\mathbf{3 8 0} \\
\text { Avg. } \mathbf{3 8 1}\end{array}$ & $\begin{array}{l}4.95 \\
5.08 \\
5.08 \\
5.01 \\
5.03\end{array}$ \\
\hline 950 & $\begin{array}{l}16.0 \\
40.0 \\
72.0\end{array}$ & $\begin{array}{r}\mathbf{5 2 0} \\
620 \\
\mathbf{5 2 0} \\
\text { Avg. } \mathbf{5 5 0}\end{array}$ & $\begin{array}{l}6.86 \\
8.19 \\
6.86 \\
7.26\end{array}$ \\
\hline 1000 & 68.0 & 1070 & $14: 11$ \\
\hline
\end{tabular}

* Parts per million parts $\mathrm{Pu}$, by weight.

(Pu-99.91 wt. \% pure, Ta-all impurities $<100 \mathrm{ppm})$
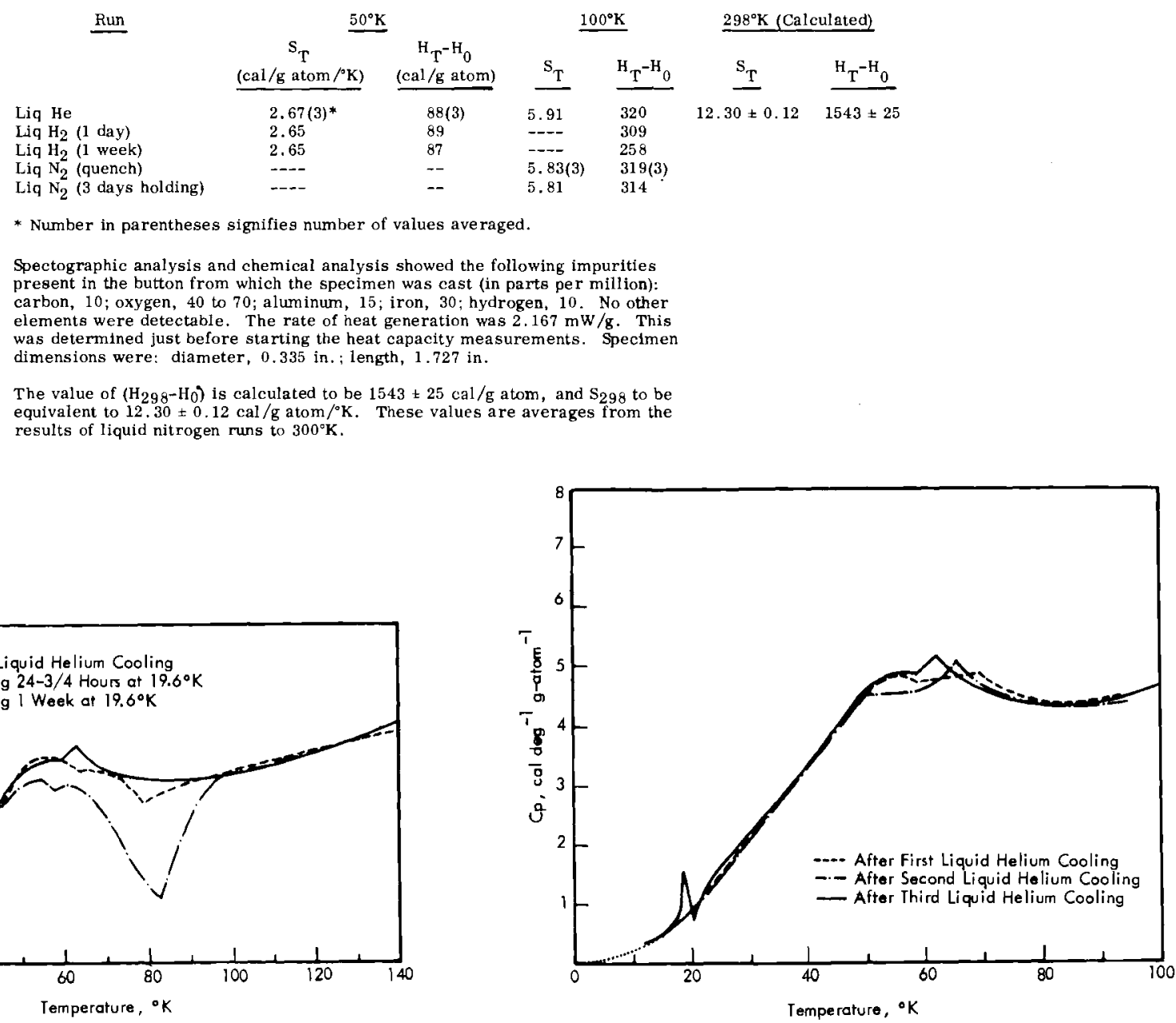

Heat Capacity Curves Below $100^{\circ} \mathrm{K}$ for Three Liquid Helium Coolings of an Flectro-Refined High Purity Plutonium Specimen. From T A, Sandenaw, J. Phys. Chem. Solids, vol, 23, p. 1243. 1962 
Heat capacity equations:

$$
\mathrm{C}_{\mathrm{p}} \cdots \boldsymbol{\gamma} \mathrm{T}+\mathrm{B} \mathrm{T}^{3}\left(11^{\circ} \mathrm{K} 10331^{\prime \prime} \mathrm{K}\right)
$$

where $\gamma$ (electronic colltribution coefficient) $1.9421 \times 11^{-2}$

$$
\mathrm{B}=5.8815 \times 10^{-5}
$$

$\boldsymbol{\sigma}$ (standard deviation) 0.13

$\mathrm{C}_{\mathrm{p}} \therefore \mathrm{A}+\mathrm{B} \mathrm{T} \cdot \mathrm{CT}^{2}\left(08^{\circ} \mathrm{K}\right.$ to $\left.300^{\circ} \mathrm{K}\right)$

where

$$
\begin{aligned}
& \text { A }=2.3180 \\
& B=2.4651 \times 10^{-2} \\
& C=-2.2813 \times 10^{-5}
\end{aligned}
$$

Limitations in the data, from the equation as given, were estimated to be (in $e^{a} 1 / \mathrm{g}$ atom $/{ }^{\circ} \mathrm{K}$ ): to, 05 at $100^{\circ} \mathrm{K}, \pm 0.13$ at $200^{\circ} \mathrm{K}$ and \pm 0.17 at $3000^{\circ} \mathrm{K}$.

From T'. A. Sandenaw. J. Phys. Chem. Solids, vol, 23, p. 1244. 196?

\begin{tabular}{|c|c|c|c|c|c|}
\hline $\begin{array}{l}\mathrm{T}, \\
{ }^{\circ} \mathrm{K}\end{array}$ & $\begin{array}{c}\mathrm{C}_{\mathrm{p}} \\
\mathrm{cal} /{ }^{\circ} \mathrm{K} \\
\end{array}$ & $\begin{array}{l}\mathrm{T}, \\
{ }^{\circ} \mathrm{K}\end{array}$ & $\begin{array}{c}\mathrm{C}_{\mathrm{p}}, \\
\mathrm{cal} /{ }^{\circ} \mathrm{K} \\
\end{array}$ & $\begin{array}{l}\mathrm{T}, \\
{ }^{\circ} \mathrm{K}\end{array}$ & $\begin{array}{c}\mathbf{C}_{\mathrm{p}}, \\
\mathrm{cal} /{ }^{\circ} \mathbf{H}\end{array}$ \\
\hline 100 & 4.56 & 170 & 5.85 & 240 & 6. \\
\hline 110 & 4.75 & 180 & 6.02 & 250 & 7.0 \\
\hline 120 & 4.95 & 190 & 6.18 & 260 & 7. \\
\hline 130 & 5.14 & 200 & 6.34 & 270 & 7.3 \\
\hline 140 & 5.32 & 210 & 6.49 & 280 & 7.4 \\
\hline 150 & 5.50 & 220 & 6.64 & 290 & 7.5 \\
\hline 160 & 5.68 & 230 & 6.78 & 300 & 7.6 \\
\hline
\end{tabular}

MOLAL, HLAT CAPACTIY OF PLUTONIM METAL, AT ROUNDED TEMPERATURES

From T. A. Sandenaw. J. Phys, Chem. Solids, vol. 23, p. 1245,1962

COMPARISON OF IIEAT CAPACITY, $C_{p}$, AND ENTHALPY,

\begin{tabular}{|c|c|c|c|c|}
\hline \multirow[b]{2}{*}{$\begin{array}{c}\text { Temperature, } \\
{ }^{n} \mathrm{~K}\end{array}$} & \multicolumn{2}{|c|}{$\mathrm{C}_{\mathrm{p}}$, cal mole ${ }^{-1} \mathrm{deg} \mathrm{K}^{-1}$} & \multicolumn{2}{|c|}{$\mathrm{H}_{\mathbf{T}}-\mathrm{H}_{0}, \mathrm{cal} / \mathrm{mole}^{*}$} \\
\hline & $\begin{array}{c}\text { a - Phase, } \\
163 \mathrm{~h} \\
\end{array}$ & $\begin{array}{c}8-P h a s c, \\
18+h \\
\end{array}$ & $\begin{array}{c}\text { a - Phase, } \\
163 \mathrm{~h} \\
\end{array}$ & $\begin{array}{c}8-\text { Phase } \\
18 .+1\end{array}$ \\
\hline 25 & 1.20 & 2.68 & 12.7 & 23.0 \\
\hline 50 & 4.21 & 4.52 & 87 & 100 \\
\hline 75 & 2.68 & 5.46 & 183 & 236 \\
\hline 100 & 4.52 & 5.78 & 258 & 379 \\
\hline 150 & 5.54 & 6.70 & 513 & 693 \\
\hline 200 & 6.35 & 7.20 & 816 & 1030 \\
\hline 273.15 & 7.40 & 7.75 & 1327 & 1570 \\
\hline 298.15 & 7.70 & 7.97 & 1519 & 1768 \\
\hline
\end{tabular}

$\mathrm{H}^{-\mathrm{H}_{0}}$, OF - PHASE, PLUTONIUM AND A $\boldsymbol{i}$-A LLOY CONTAINING 3.4 at. $\%$ ALUMINUM "SELF-DAMAGED" STATES

From C. E: Olsen et al. in Radiation Damage in Reactor Materials, p. 5.5

*After storage of self-irradiation damage by holding at $\sim 19.6^{\circ} \mathrm{K}$ for the specified times.

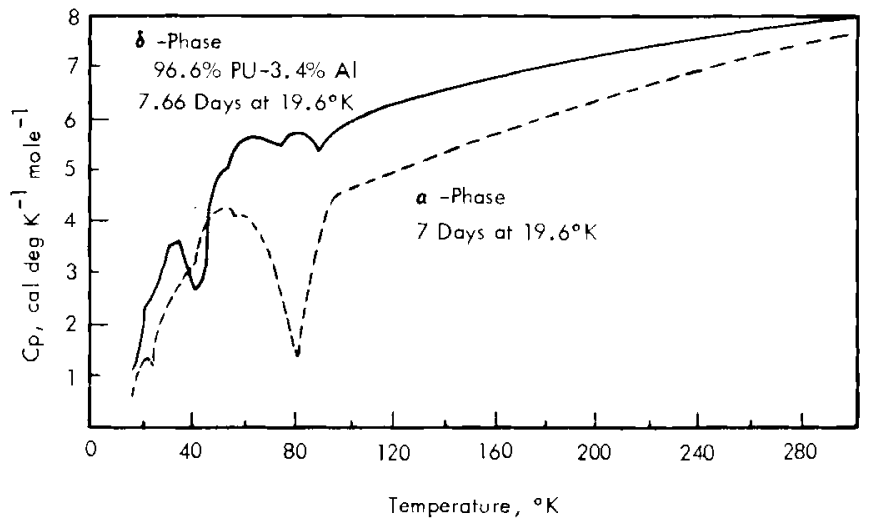

Heat capacity as a Function of Absolute Temperature for Figh-Pupity Flectro-

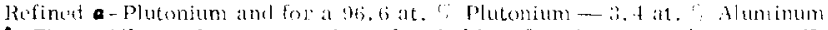
8 -Phase Alloy, (1)ata were talien after holding for about l weok alt $\sim 19.6^{\circ} \mathrm{K}$, From C. F. Olsen et all. in Radiation Inamage in Reactor Materials, p. i.3.
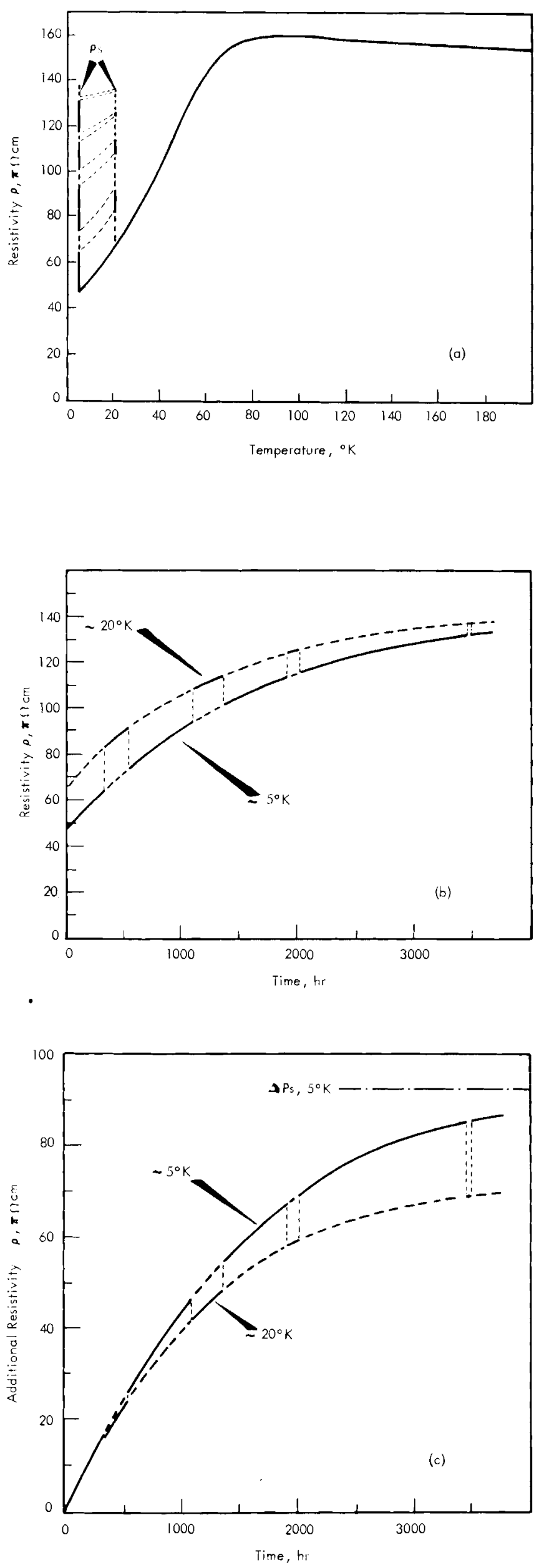

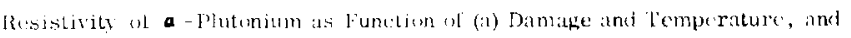
(1) Damage and Time. (c) Addtional Resistivity as Function of Time. From

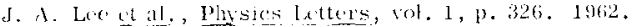




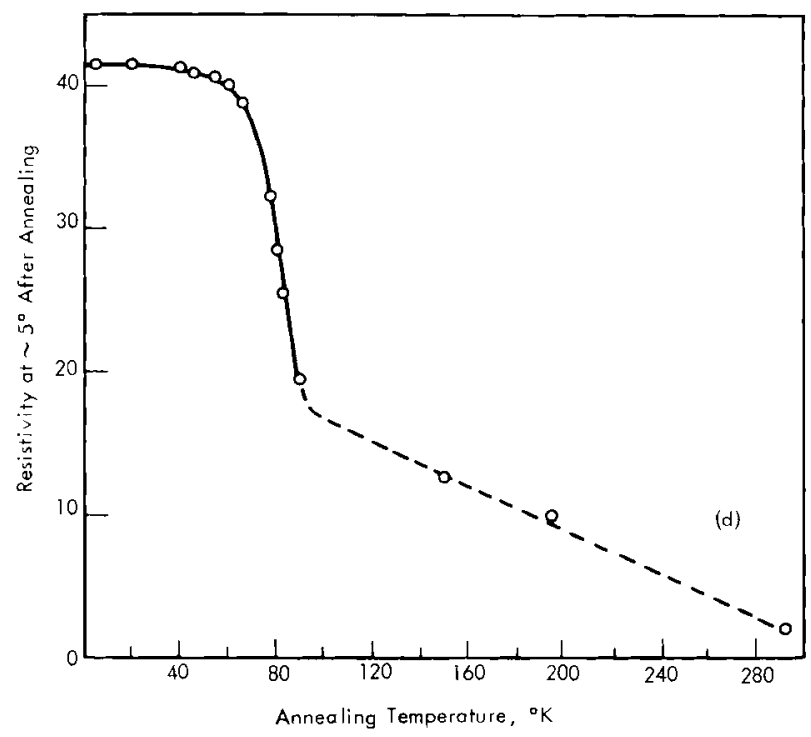

Variation of Acklitional Resistivity After Annıaling for 30 Minute Poriods with Temperature. From J. A. Lee ct al., Physies latters, vil. 1, p. $326,1 ! 662$.

The specimen which in most of the time was kept in liquid helium $\left(\sim 5^{\circ} \mathrm{K}\right)$ was on cucral neeasions temperarily transferred into a bath of liquid hedrogen $\left(20^{\circ} \mathrm{K}\right)$ and the resulting changes in resistivity are plotted in depentence on tomperature and time in (a) and (b). The following features we re noted:

1. After warmine the damalged specinen for a short while from $\sim 5^{\circ} \mathrm{K} 10 \sim 20^{\circ} \mathrm{K}$. the originat resistivity wats again recorded at $\sim 5 \mathrm{~K}$, showing that no annealing hat talien place in this temperature interval.

2. The rate of resistivity-rise at both temneratures changes with time, the resistivitics approaching almost the same saturation values $\rho_{S}$ of about 141 $\mu \mathrm{ohm} \mathrm{cm}$, as shown in (c).

:i. The rale of the resistivity-change with (ime can in first approximation be expressed as do/dt $\propto\left(p_{s}-\rho\right)$.

4. In accordance with $\stackrel{3}{-}$, it is found that the rise in resistivity $\Delta_{p}$ due to the same imount of acemulated damalge is smaller at $20^{\circ} \mathrm{K}$ than at $4^{\circ} \mathrm{K}$, as is best seen inl a plot of $\rightarrow 0$ azitinst time. (C) This means that the additional resistivit does nol olos Matthiesen's rule, and that the scittering cross section of this radiation dimige ilplears temperature dependent.

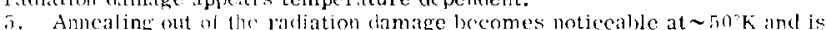
rapid $\sim \times 0^{2} \mathrm{~K}$. A spocime of a-plutonium which had accumulated an adtlitional resistivity $ذ_{\rho}$ of $t 1,1 \mu \mathrm{ohm} \mathrm{cm}$ at $\sim 7^{\circ} \mathrm{K}$ was raised to $40^{\circ} \mathrm{K}$ for a period of 30 min ancl then cooled again to $\sim 5^{\circ} \mathrm{K}$ where its resistivity was recorded. This procedure was repented at sucessively higher tenperatures up to $290^{\circ} \mathrm{K}$ and the resulting praph is show'l in (d). From d. A. Lee et it., Physics Letters, vol. 1, 1. $325,1 !(i 2$.

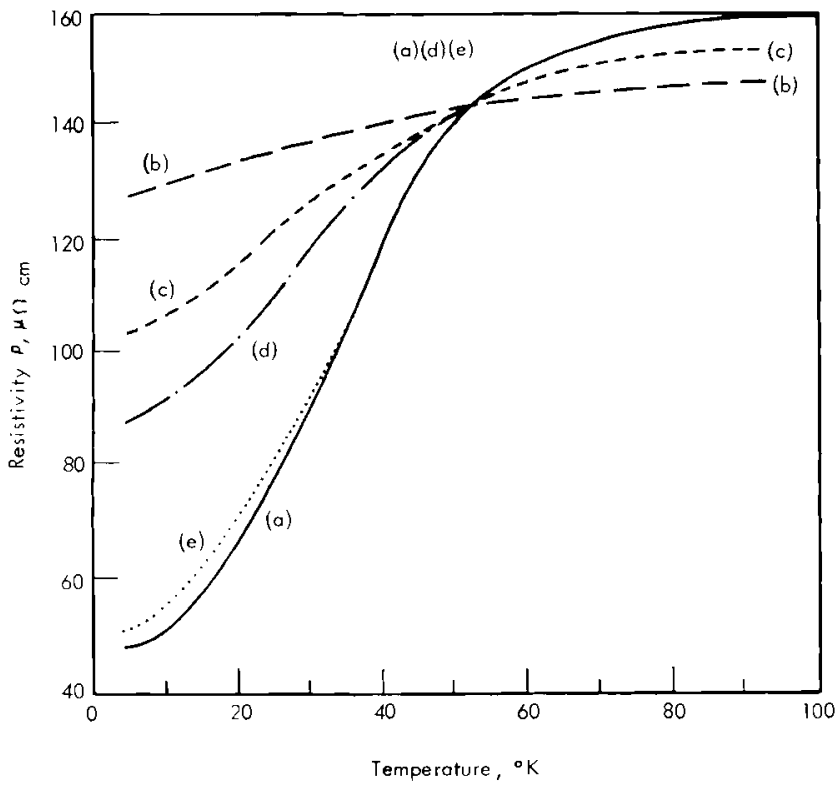

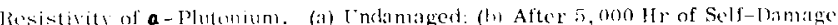

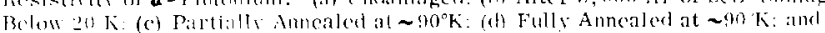

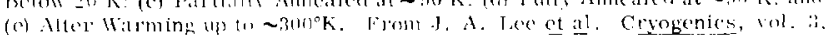
p. LT. 1!!):3.

The rise of resistivith o with holding time t follows approximately do ale $\propto\left(\rho_{-}-\rho\right)$. where $\rho_{s}$ is the saluration value.

58

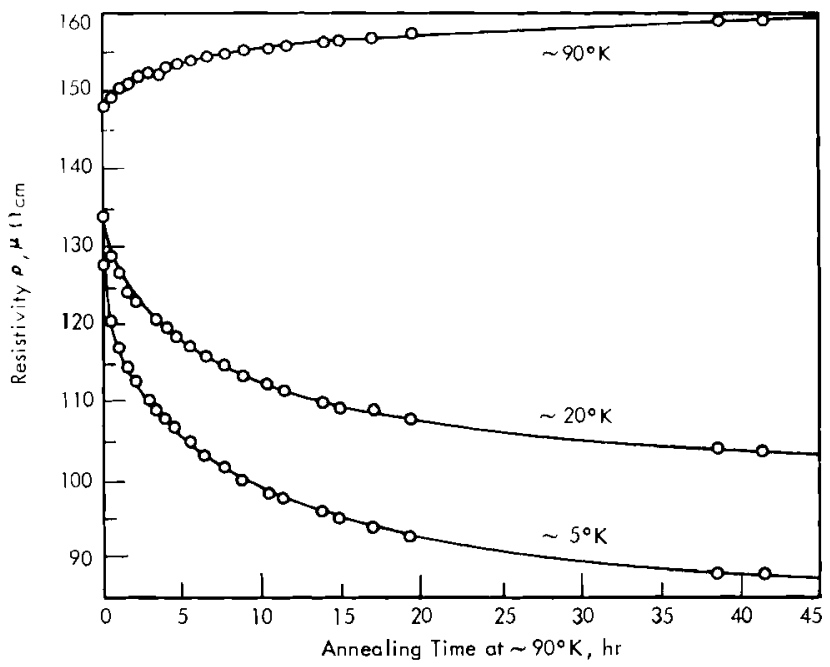

Isnthermat Anneal of $\mathrm{Pu}-2$ at $\sim 90^{\circ} \mathrm{K}$ After $5,000 \mathrm{Hr}$ at or Below $\sim 20^{\circ} \mathrm{K}$.

The initial rise was, within $3-1 / 2 \%$, the same for all specimens in the $a$-phase amounting at $\sim 5^{\circ} \mathrm{K}$ io $0,0515 \mu \mathrm{ncm} / \mathrm{hr}$ and being slightly smaller at $\sim 20^{\circ} \mathrm{K}$ Fron , I. A Lee et al. Cryogenics, vol. 3, p. 47. 1963. Similar curves appor in J. Phys. Soc. Japan, vol. 18, supplement III, pp. 312-313. 1963.

IRTF: OF JNCRFASF. IN RESIST ANCE AT $\sim 5^{\circ} \mathrm{K}$ FOR TINFS ITP TO 7000 HOURS (SLOPE OF THE CURVE OF RESISTANCF VS TIME)

From F. King et al. Acta Met., vol. 12, p. 112. 1964

\begin{tabular}{|c|c|c|c|c|}
\hline \multirow[b]{2}{*}{$\begin{array}{c}\text { Time, } \\
h r\end{array}$} & a Plutonium & \multirow[b]{2}{*}{$\begin{array}{c}\text { Normalised } \\
\text { Sinpe }\end{array}$} & \multicolumn{2}{|c|}{4 Al \&-Stabilized Plutonium } \\
\hline & $10^{-3} \begin{array}{l}\text { Slope, } \\
\mu \omega \mathrm{cm} / \mathrm{hr}\end{array}$ & & $\begin{array}{l}* \text { *lope, } \\
10^{-3} \mu \omega \mathrm{cm} / \mathrm{hr}\end{array}$ & $\begin{array}{l}\text { Normalised } \\
\text { Slope }\end{array}$ \\
\hline 11 & 52.50 & 1.000 & 11.45 & 1.000 \\
\hline trino & 33,05 & .629 & 10.025 & $(.87 \mathrm{f})$ \\
\hline 2000 & 17.35 & .330 & 9.725 & .849 \\
\hline 3100 & 8.20 & .156 & 8.46 & .739 \\
\hline 411301 & 3.71 & .0706 & f. 78 & .592 \\
\hline 5000 & 1.675 & .0319 & 5.628 & .491 \\
\hline Boon & 0.715 & .0136 & 4.395 & .384 \\
\hline $701 \%$ & 0.412 & .00785 & 3.04 .4 & .266 \\
\hline
\end{tabular}

* Alope corrected for change in absolute magnitude die to a change in form lactor of specimen. 


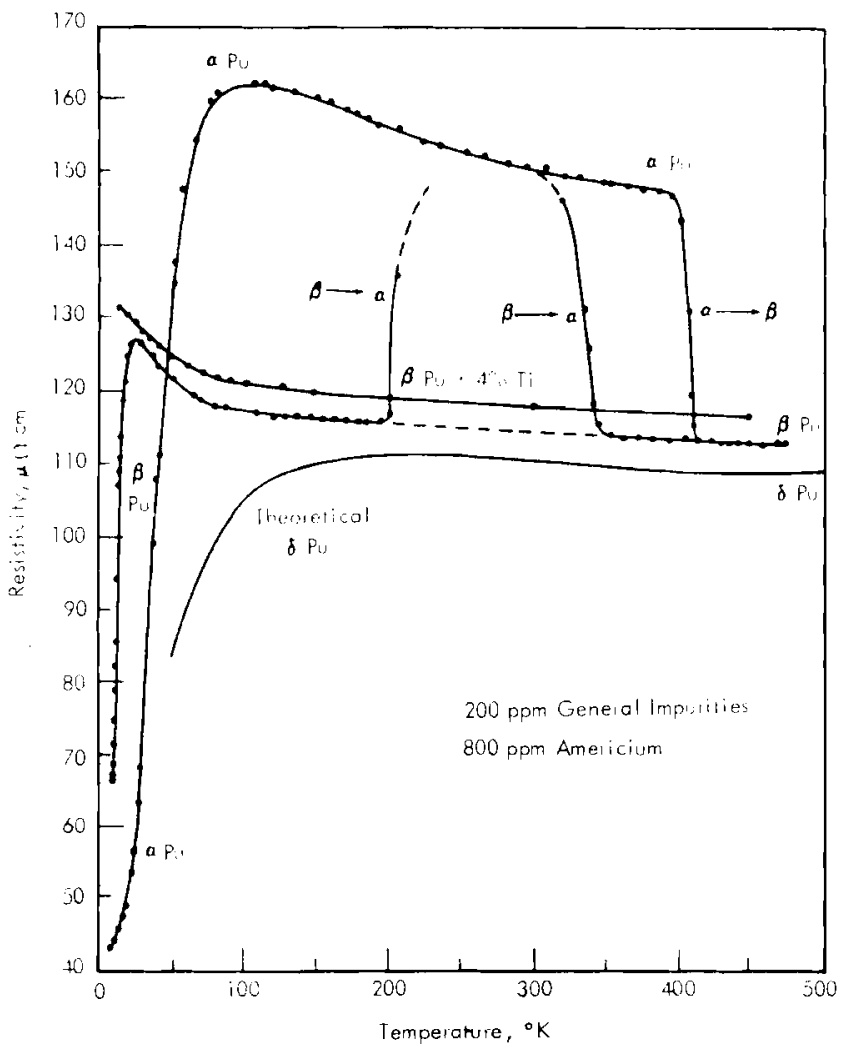

Resistivity-Temperature Values for Pure Plutonium, $\boldsymbol{\beta}$-Plase. From F. King and J. A. lee. Cryogenics, vol. 3, p. 17i. 1963.

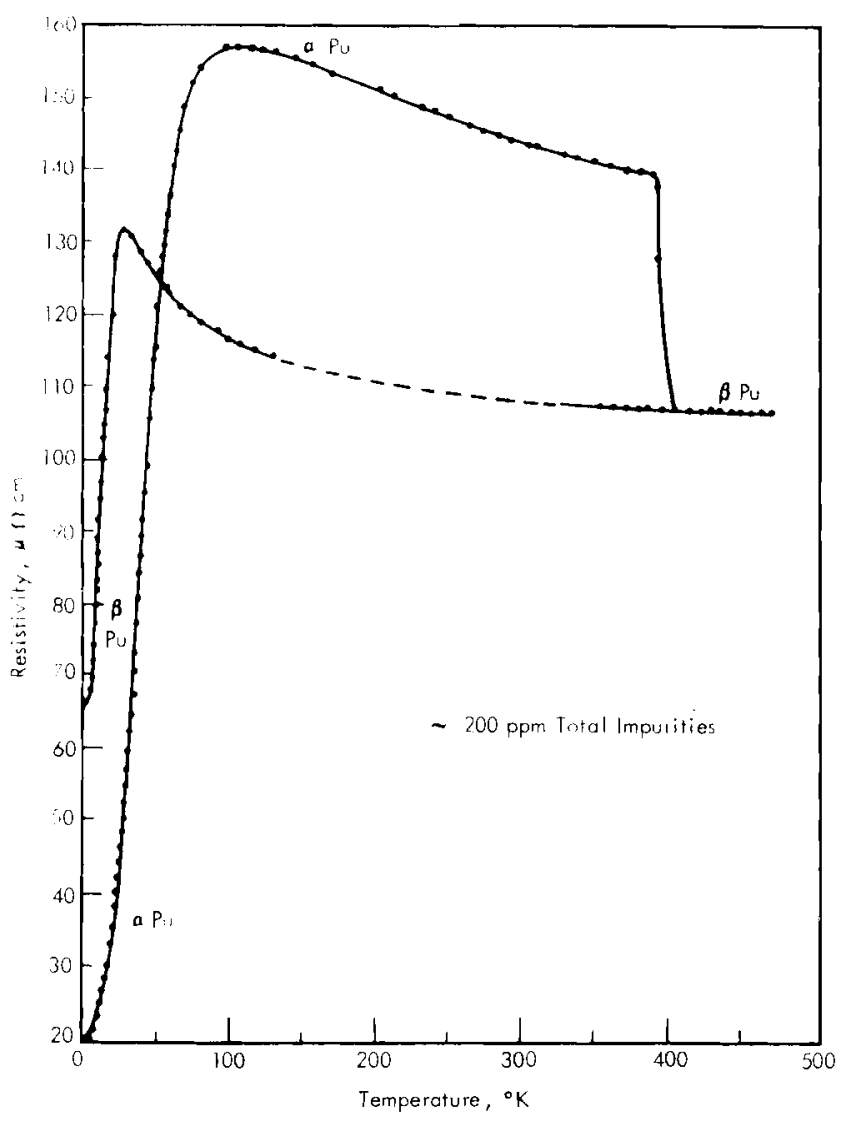

Resistivity-Temperature Values for ligh Purity Plutonium, p-Phase. From E. King and $J, A$, Lee Cryogenics, rol. $A, p .172$, 1963 

V

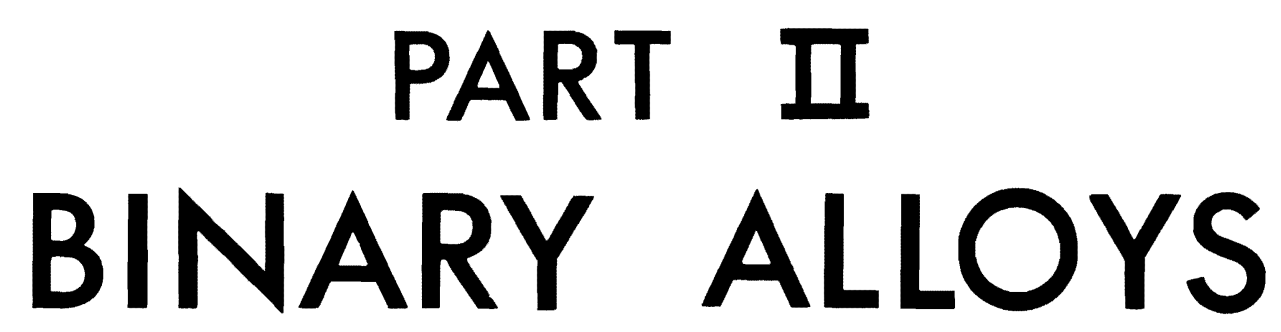




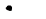


$\mathrm{PuAg}_{3}$ is hexagonal, with

a $\quad 12.730=0.003 \mathrm{~A}$

$9.402 \neq 0.005$
$-16.00 x-11.33$

Space group. $\underline{\mathrm{x}} \mathrm{P}_{3}$ (Int. Tal). 173), $\mathrm{P}_{3} / \mathrm{m}$ (Int. Tab. 176), or $\mathrm{P}_{3} 22$ (Int.

Tab, 182).

The atomic positions have not been determined.

From O. J. C. Runnalls quoted in The Metal Plutonium, p. 302

Phase Diagrams

Al 101

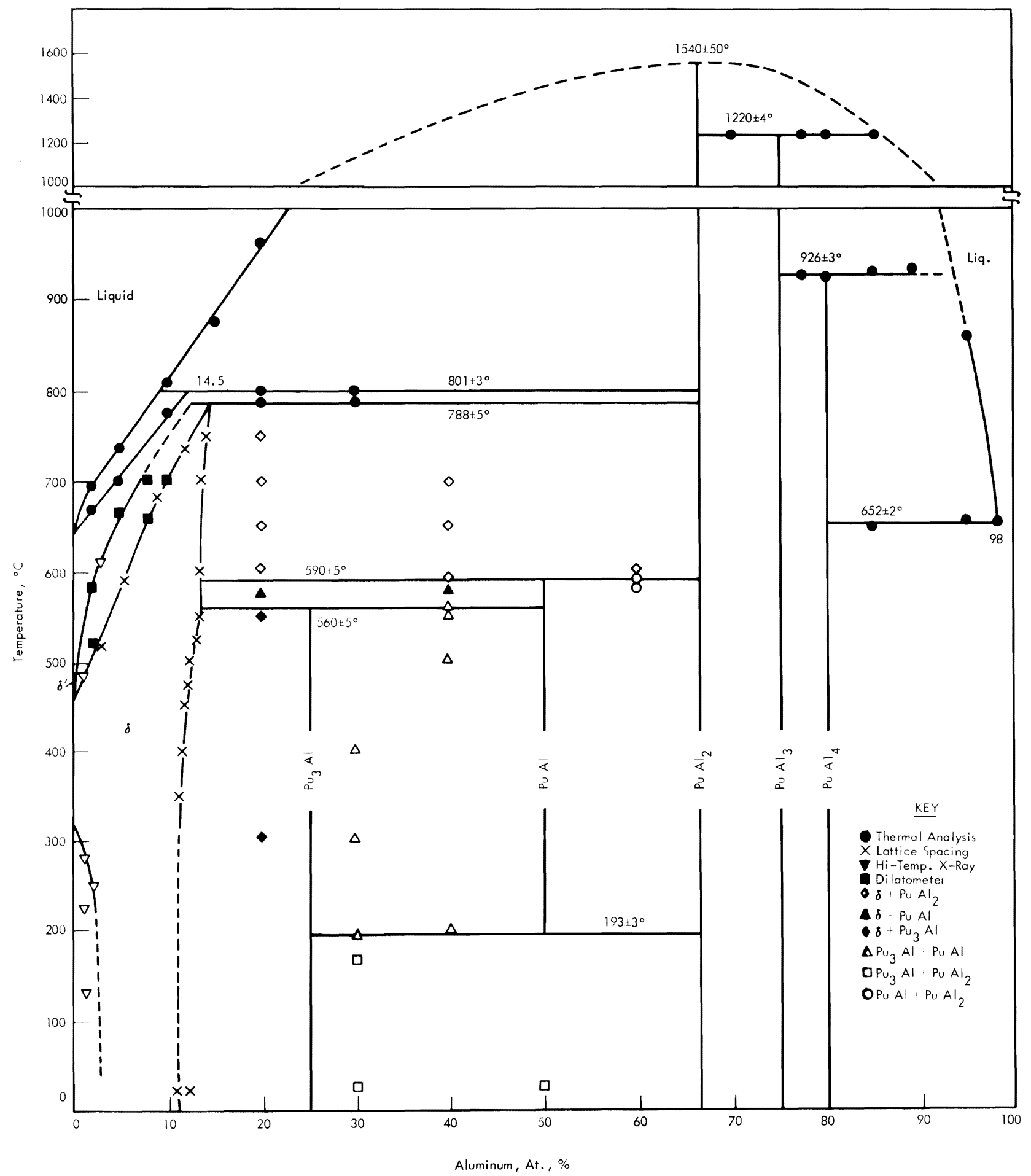

The Plutonium-Aluminum Phase Diagram.

From F. H. Ellinger et al., J. Nucl. Mater., vol. 5, p. 167. 1962. 


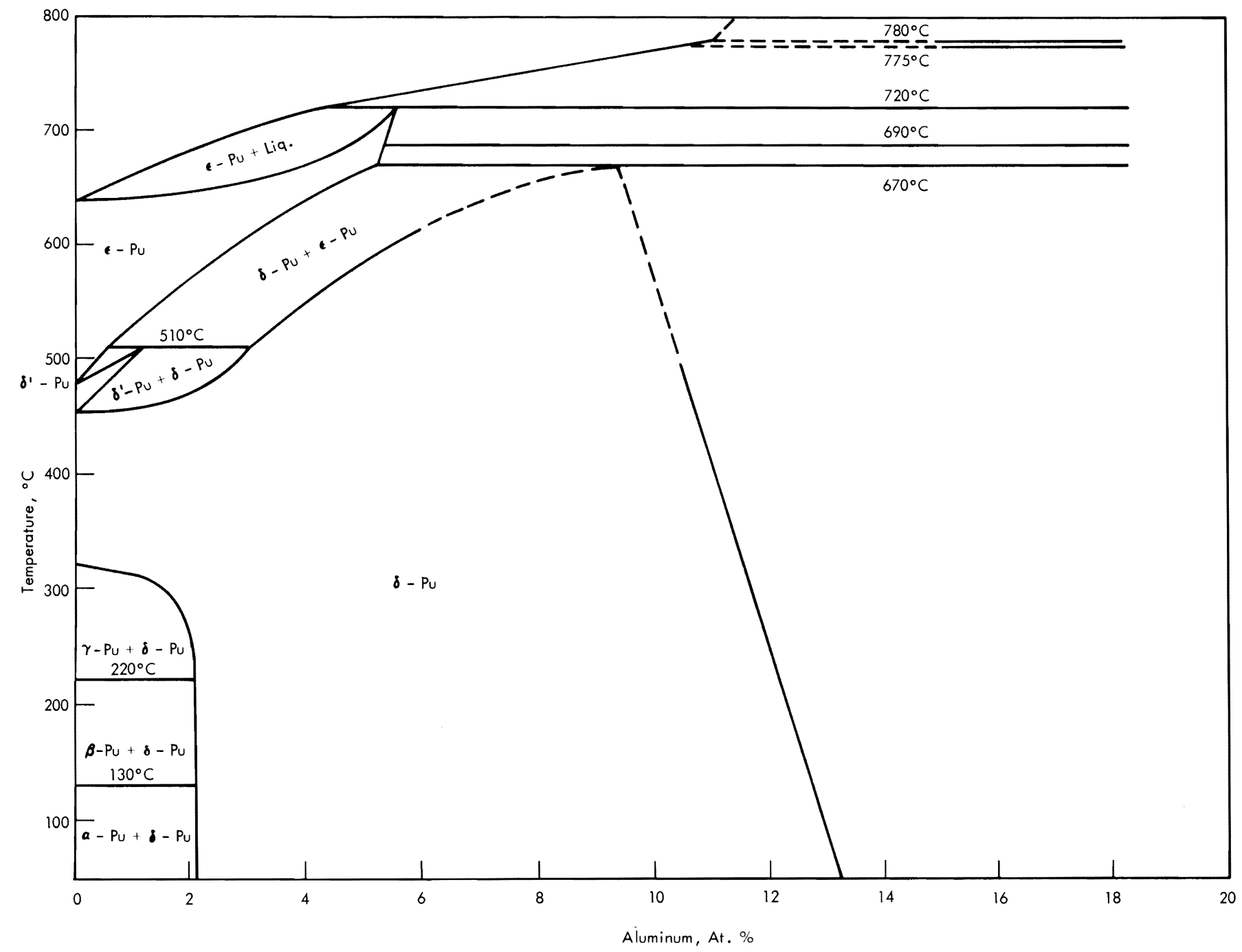

The Plutonium-Rich Fnd of the Plutonium-Aluminum System.

From M. B. Waldron et al. in Proc. Second Geneva Internat. Conf. vol. 6,

p. 165.1958 . 


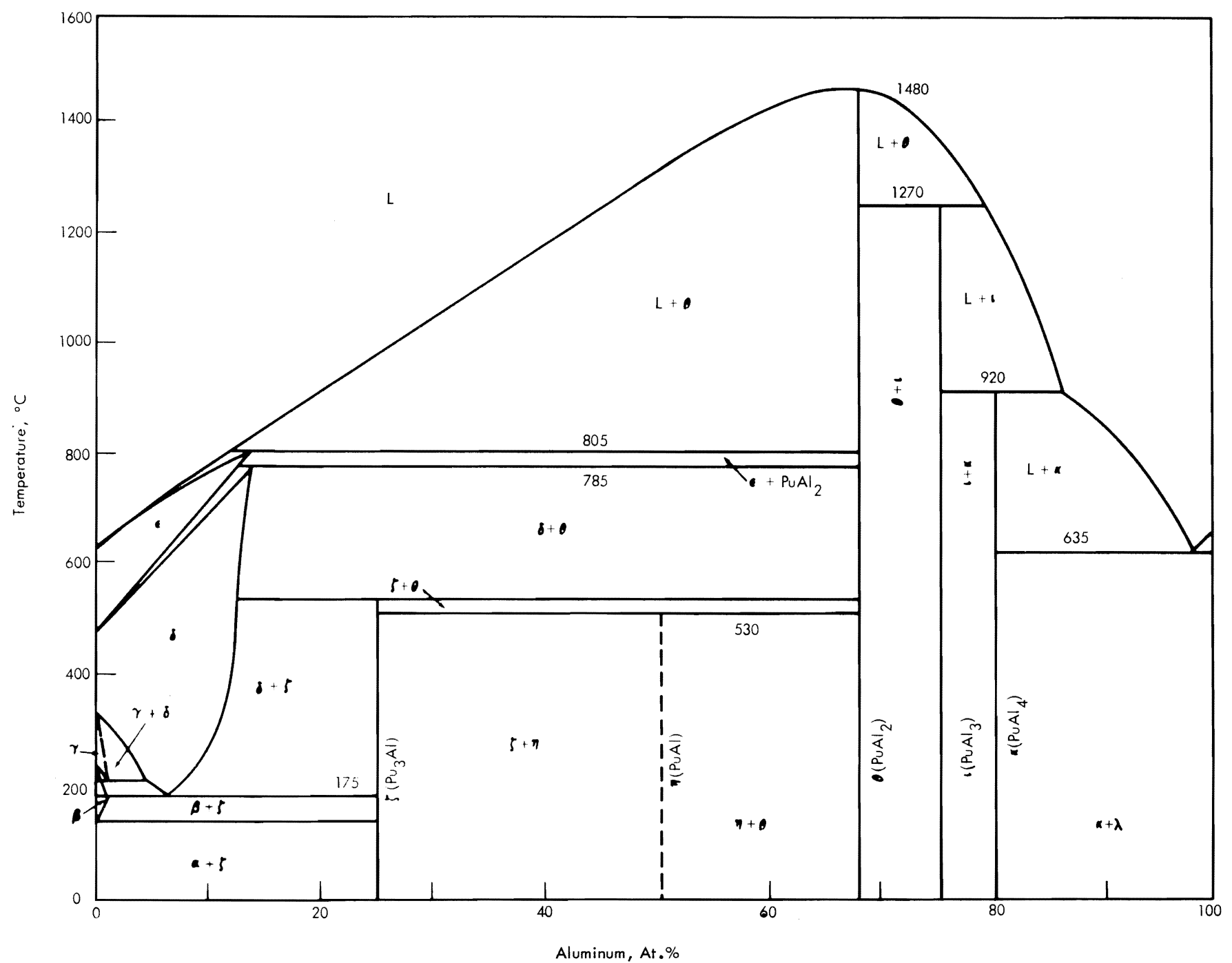

The Plutonium-Aluminum System.

From F. W. Schonfeld, in The Metal Plutonium, p. 259.

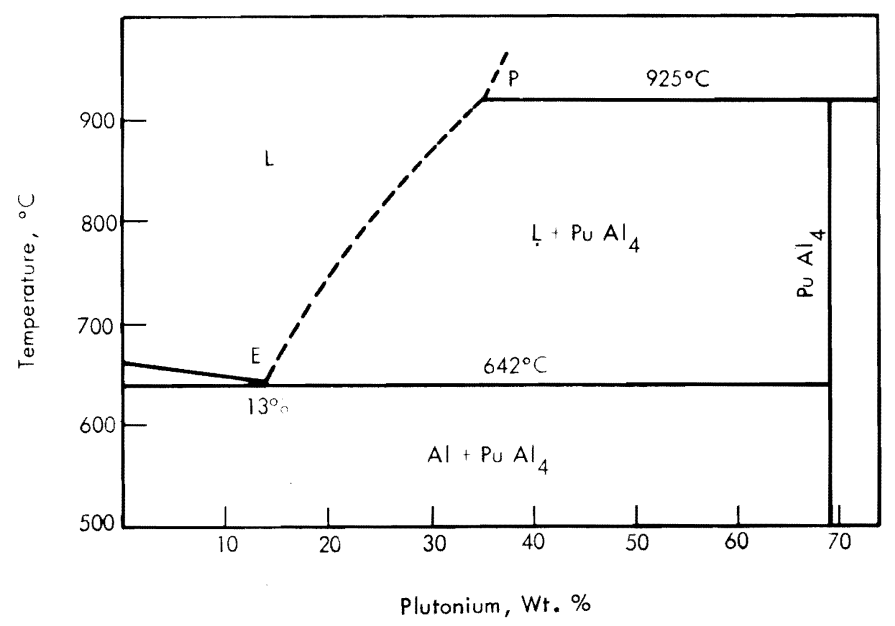

From R. Abramson et al. Proc. Second Geneva Internat. Conf. vol. 6, p. 179. 


\section{PLUTONIUM-ALUMINUM}

$\mathrm{Pu}_{3} \mathrm{Al}$

Tetragonal, partially ordered $\mathrm{SrPb}$ type (tetragonally deformed $\mathrm{AuCu}_{3}$, trpe) $\mathrm{a}=4.499 \pm 0.002, \mathrm{c} 4.538 \pm 0.002 \mathrm{~A}$ $\mathrm{a}=4.530, \mathrm{c}=4.475 \mathrm{~A}$ From A. S. Coffinberry and M. B. Waldron, Metallurgy and Fuels, series V, vol. 1, p. 390 .

Space group. $-\mathrm{P} 4 / \mathrm{mmm}$ (?) (Int. Tab. 123).

Atomic positions

$1 \mathrm{Al}$ in (a): $0,0,0$

1 Pu in (c): $1 / 2,1 / 2,0$

$2 \mathrm{Pu}$ in (e): $0,1 / 2,1 / 2: 1 / 2,0,1 / 2$

Interatomic distances

$$
\begin{array}{ll}
\mathrm{Pu}-8 \mathrm{Pu}=3.195 & \mathrm{Al}-8 \mathrm{Pu}=3.195 \\
\mathrm{Pu}-4 \mathrm{Al}=3.181 & \mathrm{Al}-4 \mathrm{Pu}=3.181
\end{array}
$$

PuAl

Body centered cuhic F. II. Ellinger et al. J. Nucl. Mater., vol. 5, p. 165. 1962

$$
\mathrm{a}=10.769 \pm 0.001 \mathrm{~A}
$$

$\mathrm{Z}=29.01(58$ atoms $)$

PuAl,

Cubic face-centered, $\mathrm{Cu}_{2} \mathrm{Mg}$ type

Pu-rich: $a=7.838=0.001 \mathrm{~A}$

Al-rich: a $7.848=0.001$

a $7.8 .31+0.005$ O. J. C Runnalls, Can. J. Chem., vol. 34, p. 133.1956 .

a $\bar{i} .832$ (Coffinherry and Waldron)

$\mathrm{Z} \quad 8, \mathrm{D}_{\mathrm{x}}=8.06$

Space group. - Fd3m (Int. Tab). 227).

Space group.-
Atomic positions

$(0,0,0: 0,1 / 2,1 / 2: 1 / 2,0,1 / 2: 1 / 2,1 / 2,0)$

SPu in (a): $0,0,0: 1 / 4,1 / 4,1 / 4$

$16 \mathrm{Al}$ in (d): $5 / 8,5 / 8,5 / 8: 5 / 8,7 / 8,7 / 8: 7 / 8,5 / 8,7 / 8: 7 / 8,7 / 8,5 / 8$

Interatomic ristances (hased $\mathrm{on} \mathrm{a}=7.848 \mathrm{~A}$ )

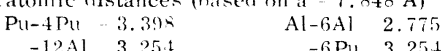

Isotypes. - $\mathrm{LaAl}_{2}, \mathrm{CeAl}_{2}, \mathrm{UAl}_{2}, \mathrm{NpAl}_{2}$.

$\mathrm{PuAl}_{3}$

Hexagonal, $\mathrm{PuAl}_{3}$ trpe (new structure type)

a $6.10=0.02 \mathrm{~A}$ (Iarson et al. Acta Cryst. , vol. 10, p. 443. 1957.)

c. $14.17 \pm 0.02$

$\mathrm{a}=6.08 \pm 0.01$ (Runnalls)

$\mathrm{c}=14.40 \pm 0.03$

$\mathrm{Z}=6, \mathrm{D}=6.67$

6.92 (Runnalls)

Space group. - $\mathrm{P}_{3} / \mathrm{mmc}_{3}$ (Int. Tab. 194)

Atomic positions

$2 \mathrm{Pu}_{\mathrm{I}}$ in $(\mathrm{b}): \pm(0,0,1 / 4)$

$4 \mathrm{PuI}_{\mathrm{II}}$ in (f): $\pm(1 / 3,2 / 3, \mathrm{z}: 1 / 3,2 / 3,1 / 2-\mathrm{z})$ with $\mathrm{z}=0.0892 \pm 0.0001$

${ }^{6} \mathrm{Al}_{\mathrm{I}}$ in $(\mathrm{h}): \pm(\mathrm{x}, 2 \mathrm{x}, 1 / 4: 2 \mathrm{x}, \mathrm{x}, 1 / 4: \mathrm{x}, \overline{\mathrm{x}}, 1 / 4)$ with $\mathrm{x}=0.5160 \pm 0.0030$

$12 \mathrm{Al}$ in $(\mathrm{k}): \pm\left(\mathrm{x}, 2 \mathrm{x}, \mathrm{z}: 2 \overline{\mathrm{x}}, \overline{\mathrm{x}}, \mathrm{z}: \mathrm{x}, \overrightarrow{\mathrm{x}}, \mathrm{z}: \overline{\mathrm{x}}, 2 \overline{\mathrm{x}}, 1 / 2+\mathrm{z} ; 2 \mathrm{x}, \mathrm{x}, 1 / 2{ }^{\circ} \mathrm{z}\right.$ :

$\begin{aligned}\mathrm{x}, \mathrm{x}, 1 / 2 \cdot \mathrm{z}) \text { with } \mathrm{x} & =0.8337 \pm 0.0040 \\ z & =0.0815 \pm 0.0007\end{aligned}$

Interatomic distances

$$
\begin{aligned}
\mathrm{Pu}_{\mathrm{I}}-\mathrm{6Al} & =3.01 \\
-6 \mathrm{Al} & =3.06 \\
\mathrm{Pu}^{\mathrm{II}}-3 \mathrm{Al} & =3.02 \\
-3 \mathrm{Al} & =3.03 \\
-6 \mathrm{Al} & =3.05
\end{aligned}
$$

$$
\begin{aligned}
\mathrm{Al}_{\mathrm{I}}-2 \mathrm{Pu} & =3.02 \\
-2 \mathrm{Pu} & =3.06 \\
-2 \mathrm{Al} & =2.76 \\
-4 \mathrm{Al} & =2.96 \\
-2 \mathrm{Al} & =3.34 \\
\mathrm{Al}_{\mathrm{II}}-2 \mathrm{Pu} & =3.01 \\
-2 \mathrm{Pu} & =3.05 \\
-2 \mathrm{Al} & =2.94 \\
-2 \mathrm{Al} & =2.96 \\
-4 \mathrm{Al} & =3.05
\end{aligned}
$$

Mean $\mathrm{Pu}-12 \mathrm{Al}-3.0 \mathrm{t}$

Atomic positions or space group. - $\mathrm{P} 6 \mathrm{~m} 2$ (Runnalls)

$1 \mathrm{Pl}$ in (a) $0,0,0$

$1 \mathrm{Pu}$ in (b) $0,0,1 / 2$

$2 P_{11}$ in (h) $1 / 3,2 / 3, z: 1 / 3,2 / 3, \bar{z}: z=1 / 6$

$2 \mathrm{Pu}$ in (i) $2 / 3,1 / 3, \mathrm{z}: 2 / 3,1 / 3, \bar{z}: \mathrm{z}=1 / 3$

$3 \mathrm{Al}$ in (i) $\mathrm{x}, \overline{\mathrm{x}}, 0 ; \mathrm{x}, 2 \mathrm{x}, 0: 2 \overline{\mathrm{x}}, \overline{\mathrm{x}}, 0: \mathrm{x}-1 / 2$

$3 \mathrm{Al}$ in (k) $\mathrm{x}, \overline{\mathrm{x}}, 1 / 2: \mathrm{x}, 2 \mathrm{x}, 1 / 2: 2 \overline{\mathrm{x}}, \overline{\mathrm{x}}, 1 / 2: \mathrm{x}-1 / 2$

GAl in (n) $\mathrm{x}, \overline{\mathrm{x}}, \mathrm{z}: \mathrm{x}, 2 \mathrm{x}, \mathrm{z}: 2 \overline{\mathrm{x}}, \overline{\mathrm{x}}, \mathrm{z}: \mathrm{x}, \overline{\mathrm{x}}, \overline{\mathrm{z}}: \mathrm{x}, 2 \mathrm{x}, \overline{\mathrm{z}} ; 2 \overline{\mathrm{x}}, \overline{\mathrm{x}}, \overline{\mathrm{z}} ; \mathrm{x}=-1 / 6, z=1 / 6$

$6 \mathrm{Al}$ in $(\mathrm{n}) \mathrm{x} 1 / \mathrm{h}, \mathrm{z}, 1 / 3$.

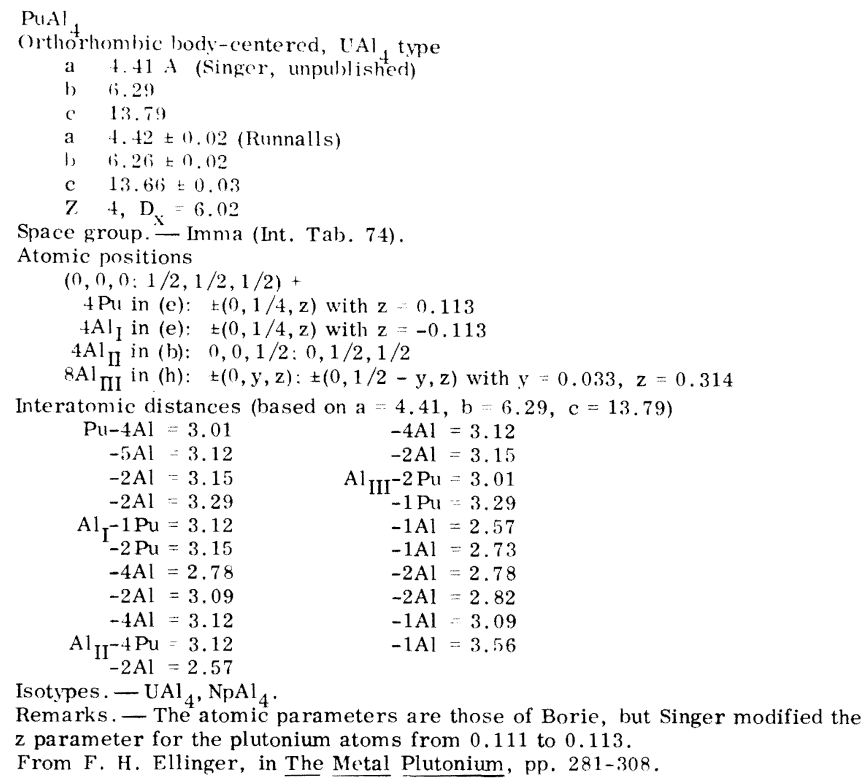

Remarks. - The atomic parameters are those of Borie, but Singer modified the $\mathrm{z}$ parameter for the plutonium atoms from 0.111 to 0.113 .

From F. H. Ellinger, in The Metal Plutonium, pp. 281-308.

\section{$\delta$-PHASE LATTICE CONSTANT/COM POSITION DATA}

$\left(23 \pm 2^{\circ} \mathrm{C}\right)$

From F. H. Ellinger et al., J. Nucl. Mater., vol. 5, p. 170. 1962.

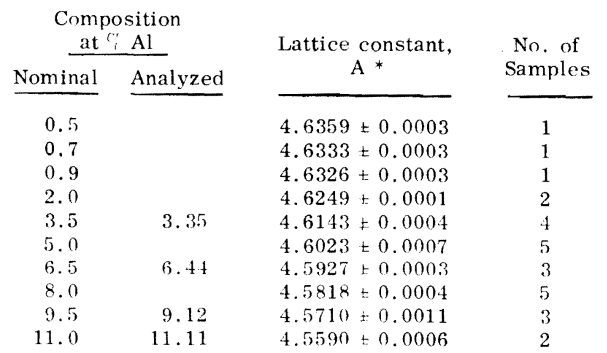

* Uncertainty is the mean deviation.

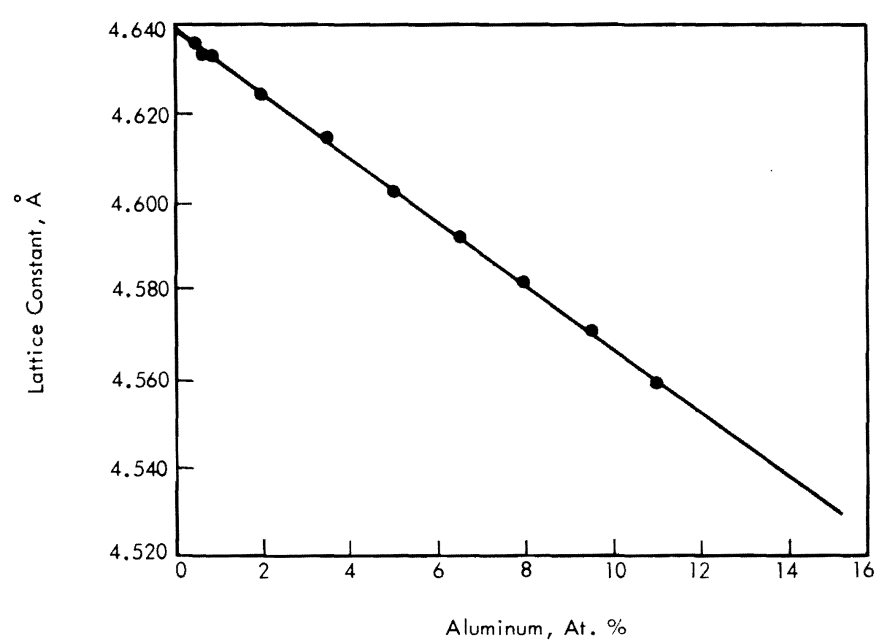

The Lattice Constant/Composition Curve of the $\delta$ Plutonium Solid Solution. From F. H. Fllinger et al., J. Nucl. Mater., vol. 5, p. 170.1962 
ROOM TEMPERATURE DENSITIES, $\mathrm{m} / \mathrm{cm}^{\prime \prime}$

From A. E. Martin and G. D Selmanoff, IAA-501. (Classifiedi)

Nominal Compositio at. $\mathrm{Al}$

\begin{tabular}{l} 
As-Ciast \\
\hline 17.58 \\
16.92 \\
16.55 \\
16.72 \\
16.74 \\
16.01 \\
16.47 \\
16.18 \\
15.93
\end{tabular}

$$
\begin{aligned}
& 17.25 \\
& 16.76 \\
& 16.05 \\
& \hdashline 15.74 \\
& 15.80 \\
& 15.60 \\
& 15.57 \\
& 15.59
\end{aligned}
$$$$
\text { Anneal in Ice Water }
$$

DENSTTIFS OF P1-Al ALLOYS HOMOGENIZED AT $450^{\circ} \mathrm{C}$ FOR 500 IIOURS, AIR COOLED TO ROOM TEMPERATURE:

From R. O. Flliott et al., J. Phys. Chem. Solids, vol. 23, p. 1036.1962 .

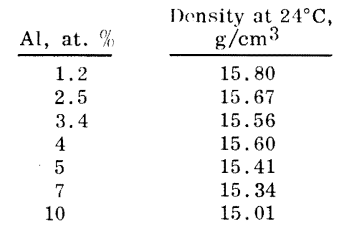

Density of as-cast 8 at. $\%$ Al-Pu alloy at room temperature $=15.235 \mathrm{~g} / \mathrm{cm}^{3}$. From T. A. Sandenaw, J. Phys. Chem. Solids, vol. 16, p. 330.1960.

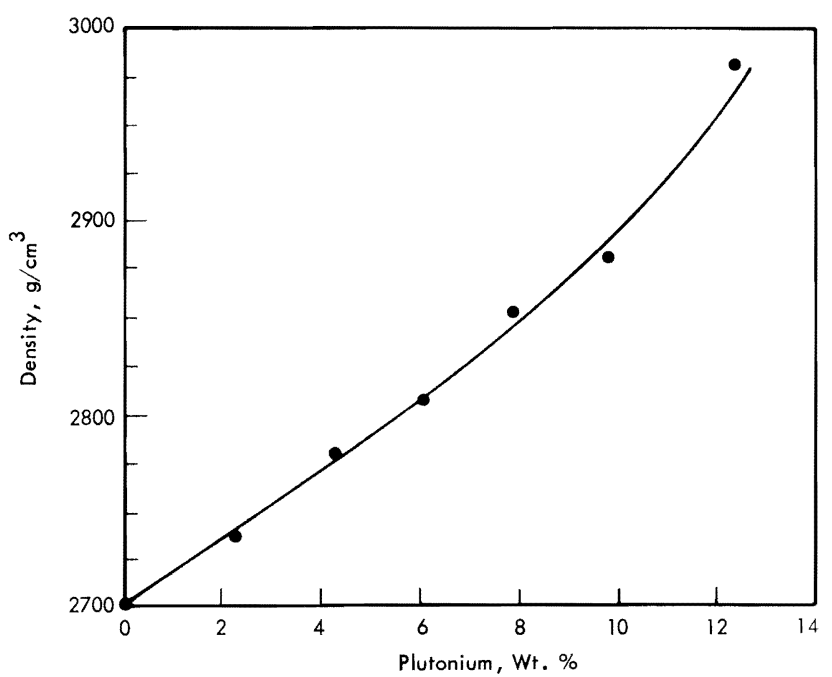

From H. R. Gardner, in Proc. Second Geneva Internat. Conf., vol. 6, p. 689.1958

Thermal Expansion

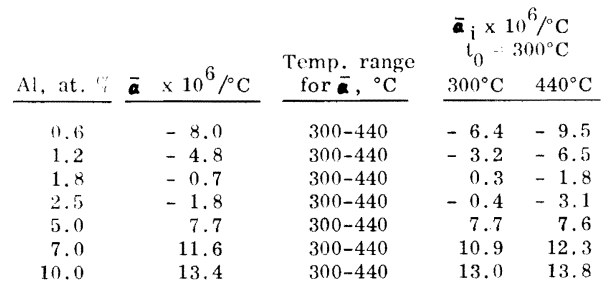

From R. O. Elliott et al., in Plutonium $\underline{1960}$, p. 150.

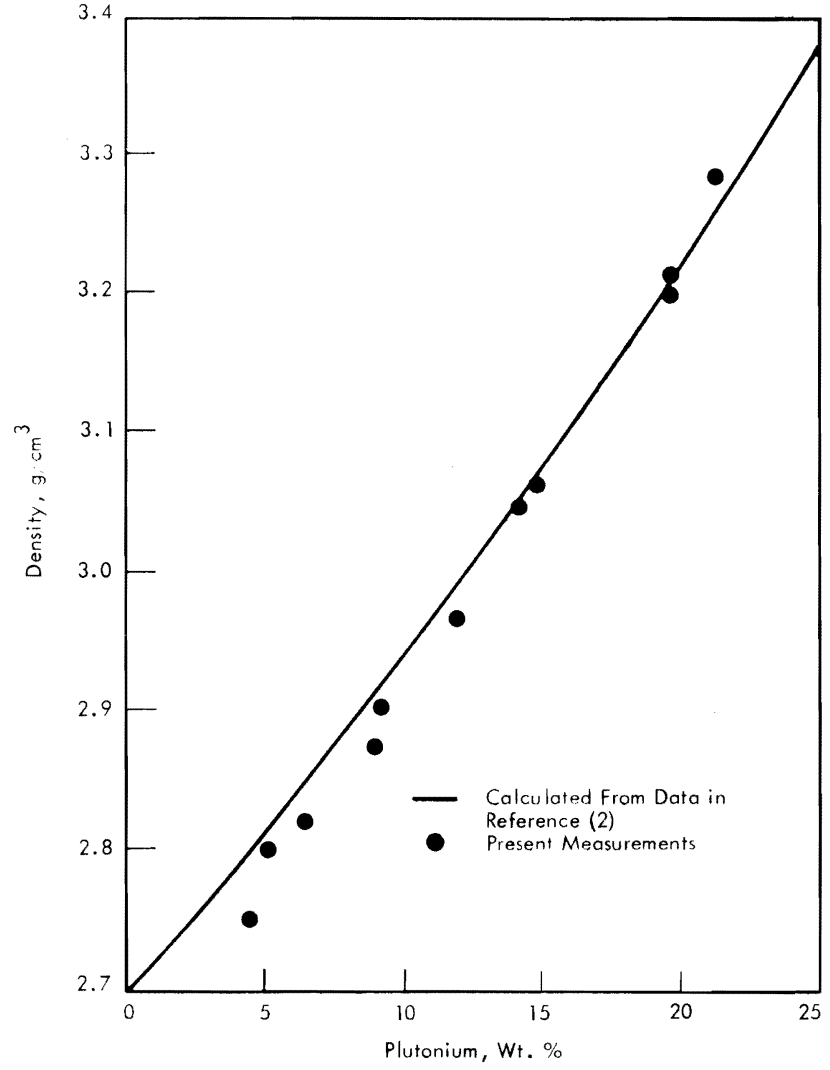

Density of Al-Pu Alloys. (1) From T. I. Jones, in Plutonium 1960, p. 726. (2) From O. J. C. Runnalls, Can. J. Chem. vol. $3 \overline{4, \text { pp. } 133}-145,1956$.

For alloys containing $\mathrm{PuAl}_{4}$ and aluminum, the following relationship between $x$, the weight fraction of plutonium, and $\bullet$, the density, has been derived:

$$
\mathrm{x}=1.24924-3.37296 \frac{1}{\mathrm{p}}
$$

From R. E. Tate, in The Metal Plutonium, p. 359

Al 202.1

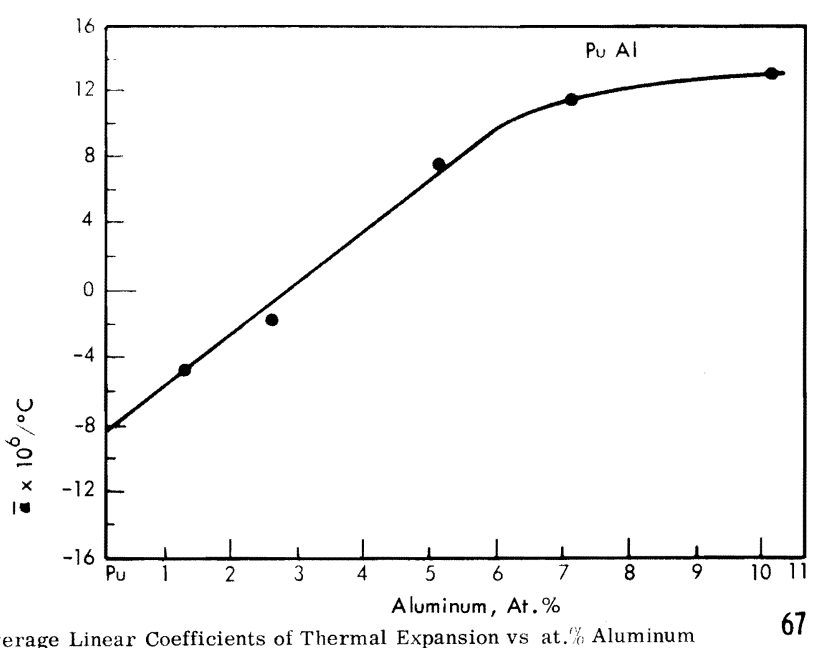

Average Linear Coefficients of Thermal Expansion vs at. Aluminum From R. O. Elliott et al., in Plutonium 1960, p. 152 


\section{THERMAL EXPANSION OF AI-PU ALLOYS}

From J. M. Taylor, Private Communication, reported lw I. 1) Thomas and (1. J. Wick in Plutonium 1960, p. .6-
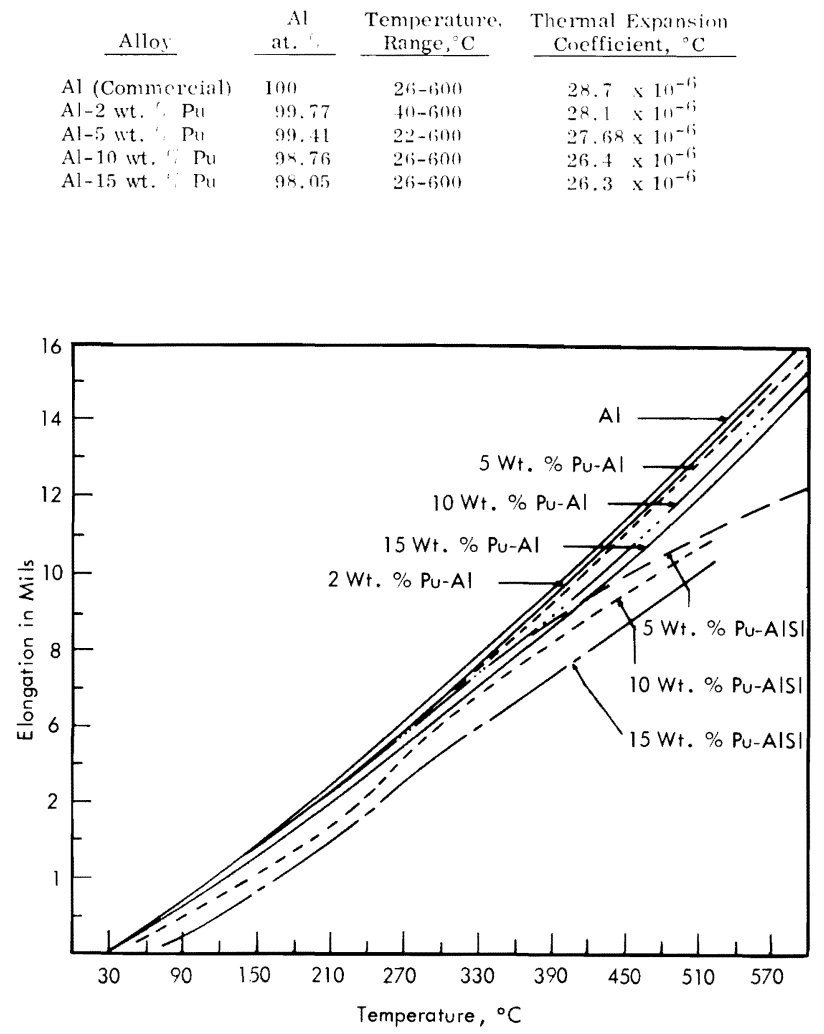

Thermal Expansion of $\mathrm{Al}-\mathrm{Pu}$ and $\mathrm{Al}-12 \mathrm{Wt}$. of $\mathrm{Si}-\mathrm{Pu}$ (1-in. specimen). From J. M. T:Ivlor. Private Communication, reported by I. D. Thomas and O. J. Wick in Plutonium 1960, p. 769.

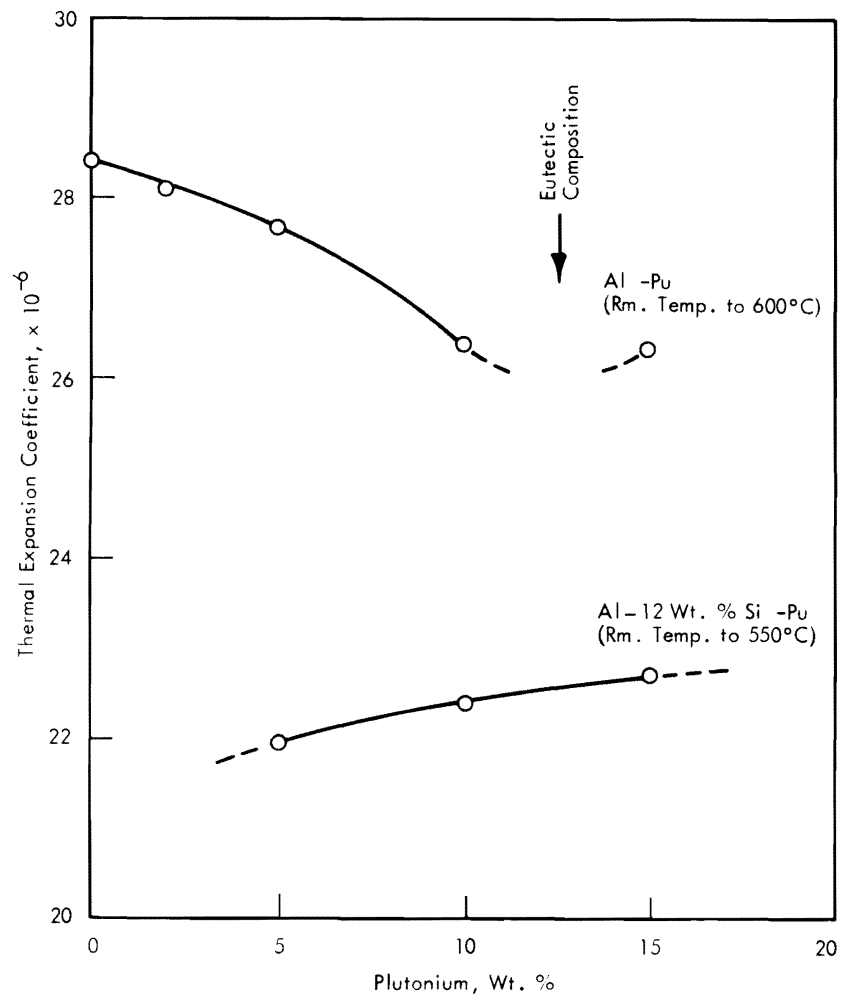

Fffect of Plutonium Comeentration on the Linear Fxpansion of Al-Pu and Al-si-Pt. Froml. 11. Taylor. Private Communication, reporterl hy 1. D. Thomas and (1. I. Wick in Plutonim 1960, 1). 769 .

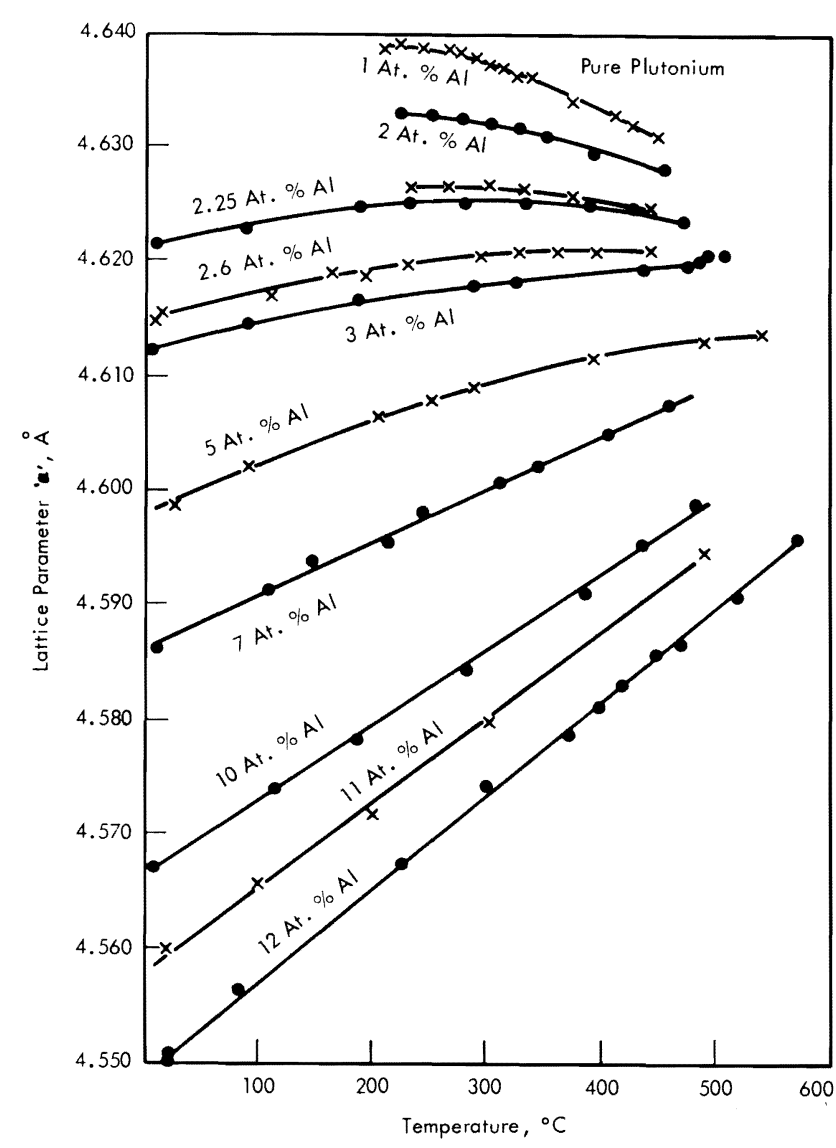

Lattice Parameter vs Temperature for $\mathrm{Pu}-\mathrm{Al} \delta$-Phase Solid Solutions. From J. A. Lee et al., in Plutonium 1960, p. 41 .

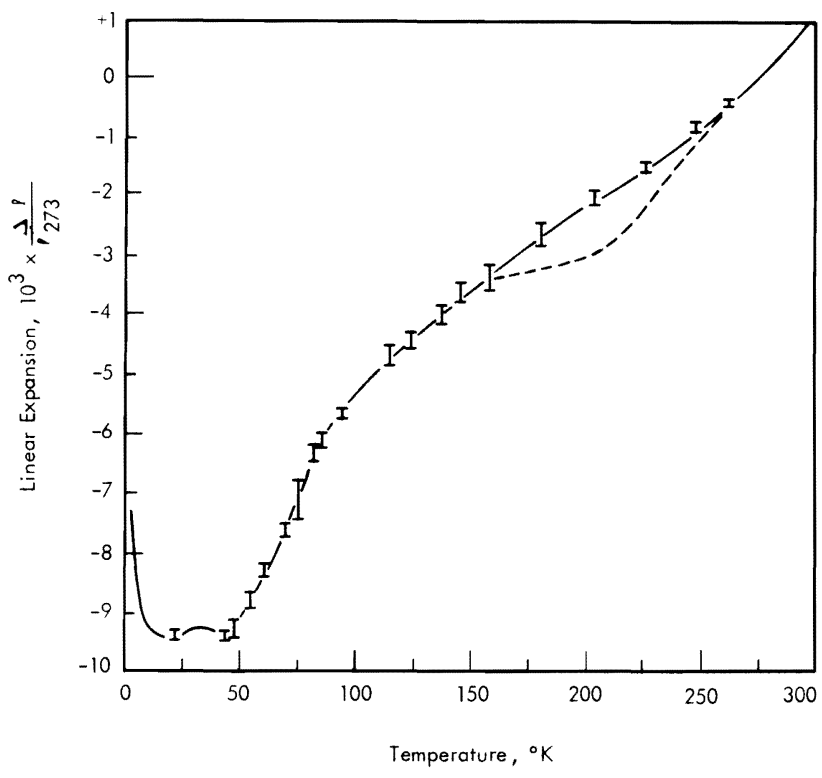

Thermal Expansion Curve inr an 8 At. Al-Pu Alloy (Stabilized Delta Phase at liom Temperature) Relative to Fused Quartz. The dashed line represents lehavior on first cooling. From T. A. Sandenaw, J. Phys. Chem. Solids, vol. 16. p. 3331 . 1960 . 


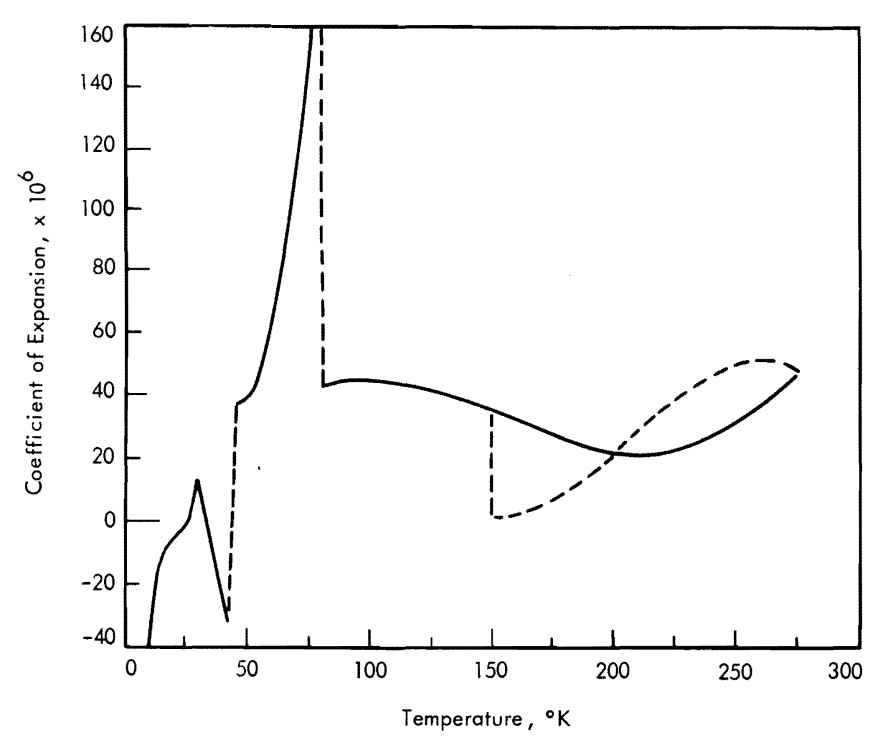

Curve of Coefficient of Thermal Expansion for an 8 At. \% Al-Pu Alloy. Dashed line at $150^{\circ} \mathrm{K}$ and above represents data of dashed line in preceding figure in the same temperature range. From T. A. Sandenaw, J. Phys. Chem. Solids, vol. 16 , p. 334 . 1960

Least squares equations for linear expansion of 8 at. \% Al-Pu alloy

The thermal expansion data for the plutonium alloy were submitted to least squares analysis. Because of the abrupt changes of slope of the curve, the data

Thermal Conductivity

\begin{tabular}{|c|c|}
\hline $\begin{array}{l}\text { Aluminum } \\
\text { Avg. at. } \% \text { Measured }\end{array}$ & $\mathrm{K}\left(\mathrm{cal} / \mathrm{cm} \quad{ }^{\circ} \mathrm{C} \quad \mathrm{Sec}\right)$ \\
\hline 0 & 0.58 \\
\hline 1.39 & 0.53 \\
\hline 5.18 & 0.47 \\
\hline 1.0 .26 & 0.28 \\
\hline 11.92 & 0.21 \\
\hline
\end{tabular}

The ratio of thermal to electric conductivity is approximately constant over the range of compositions studied.

$\mathrm{K}=5.02 \sigma \mathrm{T} \times 10^{-9}+0.05$

$$
\begin{aligned}
& \mathrm{K}=\text { thermal conductivity }\left(\mathrm{cal} / \mathrm{cm}^{\circ}{ }^{\circ} \mathrm{C}\right. \text { Sec) } \\
& \sigma=\text { electrical conductivity }\left(\mathrm{ohm}^{-1} \mathrm{~cm}^{-1}\right) \\
& \mathrm{T}=\text { absolute temperature }
\end{aligned}
$$

From R. B. Gibney. LAMS -1080 . were divided into four temperature ranges. Equations for these temperature ranges were:

$$
\begin{aligned}
& \text { Temperature Range: } 1.86-30^{\circ} \mathrm{K} \\
& \Delta 1_{\mathrm{T}}=1_{273}\left(\mathrm{~A}+\mathrm{BT}+\mathrm{CT}^{2}+\mathrm{DT}^{3}+\mathrm{ET}^{4}+\mathrm{FT}^{5}\right) \\
& \mathrm{A}=-6.0281 \times 10^{-3} \\
& \mathrm{~B}=-7.4790 \times 10^{-4} \\
& \mathrm{C}=6.6822 \times 10^{-5} \\
& \mathrm{D}=-2.9584 \times 10^{-6} \\
& \mathrm{E} \quad=6.3943 \times 10^{-8} \\
& \mathrm{~F} \quad=-5.3308 \times 10^{-10} \\
& \sigma \quad=1.6 \times 10^{-4} \\
& \text { Temperature Range: } 30-42^{\circ} \mathrm{K} \\
& \Delta{ }^{1} \mathrm{~T}=1273\left(\mathrm{~A}+\mathrm{BT}+\mathrm{CT}^{2}\right) \\
& \mathrm{A}=-1.1283 \times 10^{-2} \\
& \mathrm{~B}=1.2230 \times 10^{-4} \\
& \mathrm{C} \quad=-1.8558 \times 10^{-6} \\
& \boldsymbol{\sigma}=1.1 \times 10^{-5}
\end{aligned}
$$

Temperature Range $44-76^{\circ} \mathrm{K}$

$$
\begin{aligned}
\Delta 1_{\mathbf{T}} & =1_{273}\left(\mathrm{~A}+\mathrm{BT}+\mathrm{CT}^{2}+\mathrm{DT}^{3}\right) \\
\mathrm{A} & =-1.6472 \times 10^{-2} \\
\mathrm{~B} & =3.8990 \times 10^{-4} \\
\mathrm{C} & =-7.5351 \times 10^{-6} \\
\mathrm{D} & =5.3651 \times 10^{-8} \\
\boldsymbol{\sigma} & =3.4 \times 10^{-4}
\end{aligned}
$$

Temperature Range $78-273^{\circ} \mathrm{K}$

$$
\begin{aligned}
\boldsymbol{\Delta}_{1}{ }_{\mathrm{T}} & =1_{273}\left(\mathrm{~A}+\mathrm{BT}+\mathrm{CT}^{2}+\mathrm{DT}^{3}+\mathrm{ET}^{4}\right) \\
\mathrm{A} & =-7.3254 \times 10^{-3} \\
\mathrm{~B} & =-3.9405 \times 10^{-5} \\
\mathrm{C} & =9.8614 \times 10^{-7} \\
\mathrm{D} & =-4.8175 \times 10^{-9} \\
\mathrm{E} & =7.6700 \times 10^{-12} \\
\boldsymbol{\sigma} & =8.6 \times 10^{-4}
\end{aligned}
$$

From T. A. Sandenaw, J. Phys. Chem. Solids, vol. 16, p. 333.1960.

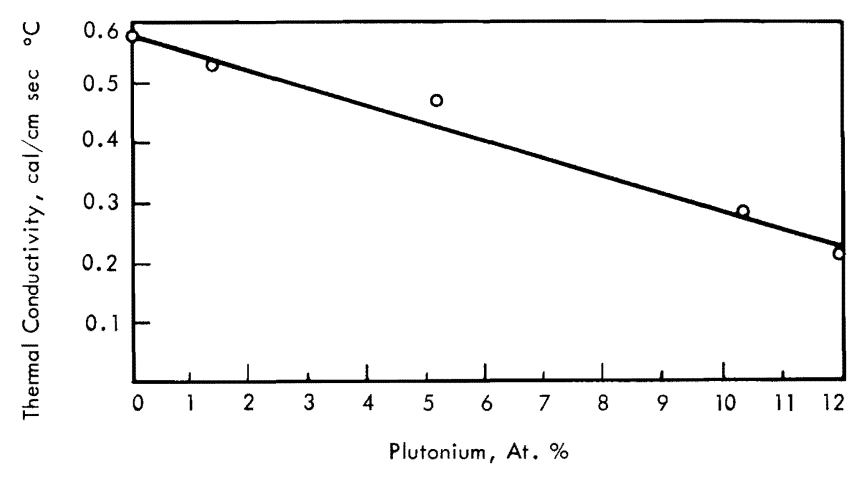

Thermal Conductivity as a Function of Atomic Percent Pu in Pu-Al Alloys. Temperature Range $=0$ to $25-30^{\circ} \mathrm{C}$. From R. B. Gibney. LAMS-1080. 


\section{Al 202.3 spexilic Heat and Heat capactity}

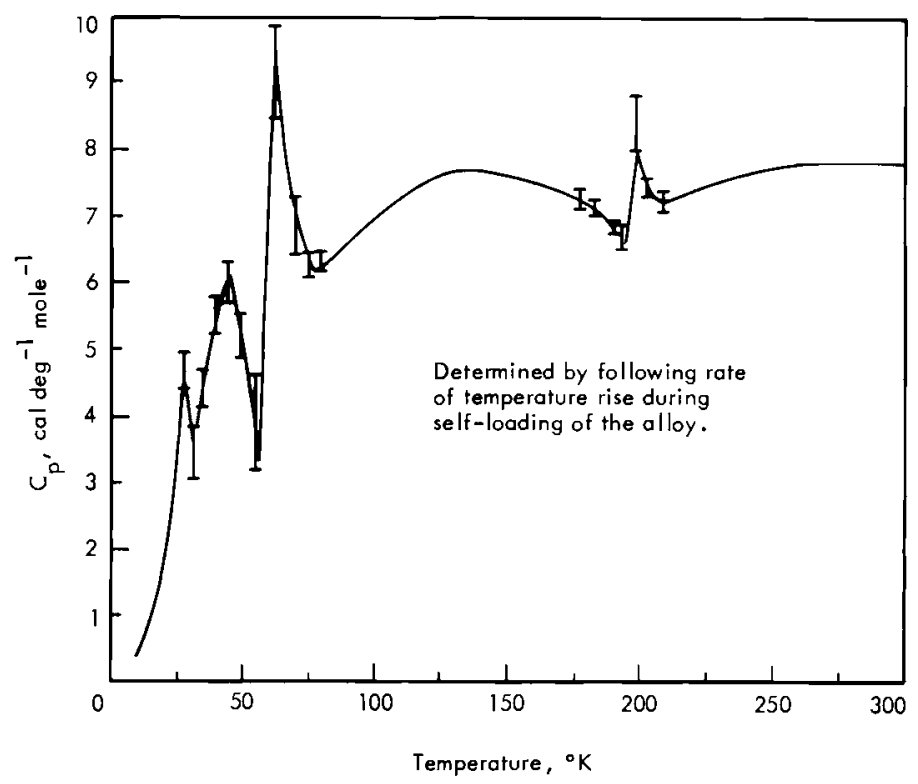

From T. A. Sandenaw, J. Phys. Chem. Solids, vol. 16, p. 330.1960.
The equations for heat capacity $\left(\mathrm{C}_{\mathrm{p}}\right)$, as a function of temperature for regions where spikes were absent, we re found to be as follows:

Temperature Range below $25,74^{\circ} \mathrm{K}$

$\mathrm{C}_{\mathrm{p}}=\boldsymbol{\gamma} \mathbf{T}+\mathrm{BT}^{3}$

${ }_{\gamma}^{\mathrm{p}}=139.3 \times 10^{-4}$

B $\therefore 2.0994 \times 10^{-4}$

$\sigma \quad$ standard deviation $=0.358$

Temperature Range: $76-193^{\circ} \mathrm{K}$

$\mathrm{C}_{\mathrm{p}}=\mathrm{A}+\mathrm{BT}+\mathrm{CT}^{2}+\mathrm{DT}^{3}$

$\mathrm{A}^{\mathrm{p}}=2.2804$

$\mathrm{B}=4.4898 \times 10^{-2}$

C $=1.7175 \times 10^{-4}$

$\mathrm{D}=-1.5012 \times 10^{-6}$

$\sigma=0.323$

Temperature Range: $208-300^{\circ} \mathrm{K}$

$\mathrm{C}_{\mathrm{p}}=\mathrm{A}+\mathrm{BT}+\mathrm{CT}^{2}$

$A^{p}=-3.8356$

$\mathrm{B}=8.5039 \times 10^{-2}$

$\mathrm{C}=-1.5484 \times 10^{-4}$

- $=0.450$

From T. A. Sandenaw, J. Phys. Chem. Solids, vol. 16, p. 333.1960

ESTIMATED HEAT CAPACITY OF ALUMINUM-STABILIZED DELTA-PHASE PLUTONIUM

From F. W. Sehonfeld, Unpublished Data, 1961.

\begin{tabular}{cc}
$\begin{array}{c}\text { Temperature, } \\
{ }^{\circ} \mathrm{K}\end{array}$ & $\begin{array}{c}\mathrm{C}_{\mathrm{p}} \\
\left.\text { (cal } /{ }^{\circ} \mathrm{C}\right) / \text { mole }\end{array}$ \\
\cline { 2 - 2 } 300 & 7.8 \\
400 & 8.3 \\
500 & 8.6 \\
600 & 8.8
\end{tabular}

\section{Al 202.4 entroy}

AVERAGED VALUES OF S AND $\triangle$ H AT SEVERAL TEMPERATURES

From T. A. Sandenaw, J. Phys. Chem. Solids, vol. 16, p. 334. 1960.

\begin{tabular}{c} 
Temperature, \\
\hline${ }^{\circ} \mathrm{K}$ \\
\hline 77.5 \\
138 \\
203 \\
262 \\
298
\end{tabular}

\begin{tabular}{|c|c|}
\hline $\mathrm{S}_{\mathrm{T}}$ & $\mathrm{H}_{\mathrm{T}}-\mathrm{H}_{0}$ \\
\hline $7.66 \pm 0.24$ & $324 \pm 8$ \\
\hline $11.70 \pm 0.17$ & $754 \pm 10$ \\
\hline $14.60 \pm 0.14$ & $1240 \pm 14$ \\
\hline $16.54 \pm 0.11$ & $1675 \pm 10$ \\
\hline $17.52 \pm 0.11$ & $1950 \pm 14$ \\
\hline
\end{tabular}

\section{Al 202.6 Heatsol transition}

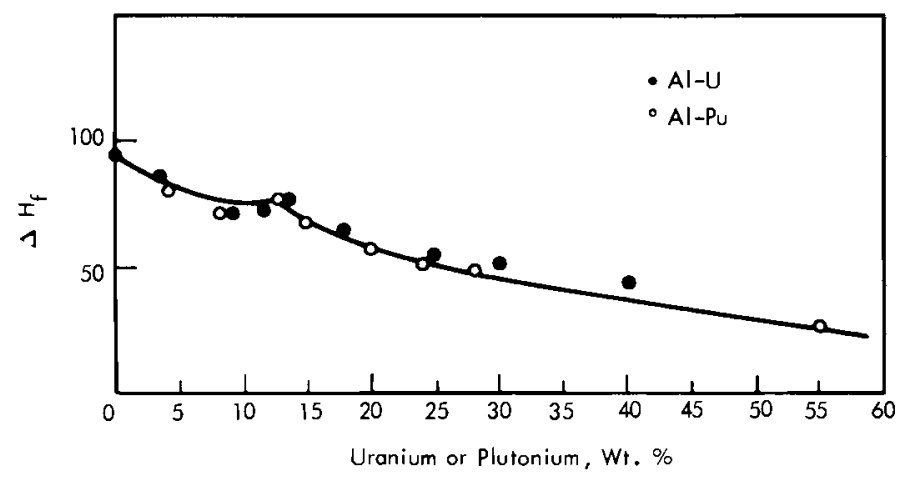

Heats of Fusion for Al-U and Al-Pu Alloys. From R. Abramson et al. in Proc.

Second Geneva Internat. Conf., vol. 6, p. 180. 1958.
ALUMINUM-PLUTONIUM ALLOYS

From R. Abramson et al. in Proc. Second Geneva 1nternat. Conf., vol. 6, p. 180, 1958 .

\begin{tabular}{ccc}
$\begin{array}{c}\text { Pu Concentration, } \\
\text { wt. } 8\end{array}$ & $\begin{array}{c}\text { Heat of Fusion, } \\
\text { cal }\end{array}$ & $\begin{array}{c}\text { Temperature of } \\
\text { Eutectic Fusion, }{ }^{\circ} \mathrm{C}\end{array}$ \\
\cline { 2 - 2 } & 94.5 & ---- \\
4 & 81.5 & 645.3 \\
8 & 71.2 & 642.9 \\
11.3 & 76.5 & 643.5 \\
12.75 & 79.4 & 642.4 \\
14.9 & $66.2^{*}$ & 640.5 \\
20 & $56.3^{*}$ & 642.9 \\
23.9 & $49.8^{*}$ & 640.5 \\
27.9 & $49.2^{*}$ & 640.5 \\
55 & $26.6^{*}$ & 646
\end{tabular}

* Heat of fusion of the eutectic mass present in the alloy. 


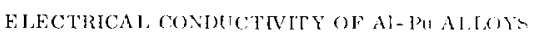

From R. B. Gibney. IAMSS-1080.
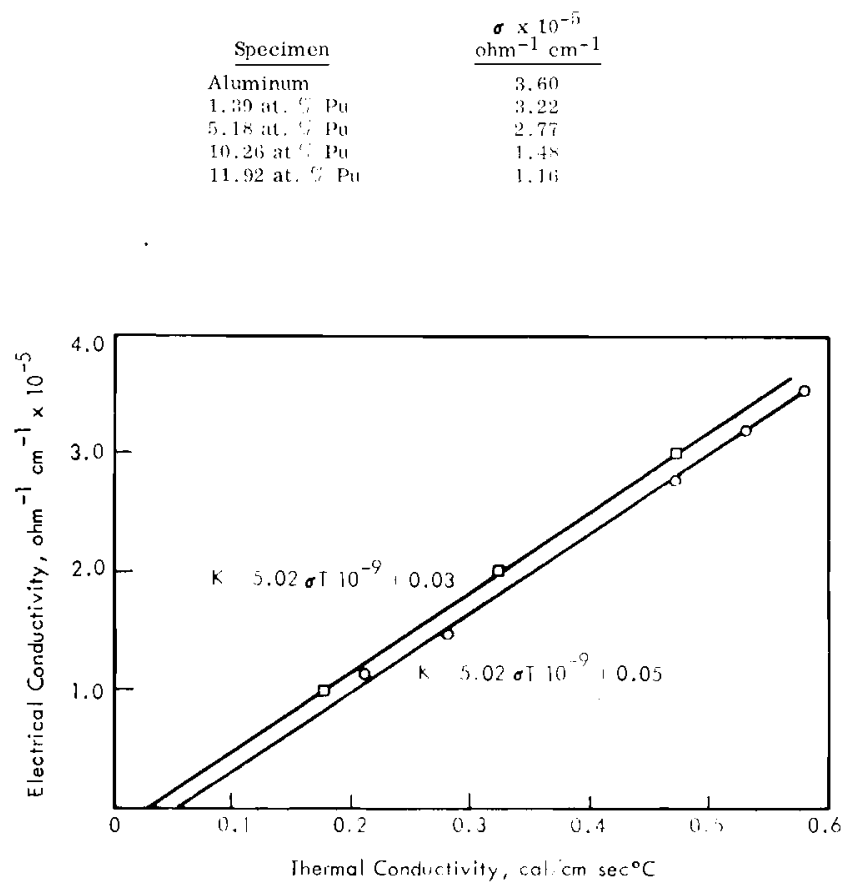

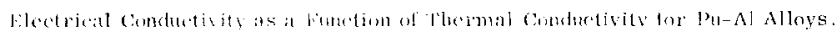

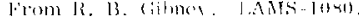

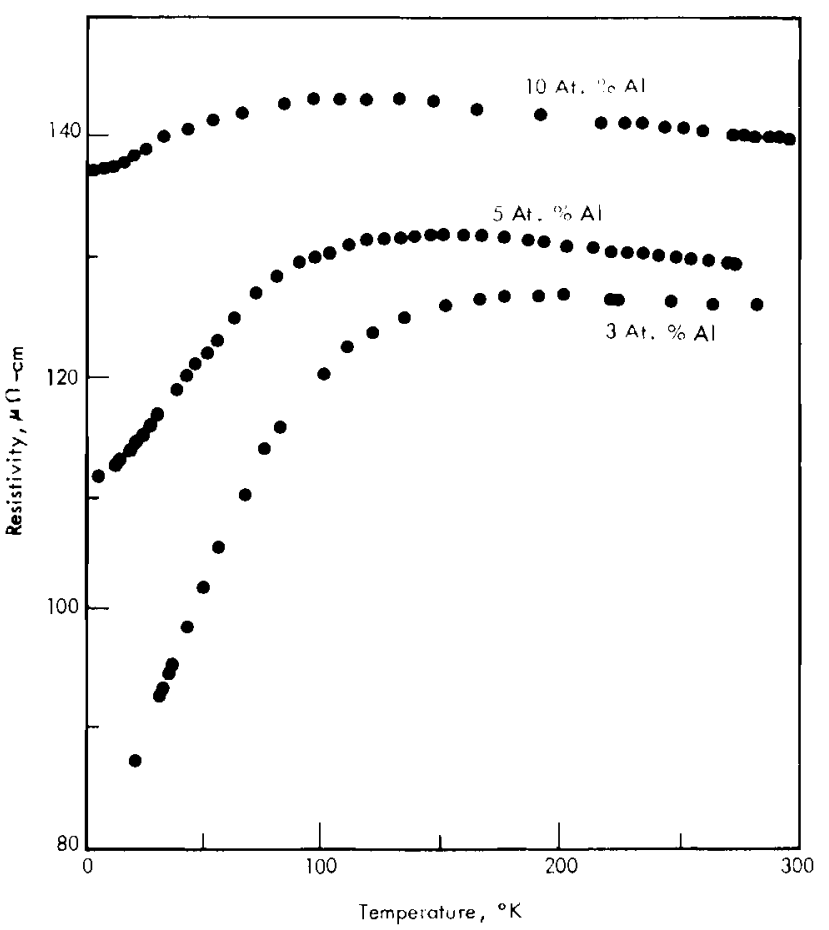

Resistivity of Three Plutonimm-Alung inum Afloys. From J. A. Late al al, Cryogenics, vol. 1. p. 52.1964 .

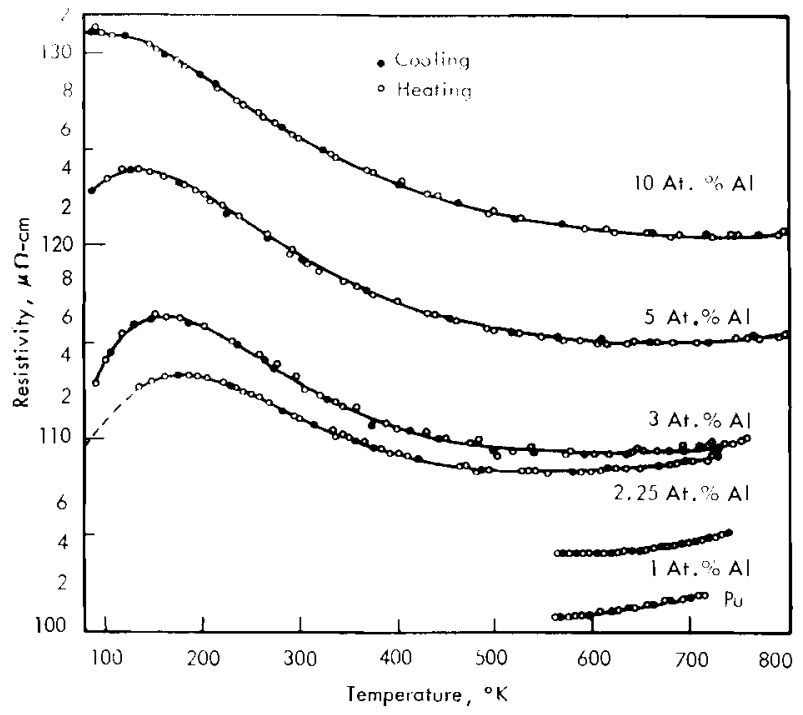

Fesistivities of Pu-Al \& - Phase Solid Sishutions. From d. A. l.ee et al, in I]

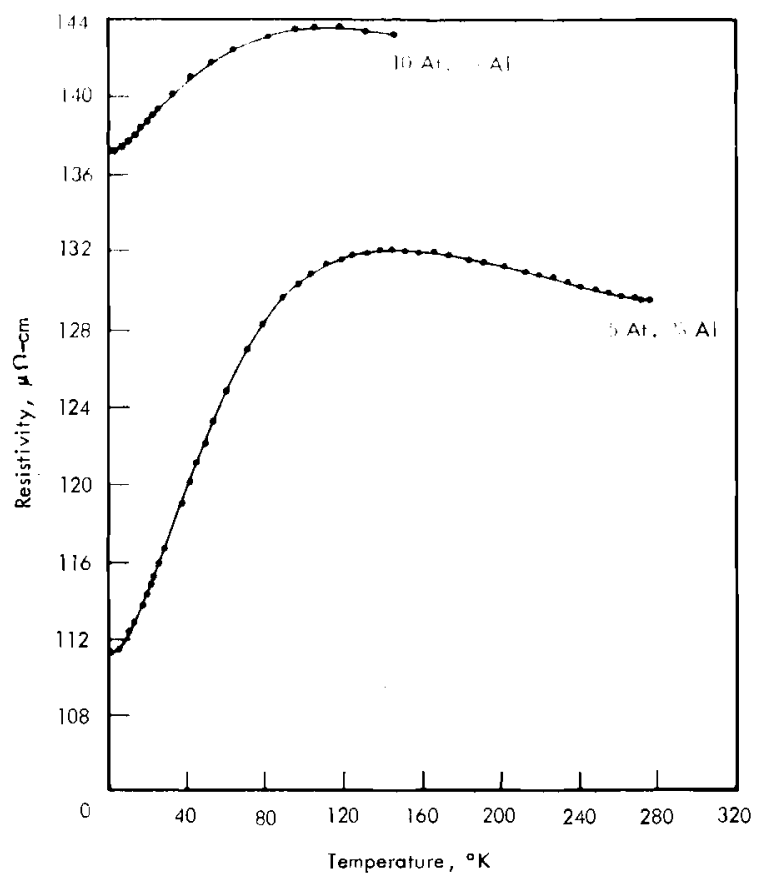

Electrical Rusistivity of 'l'wo Al-Pu -Phase Alloys. From I. A. Lee et al. in Plutonium 1960 , p. 45 . 


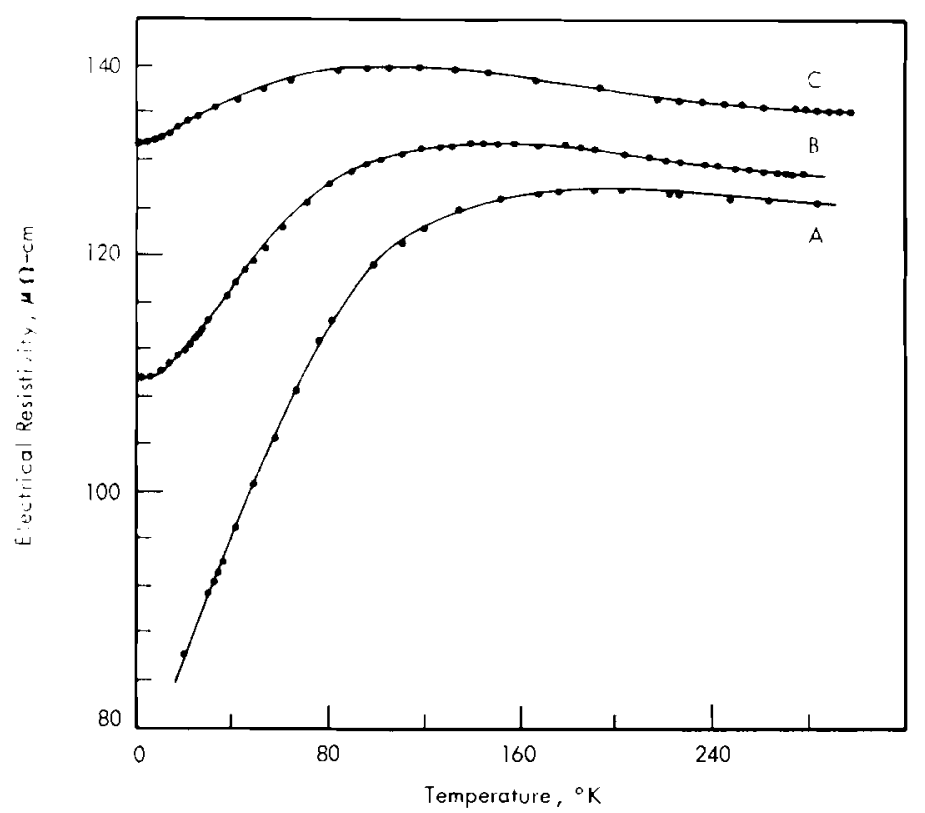

The Resistivities of Three $\delta$ - Pu - Al Alloys Below $300{ }^{\circ} \mathrm{K}$. Specimens: A, 3 at. Al: B, 5 at. $\mathrm{Al}$; $\mathrm{C}, 10$ at. i Al. From G. T. Mladen, Proc. Roy. Soc. (London), ser. A, vol. 276, p. 5f66. 1963.

The resistivity rise in $\delta$ plutonium (t at. ${ }^{\prime \prime} \mathrm{Al}$ ) held below $20^{\circ} \mathrm{K}$ was about 0.012 mem /hr.

From .J. A. Lee el al., I. Phys. Sic. Japan, rol. 1n, supplement III, p. 312. $190: 3$.

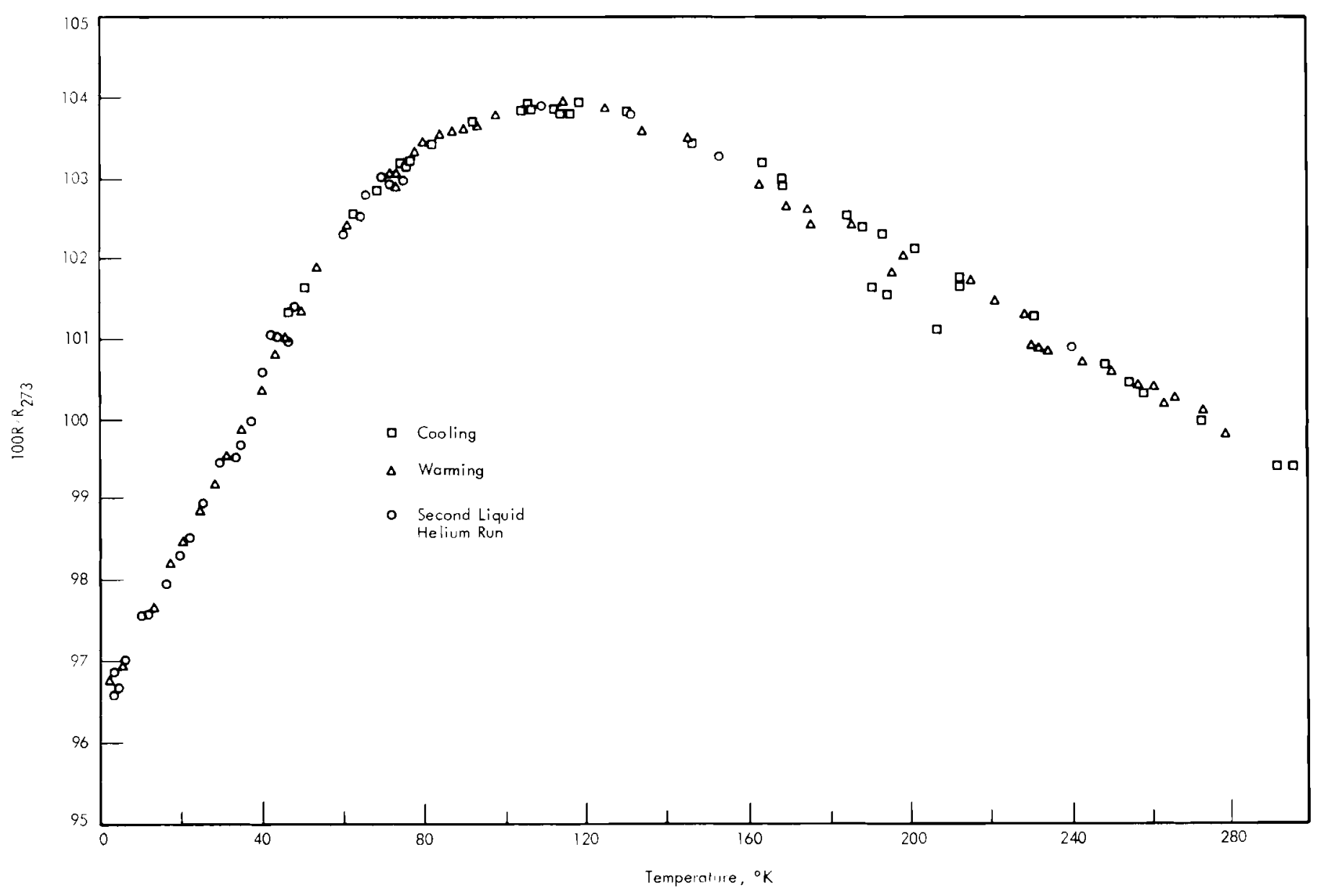

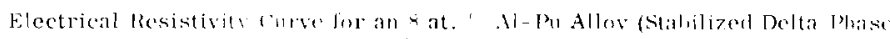

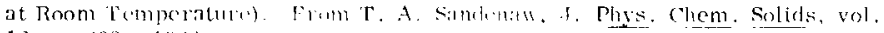

16. p. 332. 1!nit.

\section{Al 203.2 superconductivity}




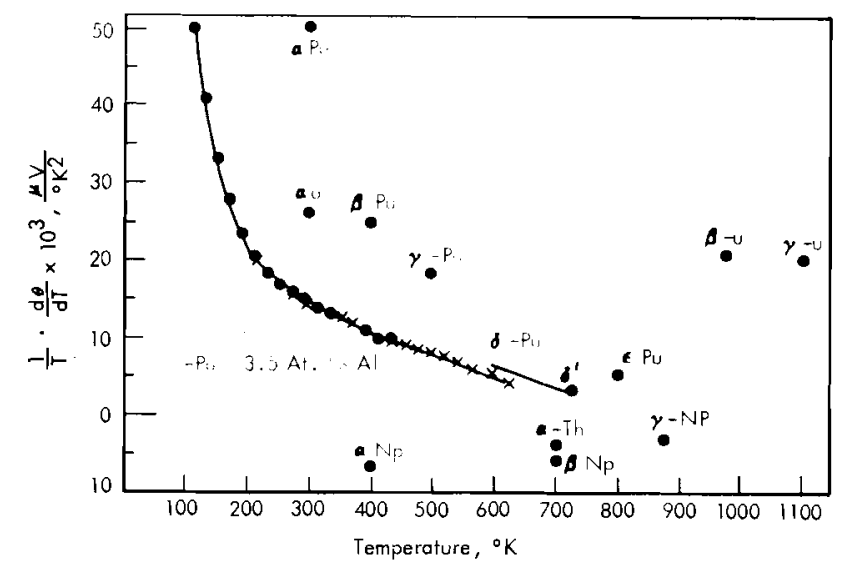

Thermoelectric Power. From J. A. Lee et al. in Plutonium 1960, p. 43.

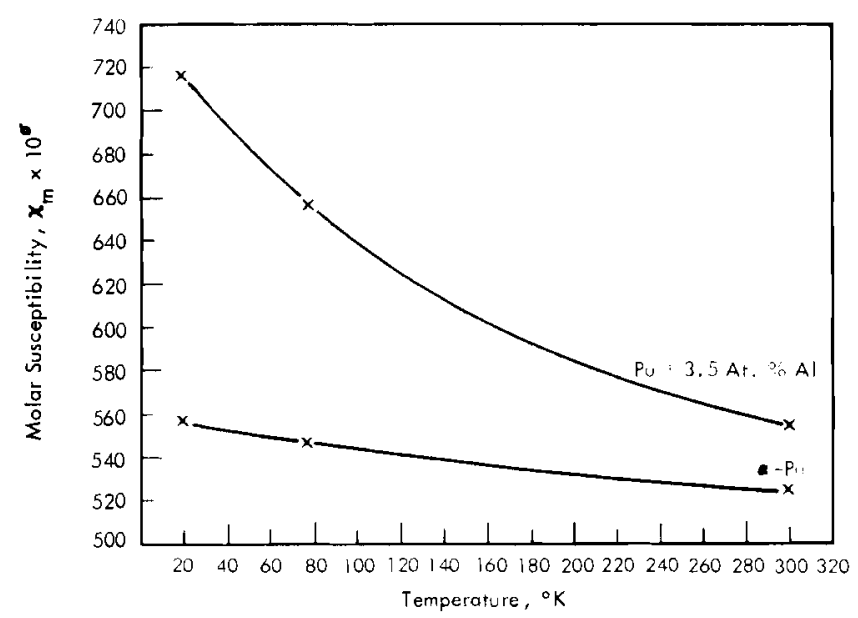

Paramagnetic susceptibilities. From d. A. leet et a1. in Plutonium 1960, p. 43.

Hall Effect

Hall effect in 3.4 at. Aluminum Delta Stabilized Plutonium

$$
\begin{array}{ll}
\text { at } 293^{\circ} \mathrm{K} & \mathrm{R}_{\mathrm{H}}=+11.1 \pm 1.0 \times 10^{-11} \mathrm{~m}^{3} \mathrm{c}^{-1} \\
\text { at } 77^{\circ} \mathrm{K} & \mathrm{R}_{\mathrm{H}}=+16.2 \pm 1.5 \times 10^{-11} \mathrm{~m}^{3} \mathrm{c}^{-1}
\end{array}
$$

From B. G. Loasby and J. C. Taylor. Proc. Phys. Soc. (London), vol. 78, p. 7771961 . 


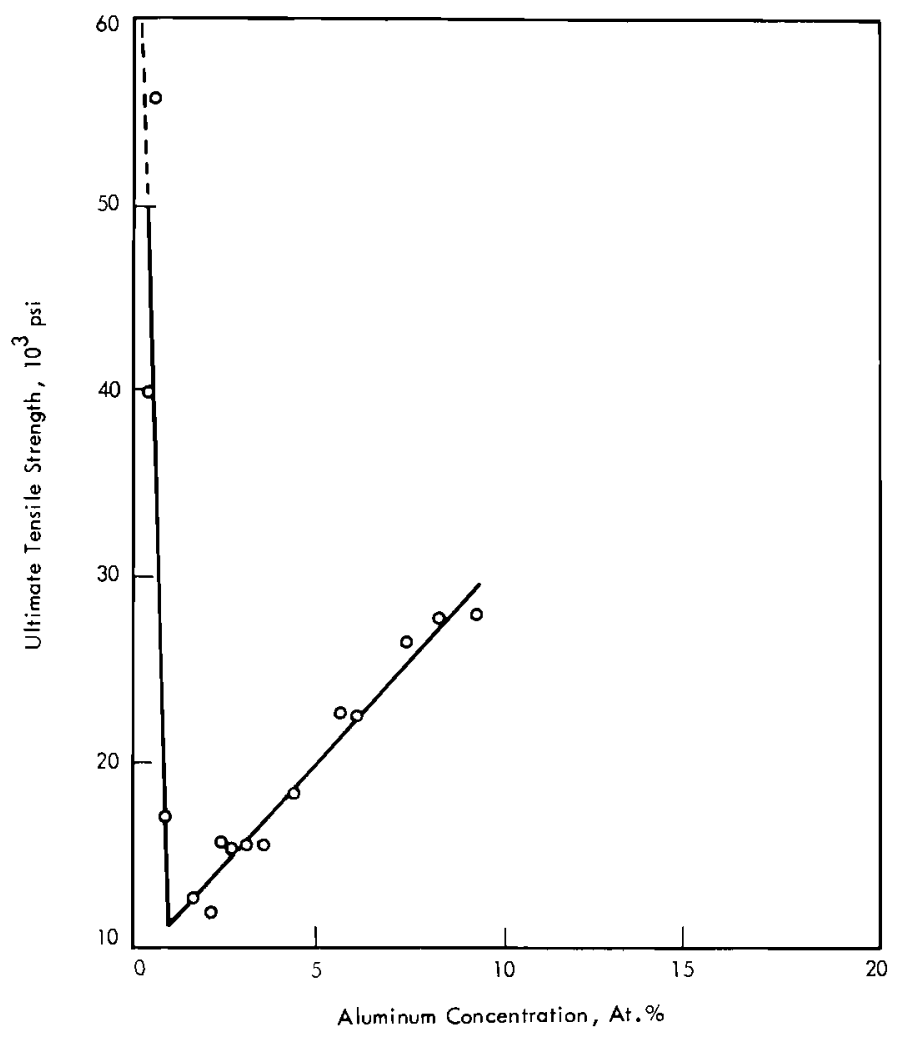

The Effect of Composition on Ultimate Strength in Pu-Al. Tested at Room Temperature. From D. C. Miller and J. S. White, Private Communication, reported by H. R. Gardner.

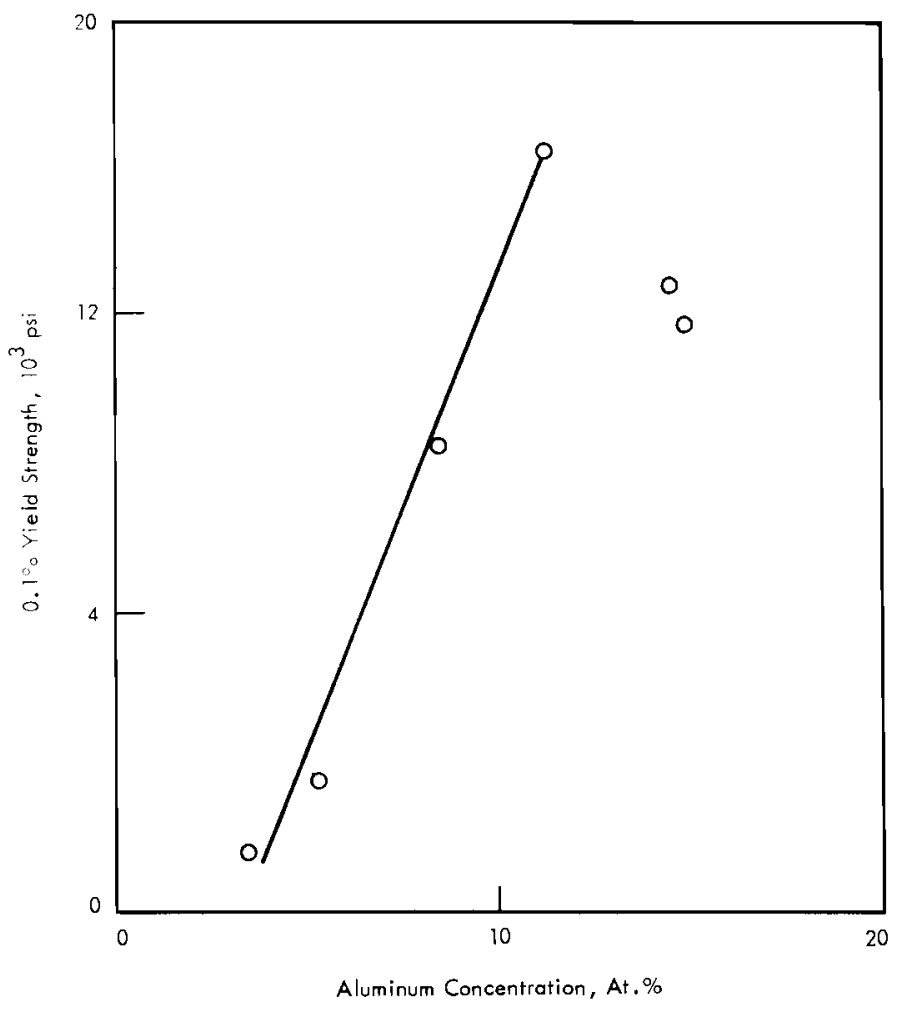

The Fffect of Composition on the $0.1 \%$ Yield Stress in Pu-Al. Tested at Room Temperature. From D. C. Miller and J. S. White, Private Communication reported by H. R. Gardner.

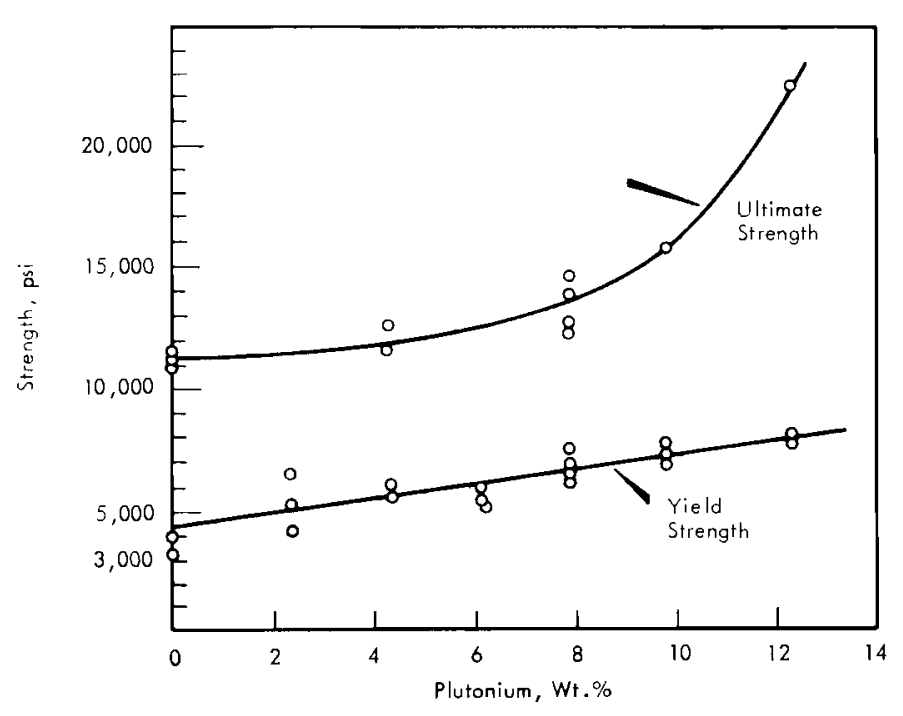

Elongation Ranges From 3 to $12 \%$ for Alloys Studied. From H. R. Gardner et al. in Proc. Second Geneva Internat. Conf., vol. 6, p. 689. 1958. 


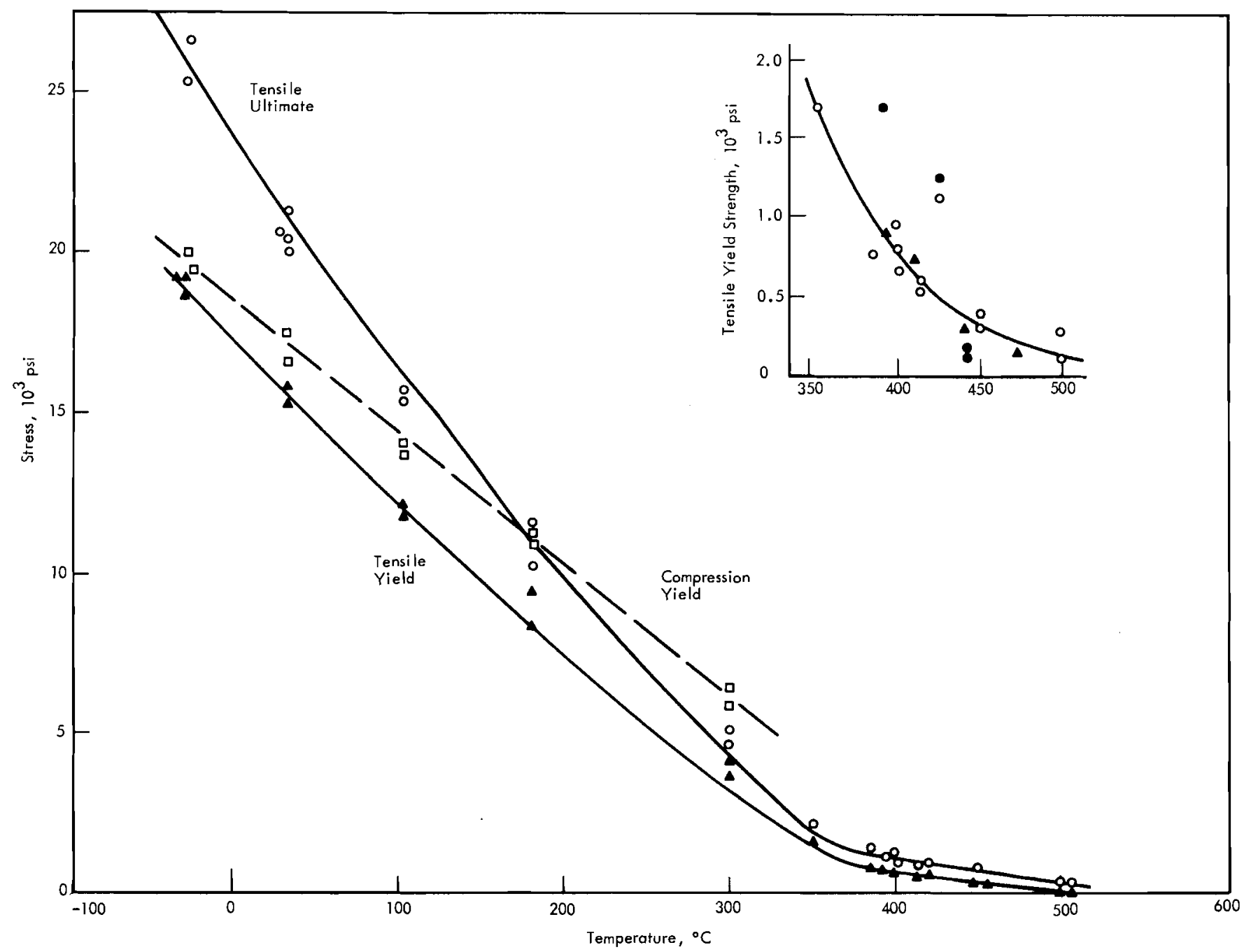

Effect of Temperature on Tensile and Compressive Properties of Plutonium -4.5 at. $\%$ Aluminum Delta-Stabilized Alloy. Testing Speed, $0.015 \mathrm{in} . / \mathrm{min}$.

$\boldsymbol{A}, \square$, O Less than $250 \mathrm{ppm}$ iron $\triangle 910 \mathrm{ppm}$ iron

- $1300 \mathrm{ppm}$ iron

From H. R. Gardner in HW-77182 and HW-78158, (Classified)

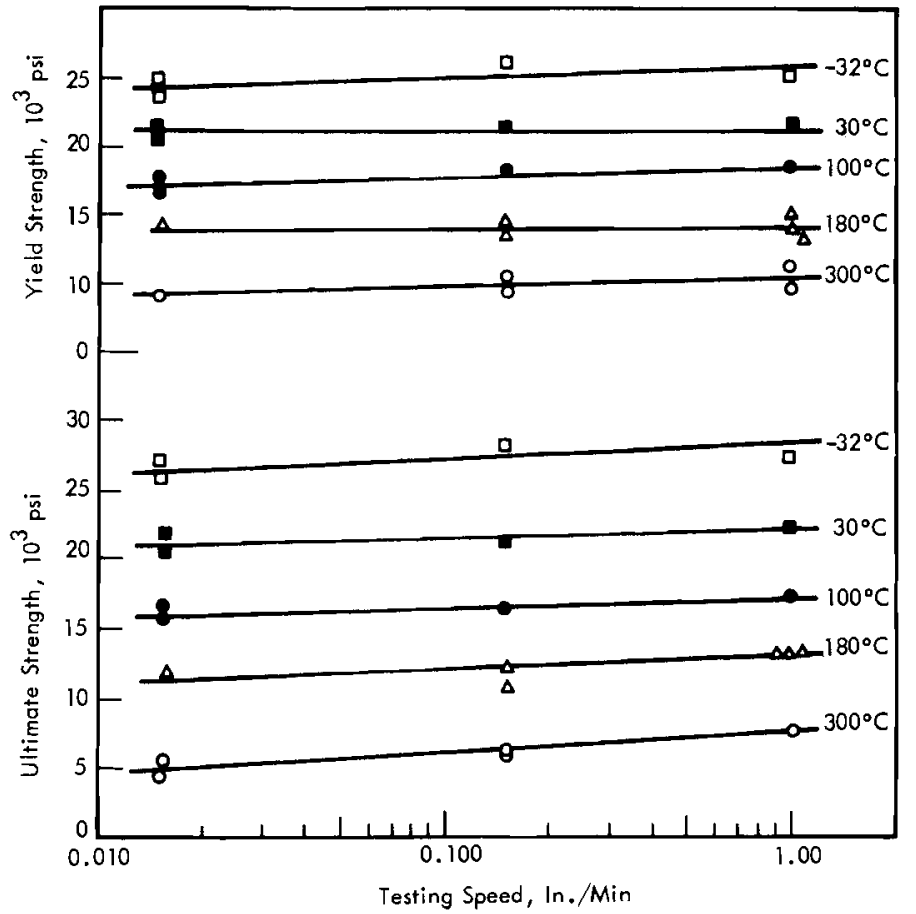

Effect of Testing Speed and Temperature on the Strength of a Plutonium-4.5 at. $\%$ Aluminum Delta-Stabilized Alloy. From H. R. Gardner in HW-77182 and HW-78158. (Classified) 


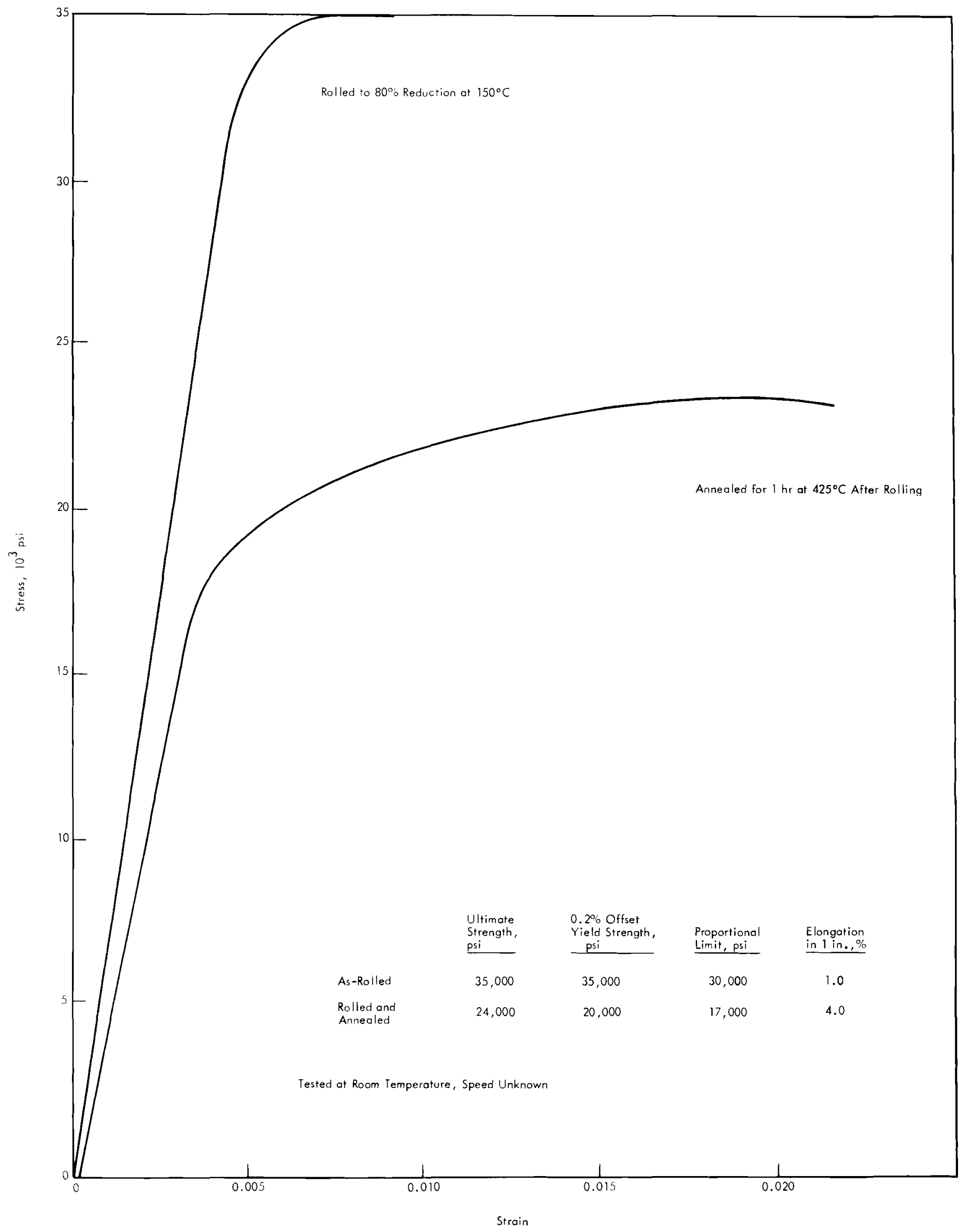


TENSILE PROPERTIFS OF PU-11.1 at. 'A AI DELTA-

STABILIZEI) ALLOY ROLLED TO $94 \%$ REDICTION

From H. R. Gardner in $11 W-78158$. (Classified)

\begin{tabular}{lccc}
\multicolumn{1}{c}{ Treatment } & $\begin{array}{c}\text { Ultimate } \\
\text { Strength, } \\
\text { psi }\end{array}$ & $\begin{array}{c}0.2 \% \text { Yicld } \\
\text { Strength, } \\
\text { psi }\end{array}$ \\
& 50,800 & & 46,300 \\
$\begin{array}{l}\text { Annealed for } 6 \text { hrs at } 650^{\circ} \mathrm{C} \\
\text { before rolling. }{ }^{* *}\end{array}$ & 50,100 & & 44,300 \\
$\begin{array}{l}\text { Anneal as above, roll, anneal at } \\
450^{\circ} \mathrm{C} \text { for } 24 \mathrm{hrs}, \text { furnace cool. }{ }^{*}\end{array}$ & 28,500 & & 16,900
\end{tabular}

* Average of two tests.

** Average of four tests.

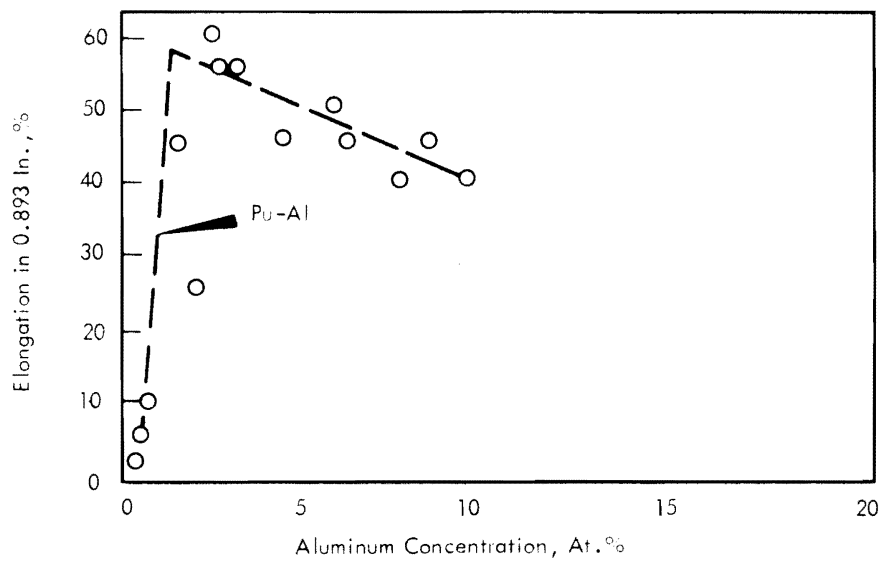

The Effect of Composition on Elongation in Pll-Al. Tested at Room Temperature From D. C. Miller and J. S. White, Private Communication, reported by H. R. Gardner.

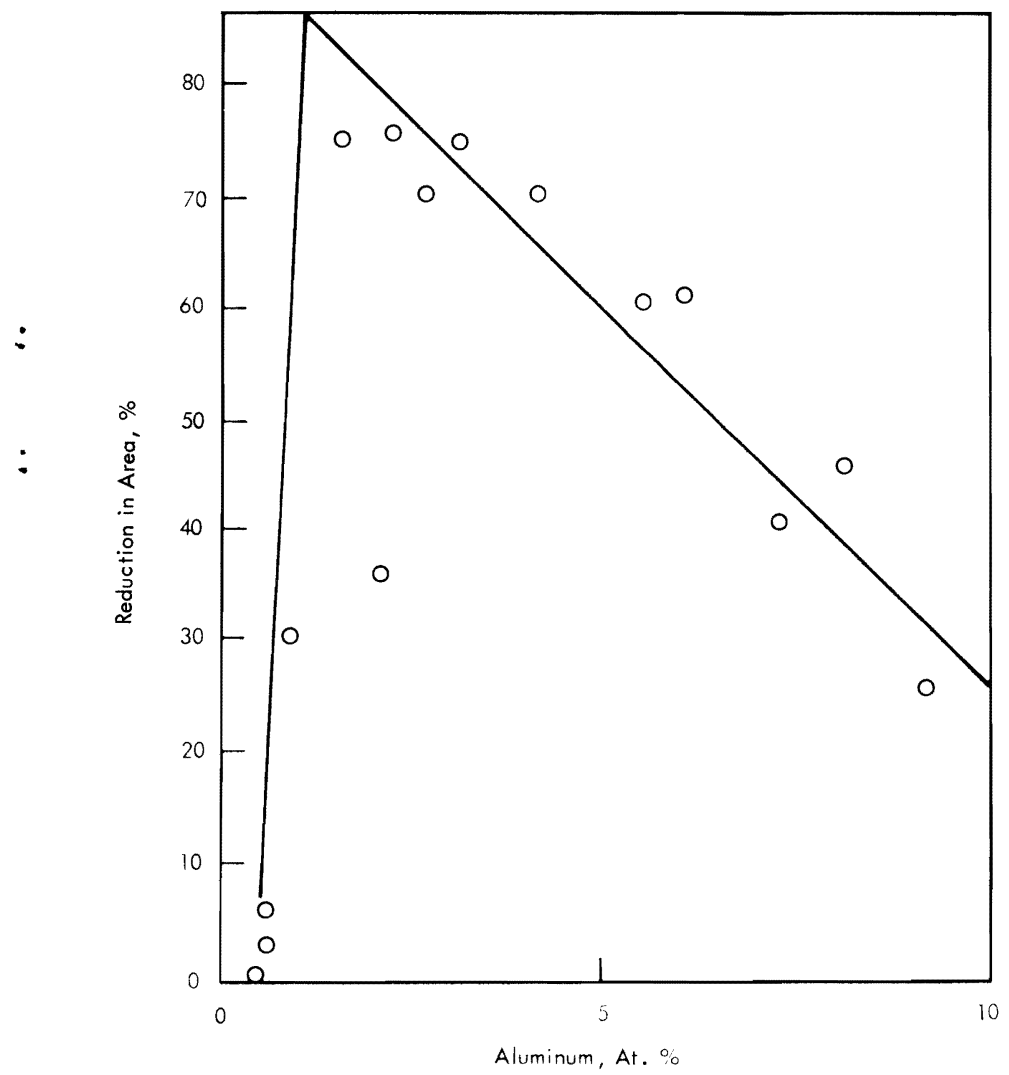

The Effect of Composition on Reduction in Area in Pu-Al. Tested at Room Temperature. From D. C. Miller and I. \&. White, Private Communication, reported by $\mathrm{H}$. R. Gardner.

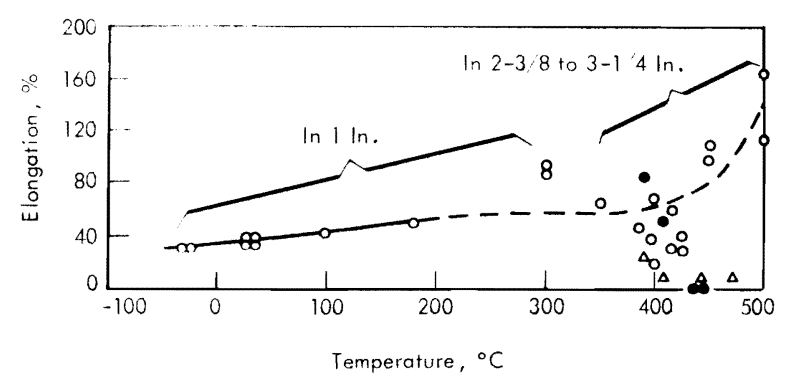

Fiffect of Temperature on Luetility of Plutonium-4.5 at. Aluminum DeltaStathilized Alloy. Testing speed, 0.015 in. /min.

O Less than $250 \mathrm{ppm}$ iron

$\Delta 910 \mathrm{ppm}$ iron

- $1300 \mathrm{ppm}$ iron

From H. R. Gardner in HW-77182 and HW-78158. (Classified)

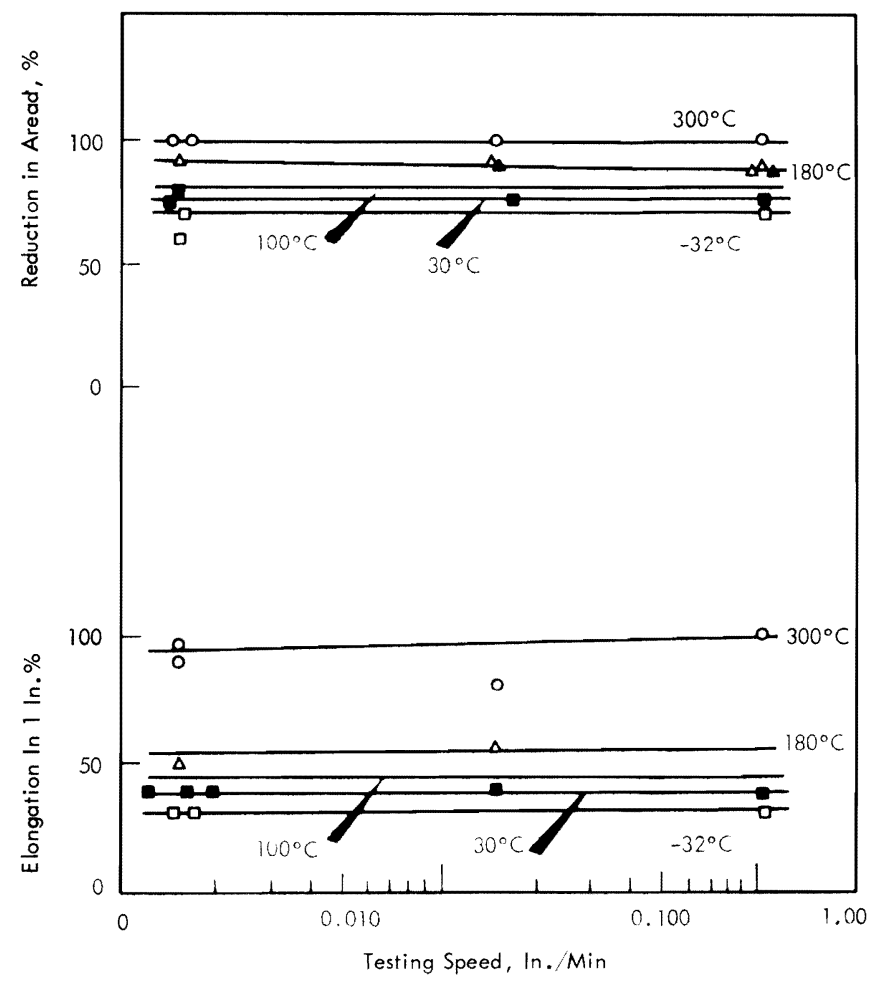

Effect of Testing Speed and Temperature on Ductility in a Plutonium-4.5 at.

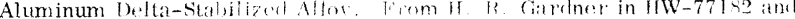
HW -78158 . (Classifice 
Al 302 compressive Properties

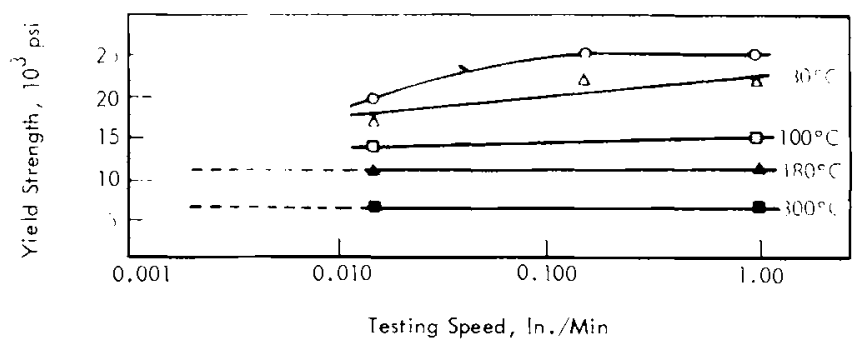

Fffect of Testing Speed and Temperature on the Compression Properties of a Plutonium-4.5 At. " Delta-Stabilized Alloy. From H, R. Gardner in HW-77182 and HW-78158. (Classified)

Shear strength of cold-rolled, delta-phase, $\mathrm{Pu}-f, 0$ at. $\mathrm{r}_{\mathrm{i}} \mathrm{Al}$ and $\mathrm{Pu}-8.0$ at. $\mathrm{Z} \mathrm{Al}$ at room temperature is approximately $16,000-18,000 \mathrm{psi}$

From F. W. Schonfeld, as reported by H. R. Gardner in Chap. 4

The Plutonium Hantilook, 1960

\section{A| 307 Hardness}

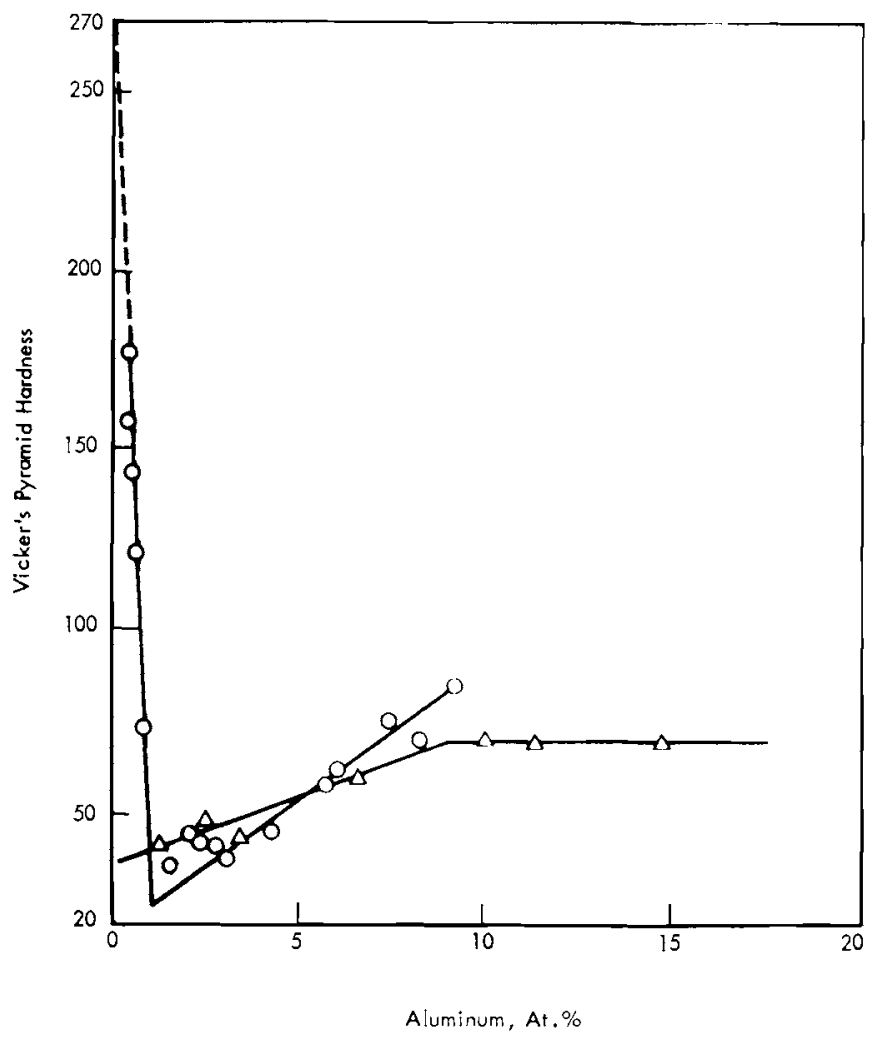

The Elfect of Composition on Hardiness in Pu-Al. Tested at Room Temperature. From D. C. Miller and J. S. White, Private Communication, reported by H. R. Gardner.

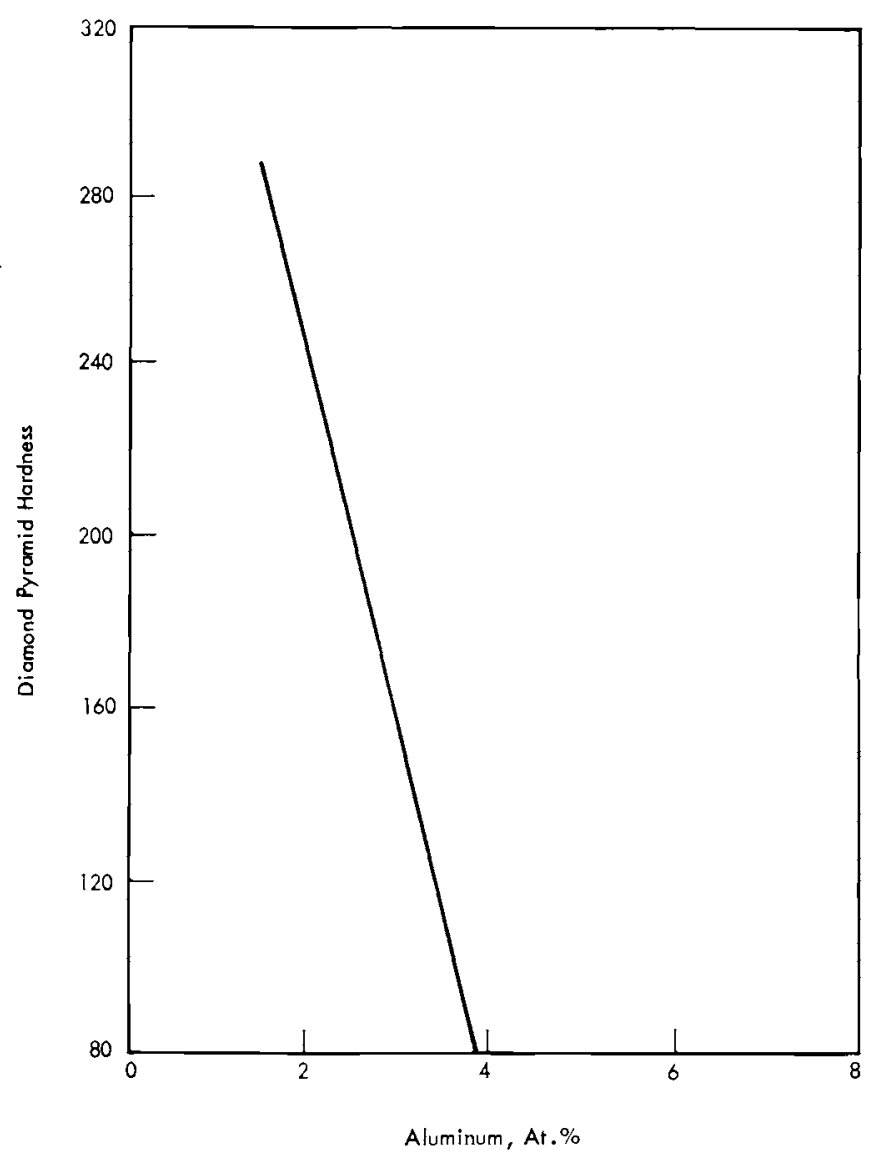

The Elfect of Concentration of Aluminum and Pressure up to About 10,000 Atm on the Hardness of Delta-Stabilized Aluminum-Plutonium Alloys. 


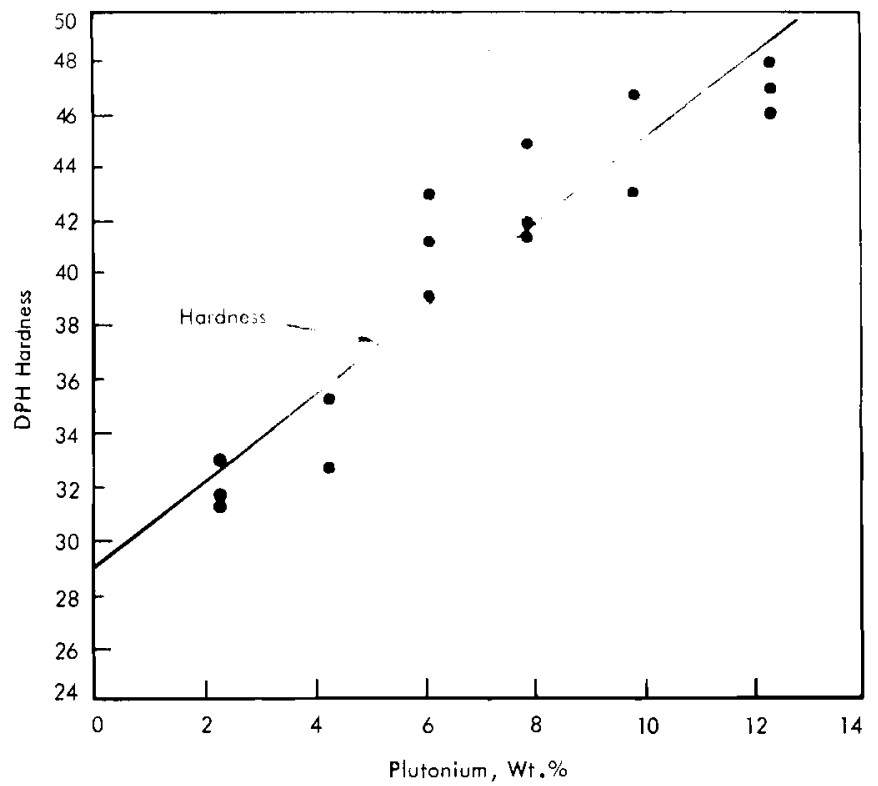

Aluminum-Plutonium Alloys (0-14 At. . Pu). From H. R. Gardner et al. in Proc. Second Geneva Internat. Conf., vol. 6, p. 689. 1958.

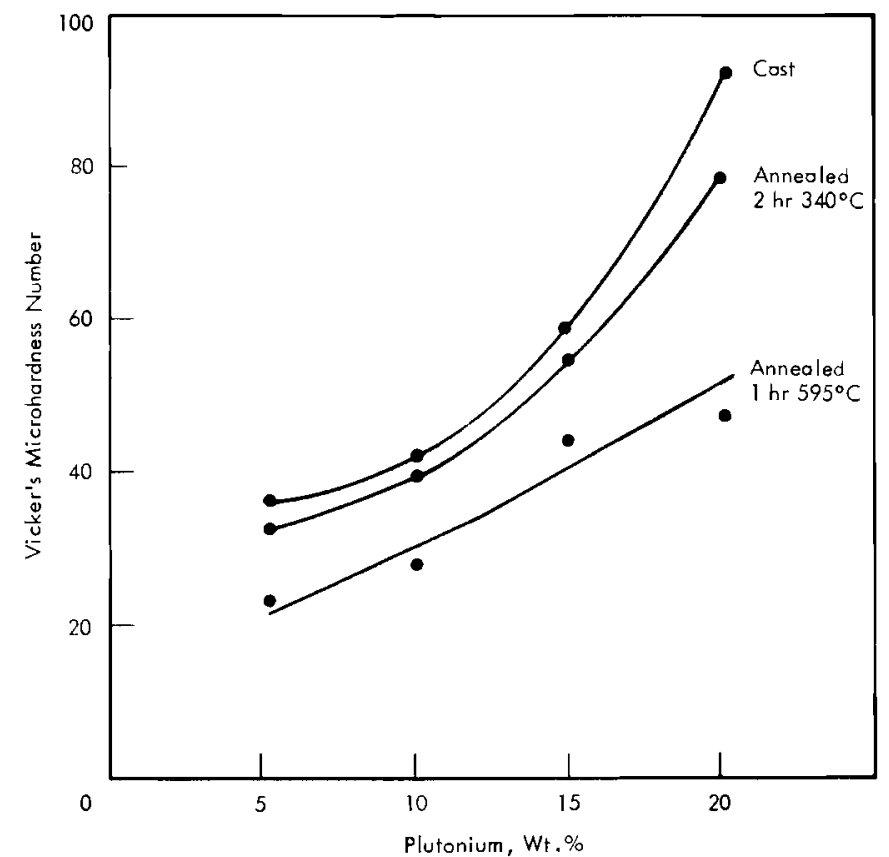

Effect of Composition on the IIardness of Cast and Annealed Al-Pu Alloys.

From T. I Jones in Plutonium 1960, p. 725.

Young's Modulus
HARDNESS OF ALIMINTM-PLITTONNTM ALLOYS IN VICKFRS UNITS BEFORE AND AFTER ANNEALING AT $600^{\circ} \mathrm{C}$

From R. Ahramson ed al. in Proc. Second Geneva Internat. Cont., wol. i. $190,181-72^{2} \quad 195 \%$

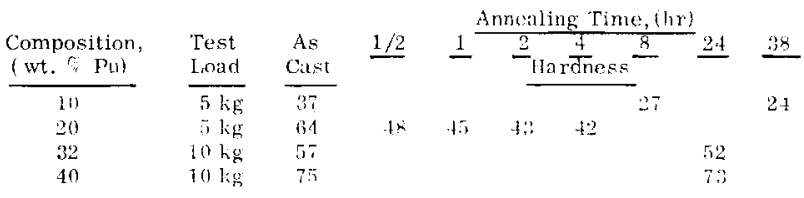

Average microhardness of $\mathrm{PuAl}_{3}$ and $\mathrm{PuAl}, \mathrm{f}$, Tinder loads of 30 and $40 \mathrm{~g}$ :

$$
\begin{array}{ll}
\mathrm{PuAl}_{4} & 380 \mathrm{~kg} / \mathrm{mm}^{2} \\
\mathrm{PuAl}_{3} & 500 \mathrm{~kg} / \mathrm{mm}^{2}
\end{array}
$$

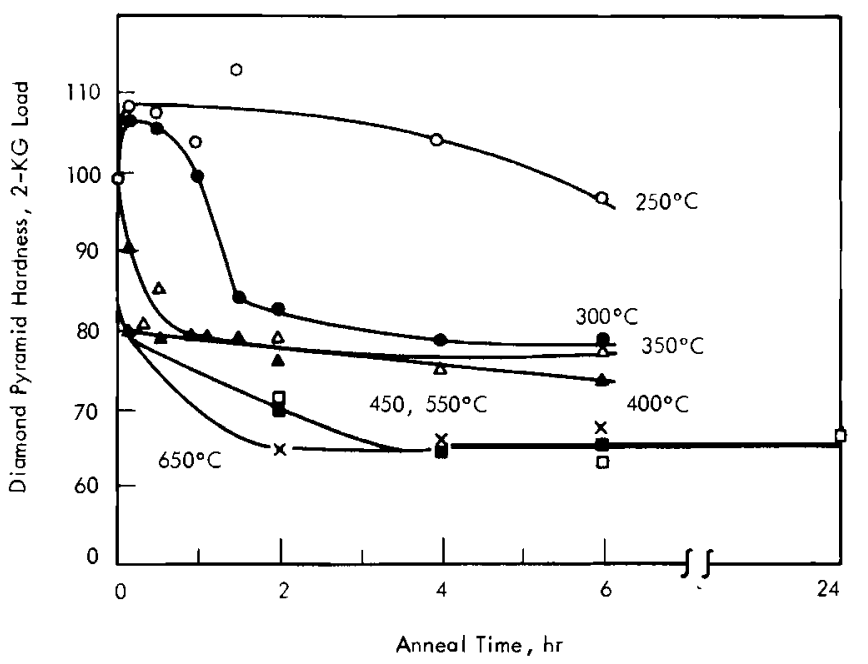

Effect of Anneal Time and Temperature on Hardness of a Plutonium-11.1 At. $\%$ Aluminum Delta-Stabilized Alloy Rolled to $94^{\circ} \%$ Reduction at Room Temperature. From H, R, Gardner in HW-78185. (Classified) 


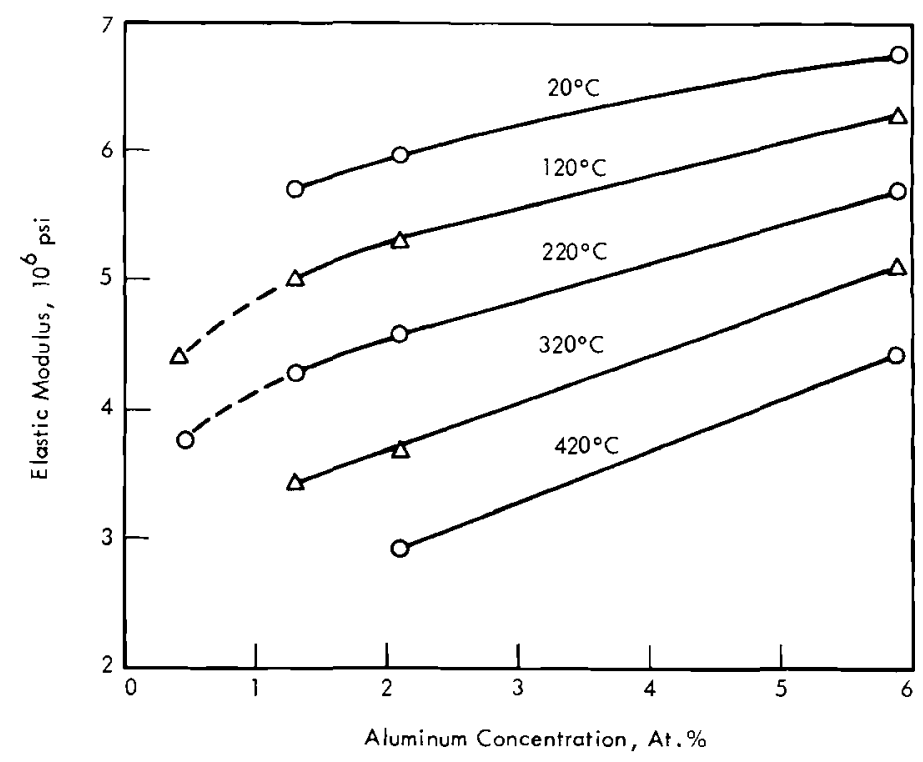

The Effect of Composition on the Elastic Modulus of As-Cast Plutonium-Aluminum Alloys. From P. F. T. Linford, Private Communication, reported by H. R. Gardner. In Chap. 4, The Plutonium Handbook, 1966

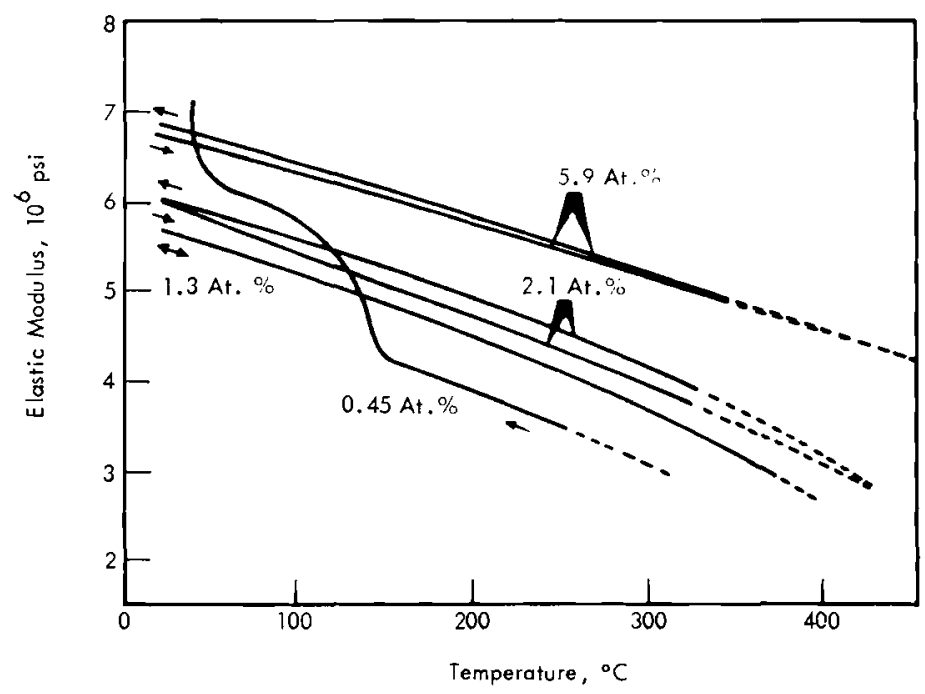

The Effect of Temperatur" on the Elastic Modulus of As-Cast Plutonium-Aluminum Alloys. From P. F. T. linford, Private Communication, reported by H. R. Gin roner.

ELASTIC MOTITIIS OF PIITONIIN-11.1 \#1, ; ALUMINUM,

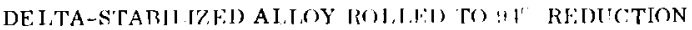

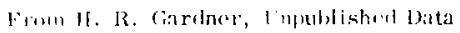

As-rolled* $^{\text {Truatment }} \quad \frac{\begin{array}{c}\text { Clastic: Modulus, } \\ 10^{6 j} \mathrm{psi}\end{array}}{11.9}$

Annealed for 6 irs at $650^{\circ} \mathrm{C}$ before rolling. ** 


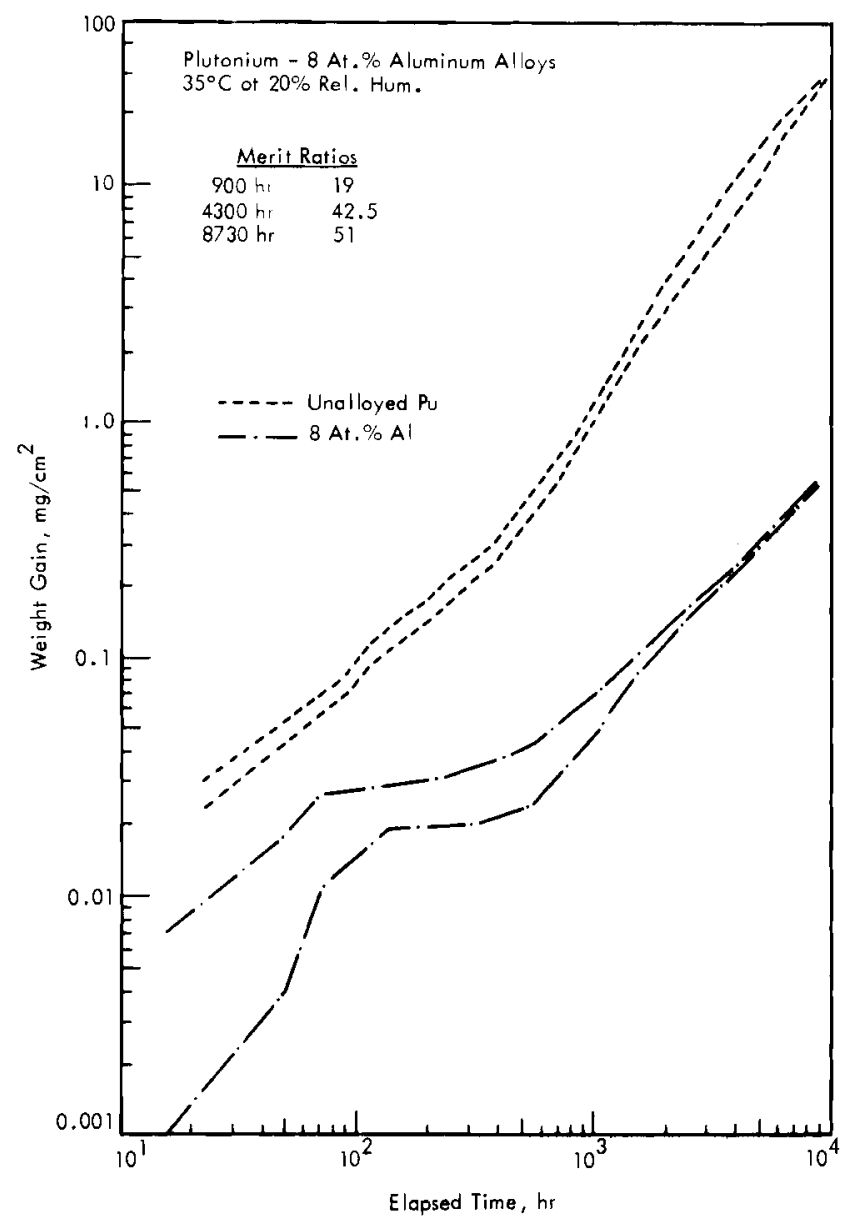

Corrosion Data for 8 At. Al-Pu Alloys, Rolled and Annealed: Exposed to Air Containing $20 \%$ Relative Humidity at $35^{\circ} \mathrm{C}$. From J. T. Waber in Proc. Second Geneva Internat. Conf, vol. 6, p. 211. 1958.

WEIGHT GAINS IN $\mathrm{mg} / \mathrm{cm}^{2}$ FOR Pu-3 At. \% Al AT 50 \% REIATIVE HUMIDITY

From J. T. Waber in Proc. Second Geneva Internat. Conf., vol. 6, p. 212,1958 .
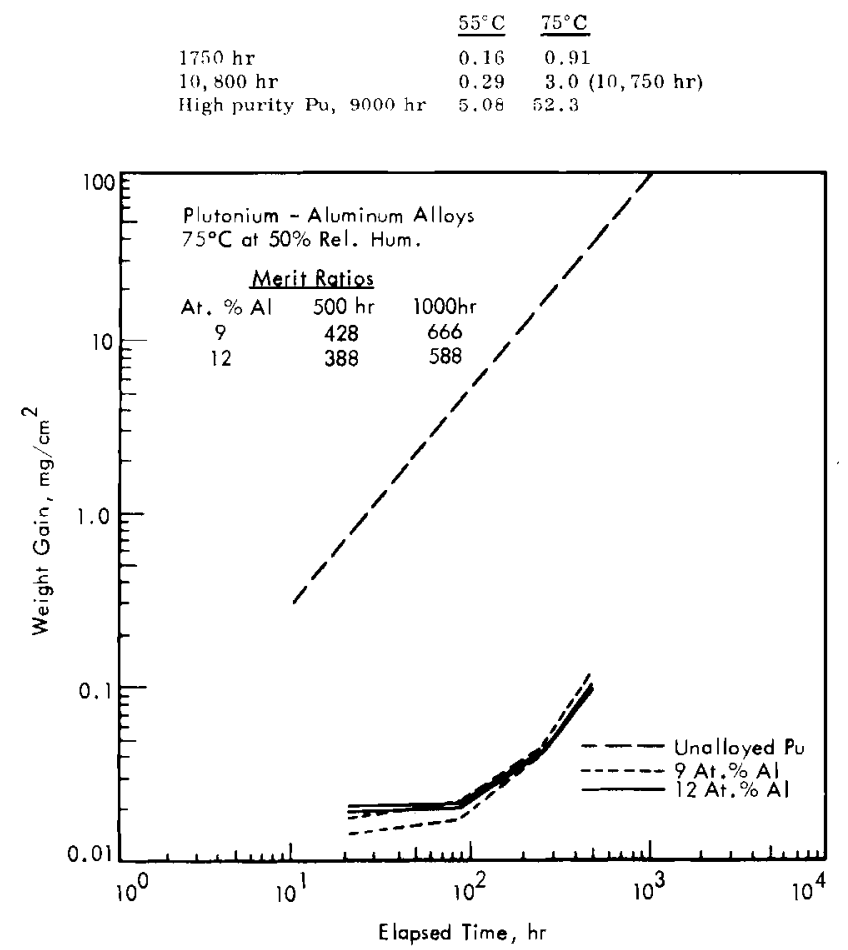

Comparison of the Corrosion Behaviors of Plutonium-Aluminum Alloys and

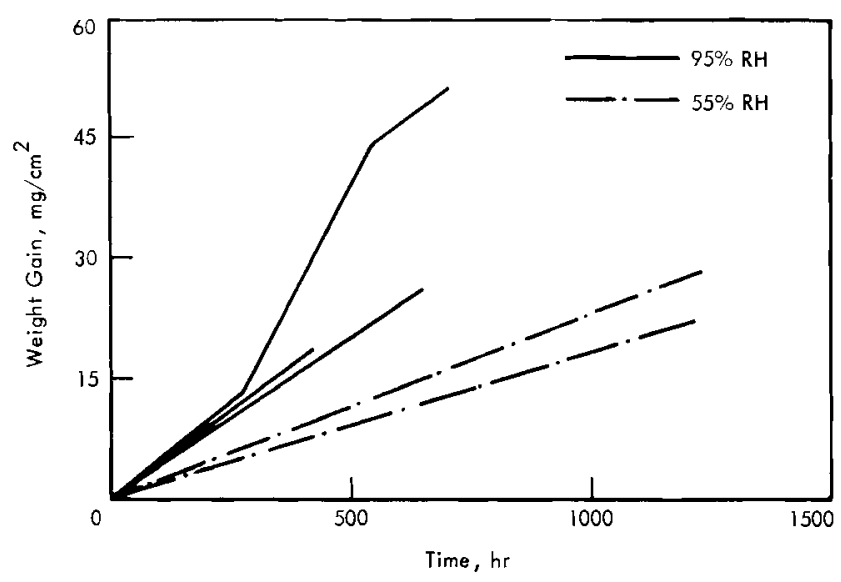

The Effect of Humidity on the Oxidation of 3.5 At. Al -Stabilized Plutonium at $90^{\circ} \mathrm{C}$. From J. F. Sackman in Plutonium 1960, p. 225.

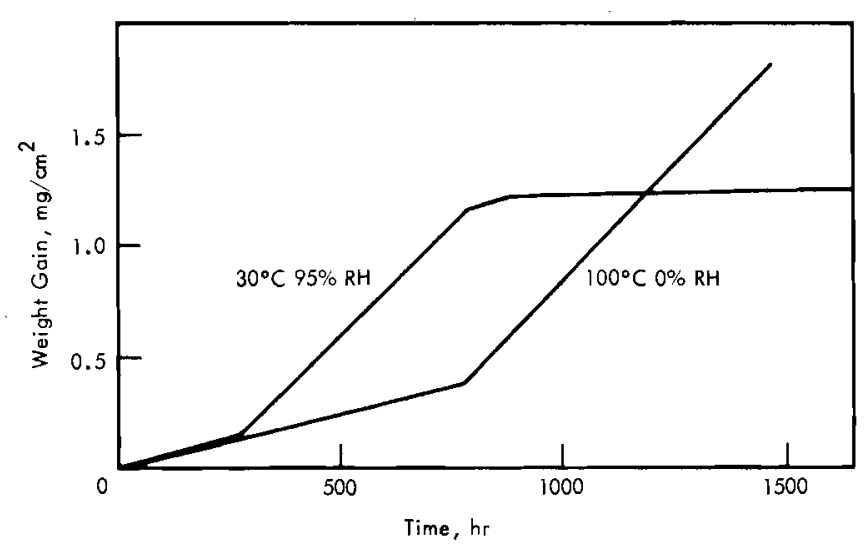

The Oxidation of 3.5 At. $\%$ Al -Stabilized Plutonium Under Mild Conditions Showing the Termination of Oxidation at $30^{\circ} \mathrm{C}$ and $90 \%$ RH. From J. F. Sackman in Plutonium 1960, p. 226.

MERTT RATIOS FOR 3,5 At. \% Al -STABILIZED Pu

From J. F. Sackman in Plutonium 1960, p. 229.

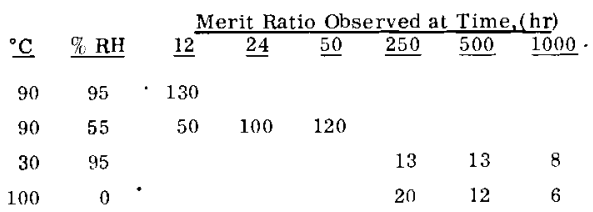

Weight increase for a-plutonium in a given time Merit Ratio $=\frac{\text { Weight increase for } \mathbf{a}-\text { plutonium }}{\text { Weight increase for }- \text { stabilized plutonium in the same time }}$ 
An aluminum alloy eontaining 11 wt. phanitum, whith consists of the intermetallic eompound PuAl ${ }_{1}$ dispersed in a matrix of aluminum. was held 1 wow

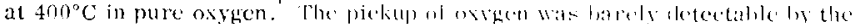

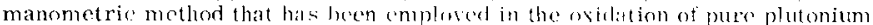
An upper limit on the amount of oxide formerl is $x \mu$. An $x$-ray dilleation

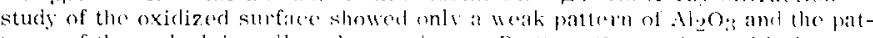

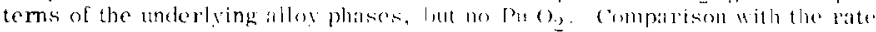

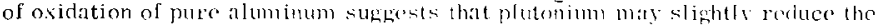
protection afforded aluminum be its asiale.

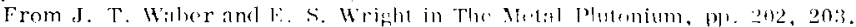

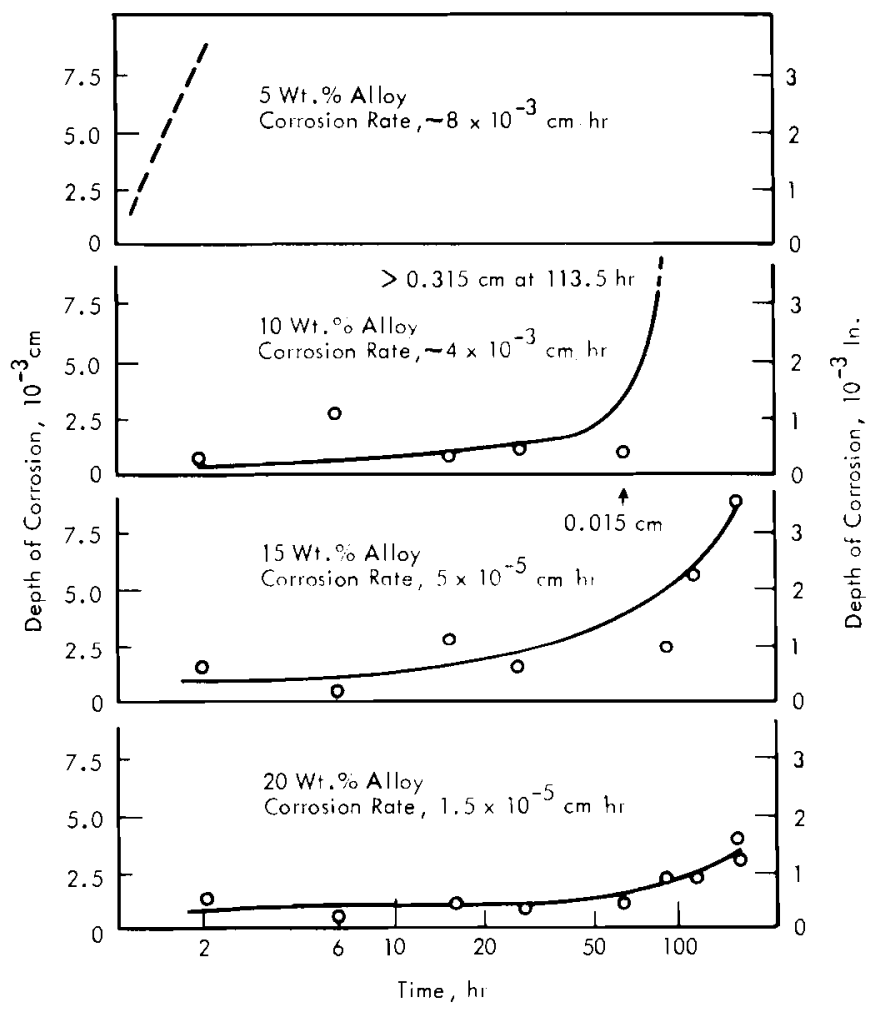

Corrosion of Al-Pu Alloys in Static Hater at 74 ' $^{2} \mathrm{C}$ and $\mathrm{nH} \overrightarrow{\mathrm{i}} . \mathrm{l}$ (in AIsI 316

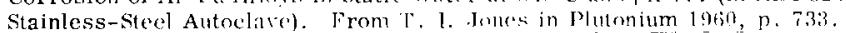

A Two-hour corrosion test was carriol out on the four alloys at $360^{\circ} \mathrm{C}$. The 5 we. "alloy was completely destroyed and the colosive attack penetrated

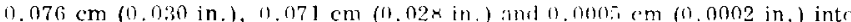
the 10,15 and 20 wt. '. alloys, resperetively.

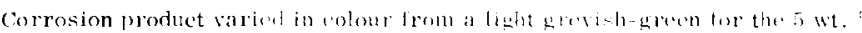

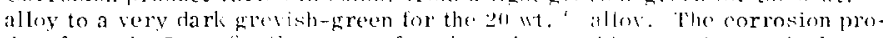
duct from the 5 wt. "aldow was soft and powdert and became ine reasingly dense with greater plutwium concontrations.

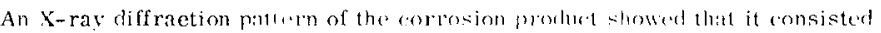

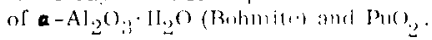

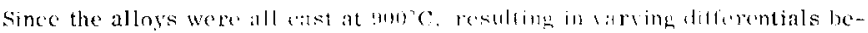

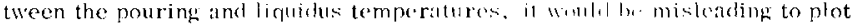

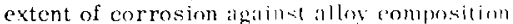

From $\Gamma$. I. Jeness in Plutunium lgan, P. 73:3. 
DESCRIPTION OF ALLOY PRE PAIRATION

From II. C. Bowen. Corrosion, vol. 17, no. T, p. 12.

\begin{tabular}{|c|c|c|c|}
\hline Alloy, wt. \% & $\begin{array}{l}\text { Max. } \\
\text { Melt, }{ }^{\circ} \mathrm{C}\end{array}$ & $\begin{array}{c}\text { Soak } \\
\text { Time, hrs } \\
\end{array}$ & $\begin{array}{c}\text { Casting, } \\
{ }^{\circ} \mathrm{C} \\
\end{array}$ \\
\hline \multicolumn{4}{|l|}{$\mathrm{Al}-\mathrm{Si}-\mathrm{Pu}$} \\
\hline $\begin{array}{rl}2 & \mathrm{Pu} \\
5 & \mathrm{Pu} \\
10 & \mathrm{Pu} \\
15 & \mathrm{Pu}\end{array}$ & $\begin{array}{l}925 \\
900 \\
900 \\
950\end{array}$ & $\begin{array}{l}2 \\
2 \\
1-1 / 2 \\
4\end{array}$ & $\begin{array}{l}700 \\
725 \\
775 \\
850\end{array}$ \\
\hline \multicolumn{4}{|l|}{$\mathrm{Al}-\mathrm{Pu}$} \\
\hline To $15 \mathrm{Pu}$ & 900 & 4 & 730 \\
\hline
\end{tabular}

RAPID HIGI TEM PERATURE ATTACK OF Al-PI AND Al-Si-PU ALLOYS IN $360^{\circ} \mathrm{C}$ WATER

From II. C. Bowen. Corrosion, vol. 17, no. 7, p. 12.

\begin{tabular}{|c|c|c|c|}
\hline $\begin{array}{c}\text { Wt. } \\
\mathrm{Pu}\end{array}$ & $\begin{array}{l}\text { Time, } \\
\text { hr }\end{array}$ & $\begin{array}{c}\text { Sample } \\
\text { Treat- } \\
\text { ment* }\end{array}$ & Results \\
\hline 13 & 24 & 1 & Etched cut end attacked. \\
\hline 13 & 24 & 2 & $\begin{array}{l}\text { Both ends attacked and } \\
3 / 4 \text { of sample destroyed. }\end{array}$ \\
\hline 10 & 24 & 1 & $\begin{array}{l}\text { Etched cut end attacked, } \\
2 \text { small spots on side } \\
\text { attacked. }\end{array}$ \\
\hline 10 & 24 & 2 & $\begin{array}{l}\text { Cut end and one side of } \\
\text { sample attacked and } 1 / 2 \\
\text { of sample destroyed. }\end{array}$ \\
\hline 6 & 12 & 1 & $\begin{array}{l}\text { Etched cut end attacked } \\
\text { and } 1 / 2 \text { of sample } \\
\text { destroyed. }\end{array}$ \\
\hline
\end{tabular}

$\begin{array}{ll}6 & 12 \\ 1.8 & 12 \\ 1.8 & 12 \\ 15^{* *} & 24 \\ 15^{* *} & 24 \\ 10^{* *} & 24 \\ 10^{* *} & 24 \\ 5 * * & 24 \\ 5 * * & 24 \\ 1.8 * * & 24 \\ 1.8 * * & 24\end{array}$

** In $\mathrm{Al}-12^{\prime \prime} \mathrm{Si}$
RAPID HIGH TEMPERATURE ATTACK BY Al-Pu ALLOYS IN $350^{\circ} \mathrm{C}$ WATER

From H. C. Bowen. Corrosion, vol. 17, no. 7, p. 12

\begin{tabular}{|c|c|c|c|}
\hline $\begin{array}{l}\text { Wt. \% } \\
\mathrm{Pu} \\
\end{array}$ & $\begin{array}{c}\text { Time, } \\
\mathrm{hr}\end{array}$ & $\begin{array}{l}\text { Sample } \\
\text { Treat- } \\
\text { ment* }\end{array}$ & Results \\
\hline 13 & 24 & 1 & $\begin{array}{l}1 / 4^{\prime \prime} \text { D spot attacked on } \\
\text { etched cut end. }\end{array}$ \\
\hline 13 & 24 & 2 & $\begin{array}{l}1 / 4^{\prime \prime} \mathrm{D} \text { spot attacked on } \\
\text { both ends. }\end{array}$ \\
\hline 10 & 24 & 1 & Not attacked. \\
\hline 10 & 24 & 2 & $\begin{array}{l}\text { Both ends and one side } \\
\text { attacked. }\end{array}$ \\
\hline 6 & 24 & 1 & Not attacked. \\
\hline 6 & 24 & 2 & $\begin{array}{l}\text { Badly attacked on cut end } \\
\text { and one side }\end{array}$ \\
\hline $\begin{array}{l}1.8 \\
1.8\end{array}$ & $\begin{array}{l}24 \\
24\end{array}$ & $\begin{array}{l}1 \\
2\end{array}$ & Destroyed. \\
\hline $\begin{array}{l}10 \\
10\end{array}$ & $\begin{array}{l}3 \\
3\end{array}$ & $\begin{array}{l}3 \\
3\end{array}$ & $\begin{array}{l}\text { Approximately } 1 / 2 \text { of } \\
\text { diameter lost }\end{array}$ \\
\hline $\begin{array}{l}1.9 \\
1.9\end{array}$ & $\begin{array}{l}3 \\
3\end{array}$ & $\begin{array}{l}3 \\
3\end{array}$ & Destroyed. \\
\hline
\end{tabular}

*Sample Treatments:

1. A 2-inch long end section was cut from a 6 -inch long cast rod. The cut end was etched in a 78 percent commercial $\mathrm{H}_{3} \mathrm{PO}_{4}, 11$ percent commercial $\mathrm{HNO}_{3}$, 11 percent commercial $\mathrm{H}_{2} \mathrm{SO}_{4}$, and $0.8 \mathrm{~g} / \mathrm{FeSo} \cdot 7 \mathrm{H}_{2} \mathrm{O}$ chemical polishing bath This was done to remove the cold work due to cutting. This left an as-cast and etched cut end on the sample.

2. A 2-inch long section was cut from the center of a 6-inch long cast rod. One end was treated in a polishing bath as above. This left a cut end and an etched cut end on the sample.

3. Two-inch long sections we ro cut from $1 / 2$-inch diameter extruded rod and tested without further treatment.

The following conclusions may be drawn from these tests:

1. Low percentage $(<6$ percent) aluminum-plutonium as-cast alloys have little resistance to rapid high temperature attack but resistance increases with increasing plutonium content up to at least 13 percent $\mathrm{Pu}$.

2. Any working of the material drastically decreases its resistance to attack as compared to as-cast material of the same Pu content.

3. Adrition of 12 percent silicon to aluminum-plutonium alloy makes the alloys up to at least 15 percent Pu resistant to attack for at least 24 hours and makes them less sensitive to working.

From H. C. Bowen. Corrosion, vol. 17, no. 7, p. 12.

\begin{tabular}{|c|c|c|c|}
\hline Treatment & $\begin{array}{c}\text { Wt. After } \\
\text { Treatment, g }\end{array}$ & $\begin{array}{c}\text { Wt. } \\
\text { Change, mg }\end{array}$ & $\begin{array}{l}\text { Apparint Corrosion } \\
\text { Rate, } \mathrm{mg} \mathrm{cm}-2 \mathrm{hr}^{-1}\end{array}$ \\
\hline Coating removed & 0.1167 & & \\
\hline $\begin{array}{l}160 \mathrm{hr}, 400^{\circ} \mathrm{C} \text { in } \mathrm{NaK} \\
\text { large container } \\
\text { pickled in } \mathrm{HCl}\end{array}$ & $\begin{array}{l}0.1144 \\
0.0785\end{array}$ & $\begin{array}{l}-2.3 \\
-35.9\end{array}$ & -0.06 \\
\hline \multicolumn{4}{|l|}{$720 \mathrm{hr}, 400^{\circ} \mathrm{C}$ in $\mathrm{NaK}$} \\
\hline $\begin{array}{l}\text { Rinserl and air } \\
\text { dried }\end{array}$ & 0.0845 & $\left({ }^{+} 6.0\right)$ & \\
\hline $\begin{array}{l}\text { Rinsed and mech- } \\
\text { anically dried }\end{array}$ & 0.0794 & +0.9 & +0.005 \\
\hline
\end{tabular}




\section{Al 500}

Interaction of eteled Al-Pu alloss with etched Zircalos-3 sheaths:

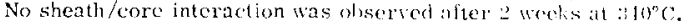

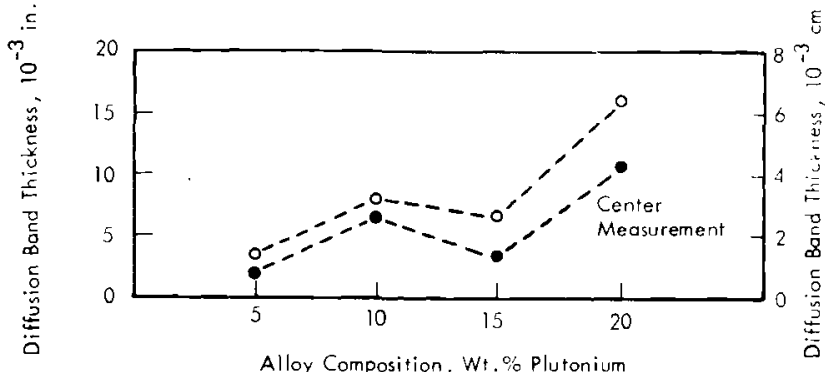

Sheath/Core Interaction After $26 \mathrm{Hr}$ at bistic. From $\mathrm{r}$. I. Jones in Plutonium 1960), p. 727 .
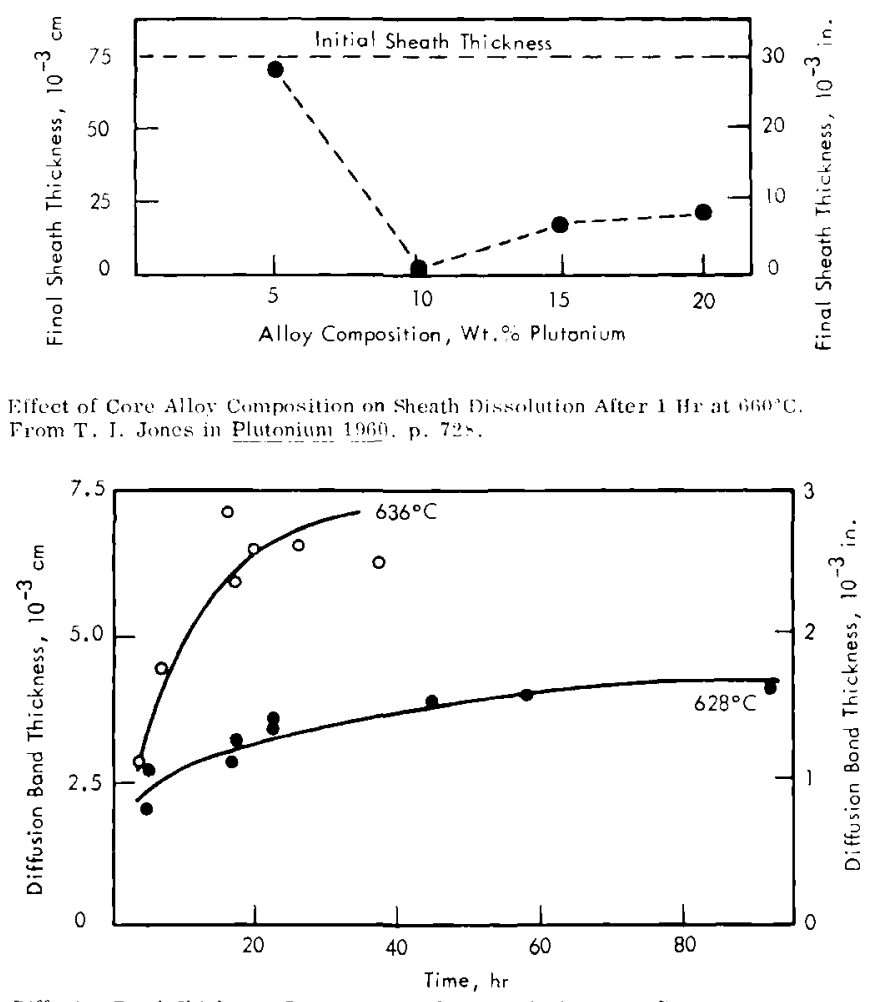

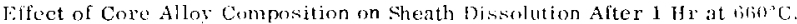
From T. I. Jones in Plutonium 1960. p. 720.

Diffusion Band Thickness Between Zircaloy-2 and Ai-10 Wt. Pu Alloy as a Funetion of Time and Temperature. From T . I. Jones in Plutonium 1930 , 17. 729

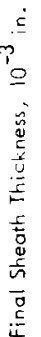

\section{Al 600}

Radiation Effects

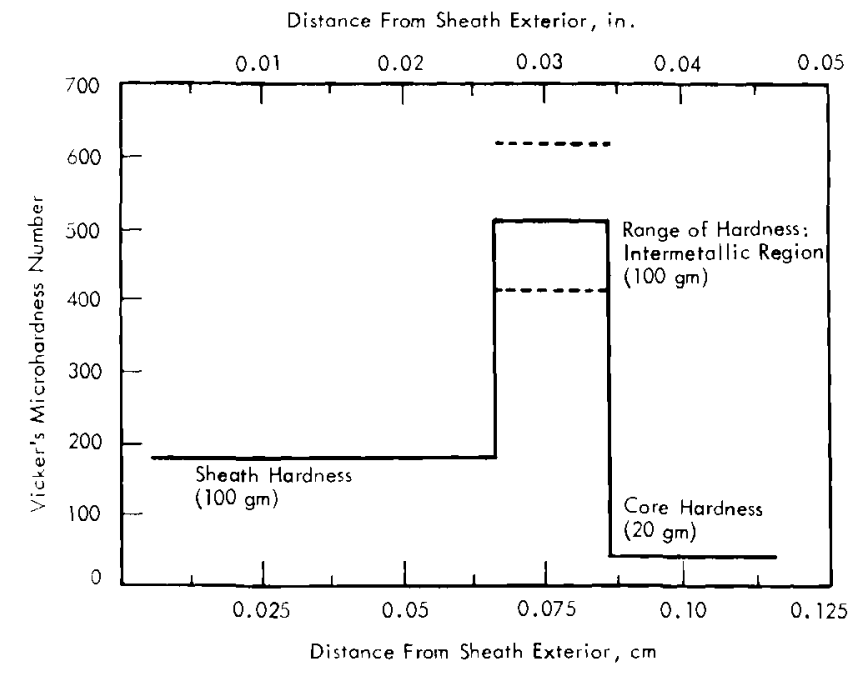

II:trincss of Diffusion Band Relative to 7.ircaloy-2 sheath and Al-10 Wt. $\mathrm{Pu}$ Alloy Core. The specimen had been heated for 5 hr at $13.38-642^{\circ} \mathrm{C}$, producing a rliffusion band $0.02 \mathrm{~cm}(0.00 \mathrm{~g}$ in.) wide. From T. I. Jones in Plutonium $19+i 1$, p. 731 ,

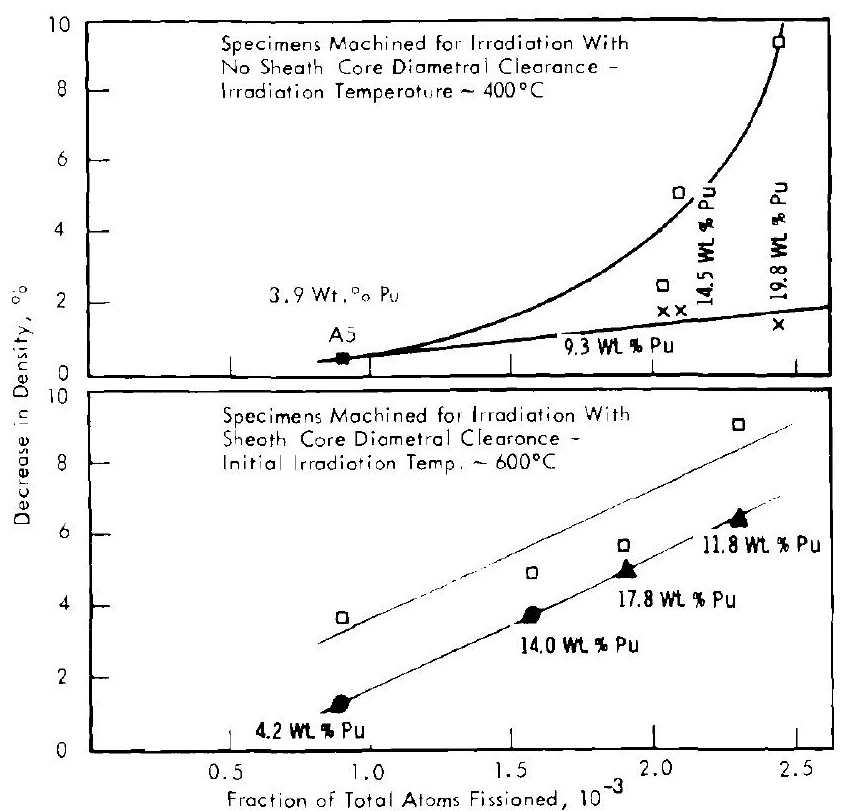



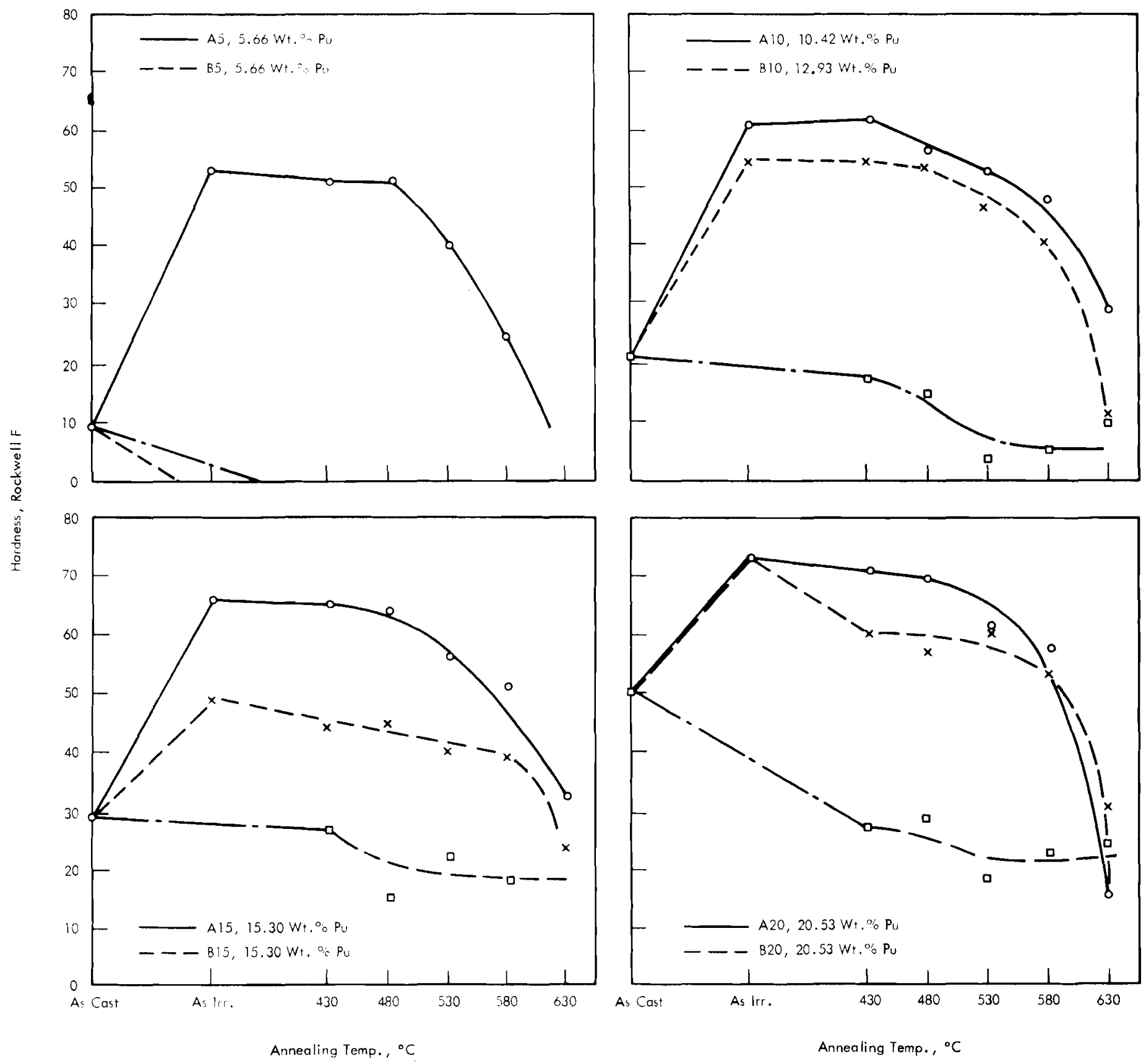

Hardness Changes After Irradiation and Postirradiation Annealing with Aluminum-Plutonium Alloys.

o- -0 Specimens machined for operation with no sheath/core diametral clearance, irradiation temperature, $350-400^{\circ} \mathrm{C}$.

$x---x$ Specimens machined for operation with sheat/core diametral clearance, initial irradiation temperature, $550-625^{\circ} \mathrm{C}$.

0- $\rightarrow$ Unirradiated alloys.

Annealing treatments were carried out for one hour at each temperature. Each point is an average of five hardness determinations.

From T. I. Jones, CRRM-1097. 


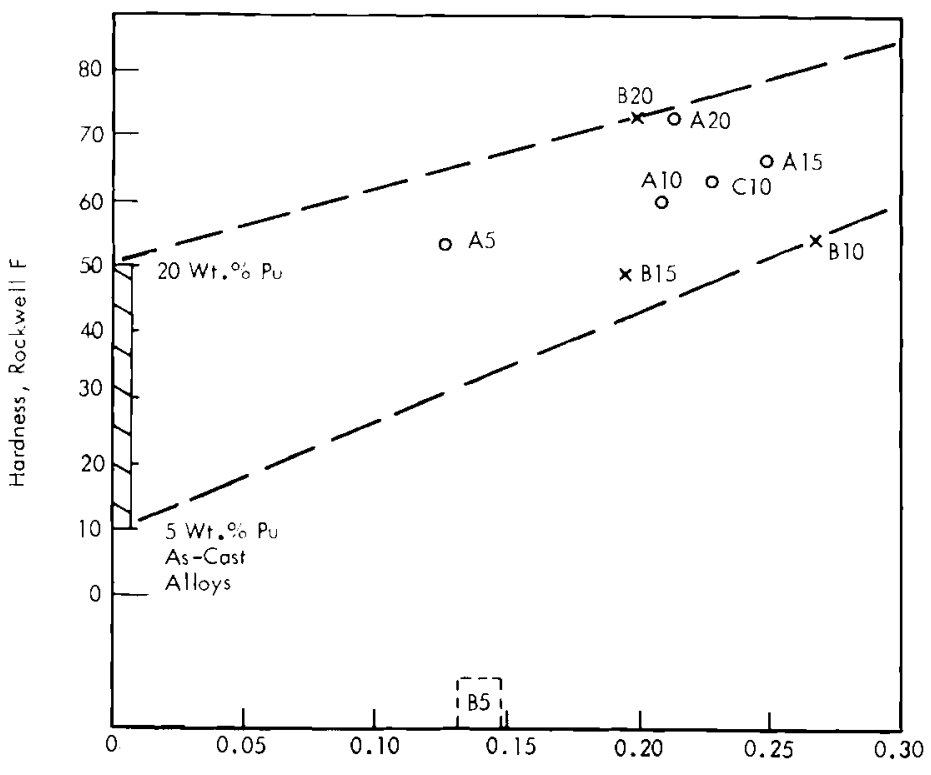

Percent of Total Atoms Fissioned

Elfect of Plutonium Fissioning on the Hardness of Aluminum-Plutonium Alloys.

- Irradiation temperature, $350-400^{\circ} \mathrm{C}$.

$\times \quad$ Initial irradiation temperature, $550-625^{\circ} \mathrm{C}$ Cinal irradiation temperature $\sim 400^{\circ} \mathrm{C}$

$\mathrm{B}_{5}$ Irradiation throughout at $\sim 625^{\circ} \mathrm{C}$

From T. I. Jones, CRRM-1097.

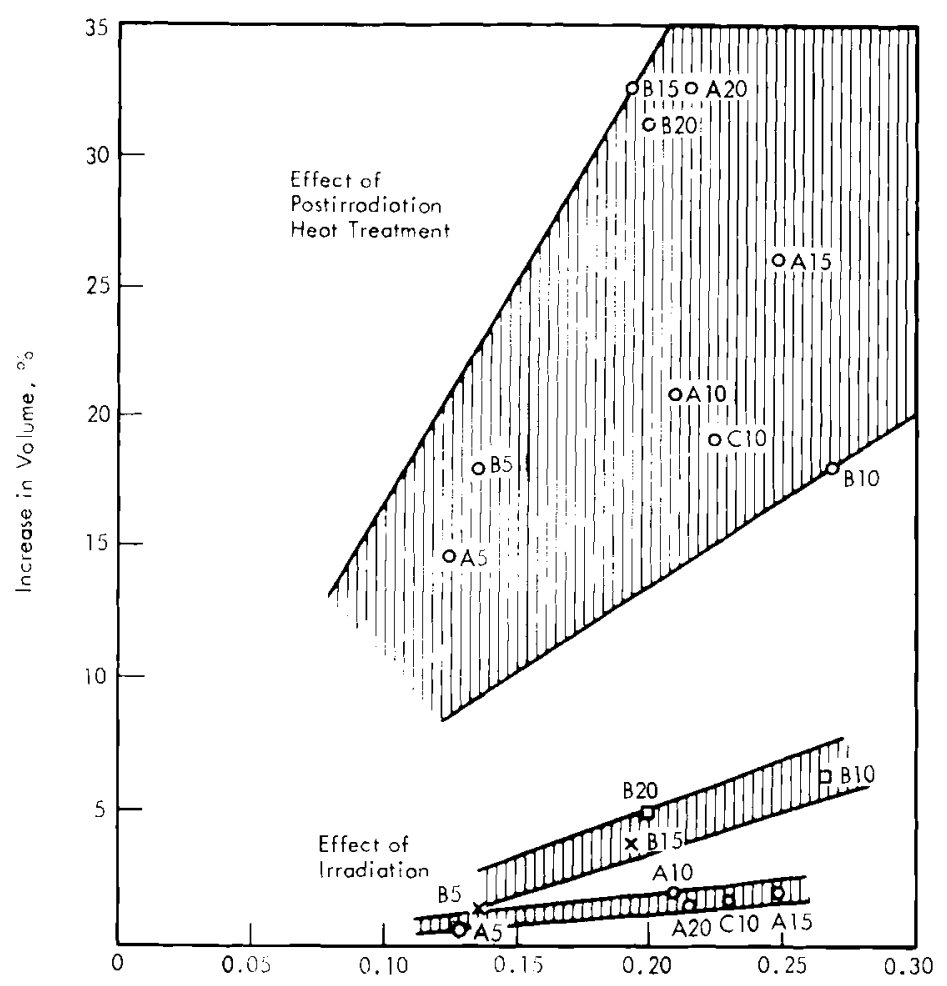

Percent of Total Atoms Fissioned

Effect of Plutonium Fissioning, Irradiation Temperature and Postirradiation Heat-Treatment at $6.60-650^{\circ} \mathrm{C}$ on the Volumes of Aluminum-Plutonium Alloys

- Specimens irradiated under restraint at $350-400^{\circ} \mathrm{C}$.

$\times$ Specimens irradiated under no restraint: initial irradiation temperatures, $550-625^{\circ} \mathrm{C}$.

- Specimens irradiated initially under no restraint at $600-625^{\circ} \mathrm{C}$ and later under restraint at $\sim 400^{\circ} \mathrm{C}$.

- Specimens postirradiation annealed at $640-650^{\circ} \mathrm{C}$, after desheathing. 

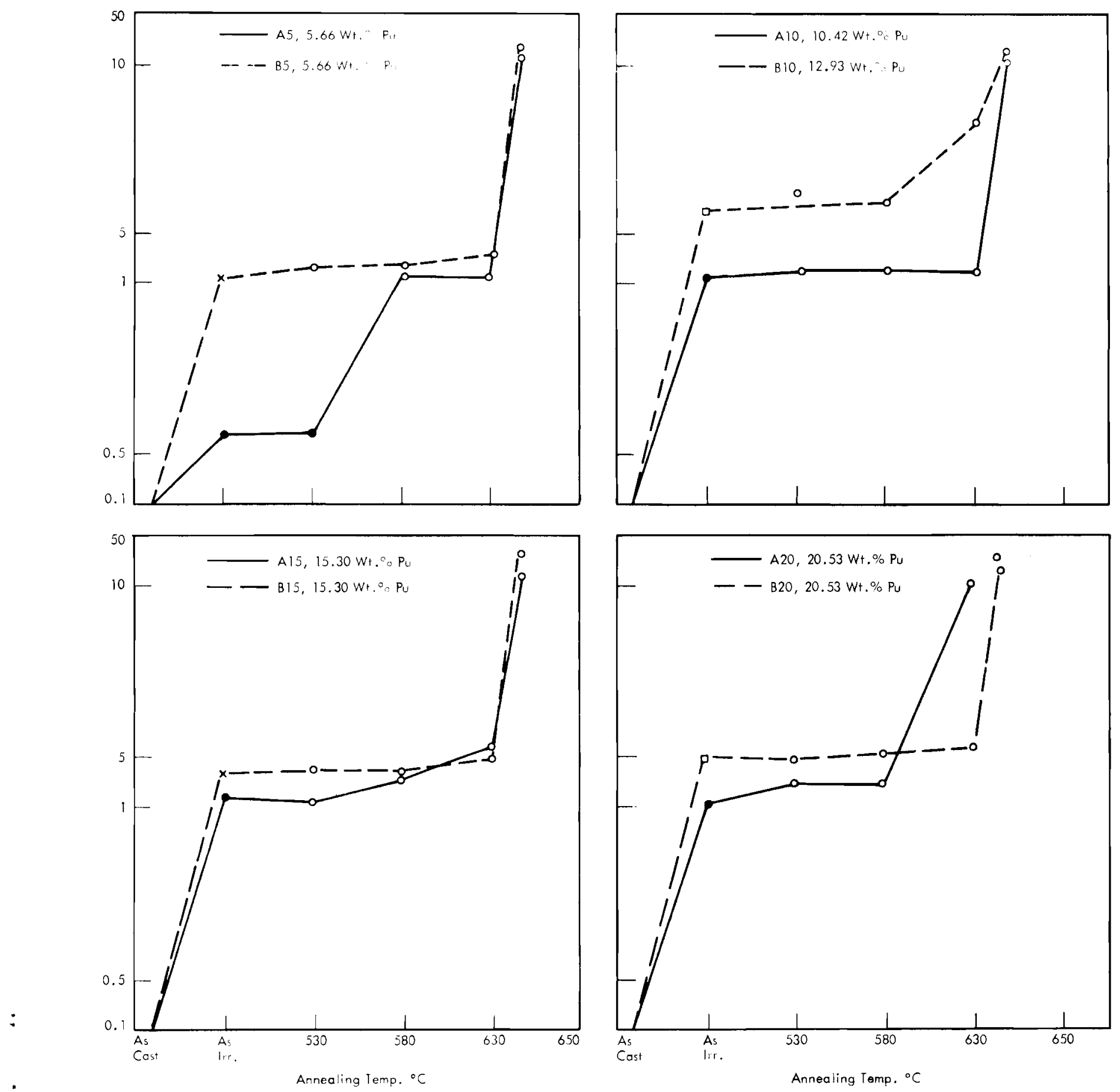

Volume Changes After Irradiation and Postirradiation Annealing with AluminumPlutonium Alloys.

- Specimens irradiated under restraint at 350 to $400^{\circ} \mathrm{C}$.

$\times$. Specimens irradiated under no restraint; initial irradiation temperature 550 to $625^{\circ} \mathrm{C}$.

- Specimens irradiated initially under no restraint at $600-625^{\circ} \mathrm{C}$ and later under restraint at $\sim 400^{\circ} \mathrm{C}$.

- Specimens postirradiation annealed for $1-\mathrm{hr}$ periods at successively higher temperatures.

From T. I. Jones, CRRM-1097. 
DATA FOR THE ALUMINIMT-CIAI) Al-5 WT. " PU ALLOY IRRADLATED TO

$11.97 \times 10^{21}$ nvt ( $(9,3$ PU ATOMS FISSIONED) ANI) TIIEX ANNEALED

15 MIN AT VARIOL'S TI:MPERATIRES From? D. A. LOm

\begin{tabular}{|c|c|c|c|c|c|}
\hline $\begin{array}{c}\text { Annealing } \\
\text { Tenperature, }{ }^{\circ} \mathrm{C} \\
\end{array}$ & $\begin{array}{c}\text { Weiglut, } \\
\qquad 5 \\
\end{array}$ & $\begin{array}{c}\text { Volune } \\
\text { en? } \\
\end{array}$ & $\begin{array}{l}\text { Density, } \\
\text { g/cms? } \\
\end{array}$ & $\begin{array}{l}\text { Change in } \\
\text { Density, }\end{array}$ & $\begin{array}{l}\text { Hardness } \\
R_{H} \\
\end{array}$ \\
\hline Preirradiation & 20.354 & 7.460 & 2.721 & & \\
\hline Postirradiation & $19.708+$ & $7.2 \times 1$ & 2.707 & & $61+$ \\
\hline 125 & $19.711)$ & $7.2 \times 1$ & 2.707 & 0 & 62 \\
\hline 150 & $19.70 \mathrm{n}$ & $7.27 x$ & 2.708 & -0.04 & 59 \\
\hline 200 & $19.719 \times$ & 7.272 & 2.710 & +0.11 & \\
\hline 250 & 19.707 & 7.275 & 2.709 & 0.07 & $6 R$ \\
\hline 300 & 19.706 & 7.274 & 2.709 & +0.07 & 57 \\
\hline 325 & 19.705 & 7.274 & 2.709 & +0.07 & \\
\hline 360 & 19.706 & 7.271 & 2.710 & +0.11 & 10 \\
\hline 420 & $19.706 \mathrm{j}$ & 7.265 & 2.712 & -0.19 & \\
\hline 465 & $19.70 x$ & 7.265 & 2.713 & 0.22 & 15 \\
\hline 496 & 19.704 & 7.260 & 2.714 & -0.26 & 1 \\
\hline 850 & 19.710 & 7.630 & $2.5 \times 3$ & -4.58 & 15 \\
\hline 575 & 19.702 & 7.628 & $2.5 \times 3$ & -4.58 & 15 \\
\hline 598 & 19.706 & 7.625 & 2.584 & -4.54 & 16 \\
\hline
\end{tabular}

* The percent change in density is expressed as the ratio of the change in density on annealing to the density of the specimen after irradiation.

+ This weight change is partially due to removal of an oxide film.

$\ddagger$ This value is the average of three or more determinations.

DATA FOR THE ALIMINUM-CLAD Al-10 WT, ; PI ALLOY IRRADIATED TO $2.14 \times 10^{21}$ nvt (75.6\% PU ATOMS FISSIONED) AND THEN ANNEALED 15 MIN AT VARTOIS TEMPERATURES From B. A. LOOMis, ANL-6651.

\begin{tabular}{|c|c|c|c|c|c|}
\hline $\begin{array}{c}\text { Annealing } \\
\text { Temperature, }{ }^{\circ} \mathrm{C} \\
\end{array}$ & $\begin{array}{c}\text { Weight, } \\
\text { :2 }\end{array}$ & $\begin{array}{l}\text { Volume, } \\
\mathrm{cm}^{?}\end{array}$ & $\begin{array}{l}\text { Density, } \\
5 / \mathrm{cm}^{3}\end{array}$ & $\begin{array}{l}\text { Change in } \\
\text { Density } * ?\end{array}$ & $\begin{array}{l}\text { Hardiness, } \\
\mathrm{R}_{\mathrm{I}}\end{array}$ \\
\hline Proirradiation & 20.670 & 7.660 & 2.731 & & \\
\hline Postirradiation & $20.599 \dagger$ & 7.572 & 2.720 & & $52 t$ \\
\hline 125 & $20.5 \leqq 9$ & 7.571 & 2.721 & .0 .04 & \\
\hline 150 & $20.5 ! 2$ & 7.570 & 2.720 & $n$ & 59 \\
\hline 200 & 20.592 & $7.56 \pi$ & 2.721 & -0.04 & \\
\hline 250 & 20.590 & 7.568 & 2.721 & $\cdot 0.04$ & 53 \\
\hline 275 & 20.585 & 7.567 & 2.721 & +0.04 & 15 \\
\hline .325 & 20.588 & 7.548 & 2.721 & .17 .04 & \\
\hline 375 & $20.5 \times 6$ & 7.562 & 2.722 & 0.07 & 15 \\
\hline 410 & 20.586 & 7.561 & 2.723 & .0 .11 & 1.7 \\
\hline 453 & 20.586 & 7.560 & $2.72: 3$ & -0.11 & \\
\hline 487 & 20.586 & 7.561 & 2.723 & $-n .11$ & \\
\hline 499 & 20.588 & 7.544 & 2.729 & +0.33 & 15 \\
\hline 521 & 20.590 & 7.554 & 2.726 & $\cdot 0.22$ & \\
\hline 5,50 & 20.587 & $7.5666^{\circ}$ & 2.721 & -0.04 & \\
\hline 574 & $211.5 \mathrm{NH}$ & 7.572 & 2.719 & -0.04 & \\
\hline 605 & 20.589 & 7.607 & 2.707 & -0.48 & 15 \\
\hline
\end{tabular}

* The percent change in density is expressed as the ratio of the change in density on annealing to the density of the specimen after irradiation.

$\uparrow$ This weight change is partially due to removal of an oxide film.

$t$ This value is the average of three or more determinations.

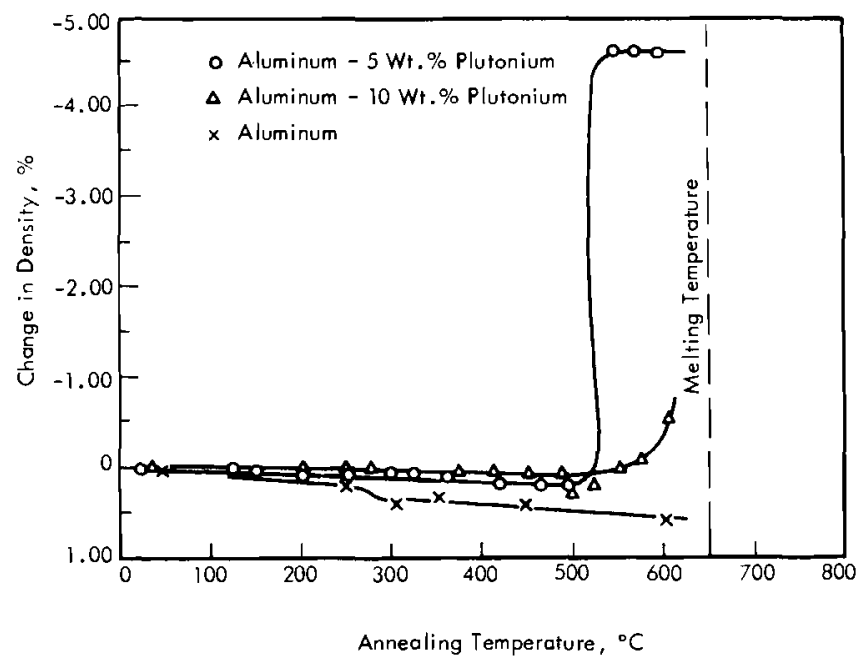

Change in Density of Irradiated Aluminum-Clad Al-5 Wta Pu and Al-10 Wt. Pu Alloys and of Rolled Aluninum on Annealing 15 Min at Successively Higher Temperatures. From B. A. Lomis, ANL-(365)

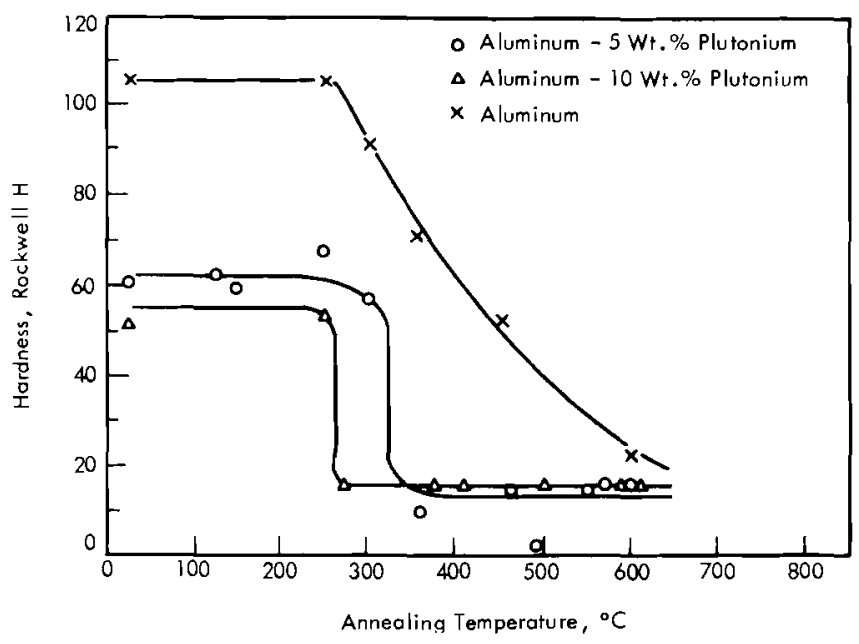

Change in the Room-Temperature Hardness of Irradiated Aluminum-Clad A1-5 Ch't 15. $\mathrm{M}$ in at Successively Higher Temperatures. From B. A. Loomis, ANL-6651. 
PLLTONIUM-GOLD

Plutonium-gold intermetallic phases are known to exist, but they have not yet been identified.

From F. H, Ellinger in The Metal Plutonium, p. 291

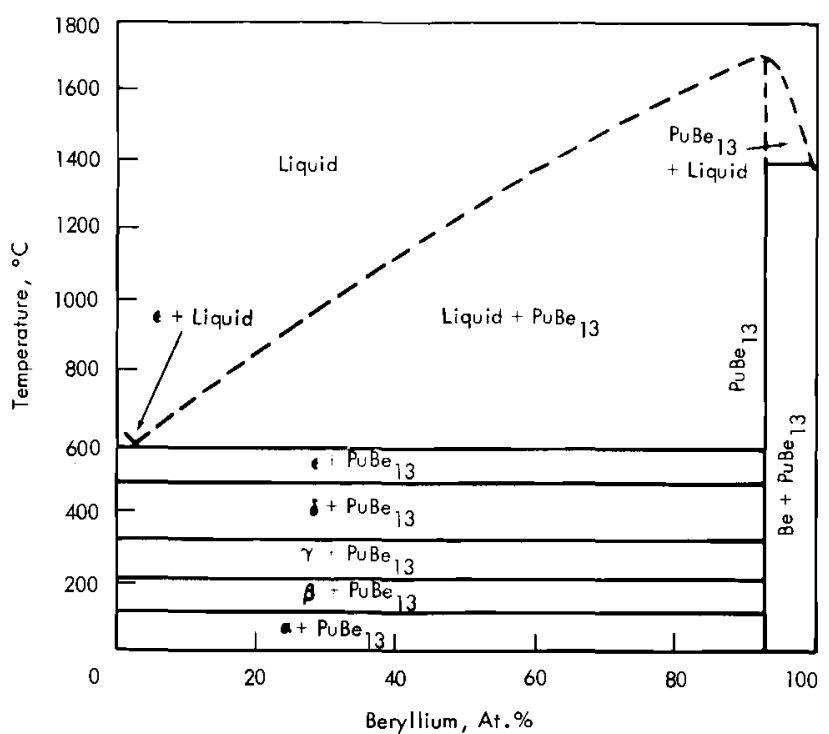

Constitutional Diagram for the System Plutonium-Beryllium. From F. W. Schonfeld et al. in Metallurgy and Fuels, series V, vol. 2, p. 580

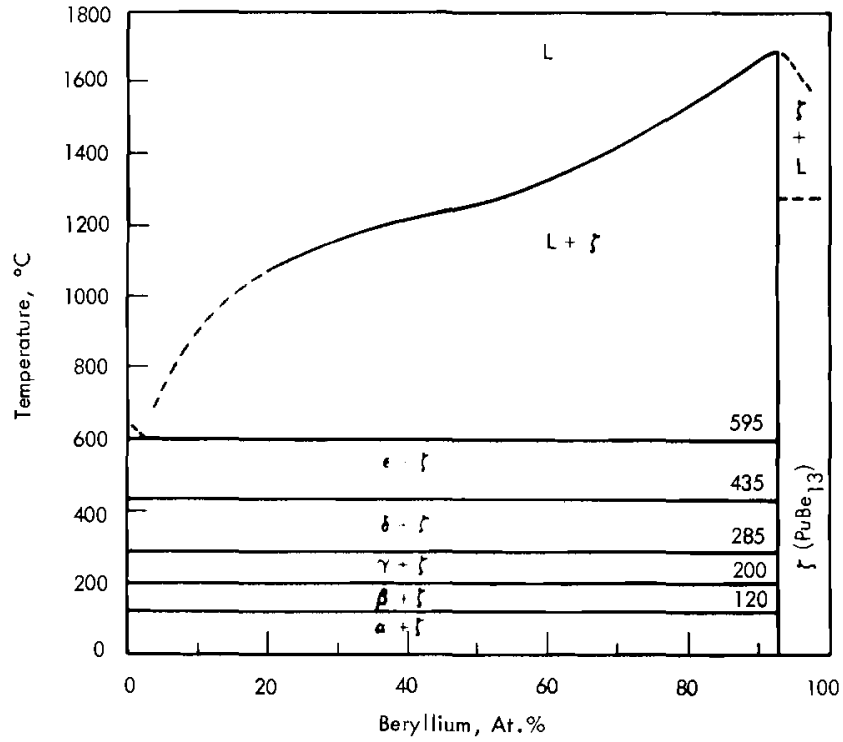

The Plutonium-Beryllium System

From F. W. Schonfeld in The Metal Plutonium, p. 256.

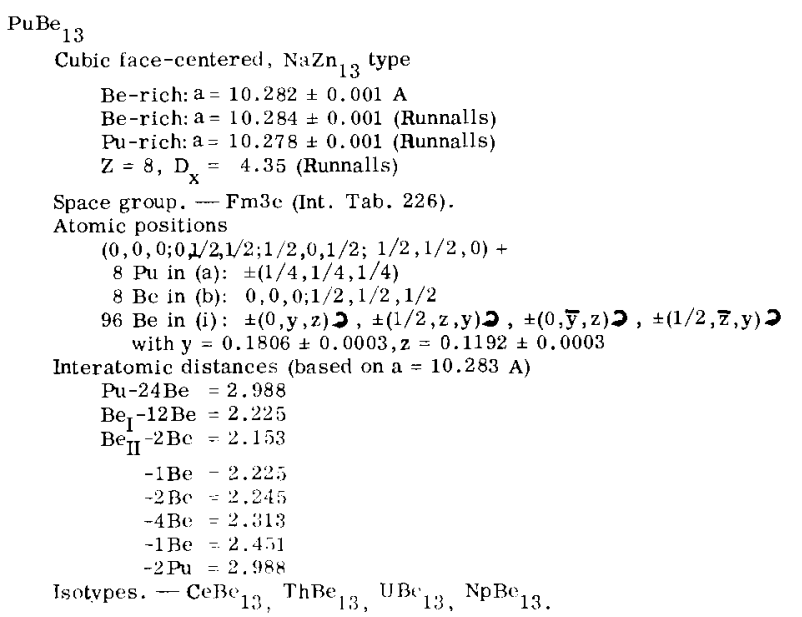

Romarks, - The atomic parameters usuif for calculating the interatomic distances are those of $\mathrm{NaZn}_{13}$ taken from the work of D.P. Shoemaker,

R. E. Marsh, F. J. Ewing, and L. Pauling. (Acta Cryst., vol, 5, p. 637. 1952.) 
Mass susceptibility at $20^{c} \mathrm{C}, \mathrm{x}_{\mathrm{H}} \times 10^{\prime \prime}-2 .+$ cinu/g

Temperature dependenee of susceptibility: $x_{n} \times 10^{6 i}=\frac{9011}{T: \times 0}$

Magnetic moment, $M_{B}=1.7$

From S. T. Konobeevsky at al. in Proc. Sceond Geneva Internat. Conf., vol. 6, p. $19 \%$.

\section{Be 307}

Hardness

Microhardness of $\mathrm{PuBe}_{13}=947 \mathrm{~kg} / \mathrm{mm}^{2}$

From S. T. Konobeevsky, AEC-tr-2345 P1. 2, p. 207.

\section{Bi 101 nnass niagrams}

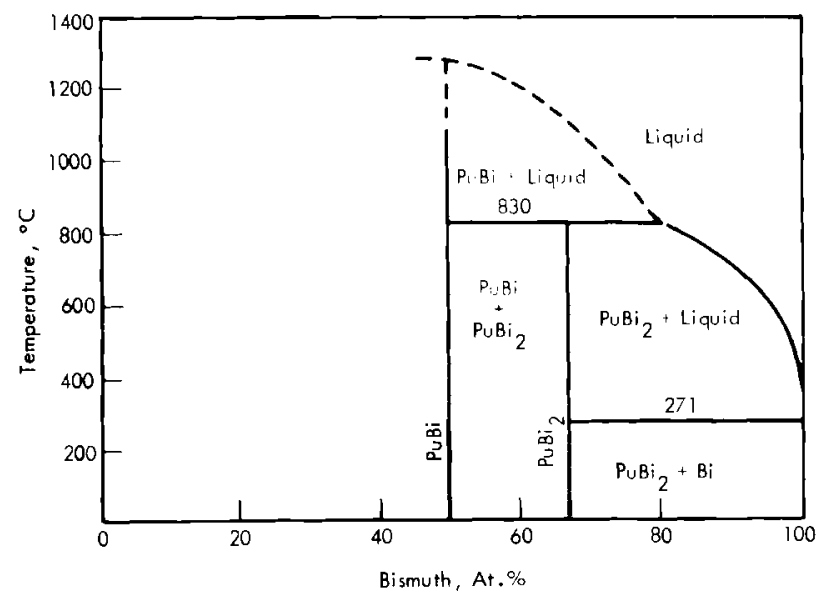

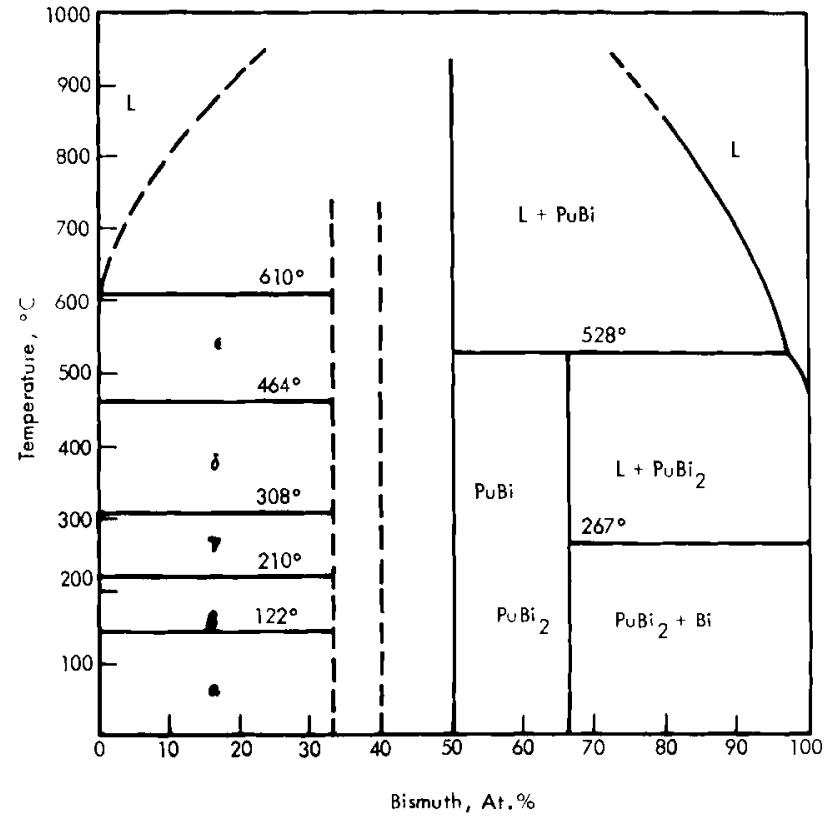

From A. A. Bochvar et al. in Proc. Second Geneva Internat. Conf., vol. 6 , p. 189.1958 .

Bi 103 crystal structure

$\mathrm{PuBi}_{2}$ is pyrophoric and a satisfactory diffraction pattern of it has not been obtained. Its composition is basect upon metallographic and thermalanalysis observations.

From F. H. Fllinger in The Metal Plutonium. P. 287. 


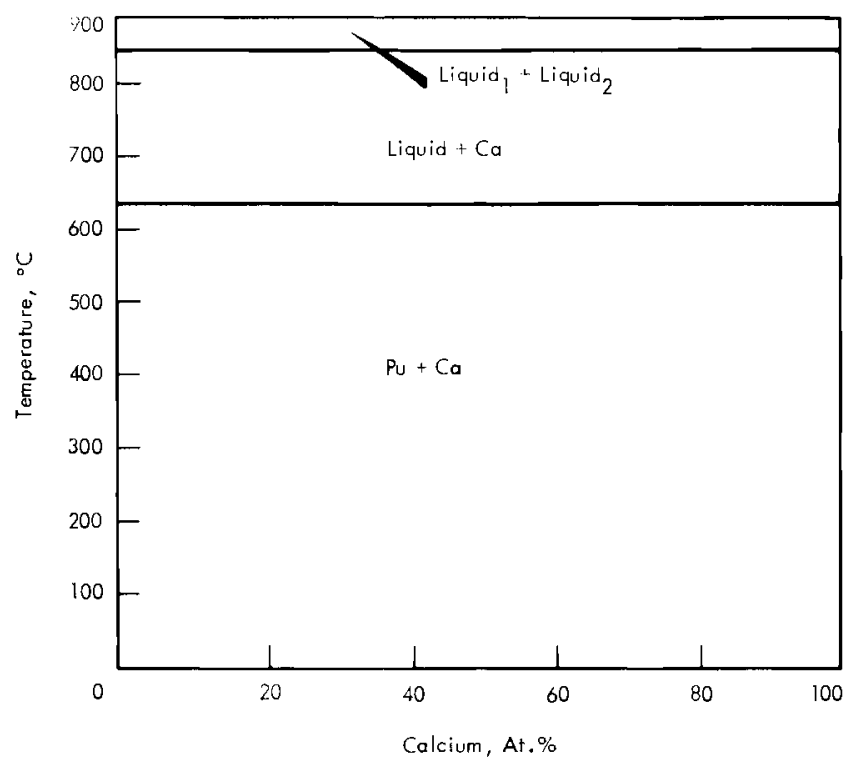

Constitutional Diagrani for the System Plutonium-Calcium.

from F. W. bchonfekl ot al. in Metallurgy and Fuels, Series V, vol. 2, p. 542 .

Phase Diagrams

Ce 101

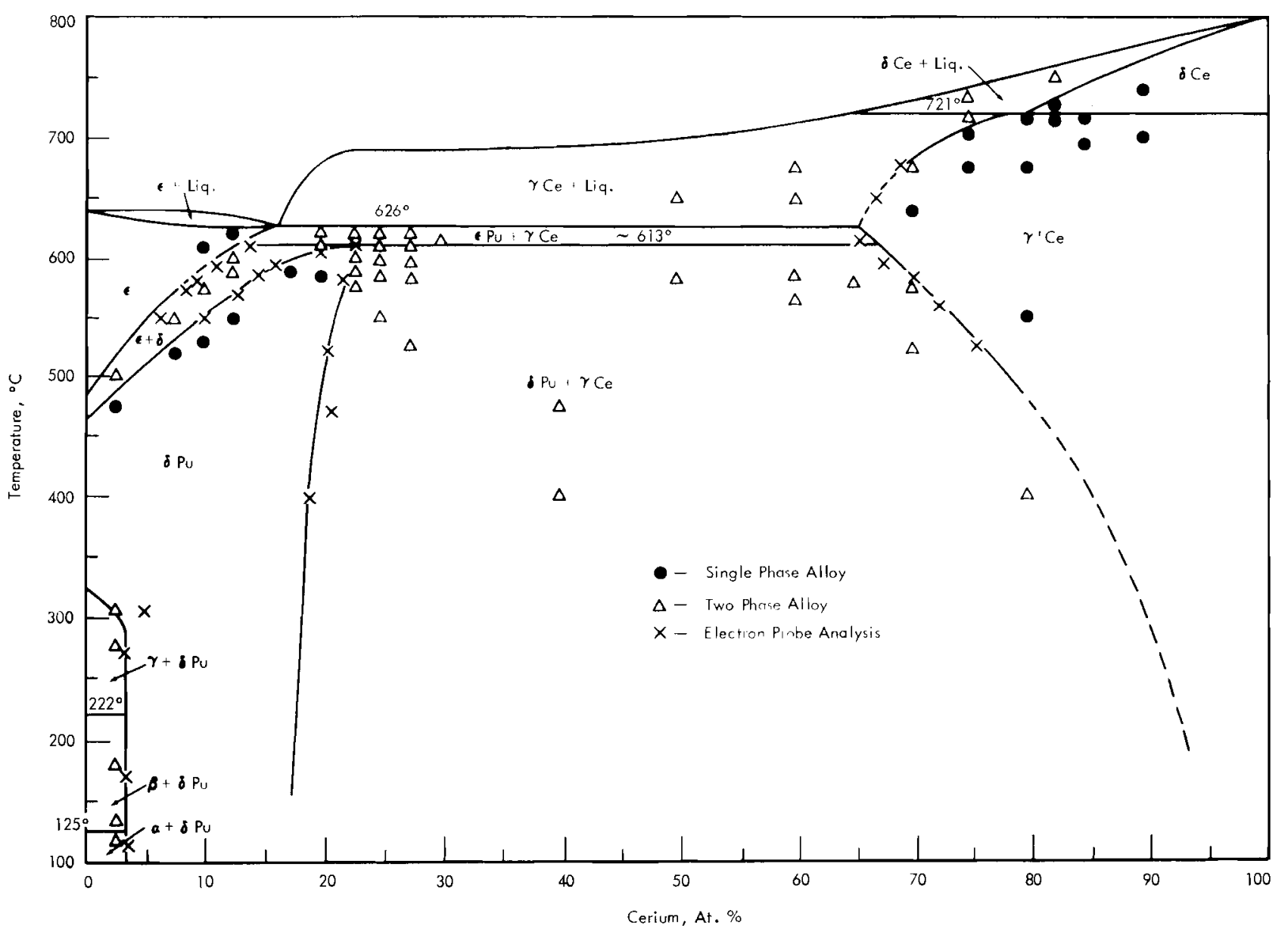




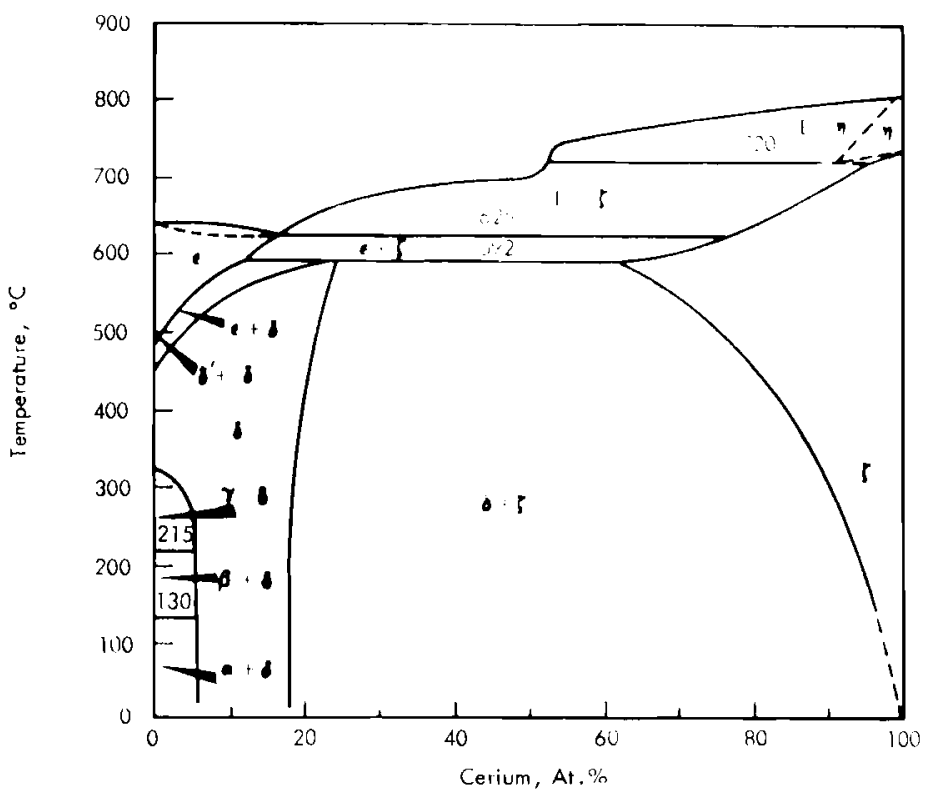

The Plutonium-Cerium System.

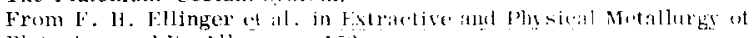

Plutonimu and Its Alloy s. B. 1,is.

\section{$\mathrm{Ce} 102$ Transtormation kinetics}

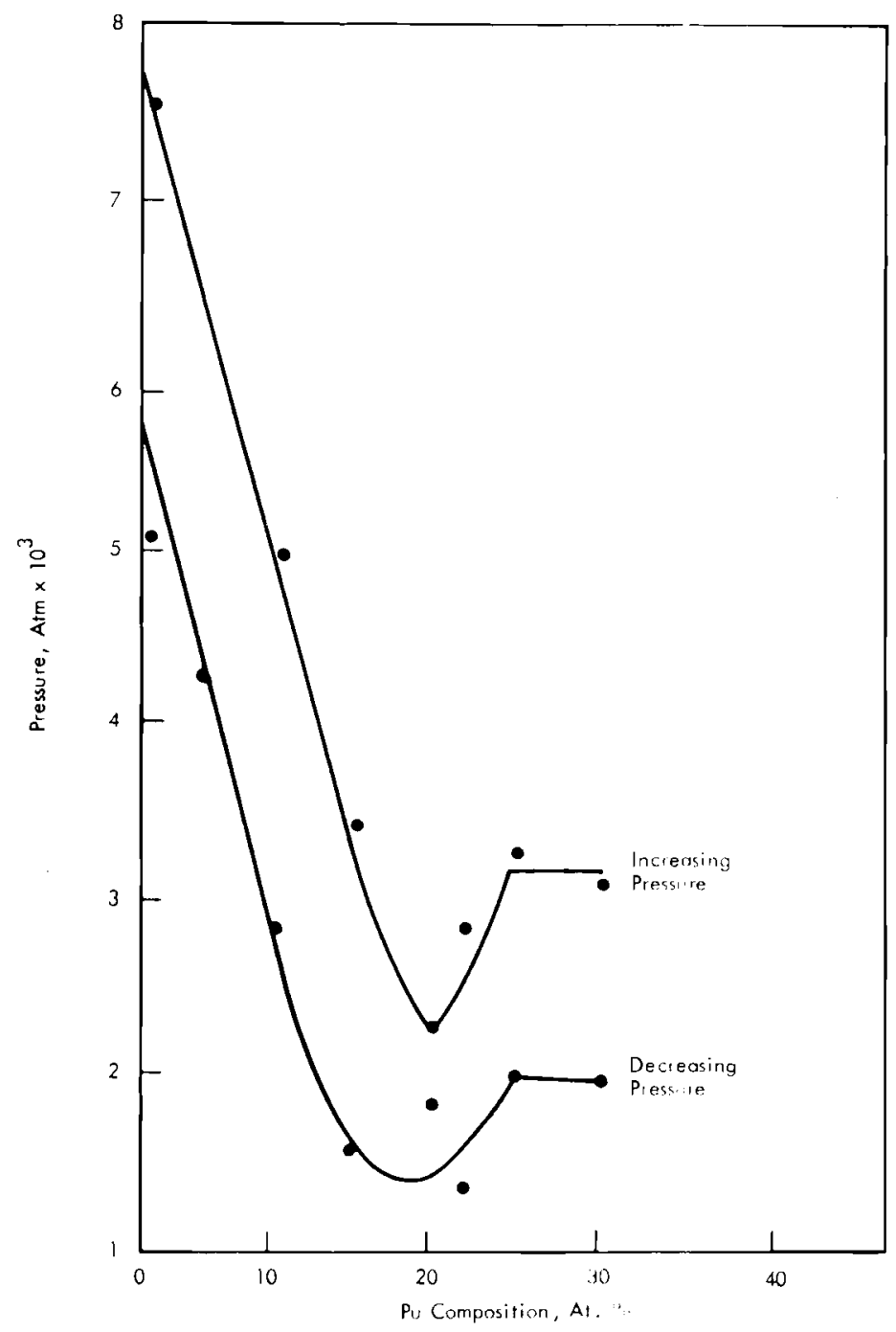

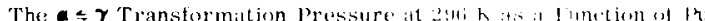

Concentrittion.

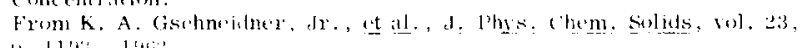


UNI'J' CI:TL, DIMENSIONS*

From W. H. Zachariasen and H. H. Hilinger, Acta Cryst., vol. 16, p. 371 .

\begin{tabular}{|c|c|c|c|c|c|}
\hline Sample & $\begin{array}{c}\text { Temper }- \\
\text { ature } \\
\end{array}$ & $: 1, \Lambda$ & b, A & $\mathrm{e}, \mathrm{A}$ & $\boldsymbol{\beta},{ }^{\circ}$ \\
\hline Pure Pu & $93^{\circ} \mathrm{C}$ & 9.227 & 10.449 & 7.824 & 92.54 \\
\hline Pure Pu & 190 & 9.284 & 10.463 & 7.859 & 92.13 \\
\hline $99 \mathrm{Pu}-1 \mathrm{Ce}$ & 133 & 9.251 & 10.436 & 7.834 & 92.35 \\
\hline $99 \mathrm{Pu}-1 \mathrm{Ce}$ & 172 & 9.260 & 10.449 & 7.846 & 92.24 \\
\hline $99 \mathrm{Pu}-1 \mathrm{Ce}$ & 202 & 9.286 & 10.451 & 7.852 & 92.06 \\
\hline
\end{tabular}

*Calculated density for pure $P u$ is $17.70 \mathrm{~g} \cdot \mathrm{cm} .^{-3}$ at $190^{\circ} \mathrm{C}$. Precision is $\pm 0.002 \mathrm{~A}$ for $\mathrm{a}, \mathrm{b}, \mathrm{c}$, and $\pm 0.02^{\circ}$ for $\boldsymbol{\rho}$.

PRINCIPAL IINEAR COEFFICIENTS OF

THFRMAL, EXPANSION $\left(\times 10^{6}\right)$

From W. H. Zachariasen and F. H. Ellinger, Acta Cryst., vol. 16, p. 371 .

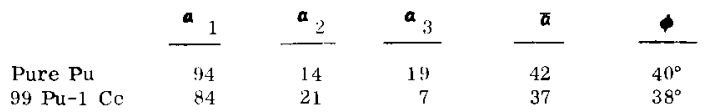

Cerium Alloys

Linear expansion $\left(\mathrm{per}{ }^{\circ} \mathrm{C}\right.$ ) for $\boldsymbol{\beta} \mathrm{Pu}-1$ at. $\mathrm{Cc}$ at $133-202^{\circ} \mathrm{C}$ :

$$
\begin{aligned}
& a_{a} \times 10^{6}=55 \\
& a_{b} \times 10^{6}=21 \\
& a_{c} \times 10^{6}=33
\end{aligned}
$$

Angular change $\left(\right.$ per ${ }^{\circ} \mathrm{C}$ ) at $133-202^{\circ} \mathrm{C}: \Delta \boldsymbol{\beta} /{ }^{\circ} \mathrm{C}=-73 \times 10^{-6}$

From W. D. Wilkinson in Extractive and Physical Metallurgy of Plutonium and Its Alloys, D. 16. Also, W. H. Zachariasen and

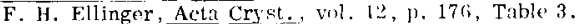

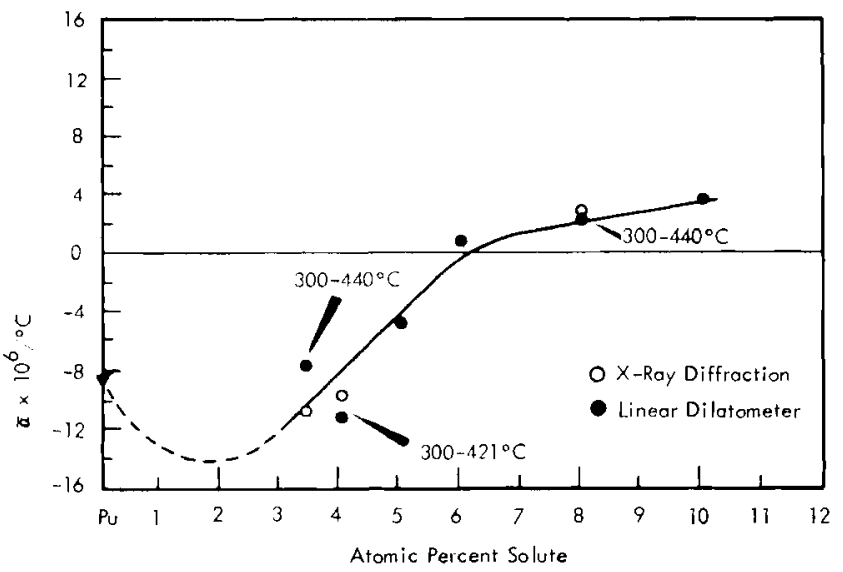

Average Linear Coefficients of Thermal Expansion vs at. 范 Solute For Binary Pu-Ce $\boldsymbol{b}$-phase Solid Solution Alloys. From R. O. Elliott et al in Plutonium 1960, p. 152.

Electron-to-atom ratios and concentrations at which the thermal expansion of cerium-plutonium alloys are zero:

e/a Ratio $=4.74$

at. $\mathrm{Ce}=6.3$

at. Cefor $=6.2 \pm 1.7$

constant $\mathrm{e} / \mathrm{a}$ $+.74 \pm 0.02$ 


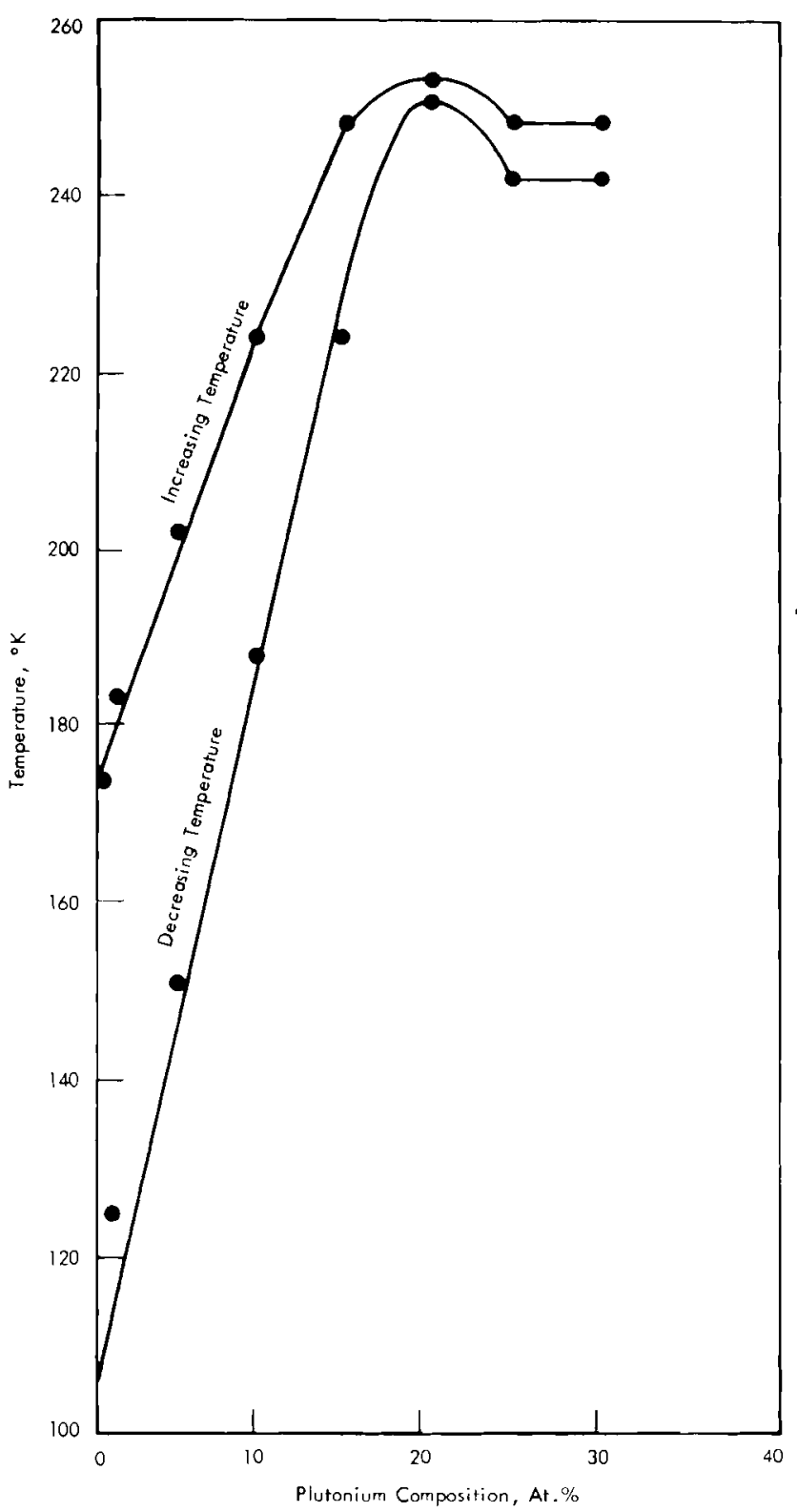

The $\Rightarrow \gamma$ Transformation Temperature as a Function of pu Concentration at $1 \mathrm{~atm}$. From K. A Gischneidiner, Jr, et al. J. Phys. Chem. Solids. vol. 23 , p. 1195 . 196:?

\section{Ce 202.6 Heass of transition}

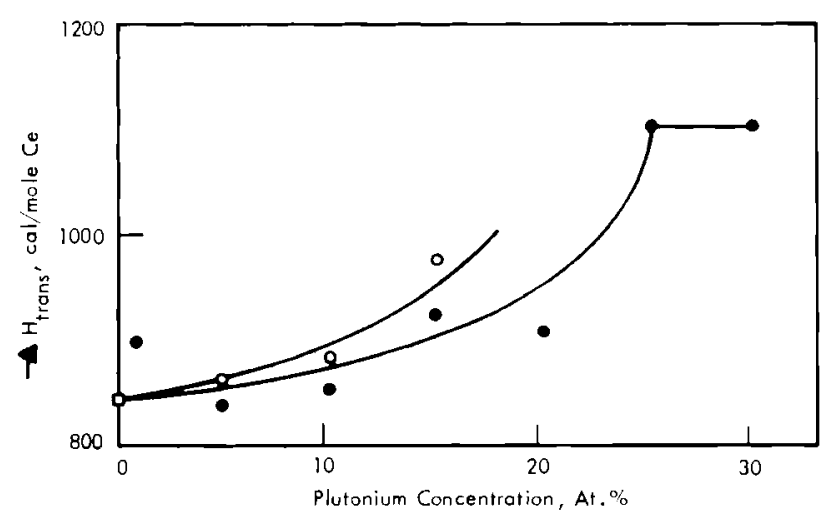

The Heat of Transformation Per Mole of Cerium Versus the Pu Con-

centration at $296^{\circ} \mathrm{K}$. From K. A. Gschneidner. Ir. . 1. J. Phys 


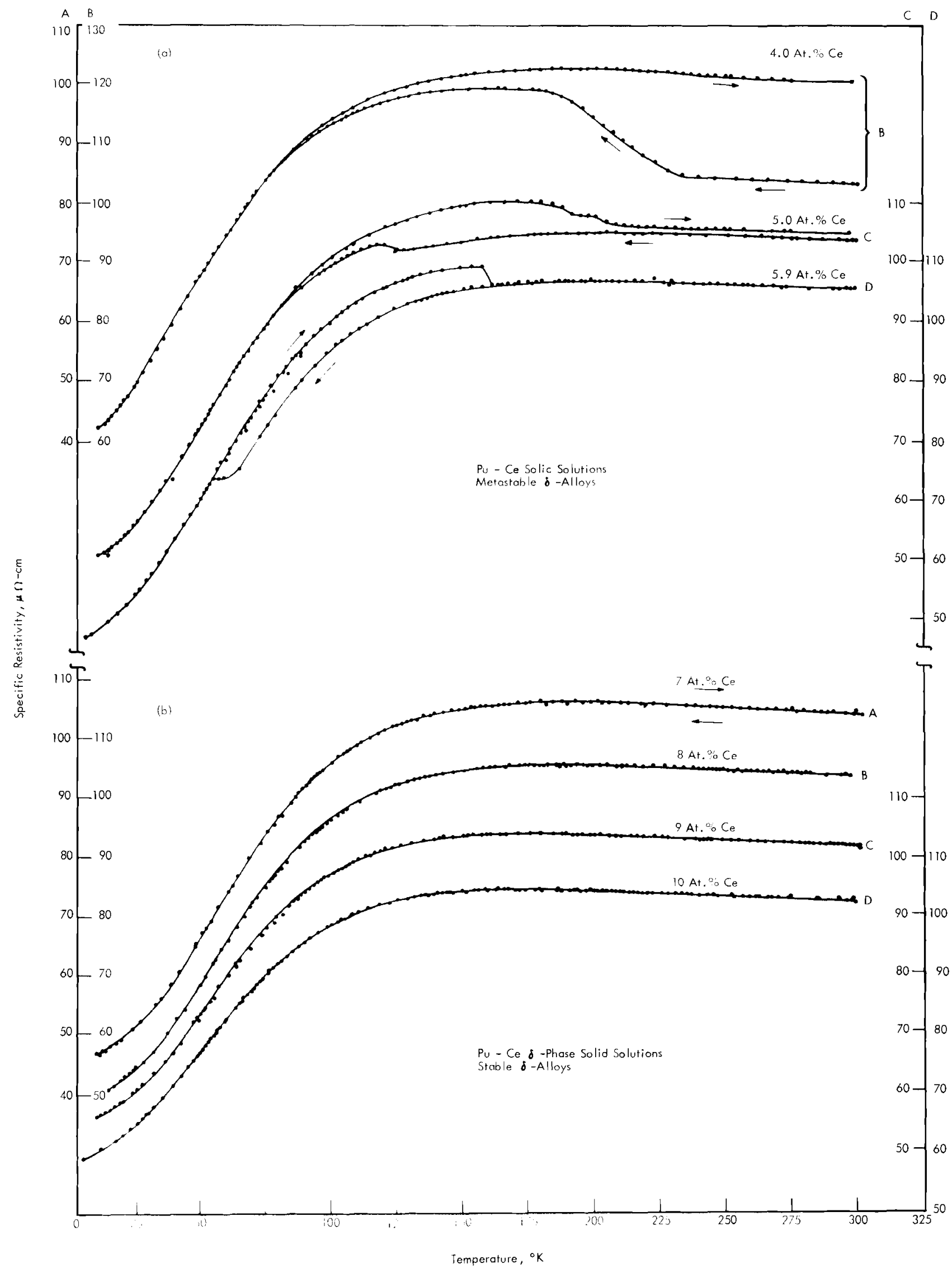

Specific Resistivity in $\mu \cap-c m$ as a Function of Alssolute Temperature: 


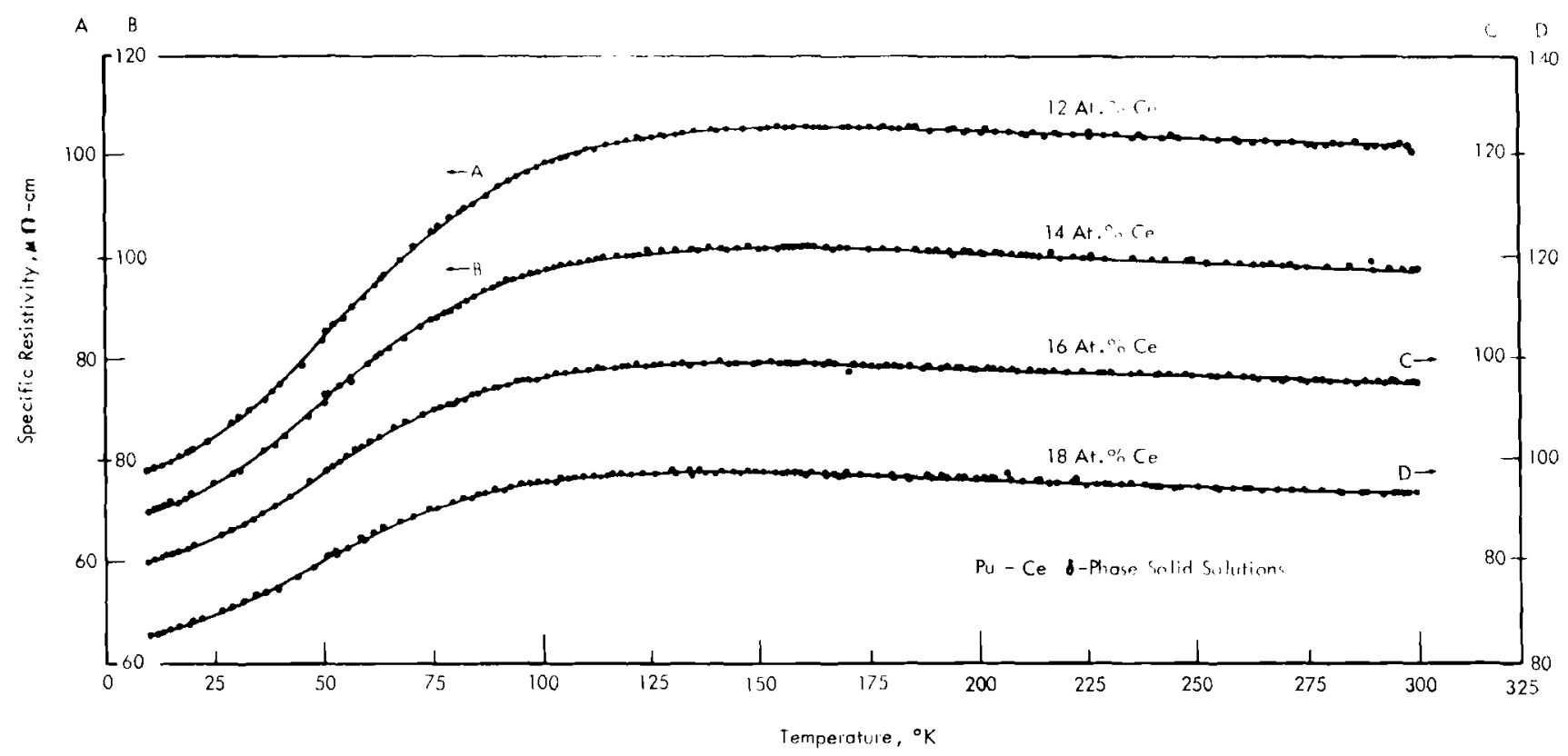

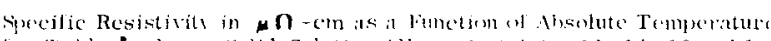

tor Stable b-phalse'. Soldid-Solution Alluts contitining 12, 14, 16 and 1 th

at. Corjum.

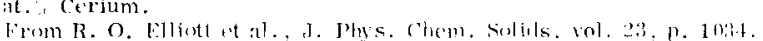

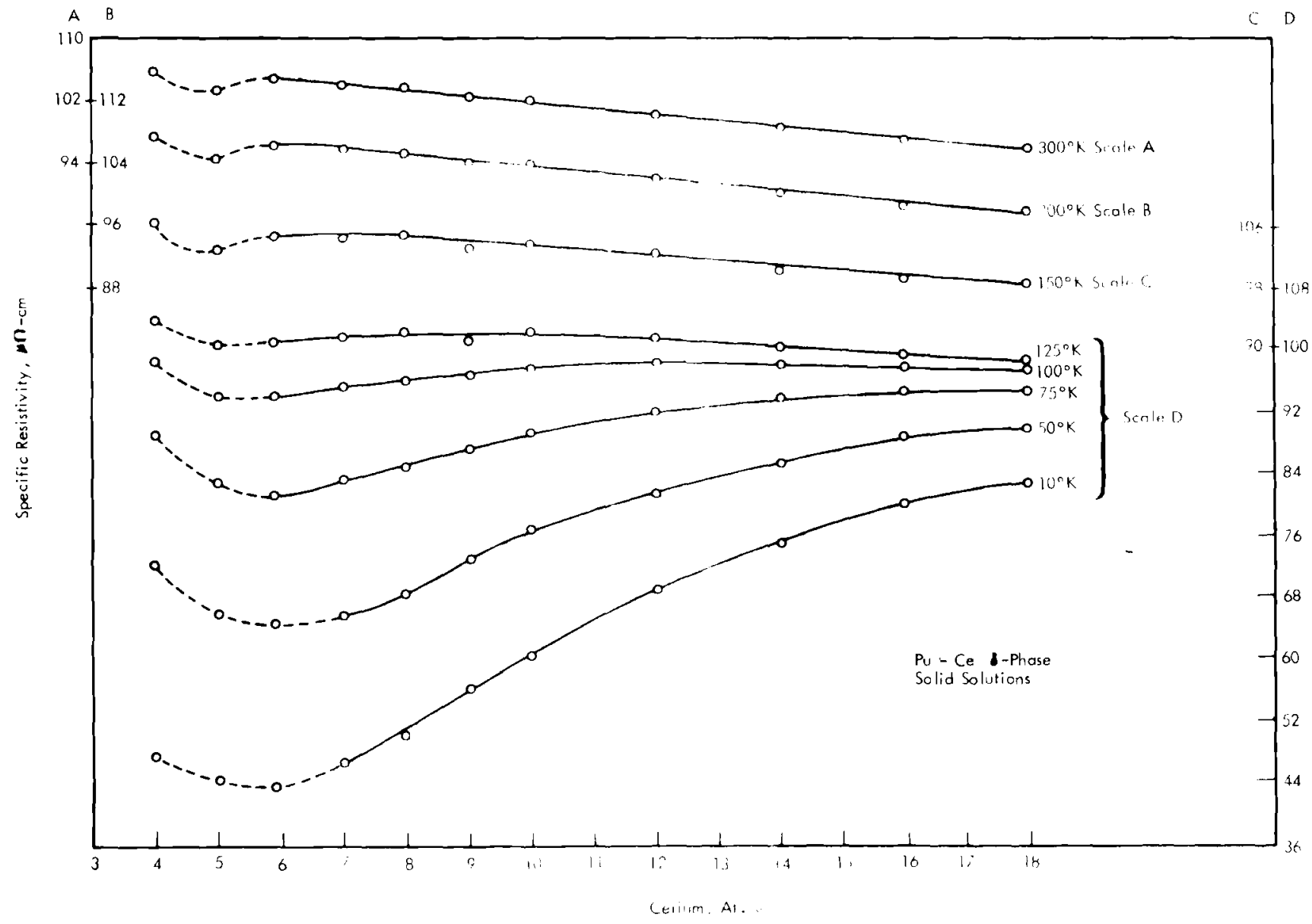

Isothermal plots of sixerific Resistivity in $\mu(1-6)$ as a finction ol

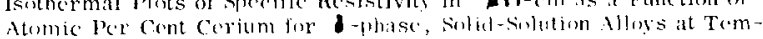

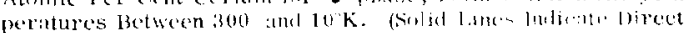

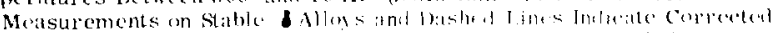

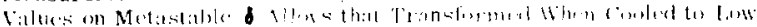

Tomeratures.

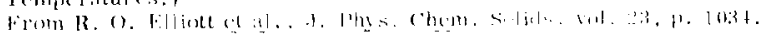

1!mi: 


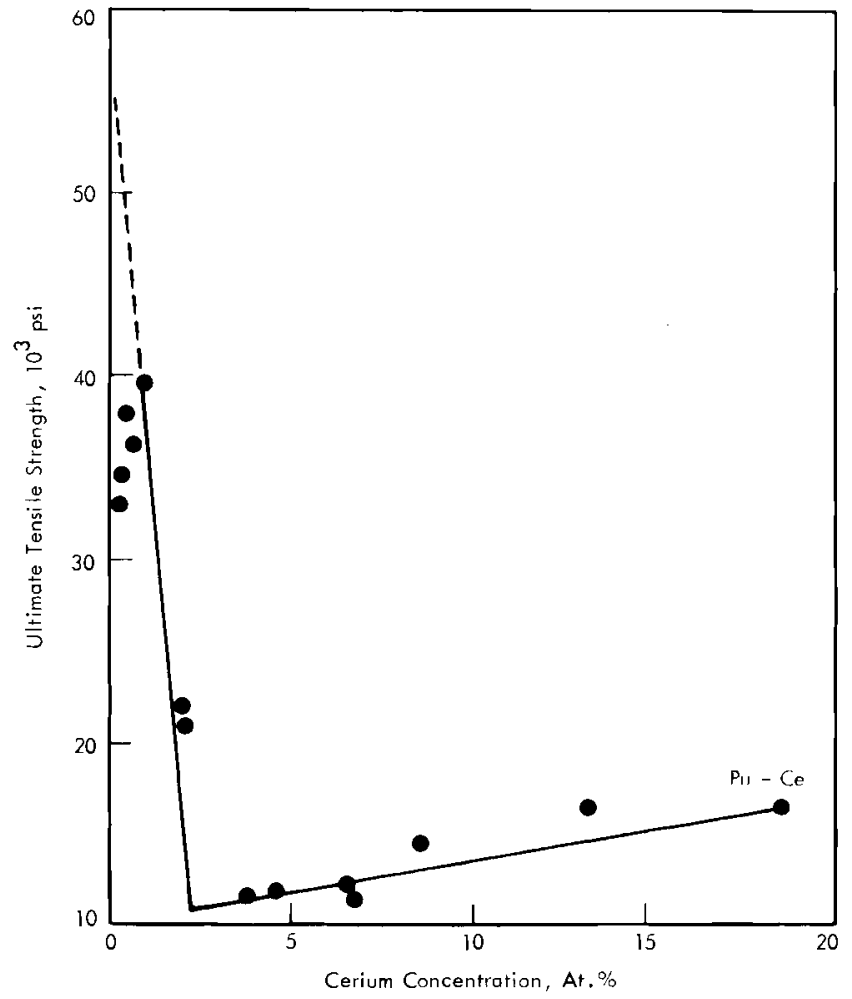

The Effect of Composition on Ultimate Strength in Plutonium-Cerium Alloys. Tested at Room Temperature.

From D. C. Miller and J, S. White, Private Communiention, reported by II. R. Gindner.

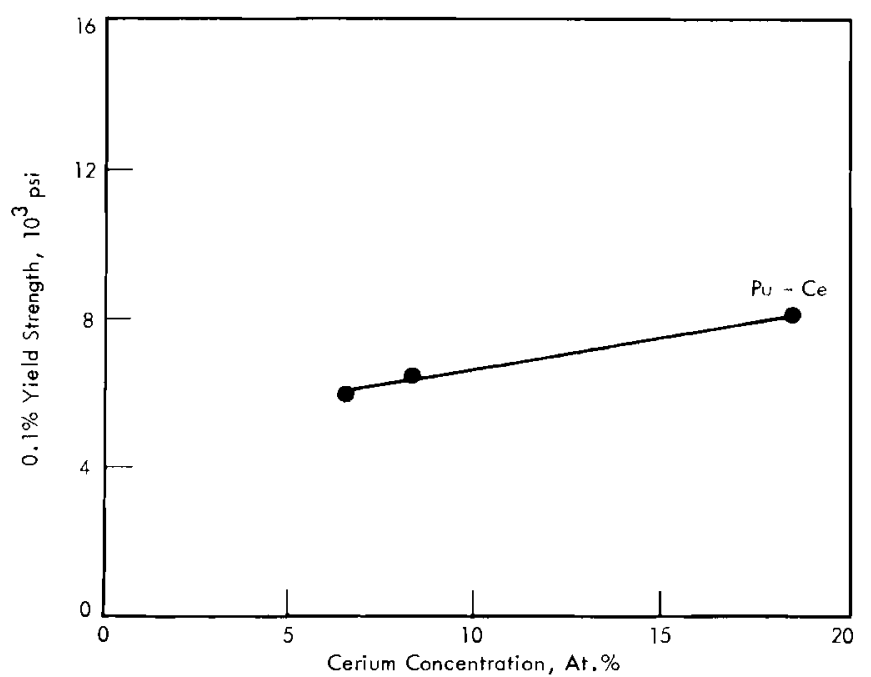

The Effect of Composition on the $0.1 \%$ Yield Stress in Plutonium-

Cerium Allows. Tested at Room Temperature.

From D. C. Miller and J. $\therefore$. White, Private Communjeation, reporeterl

by l1. R. Gariner.

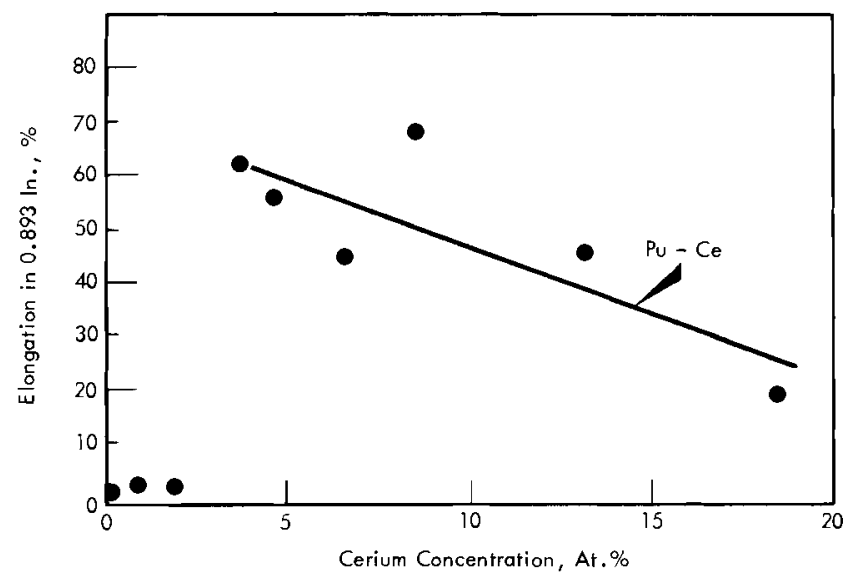

The Effect of Composition on Elongation in Plutonium-Cerium Alloys. Tested at Rom Temperature.

From D. C. Miller and J. S. White, Private Communication, reported by H. R. Gardner.

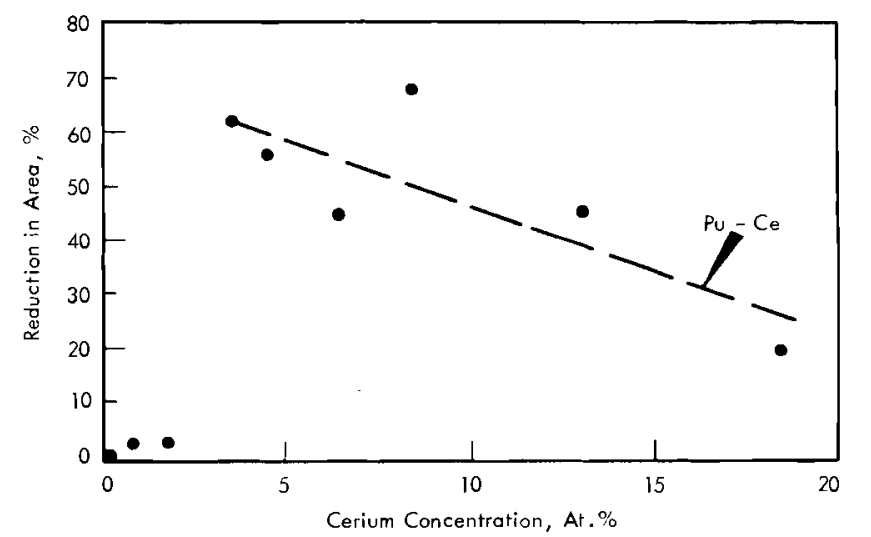

The Effect of Composition on Reduction in Area in Plutonium-Cerium Alloys. Tested at Room Temperature.

From D. C. Miller ani J. S. White, Private Communication, reporter by II. R. Gardner. 


\section{$\mathrm{Ce} 307$ Hardness}

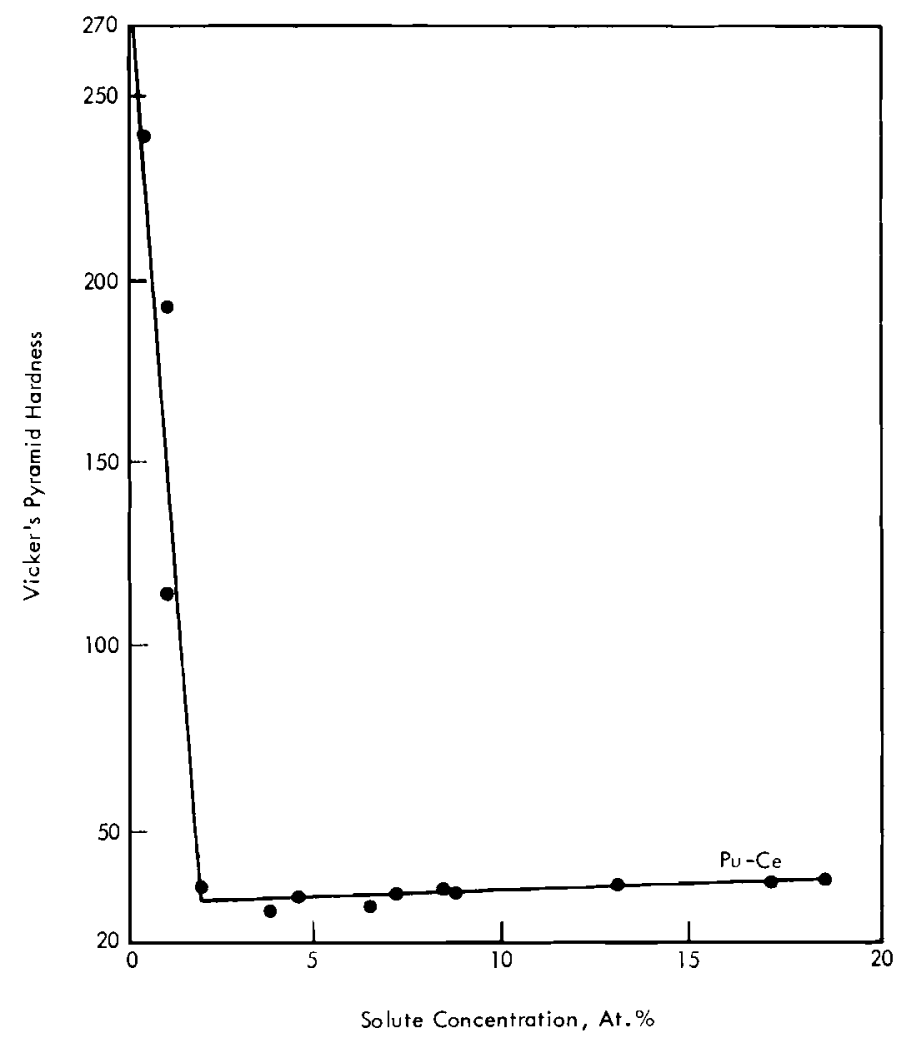

The Effect of Composition on Hardness in Pu-Ce. Tested at Room Temperature. From D. C. Miller and J. S. White, Private Communication, reported by H. R. Gardner.

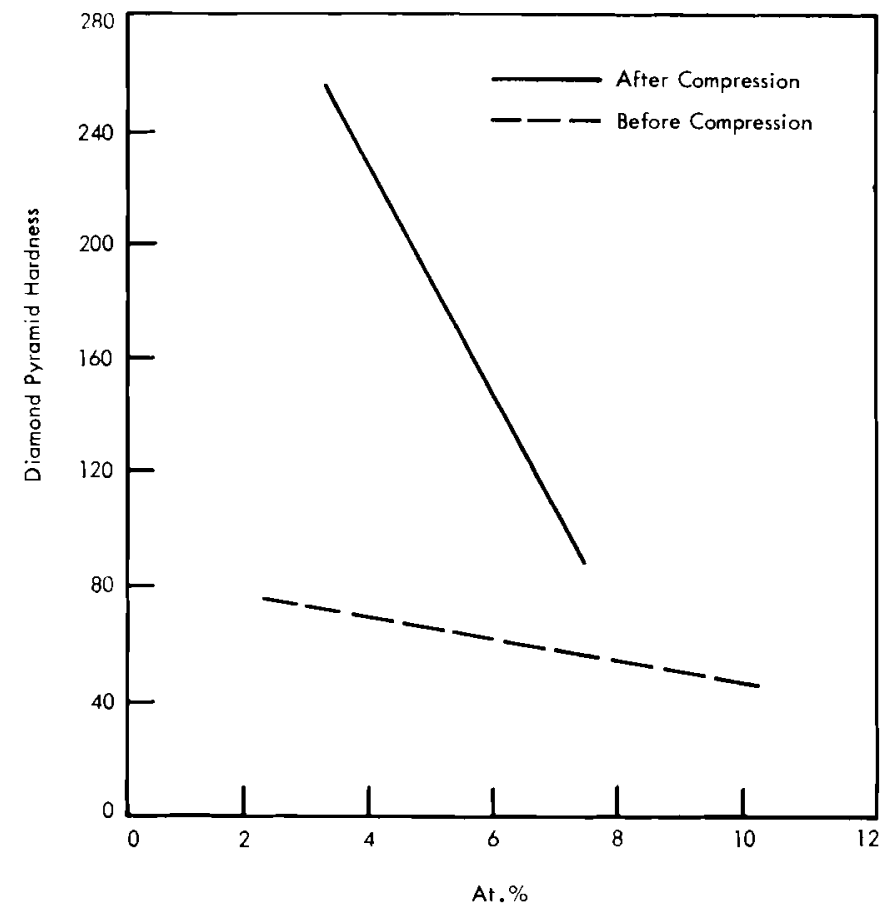

The Fffect of Concentration of Cerium and Pressure up to Ahout 10,000 atm on the Ifardness of Delta-Stabilized Plutonium Alloys. From $R . O$. Flliott and K. A. Gschneidner, Jr., in Fxtractive and Physical Metallurgy of Plutonium and Its Alloys, p. 259.

\section{Ce 308.1 roung's Modulus}

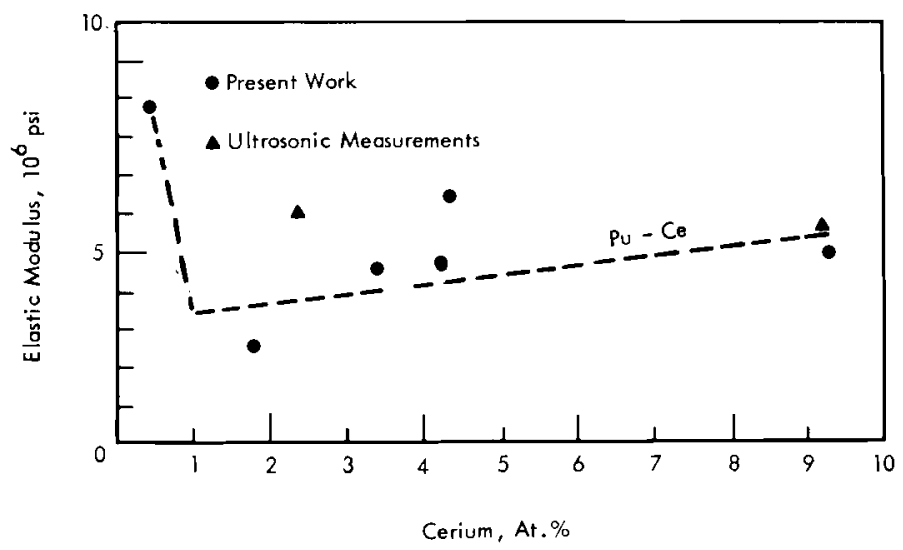

The Variation of Young's Morlulus with Composition for I'lutoniumCerium Alloys. Tested at Room Temperature.

From D. C. Miller and J. S. White, Private Communication, reported

by H. R. Gardner in Chap. 4, The Plutonium Hanllorek, 1966. 

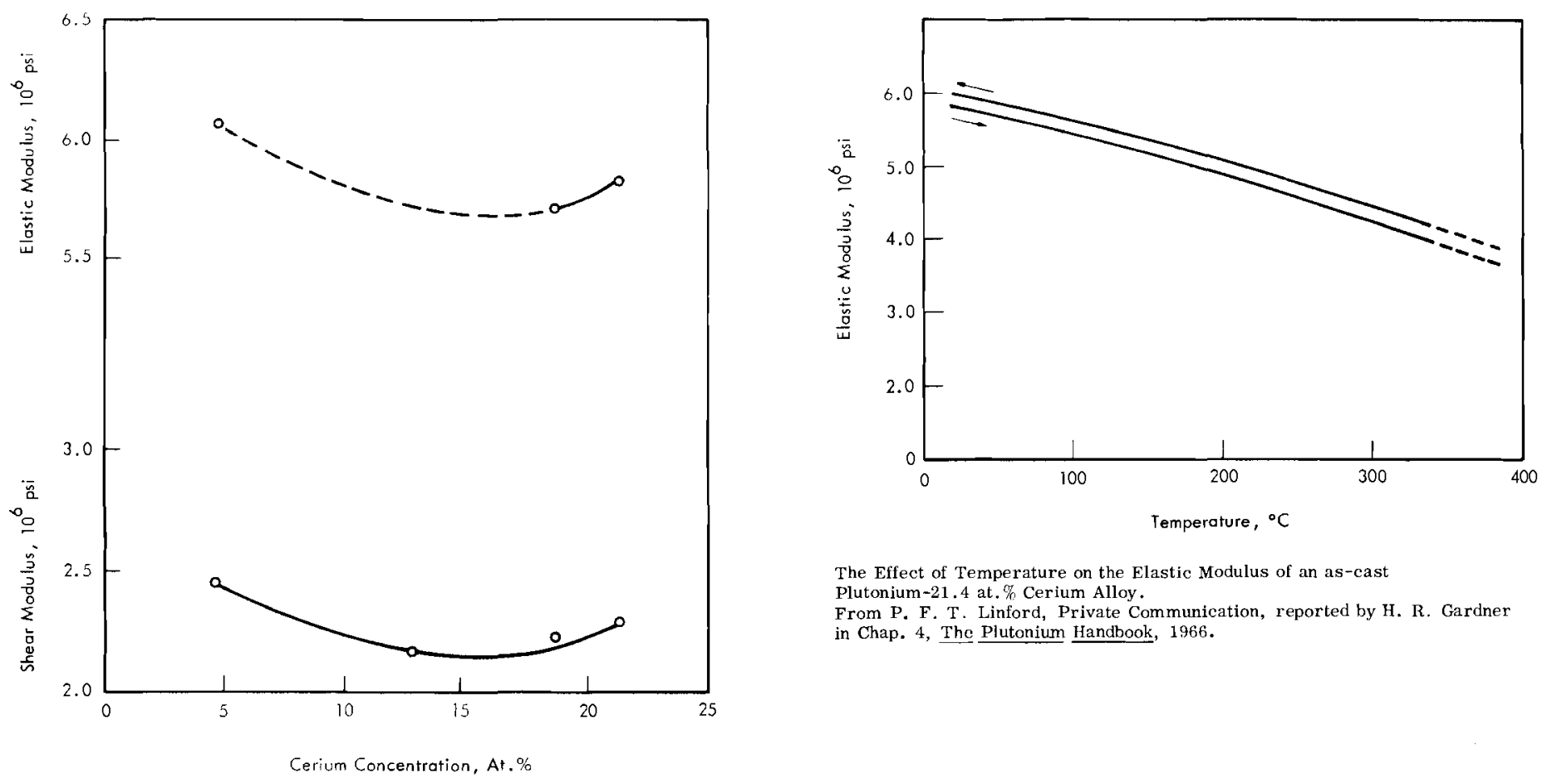

The Effect of Temperature on the Elastic Modulus of an as-cast Plutonium-21.4 at. $\%$ Cerium Alloy.

From P. F. T. Linford, Private Communication, reported by H. R. Gardner in Chap. 4, The Plutonium Handbook, 1966.

The Effect of Composition on the Modulus of Elasticity and Modulus

of Rigidity of as-cast Plutonium-Cerium Alloys at Rnom Temperature.

From P. F. T. Linford, Private Communication, reported by H. R.

Gardner in Chap. 4, The Plutonium Handbook, 1966 .

Air

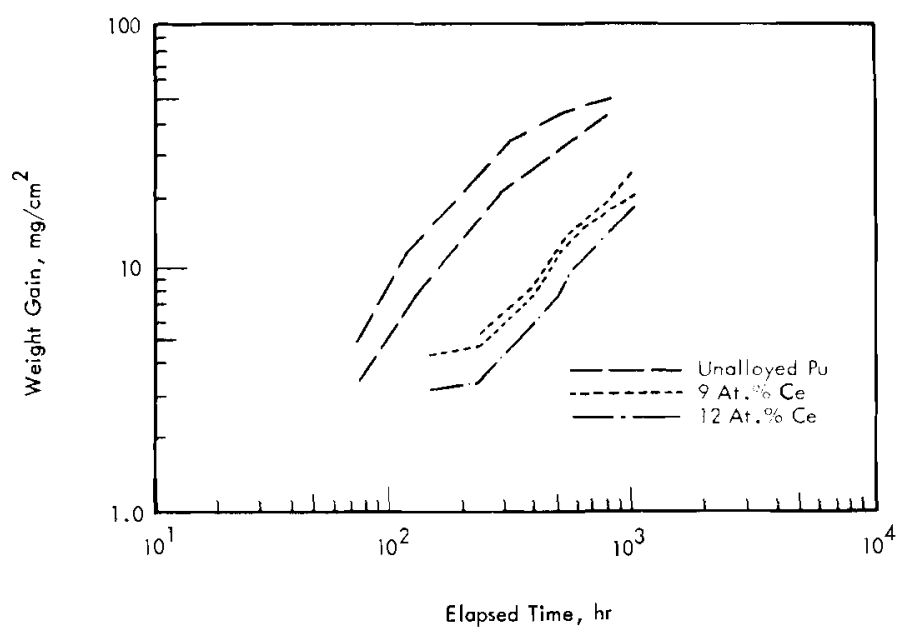

Comparison of the Corrosion Behaviors of Plutonium-Cerium Alloys and Unalloyed Plutonium Exposed to Air at $75^{\circ} \mathrm{C}$ and $50 \%$ Relative Humidity.

From J. T. Waber et al., J. Nucl. Mater, vol. 3, p. 205.1961. 


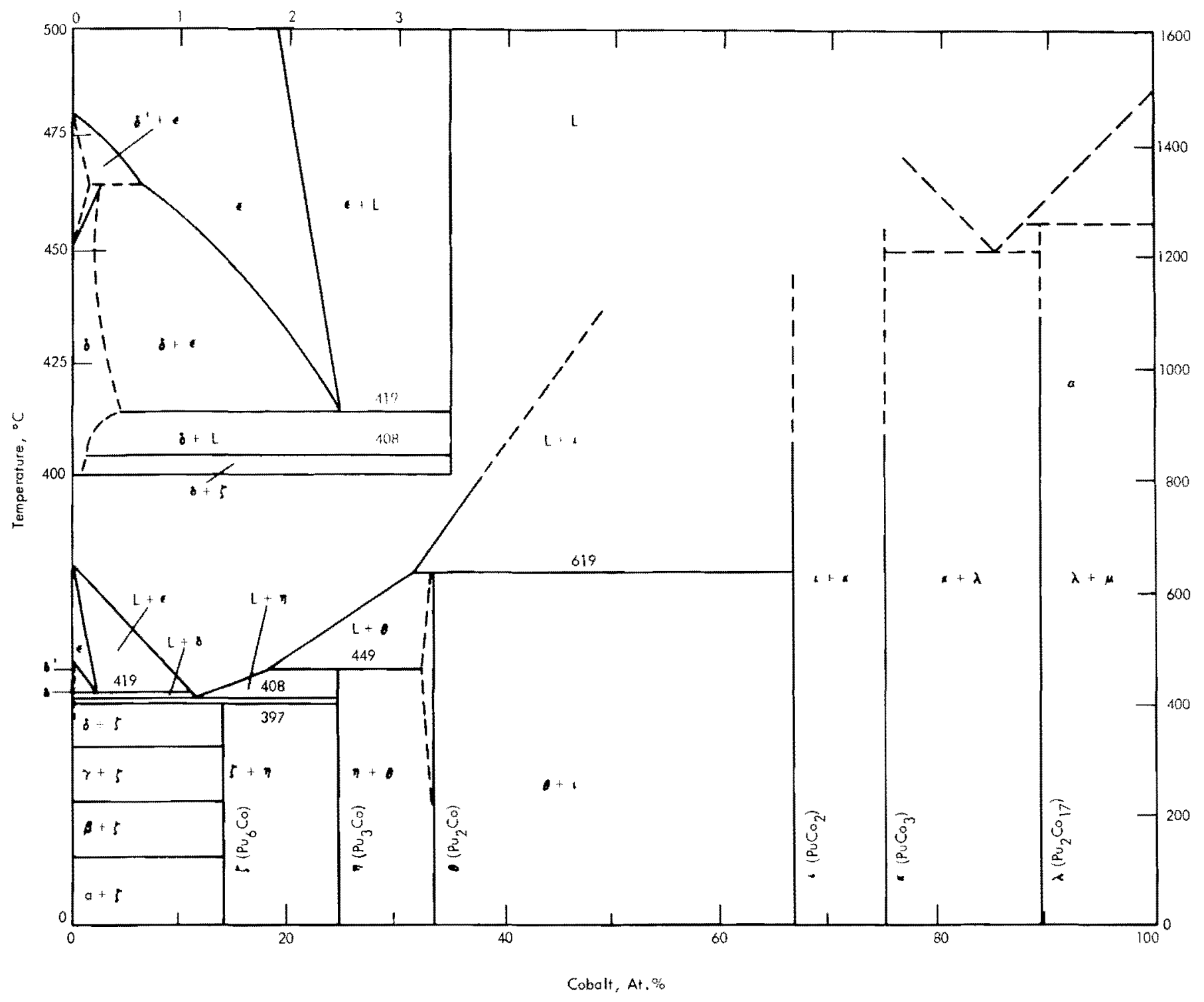

The plutummm Cobalt System

From $F, W$, Schonfeld in The Metal Plutonium, p. 242. 


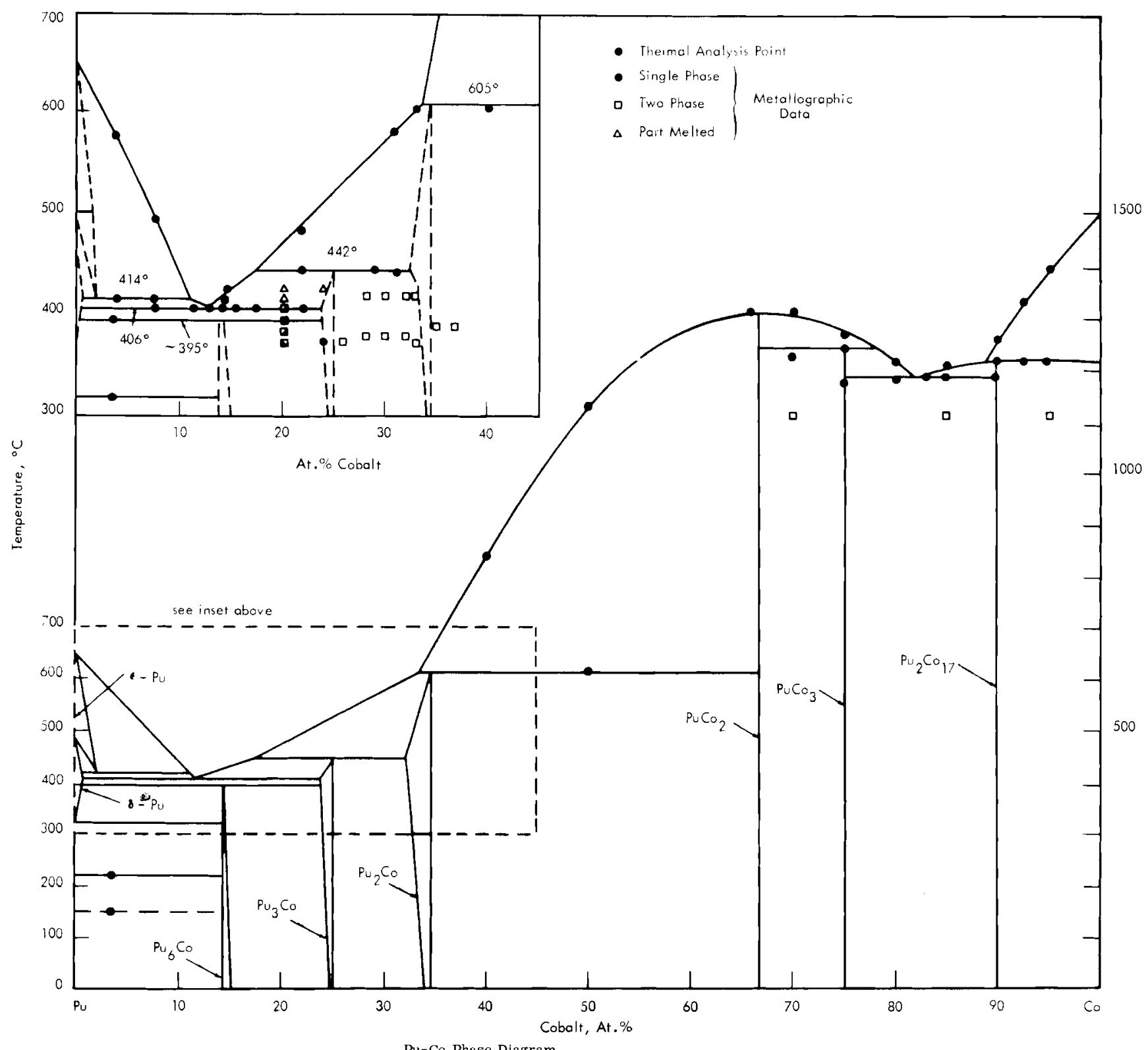

Pu-Co Phase Diagram.

From D. M. Poole et al. in Plutonium 1960, p. 274.

:

\section{Crystal Structure}

$\mathrm{Pu}_{\mathrm{fi}_{\mathrm{i}}} \mathrm{Co}$

Tetragonal body-centered, $\mathrm{U}_{6} \mathrm{Mn}$ type

$a=10.46 \pm 0.02 \mathrm{~A}$

$\mathrm{c}=\overline{\mathrm{s}} \mathrm{3} .33 \pm 0.01$

$a=10.475 \pm 0.005$ From D. M. Poole et al. in plutonium

1960, p. 276

$\mathrm{c}=5.340 \pm 0.005$

$Z=4, D=17.00$

Space group. - I4/mem (Int. Tab. 140).

Atomic positions. - Sec Pu $\mathrm{Fe}$.

Interitomic distances (based on $a=10.46, c=5.33 \mathrm{~A}$ )

$\mathrm{Pu}_{\mathrm{I}}-1 \mathrm{P} \mathrm{u}_{\mathrm{I}}=2.7 \mathrm{i}$

$$
\begin{aligned}
& -2 \mathrm{Pn}_{\mathrm{II}}=2.8 \mathrm{i} \\
& -4 \mathrm{Pu}_{\mathrm{I}}=3.30 \\
& -4 \mathrm{Pu}_{\mathrm{II}}=3.34 \\
& -2 \mathrm{Pu}_{\text {II }}=3.43 \\
& -1 \mathrm{Pu}_{\mathrm{I}}=2.86 \\
& -2 \mathrm{Pu}_{\text {II }}=3.14 \\
& -2 \mathrm{Pu} I=3.34 \\
& -2 \mathrm{Pu}_{\mathrm{II}}=3.4 \mathrm{l} \\
& -1 \mathrm{Pu} \mathrm{I}_{\mathrm{I}}=3.44 \\
& -21 u_{I I}=3.51
\end{aligned}
$$$$
\mathrm{Pu}_{\mathrm{II}}-1 \mathrm{Pu}_{\mathrm{II}}=2.73
$$

$$
\begin{aligned}
-2 \mathrm{Co} & =2.81 \\
\mathrm{Co}-2 \mathrm{Co} & =2.66 \\
-8 \mathrm{Pu} & =2.81
\end{aligned}
$$

Average $\mathrm{Pu}-\mathrm{Pu}$ distance $=3.24$ Isotypes. $-\mathrm{U}_{6} \mathrm{Co}$.

$\mathrm{Pu}_{3} \mathrm{CO}$

Orthorhombic

a $=3.470 \mathrm{~A}$ (Elliott and Larsen)

$\mathrm{b}=10.939$

$\mathrm{c}=9.196$

$Z=4, D_{x}=14.76$

Space group. - Probably Cmcm (Int. Tab, 63).

$$
\left.\begin{array}{l}
a=3.501 \pm 0.003 \mathrm{~A} \\
b=11.03 \pm 0.03 \\
c=9.23 \pm 0.015
\end{array}\right\} \text { Pu-rich }
$$




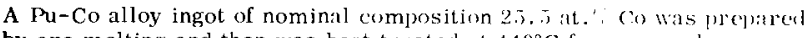
by arc melting and then was heat treated at $440^{\circ} \mathrm{C} f(r)$ one week.

$$
\begin{aligned}
& \mathrm{a}=3.475 \pm 0.004 \mathrm{~A} \quad\left(\lambda \mathrm{MoK} \mathrm{K}_{1}=0.7092(; \mathrm{A})\right. \\
& \mathrm{b}=10.976 \pm 0.010 \\
& \mathrm{c}=9.220 \pm 0.008 \\
& \mathrm{~d}_{0}=14.82 \mathrm{~g} \cdot \mathrm{cm}^{-3} \\
& \mathrm{~d}_{\mathrm{c}}=14.65 \mathrm{~g} \cdot \mathrm{cm}^{-3}
\end{aligned}
$$

\begin{tabular}{|c|c|c|c|}
\hline Iru(1)-2 Iru(1) & $3.48 \mathrm{~A}$ & Pu(2)-2 I & $3.36 \mathrm{~A}$ \\
\hline Pu(1)-4Iru(2) & 3.36 & $P^{\prime} \mathrm{u}(2)-2 \mathrm{P} u(1)$ & 3.40 \\
\hline Iu(1)-4Pu(2) & 3.40 & $P u(2)-1$ Pu(1) & 3.65 \\
\hline Pu(1)-2 Pu(2) & 3.65 & Pu(2)-1 Pu(2) & 3.08 \\
\hline $\mathrm{Pu}(1)-2 \mathrm{Co}$ & 2.40 & Pu (2)-2 Pu(2) & 3.28 \\
\hline \multirow[t]{2}{*}{ Pu(1)-1 Co } & 3,29 & Pu (2) -2 I u (2) & 3.48 \\
\hline & & $P u(2)-11$ Pu(2) & 3.59 \\
\hline$\left.C_{0}\right)-2 P u(1)$ & 2.40 & 'ru(2)-2Co & 2.69 \\
\hline Co-1 Iru (1) & $3.2 ! 9$ & $P u(2)-1 \mathrm{Co}$ & 3.24 \\
\hline Co-4lu(z) & ב. (is) & & \\
\hline $\operatorname{Co}-2 \ln (2)$ & 3.24 & & \\
\hline $\mathrm{Co}-2 \mathrm{Co}$ & $3.47 i$ & & \\
\hline
\end{tabular}

Interatomic distances in $\mathrm{Pu}_{3} \mathrm{C}()$.

The standard deviation of Tht-]h distances is $0.02 \mathrm{~A}$ and for Pu-Co distanees $0.06 \mathrm{~A}$

From A. C. Larson tet al, , Ateta Cryst, vol. 16, pp. 835, 836.

$\mathrm{Pu}_{i 2} \mathrm{Co}$

Hexagonal, $\mathrm{Fe}_{2}$ P type

$$
\begin{aligned}
& \text { Co-rich: } a=7.763 \pm 0.002 \mathrm{~A} \quad \mathrm{c} / \mathrm{a}=0.470 \\
& \mathrm{c}=3.648 \pm 0.003 \quad \mathrm{c} / \mathrm{a} \cdot 0.449 \text { From A. S. } \\
& \text { Pu-rich: }:=7.902=0.004 \quad \text { Coffinberry and } F \text {. H. Ellinger } \\
& c=3.549 \pm 0.002 \quad \text { in Proc. First Geneva Internat. } \\
& \text { Conf., vol. 9, p. } 139 \text {. } 1958 .
\end{aligned}
$$

Space group. - P321 (Int. Tab. 150).

Atomic positions

$\mathrm{CO}_{\mathrm{I}}$ in $(\mathrm{b}): 0,0,1 / 2$

$2 \mathrm{Co}$ in (d): $1 / 3,2 / 3,7 ; 2 / 3,1 / 2, \bar{z}$ with $\mathrm{z}=1 / 8$

$3 \mathrm{Pu}_{\mathrm{I}} \mathrm{U}_{\text {in }}(\mathrm{e}): \mathrm{x}, 0,0 ; 0, \mathrm{x}, 0 ; \overline{\mathrm{x}}, \overline{\mathrm{x}}, 0$ with $\mathrm{x}=-0.26$

$3 \mathrm{Pu}_{1}$ in (f): $x, 0,1 / 2 ; 0, x, 1 / 2 ; \bar{x}, \bar{x}, 1 / 2$ with $x=0.40$

Interatomic distances (hased on $a-7.763, c=3.648 \mathrm{~A}$ )

$$
\begin{aligned}
& \mathrm{Pu}_{\mathrm{I}}-2 \mathrm{Pu} \text { II }=3.21 \\
& 4 \mathrm{Pu}_{\mathrm{II}}=3.28 \\
& -2 \mathrm{Pu}_{\mathrm{I}}=3.50 \\
& -2 \mathrm{Co}_{\mathrm{I}}=2.72 \\
& -2 \mathrm{Co}_{\mathrm{Il}}=2.95 \\
& \mathrm{Pu}_{\mathrm{II}}{ }^{-2 \mathrm{Pu}} \mathrm{I}=3.2 \mathrm{1} \\
& -4 \mathrm{Pu}{ }_{\mathrm{I}}=3.28 \\
& -1 \mathrm{Co}_{\mathrm{I}}=3.11 \\
& -2 \mathrm{Co}_{\mathrm{II}}=2.74 \\
& \mathrm{Co}_{1}-6 \mathrm{Pu}_{1}=2.72 \\
& -3 \mathrm{Pu}_{\mathrm{I}}=3.1 \mathrm{I} \\
& { }^{\mathrm{Co}} \mathrm{U}^{-3 \mathrm{Pu}} \mathrm{I}=2.95 \\
& -3 \mathrm{Pu}_{\mathrm{II}}=2.74
\end{aligned}
$$

$\operatorname{Iru}(\stackrel{3)}{\longrightarrow}$

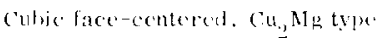

Pu-rich: a $7.0 \times 1+0.001$ From A. S. Coffinberry and $F . H$

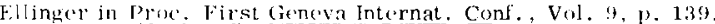

$a 7.07 .5 \pm 0.005$
$a-7.005-7.053$

$\therefore=7.005-7.025$

$$
\begin{aligned}
Z=8, \mathrm{D}_{\mathrm{x}} & =13.3 . \overline{3} \\
\mathrm{D}_{\mathrm{x}} & =133.39 \text { From (). J. C. Runnalls, Can. J. Chem., }
\end{aligned}
$$
vol. $33, \mathrm{t}, 140$.

Snace group. - F (13m (1nt. Tab. 227).

Atomic positions. - See PUAl,

Interatomic distances

$$
\mathrm{Pu}-4 \mathrm{Pu}=3.066
$$

$\mathrm{Co}-6 \mathrm{Co}=2.503$

$$
-12 \mathrm{Co}=2.036
$$

Isotypes. - $\mathrm{I}^{\mathrm{C}} \mathrm{Co}_{2}, \mathrm{CeCo}_{2}$.

$\mathrm{PuCO}_{3}$

Rhombohedral, PuNi ${ }_{3}$ type $\underline{1960}, p .276$

$=33^{\circ} 40^{\prime} \pm 4^{\prime}$

or Hexigonal

$a=5.003 \pm 0.007 \mathrm{~A}$

$c=24.42 \pm 0.06$

$\mathrm{Pu}_{2} \mathrm{Co}_{17}$

Hexagonal, ' $\mathrm{Th}{ }_{2} \mathrm{Ni}_{17}$ type

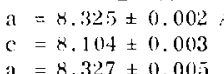

$\mathrm{a}=8.327 \pm 0.005 \quad \mathrm{Fr} \times m$ D. M. Prosle ct al, in Plutonium

1960, p. 276

$\mathrm{c}=8.1117 \pm 0.005$

$$
\text { 7. }=2, \mathrm{D}_{\mathrm{x}}-10.10
$$

Space group. - $\mathrm{PG}_{3} / \mathrm{mmc}$ (Int. Tah. 19) 1 ).

$$
\begin{aligned}
& 2 \mathrm{Pu}_{\mathrm{I}} \text { in (b): } \pm(0,0,1 / 4) \\
& 2 \mathrm{Pu}_{\mathrm{II}} \text { in (d): } \pm(1 / 3,2 / 3,3 / 4) \\
& 6 \mathrm{Co} \text { in (g): } 1 / 2,0,0 ; 0,1 / 2,0: 1 / 2,1 / 2,0 ; 1 / 2,0,1 / 2 \text {; } \\
& 0,1 / 2,1 / 2 ; 1,2,1 / 2,1 / 2 \\
& 12 \mathrm{Co} \text { in }(\mathrm{j}): \pm(\mathrm{x}, 0,1 / 4) ; \pm(0, \mathrm{x}, 1 / 4) ; \pm(\mathrm{x}, \mathrm{x}, 1 / 1) ; \pm(\bar{x}, 0,1 / 4) \\
& \pm(0, \bar{x}, 1 / 4) ; \pm(\vec{x}, \bar{x}, 1 / 4) \text { with } x-1 / 3 \\
& 12 \mathrm{Co} \text { III } \text { in }(k): \pm(x, 3 x, 0) ; \pm(\bar{x}, x,(0) ; \pm(2 x, x, 0) ; \pm(x, 2 x, 1 / 2) \\
& 4 \mathrm{CO}_{\mathrm{IV}} \text { in (f): } \pm(1 / 3,2 / 3,7): \pm(1 / 3,2 / 3,1 / 2-7) \text { with } 7.0 .11
\end{aligned}
$$

Remarks. - The atomic parameters are those of $\mathrm{Th}_{2} \mathrm{Ni}_{17}$ as determined by Florio et al.

Where no other reference is indicated, data are from $F$. H, Ellinger in The Metal Plutonium, pp. $28 x, 289$. 
PARAMAGNETIC SUSCEPTIBILITIF, OF PLUTONIUM- COBALT COMPOUNDS

From S. T. Konobeevsky et al, in Proc. Sccond Geneva Internat. Conf., vol, 6, p. 195. 1958.

\begin{tabular}{|c|c|c|c|}
\hline \multirow[b]{2}{*}{ Compound } & \multirow[b]{2}{*}{ Structure } & \multicolumn{2}{|c|}{$\begin{array}{l}\text { Paramagnetic Susceptibility } \\
\text { at } 20^{\circ} \mathrm{C}\end{array}$} \\
\hline & & \begin{tabular}{l}
\multicolumn{1}{c}{ Mass } \\
$x_{g} \times 10^{6}$ \\
emu g \\
\end{tabular} & $\begin{array}{l}\text { Atomic } \\
x_{\mathrm{a}} \times 10^{6} \\
\text { emu g-at. }\end{array}$ \\
\hline $\mathrm{Pu} \quad(\boldsymbol{a})$ & Orthorhombic. & 2.4 & 570 \\
\hline $\mathrm{Pu}_{6} \mathrm{Co}$ & be, tetragonal, type $\mathrm{U}_{6} \mathrm{Mn}$ & 2.3 & 490 \\
\hline $\mathrm{Pu}_{3} \mathrm{Co}$ & -- & 2.5 & 480 \\
\hline $\mathrm{Pu}_{2} \mathrm{Co}$ & Hexagonal, type $\mathrm{Fe}_{2} \mathrm{P}$ & 2.4 & 430 \\
\hline $\mathrm{PuCo}_{2}$ & fcc, type $\mathrm{MgCu}_{2}$ & 10.5 & 1250 \\
\hline
\end{tabular}

*The average susceptibility per gram atom is calculated according to the formula $\boldsymbol{X}_{\mathrm{a}}=\boldsymbol{x}_{\mathrm{g}}(\boldsymbol{A}+\boldsymbol{\beta}$ ) where the expression in brackets stands for the mean atomic weight.

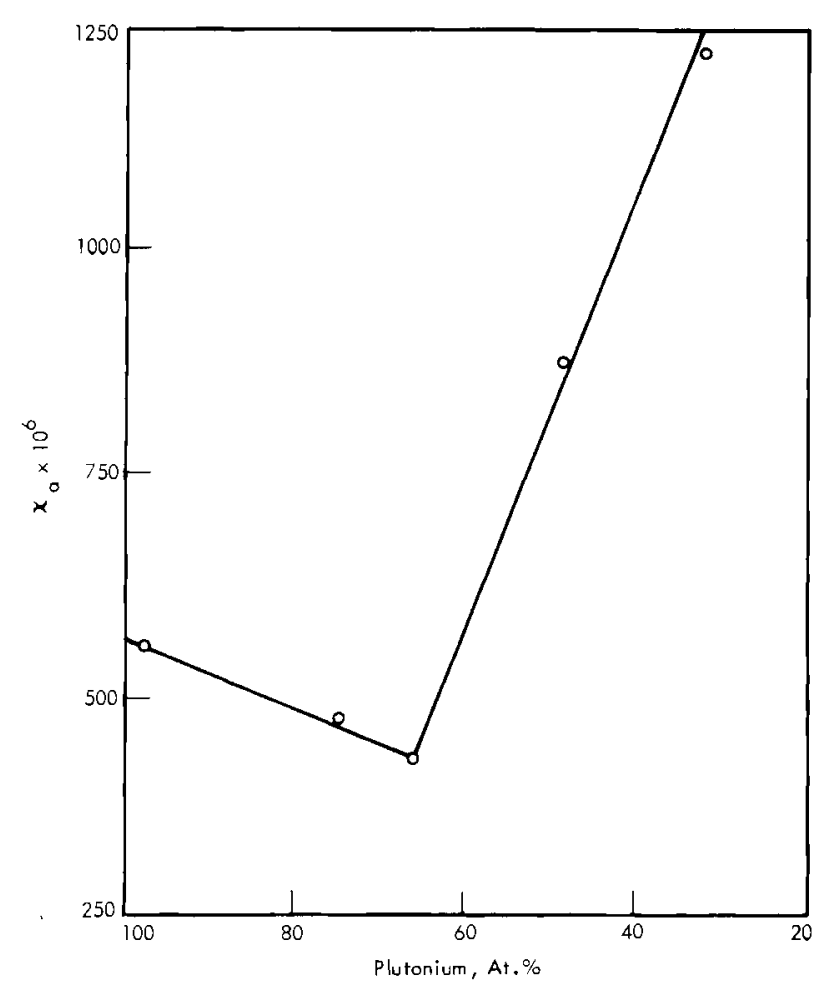

Paramagnetic Susceptibility of Plutonium Alloys with Cobait at Room Temperature. From S. T. Konobeevsky et al. in Proc. Second Geneva Internat. Conf., vol. 6, p. $195,1958$.

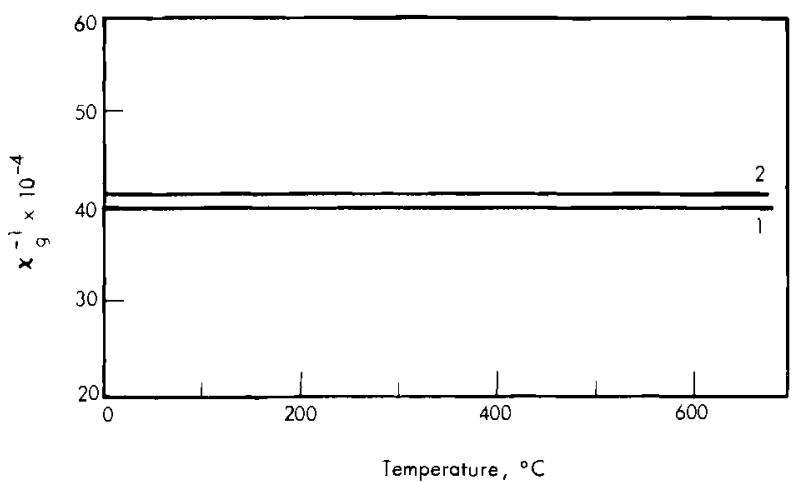

Temperature dependence of paramagnetic susceptibility of Certain PIutonium Compounds; $1, \mathrm{Pu}_{3} \mathrm{CO} ; 2, \mathrm{Pu}_{2} \mathrm{Co}$.

From S. T. Konobeevsky et al, in Proc. Second Geneva Internat. 


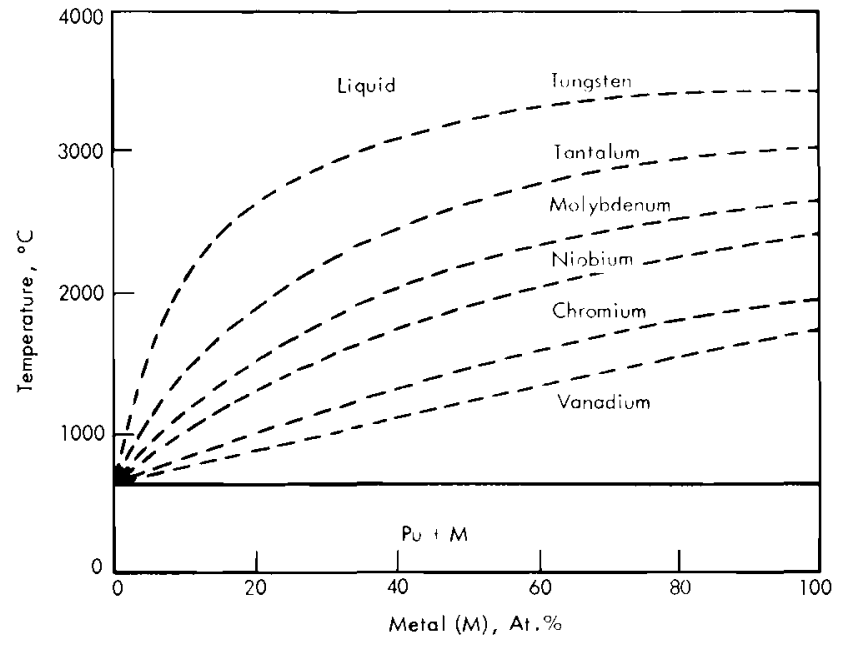

Constitutional Diagram for the Systems Plutonium-Vanadium, Plutonium-Chromium, plutonium-Niobium, Plutonium-Molybrlenum, P]utonium-Tantalum, and plutonium-Tungsten.

Fom A. S. Coffinberry at al. in Reactor Handbook, and ed., vol. 1 1). 273 .

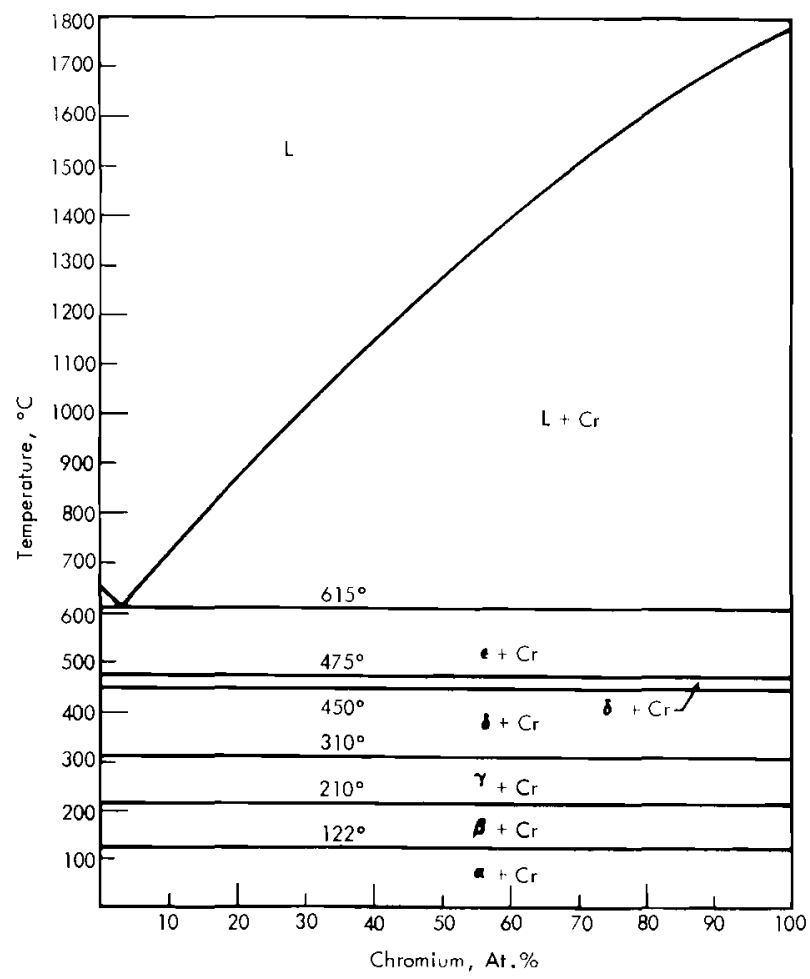

The Plutonium-Chromium System. From F. W. Schonfeld in The Metal Plutonium, p. 260.

\section{Cu 101 prase oiagrans}
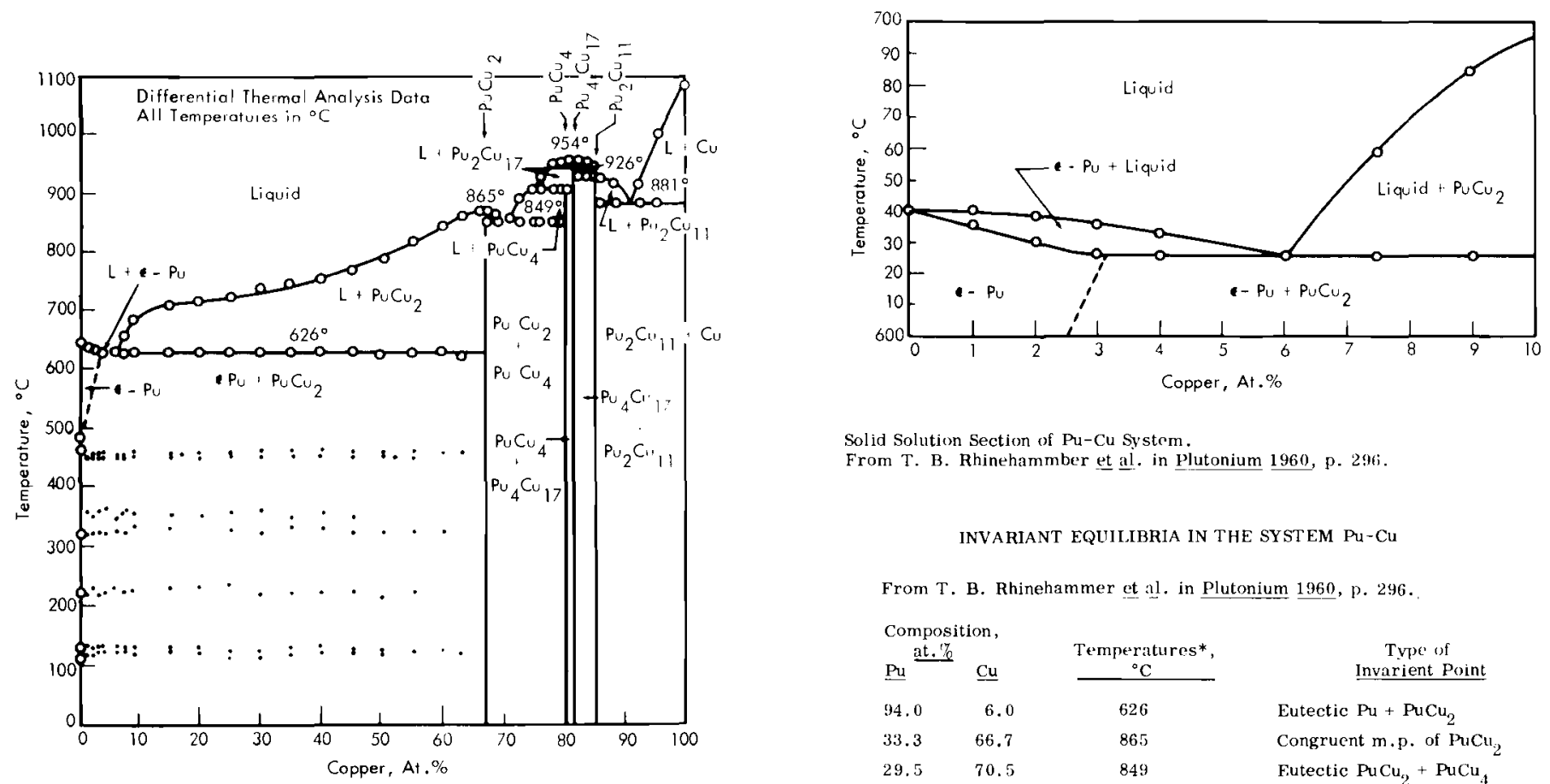

Solid Solution Section of Pu-Cu System.

From T. B. Rhinehammber et $\underline{\text { al. }}$. in p'lutonium 1960, p. 296

INVARIANT EQUILIBRIA IN THE SYSTEM Pu-Cu

From T. B. Rhinehammer et al. in Plutonium 1960, p. 296.

\begin{tabular}{|c|c|c|c|}
\hline \multicolumn{2}{|c|}{$\begin{array}{c}\text { Composition, } \\
\text { at. } \%\end{array}$} & $\begin{array}{l}\text { Temperatures*, } \\
{ }^{\circ} \mathrm{C} \\
\end{array}$ & $\begin{array}{c}\text { Type of } \\
\text { Invarient Point }\end{array}$ \\
\hline 94.0 & 6.0 & 626 & Eutectic $\mathrm{Pu}+\mathrm{PuCu}_{2}$ \\
\hline 33.3 & 66.7 & 865 & Congruent $\mathrm{m}$. p. of $\mathrm{PuCu}_{2}$ \\
\hline 29.5 & 70.5 & 849 & Futectic $\mathrm{PuCu}_{2}+\mathrm{PuCu}_{4}$ \\
\hline 25.0 & 75.0 & 906 & Peritectic point \\
\hline 20.0 & 80.0 & 906 & Incongruent m.p. of $\mathrm{PuCu}_{4}$ \\
\hline 19.2 & 80.8 & 954 & Congruent m.p. of $\mathrm{Pu}_{4} \mathrm{Cu}_{17}$ \\
\hline 15.4 & 84.6 & 926 & Incongruent $\mathrm{m}$. p. of $\mathrm{Pu}_{2} \mathrm{Cu}_{11}$ \\
\hline 14.5 & 85.5 & 926 & Peritectic point \\
\hline 9.0 & 91.0 & 881 & Eutectic $\mathrm{Pu}_{2} \mathrm{Cu}_{11}+\mathrm{Cu}$ \\
\hline
\end{tabular}

Proposed Pu-cu binliny Phase Diagram (by Differential Thermal 
DIFFERENTIAL THERMAL ANALYSIS OF Pu-Cu SYSTEM

From T. B. Rhinehammer et al. in Plutonium 1960, p. 297.

\begin{tabular}{|c|c|c|c|c|c|}
\hline \multicolumn{2}{|c|}{$\begin{array}{l}\text { Cumposition, } \\
\text { at. \% } \\
\end{array}$} & \multirow[b]{2}{*}{ Eutectic } & \multicolumn{2}{|c|}{ Transitions, ${ }^{\circ} \mathrm{C}$} & \multirow[b]{2}{*}{ Solidus } \\
\hline Pu & $\overline{\mathrm{Cu}}$ & & Peritectic & Liquidus & \\
\hline 99.0 & 1.0 & --- & --- & 640 & 636 \\
\hline 98.0 & 2.0 & -- & --- & 639 & 630 \\
\hline 97.0 & 3.0 & --- & $\cdots-$ & 635 & 627 \\
\hline 96,0 & 4.0 & 626 & $\cdots$ & 633 & -- \\
\hline 94.0 & 6.0 & 625 & --- & 626 & --- \\
\hline 92.5 & 7.5 & 626 & $\ldots$ & 660 & -- \\
\hline 91.0 & 9.0 & 625 & -- & 687 & --- \\
\hline 85.0 & 15.0 & 626 & -- & 708 & --- \\
\hline 80.0 & 20.0 & 625 & $\ldots$ & 716 & $-\ldots$ \\
\hline 75.0 & 25.0 & 626 & $-\infty$ & 722 & -- \\
\hline 70.0 & 30.0 & 626 & $\cdots$ & 734 & --- \\
\hline 65.0 & 35.0 & 626 & $-\infty$ & 742 & --- \\
\hline 60.0 & 40.0 & 626 & -- & 756 & --- \\
\hline 55.0 & 45.0 & 627 & $\cdots$ & 769 & --- \\
\hline 50.0 & 50.0 & 624 & --- & 787 & -- \\
\hline 45.0 & 55.0 & 625 & --- & 819 & -- \\
\hline 40.0 & 60.0 & 626 & -- & 841 & --- \\
\hline 37.0 & 63.0 & 620 & --- & 855 & $-\cdots$ \\
\hline 34.0 & 66.0 & $\ldots$ &.- & 865 & --- \\
\hline 33.0 & 67.0 & 847 & -- & 864 & --- \\
\hline 31.5 & 68.5 & 849 & -- & 862 & --- \\
\hline 29.0 & 71.0 & 849 & --- & 854 & -- \\
\hline 27.5 & 72.5 & 849 & $-\cdots$ & 891 & --- \\
\hline 25.0 & 75.0 & 849 & $\cdots$ & 906 & --- \\
\hline 24.0 & 76.0 & 849 & 908 & 926 & -- \\
\hline 22.0 & 78.0 & 854 & 906 & 948 & $-\cdots$ \\
\hline 21.0 & 79.0 & 850 & 907 & 951 & --- \\
\hline 20.0 & 80.0 & 854 & 906 & 952 & --- \\
\hline 19.2 & 80.8 & -- & $\ldots$ & 954 & --- \\
\hline 18.2 & 81.8 & --- & 928 & 954 & --- \\
\hline 16.7 & 83.3 & -- & 926 & 952 & --- \\
\hline 16.0 & 84,0 & $\cdots$ & 928 & 951 & -- \\
\hline 15.39 & 84.61 & --- & 926 & 941 & -- \\
\hline 14.5 & 85.5 & 881 & --- & 926 & --- \\
\hline 12.0 & 88.0 & 881 & $\cdots$ & 916 & -- \\
\hline 8.0 & 92.0 & 880 & -- & 916 & --- \\
\hline 5.0 & 95.0 & 881 & -- & 1002 &.- \\
\hline
\end{tabular}

SOLID PHASE TRANSITIONS

From T. B. Rhinehammer et a1. in Plutonium 1960, p. 298.

\begin{tabular}{|c|c|c|c|c|c|c|c|c|}
\hline \multicolumn{2}{|c|}{$\begin{array}{c}\text { Composition, } \\
\text { at. }\end{array}$} & \multirow[b]{2}{*}{ Other } & \multicolumn{5}{|c|}{ Transitions, ${ }^{\circ} \mathrm{C}$} & \multirow[b]{2}{*}{$1+$} \\
\hline $\mathrm{Pu}$ & $\overline{\mathrm{Cu}}$ & & $a-p$ & $\boldsymbol{\beta}-\boldsymbol{\gamma}$ & $\gamma-8$ & Other & $1-b^{\prime}$ & \\
\hline 99.0 & 1.0 & 118 & 134 & 218 & 317 & 358 & 439 & 453 \\
\hline 98.0 & 2.0 & 121 & 129 & 229 & 321 & 350 & 447 & 454 \\
\hline 97.0 & 3.0 & 124 & 132 & 216 & 319 & 360 & 445 & 457 \\
\hline 96.0 & 4.0 & 124 & 134 & 222 & 321 & 365 & 450 & 457 \\
\hline 94.0 & 6.0 & 124 & 130 & 227 & 322 & 346 & 447 & 453 \\
\hline 92.5 & 7.5 & 122 & 132 & 222 & 319 & 358 & 448 & 452 \\
\hline 91.0 & 9.0 & 122 & 132 & 226 & 336 & 353 & 450 & 457 \\
\hline 85.0 & 15.0 & 120 & 128 & 230 & 327 & 351 & 148 & 457 \\
\hline 80.0 & 20.0 & 122 & 128 & 232 & 325 & 350 & 447 & 454 \\
\hline 75.0 & 25.0 & 110 & 122 & 234 & 324 & 355 & 448 & 457 \\
\hline 70.0 & 30.0 & 116 & 124 & 220 & 322 & 338 & 450 & 458 \\
\hline 65.0 & 35.0 & 122 & 128 & 222 & 331 & 346 & 452 & 462 \\
\hline 60.0 & 40.0 & 122 & 128 & 222 & 329 & 353 & 450 & 462 \\
\hline 55.0 & 45.0 & 118 & 126 & 222 & 324 & 348 & 448 & 459 \\
\hline 50.0 & 50.0 & 116 & 122 & 212 & 324 & -- & 448 & 457 \\
\hline 45.0 & 55.0 & $\cdots$ & 122 & 222 & 326 & --- & 445 & 457 \\
\hline 40.0 & 60.0 & --- & 122 & --- & 324 & --- & -- & 455 \\
\hline 37.0 & 63.0 & $\ldots$ & 122 & --- & -- & -- &.-- & 455 \\
\hline
\end{tabular}




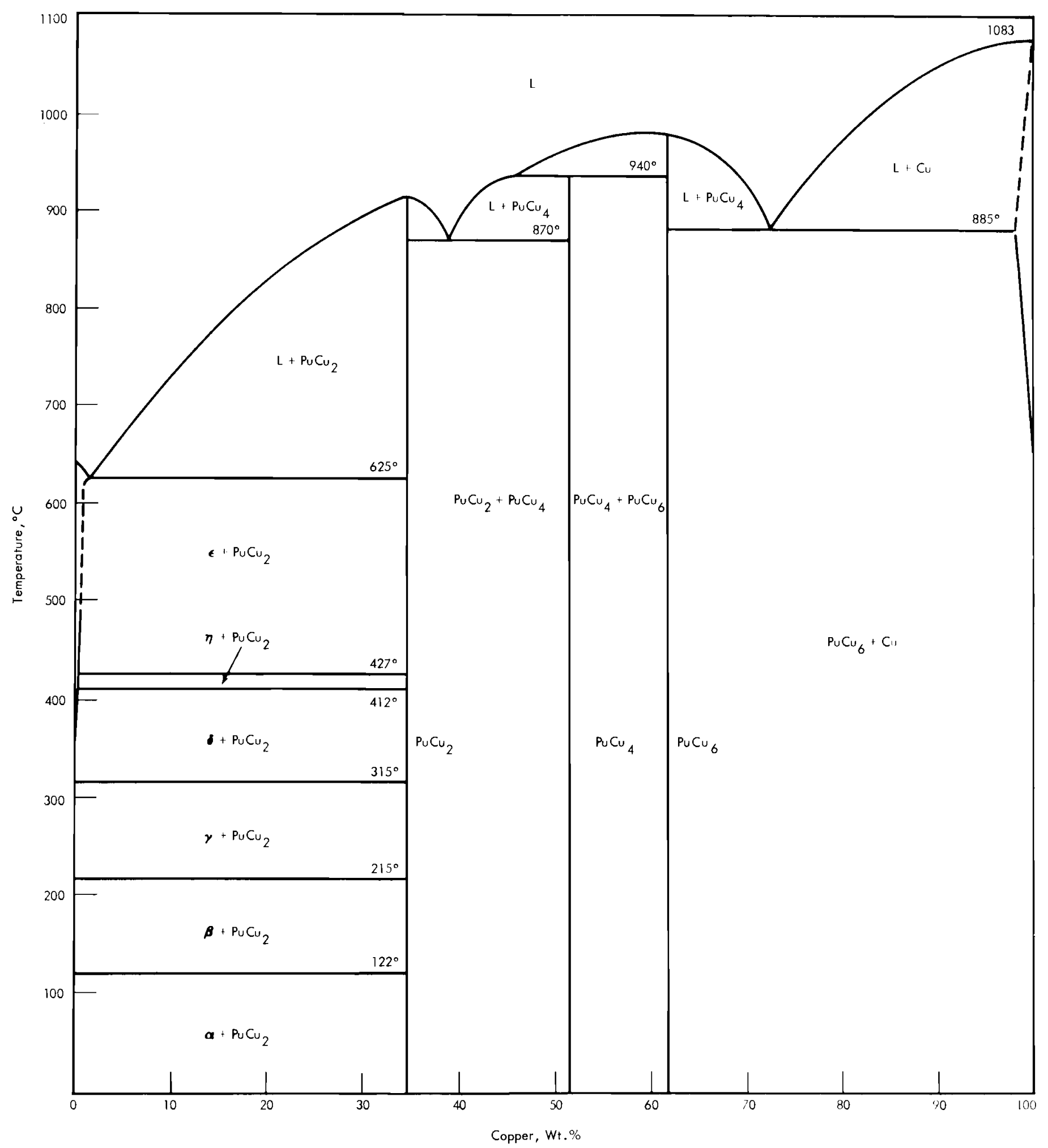

The Plutonium-Copper System. From F. W. Schonfeld in The Metal Plutonium, p. 261. 
Fe 101 Phase Diagrams

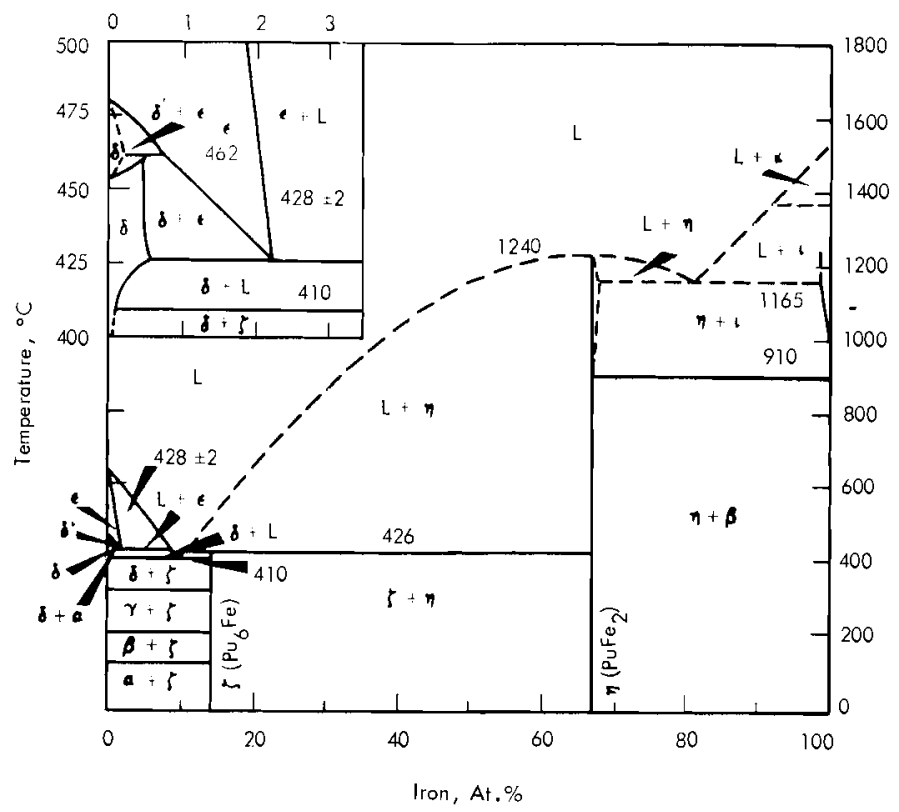

The Plutonium-Iron Srstem

From F, W. Schonfeld in The Metal plutonium, p. 243.

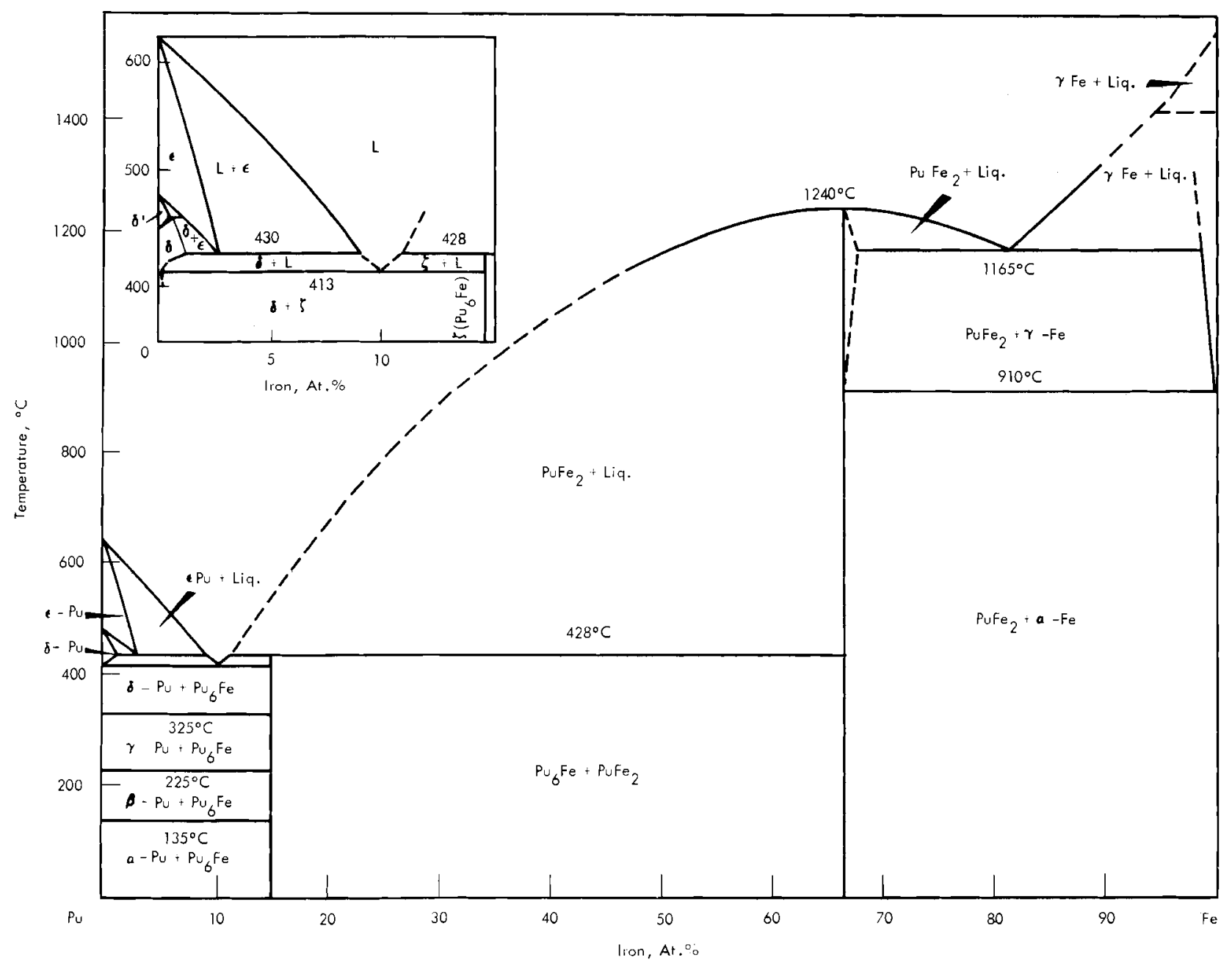


$\mathrm{Pu}_{6} \mathrm{Fe}$

$$
\begin{aligned}
\text { Tetragonal bocdy-centered, } \mathrm{U}_{6} \mathrm{Mn} \text { type } \\
\mathrm{a}=10.404 \pm 0.004 \mathrm{~A} \\
\mathrm{c}=5.355 \pm 0.002 \\
\mathrm{a}=10.40 \pm 0.02 \\
\mathrm{c}=5.345 \pm 0.005 \\
\mathrm{a}=10.4013 \\
\mathrm{c}=5.347 \\
\mathrm{z}=4, \mathrm{D}_{\mathrm{X}}=17.07
\end{aligned}
$$

Space group, - 14/mem (Int. Tab. 140). Atomic positions

$(0,0,0 ; 1 / 2,1 / 2,1 / 2)+$

$4 \mathrm{Fe}$ in $(2): \pm(0,0,1 / 4)$

$8 \mathrm{Pu}_{\mathrm{I}}$ in $(\mathrm{h}): \pm(\mathrm{x}, 1 / 2+\mathrm{x}, 0) ; \pm(1 / 2-\mathrm{x}, \mathrm{x}, 0)$ with $\mathrm{x}=0.407$

16 Pru $_{\text {II }}$ in $(\mathrm{k}): \pm(\mathrm{x}, \mathrm{y}, 0) ; \pm(\mathrm{y}, \overline{\mathrm{x}}, 0) ; \pm(\mathrm{x}, \overline{\mathrm{y}}, 1 / 2) ; \pm(\mathrm{y}, \mathrm{x}, 1 / 2)$

Interatomic distances (based on $\mathrm{a}=10.404, \mathrm{c}=5.355$ )

$$
\begin{aligned}
\mathrm{Pu}_{\mathrm{I}}-1 \mathrm{Pu} & =2.74 \\
-2 \mathrm{Pu}_{\mathrm{II}} & =2.85 \\
-4 \mathrm{Pu}_{\mathrm{I}} & =3.30 \\
-4 \mathrm{Pu} & =3.35 \\
-2 \mathrm{Pu} & =3.41 \\
\mathrm{Pu}_{\mathrm{II}}-1 \mathrm{Pu} & =2.71 \\
-1 \mathrm{Pu} & =2.85 \\
-2 \mathrm{Pu} & =3.14 \\
-2 \mathrm{Pu} & =3.35 \\
-1 \mathrm{Pu}_{\mathrm{I}} & =3.41 \\
-2 \mathrm{Pu} & =3.42 \\
-2 \mathrm{Pu} & =3.49 \\
2 \mathrm{Fe} & =2.81 \\
\mathrm{Fe}-2 \mathrm{Fe} & =2.68 \\
-8 \mathrm{Pu} & =2.81
\end{aligned}
$$$$
\text { Average } \mathrm{Pu}-\mathrm{Pu} \text { distance }=3.23
$$

Isotypes. - $\mathrm{U}_{6} \mathrm{Fe}$.

Rematks. - The alomic parameters given for: $\mathrm{Pu}_{6} \mathrm{Fe}$ are those dedermined by Bakenzigere at al. for I Mn.

Thermal expansion, - The average enetficients of line ar expansion of $P^{2} \mathrm{u}_{6} \mathrm{FC}$ parallel to the axes of the unit cell between room temperature and $400^{\circ} \mathrm{C}$ hilus been found an b

$$
\begin{aligned}
& \text { a. }(73 \pm 2) \times 10^{-6 / 0} / 0 \\
& \bar{c} \cdot(-10 \pm 1) \times 10^{4 i}
\end{aligned}
$$

$\ln _{6}$ Fr- ${ }^{2}$ Fe SOLID SOLUTIONS

$\mathrm{Pu}_{6} \mathrm{Fe}^{2}$ and $\mathrm{C}_{6} \mathrm{Fe}$ were found to be miscible in all proportions. Although it is difficult to prepare single-phase alloys of the ternary comprsition (Pu, U $)_{6} \mathrm{Fe}$, the powder photographs to show essentially a single phase, and there is a uniform change in the unit-eell dimensions between $\mathrm{Pu}_{6} \mathrm{Fe}$ and $\mathrm{U}_{6} \mathrm{Fe}$.

From F. H. Filinger in The Metal plutonium, pp. 292, 293

: $\quad$ prise $_{2}$

Cubis lace-centered, $\mathrm{Cu}_{2}, \mathrm{Mg}$ trpe

$$
\begin{array}{rl}
\text { Mu-rich: } & a=7.191 \pm 0.001 \text { A } \\
& a=7.150 \pm 0.005 \text { (Runnalls) } \\
& a=7.190 \pm 0.005 \text { (Runnalls) } \\
\text { Pu-rich: } a=7.18 \pm 0.01 & a=7.178 \\
\eta=4, D_{\mathrm{X}} & 12.53
\end{array}
$$

Space group. - Fidizm (Int. Tab). 227).

Atomic positions. - See $\mathrm{PuAl}_{2}$.

Interitomic distances (based on $\mathrm{a}=7,191$ )

$$
\begin{aligned}
P u-4 P u & =3.114 \\
-12 F e & =2.9 \times 1 \\
F(P-6 F & =
\end{aligned}
$$

Isotypes. $-\mathrm{CeFc}_{2}, \operatorname{Gr} \mathrm{Fc}_{23}, \mathrm{l}_{2} \mathrm{Fe}_{2}$.

$\mathrm{PuIC}_{2}-\mathrm{LFC}_{2}$, solid Solutions

Pulte, and LiFe, itre mutually soluble in any proportion. The variation it the unit 'ell dimension from $\mathrm{PrFe}_{2}$, to $\mathrm{CFe}_{2}$, is shown in Figure 2. From F. H. Fllinger in The Metal I'lutonium, p. 294.
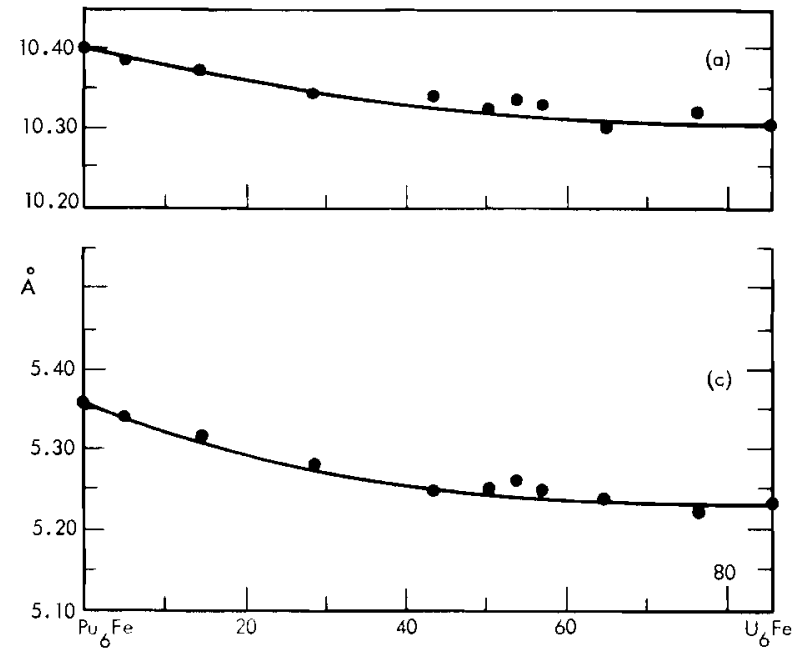

Atomic Percent $U$

Unit-Cell Dimensions Versus Composition of $\mathrm{Pu}_{6} \mathrm{Fc}-\mathrm{L}_{6} \mathrm{FC}$ Solid Solution. From F. 11. Fillinger in The Metal Plutonium, p. $2 \$ 4$.

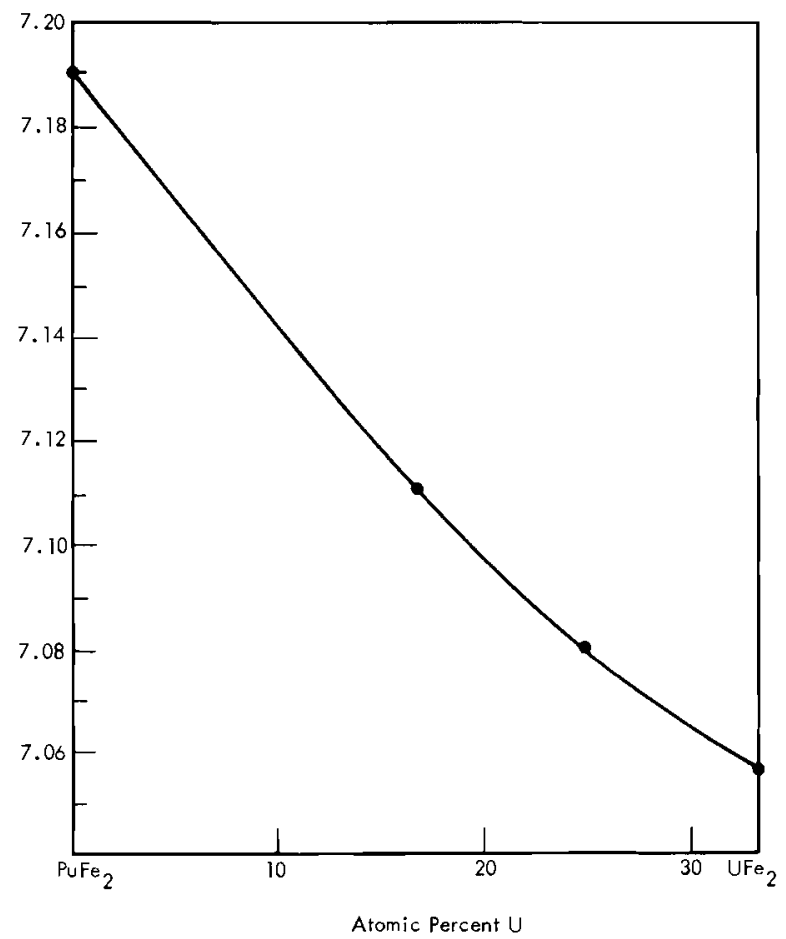

Unit-Cell Dimensions Versus Composition of $\mathrm{PuFe}_{2}-\mathrm{UFe}_{2}$ Solid Solution, From F. H. Ellinger in The Metal Plutonium, p. 294.

$\mathrm{PuFe}_{2}$ is face centered cubic with $\mathrm{a}_{0}=7.164 \mathrm{kx}(7.178 \mathrm{~A})$

It is isostructural with $\mathrm{MgCu}_{2}$.

It has 8 formula weights/unit cell.

Calculated density $=12.59 \mathrm{~g} / \mathrm{cm}^{3}$.

$31.8 \mathrm{wt} . \% \mathrm{Fe}$ $\mathrm{Pu}_{6} \mathrm{Fe}$ is tetragonal with $\mathrm{a}_{0}=10.382 \mathrm{kx}(10.403 \mathrm{~A})$ and $\mathrm{c}=5.336 \mathrm{kx}$ $(5.347 \mathrm{~A})$

It contains 4 formulit weights/unit cell.

Calculated density $=17.1 \mathrm{~g} / \mathrm{cm}^{3}$. 3.75 wt. 
IXIT:C

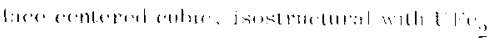

a range of compusitionte

lattice constants:

calculinted density
(it) $7.15,11+11.106534$
L.2. $7.4 \mathrm{~g} / \mathrm{cm}^{3}$
(b) $7.190+11.100,4$
$12.5 .3 \mathrm{~g} / \mathrm{cm}^{3}$

$\mathrm{MgCu}_{1}$, structur', space group $\mathrm{Fd} 3 \mathrm{~m}$

r formula weights/unit cell

From O. J. C. Rumuals, Can. J. Chem., vol. 34, p. 140. (Selected pirts of table VI).

INTERATOMTIC DISTANCFS IN THF COMPOUNDS PUFE

\begin{tabular}{|c|c|c|c|c|}
\hline \multirow[b]{2}{*}{$\mathrm{At} \mathrm{cm}$} & \multirow[b]{2}{*}{ Noishbor } & \multirow[b]{2}{*}{$\begin{array}{l}\text { Number of } \\
\text { Noighbors }\end{array}$} & \multicolumn{2}{|c|}{ Distance A } \\
\hline & & & (a) & (b) \\
\hline Ine & $P$ w & 4 & 3.10 & 3.12 \\
\hline & Fes & 12 & 2.96 & 2.98 \\
\hline $\mathrm{Fe}$ & $\mathrm{Fe}$ & 6 & 2.53 & 2.54 \\
\hline & $\mathrm{Pu}$ & ij & 2.96 & 2.98 \\
\hline
\end{tabular}

From 0. 1. C. Runnals, Can. J. (chem., wol. 34, p. 140. (Selected

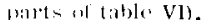

CRYSTA IOCRATIY GF PLUTONIUM-IRON SYSTEM

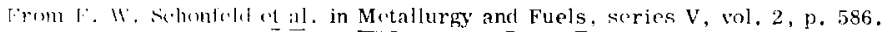

\begin{tabular}{|c|c|c|c|c|c|c|}
\hline Comporind & Турх & Crystal Jattice & $\begin{array}{r}\text { Unit Cell } \\
\text { Dimensions, } \\
\mathbf{A} \\
\end{array}$ & $\begin{array}{c}\text { Number } \\
\text { of Mole- } \\
\text { cules } \\
\end{array}$ & $\begin{array}{l}\text { Space } \\
\text { Group }\end{array}$ & $\begin{array}{l}\text { Calculated } \\
\text { Density } \\
\mathrm{g} / \mathrm{cm}^{3} \\
\end{array}$ \\
\hline $\ln _{6 ;} F$ & $V_{10} \mathrm{Nnn}_{n}$ & $\begin{array}{c}\text { Body-centered } \\
\text { tetragonal }\end{array}$ & $\begin{array}{l}a=10.41 \\
c=5.359\end{array}$ & 4 & $14 / \mathrm{mcm}$ & 17.04 \\
\hline $\mathrm{PuF}_{2}$ & $\mathrm{Cu}_{2} \mathrm{M}_{\mathrm{r}}$ & $\begin{array}{l}\text { Face-centered } \\
\text { cubic }\end{array}$ & 7.19 & 8 & $\mathrm{Fd} 3 \mathrm{~m}$ & 12.53 \\
\hline
\end{tabular}

Fe 201 Density

The density of a Pru-7.93 it .' Fe alloy was determined to be:

$$
\text { - }[16.88-(1.369) \mathrm{T}] \pm 0.01 \div 2 \mathrm{~g} / \mathrm{cm}^{3}
$$

The apparatus and procedure used are deseribed in: 1.A-2358,

"The Density of Liquid Plutonium Metal", C. E. Olsen, T. A. Sandenaw,

and c. C. Herrick, May 1959.

From C. E. Olsen et al. $1 A-2338$.

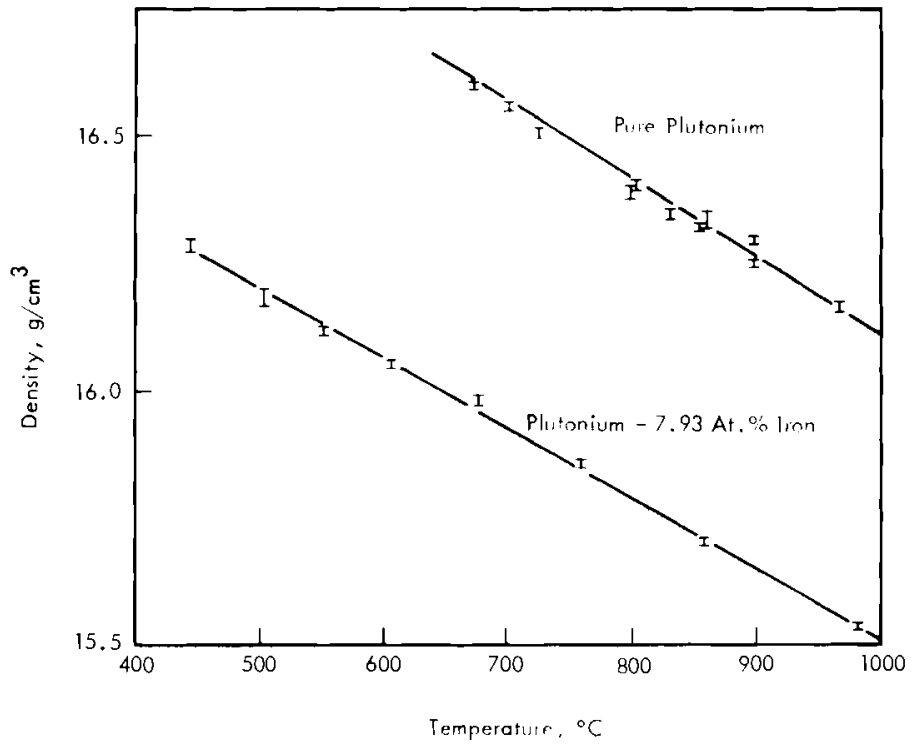

Density v Temperature of I'lutonium-Iron Alloy. from $\mathrm{C}$. $\because$. (lsen et al, LA-2358.

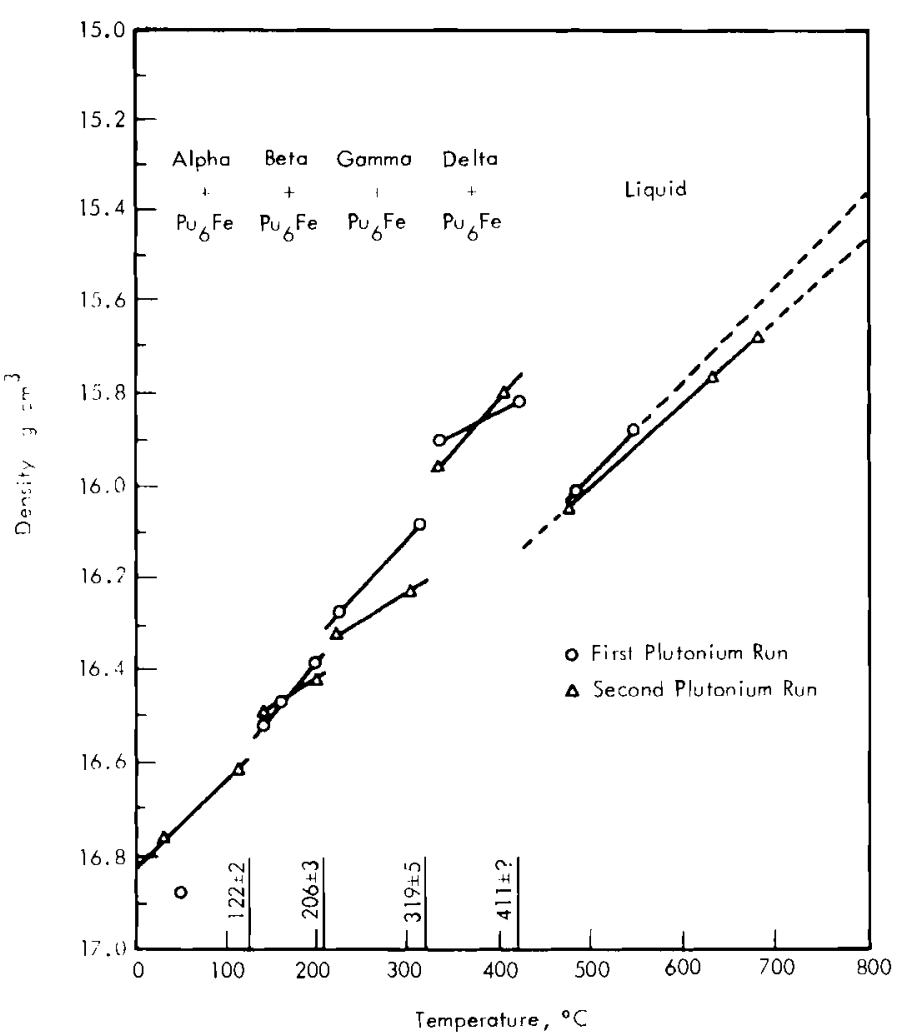

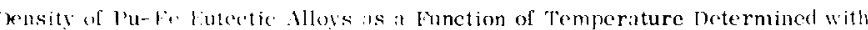
the Nik Volumeter. lirom F. W. Knight in Plutonium 1960, 1. 692. 


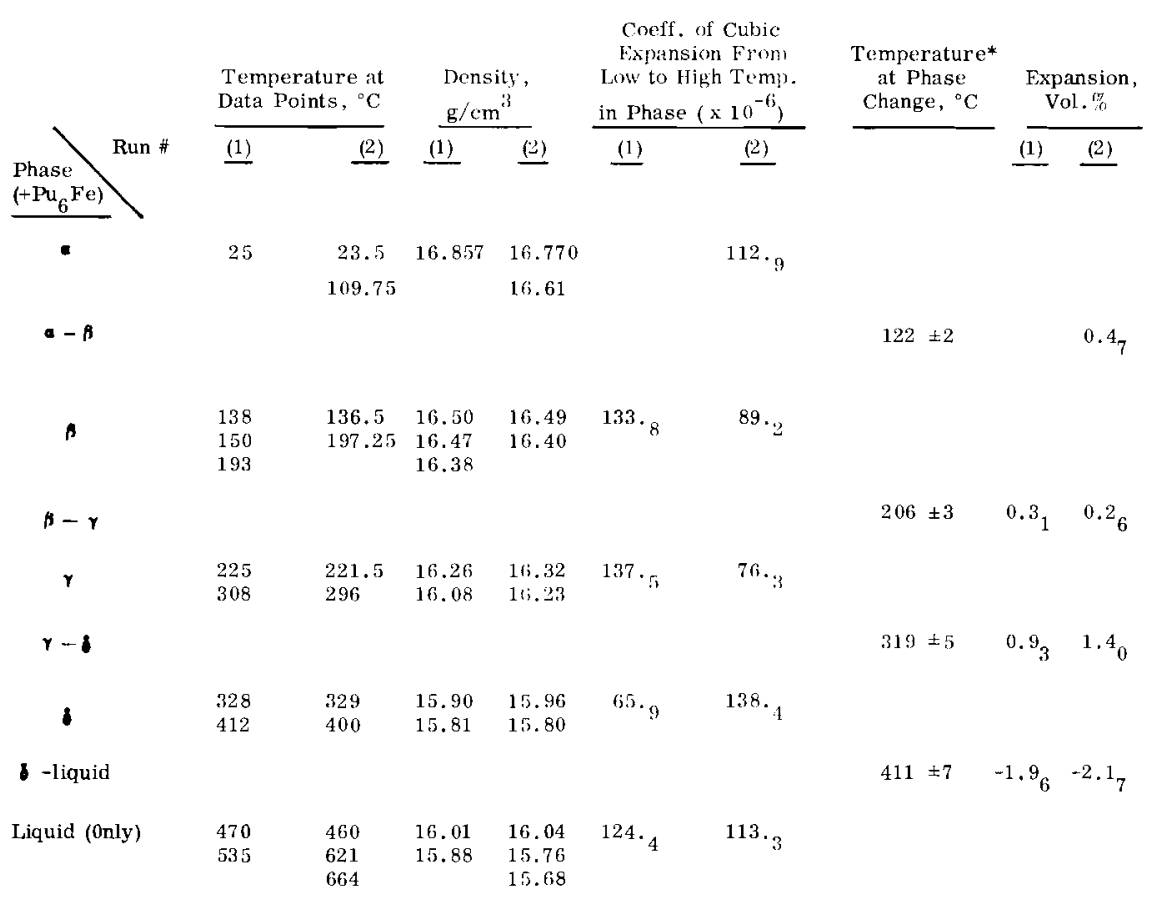

(1) Run \#1 =2/36 wt. \% Fe, (2) Run $\# 2=2.35$ wt. \% Fe. From F. W. Knight in Plutonium 1960, p. 693. * From A. S. Coffinberry and M. B. Waldron in Metallurgy and Fuels, series V, vol. $1, \frac{1}{\text { p. } 372 . ~}$

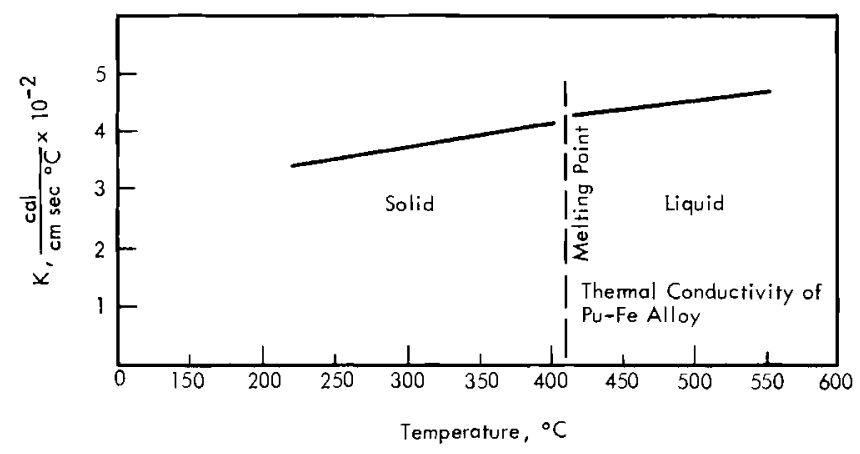

Thermal Conductivity of Pu-Fe Futectic. The Temperature Coefficient of Conductivity for the Solid State is $13 \times 10^{-4} /{ }^{\circ} \mathrm{C}$, for the Liquid State, $7.0 \times 10^{-4} /{ }^{\circ} \mathrm{C}$. From J. E. Deverall, LA-2269. 


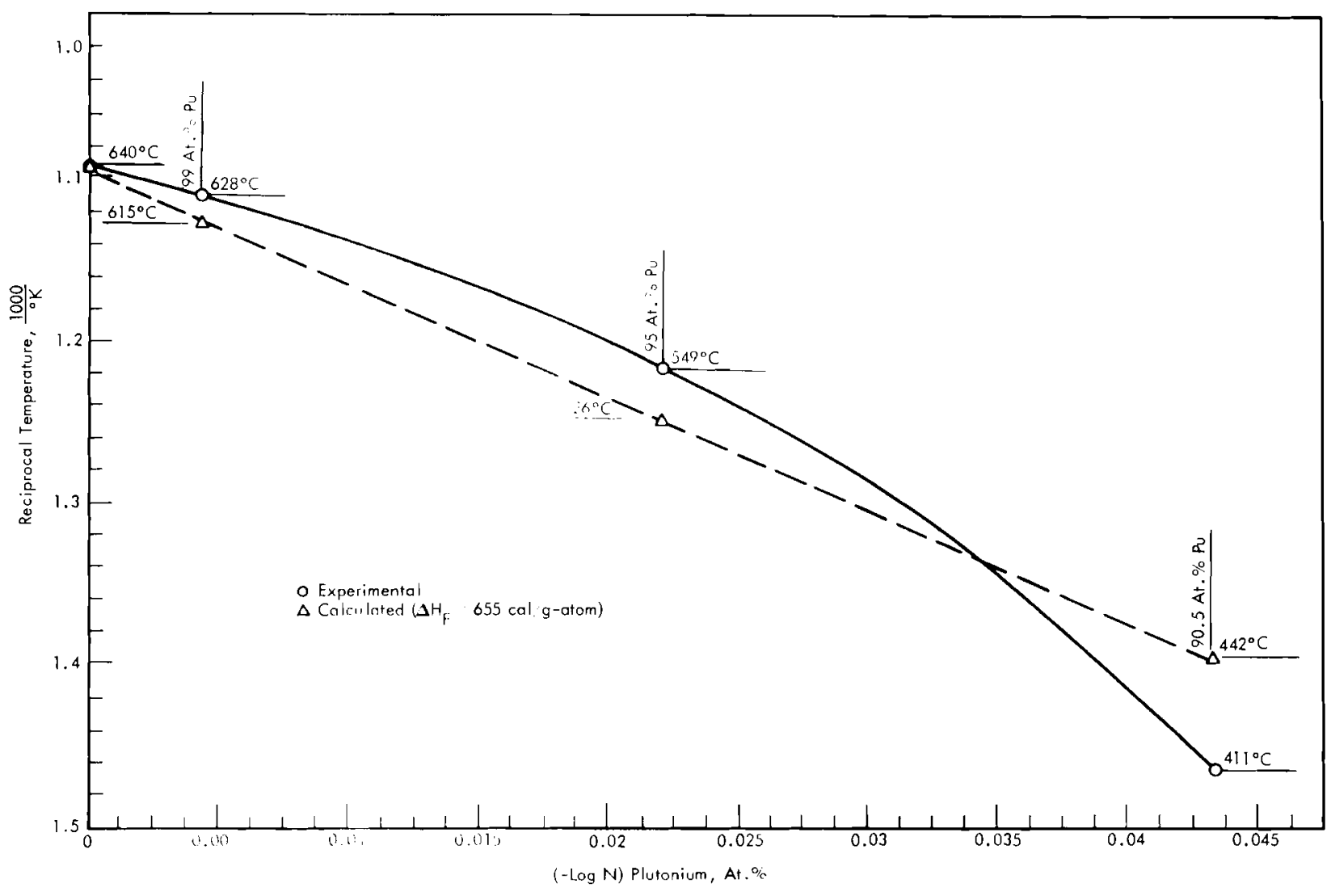

Fe 204.1 Magnetic Susceptibility

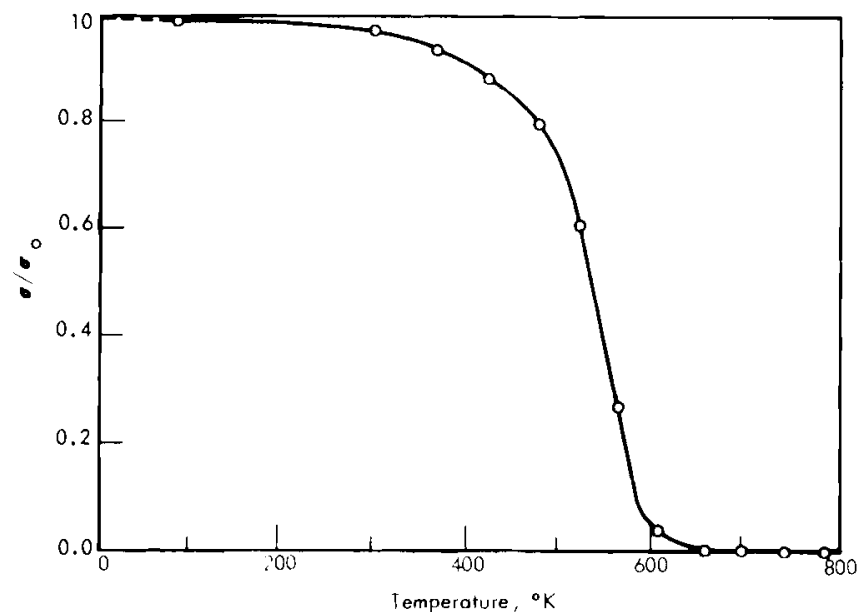

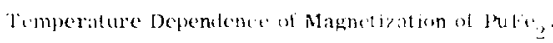

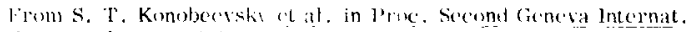

(coml, vol. ti, p. 19t, la,

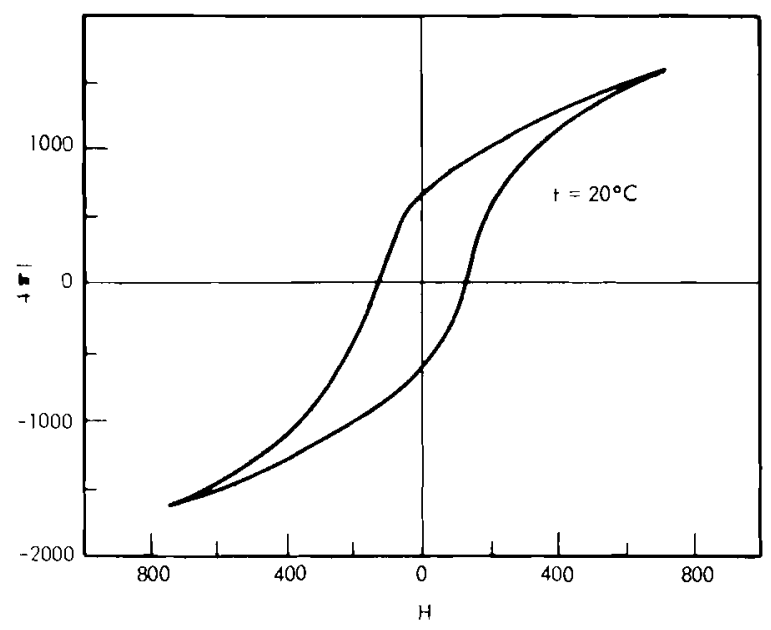

Hysteresis curve tor $\mathrm{PuFe}_{2}$.

Fron $S$, T. Konobeevsky el al, in Jroc. Second Geneva Internat. Conit. vol. F, p. 197. 1958 . 
DEPENDENCE OF VISCOSITY UPON TEMPERATURE FOR PU-Fe ALIOY

From L. J. Wittenberg et al. in Plutonium 1960, p. 682 .

\begin{tabular}{|c|c|c|c|c|c|}
\hline Run No. & Temperature, & $\begin{array}{c}\text { Logarithmic } \\
\text { Decrement, } \\
\delta_{1}-\delta_{e} \\
\end{array}$ & $\begin{array}{l}\text { Period, } \\
\text { sec }\end{array}$ & $\begin{array}{l}\text { Density, } \\
\mathrm{g} / \mathrm{cm}^{3}\end{array}$ & $\begin{array}{l}\text { Viscosity, } \\
\text { cP }\end{array}$ \\
\hline 1 & 707 & 0.084301 & 11.244 & 15.63 & 7.36 \\
\hline 1 & 654 & 0.087085 & 11.254 & 15.72 & 8.84 \\
\hline 1 & 603 & 0.090385 & 11.273 & 15.80 & 10.2 \\
\hline 1 & 553 & 0.093535 & 11.298 & 15.88 & 11,4 \\
\hline 2 & 808 & 0.069602 & 11.304 & 15.37 & 6.14 \\
\hline 2 & 759 & 0.071501 & 11.308 & 15.53 & 6.57 \\
\hline 2 & 706 & 0.074228 & 11.310 & 15.63 & 7.51 \\
\hline 2 & 656 & 0.076533 & 11.320 & 15.72 & 8.71 \\
\hline 2 & 604 & 0.078925 & 11.332 & 15.80 & 9.64 \\
\hline 3 & 597 & 0.052195 & 2.720 & 15.80 & 10.3 \\
\hline 3 & 555 & 0.055281 & 2.724 & 15.88 & 11.9 \\
\hline 3 & 532 & 0.058358 & 2.725 & 15.92 & 13.4 \\
\hline 3 & 511 & 0.059846 & 2.731 & 15.96 & 14.7 \\
\hline 3 & 494 & 0.062538 & 2.739 & 16.00 & 16.5 \\
\hline 3 & 474 & 0.064760 & 2.747 & 16.02 & 18.5 \\
\hline 3 & 451 & 0.067860 & 2.755 & 16.06 & 20.8 \\
\hline 3 & 433 & 0.069834 & 2.757 & 16.10 & 22.4 \\
\hline
\end{tabular}

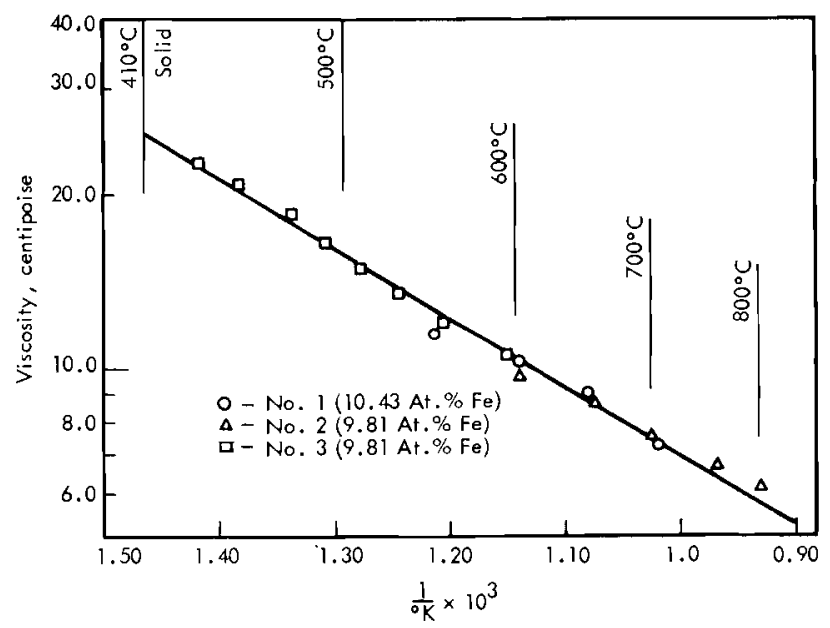

Viscosity of Plutonium Iron Eutectic Alloy as a Function of Absolute Temperature.

From L. J. Wittenberg et al. in Plutonium 1960, p. 683.

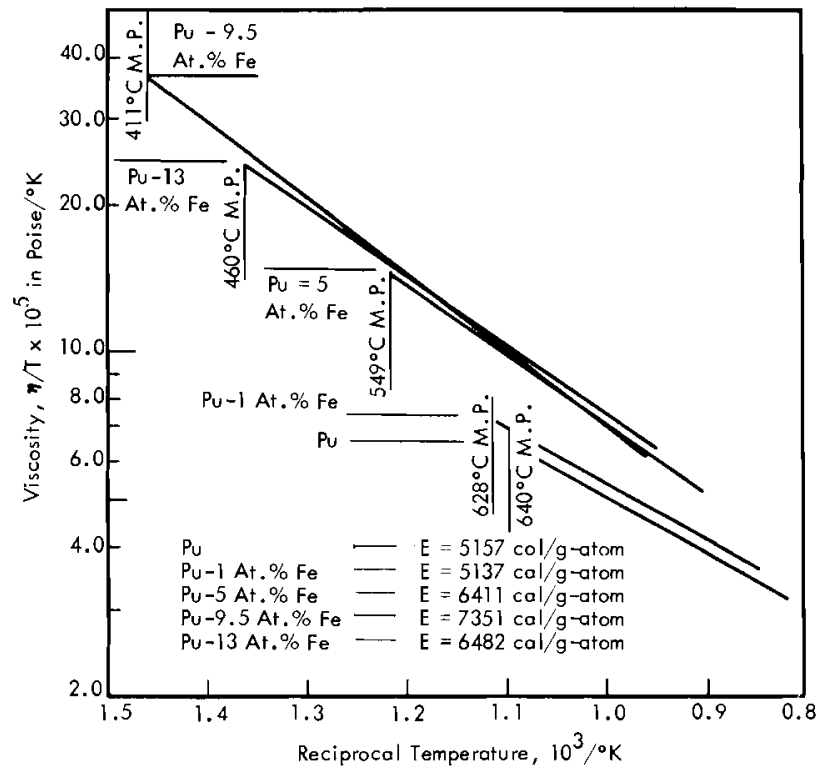

Viscosity of Plutonium-Iron Alloys as a Function of Reciprocal Absolute Temperature.

From L. J. Wittenberg and G. R. Grove, MLM-1184.
From L. J. Wittenberg and G. R. Grove, MLM-1184.

\begin{tabular}{|c|c|c|c|c|}
\hline Composition & $\underline{\mathrm{m}}$ & $\underline{b}$ & $\begin{array}{c}\text { Standard } \\
\text { Deviation, } \\
\text { poise } \\
\end{array}$ & $\begin{array}{c}\text { Activation } \\
\text { Energy. } \\
\text { cal/g-atom }\end{array}$ \\
\hline $\mathbf{P u}$ & 1.1270 & 0.4191 & \pm 0.0035 & 5157 \\
\hline 99 at. $\% \mathrm{Pu}-1$ at. $\% \mathbf{F e}$ & 1.1226 & 0.3898 & \pm 0.0012 & 5137 \\
\hline 95 at. $\% \mathrm{Pu}-5$ at. $\% \mathrm{Fe}$ & 1.4013 & 0.5480 & \pm 0.0013 & 6411 \\
\hline $\begin{array}{l}90.5 \text { at. } \% \mathrm{Pu}-9.5 \text { at. } \% \\
\mathrm{Fe}\end{array}$ & 1.5565 & 0.7096 & \pm 0.0044 & 7351 \\
\hline 87 at. $\% \mathrm{Pu}-13$ at. $\% \mathrm{Fe}$ & 1.4166 & 0.5478 & \pm 0.0032 & 6482 \\
\hline
\end{tabular}




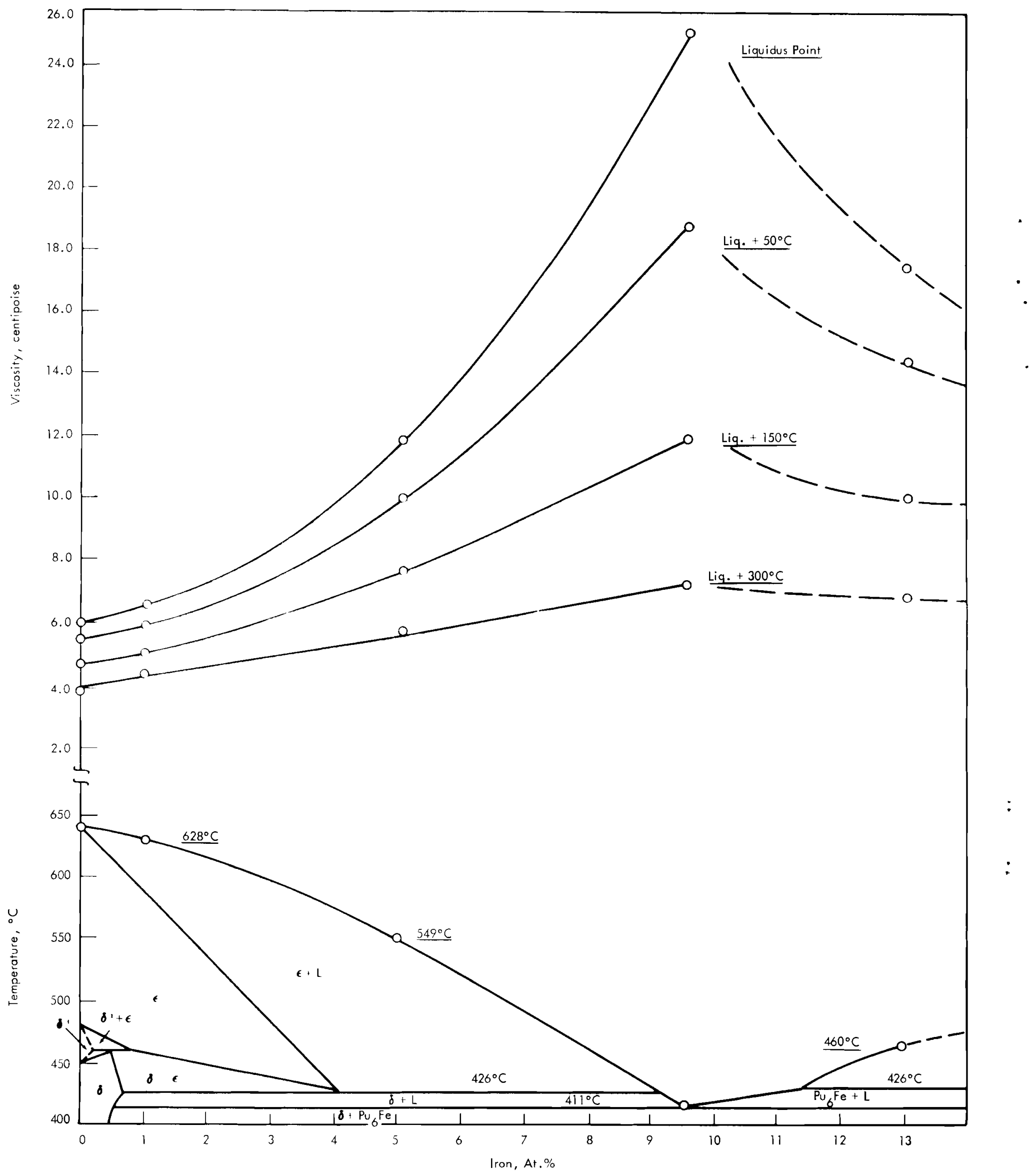

Viscositics of Plutonium-Iron Molten Alloys as a Function of Iron Concentrition and Super-Heating Temperature.

From L. J. Wittenberg and G. R. Grove, MLM-1184. 
TENSILF AND IHARINNESS PROPERTIES OI" PLUTONIUM-IRON A LLOYS*

From II. R. Garincr in HW-66985 (Classified)

\begin{tabular}{|c|c|c|c|c|c|c|c|c|}
\hline $\begin{array}{l}\text { Won, } \\
\text { at. }\end{array}$ & $\begin{array}{l}\text { Ultimate } \\
\text { Strength, } \\
\text { psi } \\
\end{array}$ & $\begin{array}{c}\text { Yield Strength } \\
0.01 \% \text { Off set, } \\
\text { psi } \\
\end{array}$ & $\begin{array}{l}\text { Modulus of } \\
\text { Elasticity, } \\
10^{6} \mathrm{psi} \\
\end{array}$ & $\begin{array}{c}\text { Elonga- } \\
\operatorname{tion} \mathbf{1} \\
\vdots\end{array}$ & $\begin{array}{l}\text { Reduction } \\
\text { In Arca, } \\
\end{array}$ & $\begin{array}{l}\text { Test } \\
\text { Temp., } \\
{ }^{\circ} \mathrm{C} \\
\end{array}$ & $\begin{array}{c}\text { Density, } \\
\mathrm{g}^{3} / \mathrm{cm}^{3} \\
\end{array}$ & $\begin{array}{c}\text { Diamond Py ramid } \\
\text { Hardness, } \\
\text { 10-Kg Load }\end{array}$ \\
\hline 7.0 & 54,500 & 26,700 & 7.1 & 0.23 & 0 & 30 & 17.54 & 218 \\
\hline 7.0 & 29,700 & - & - & - & - & 125 & 17.54 & - \\
\hline 7.0 & 24,500 & 8,720 & 3.8 & 0.59 & 0 & 127 & 17.54 & - \\
\hline 8.7 & 35,900 & 25,700 & 6.7 & 0.044 & 0 & 30 & 17.07 & 202 \\
\hline 8.7 & 34,100 & 10,750 & 5.0 & 0.43 & 0 & 127 & 17.07 & - \\
\hline 8.7 & 14,150 & - & - & $<1.0$ & 0 & 220 & 17.07 & - \\
\hline 8.7 & 4,260 & - & - & 12.5 & 5.9 & 318 & 17.07 & - \\
\hline
\end{tabular}

*All tensile specimens 0.250 -in.diameter. Testing speed: $0.015 \mathrm{in.} / \mathrm{min}$

f Gage length 1 in.

\section{Compressive Properties}

Ultimate compression strength of two specimens of 8.7 at. $\%$ Fe $=$

112,000 and $115,000 \mathrm{psi}$. Total deformation at failure $=6.7 \%$ and

$7.3 \%$.

Hardness

Fe 307

ROOM TEMPERATURF: HARINESS FIGLRFS FOR PLLTONIUM A ILOYS

From D. M. Poole et al. in Plutonium 1960, p. 640.

\begin{tabular}{|c|c|c|}
\hline $\begin{array}{l}\text { Alloy Composition, } \\
\text { at. }\end{array}$ & Heat Treatment & $\begin{array}{l}\text { Vickers Hardness } \\
\text { Number } \\
\text { VHIN } \\
\end{array}$ \\
\hline$F\left(-2.5 P_{4}\right.$ & Are cast & 106 \\
\hline $\mathrm{Fa}-{ }_{3} \mathrm{Pu}$ & Arc cast & 184 \\
\hline $\mathrm{Fe}-7.5 \mathrm{Pu}$ & Are cast & 223 \\
\hline $\mathrm{Fo}-10 P_{1}$ & Are cast & 268 \\
\hline $\mathrm{TC}-12,5 \mathrm{Pu}$ & Arc cast & 286 \\
\hline $\mathrm{Fe}-15 \mathrm{Pu}$ & Arc cast & 318 \\
\hline $\mathrm{F} \bullet-20 \mathrm{Pu}$ & Arc cast & 423 \\
\hline $\mathrm{Fe}-10 \mathrm{Pu}$ & $1000^{\circ} \mathrm{C} 2$ days & 222 \\
\hline $\mathrm{Fe}-15 \mathrm{P}_{1}$ & $1000^{\circ} \mathrm{C} 2$ days & 293 \\
\hline $\mathrm{Ft}-20 \mathrm{Pu}$ & $1000^{\circ} \mathrm{C} 2$ days & 365 \\
\hline
\end{tabular}

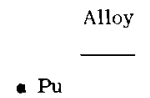

Compound Pu $\mathrm{Fe}(14.3$ at.\%)

Compound PuFe ${ }_{2}$ (166.4 at. \%)

Eutectic $(2.5 \mathrm{wt} . \% \mathrm{Fe})(10$ at. \%)

Eutectic (50 wt.\% Fe) (81.1 at. \%)

From S. T. Konobeevsky, AEC-tr-2435 Pt. 2, p. 211.

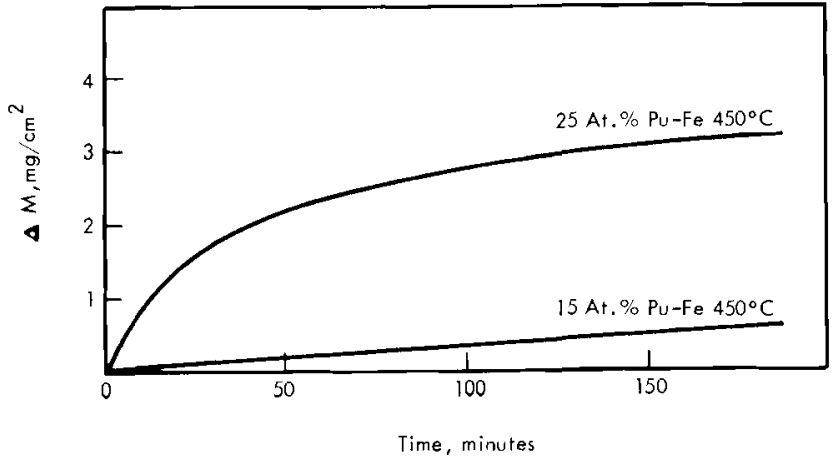




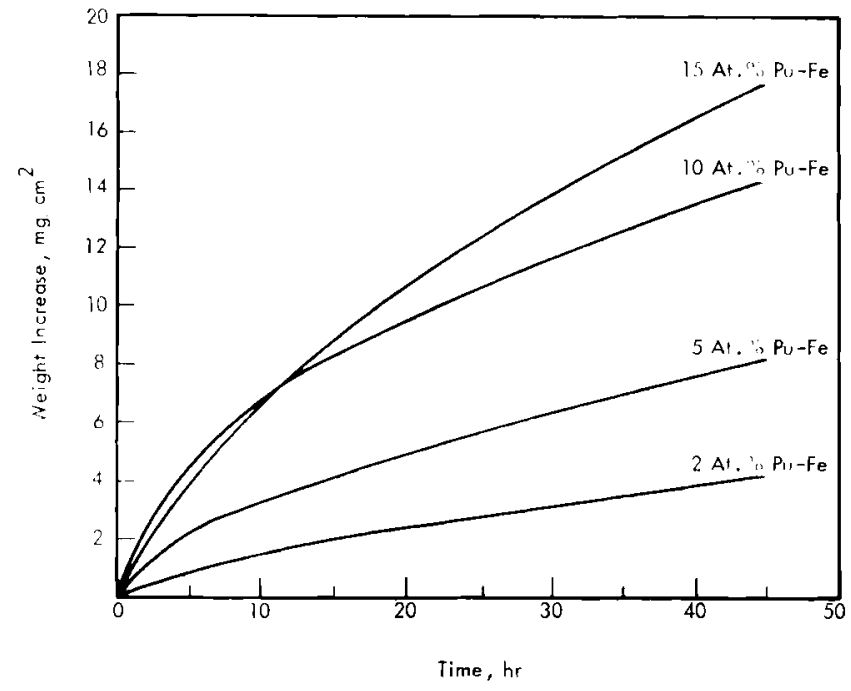

Oxidation of Plutonium-Iron Alloys in Dry $\mathrm{CO}_{2}$ at $600^{\circ} \mathrm{C}$.

From M. J. F. Notlcy et al. , AERE-R-4070.

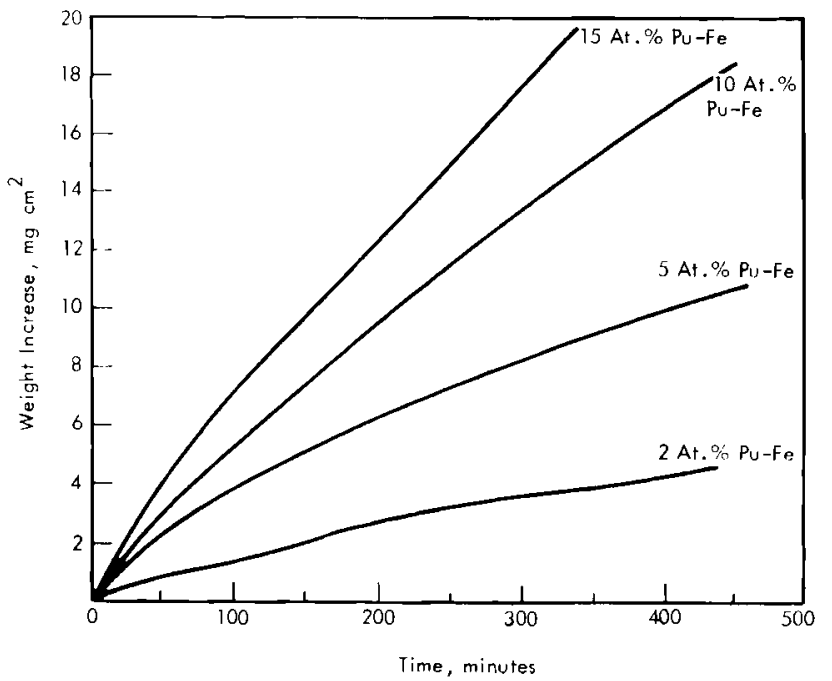

ORidation of l'lutonium-lron Alleys in Dry $\mathrm{CO}_{2}$, at $7000^{\mathrm{n}} \mathrm{C}$.

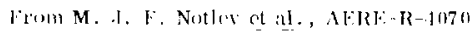

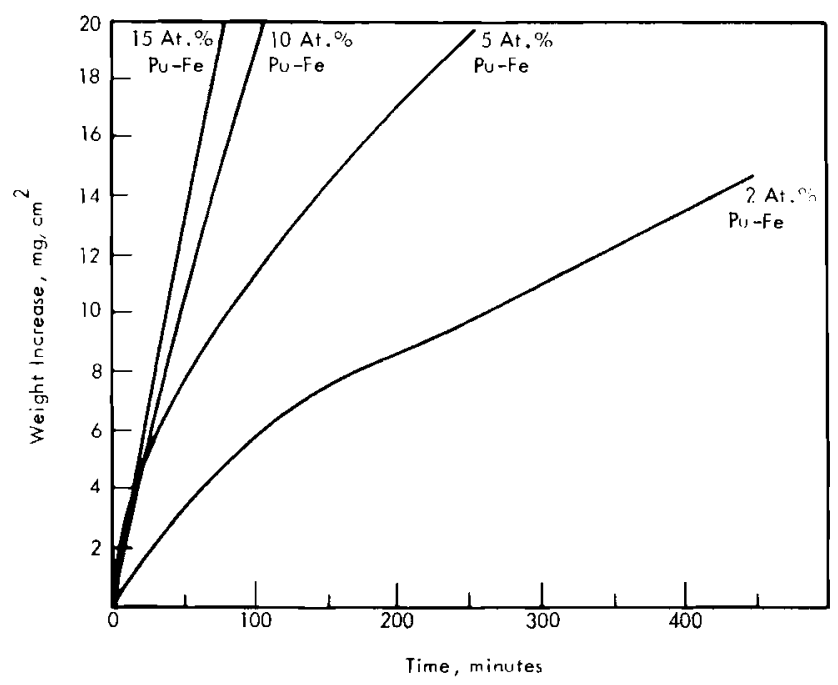

Oxidation of Plutonium-Iron Alloys in Dry CO, at $800^{\circ} \mathrm{C}$

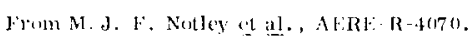

OXIDATION RATFS, PIIYSICAL APPEARANCE AND STRI 'CTLIRE OF OXIDES OBTAINED FROM

PIJ'TONILM-IRON ALI OYYS OXIDIZED IN DRY CO, AT $; \% 00,700$ AND $800^{\circ} \mathrm{C}$

From M. J. F. Notley et al, A ERE-13-4070.

\begin{tabular}{|c|c|}
\hline $\begin{array}{l}\text { Alloy. } \\
\text { itt. " Pu } \\
\text { In Fe }\end{array}$ & $\begin{array}{c}\text { Temperature. } \\
=\mathrm{C} \\
\end{array}$ \\
\hline 2 & 600 \\
\hline 5 & 600 \\
\hline 10 & 600 \\
\hline 15 & 500 \\
\hline 2 & 700 \\
\hline 5 & 700 \\
\hline 10 & 700 \\
\hline 15 & 700 \\
\hline 2 & 800 \\
\hline.$\overline{ }$ & P:10 \\
\hline 10 & suo \\
\hline
\end{tabular}

\begin{tabular}{|c|c|c|c|}
\hline $\begin{array}{l}\text { Lincar Rute, } \\
\mathrm{mg} / \mathrm{can}^{2} \\
/ \mathrm{hr} \\
\end{array}$ & $\begin{array}{c}\text { Constituents } \\
\text { Irentified By X-ray } \\
\text { Analysis } \\
\end{array}$ & $\begin{array}{l}\text { Micrograph } \\
\text { Figure } \\
\end{array}$ & Appearance \\
\hline 0.072 & & 19 & $\begin{array}{l}\text { Dull grey adherent } \\
\text { oxide layer }\end{array}$ \\
\hline $0.12 f i$ & & & $\begin{array}{l}\text { Dull grey adherent } \\
\text { oxide layer }\end{array}$ \\
\hline 0.148 & & & $\begin{array}{l}\text { Dull grey adherent } \\
\text { oxide layer }\end{array}$ \\
\hline 0.220 & & & $\begin{array}{l}\text { Dull grey adherent } \\
\text { oxide layer }\end{array}$ \\
\hline 0.432 & $\mathrm{Pu}_{2} \mathrm{O}_{3}, \mathrm{FeO}$ & 21 & Dull grey oxide. \\
\hline 0.792 & $\mathrm{PuO}_{2}, \mathrm{Pu}_{2} \mathrm{O}_{3}, \mathrm{FeO}$ & 17.20 & $\begin{array}{l}\text { darker than at } 600^{\circ} \mathrm{C} \\
\text { and more easily }\end{array}$ \\
\hline 1. 83315 & 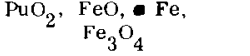 & & detached \\
\hline $3 .(B)$ & $\mathrm{PuO}_{2}, \underset{\underset{F e}{F}, \mathrm{O}_{4}}{\mathrm{FeO}}$ & & $\begin{array}{l}2 / 3 \text { surface bright and } \\
\text { metallic, remainder dark grey }\end{array}$ \\
\hline $1.476 i$ & $\begin{array}{c}\mathrm{l}^{\mathrm{u}} \mathrm{O}_{2}, \\
\mathrm{FeO}_{3} \text {, truee } \\
\mathrm{Fe}_{3} \mathrm{O}_{4}\end{array}$ & & $\begin{array}{l}\text { Bright surface covered } \\
\text { in dark friable oxirle }\end{array}$ \\
\hline $2.71 x$ & - & & \\
\hline 9.3130 & $\mathrm{PuO}_{2}, \mathrm{FeO}$, some a $\mathrm{Fe}$ & & Hard scale. some cracking \\
\hline
\end{tabular}




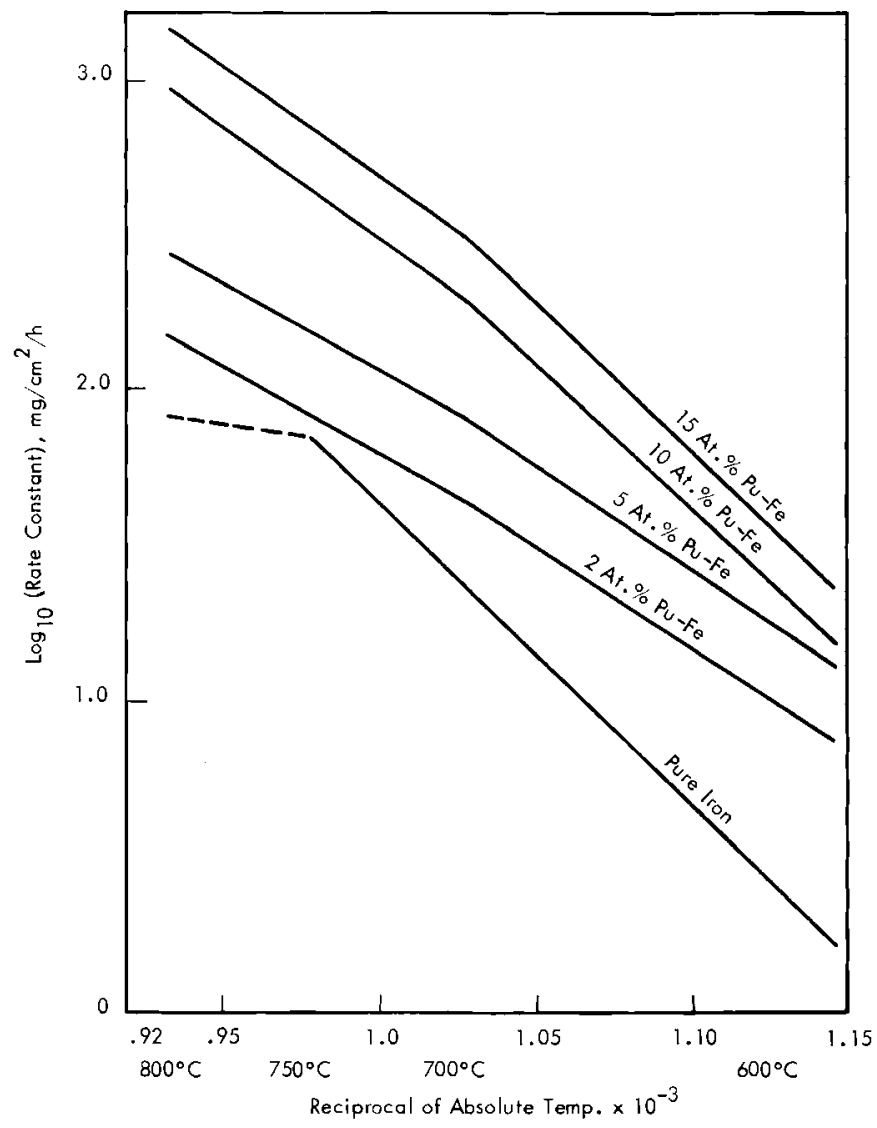

Arrhenius Ptot of Oxidation of Plutonium-Iron Alloys in Dry $\mathrm{CO}_{2}$.

From M. J. F. Notley at it., AERF-R-4070.

\section{Corrosive Properties}

Ta MASS TRANSFER RATES IN CONCENTRATED PU FUELS

From C. C. Burwell et al., Nucl. Sci, Eng., vol. 14, p. 130.

Average Attack Rate. Mils Per Year

$\underline{\text { Fuel Additive }}$\begin{aligned} & No. Temp. Temp. Temp. \\ & \hline\end{aligned}

PuFe (cast iron) None $16 \quad 000011$

PuFc (pure iron) None

PuFe (pure iron) 500 to 2000 ppm C

PuFe (pure iron) 500 to 5000 $\mathrm{Ppm} \mathrm{Si}$

PuFe (pure iron) $5000 \mathrm{ppm} \mathrm{M}$

INTERGRANUA LAR PENETRATION OF Ta VS. THF

ADDITION OF C TO THE PU-Fe F'UEL (200 HR AT $\left.750^{\circ} \mathrm{C}\right)$

From C. C. Burwell et al., Nucl. Sci. Eing., vol. 14, p. 131.

\begin{tabular}{|c|c|c|c|c|}
\hline Fuel & Additive & $\begin{array}{l}\text { No. } \\
\text { Tests } \\
\end{array}$ & $\begin{array}{l}\text { Av. no. of } \\
18 \text { sir bends } \\
\text { to tailure } \\
\end{array}$ & $\begin{array}{l}\text { Results of } \\
\text { radioauto- } \\
\text { graphy } \\
\end{array}$ \\
\hline Pu-Fo' (cast iron) & $\begin{array}{l}\text { PuC to give } \\
1000 \mathrm{ppm} \mathrm{C}\end{array}$ & 1 & $151 / 2$ & Negative \\
\hline$P_{1}-F_{C}($ cast iron) & None & 2 & 1 & - \\
\hline Pu-Fe (pure iron) & None & 3 & 2 & Positive \\
\hline$P_{11}-F_{t}$ (pure iron) & $\begin{array}{l}\text { Graphite rol } \\
\text { to give } 1000 \\
\text { []) C }\end{array}$ & 3 & $131 / 2$ & Nopative \\
\hline$\Gamma_{1}-F_{0}($ pure iron) & $\begin{array}{l}\text { PuC to give } \\
1000 \text { ppm C: }\end{array}$ & 1 & $71 / 2$ & - \\
\hline$P n-l ' c$ (pure iron) & $\begin{array}{l}\text { Premixed } \\
\text { with Puc to } \\
\text { wive } .70 \text { plpm }\end{array}$ & 1 & [3 3 & Nergative: \\
\hline
\end{tabular}

A positive result for mationutography means that l'u penetrated the capsule wall during test. In bend testing a result over 11 indicates little or no intergranular attack of the metal.
Fe 500

INTERGRANULAR PENETRATION OF Ta AT $62: 5^{\circ} \mathrm{C}$ VS. ADDITIONS OF C AND Si TO Pu-Fe FUEL

From C. C. Burwell et al., Nucl. Sci. Eng., vol. 14, p. 132.

\begin{tabular}{|c|c|c|c|c|}
\hline Additive & $\begin{array}{r}\text { No. } \\
\text { Tests } \\
\end{array}$ & $\begin{array}{c}\text { Time, } \\
\mathrm{Hr} .\end{array}$ & $\begin{array}{c}\text { Results Of } \\
\text { Radioautograph } \\
\end{array}$ & $\begin{array}{l}\text { No. } 180^{\circ} \\
\text { Bends To } \\
\text { Failure }\end{array}$ \\
\hline None & 2 & 1026 & Positive & $1 / 2$ \\
\hline PuC $(300 \mathrm{ppm} \mathrm{C})$ & 2 & 1026 & Positive & $1 / 2$ \\
\hline $\begin{array}{l}\text { PuC premixed to give } \\
300 \mathrm{ppm} \mathrm{C}\end{array}$ & 1 & 1026 & Positive & $1 / 2$ \\
\hline 0.7 wt. si added & 1 & 1026 & Positive & $31 / 2$ \\
\hline 0.7 wt. $.5 \mathbf{s}$ added & 1 & 1026 & $\begin{array}{l}\text { Negative (lest } \\
\text { continued) }\end{array}$ & - \\
\hline 0.7 wt. Si prealloyed & 1 & 1026 & $\begin{array}{l}\text { Negative (test } \\
\text { continued) }\end{array}$ & - \\
\hline 0.7 wt. Si added & 1 & 1526 & Posilive & $51 / 2$ \\
\hline $0.7 \mathrm{wt} . \%$ Si prealloyed & 1 & 1526 & Positive & $71 / 2$ \\
\hline
\end{tabular}

INTERGRANULAR PENETRATION OF Ta AT 62: $5^{\circ}$ AND $750^{\circ} \mathrm{C}$ VS. ADDITIONS OF Si AND Al TO PU-Fe EUTECTIC

From C. C. Burwell et al., Nucl. Sci. Eng., vol. 14, p. 132.

\begin{tabular}{|c|c|c|c|c|}
\hline \multirow[b]{2}{*}{ Adifitive } & \multicolumn{2}{|c|}{$1000 \mathrm{hr}$ at $625^{\circ} \mathrm{C}$} & \multicolumn{2}{|c|}{$200 \mathrm{hr}$ at $750^{\circ} \mathrm{C}$} \\
\hline & Radioautograph & $\begin{array}{c}\text { Number } \\
\text { Of } 180^{\circ} \\
\text { Bends To } \\
\text { Failure }\end{array}$ & Radioautograph & $\begin{array}{c}\text { Number } \\
\text { Of } 180^{\circ} \\
\text { Bends To } \\
\text { Failure }\end{array}$ \\
\hline $0.4 \mathrm{wt} . \mathrm{Si}$ & Positive & 10 & Positive & - \\
\hline $0.7 \mathrm{wt} . \mathrm{Si}$ & Negative & $14 \mathrm{l} / 2$ & Negative & $131 / 2$ \\
\hline $1.5 \mathrm{wt} . \mathrm{Si}$ & Positive & 3 & Positive & 11 \\
\hline $\begin{array}{r}0.7 \mathrm{wt}, \mathrm{Si} \\
0.7 \mathrm{wt}\end{array}$ & Negative & 12 & Negative & 5 \\
\hline $0.7 w \mathrm{t} . \% \mathrm{~A} 1$ & Negative & $111 / 2$ & Negative & - \\
\hline $0.3 \mathrm{wt} .: \mathrm{Al}$ & Negative & $111 / 2$ & Positive & 12 \\
\hline
\end{tabular}


IN TERGRANULAR PENETRATHON OF THE CAPSULE GAS PHASE vs. ADDITIONS OF Ca OR Mg TO THE Pu-Fe FUEL $\left(200 \mathrm{hr}^{\circ}\right.$ AT $\left.750^{\circ} \mathrm{C}\right)$

From C. C. Burwell et al., Nuei. Sci. Eng., vol. 14, 1. 1.32.

\begin{tabular}{|c|c|c|c|}
\hline Fuel System & Composition & $\begin{array}{c}\text { No. Of } \\
180^{\circ} \\
\text { Bend To } \\
\text { Failure } \\
\text { In The } \\
\text { Gas Zone }\end{array}$ & $\begin{array}{l}\text { Results of } \\
\text { Radioautograph }\end{array}$ \\
\hline $\begin{array}{l}\text { Pu-Fe prealloyed } \\
\text { with } \mathrm{Mg} \text { and PuC }\end{array}$ & $\begin{array}{l}700 \mathrm{ppm} \mathrm{C}, 350 \\
\text { ppm Mp }\end{array}$ & 1 & Positive \\
\hline $\begin{array}{l}\text { Pu-Fe plus a layer } \\
\text { of } \mathrm{Na}-\mathrm{Ca}\end{array}$ & $\begin{array}{l}3 \text { at. \% Ca in } \\
\text { No layer }\end{array}$ & $1 / 2$ & Positive \\
\hline$M_{n}$ without $P_{1}-F_{c}$ & & 10 & Not applicable \\
\hline $\begin{array}{l}\text { Pu-Fe plus a Ca- } \\
\text { Mg layer }\end{array}$ & $\begin{array}{l}73 \mathrm{at} . \% \mathrm{Cat} \\
\text { eutectic }\end{array}$ & $1 / 2$ & Pesilive \\
\hline
\end{tabular}

CUMU LATIVE PERCENTAGE OF CAPSULES OF Ta + $0.1 \%$ W LEAKING AS A FUNCTION OF THE PRESENCE OR ABSENCE OF ADDED CALCIUM AND MAGNESIUM IN THE FUF

(The capsules had been annealed in a vacuum at $1300^{\circ} \mathrm{C}$ for $1 \mathrm{~h}$. The basic luel was Pu-Fe alloy of high purity.

The limits of detection were $10 \mathrm{ppm}$ for Ca and $5 \mathrm{ppm}$ for Mg.)

FWin 13. J. Thamer in Corrosion of Reactor Materials, vol. II, p. 2!!9.

Additive Treatmont

\begin{tabular}{|c|c|c|c|c|c|}
\hline \multirow{2}{*}{ Adderl: } & & \multirow{2}{*}{$\frac{700 \mathrm{~h}}{67 \%}$} & \multirow{2}{*}{$\frac{1400 \mathrm{~h}}{100}$} & $2100 \mathrm{~h}$ & $2800 \mathrm{~h}$ \\
\hline & $\begin{array}{c}15-160 \mathrm{pm} \mathrm{Mg} \\
<10-150 \text { [1]m Ca }\end{array}$ & & & Discor & 1-n \\
\hline \multicolumn{6}{|c|}{$\begin{aligned} \text { Sime, then remova } 7 \\
\\
<5 \mathrm{ppm} \mathrm{M}_{\mathrm{H}} \\
<10 \mathrm{ppm} \mathrm{Ci}\end{aligned}$} \\
\hline Basic $f$ & $\begin{array}{r}20-30 \mathrm{pmm} \mathrm{Mg} \\
<10 \mathrm{ppm} \mathrm{Ca}\end{array}$ & 0 & 0 & 0 & 33 \\
\hline
\end{tabular}

\section{TANTALUM MASS TRANSFER RATES OF FUELS}

CONSIDERED FOR USE IN IAAMPRE

From C. C. Burwell et gl., Trans. Am. Nucl. Soc., vol, 4, p. $34 \mathrm{i}$.

\begin{tabular}{|c|c|c|c|c|}
\hline $\begin{array}{r}\text { Eutectic } \\
\text { Fucls } \\
\end{array}$ & $\begin{array}{r}\text { No. } \\
\text { Tests } \\
\end{array}$ & 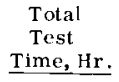 & $\begin{array}{c}\text { Ave. Mass } \\
\text { Transfer } \\
\text { Rate, Mills/yr } \\
\end{array}$ & $\begin{array}{l}\text { Temp., } \\
{ }^{\circ} \mathrm{C} \\
\end{array}$ \\
\hline $\begin{array}{l}\text { Pu-Fe (made with } \\
\text { cast iron) }\end{array}$ & (3) & 6722 & 0.5 & 7000 \\
\hline $\begin{array}{l}\text { Pu-Fe (made with } \\
\text { cast iron) }\end{array}$ & 11 & 2832 & 13 & 800 \\
\hline $\begin{array}{l}\text { Pu-Fe (made with } \\
\text { pure iron) }\end{array}$ & 8 & 1901 & 24 & 700 \\
\hline
\end{tabular}

TANTALUM MASS TRANSFER RATES OF

Pu-Fe FUTECTIC FUELS WITH ADDITIONS

OF C, Mn, AND Si

From C. C. Burvell et al., Trans. Am. Nucl. Soc., vol. 4, p. 347.

\begin{tabular}{|c|c|c|c|c|}
\hline Fuel & $\begin{array}{l}\text { No. } \\
\text { Tests }\end{array}$ & $\begin{array}{c}\text { Total } \\
\text { Test } \\
\text { Time, } \mathrm{Hr} .\end{array}$ & $\begin{array}{c}\text { Ave. Mass } \\
\text { Transfer } \\
\text { Ratc, Mills/yr }\end{array}$ & $\begin{array}{c}\text { Temp. } \\
{ }^{\circ} \mathrm{C}\end{array}$ \\
\hline $\mathrm{Pu}-\mathrm{Fe}$ (cast iron) & $\begin{array}{l}5 \\
4\end{array}$ & $\begin{array}{l}1142 \\
1230\end{array}$ & $\begin{array}{l}0 \\
0.4\end{array}$ & $\begin{array}{l}700 \\
750\end{array}$ \\
\hline $\mathrm{Pu}-\mathrm{Fe}$ (pure iron) & $\begin{array}{l}8 \\
3\end{array}$ & $\begin{array}{r}1901 \\
601\end{array}$ & $\begin{array}{r}24 \\
>31\end{array}$ & $\begin{array}{l}700 \\
750\end{array}$ \\
\hline $\begin{array}{l}\text { Pu-Fe (pure iron) } \\
+500-2000 \text { ppm C }\end{array}$ & $\begin{array}{l}5 \\
5\end{array}$ & $\begin{array}{l}1137 \\
1227\end{array}$ & $\begin{array}{l}0 \\
0\end{array}$ & $\begin{array}{l}700 \\
750\end{array}$ \\
\hline $\begin{array}{l}\text { Pu-Fe (pure iron) } \\
+500-5000 \mathrm{ppm} \mathrm{Si}\end{array}$ & $\begin{array}{l}3 \\
2\end{array}$ & $\begin{array}{l}648 \\
500\end{array}$ & $\begin{array}{r}3 \\
14\end{array}$ & $\begin{array}{l}700 \\
750\end{array}$ \\
\hline Pu-Fe (pure iron) & 1 & 149 & 11 & 700 \\
\hline
\end{tabular}

Control of intergranular attack of tantalum by modifications to the Pu-Fe eutectic fuel is promising but still in the experimental stage. The major effects of fuel modifications observed so far are:

1. The addition of $500 \mathrm{ppm}$ carbon to the Pu-Fe fuel reduces intergranular attack of tantalum at $750^{\circ} \mathrm{C}$ but is ineffective or even detrimental at $6255^{\circ} \mathrm{C}$. 2. The addition of 0.4-1.5 wt.\% silicon to the Pu-Fe fuel eliminated Intergranular attack of tantilum in 200 hour tests at $750^{\circ} \mathrm{C}$. At $625^{\circ} \mathrm{C}$ the addition of $0.7 \mathrm{wt} . \%$ silieon to the fuel reduced the rate of intergranular

attack (by approximately a factor of 2 ), but did not eliminate it.
3 . The addition of $0.3-0.7$ t. aluminum to the fuel appears to reduce the 3. The addition of $0.3-0.7$ ut. alu

4. The addition of $500 \mathrm{ppm}$ oxygen to the fuel does not significantly affect

4. The addition of $500 \mathrm{ppm}$ oxygen to the fuel does not

the fuel properties with respect to intergranular attack.
5 . Small amounts $(<10 \mathrm{ppm})$ of calcium and magnesium in the fuel are 5. Small amounts $(<10 \mathrm{ppm})$ of calcium and magnesium in the fuel are detrimental in that they facilitate fuel-capsule wetting and thus aid exposure
of the tantalum above the fuel level to plutonium. From C. C. Burwell et al. of the tantalum above the fuel level to plutor
Trang. An. Nucl. Soc., vol. 4 , p. 348 .
(OMPARATIVI: RATES OF MASS TRANSFER OF TANT ALUM FOR VARIOUS FLELS ANI) FIEL ADDITIVES

$$
\text { Inits: } \mathrm{mil} / \mathrm{yr} \text {. Sensitivity: } 0.2 \mathrm{mil} / \mathrm{yr} \text { * }
$$

From 13. J. Thamer in Currosion of Reactor Materials, vol. II, p. 298.

\begin{tabular}{|c|c|c|}
\hline Fuel & Hot Zone at $700^{\circ} \mathrm{C}$ & Hot Zone at $750^{\circ} \mathrm{C}$ \\
\hline$P_{1}-F^{2}$ (pure iron) & 24. & $>31$ \\
\hline mal- Fe (wast iron) & 0.3 & 0.4 \\
\hline $\begin{array}{l}{\left[Y_{1}-F(\text { o (pure iron) }\right.} \\
-3(111-2000 \text { ppm C }\end{array}$ & 0. & 0. \\
\hline 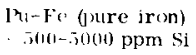 & 3. & 14. \\
\hline 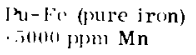 & 11. & --- \\
\hline
\end{tabular}

\section{EFFECTS OF TANTALUM STRUCTURE ON CORROSION}

Excellent corrosion resistance was observed in some early "pure" tantalum capsules from Metallwerk Plansee A.G., in which the fine, heavily worked structure had the form of platelets oriented parallel to the surfaces (Fig. 10), ind is charncteristic of ironed material. It is postulated that this structure presented a minimum of grain boundary area to the fuel and created a tortuous path of penetration through the wall (Fig. 11). In deep-drawn tantalum capsules the platelets were oriented radially, and the rapid penetration by plutonium was ascribed to the direct paths existing through the material (Fig. 12). The ratio between penctrations in the radial and Iongritudinal directions, respectively, was actually observed to be proportional to the number of grain boundaries of those orientations originally present in the tantalum. These conclusions led to the early specification of impact extrusiun and ironing instead of decp thrawing.

Iromert ennsules, luwever, still exhibited a point of weakness at the juncture betwen the worked structure and the heat-affected zone associated with the werli at the cas. Fuel alloys shewed a tendency to penetrate at this point, the'n to creep dounward through the worked strueture. The advaneing plutonium caused recrystallization of the tantalum, presumably through dissolution of strained crystals and precipitation of unstrained tantalum. This recrystallization in the presence of fuel is complete within a few hours at $70{ }^{\circ} \mathrm{C}$, which is hundreds of times faste than recrystallization of intact metal at the same temperature. Figure 13 shows a specinen penetrated by fuel alloy and reciystallized, while a small area still unaffected shows the unaltereat heavily ironed tantalum structure.

There is evidence that penetration at the heat-affected zone may result either from internal energy differences between the general worked structure and the annealed structure near the weld, or from diffusion of copper from the ebill block in which the capsule is clamped during welding. Diffusion of some such deleterious substance as thorium from the welding electrode into some such deleterious substance as thorium from the welding electrode into the heat-affected zone has also been suggested. The results of independent studies of the effects of carbon and nitrogen, and the practice of purging and thorough blanketing with helium and argon of selected purities before welding, seem to have eliminated the possibility of interstitial elements as primary causes of this attack.

The hypothesis of an internal energy difference as the cause of attack at the heit-affected rone is strengthened by the fact that capsules annealed before loading and welding have better corrosion resistance than those with any worked structure. Treatment at the minimum annealing conditions of one hour at $1050^{\circ} \mathrm{C}$ doubled the time required for plutonium penetration.

Annealing at higher temperatures leads naturally to the growth of larger and more symmetrical crystals. It would seem reasonable that this further grouth past a visually complete anneal should involve minor changes in internal enero, probably having no effect on corrosion. The experimental fact is, however, that at least up to $1450^{\circ} \mathrm{C}$, higher annealing temperatures fact is, hover, that at least up to $1450^{\circ} \mathrm{C}$, higher annealing temperatures result in progressively improved corrosion resistance, as shown in Table Nucl. Sci. Eng., vol, 14, pp. 115-188.

\section{PERCENT OF Ta-0.1\% W TEST CAPSULES FAILING AS A FUNCTION OF ANNEALING TEMPERATURE AND} EXPOSURE TIME AT $625^{\circ} \mathrm{C}$

From R. M. Bidwell "Effects of Tantalum Structure on Corrosion" et al., Nucl. Sci. Eng., vol. 14, p. 120.

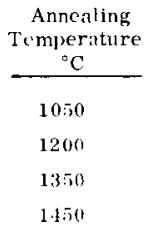

Cumulative Percent Friling in Given Time

\begin{tabular}{rrrr}
$700 \mathrm{hr}$ & $1400 \mathrm{hr}$ & $\underline{2100 \mathrm{hr}}$ & $\underline{2800 \mathrm{hr}}$ \\
\cline { 2 - 3 } 100 & --- & $-\cdots$ & -- \\
50 & 100 & $\cdots-$ & $-\cdots$ \\
0 & 25 & 75 & $\cdots$ \\
0 & 0 & 0 & 0
\end{tabular}




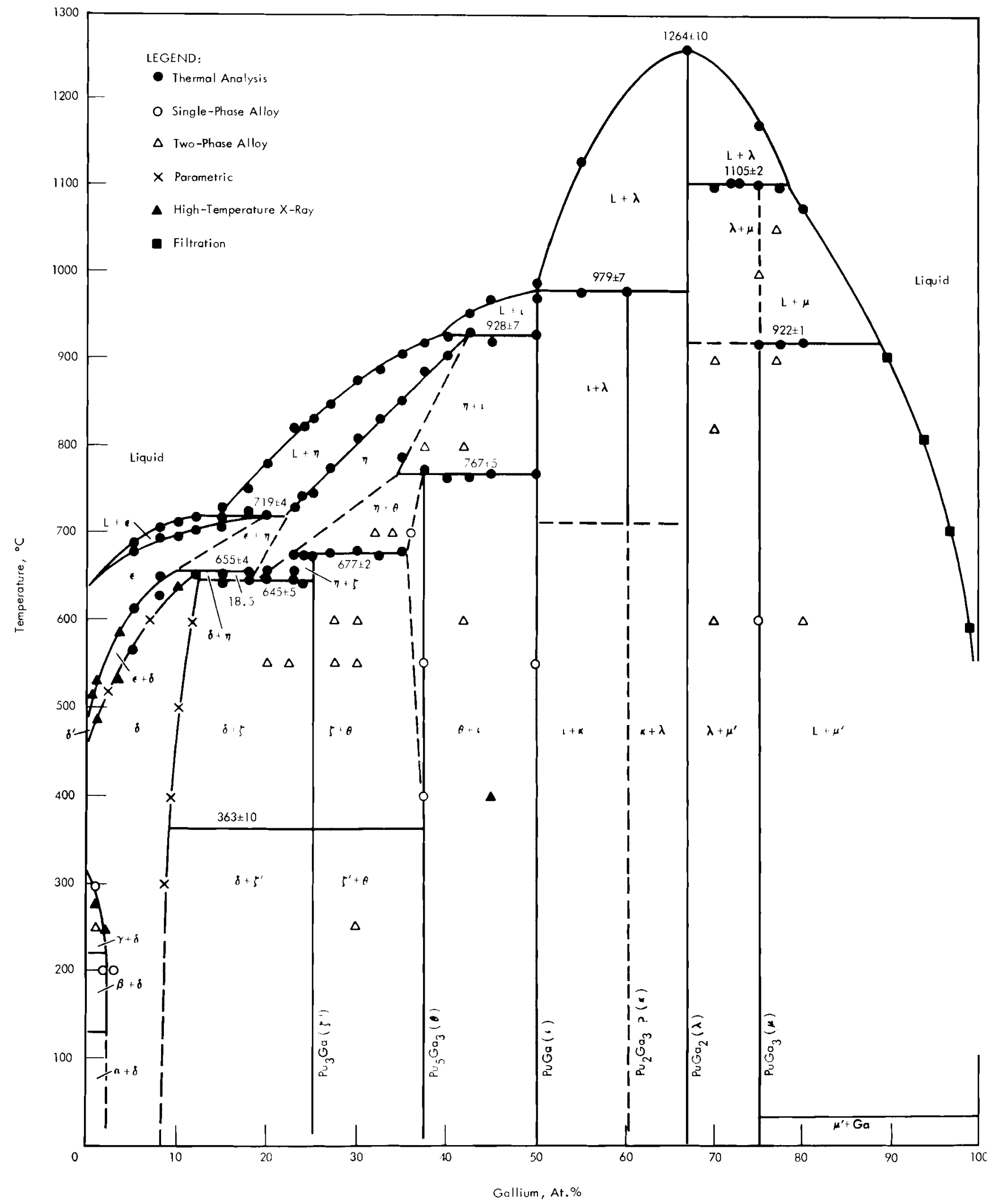

The Plutonium-Gallium Equilibrium Diagram.

From F. H. Fllinger et al. I. Nuel. Mater., vol. 12, p. 228. 1964. 
Ga 103 crystal structure

PLUTONIUM-GAILIUM INTERMEITATE PIIASFS - CRYSTAF. STRICTIIRE ANI HARDNESS IMYTA

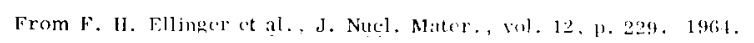

\begin{tabular}{|c|c|c|c|c|c|c|}
\hline Phase & $\begin{array}{c}\text { Structure } \\
\text { Type }\end{array}$ & $\begin{array}{l}\text { Space I attice } \\
\text { And } \\
\text { Space Group }\end{array}$ & $\begin{array}{c}\text { Unit Cell } \\
\text { Dimensions, A }\end{array}$ & $\begin{array}{l}\text { Formula } \\
\text { Linits } \mathrm{P}^{\mathrm{P}} \times \mathrm{r} \\
\text { Init cocll } \\
\end{array}$ & $\begin{array}{c}\text { Calculaterl } \\
\text { Density. } \\
\text { gr/emi }\end{array}$ & $\begin{array}{c}\text { Mirerohardincss, } \\
\text { DIHN, } \\
k \mathrm{~kg} / \mathrm{mm}^{2}\end{array}$ \\
\hline $\mathrm{Pu}_{3} \mathrm{Ga}(5)$ & $\mathrm{AuCu}_{3}$ & $\begin{array}{l}\text { Cubic } \\
\text { Pm3m }\end{array}$ & $a-4.507+0.0102$ & 1 & 14.27 & 155.149 \\
\hline $\mathrm{Pu}_{3} \mathrm{Ga}\left(5^{\prime}\right)$ & $\mathrm{SrPb}_{3}$ & $\begin{array}{r}\text { Tetragonal } \\
\text { P4/mmm }\end{array}$ & $\begin{array}{l}a-4.469+0.001 \\
c-4.527 \pm 0.002\end{array}$ & 1 & 14.45 & \\
\hline $\mathrm{Pu}_{5} \mathrm{Ga}_{3}(0)$ & $\begin{array}{c}\mathrm{W}_{5} \mathrm{SI}_{3} \\
(?)\end{array}$ & $\begin{array}{c}\text { Tetragonal } \\
\text { I } 4 / \mathrm{me} \text { in }\end{array}$ & 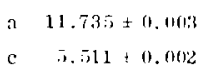 & 1 & $12.2 ! 1$ & $250-\vdots k 1$ \\
\hline PuGa (1) & & $\begin{array}{l}\text { Tetragonal } \\
\text { I..... }\end{array}$ & $\begin{array}{l}a \quad 6.640+0.001 \\
\text { c } \quad 8.066+0.004\end{array}$ & $\dot{s}$ & 11.53 & $12.3-216$ \\
\hline$\left(\mathrm{Pu}_{2} \mathrm{Gra}_{3} ?\right)$ & $x$ & & & & & $357-578$ \\
\hline $\operatorname{MiGa}_{2}(\lambda)$ & $\operatorname{AlB}_{2}$ & $\begin{array}{l}\text { Hexagenal } \\
\text { Dfi } / \text { mnnm }\end{array}$ & $\begin{array}{ll}1 & 4.248+0.001 \\
\therefore & 1.120+0.0101\end{array}$ & 1 & 9.76 & $250-471$ \\
\hline $\operatorname{mug}_{3}(\mu)$ & & & & & & $2(i f i-46 i-4$ \\
\hline${ }^{\prime \prime \prime I G: I}{ }_{3}\left(\mu^{*}\right)$ & $\mathrm{Ni}_{3} \mathrm{Sn}_{n}$ & 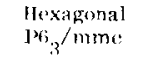 & $\begin{array}{l}\text { a } \quad 6.300+0.001 \\
6 \quad 4.51+ \pm 0.001\end{array}$ & $\ddot{-}$ & 9.59 & \\
\hline
\end{tabular}

\section{Ga 201 Density}

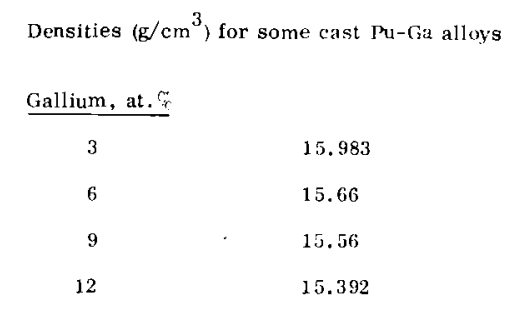

WASH-915 (Classified),

Ga $301 \quad$ Tensile Properties

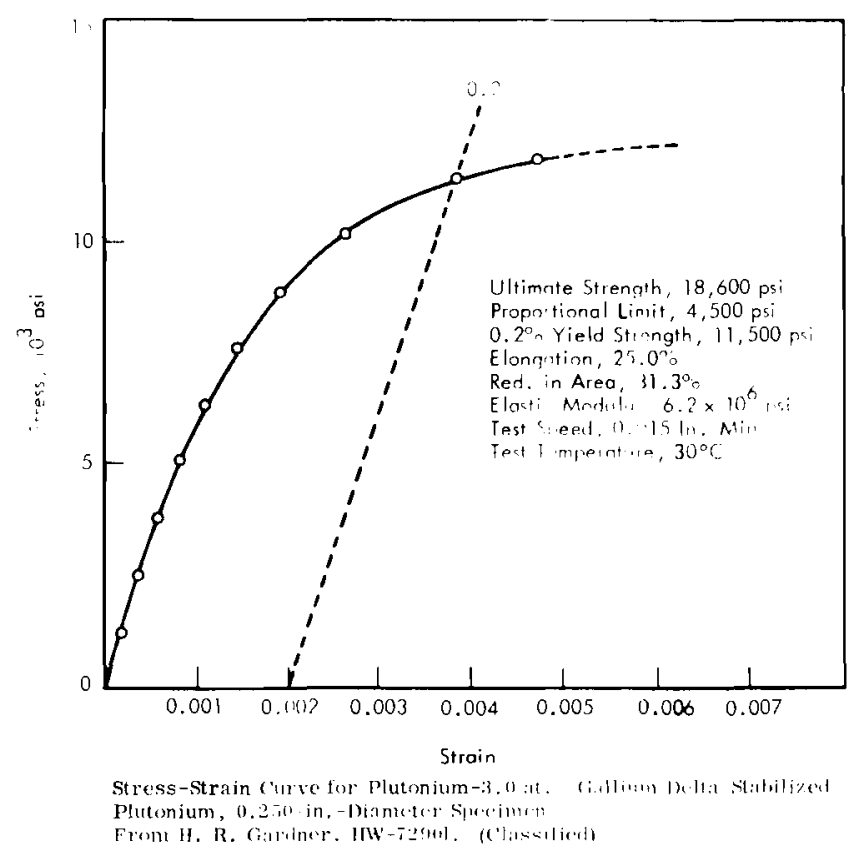



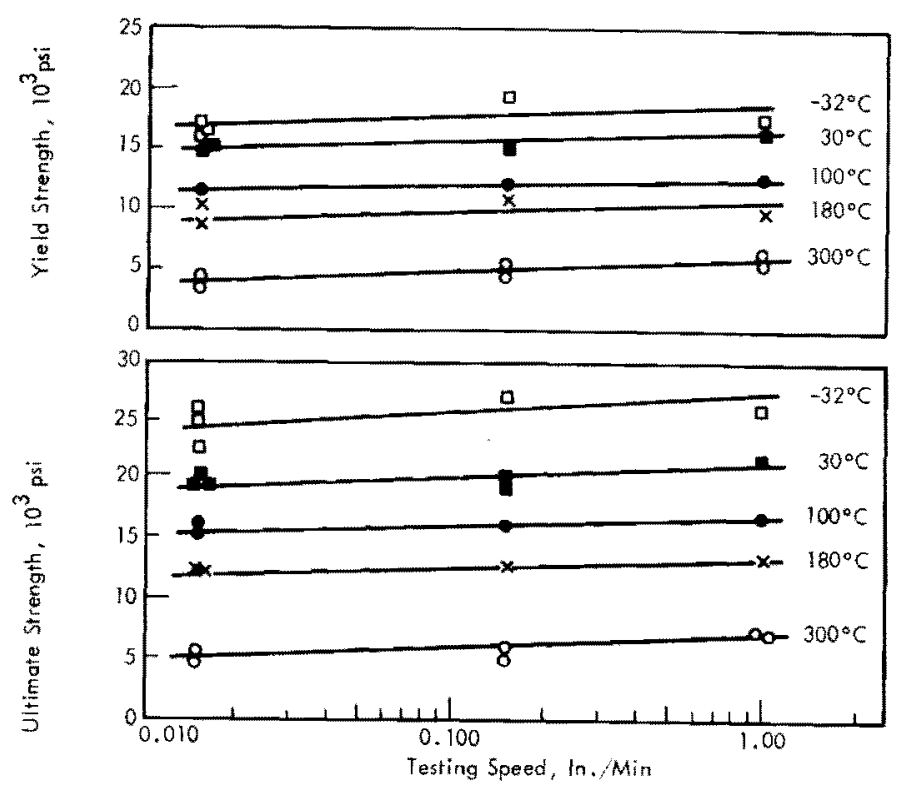

Effect of Testing speed and Temperature on Trensile Strength in a Plutonium 4.0 at. Gallium Delta - Stabilized Altịy.

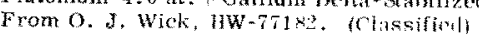

\section{Ga 302 conpressive Properties}

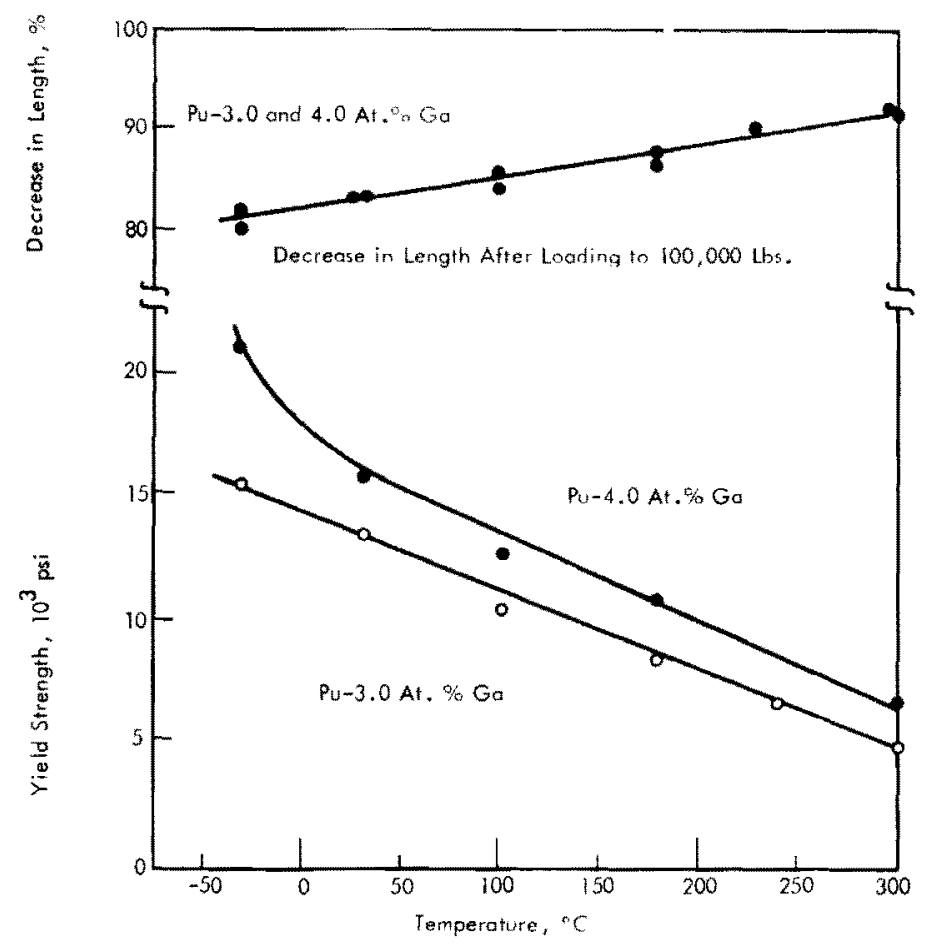

Effect of Temperature on Compression properties of Alloys. Testing Speed, $0.015 \mathrm{in} . / \mathrm{min}$.

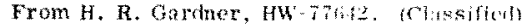

122
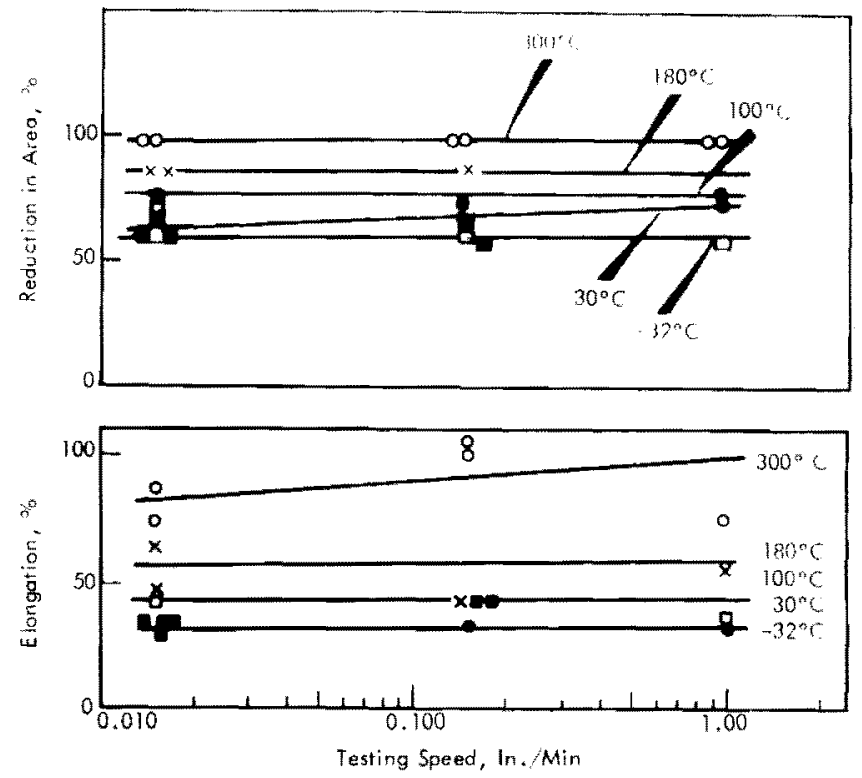

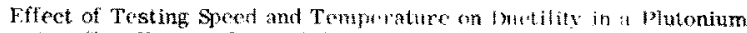
4.0 at. Gallium Delta-Stabilizal Vlw.

From O. J. Wiek, HW-77182. (Classiliond)

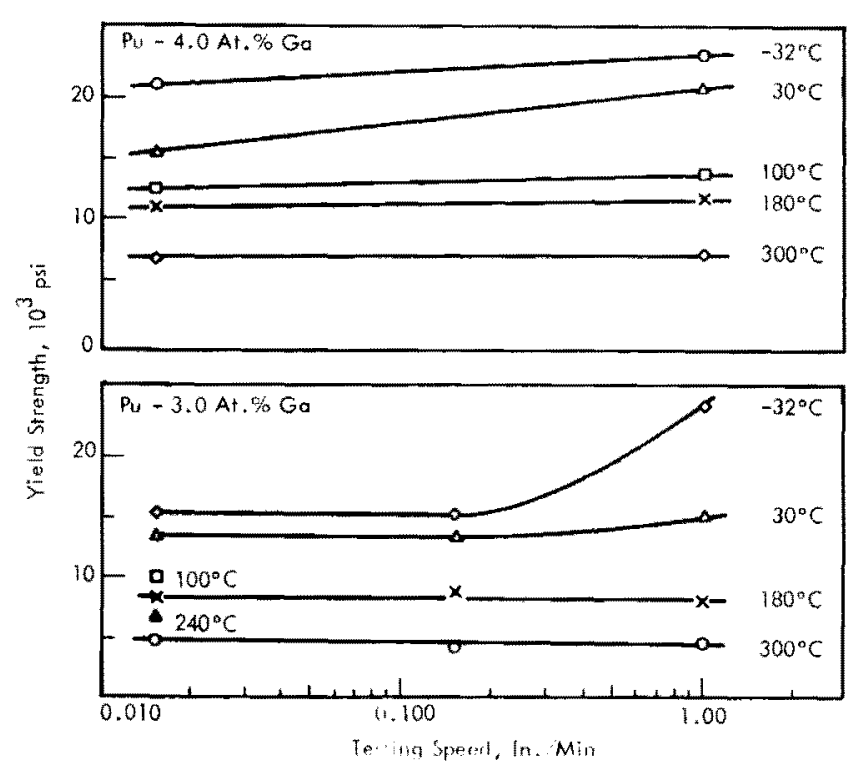

Fffect of Testing speed on Compresive Yold strength of nlays. From H. R. Gardner, HW 776.42, (C)iskifitt) 
$\mathrm{Pu}_{3} \mathrm{Ge}$ and $\mathrm{Pu}_{3} \mathrm{Ge}_{2}$

These two plutonium-rich phases have been observed by means of X-rity and metallographic methods, but their crystil structures are not known. The composition $F^{2} \mathrm{G}_{3} \mathrm{Ge}$ is fairly certain, but the

composition $\mathrm{Pu}_{3} \mathrm{Gre}_{2}$, has not been proved.

The other Pu-Ge intermetallies are as follows: $\mathrm{Hu}_{2} \mathrm{Ge}_{3}$

Hexagonal, pseudo-AlB $B_{2}$ type

a $=3.175 \pm 0.002 \mathrm{~A}$

$\mathrm{c}=4,198 \pm 0.002$

7. $:=1 / 2, D_{x}=10.06$

Sisace group. $-\mathrm{P} 6 / \mathrm{mmm}$ (Int. Tit). 1!1)

Atomic positions

1 Pu in (a): $0,0,0$

1.5Ge in (t): $1 / 3,2 / 3,1 / 2 ; 2 / 3,1 / 3,1 / 2$

Interatomic distances

$\mathrm{P}$ ru $-12 \mathrm{Ge}=3.11$

$-6 \mathrm{Pu}=3.98$

$\mathrm{Ge}-3 \mathrm{Ge}=2.29$

Remarks. - See $\mathrm{Pu}_{2} \mathrm{Si}_{3}$.

PriGe $_{2}$

Tetragonal body-centered, $\mathrm{ThSi}_{2}$ typo

Ge-rich: $\mathrm{a}=4.102 \pm 0.002 \mathrm{~A}$

$$
\begin{aligned}
& \mathrm{c}=13.81 \pm 0.01 \\
& \mathrm{Z}=4, \mathrm{D}=10.98
\end{aligned}
$$

Suace group. - I4/amd (Int, Tab. 141).

Atomic positions. - Sce irusi.

Density

Density

2 it. $\%$ Ge, as cast: $17.87 \mathrm{~g} / \mathrm{cm}^{3}$ (average)

From A. E. Martin and G. D. Selmanoff, LA-501. (Classified)

PuGe $_{3}$

Isotypes. - $\mathrm{UGe}_{3}$.

Reactions with Gases - Air

$\mathrm{Hf} \quad 401.1$

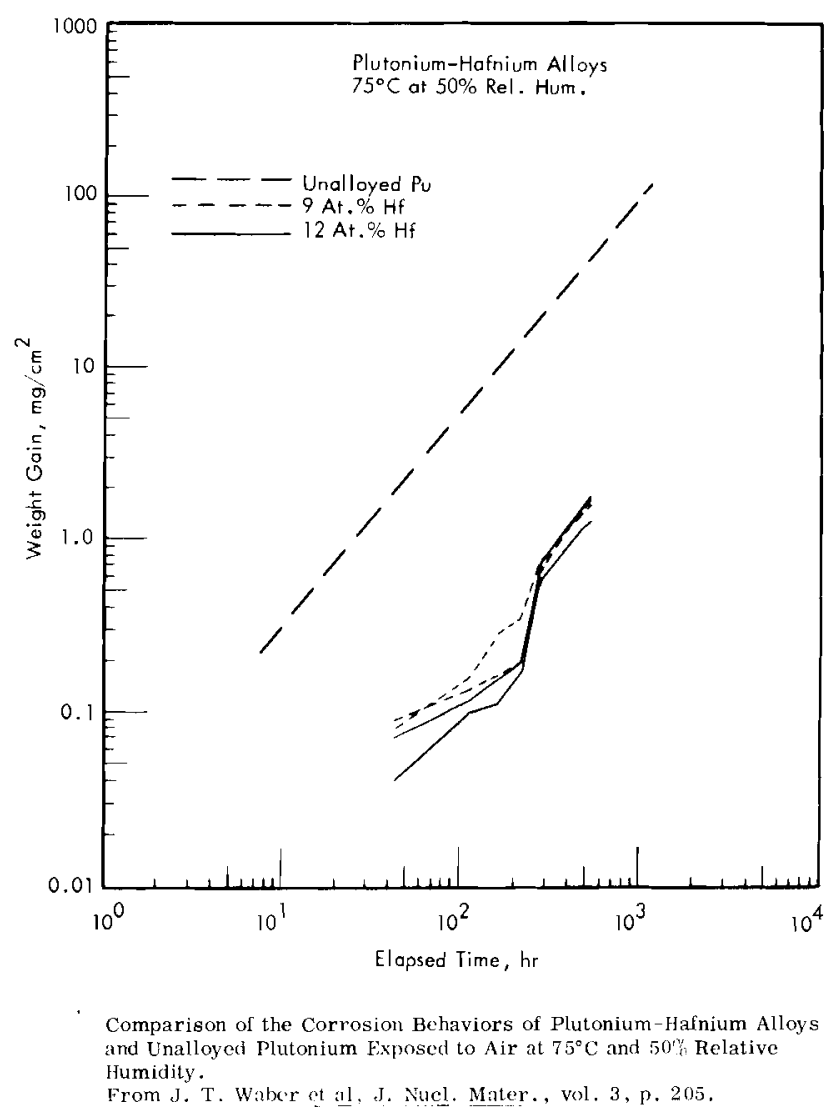

Ge 201

Cubic primitive, $\mathrm{AuCu}_{3}$ type

$$
\begin{aligned}
& \mathrm{a}=4.223 \pm 0.001 \mathrm{~A} \\
& \mathrm{Z}=1, \mathrm{D}_{\mathrm{X}}=10.07
\end{aligned}
$$

Space group. - Pm3m (Int, Tab. 221).

mic positions
$1 \mathrm{Pu}$ in (a): 0,0

3Ge in (c): $0,1 / 2,1 / 2 ; 1 / 2,0,1 / 2 ; 1 / 2,1 / 2,0$

Interatomic distances

$\mathrm{Fu}-12 \mathrm{Ge}=2.99$

$\begin{aligned} \mathrm{Ge}-8 \mathrm{Ge} & =2.99 \\ -4 \mathrm{Pu} & =2.99\end{aligned}$

From F. H. Ellinger in The Metal Plutonium, pp. 290, 291. Also

from Proc, Eirst Geneva Internat. Conf., vol. 9, pp. 139, 140. 1956.

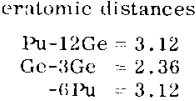

$$
\begin{aligned}
\mathrm{Ge}-3 \mathrm{Ge} & =2.36 \\
-6 \mathrm{I} d & =3.12
\end{aligned}
$$




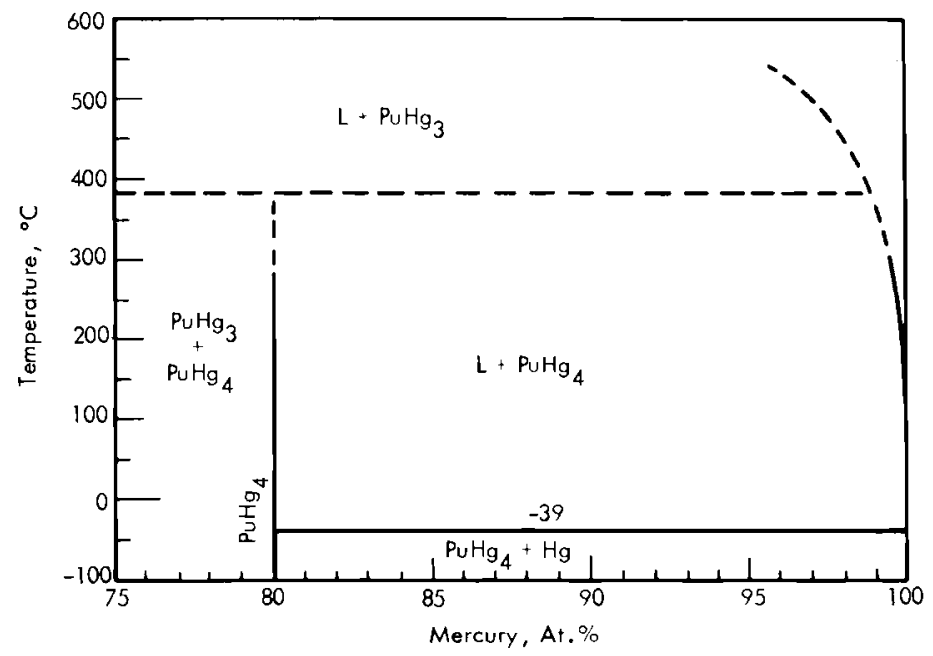

The High-Mercury Fnd of the Plutonium-Mercury System. From F. W. Schonfeld in The Metal Plutonium, p. 248 .

$$
\text { PuH }_{\mathrm{G}_{3}}
$$

Apparently this intermetallic is isostructural with hexagonal UHg, but a . satisfactory diffraction pattern of $\mathrm{PuHg}_{3}$ has never heen obta ined.

$\mathrm{PuH}_{\mathrm{G}_{4}}$

This intermetallic is isostructural with $\mathrm{UHg}_{4}$. The dimension of the pseudocube is $\mathrm{a}: 3,61 \pm 0.01 \mathrm{~A}$.

From F. II. Ellinger in The Metal Plutonium, p. 296

\section{In 103 crystal strucure}

$$
\mathrm{Pu}_{3} \text { In }
$$

Cubic primitive, $\mathrm{AuCu}_{3}$ type

$a=4.703 \pm 0.002 \mathrm{~A}$

$\mathrm{z}=1, \mathrm{D}_{\mathrm{x}}=13.3$

Space group. - Pm3m (Int. Tab. 221).

Atomic positions. - See $^{2}{ }^{2} \mathrm{Cre}_{3}$.

Interatomic distances

Pu-8I $\mathbf{u}=3.32$

$-4 \operatorname{In}=3.32$

$\mathrm{In}-12 \mathrm{Pu}=3.32$

Remarks. - Although $\mathrm{I}_{3} \mathrm{u}_{3}$ in is described above as having the $\mathrm{AuCu}_{3}$ type of structure, i.e., fully ordered, it has not been observed with complete ordering of the atoms. The powder patterns of the alloys that were examined showed weaker super-lattice lines than would be required by complete ordering. It is believed. however, that proper thermal treatment of $\mathrm{Pu}_{3}$ In would result in it fully oldered structure.

From F. H. Fllinger in The Metal Plutonium, p, 292.

Density of as cast $3 \mathrm{at} . ;$ In $=10.80 \mathrm{~g} / \mathrm{cm}^{3}$

From A. E. Martin and G. D. Selmanoff, IA-501. (Classified) 


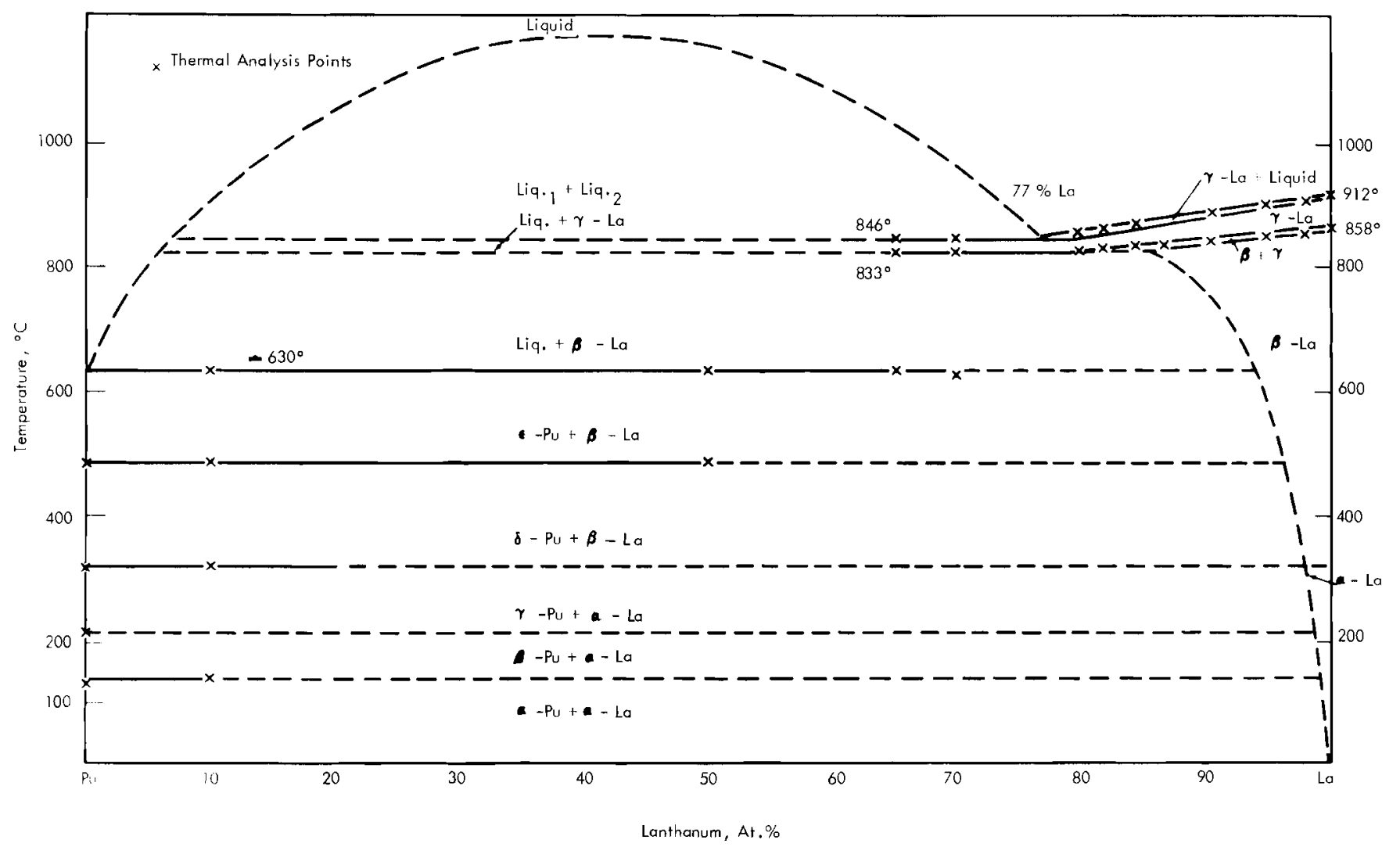

Tentative Phase Diagram of the Pu-La System.

From D. M. Poole et al. in Plutonium 1960, p. 279.

Phase Diagrams

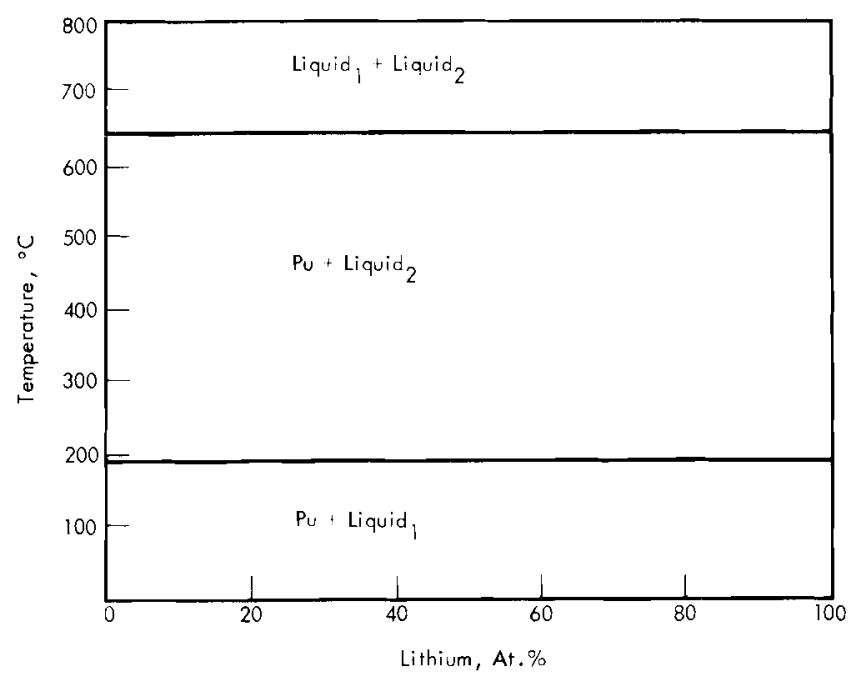

Constitutional Diagram for the System I'lutonium-1,ithium.

From F. M. Schonfeld et al in Metallurgy and Fucls, series V, vol. $2, p, 587$. 


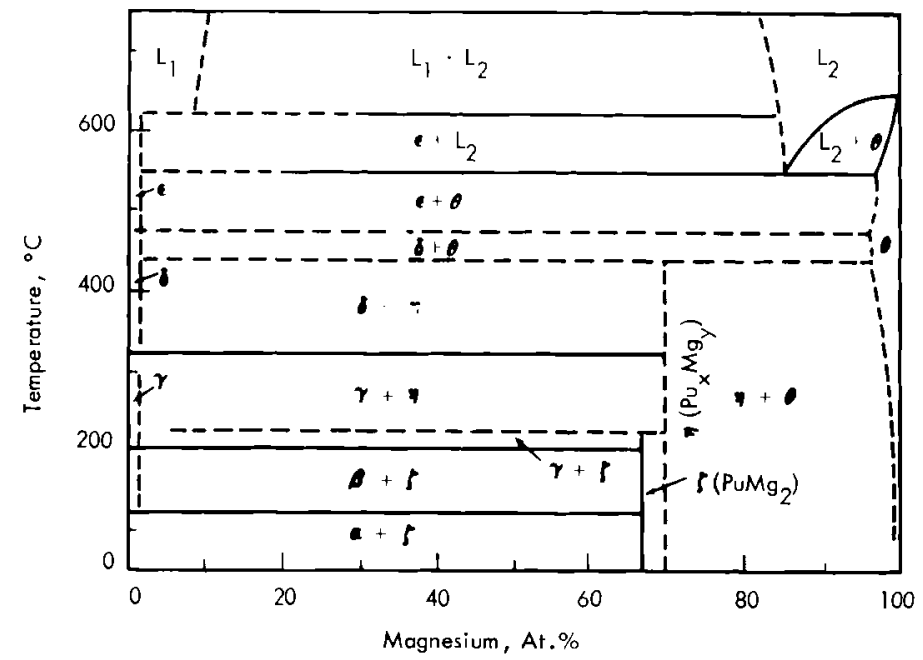

The Plutonium-Magnesium System.

From F. W. Schonfeld in The Metal Plutonium, p. 244.

$\mathrm{Mg} 103$ constal structure

PuMgig

Cubic face-centered, $\mathrm{CaF}_{2}$ type

$$
\begin{aligned}
& \mathrm{a}=7.34 \pm 0 .(11 \mathrm{~A} \\
& z=4, \mathrm{D}_{\mathrm{x}}=4.83
\end{aligned}
$$

Space group. - Fm3m (Int. Tab. 225).

Atomic positions. - See PuH ${ }_{2}$.

Interatomic distances

$$
\text { Pu- } 8 \mathrm{Mg}=3.18 \quad \mathrm{Mg}_{\mathrm{g}}-4 \mathrm{PMI}^{\mathrm{N}}-3 . \mathrm{I8}
$$

Remarks. - PuMg, is formed by a reaction in the solid state, and its diffraction pattern has never been obtained free from the super-

imposed patterns of other phases. Farlier it was reported (1) as

possibly being lu $\mathrm{Mg}$, but re-evaluation of the data indicates that the composition PuMğ, is more probably correct.

$\operatorname{PuMg}_{\mathrm{x}}$ (Where $\mathrm{x}$ is Approximately 2)

Hexagonal

$$
\begin{aligned}
& \mathrm{a}=13.8+0.1 \mathrm{~A} \\
& \mathrm{c}=9.7+0.1
\end{aligned}
$$

Like $\mathrm{PuMg}_{2}$, his intermetallic is formed by a peritectoid reaction, and its approximate composition was determined by microstructural studies.

From F. H. Fllinger in The Metal Plutonium, p. 29.5. 


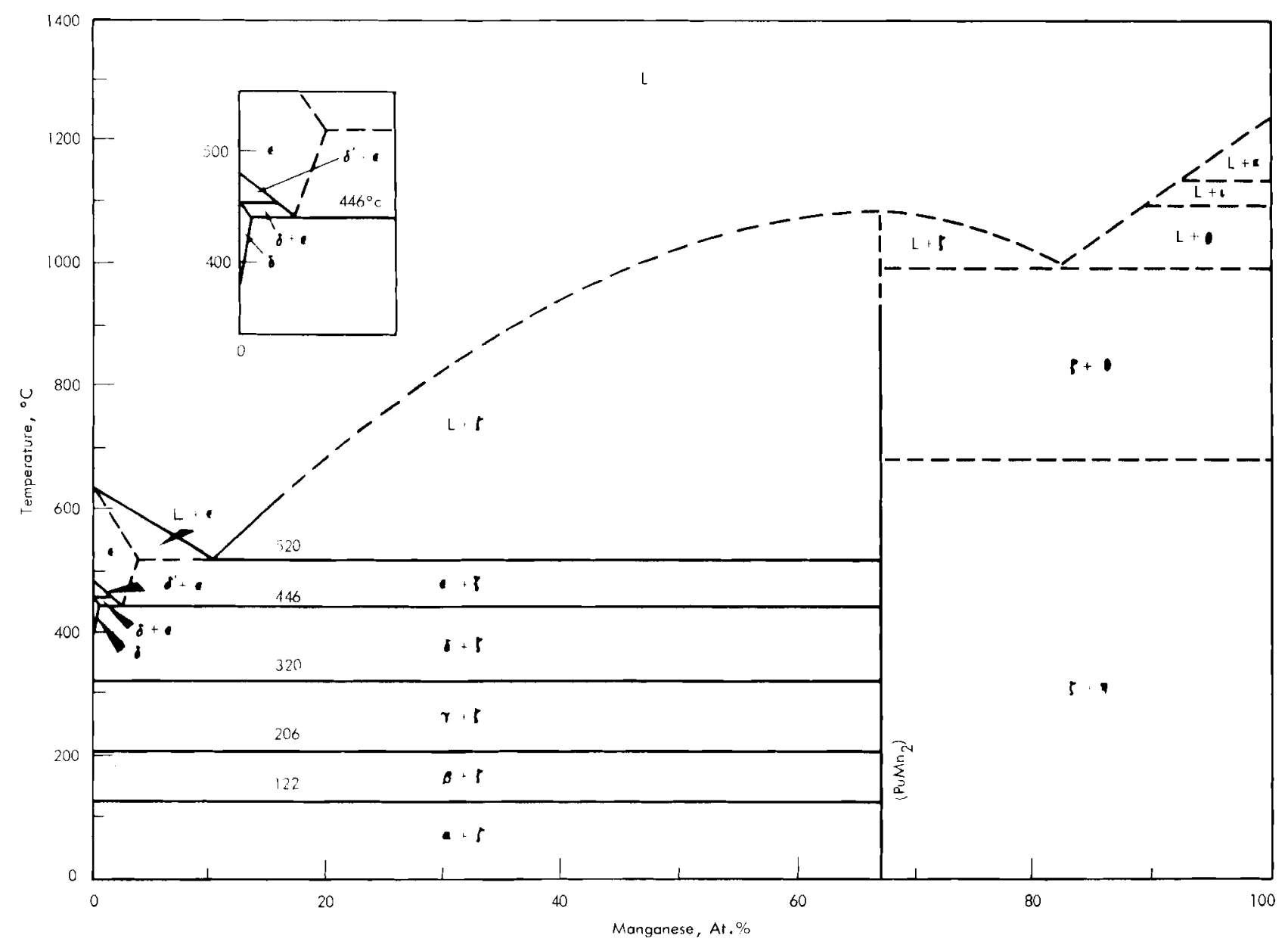

The plutonium-Manparese System.

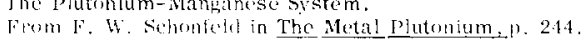

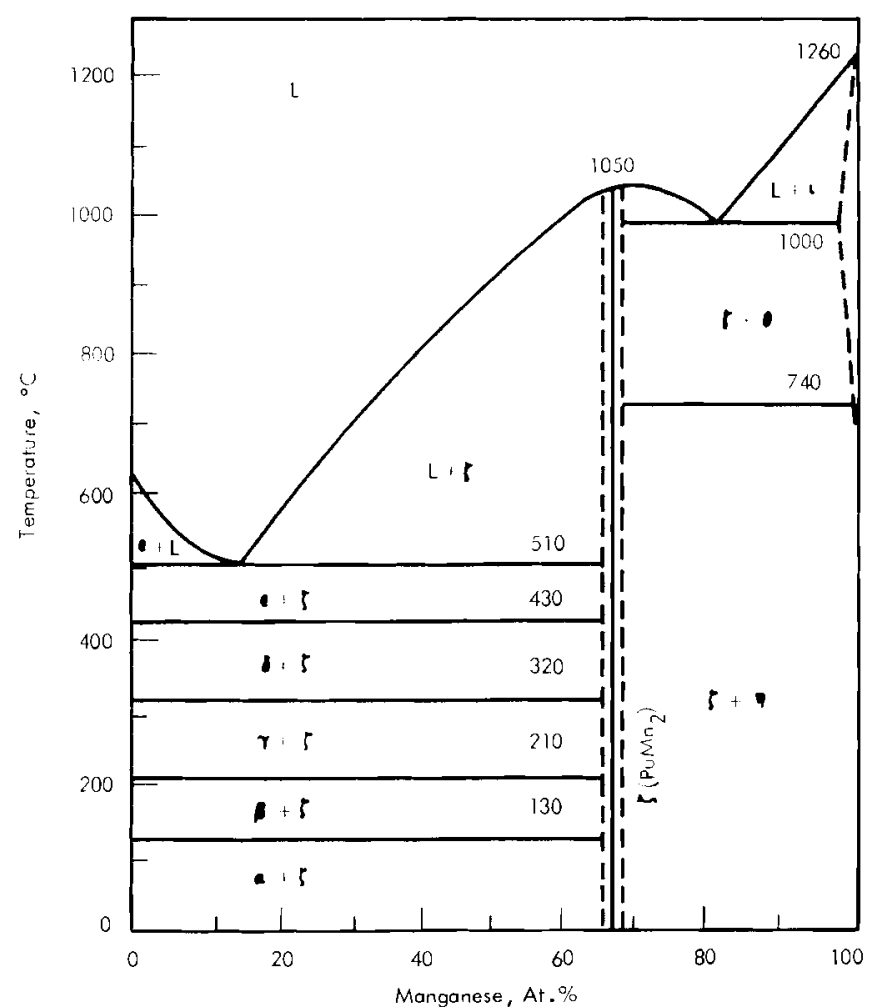

The Plutonjum-Nanganese System. 
Mn 201 Dessity

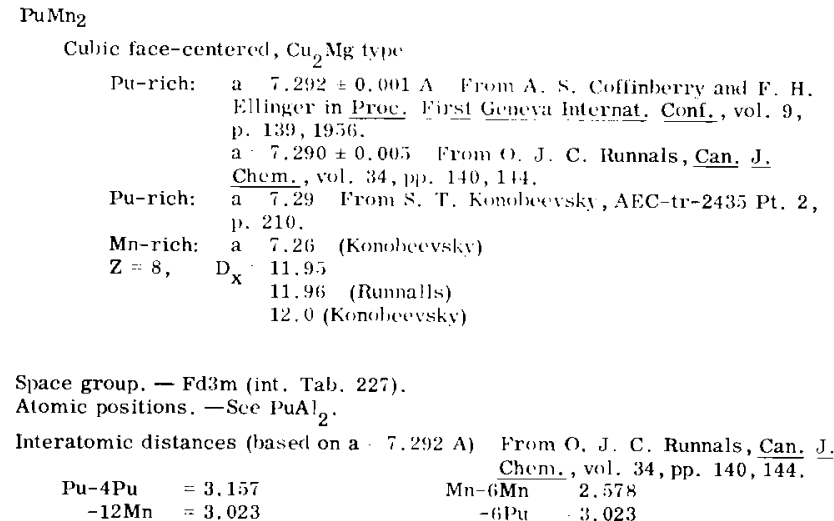

From F. H. Ellinger in The Metal plutonium, pp. 295, 296.

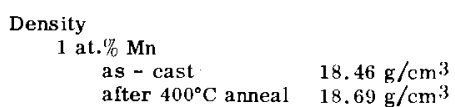

From A. E. Martin and G. D. Selmanoff, LAA-ino1. (Classified)

\section{Mn 204.1}

Magnetic Susceptibility

Paramagnetic susceptibility of $\mathrm{PuMn}_{2}$ at $20^{\circ} \mathrm{C}$

$$
\begin{array}{cc}
\mathbf{x}_{\mathrm{g}}^{\text {Mass }} \times 10^{6}, & \text { Atomic } \\
\mathrm{emu} \mathrm{g}^{-1} & \mathbf{x}_{\mathrm{a}} \times 10^{60} \\
7.8 & \text { emu g-at. }
\end{array}
$$

$\mathbf{X a}=\mathbf{X g}(\mathbf{a A}+\boldsymbol{B})$ where the expression in brackets stands for the mean atomic weight.

From S. T. Konobeevsky et al. in Proc. Second Geneva Internat. Conf., vol. 6 , p. 195 .

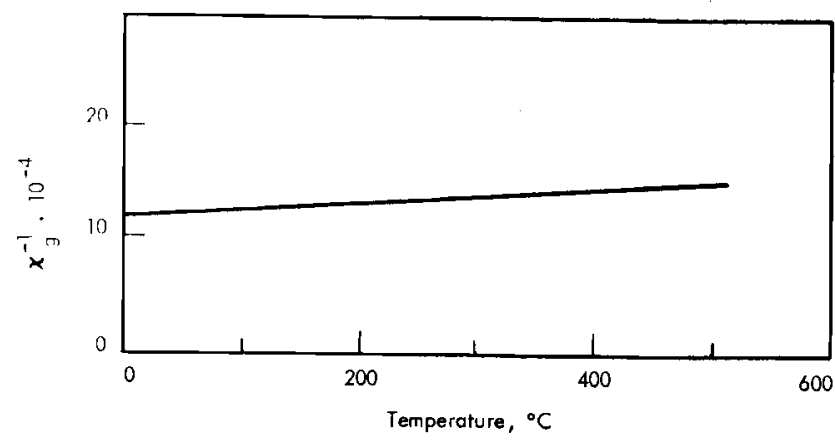

Temperature Dependence of Paramagnetic Susceptibility of PuMn, From S. T. Konobeevsky et al. in Proc. Second Geneva Internat. Gonf., vol, 6, p. 195

\section{Mo 101 Phase Diagrams}

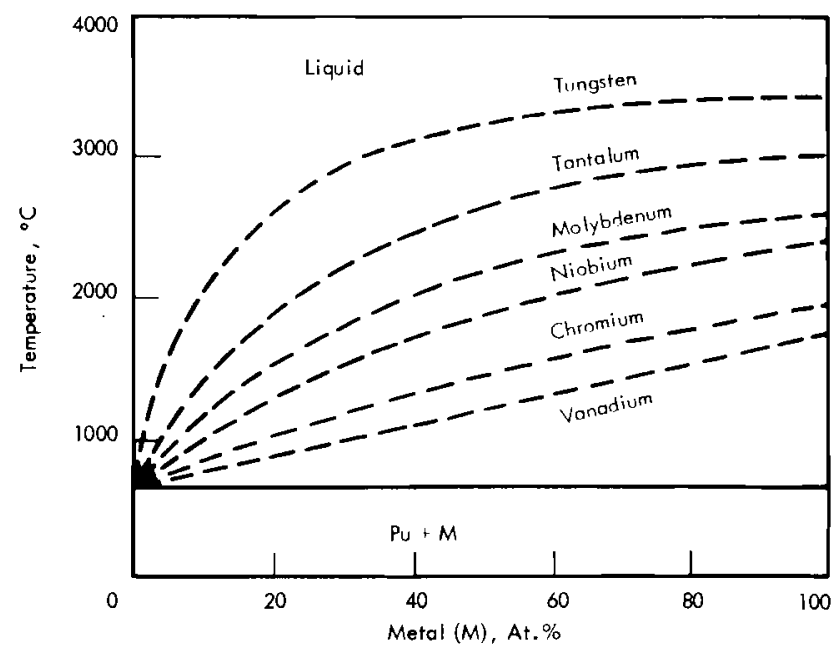




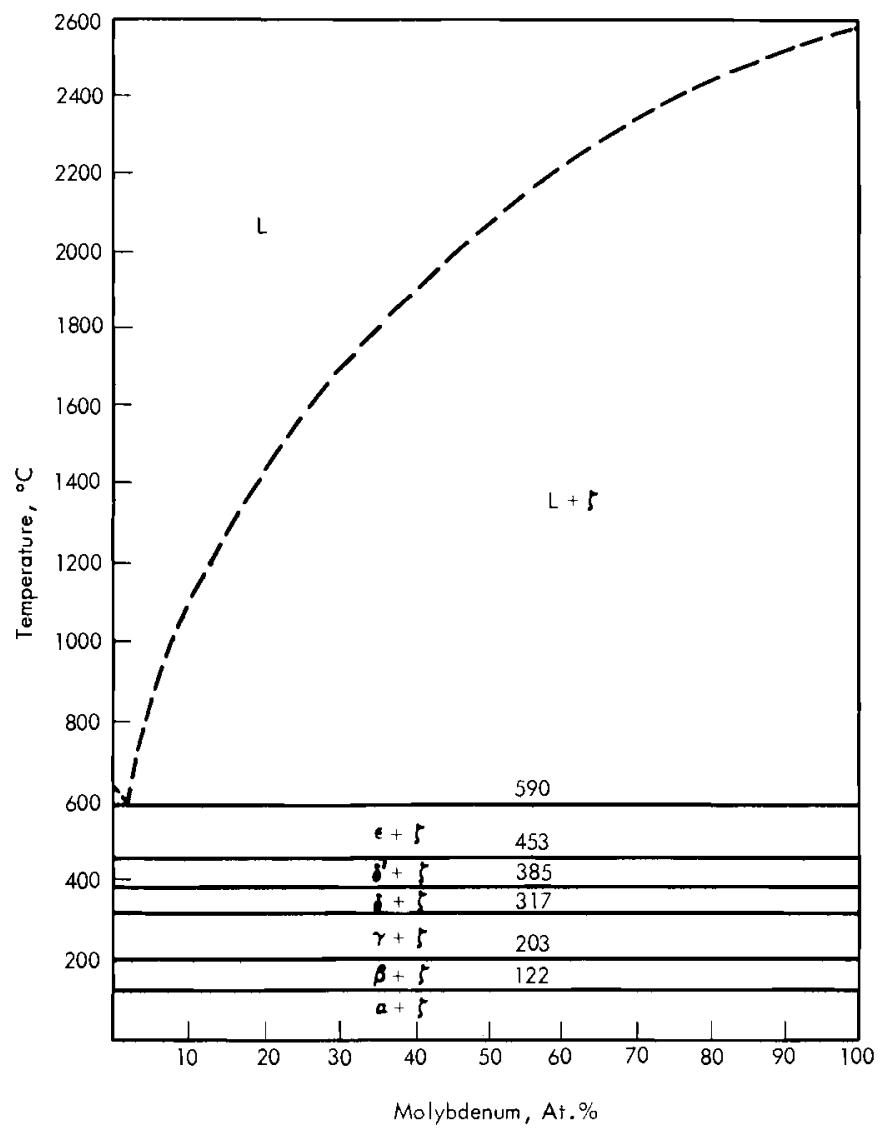

The Plutonium-Molyhdenum System.

From F. W. Schonfeld in The Metal Plutonium, p. 261.

Phase Diagrams

$\mathrm{Ni} 101$

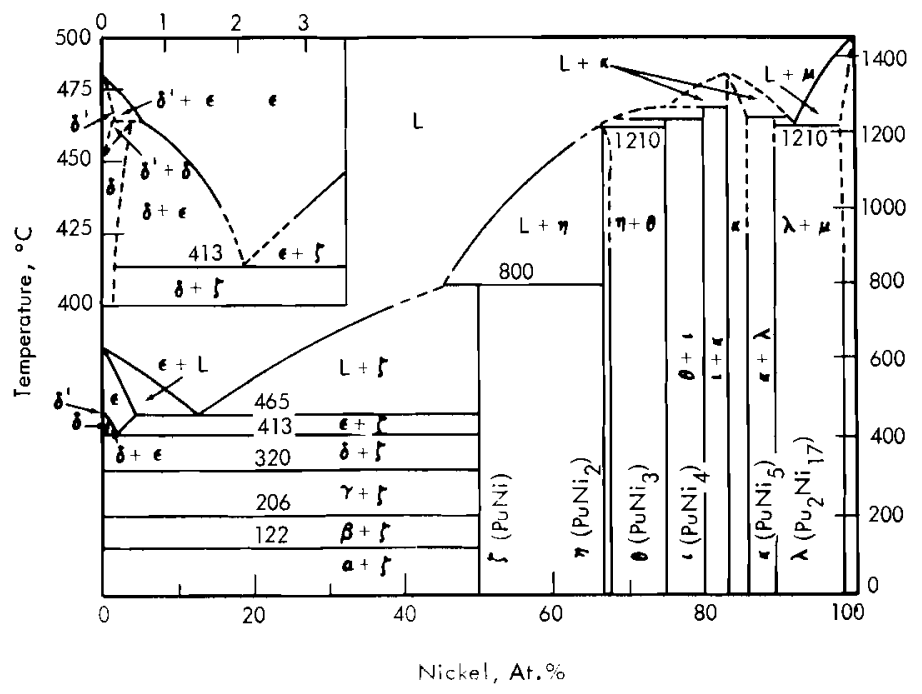

The plutonium-Nides] Sistem.

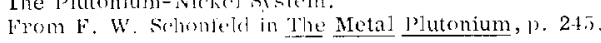




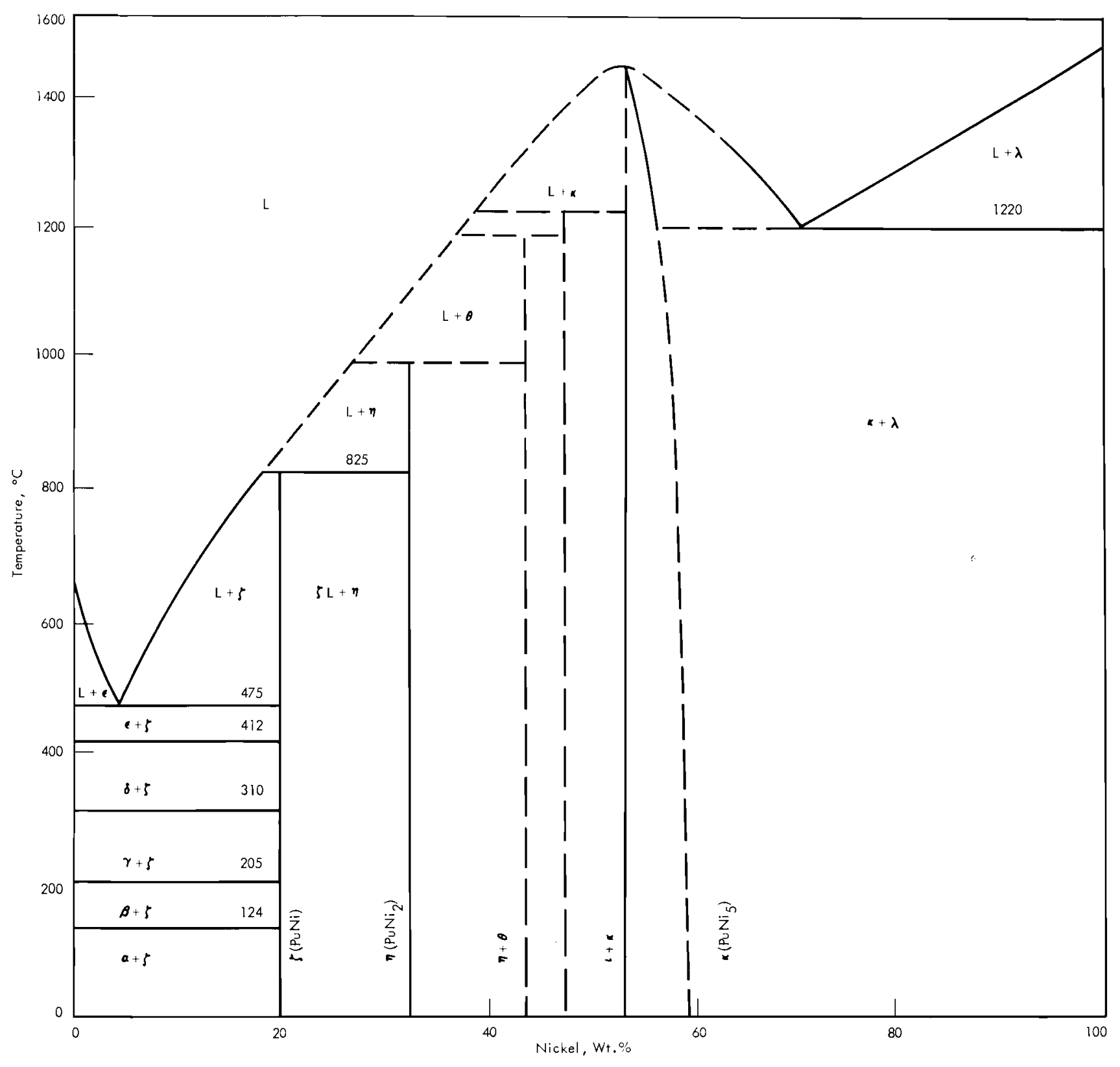

The plutonium-xickel system.

From F. W. Schonfeld in The Metal plutonium, p. 258.

\section{Ni 103 crystal structure}

\section{CRYSTALLOGRAPHY OF PLUTONIUM-NICKEL SYSTEM}

From F. W. Schonfeld et al. in Metallurgy and Fuels, series V, vol, 2, p. 591.

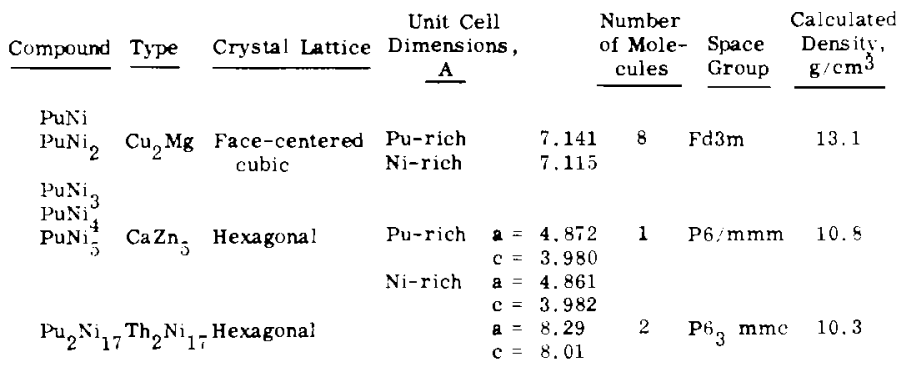

PuNi

"1.thorhonbic Til trpe

$: \quad 3.59 \pm 0.61,1, \quad 10.21=0.02, c-4.22 \pm 0.01 \mathrm{~A}$.

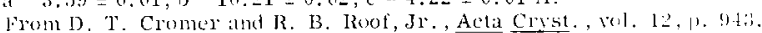

Z. $4,19+12,9$

Space gioup Cmem

Each atom has seven neighbors of the ouposite kind, 4 at 2.85 , two at

$2.8 \mathrm{~s}$ and one at $2.86 \mathrm{~A}$. In addition, the $\mathrm{Ni}$ atom has two Ni neighbors

at 2. 6t $\mathrm{A}$ and the $\mathrm{p} u$ has eight Pu neighbors, two at 3.54 and six at $3.59 \mathrm{~A}$.

The estimated standard deviations are $0.02 \mathrm{~A}$ for Pu-Pu, $0.04 \mathrm{~A}$ for

Pu-Ni and 0, 0; $\mathrm{A}$ for Ni-Ni distances.

$\mathrm{PuNi}_{2}$

Culsic face-rentered, $\mathrm{Cu}_{2} \mathrm{Mg}$ type

Pu-lich: $a=T .1+1 \pm 0.001 \mathrm{~A}$

Ni-rich: a $\quad \mathrm{i} .11 \mathrm{j} \pm 0.001$

$a=T .16 \pm 0.01$ (Runnalls)

$a-i .1+$ (Konobeersky)

$$
\mathrm{Z} \rightarrow, \mathrm{I})^{\mathrm{a}}=13.1
$$

Space groups, - Fol:3m (Int, Tab. 227),

Atonie positions. - See PuAl,

Interatomie distances (based on $\mathrm{a}=\overline{7} .115 \mathrm{~A}$ )

$$
\begin{array}{rlrl}
P_{t !-41} u & 3.081 & \mathrm{Ni}-6 \mathrm{Ni} & =2.516 \\
-12 \mathrm{X} & 2.950 & -6 \mathrm{Pu} & =2.9 .50
\end{array}
$$

lsotypes. - $\mathrm{LaNi}_{2}, \mathrm{CeNi}_{2}, \mathrm{PrNi}_{2}$.

Fon F, H, l:1linger in The Hetal plutonium, !) 296. 
PuNi:3 $_{3}$

This compound has been found to have a rhombohedral unit eell. It.s structure is being deternined b $\mathrm{I}$ ), T. Cromer.

\section{$\mathrm{PuNi}_{4}$}

The crystal structure of $\mathrm{PuNi}_{4}$ is not known, but its diffraction pattern suggests a close relationship to the $\mathrm{P}^{2} \mathrm{Ni}_{\text {, }}$ structure.

$\mathrm{l}^{\mathrm{U}} \mathrm{Ni}_{5}$

Hexagonal, CaZn type

Pu-rich: $\mathrm{a}=4.872 \pm 0.002 \mathrm{~A}$

$\mathrm{c}-3.980 \pm 0.001$

Ni-rich: $\quad \mathbf{a}=4.861 \pm 0.002$

$\mathrm{c}=3.982 \pm 0.001$

$\mathrm{a}=4.875 \pm 0.005$ (Runnalls)

$\mathrm{Z}=1, \mathrm{D}_{\mathrm{x}}^{\mathrm{C}}=10.8$

Space group. $\mathrm{X}_{\mathrm{Ph}} / \mathrm{mmm}$ (Tnt. Tab. 191).

Atomic positions

$1 \mathrm{Pu}$ in (a): $0,0,0$

$2 \mathrm{Ni}_{\mathrm{I}}$ in $(\mathrm{c}): 1 / 3,2 / 3,0 ; 2 / 3,1 / 3,0$

$3 \mathrm{Ni}_{\mathrm{II}}$ in $(\mathrm{g}): 1 / 2,0,1 / 2 ; 0,1 / 2,1 / 2 ; 1 / 2,1 / 2,1 / 2$

Interatomic distances (based on a $=4.861, \mathrm{c}=3.982 \mathrm{~A}$ )

$$
\begin{aligned}
& P \mathrm{U}-6 \mathrm{Ni} \mathbf{I}=2.81 \\
& \begin{aligned}
\mathrm{Ni}_{\mathrm{I}}-3 \mathrm{Pu} & =2.81 \\
-6 \mathrm{Ni} & =2.44
\end{aligned} \\
& -12 \mathrm{Ni}_{\mathrm{II}}=3.14 \\
& -3 \mathrm{Ni} \quad \cdot 2.81 \\
& \mathrm{Ni}^{-4 \mathrm{Pu}}=3.14 \\
& -4 \mathrm{Nj}_{\mathrm{I}}=2.44 \\
& { }^{-4 \mathrm{Nj}} \mathrm{II} \quad=2.44
\end{aligned}
$$

$$
\mathrm{Pu}_{2} \mathrm{Ni}_{17}
$$

Isotypes. - $\mathrm{LaNi}_{5}, \mathrm{CeNi}_{5}, \mathrm{PrNi}_{5}, \mathrm{GdNi}_{5}, \mathrm{ThNi}_{5}$.

$$
\begin{gathered}
\text { Hexagonal, } \mathrm{Th}_{2} \mathrm{Ni}_{17} \text { type } \\
\mathrm{a}=8.29 \pm 0.02 \mathrm{~A} \\
\mathrm{c}=8.01 \pm 0.02
\end{gathered}
$$$$
\mathrm{Z}=2, \mathrm{D}_{\mathrm{x}}=10.3
$$

Space group. $-\mathrm{P}_{3} / \mathrm{mmc}$ (Int. Tab. 194).

Atomic positions. - See $\mathrm{Pu}_{2} \mathrm{Co}_{17}$.

From F. H. Ellinger in The Metal Plutonium, pp, 296, 297.

In the T1I structure there are four formula units per unit cell with the larger atom ( $\mathrm{Pu}$ in this case) in the set $4 \mathrm{c}$ with $\mathrm{y} \approx 0.14$, and the smaller atom in the same set with $y \approx 0,42$. The calculatcl rlensity of PuNi is thus $12.9 \mathrm{~g} \mathrm{~cm}^{-3}$.

From D, T. Cromer and R. B. Roof, Jr., Acta Cryst., vol. 12, p. 942. 1959

$\mathrm{I}^{\mathrm{u}} \mathrm{Ni}_{2}$ is face-centered cubic with $\underline{\mathrm{a}} \cdot 7.13 \mathrm{kx}$.

It is of the $\mathrm{MgCu}_{2}$ type structure, with

8 formula weights/unit cell.

\begin{tabular}{|c|c|c|c|}
\hline \multirow[b]{2}{*}{ Aton $\mathrm{x}$} & \multirow[b]{2}{*}{ Neightor } & \multicolumn{2}{|c|}{ Distance, A } \\
\hline & & $\begin{array}{l}\text { Number of } \\
\text { Neighlers }\end{array}$ & $\mathrm{PuNi}_{2}$ \\
\hline \multirow[t]{2}{*}{ Pu } & $\mathrm{Pu}$ & 4 & 3.10 \\
\hline & $\mathrm{X}$ & 12 & 2.96 \\
\hline \multirow[t]{2}{*}{$\mathrm{X}$} & $\mathrm{X}$ & 6 & 2.53 \\
\hline & p'u & 6 & 2.96 \\
\hline
\end{tabular}

From S. T. Konobeevsky, AEC-tr-2435 Pt. 2, p. 212.

$\mathrm{PuNi}_{2}$

Face-centered cubic, isostructural with $\mathrm{UFe}_{2}$

Latice constant $7,16 \pm 0.01 \mathrm{~A}$

Calculated density $12.90 \mathrm{~g} / \mathrm{cm}^{3}$

$\mathrm{MgCl}_{2}$ lattice structure, space group $\mathrm{Fd} 3 \mathrm{~m}$

8 formula weights/unit cell

From O. J. C. Runnalls, Can. I. Chem., vol. 34, p. 144, 1956.
From O. J. C. liunnalls, Can. J. Chem., vol. 84, p. 1t0. 19:6

$\mathrm{PuNi}_{-}$

A powder photograph of a Pu-Ni alloy near the conıosition PuNi, $45 \mathrm{wt}$. $\mathrm{Pu}$, showed a single phase which was indexed on a hexagonal lattice with dimensions

$$
\begin{aligned}
& \mathrm{a}_{0} \quad 4.875 \pm 0.005 \mathrm{~A}, \\
& \mathrm{c}_{0} \quad 3.970 \pm 0.00 \overline{\mathrm{A}} .
\end{aligned}
$$

\begin{tabular}{|c|c|c|c|}
\hline Alom & Neighbor & $\begin{array}{r}\text { Number or } \\
\text { Neighlyors }\end{array}$ & Distance A \\
\hline \multirow[t]{2}{*}{$P_{1}$} & $\mathrm{Ni}_{I}$ & 6 & 2.82 \\
\hline & ${ }^{\mathrm{Ni}} \mathrm{II}$ & 12 & 3.14 \\
\hline \multirow[t]{2}{*}{${ }^{\mathrm{Ni}} \mathbf{I}$} & $\begin{array}{l}\mathrm{Pu} \\
\mathrm{Ni}\end{array}$ & $\begin{array}{l}3 \\
3\end{array}$ & $\begin{array}{l}2.82 \\
2.82\end{array}$ \\
\hline & ${ }^{\mathrm{Ni}} \mathrm{II}$ & 6 & 2.44 \\
\hline \multirow[t]{2}{*}{${ }^{\mathrm{Ni}}$} & $\begin{array}{l}\mathrm{Pu} \\
\mathrm{Ni}\end{array}$ & $\begin{array}{l}4 \\
4\end{array}$ & $\begin{array}{l}3.14 \\
2.44\end{array}$ \\
\hline & $\mathrm{Ni}_{\mathrm{TI}}$ & 4 & 2.44 \\
\hline
\end{tabular}

The X-ray data resembled closely those for the compound $\mathrm{ThNi}_{5}$, which has the $\mathrm{CaZn}_{5}$ structure (6).

Thus, PuNi ${ }_{5}$ may be described by the $\mathrm{CaZn}_{5}$ structure with the following atomic positions in space group $\mathrm{P} 6 / \mathrm{mmm}$ :

$$
\begin{array}{ll}
1 \mathrm{lul}^{\mathrm{u}} & \text { in } 0,0,0 \\
2 \mathrm{Ni} & \text { in } 1 / 3,2 / 3,0 ; 2 / 3,1 / 3,0 \\
3 \mathrm{Ni}_{\mathrm{II}} & \text { in } 1 / 2,0,1 / 2 ; 0,1 / 2,1 / 2 ; 1 / 2,1 / 2,1 / 2
\end{array}
$$

The calculated density is $10.82 \mathrm{gm} . \mathrm{cm}^{-3}$.

Interatemic distances are bister

From O. J. C. Runnalls, Can. J. Chem., vol. 3t, p. 140. 1956 .

INTERATOMIC DTSTANCES IN PUNi

From O. J. C. Runnalls, Can. J, Chem., vol, 34, p. 142. 1956.

Cromer and Olsent then published a rhombohedral cell for PuNi ${ }_{3}$ with a $\cdots 6.22$ and $\cdots 33^{\circ} 44^{\prime}$ but this did not fit our pattern of $\mathrm{PuCo}_{3}$; the nonprimitive hexagonal cell $(\mathrm{a}=5.5 .00, \mathrm{c}=24.35 \mathrm{~A})$ mentioned in the text of reference $t$ dirl fit out pattern, however. These two cells are not equivalent and it appeared that the rhombohedral cell of PuNi ${ }_{3}$ should have been reported as a: $8.615,-33^{\circ} 44^{\prime}$, in which case agreement with our patterns was obtained . The first 21 ohserved lines of the pattern of $\mathrm{P}^{\mathrm{u}} \mathrm{Co} \mathrm{Co}_{3}$ agreed very we]l with the first 28 calculated lines (including 6 overlaps) tor a cell 8. (35) $\mathrm{A}, \cdots 33^{\circ} 40^{\circ}$, but the higher angle lines showed less satisfactory agreement.

4 Cromer D. T. and Olsen C. E. 'The Crystal Structures of $\mathrm{PuNi}_{3}$ and $\mathrm{CeNi}_{3}$.' Acta Cryst. 12 689-94 (1959).

1 Cromer and Olsen are aware of the error and state that the a parameter of the rhomhohedral cell should in fact be reported as $8.615 \mathrm{~A}$ in their paper. From D. M. l'oole et al. in Plutonium 1960, p. 277.

$\mathrm{PuNi}_{3}$

\begin{tabular}{|c|c|c|c|}
\hline Atom & Neighbor & Number & Distance \\
\hline \multirow[t]{3}{*}{$\mathrm{P}^{\prime} \mathrm{u}_{1}$} & $\mathrm{Pu}_{2}$ & 2 & $3.44 \mathrm{~A}$ \\
\hline & $\mathrm{Ni}_{2}^{2}$ & 6 & 2.89 \\
\hline & $\mathrm{Ni}_{3}^{2}$ & 12 & 3.21 \\
\hline \multirow{5}{*}{$\mathrm{Pu}_{2}$} & $\mathrm{Pu} \mathrm{u}_{1}$ & 1 & 3.44 \\
\hline & $\mathrm{Pu}_{2}^{1}$ & 3 & 3.14 \\
\hline & $\mathrm{Ni}_{1}^{2}$ & 3 & 2.95 \\
\hline & $\mathrm{Ni}_{3}^{1}$ & 3 & 3.02 \\
\hline & $\mathrm{Ni}_{3}^{3}$ & 6 & 2.88 \\
\hline \multirow{2}{*}{$\mathrm{Ni}_{1}$} & $\mathrm{Pu}_{2}$ & 6 & 2.95 \\
\hline & $\mathrm{Ni}_{3}^{2}$ & 6 & 2.50 \\
\hline \multirow{3}{*}{$\mathrm{Ni}_{2}$} & $\mathrm{Pu}_{1}$ & 3 & 2.89 \\
\hline & $\mathrm{Ni}_{2}{ }_{2}$ & 3 & 2.89 \\
\hline & $\mathrm{Ni}_{3}^{2}$ & 6 & 2.50 \\
\hline \multirow[t]{6}{*}{$\mathrm{Ni}_{3}$} & $\mathrm{Pu}_{1}$ & 2 & 3.21 \\
\hline & $\mathrm{Pu}_{0}$ & 1 & 3.02 \\
\hline & $\mathrm{Pu}_{2}^{2}$ & 2 & 2.88 \\
\hline & $\mathrm{Ni}_{1}^{2}$ & 1 & 2.50 \\
\hline & $\mathrm{Ni}_{2}^{1}$ & 2 & 2.50 \\
\hline & $\mathrm{Ni}_{3}^{2}$ & 4 & 2.50 \\
\hline
\end{tabular}

The structure of $\mathrm{PuNi}_{3}$ and the structure and composition of $\mathrm{CeNi}_{3}$ have been determined by single crystal X-ray methods. $\mathrm{PuNi}_{3}$ has three formula units in a rhombohedral unit ccll with $a=6.22 \mathrm{~A}$ and $=33^{\circ} 44^{\prime}$, probable space group $\mathrm{R} 3 \mathrm{~m}$. Calculated density $=11.8 \mathrm{~g} \mathrm{~cm}-3$ (measured density - 11.8)

with $1 \mathrm{Pu}$ in $1 \mathrm{a}(0,0,0), 2 \mathrm{Pu}$ in $2 \mathrm{c}(\mathrm{x}, \mathrm{x}, \mathrm{x})$ with $\mathrm{x} \approx 1 / 7$; $1 \mathrm{Ni}$ in $1 \mathrm{~b}(1 / 2,1 / 2,1 / 2), 2 \mathrm{Ni}$ in $2 \mathrm{c}(\mathrm{x}, \mathrm{x}, \mathrm{x})$ with $\mathrm{x} \approx 1 / 3$; and $6 \mathrm{Ni}$ in $6 \mathrm{~h}(\mathrm{x}, \mathrm{x}, \mathrm{z})$ with $\mathrm{x} \approx 7 / 12$ and $\mathrm{z}-1 / 12$

From D. T. Cromer and C. E. Olsen, Acta Cryst., vol. 12, pp. 689, 690.

RESULTS OF THE LEAST-SQUARES REFINEMENT OF $\mathrm{PuNi}_{3}{\text { WITH } \boldsymbol{A f}_{\mathrm{Pu}}}_{\mathrm{Pu}}=-20$ ELECTRONS

From D. T. Cromer and C. E. Olsen, Acta Cryst, , vol. 12, p. 691.

\begin{tabular}{ccccc} 
Atom & \multicolumn{1}{c}{$\mathrm{x}$} & $\mathrm{y}$ & $\mathrm{z}$ & $\mathrm{B}\left(\mathrm{A}^{2}\right)$ \\
\cline { 2 - 5 } $\mathrm{Pu}_{1}$ & 0 & 0 & 0 & $0.37 \pm 0.11$ \\
$\mathrm{I}_{1} \mathrm{u}_{2}$ & 0 & 0 & $0.1414 \pm 0.0002$ & $0.59 \pm 0.08$ \\
$\mathrm{Ni}^{2}$ & 0 & 0 & $1 / 2$ & $0.58 \pm 0.30$ \\
$\mathrm{Ni}_{2}$ & 0 & 0 & $0.3336 \pm 0.0005$ & $0.45 \pm 0.20$ \\
$\mathrm{Ni}_{33}$ & $0.5002 \pm 0.0013$ & $-\mathrm{x}$ & $0.0829 \pm 0.0002$ & $1.10 \pm 0.11$
\end{tabular}

INTERATOMIC DISTANCES IN PUNi

From D. T. Cromer and C. E. Olsen, Acta Cryst., vol. 12, p. 694 .

These structures are both derived from stacking single layers of the $\mathrm{MNi}_{5}$ structure ( $\mathrm{CaCu}_{5}$-type) and double layers of the $\mathrm{MNi}_{2}$ structure $\left(\mathrm{Cu}_{2} \mathrm{M}\right.$-type $)$. 

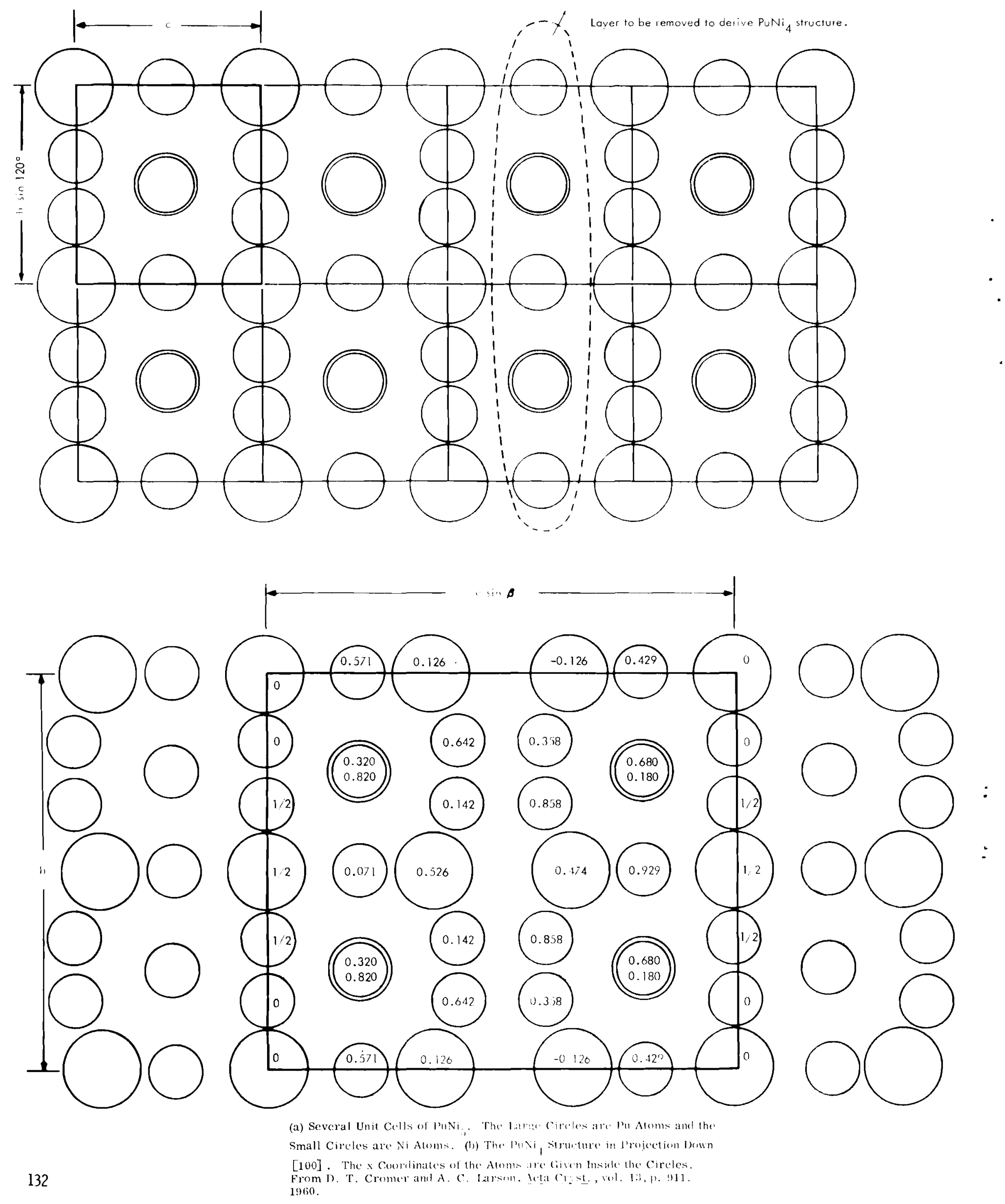
$\mathrm{PuNi}_{4}$

The structure of $\mathrm{PuNi}_{4}$ has been lictermined by single crystal $\mathrm{X}$-ray methods. The unit cell is mono-clinic, space group $\mathrm{C} 2 / \mathrm{m}$ with

$$
\mathrm{a}=4.87, \mathrm{~b}=8.46, \mathrm{c}=-10.27 \mathrm{~A}, \boldsymbol{\beta}=100^{\circ} .
$$

There are six formula units per unit cell. Two of the six Pu atoms have a atoms have a coordination somewhat similar to the $\mathrm{p}_{\mathrm{u}}$ in $\mathrm{PuNi}_{5}$. The $\mathrm{PuNi}_{4}$ structure can be ohtained by removing the $\mathrm{Ni}$ atoms at $\mathrm{z}=1 / 2$ from every third unit cell of $\mathrm{p}_{u \mathrm{Ni}_{5}}$ and by slightly shifting the remaining atoms. Calculated density is therefore $11.3 \mathrm{~g} \mathrm{~cm}^{-3}$.

From D. T. Cromer and A. C. Larson, Acta Cryst., vol. 13, p. 909. 1960. FINAL IHAST-SQUARES PARAMETERS FOR PUNi

From D. T. Cromer and A. C Lirson, Acta Cryst., vol. 13, p. 910.1960.

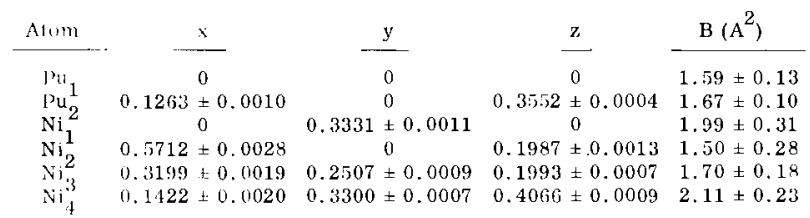

INTERATOMIC DISTANCES IN PuNi ${ }_{4}$

The number of neighbors of a given kind is in parentheses

Errm 1). T. Cromer and A. C. Larson, Acta Crysl., vol. 13, p. 909.1960.

$\begin{array}{rl}\mathrm{Pu}_{1}-\mathrm{I}^{\mathrm{P} \mathrm{u}_{2}} & 3.59(2) \mathrm{A} \\ -\mathrm{Ni}{ }^{2} & 3.15(2) \\ -\mathrm{Ni} & 3.16(2) \\ -\mathrm{Ni} 1_{2} & 2.81(4) \\ -\mathrm{Ni}_{2} & 2.82(2) \\ -\mathrm{Ni}_{3}^{2} & 3.16(4) \\ -\mathrm{Ni}_{3}^{3} & 3.17(4)\end{array}$

$\begin{array}{rl}\mathrm{Pu}_{2}-\mathrm{Pu}_{2} & 3.59(1) \mathrm{A} \\ -\mathrm{Pu}_{1} & 3.41(1) \\ -\mathrm{Ni}^{2} & 2.89(1) \\ -\mathrm{Ni} & 2.91(1) \\ -\mathrm{Ni} & 2.90(1) \\ -\mathrm{Ni}_{3} & 2.91(1) \\ -\mathrm{Ni}_{3} & 2.84(2) \\ -\mathrm{Ni}_{4} & 2.86(2) \\ -\mathrm{Ni}_{4} & 2.89(2) \\ -\mathrm{Ni}_{4} & 2.89(2)\end{array}$

Magnetic Susceptibility

PARAMAGNETIC SUSCEPTIBILITIES OF PLUTONIUM-NICKEL COMPOUNDS From S. T. Konobeevsky et al. in Proc. Second Geneva Internal. Conf., vol. $6,1,195.1958$.

\begin{tabular}{|c|c|c|c|}
\hline $\begin{array}{r}\mathrm{Ni}_{1}-\mathrm{Pu}_{-} \mathrm{Pu}_{1} \\
-\mathrm{Pu} 1 \\
-\mathrm{Pu}_{2}^{2} \\
-\mathrm{Ni}_{2}^{2} \\
-\mathrm{Ni}_{2}^{2} \\
-\mathrm{Ni}_{4}^{3} \\
-\mathrm{Ni}_{4}^{3}\end{array}$ & $\begin{array}{l}3.15(1) \text { A } \\
3.16(1) \\
2.89(1) \\
2.91(1) \\
2.45(2) \\
2.43(2) \\
2.45(2) \\
2.55(2)\end{array}$ & $\begin{aligned} \mathrm{Ni}_{2} & -\mathrm{pu}_{1}{ }_{1} \\
& -\mathrm{Pu}_{1} \\
& -\mathrm{Ni}_{1}{ }_{1} \\
& -\mathrm{Ni}_{1} \\
& -\mathrm{Ni}_{2}^{2} \\
& -\mathrm{Ni}_{3} \\
& -\mathrm{Ni}_{3}{ }_{3}\end{aligned}$ & $\begin{array}{l}2.81(2) \mathrm{A} \\
2.82(1) \\
2.45(2) \\
2.81(2) \\
2.82(1) \\
2.45(2) \\
2.46(2)\end{array}$ \\
\hline 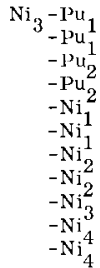 & $\begin{array}{l}3.16(1) \text { A } \\
3.17(1) \\
2.90(1) \\
2.91(1) \\
2.43(1) \\
2.45(1) \\
2.45(1) \\
2.46(1) \\
2.43(2) \\
2.51(1) \\
2.52(1)\end{array}$ & $\begin{aligned} \mathrm{Ni}_{4} & -\mathrm{Pu}_{2} \\
& -\mathrm{Pu}_{2} \\
& -\mathrm{Pu}_{2} \\
& -\mathrm{Pu}_{2} \\
& -\mathrm{Ni}_{2} \\
& -\mathrm{Ni}_{1} \\
& -\mathrm{Ni}_{3} \\
& -\mathrm{Ni}_{4}^{3} \\
& -\mathrm{Ni}_{4} \\
& -\mathrm{Ni}_{4} \\
-\mathrm{Ni}_{4} & \end{aligned}$ & $\begin{array}{l}2.84(1) \text { A } \\
2.86(1) \\
2.89(1) \\
2.89(1) \\
2.55(1) \\
2.51(1) \\
2.52(1) \\
2.43(1) \\
2.55(1) \\
2.79(2) \\
2.88(1)\end{array}$ \\
\hline
\end{tabular}

$\mathrm{Pu}_{2} \mathrm{Ni}_{17}$

Since $\mathrm{PuNi}_{5}$ proved to be isostructural with $\mathrm{ThNi}_{5}$, a search was made for a $\mathrm{Pu}-\mathrm{Ni}$ compound isomorphous with $\mathrm{Th}_{2} \mathrm{Ni}$. A phase with an $\mathrm{X}$-ray pattern similar to, but more complex than, $\mathrm{PuNi}_{5}$ was observed in alloys containing 30-35 wt. $\mathrm{Pu}$. This pattern was solved using a hexagonal lattice of dimensions

$$
\begin{aligned}
& \mathrm{a}_{\mathrm{o}}=8.30 \pm 0.01 \mathrm{~A} \\
& \mathrm{c}_{\mathrm{o}}=8.00+0.01 \mathrm{~A},
\end{aligned}
$$

The isomorphous phase $\mathrm{Th}_{2} \mathrm{Ni}_{17}$ has $\mathrm{a}_{0}=8.37 \mathrm{~A}$ and $\mathrm{c}_{0}=8.14 \mathrm{~A}(6)$. Since the intensities in the powder patterns of $\mathrm{Th}_{2} \mathrm{Ni}_{17}$ and $\mathrm{Pu}_{2} \mathrm{Ni}_{17}$ are closely related it seems reasonable to assign the $\mathrm{Th}_{2} \mathrm{Ni}_{17}$ structure to $\mathrm{Pu}_{2} \mathrm{Ni}_{17}$. The following approximate atomic positions apply in space group $\mathrm{P}_{3} / \mathrm{mmc}$, for two molecules per unit cell:

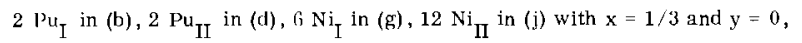

$12 \mathrm{Ni}_{\mathrm{IIl}}$ in (k) with $\mathrm{x}=1 / 6$ and $\mathrm{z}=0$, and $4 \mathrm{Ni}_{\mathrm{IV}}$ in (f) with $\mathrm{z}=0.11$.

From O. J. C. Runnalls, Can. J. Chem., vol. 34, pp. 140, 142. 1956.

Ni 204.1

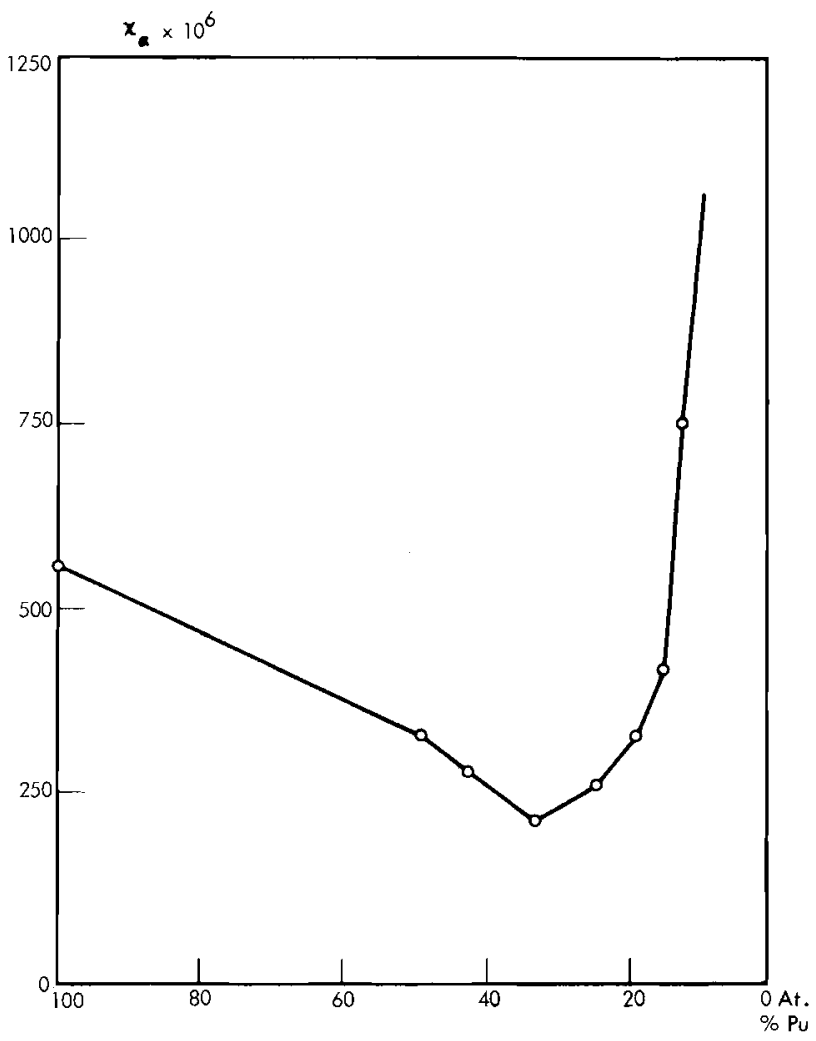

Paramagnetic Susceptibility of Plutonium Nickel Alloys at Room Temperature. From S. T. Konobeevsky et al. in Proc. Second Gemperature. From S. T. Konobeevsky $\frac{\mathrm{et}}{19} \overline{\frac{a}{8}}$ 


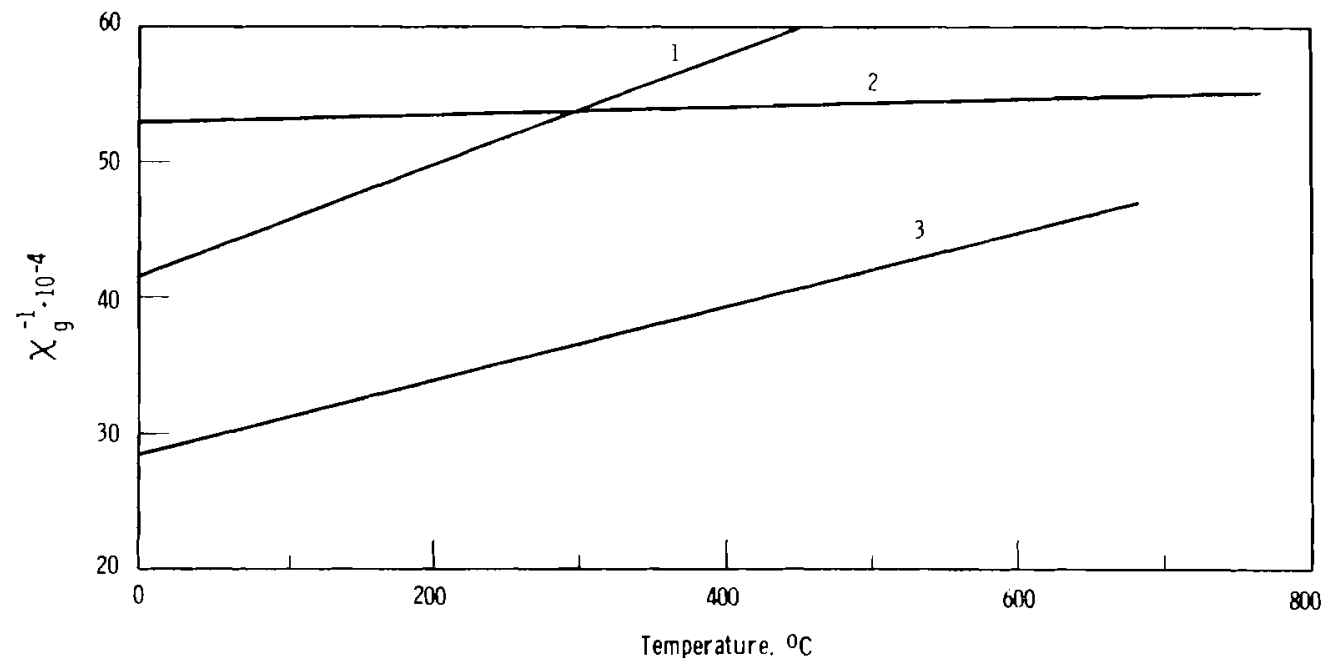

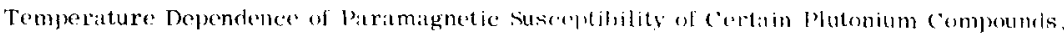

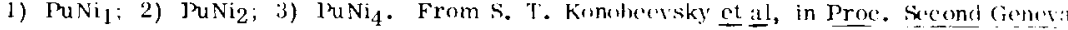
Intornit. Conf., vol. 6, p. 195. 195k.

\section{$\mathrm{Ni} 301$ Tensile properties}

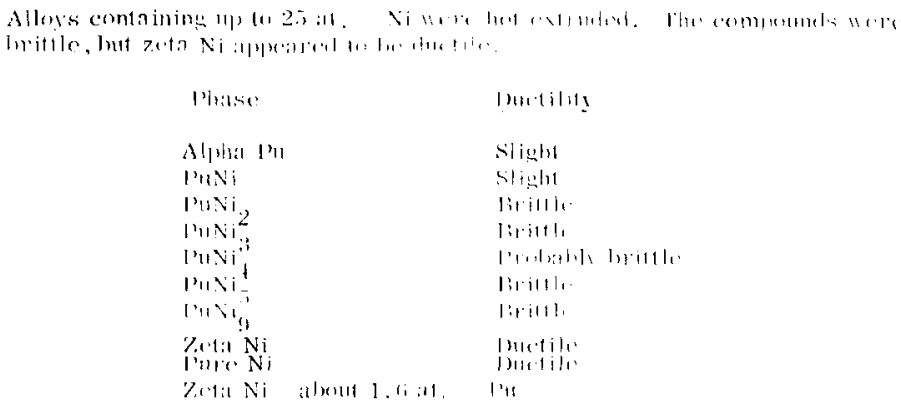

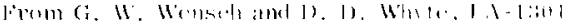

\section{Ni 307 Harresess}

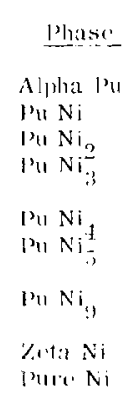

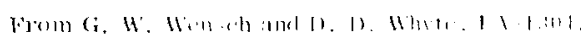

$$
\begin{aligned}
& \text { Bumalis. } \\
& \text { Slight fuclilin } \\
& \text { stishl letedilil. } \\
& \text { birite } \\
& \text { [3. } \\
& \text { L'alable levirle } \\
& \text { Irritll. } \\
& \text { I3irila. } \\
& \text { I) } 1110 \\
& \text { [rus. il. }
\end{aligned}
$$




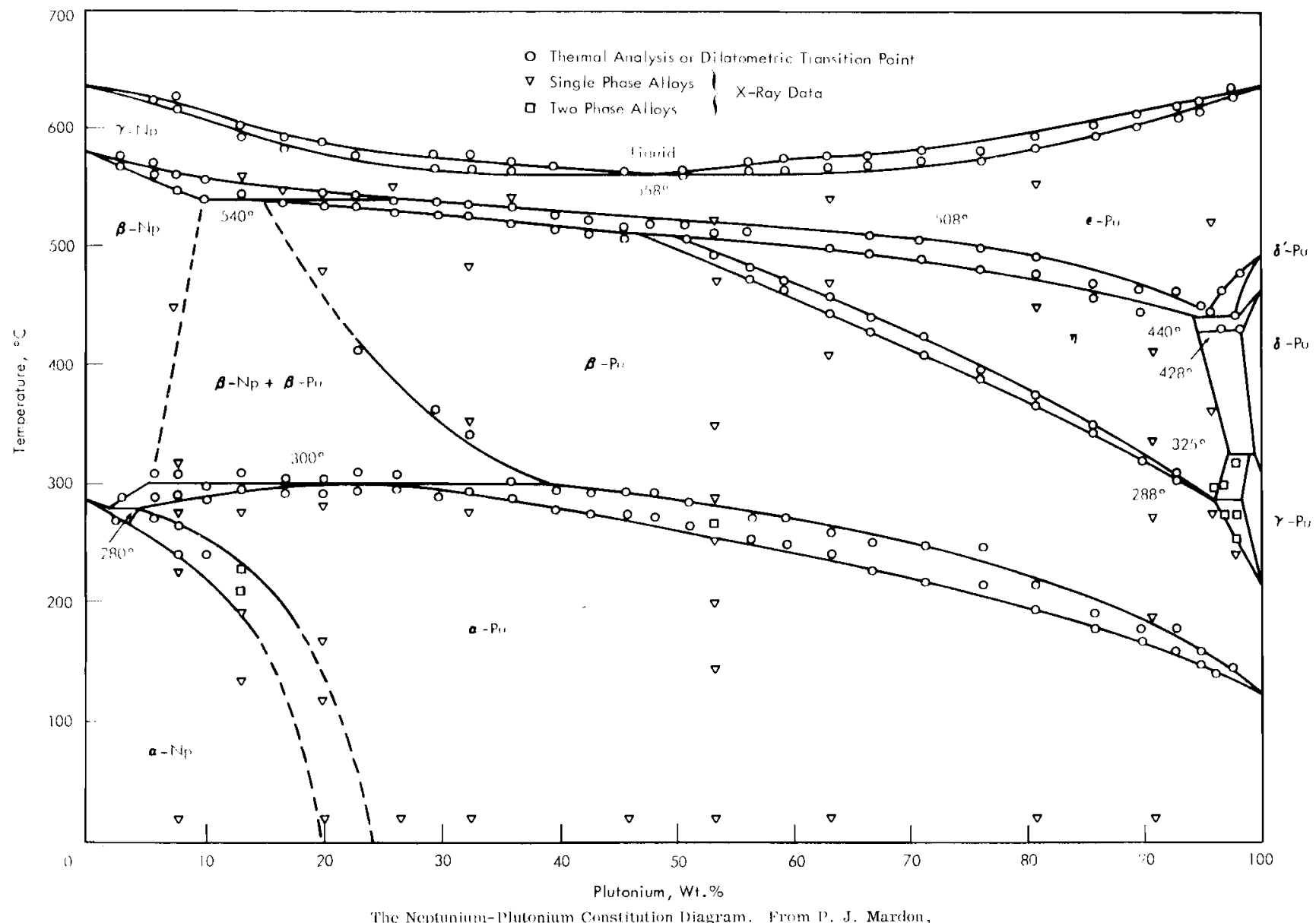

The Nepturium-luturonium Canstitution Jiagram. Flom [2. J. Mardon,

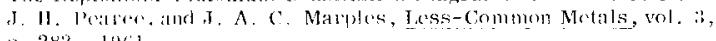

1). 2 2,2. 1 !ril.

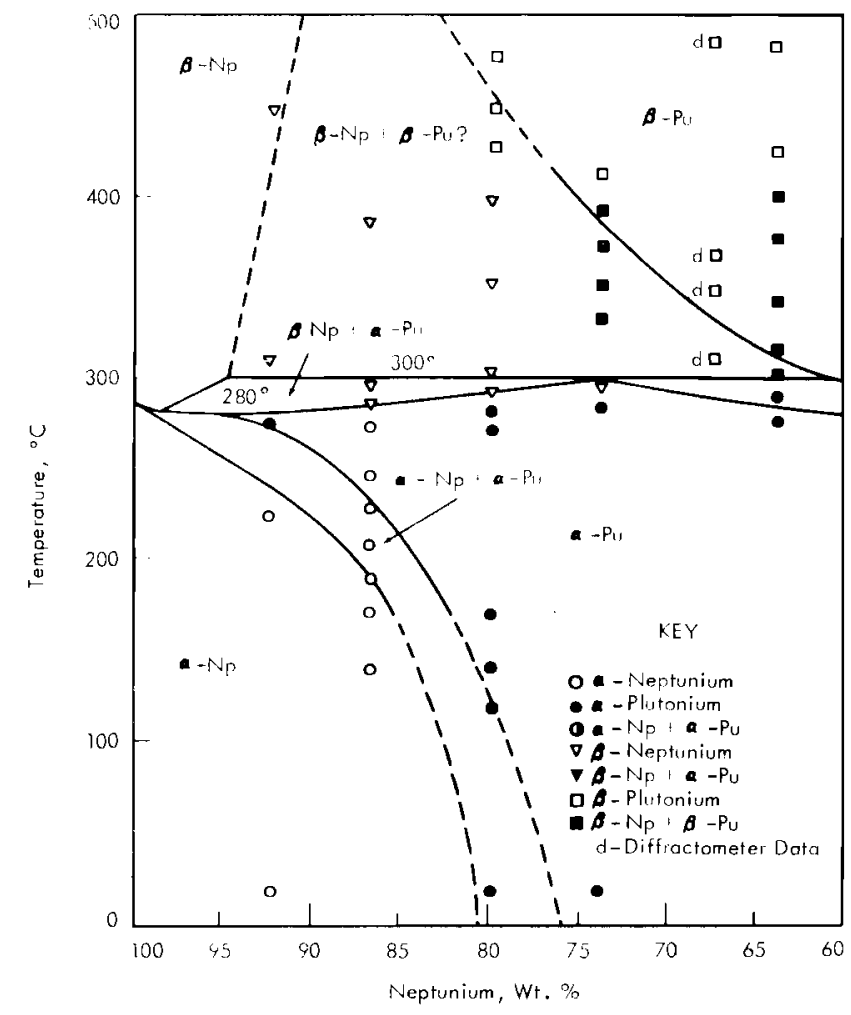

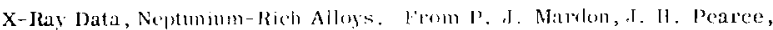

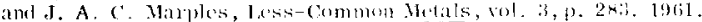

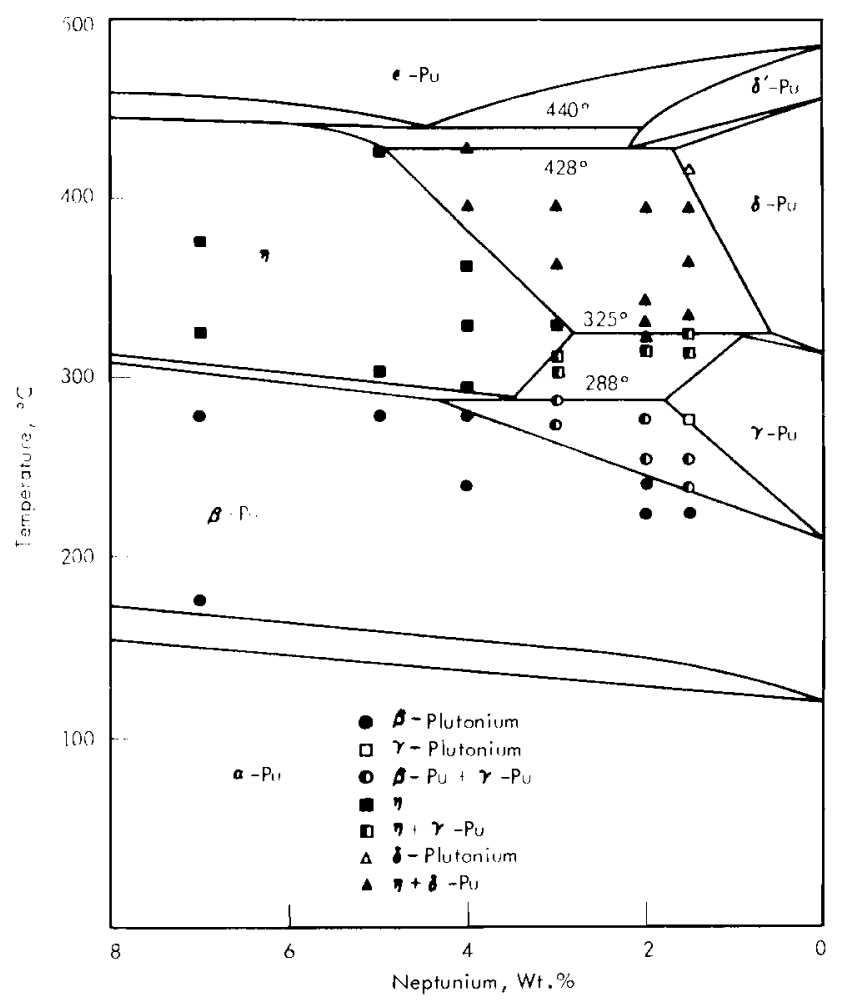

X-Ra bata, Plutomium-Rich Allows. From P. J. Mardon, J. H. Pearee,

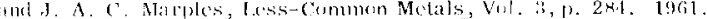




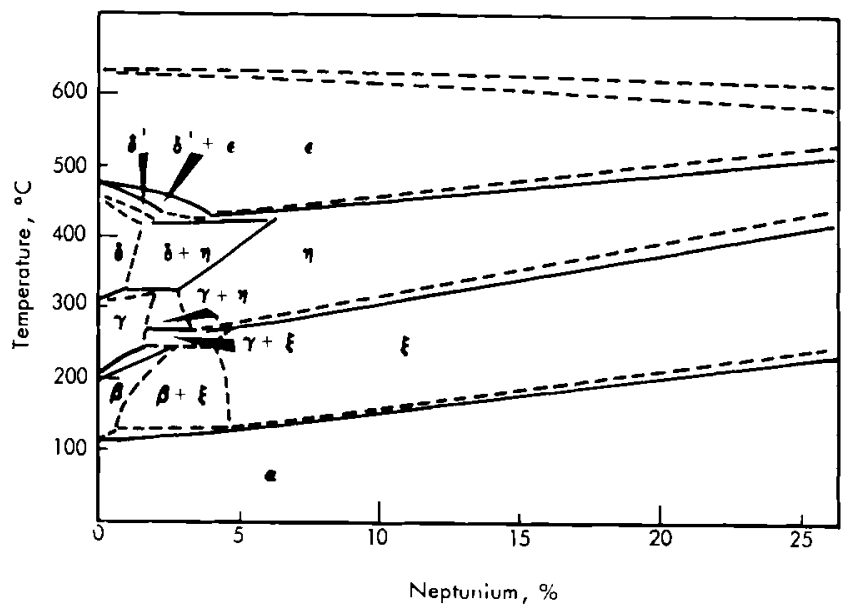

The Pu-Np Phase Diagram (From Dilatometric Results). From R. G. Cope et al. in Plutonium 1960, p. 288 .

\section{Np 103 crrstat structure}

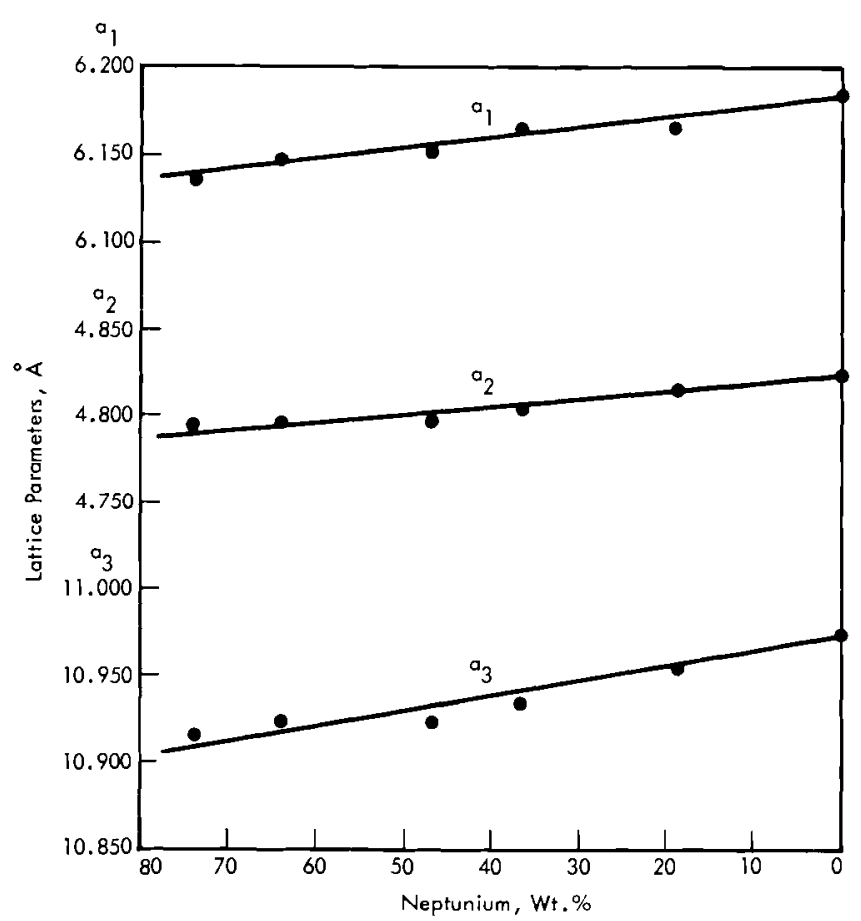

Lattice Parameter - Composition Curves, -Plutonium Field $\left(20^{\circ} \mathrm{C}\right)$.

From P. J. Mardon, J. H. Pearce, and J. A. C. Marples, Less-Common Metals, vol. 3,p. 284. 1961.

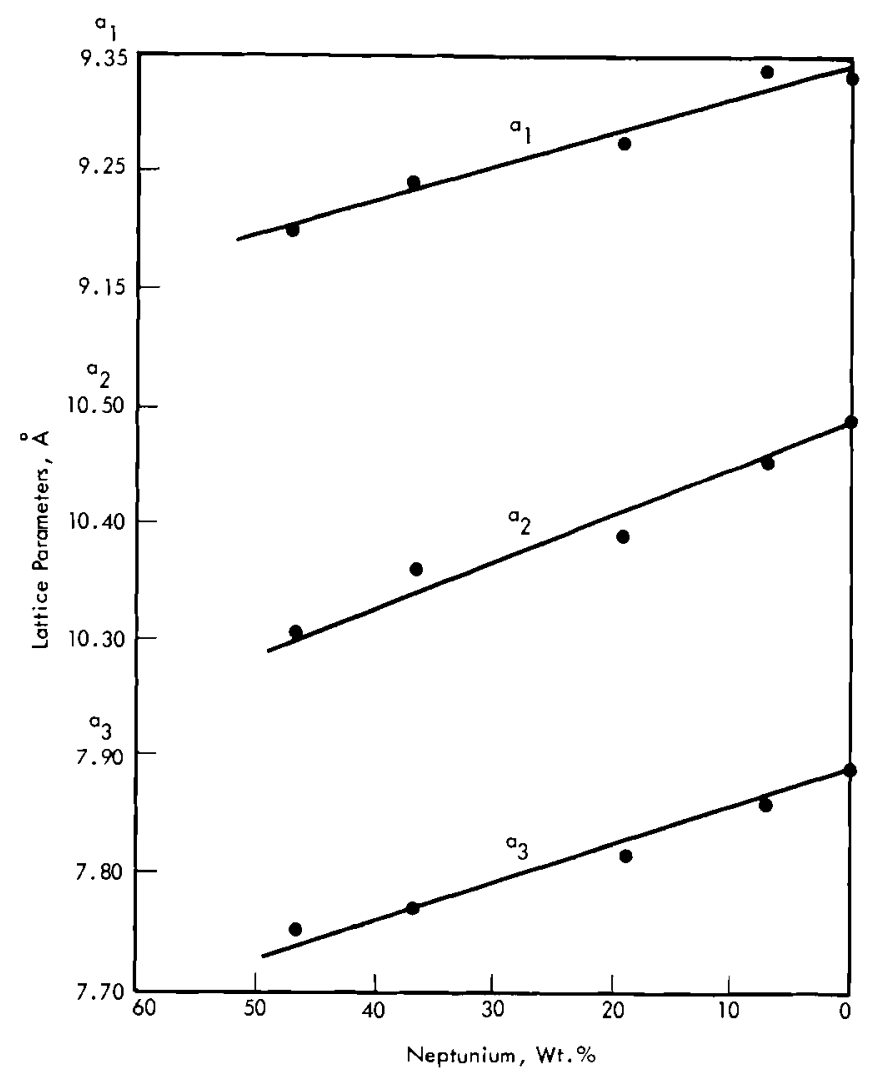

Lattice Parameter - Composition Curves, -Plutonium Field $\left(290^{\circ} \mathrm{C}\right)$

From P. J. Mardon, J. H. Pearce, and J. A. C. Marples, Less-Common Metals, vol. 3. p. 285,1961 


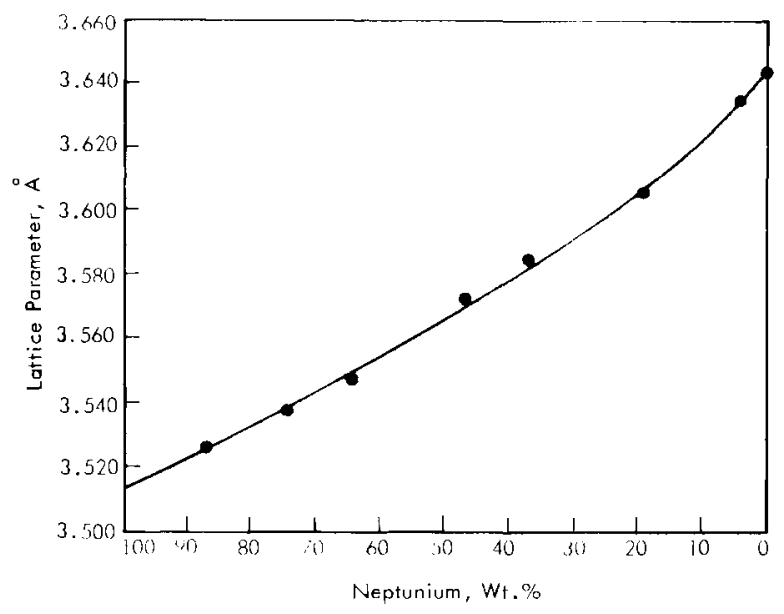

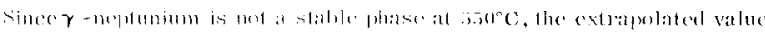

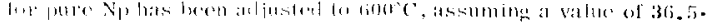

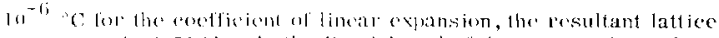

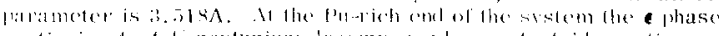

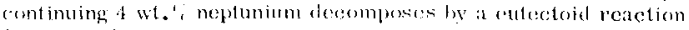

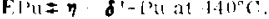

Als intermediate phase exists at high femperatures at the Pu-rich end of the $\mathrm{Ni}-\mathrm{Pu}$ system. Its crysial structure is complex. An orthorhombic cell has linen assigned to it with a $10.86 \mathrm{~A}, \mathrm{~b}-10.67 \mathrm{~A}, \mathrm{c}=10.43 \mathrm{~A}$ for a 19 wt. \% Siralloy at $375^{\circ} \mathrm{C}$. Density at $150 \mathrm{C}$ was $17.56 \mathrm{~g} / \mathrm{cm}^{3}$ which corresponds to it atoms per unit coll.

Frem D'. J. Mardon, J. II. P'Alece, and I. A. C. Marplos, Iess-Common Metals, vol, 3, p. $24 i, 1961$

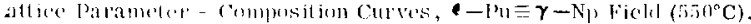

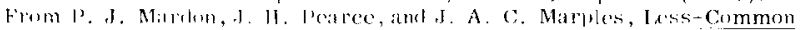

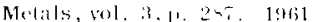

Electrical Conductivity

Np 203.1

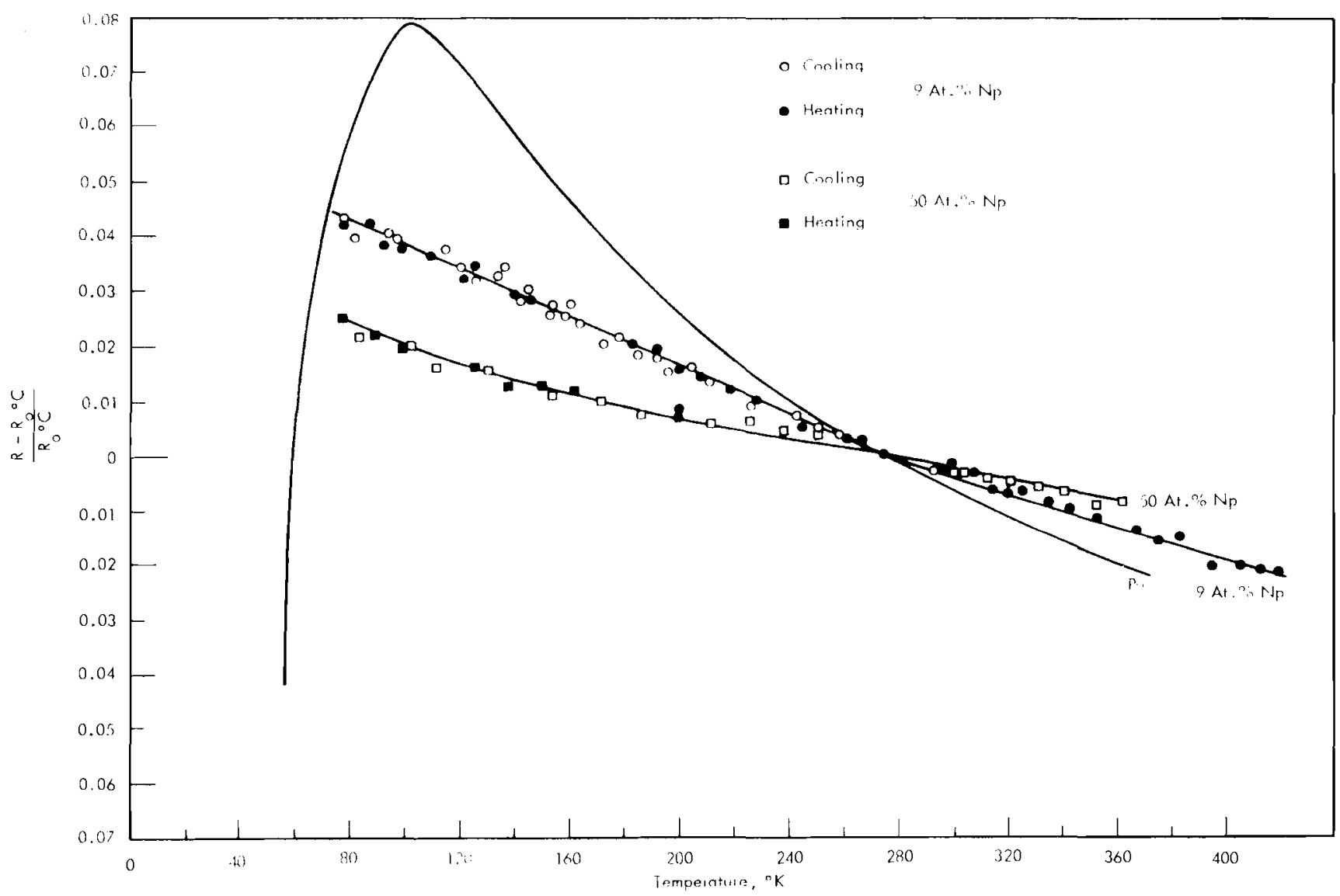

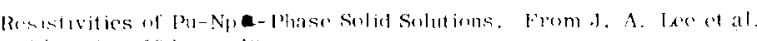

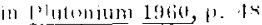




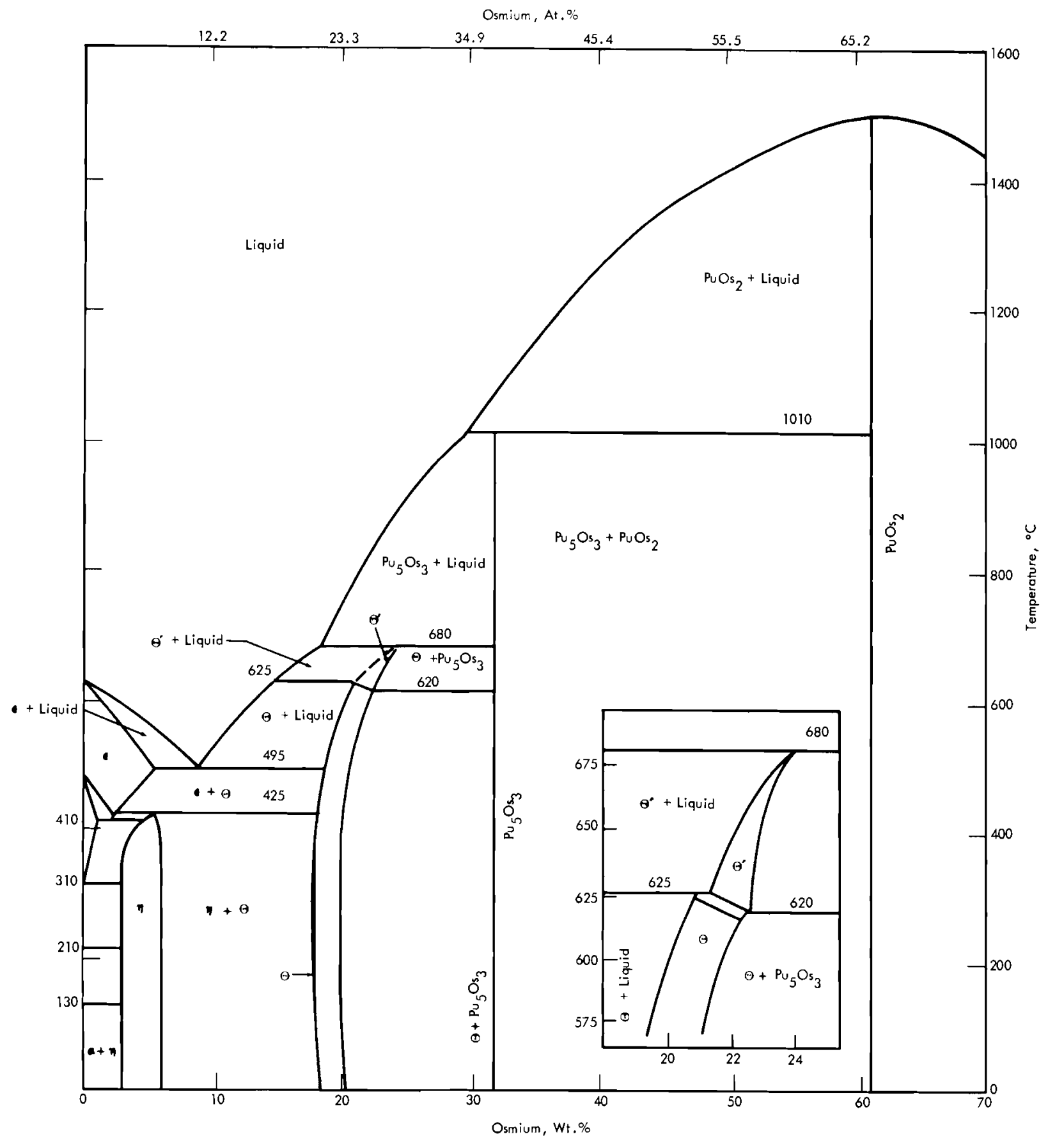

From F. W. Schonfeld et al, in Metallurgy and Fuels, series V, vol. 2, p. 591.1959.

\section{Os 103 crystal structure}

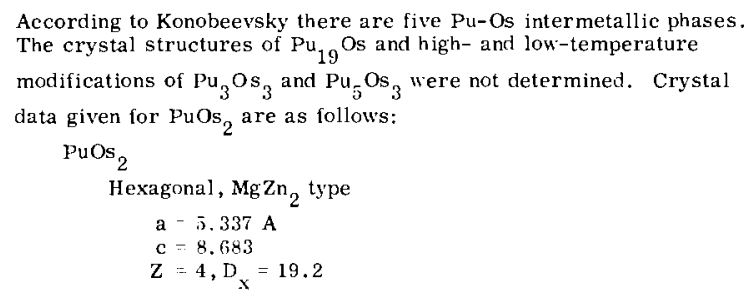




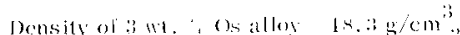

Calculated density of $1 \operatorname{los}_{2}, 19.2 \mathrm{k} / \mathrm{cm}^{2}$

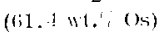

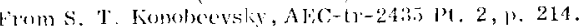

Mechanical Properties

Os 300

Pu-OS alloys in the range 2.7-6i wt. ', Os have good plastic and mochanical propertices.

Foum S. T. Konolyeevsky, AEC-11-2135 I't. 2, p, 214

Phase Diagrams

$\mathrm{Pb} 101$

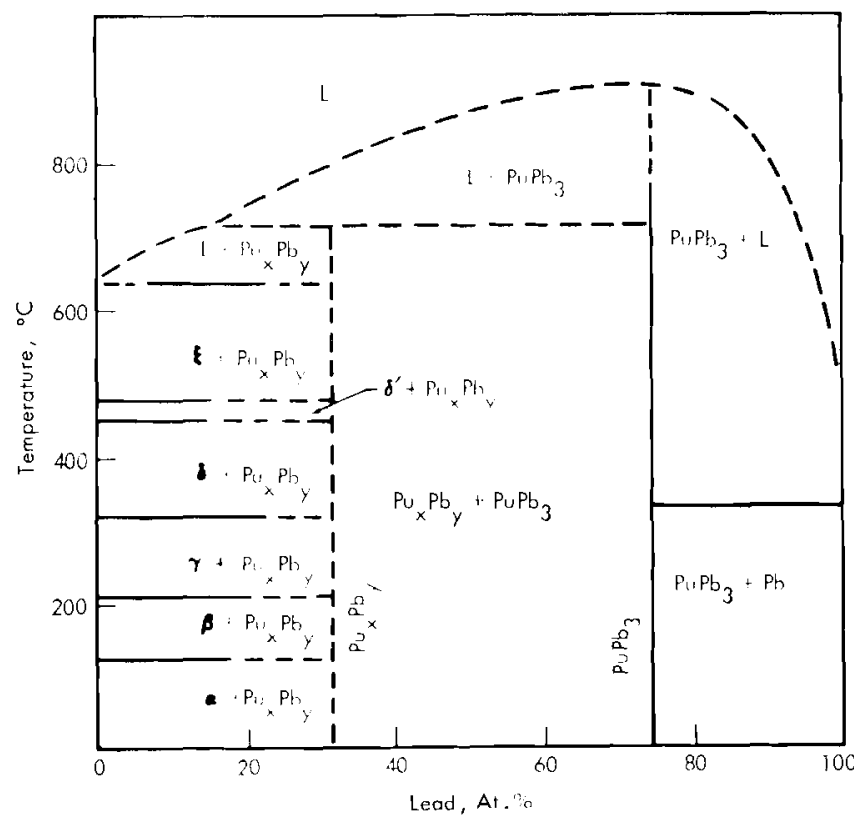

Constitutional Diagram for the Srstem Plutonium-Icad.

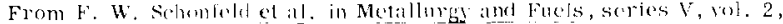

P. .36, 19.in.

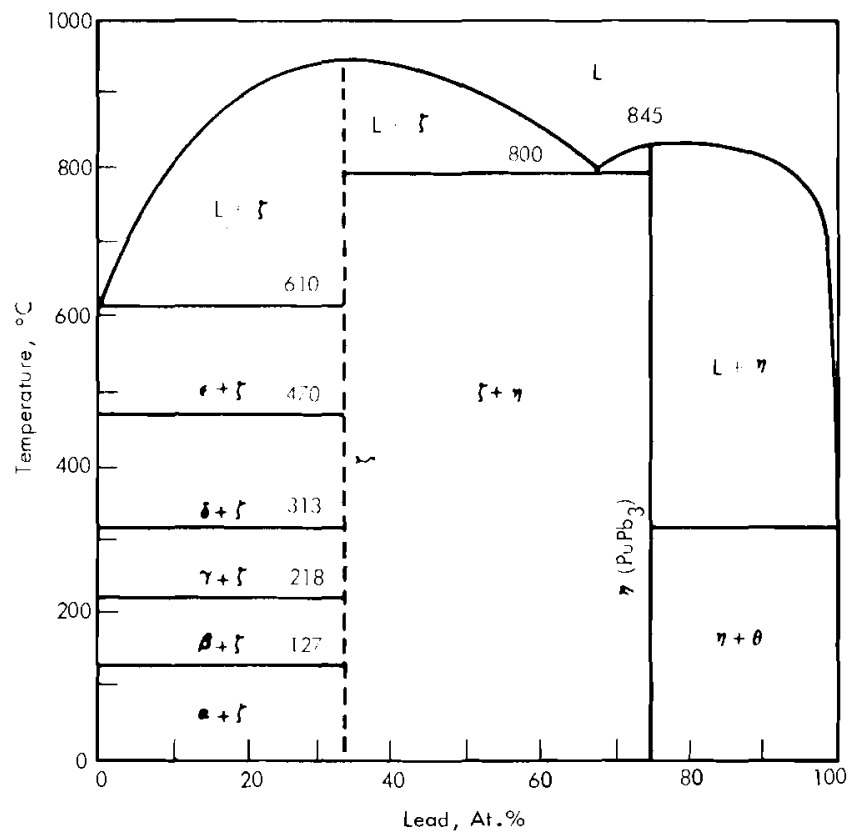

The Plutonium-leart stutem. From $k$. W. Schonfeld in The Metal plutonium, 1). $2 \pi \%$.

\section{Crystal Structure}

${ }^{\prime \prime}\left(1 P^{\prime}\right)_{3}{ }_{3}$

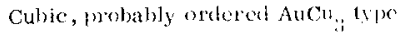

a $4.808+0.0101 \mathrm{~A}$

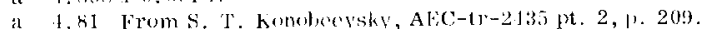

7. 1,1$) \times 12.46 \mathrm{j}$

Space yroup. - Pm3m (Int. Tab. 221)

Atomic positions. - Ste Pulic...

Interatumic distances

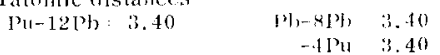

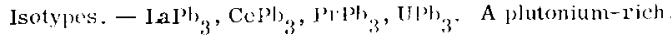




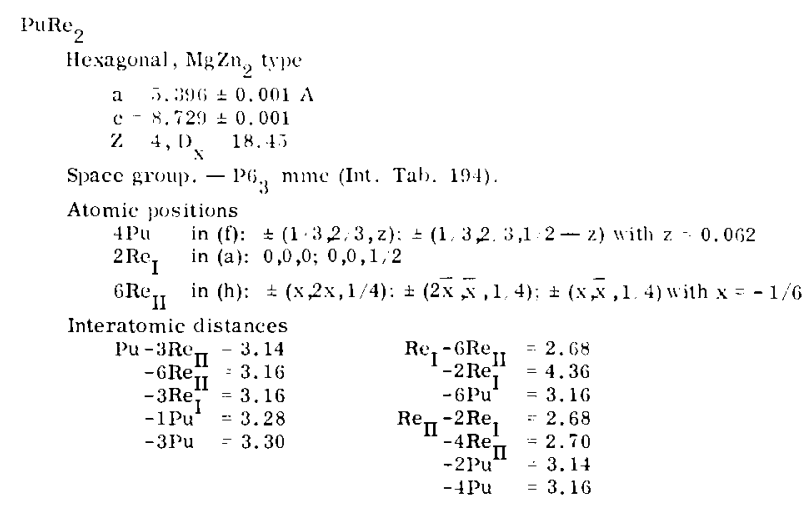

From F. H. Ellinger in The Metal Plutonium, pp. 299, 300.

\section{Ru 101 structural Properties}

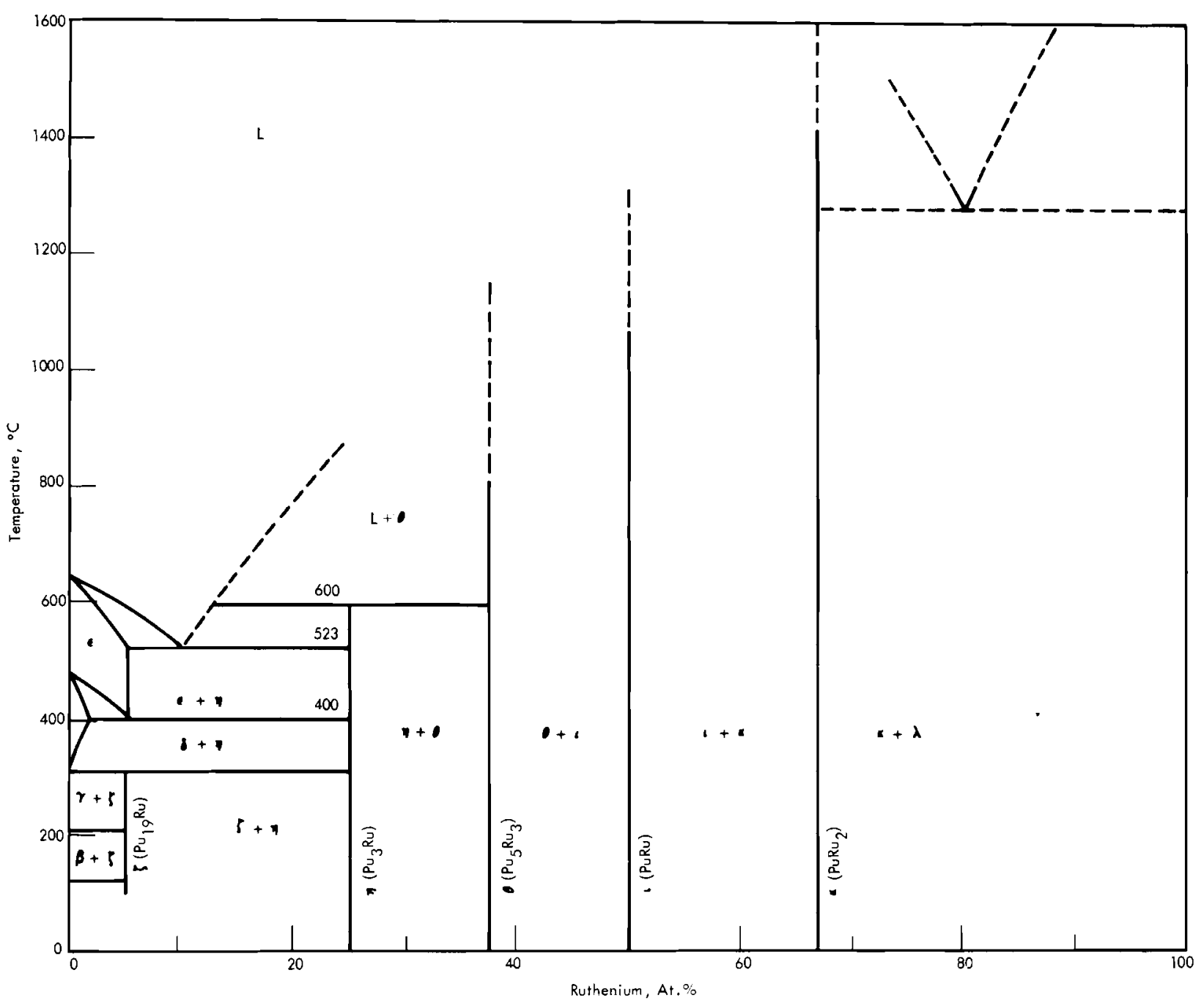


The existence of two plutonium-rich internetallics in this system hits leem established by $X$-ray' and metallographic observations, but the crystal structures are not known. Their compositions fall in the range of $30-40$ at." silicon. Other plutonium-silicon intermetallies are as follows:

Pusi

$$
\begin{gathered}
\text { Orthorhombic, leB tyle } \\
a=5.727 \pm 0.000 \mathrm{~A} \\
b-7.933 \pm 0.003 \\
\mathrm{c}-3.847 \pm 0.001
\end{gathered}
$$

$\mathrm{Z}-4, D_{\mathrm{x}}=10.1$

Sirace group. - Plonm (Int. Tab. (i2).

Atomic positions

4l'u in $(\mathrm{c}): \pm\left(\mathrm{x}_{1}, y_{1}, 1 / 4\right) ; \pm\left(1 / 2-\mathrm{x}_{1}, \mathrm{y}_{1},+1 / 2,1 / 4\right)$

with $x_{1}-0.125, y_{1}-0.180$

\begin{tabular}{|c|c|c|}
\hline$P u-2 S i$ & 2.95 & $S i-2 S i$ \\
\hline$-2 \mathrm{Si}$ & 3.01 & $-2 \mathrm{Pu}=2.95$ \\
\hline$-1 \mathrm{Si}$ & 3.03 & $-2 \mathrm{Pu}=3,01$ \\
\hline$-1 \mathrm{~S} i-$ & 3.07 & $-1 \mathrm{Pu}-3.03$ \\
\hline$-1 \mathrm{Si} \ldots$ & 3.18 & $-1 \mathrm{Pu} \quad 3.07$ \\
\hline$-4 \mathrm{P}^{\mathrm{U}^{-}}$ & 3. 62 & $-1 \mathrm{Pu}=3.18$ \\
\hline
\end{tabular}

$4 \mathrm{Si}$ in (c): $\pm\left(\mathrm{x}_{2}, y_{2}, 1 / 4\right) ; \pm\left(1 / 2-\mathrm{x}_{2}, y_{2}+1 / 2,1 / 4\right)$

with $x_{2}=0.611, y_{2}=0.028$

Interatomic clistancos

Isotypes. - ThSi, USi

IRemarks. - The atumic parameters are those of USi, taken from Zacharissen.

$\mathrm{Pu}_{2} \mathrm{Si}_{3}$

Hexagonal, pseudo- $\mathrm{AlB}_{2}$ type

$a=3.876 \pm 0.002$

c $-4.090 \pm 0.002$

$\mathrm{Z}=1 / 2, \mathrm{D}_{\mathrm{x}} \cdots 8.77$

\section{Sn 101}

Phase Diagrams

A eutectic occurs between $\mathrm{Pu}$ and $\mathrm{Pu}_{4} \mathrm{Sn}(?)$ at about 13 at $\mathrm{Sn}$

From A. S. Coffinberry et al. in Reactor Handbook, 2nd ed, , vol. 1, p. 287.

\section{Sn 103 crystal Strucuture}

Th 101

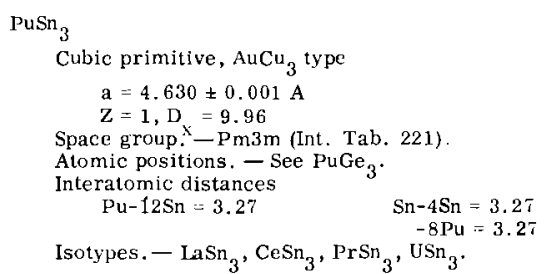

Phase Diagrams

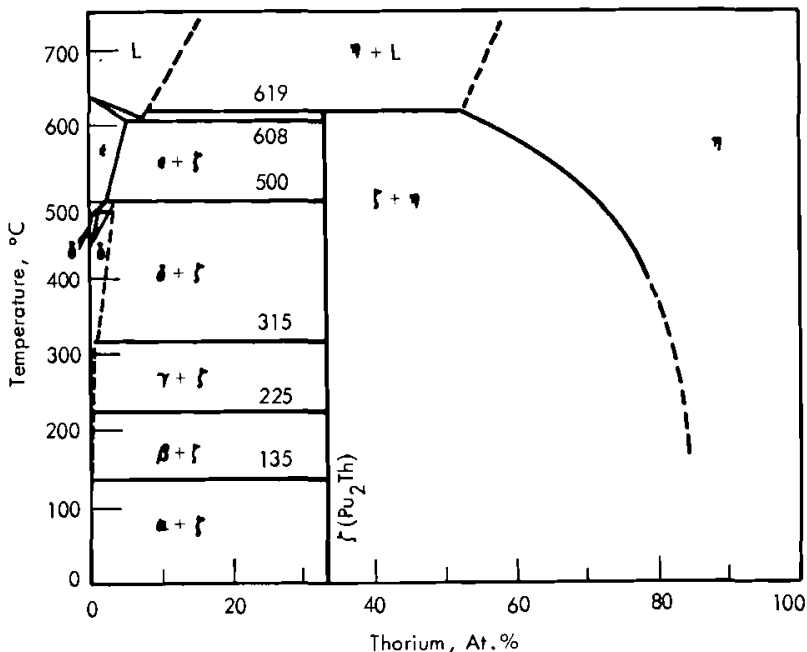

The Plutonium-Thorium System. From $F, W$. Schonfeld et al. in Metallurgy and Fuels, scries V, vol. 2, p. j94. (Same as Fig. 13 


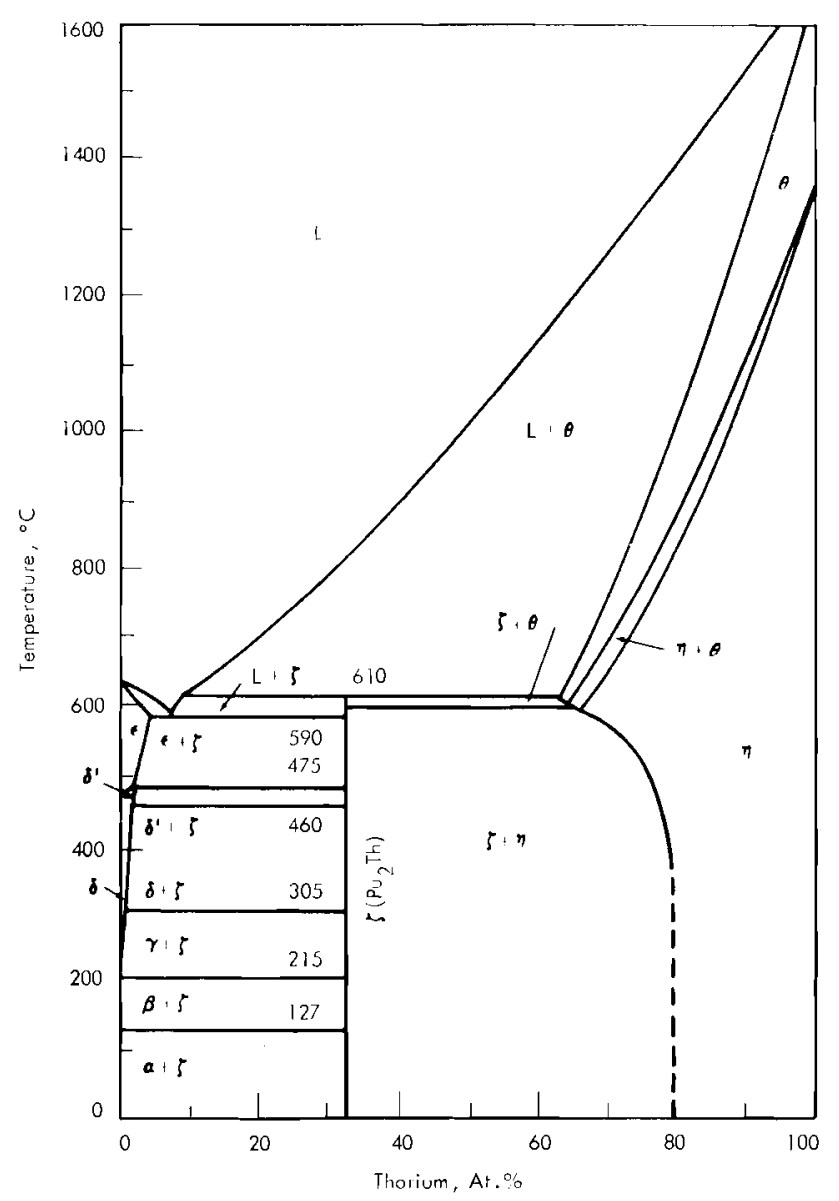

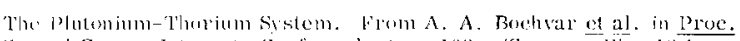

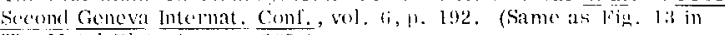

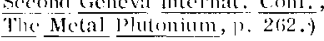

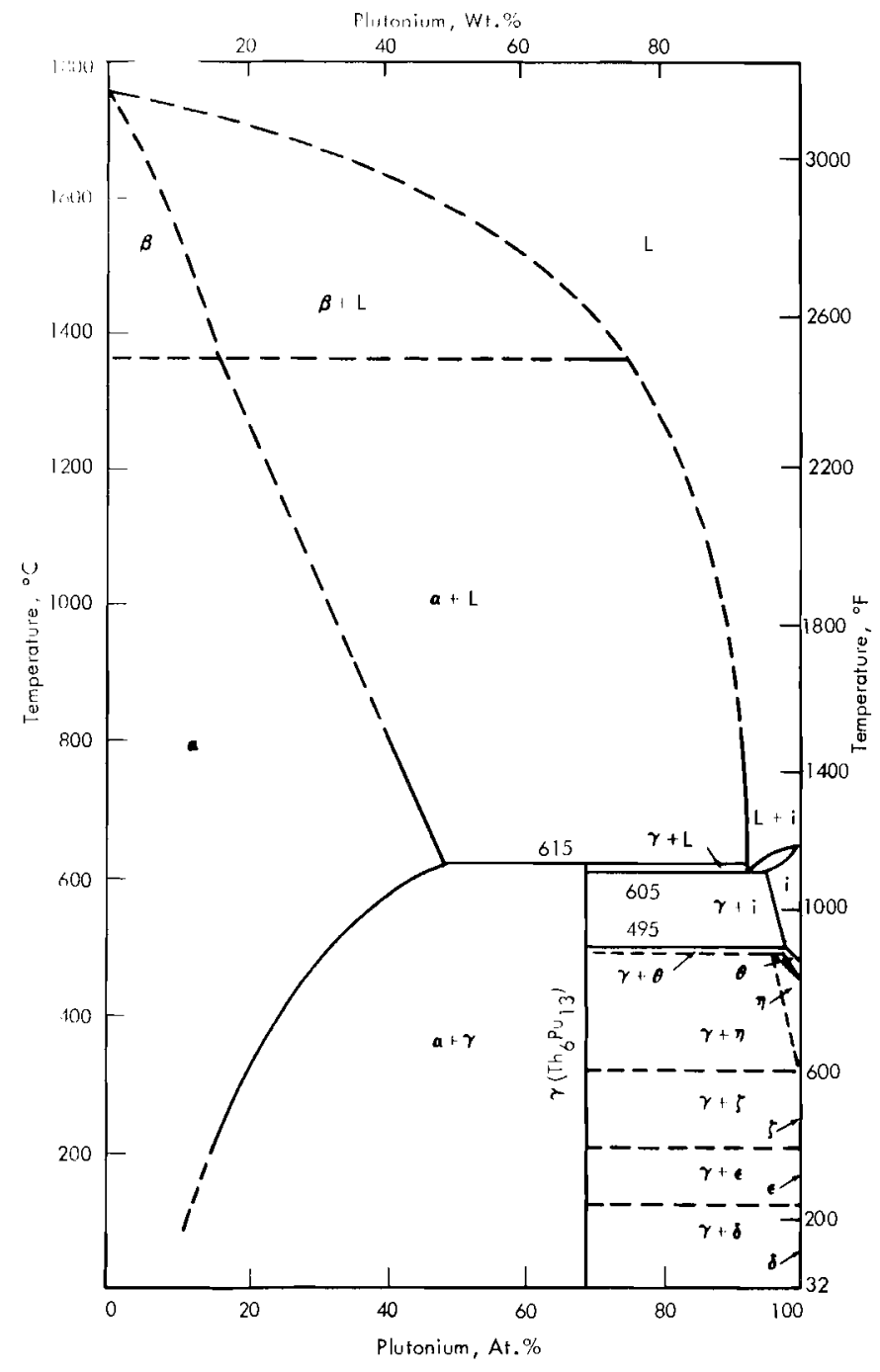

Frm A. A. Bauce and $1 \%$. $\Lambda$. Rough in Metallurgy and frucls, series $V$, W.1. 2, 1. Gith.

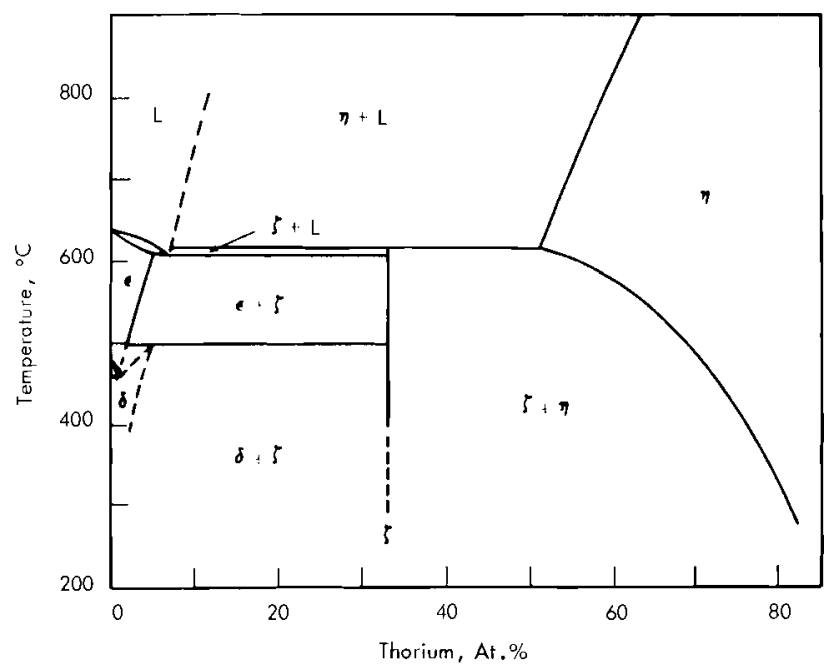

The Plutonium-Thorium System. lirom D. M. Poole, G. K. Williamson, and J. A. C. Majples, J. Inst. Metals, vol. 86, p. 173. 1957-19ij8.

(Same as $r$ ig. 10 in The Netal Plutonium, p. 231.)

Crystal Structure

Th 103

$\mathrm{Pu}_{2} \mathrm{Th}$ (or $\mathrm{Pu}_{1,} \mathrm{Th}_{6}$, according to Harwell) is the only intermcliate phase in the plutonium-thorium system. Its crystal structure is not linown, but the Harwell grou], have tentiatively reported an orthorhombic unit cell containing six formula units and having the dimensions

$\because 1.320=0.007 \mathrm{~A}$

b. $-8.164 \pm 0.00 \% \mathrm{~A}$

$\mathrm{c}=6.681 \pm 0.00 \mathrm{~J} A$

F'rom F, It. Ellinger in The Metal plutenium, p. 303. a $=9.820 \pm 0.005 \mathrm{~A}, \mathrm{~b}-8.164 \pm 0.005 \mathrm{~A}$, and $\mathrm{e}=6.681 \pm 0.005 \mathrm{~A}$. If this phase has the formula $\mathrm{P}_{13} \mathrm{Th}_{6}$ (31.6 per cent Th), good agreement is founc

Iirom M. B. Waldron in The Metal plutonium, p. 233 
Th 201 Density

DENSITY AND SPECIFIC VOLUME OF CRYSTAL BAR THORILM AND THORIUM-PLUTONTUM ALLOYS

From B. Blumenthal, ANL-6671. 1963.

\begin{tabular}{|c|c|c|c|c|c|}
\hline Number & $\begin{array}{l}\text { Nom } \\
\text { Th }\end{array}$ & $w_{u}$ & $\begin{array}{l}\text { Density } \\
\text { at } 25^{\circ} \mathrm{C}, \\
\text { g/ } / \mathrm{cm}^{3}\end{array}$ & $\begin{array}{c}\text { Mean Vialue } \\
\text { at } 2.2^{\circ} \mathrm{C}, \\
\text { g/cm." }\end{array}$ & $\begin{array}{c}\text { Sipece volume } \\
\text { at } 2 . \mathrm{J}^{\circ} \mathrm{C}, \\
\mathrm{cm}^{3} / \mathrm{g}\end{array}$ \\
\hline Bi72 & 100 & 0) & $\begin{array}{l}11.71: 5 \\
11.717\end{array}$ & 11.71, & $0.0 \times .536$ \\
\hline $\mathrm{B} 760$ & 90 & 10 & $\begin{array}{l}11.913 \\
11.916\end{array}$ & 11.914 & $0.0 \times 3913$ \\
\hline$B 767$ & 80 & 20) & $\begin{array}{l}12.134 \\
12.127\end{array}$ & 12.131 & 0.08244 \\
\hline B7fix & 70 & 30 & $\begin{array}{l}12.367 \\
12.374\end{array}$ & 12.371 & 0.08084 \\
\hline B7 69 & 60 & 40 & $\begin{array}{l}12.741 \\
12.746\end{array}$ & 12.744 & 0.07847 \\
\hline $\mathrm{B} 770$ & 50 & .0 & $\begin{array}{l}13.139 \\
13.139\end{array}$ & 13.139 & 0.07611 \\
\hline B773 & 40 & 60 & $\begin{array}{l}13.660 \\
13.670\end{array}$ & 13.665 & 0.07318 \\
\hline
\end{tabular}

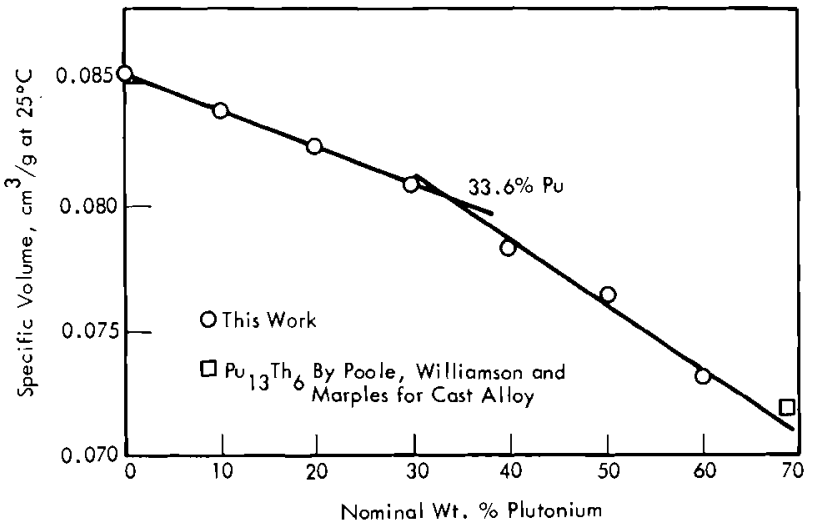

DENSITY OF Th-PU ALLOYS

l'rom Baumruclice and kelman as reported by L. T. Lloyd in WASH-703. 1957.

\begin{tabular}{cc}
$\begin{array}{c}\text { Nominal Composition } \\
\text { Wt. } \quad \text { Th }\end{array}$ & $\begin{array}{c}\text { Density } \\
\mathrm{g} / \mathrm{cm}^{3}\end{array}$ \\
\hline 95 & 11.64 \\
90 & 11.86 \\
80 & 12.02 \\
7.5 & 12.05 \\
70 & 12.25 \\
60 & 12.51
\end{tabular}

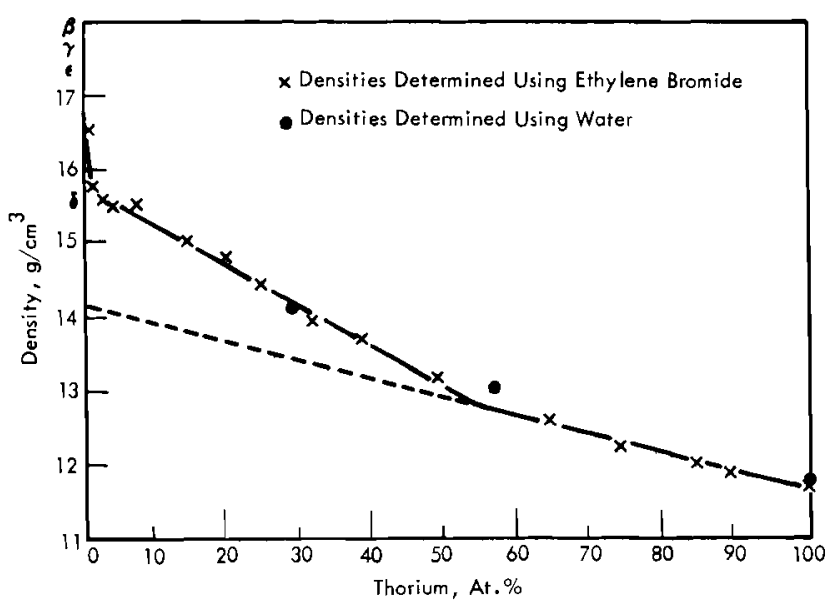

Density Values of As-Cast Plutonium-Thorium Alloys Plotted Against Composition. From D. M. Poole, G. K. Williamson, and J. A. C. Marples, J. Inst. Metals, vol. 86, p. 176, 1957-1958. (Same as Fig.

Specilic Volume of Arc-Melted High-Pu1

From

\section{Th 202.1}

AVERAGE LINEAR THERMAL EXPANSION COEFFICIENTS

$$
25-391^{\circ} \mathrm{C}
$$

From L. T. Lloyd in WASH-703. 1957 ,

\begin{tabular}{cc}
$\begin{array}{c}\text { Composition, } \\
\text { Wt.\% Th }\end{array}$ & $\begin{array}{c}\text { Expansion Coefficient, } \\
\mathbf{a} \times 10^{-6} /{ }^{\circ} \mathrm{C}\end{array}$ \\
\cline { 2 - 2 } 75 & \\
80 & 9.33 \\
90 & 9.75 \\
95 & 12.08 \\
& 10.16
\end{tabular}

\section{Th 307 Hardness}

ROOM TEMIPERATURE HARDNESS OF ARC CAST

$$
\text { Th-I } \mathrm{P} \text { A LLOYS }
$$

From P. M. French as reported by H. R. Gardner in Chapter 4, The I'lutonium Handbook, 196 (iti.

\begin{tabular}{cc} 
Composition, & Vickers Hardness Number, \\
\cline { 2 - 2 } .11. Pu & VHN \\
\hline 111 & 10.5 \\
211 & 130 \\
301 & 148 \\
40 & 133
\end{tabular}

HARDNESS OF Th-Pu ALLOYS

From Baumrucker and Kelman as reported by L. T. LJoyd in WASH-703.

\begin{tabular}{|c|c|}
\hline $\begin{array}{c}\text { Nominal Composition, } \\
\text { Wt. } \mathrm{Pu}\end{array}$ & $\begin{array}{l}\text { Hardness } \\
\text { Rockwell } \mathrm{F}\end{array}$ \\
\hline$j$ & $71-79$ \\
\hline 10 & $82-86$ \\
\hline 20 & $91-97$ \\
\hline 2,5 & $93-99$ \\
\hline 30 & $96-102$ \\
\hline 40 & $99-102$ \\
\hline
\end{tabular}
1957 . 


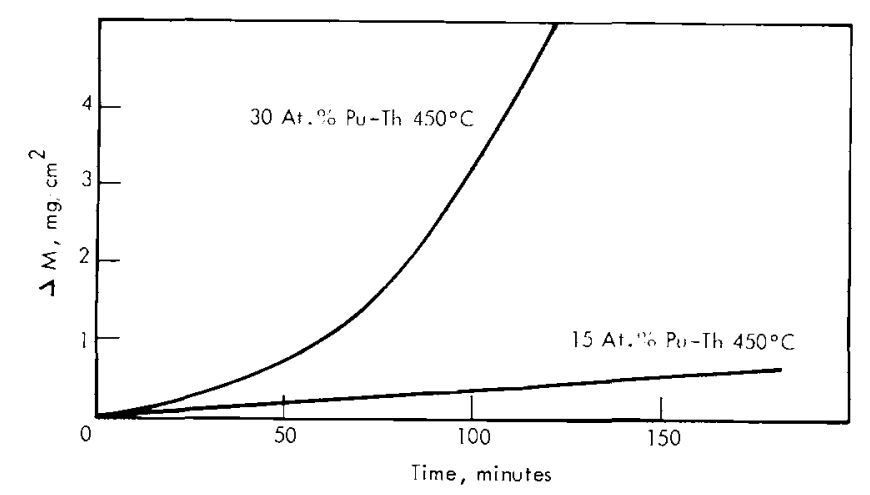

Pu-Tlt Oxidation in Air Dried Over Magnesium PerehIorate.

From 1). M. Poote at al. in plutonium 1960, 1). 629 .

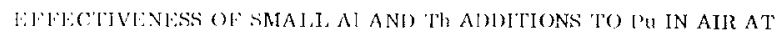

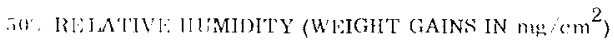

from I. T. Walwe in Proce. Serend Geneva Internat. Cont, vol, d, 1). $2 t^{2} \cdot 19.2 x$.

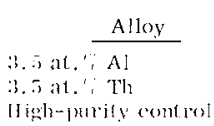

Test Temperature, Test Temperature,

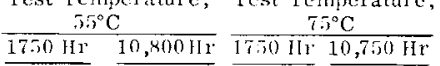

0.14

2.06

i. 2 !
$\therefore .91$
$\therefore .0 K^{*}$

$0.91 \quad 3.0$

\begin{tabular}{rr}
3.91 \\
10.1 & 16.7 \\
\hline
\end{tabular}

* Control samples afiel ?hon hr in the same test.

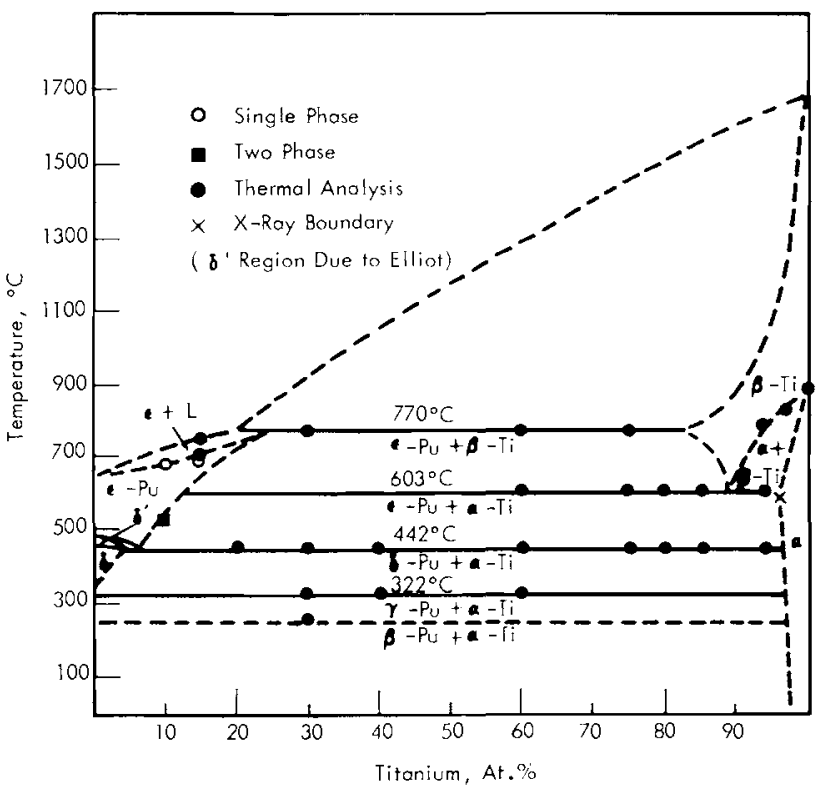

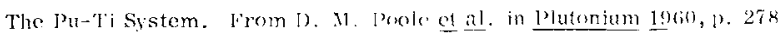




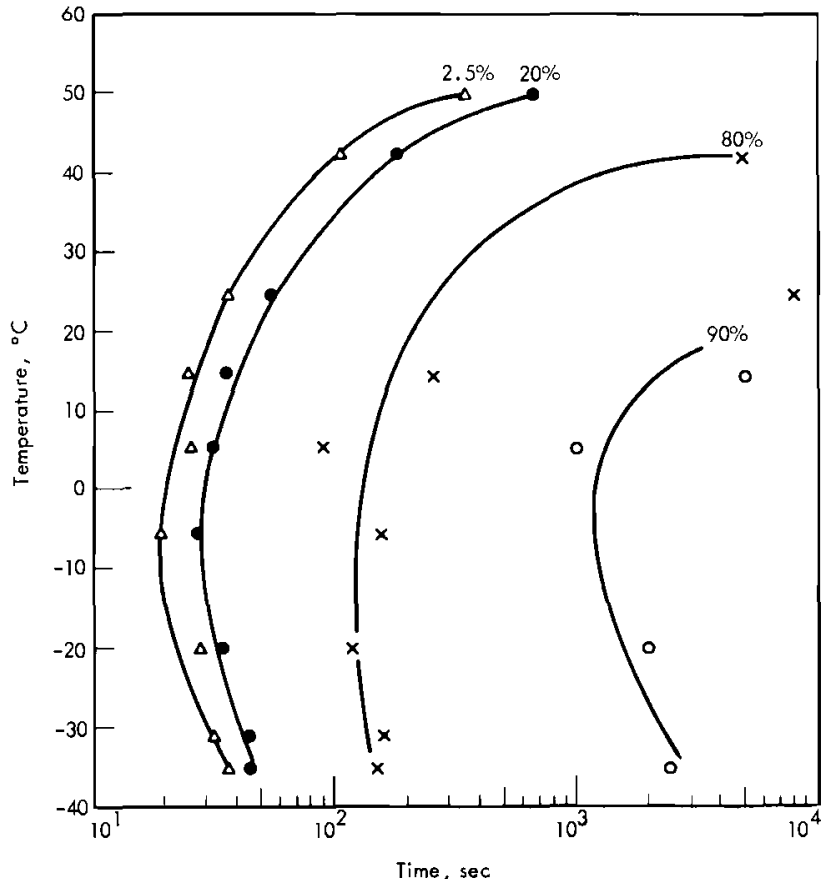

TTT Curve for the $\boldsymbol{\beta} \rightarrow \boldsymbol{a}$ Transformation in a Pu-0.23 at. \% Ti Alloy.

From I. G. T. Davy and J. S. White, J. Nucl. Mater, , vol. 12, p. 223. 1964.

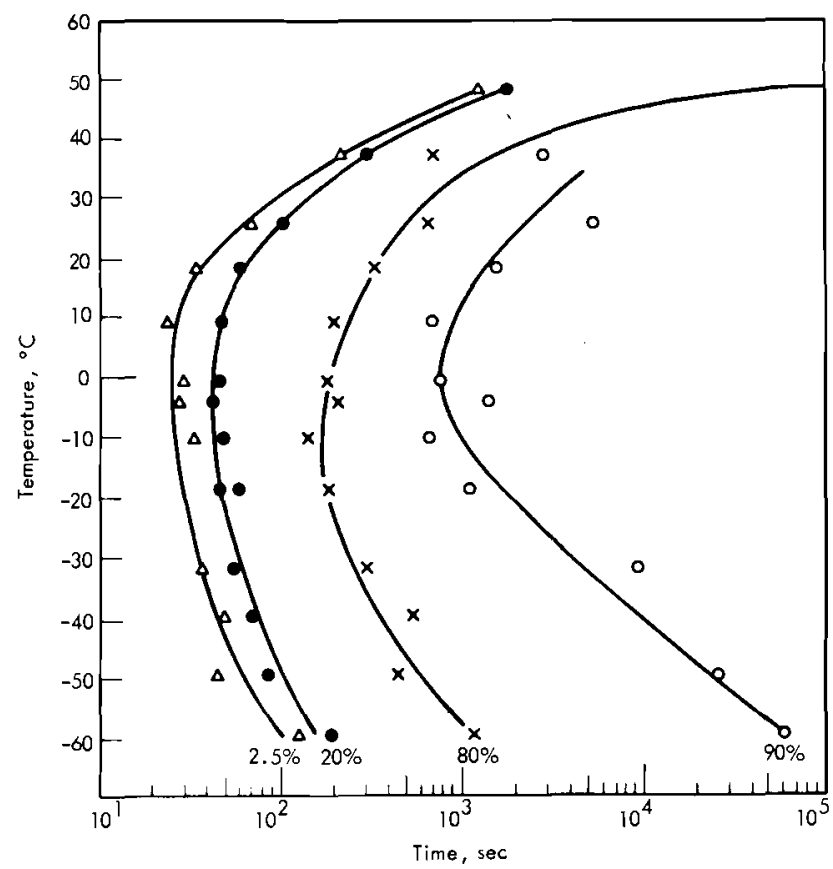

TT' Curve for the $\boldsymbol{\rho} \rightarrow \boldsymbol{a}$ Translornation in a Pu-0.82 at. " Ti Alloy. From L. G. T. Davy and J. S. White, J. Nucl. Muter., vol. 12, p. 223 , 1964 


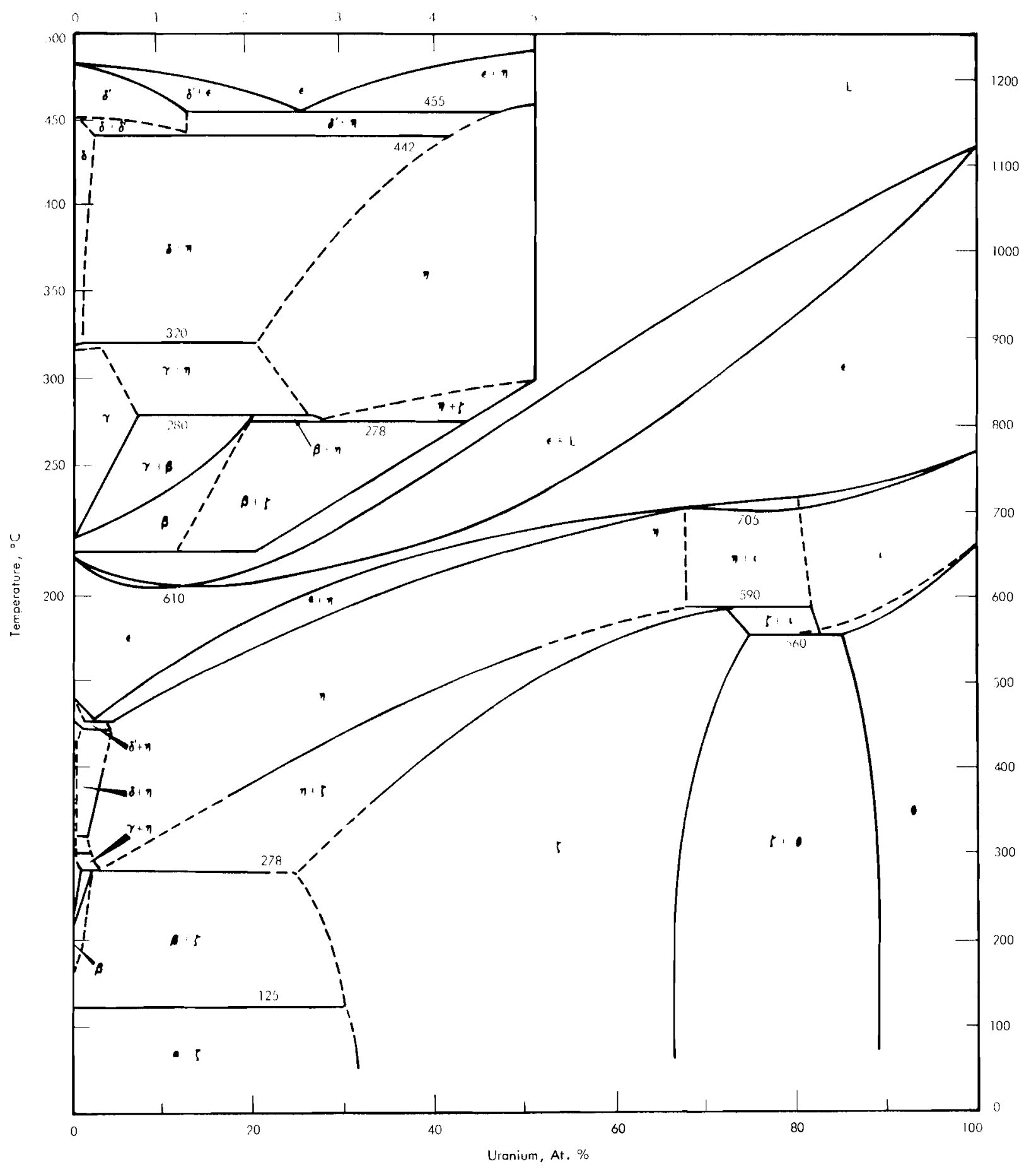

The Plutonium-Uranium System. From [: H. Ellinger, R. O. Elliott, 


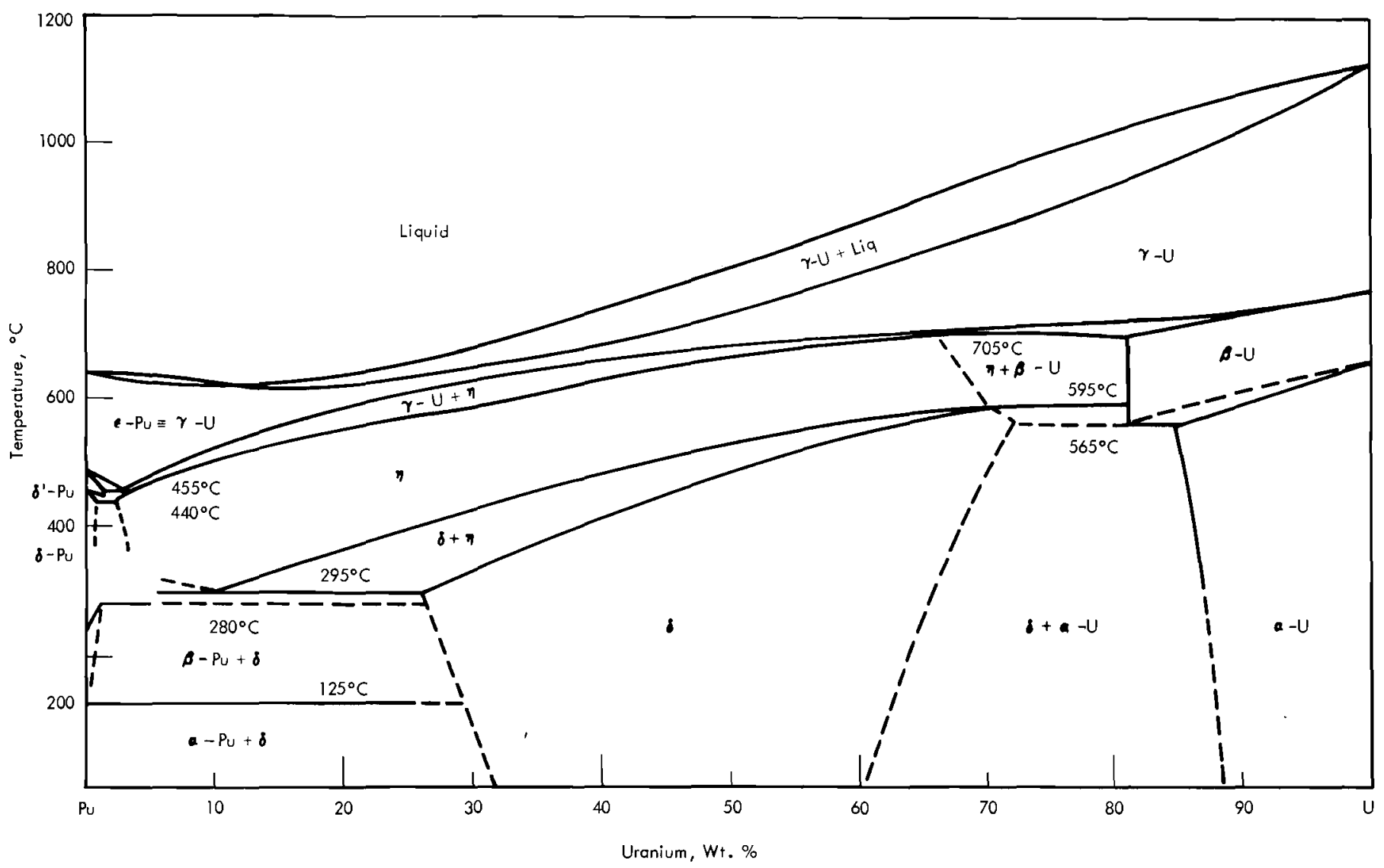

The Plutonium-Uranium System. From M. B. Waldron et al. in Proc. Second Geneva Internat. Conf., vol. 6, p. 168. 1958 .

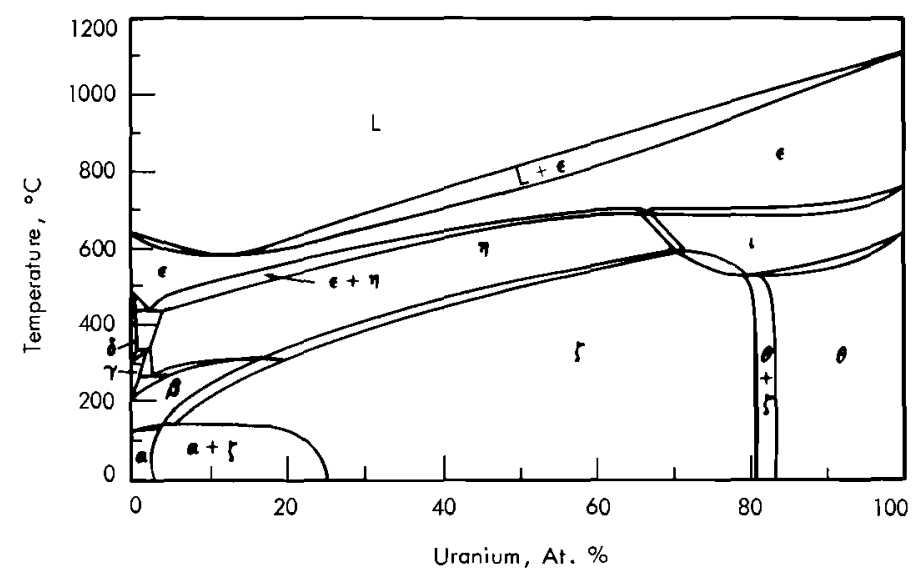

The Plutonium-Uranium System. From F. W. Schonfeld in The Metal Plutonium, p. 262

\section{U 103 crrstal structure}

UNIT CELL DIMENSIONS*

From W. H. Zachariasen and F. H. Ellinger, Acta Cryst., vol. 16, p. 371. 1963 .

\begin{tabular}{|c|c|c|c|c|c|}
\hline Sample & $\begin{array}{l}\text { Temper- } \\
\text { ature }\end{array}$ & $\mathrm{a}, \mathrm{A}$ & $\mathrm{b}, \mathrm{A}$ & $\mathrm{c}, \mathrm{A}$ & $\boldsymbol{\beta}$, \\
\hline Pure Pu & $93^{\circ} \mathrm{C}$. & 9.227 & 10.449 & T.824 & 92.54 \\
\hline Pure Pu & 190 & 9.284 & 10.463 & 7.859 & 92.13 \\
\hline $98 \mathrm{Pu}-2 \mathrm{U}$ & 83 & 9.215 & 10.418 & $T .815$ & 92.52 \\
\hline $98 \mathrm{Pu}-2 \mathrm{U}$ & 139 & 9.242 & 10.433 & 7.824 & 92.36 \\
\hline $98 \mathrm{Pu}-2 \mathrm{U}$ & 186 & 9.264 & 10.439 & T. 841 & 92.22 \\
\hline $98 \mathrm{Pu}-2 \mathrm{U}$ & 2,52 & 9.302 & 10.444 & T.859 & 91.97 \\
\hline
\end{tabular}

$*$ Precision is $\pm 0.002 \mathrm{~A}$ for $\mathrm{a}, \mathrm{b}, \mathrm{c}$, and $\pm 0.02^{\circ}$ for $\boldsymbol{A}$. 
$\operatorname{ctat}(\eta)^{*}$

compesition limise $2-70$ in 21

mol stable at reom 1 emiperaturs

['ontative lonit ree]

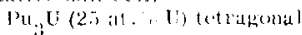

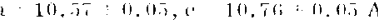

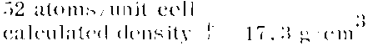

$\sin (5)$

Maxinum amposition linits $2 \ddot{-7}-74$ at. $\mathbf{V}$

Room temperature onmosition limits 33-6is at. $\mathbf{U} \pm$

Tentative unit colls:

(1) simple culise* - or lodragomal with axial ration of unit at boom temperatul's

PuU contitins -jo aloms/unit coll

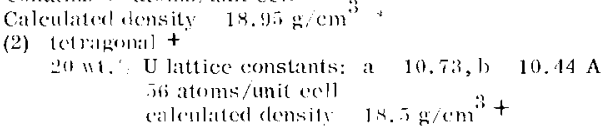

* Fom A. S. Colliulerry and M. B. Waldron in Metallurgy and Fuels, scrics V, wol. 1 , 19). $396,403.1960$

+ From A. A. Berluvar et al, in Proe. Second Geneva Internat. Conf., vol, 6, p. $192.19 \mathrm{ir}$.

\pm l'rom F. H. E:1linger in The Metal plutonium, p. 304

I'ronn F. H. Klinger et al., J. Nucl. Mater., vol. 1, p. 239. 1959.

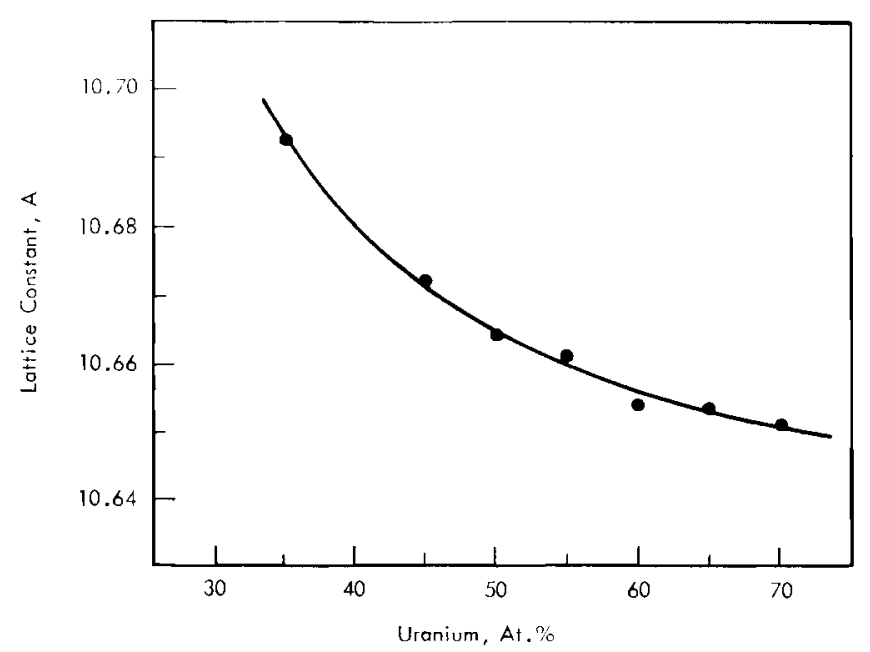

Iattice Constant Vs. Commosition of the Zeta Phase.

From l'. II. Fllinger dil., J. Nucl. Mater., vol. 1, 1) 239.1959

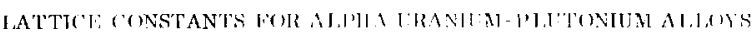

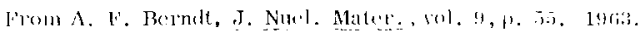

\begin{tabular}{|c|c|c|c|c|c|c|}
\hline \multirow[b]{2}{*}{ 1'ut,W1. } & \multirow[b]{2}{*}{$\because 0$} & \multicolumn{2}{|c|}{ Lattice Constants, $\wedge$} & \multirow[b]{2}{*}{$\underline{i_{0}}$} & \multicolumn{2}{|c|}{ Protalule Firror $\times 10^{5}, \mathrm{~A}$} \\
\hline & & $\underline{n_{0}}$ & "11 & & l, & $c_{0}$ \\
\hline 0 & 2. 8.336 & $\therefore$, Mriss & 1.90 .58 & 2 & 3 & 2 \\
\hline 2.06 & $2 . \times 547$ & 5.4157 & $1.050 x$ & 3 & 4 & 3 \\
\hline $3 . \times 4$ & $2, \operatorname{sing}$ & ... $81: 41$ & 1.0563 & 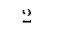 & 1 & 3 \\
\hline (1.06i & $2.85 \div 1$ & $5.81 ; 2 \%$ & $1.9 .5 \%$ & 2 & $: 3$ & 2 \\
\hline$\therefore . s 0 t$ & 2.4 .593 & $5 . M 4010$ & 1.1.178 & 2 & 1 & 3 \\
\hline 11.30 & 2. N6stas & $\therefore .8579$ & 1.94111 & 2 & 3 & :" \\
\hline $13.11+$ & 2.4615 & $\therefore .8774$ & 1.9415 & 2 & 2 & 2 \\
\hline 15.22 & $2.80^{2} 1$ & ล. & 1.94027 & $\therefore$ & $:$ & 4 \\
\hline 17.48 & 2. $963: 17$ & $\therefore, 8=60$ & $1.96 i 3$. & 12 & $T$ & 11 \\
\hline $19.66 \mathrm{j}$ & 2. $4(1: 30)$ & 万. K. & 1.148:32 & $x$ & 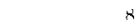 & i \\
\hline 21.70 & 2. 80112 & $\therefore .4,060$ & 1. $14 i_{2}(i$ & ais & $1: 8$ & 31 \\
\hline $13.11+ \pm$ & 2. N(it): & $\therefore .8-41$ & $1.94 ; 27$ & 3 & $: 3$ & $: 3$ \\
\hline 15. $22=$ & 2. & 5.8560 & 1,961 & $\because$ & 11 & 1 \\
\hline
\end{tabular}

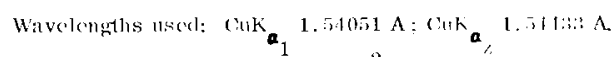

bach rellection weis!ltel ats $1 / \sin ^{2} 2 \theta$.

Corecention term $\sin ^{2} 2 \theta\left(\frac{1}{\sin \theta}+\frac{1}{\theta}\right)$.

For each case the proper correction lerm was cheseill as the one which

minimized the systcmatic erross as evidencorl an a $\sin ^{2} \theta_{\text {cal }}-\sin ^{2} \theta_{\text {olus }}$

vs, $\theta$ plot and which minimized the calculiticd pootialyle corrors.

*Average of analyout and weisheri-sul values.

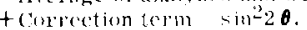

+ Speciments annealat for 116 bo. 


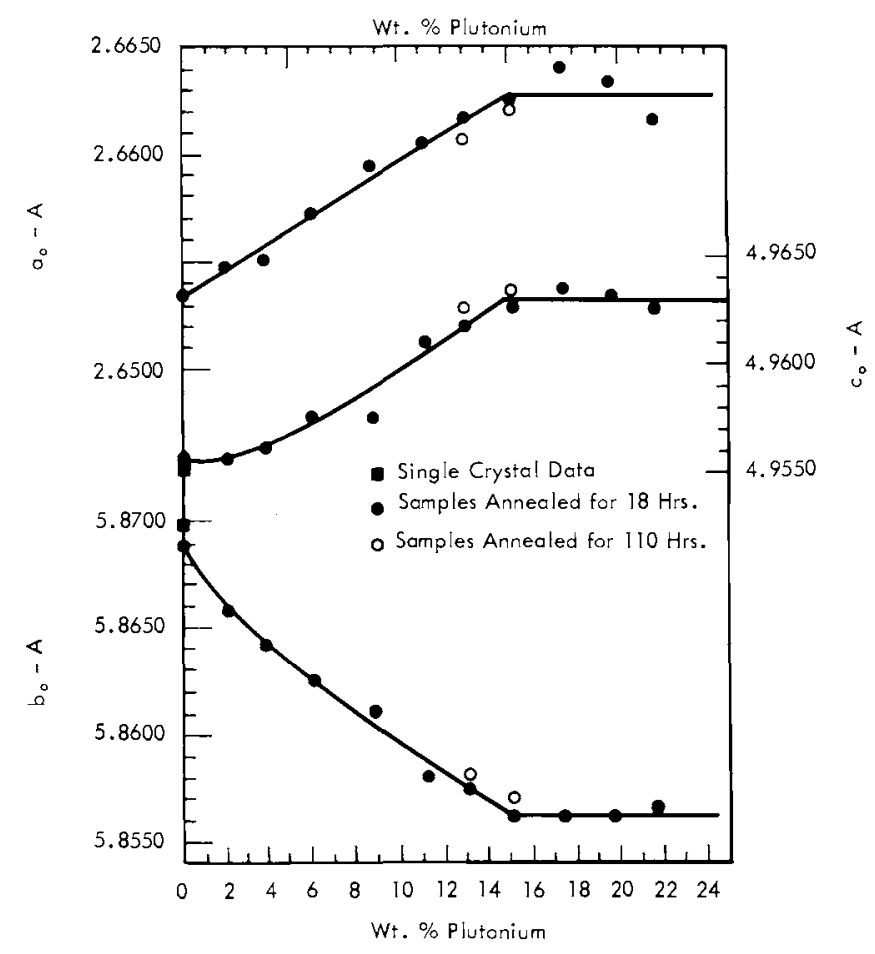

Iattice Cunstants vs. Composition Curves for Alpha Uranium-Plutonium A!loys. From A. F. Bemdt, J. Nucl. Mater, vol. 9, p. 55. 1963.

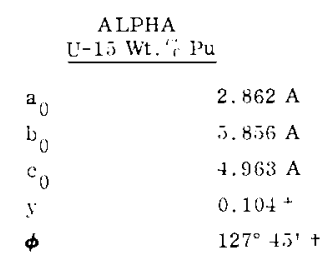

+ Esitinated values.

From A. F. Bernol, I. Nucl. Mater, vol, 9, p. 57. 1963.

\section{DENSITIES OF ALLOYS}

A. E. Martin and G. D. Selmanoff, LA-501. 1945. (Classified)

\begin{tabular}{|c|c|c|}
\hline & \multicolumn{2}{|c|}{ DENSITIES } \\
\hline $\begin{array}{c}\text { Nominal } \\
\text { Composition } \\
\text { in al. ? }\end{array}$ & As Cast & $\begin{array}{l}\text { After } \\
400^{\circ} \mathrm{C} \\
\text { Annca }\end{array}$ \\
\hline 1 ' Lranium & 1.8 .6 .5 & 18.46 \\
\hline 4" Uranium & 19.37 & --- \\
\hline 4. Urantum & 19.3 .2 & --- \\
\hline 8, Uranium & 19.10 & $--\cdots$ \\
\hline 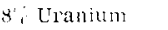 & 19.06 & ---- \\
\hline 1.7 Uranitum & 18.0 & $-\ldots$ \\
\hline 1.), Uranium & 17.95 & 17.99 \\
\hline $2.5:$ Lranium & 18.07 & $-\cdots$ \\
\hline 2\%, Uraıium & 18.07 & --.-- \\
\hline
\end{tabular}

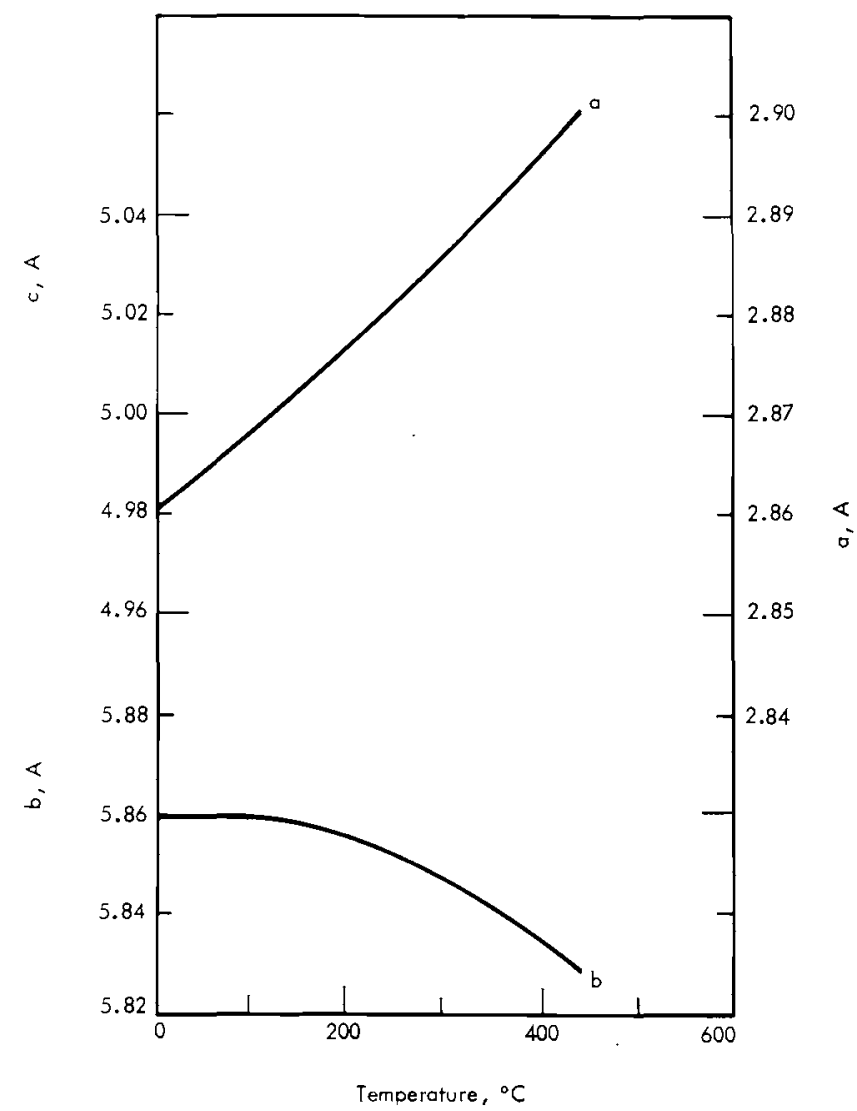

Temperature Dependence of Axial Lattice Parameters of the Phase of Uranium Alloy Containing 15 at. \% of Plutonium.

From S. T. Konobeevsky et al. in Proc. Second Geneva Internat. Conf., vol. G, P. 198.1958.

$$
\begin{aligned}
& a=2.861\left(1+26.2 \times 10^{-6} \mathrm{t}+1.4 \times 10^{-8} \mathrm{t}^{2}\right) \mathrm{A} \\
& \mathrm{b}=5.859\left(1+1.9 \times 10^{-6} \mathrm{t}-3.0 \times 10^{-8} \mathrm{t}^{2}\right) \\
& \mathrm{c}=4.956\left(1+25.2 \times 10^{-6} \mathrm{t}+1.6 \times 10^{-8} \mathrm{t}^{2}\right)
\end{aligned}
$$

$\mathrm{a}, \mathrm{b}$, and $\mathrm{c}=$ lattice parameters of $a$ phase in $\mathrm{A}, \mathrm{t}=$ temperature, ${ }^{\circ} \mathrm{C}$ at $25^{\circ} \mathrm{C}, \mathrm{a}=2.863$

$$
\begin{aligned}
& \mathrm{b}=5.860 \\
& \mathrm{c}=4.958
\end{aligned}
$$

From S. T. Konobeevsky et al. in Proc. Second Geneva Internat. Conf., vol. 6, p. 197. 1958 .

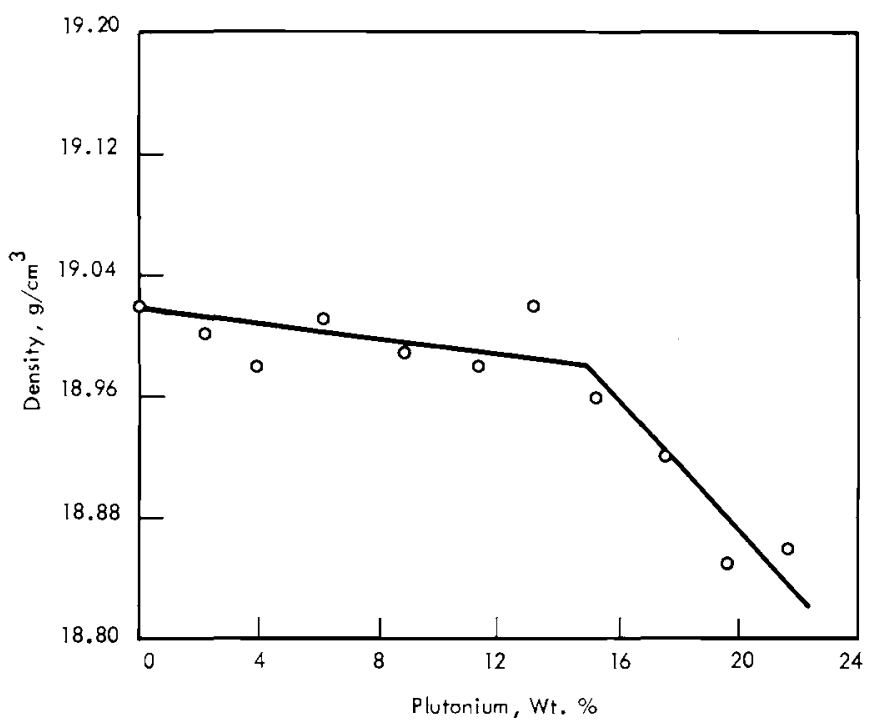

As-Cast Density vs. Composition for Uranium-Plutonium Alloys. From A. F. Berndt, J. Nucl. Mater, vol. 9, p. 54. 1963. 


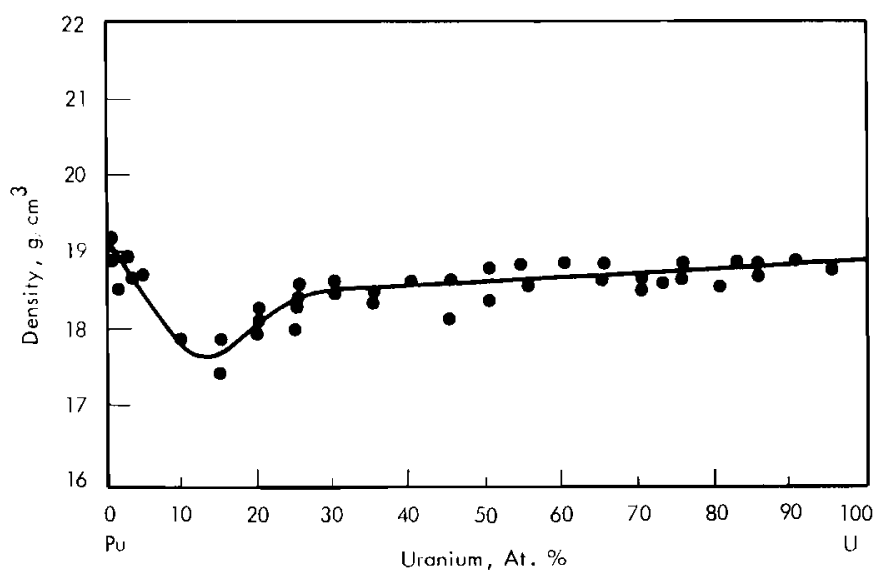

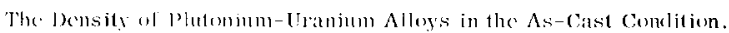

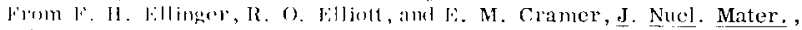
vol. $1,13.242 .19,19$

\section{Thermal Expansion}

PRINCTPAI, IINFAR COLFF FICIENTS OF TIHIRMAL EXPANSION (x 106)

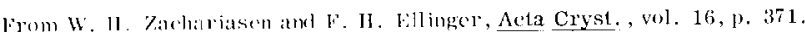
$191 ; 3$.

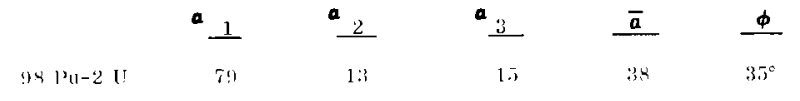

TIIERMAL, FXI'ANSION (OOEFICIEN'T'S FOR URANIUM- I'IUTONIUM ALLOYS (lZOOM TFMINRATURL: TO $\left.300^{\circ} \mathrm{C}\right)$

from W. D. Wilkinson and $\mathrm{l}$. R. Kelman in AN1-5439. 195.).

\begin{tabular}{|c|c|c|c|}
\hline \multirow{2}{*}{$\begin{array}{c}\text { Alloy Composition, } \\
\text { W1." Plutomiun }\end{array}$} & \multirow[b]{2}{*}{ Condition } & \multicolumn{2}{|c|}{$\begin{array}{c}\text { Average linenr Thermal } \\
\text { lixpansion Cocficient } \\
\vec{a} \times 10^{-6} / 0 \mathrm{C}\end{array}$} \\
\hline & & Longitudinal & Rudial \\
\hline 10 & Extruded & 11 & 25 \\
\hline 15 & Lixtruded & 16.6 & 6 \\
\hline 1.5 & Cast & $10.4-13.3$ & -. \\
\hline 20 & lixtruded & 21 & 8 \\
\hline
\end{tabular}

The ardition of (1p) to 20 wt " plutonium (auses an increase in the longitudinal

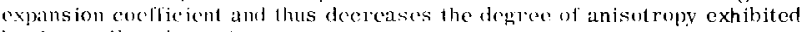
by the unaloged manium.

From A. S. Coflinberyet al. in Reactor Handlook, 2nd ed, , vol. 1, p. 280 I)
The dip in the ral've: it the plutonium-rich compositions is attributed to the

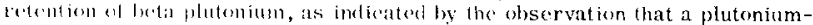

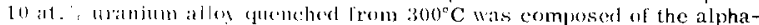

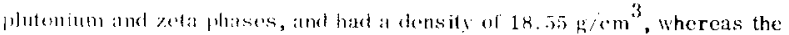

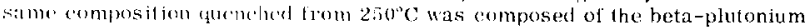
and \%edt phases, and had a density of $17.94 \mathrm{~g} / \mathrm{cm}^{3}$. From F. H. Ellinger, R. O. lillintt, and L. MT. Cramer, J. Nuel. Mater., vol. 1, p. 242. 1959 .

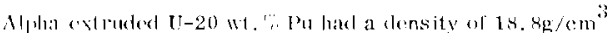

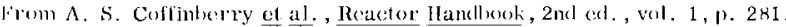
(1) (iol.
THERMAL EXPANSION COEFFICIENTS - FF FECT OF ALLOYING

From S. T. Konobecvsky et al. in Proc. Second Geneva Internat. Conf., vol. 6, p. 197. 1958 .

Alpha Phase Uranium - 15 at. "'Plutunium Alloy

\begin{tabular}{|c|c|c|c|}
\hline $\begin{array}{l}\text { Temperature } \\
\text { Range, }{ }^{\circ} \mathrm{C}\end{array}$ & $a_{a} \times 10^{\circ}$ & $a_{1}, \times 10^{6}$ & $a_{c} \times 10^{6 j}$ \\
\hline $20-300$ & $30 \div 4$ & $-8: 1: 1$ & $2 n \pm 4$ \\
\hline $20-400$ & $32+1$ & $-12+4$ & $32+1$ \\
\hline
\end{tabular}

Thermal Conductivity 


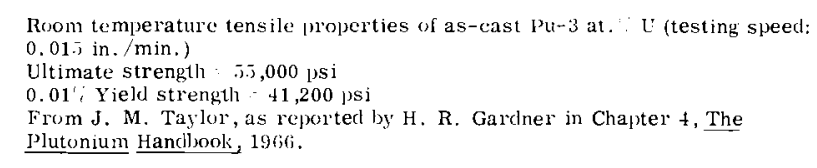

TENSILE: PROPERTIES OF INJECTION CAST Pu - 90 Wt. $:$ U AS A FUNCTION OF TEMPERATURE TESTING SPEED, 0.04i, MN MIN.

From H. E. Savage and E. R. Gilbert, as reported by H. R. Gardner in Chapter 4, The Plutonium Handbook, 1966 .

\begin{tabular}{|c|c|c|c|c|c|}
\hline $\begin{array}{c}\text { Temp., } \\
{ }^{\circ} \mathrm{C} \\
\end{array}$ & $\begin{array}{l}\text { Ultimate } \\
\text { Strength, } \\
\text { psi } \\
\end{array}$ & $\begin{array}{c}0.29, \text { Yield } \\
\text { Strength, } \\
\text { psi }\end{array}$ & $\begin{array}{l}\text { Reduction } \\
\text { in Area, } \\
\end{array}$ & $\begin{array}{l}\text { Elongation } \\
\text { in } 1 \text { in. } \% \\
\end{array}$ & $\begin{array}{l}\text { Elastic } \\
\text { Modulus, } \\
10^{6} \text { psi } \\
\end{array}$ \\
\hline $23 . \overline{3}$ & $37,500 \pm 1 \%$ & --- & $<1$ & $0.625 \pm 5 \% *$ & $19.0 \pm 1 \%$ \\
\hline 305 & $32,700 \pm 1 \%$ & $\ldots$ & $<1$ & $<1$ & -- \\
\hline 454 & $23,200 \pm 1 \%$ & $21,000 \pm 1 \%$ & $<1$ & $0.900 \pm 5 \% *$ & $15.5 \pm 8 \%$ \\
\hline 502 & $18,900 \pm 1 \%$ & $16,900 \pm 1 \%$ & $<1$ & $0.725 \pm 5 *$ & $13.0=8 \%$ \\
\hline $548+$ & $11,600 \pm 1 \%$ & $10,500 \pm 1 \%$ & $\sim 1$ & $6.3 \pm 5 \%$ & $9.20 \pm 8 \%$ \\
\hline 550 & $13,300 \pm 1$ & $13,300 \pm 1$ & $<1$ & $0.700 \pm 5 \%$ & $14.4 \pm 8 \%$ \\
\hline 589 & $14,800 \pm 1 \%$ & -- & $<1$ & $0.300 \pm 5 \% *$ & $22.3 \pm 8 \%$ \\
\hline 651 & $9,600 \pm 1$ & -.. & $<1$ & $0.275 \pm 5 \%$ & $7.31 \pm 8 \%$ \\
\hline 700 & $8,290 \pm 1 \%$ & $8,100 \pm 1 \%$ & $<1$ & $0.687 \pm 5 c^{*}$ & $1.00 \pm 8 \%$ \\
\hline
\end{tabular}

* Fracture strain includes elastic-plastic components. + High Purity.

\section{Compressive Properties}

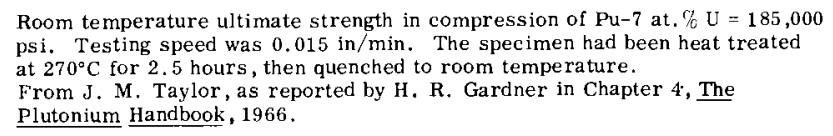

From H. E. Savage and E. R. Gilbert, as reported by H. R. Gardner in Chapter 4 , The Plutonium Handbook, 1966.

\begin{tabular}{|c|c|}
\hline $\begin{array}{c}\text { Temp. } \\
{ }^{\circ} \mathrm{C} \\
\end{array}$ & $\begin{array}{l}\text { Strain Rate in Terms of } \\
\text { Stress }(\sigma), \mathrm{Sec}^{-1}\end{array}$ \\
\hline 409 & $1.67 \times 10^{-8} \mathrm{e}^{0.498 \sigma}$ \\
\hline 448 & $1.15 \times 10^{-10} \mathrm{e}^{0.565 \sigma}$ \\
\hline 551 & $3.67 \times 10^{-9} \mathrm{e}^{1.20 \sigma}$ \\
\hline 300 & $4.67 \times 10^{-12} \cdot 5.04$ \\
\hline 550 & $5.22 \times 10^{-8} \sigma^{3.68}$ \\
\hline
\end{tabular}

$\begin{array}{ll}\text { Stress, psi } & \frac{\text { Testing Conditions }}{\text { Strain Rate, } \mathrm{Sec}^{-1}} \\ 8 \text { to } 17 & 1 \times 10^{-8} \text { to } 8 \times 10^{-7} \\ 7 \text { to } 12 & 7 \times 10^{-9} \text { to } 1 \times 10^{-7} \\ 2.5 \text { to } 5 & 7 \times 10^{-8} \text { to } 3 \times 10^{-6} \\ 6 \text { to } 10 & 7 \times 10^{-8} \text { to } 5 \times 10^{-7} \\ 2.5 \text { to } 5 & 1 \times 10^{-6} \text { to } 3 \times 10^{-5}\end{array}$

Fracture Strain, \%

* Specimen failed prematurely at a defect.

In general the hardness of the binary alloys increases with increasing plutonium content. From 5 to $15 \mathrm{wt}$. plutonium, the binary alloys increase in hardness from about 59 to 67 Rockwell $\mathrm{A}$ (210 to $250 \mathrm{DPHN})$. The extruded $20 \mathrm{wt}$. ? plutonium alloy has a hardness of about 77 Rockwell A (280 DPHN). From A. S. Coffinberry et al. in The Reactor Handbook, 2nd ed, , vol. 1,

p. 281.1960.

MICROHARDNESSES OF PLUTONIUM-URANIUM PHASES From F, H. Ellinger, R. O. Elliott, and E. M. Cramer, $\underline{J}$. Nucl. Mater., vol, 1, p. 242, 1959 .

\begin{tabular}{|c|c|}
\hline $\begin{array}{c}\text { Alloy } \\
\text { Composition } \\
\text { (at. \% U) }\end{array}$ & $\begin{array}{c}\text { Diamond Pyramid } \\
\text { Hardness Number, } \\
(2 \bar{j}-\mathrm{g} \text { Load }) \\
\end{array}$ \\
\hline $0-5$ & $200-240$ \\
\hline $10-25$ & $140-165$ \\
\hline $40-7 j$ & $275-450$ \\
\hline $30-8$ & $200-300$ \\
\hline $7 \bar{s}-90$ & $195-300$ \\
\hline $82-100$ & $160-210$ \\
\hline
\end{tabular}




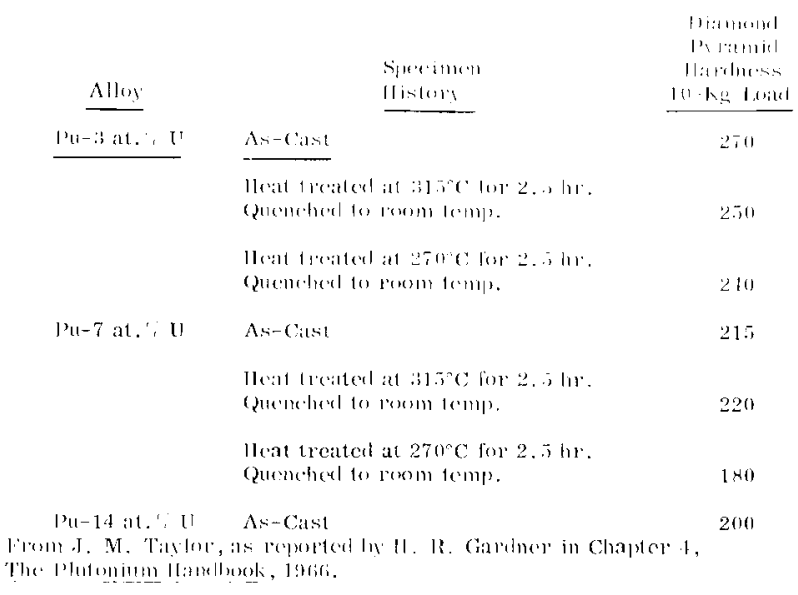

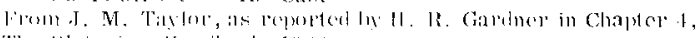

Elastic Properties

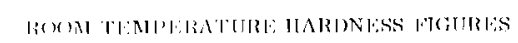

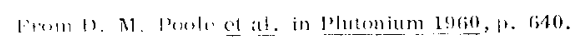

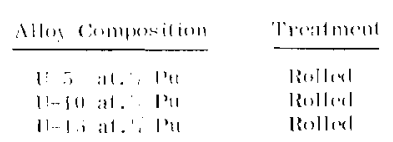

Viekeles llariness Number

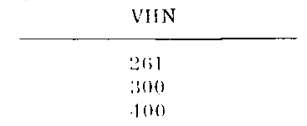

\section{U 308}

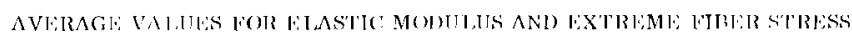

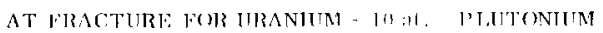

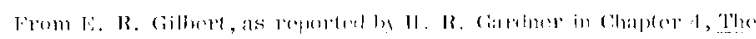

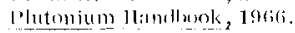

$\frac{\text { Speneimen Condetion }}{\text { As-Cast }}$

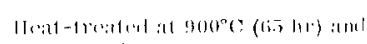
$1.006(2111)$.

Sien and of sertion 1:301.

Reactions with Gases - Air

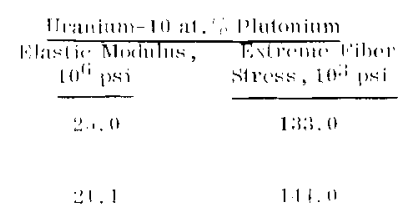

21.1

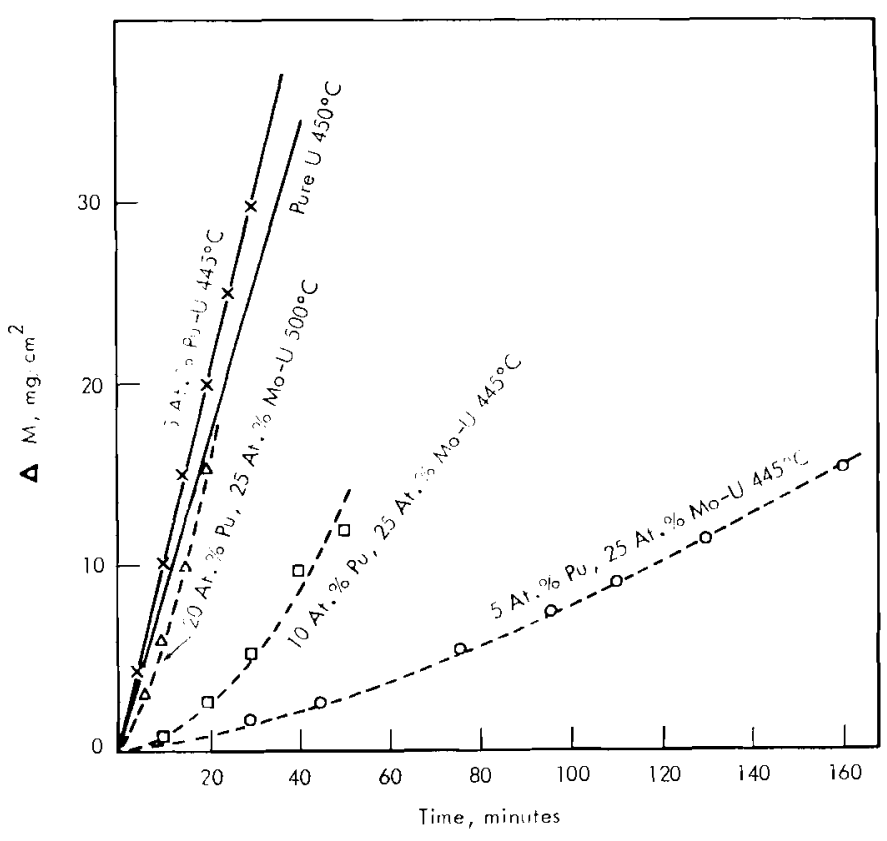

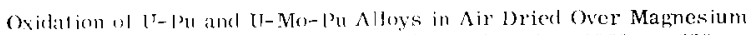

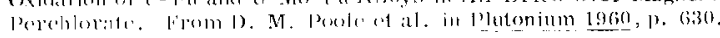


OXIDATION RATES, LATTICE PARAMETEIR MEASUREMENTS AND STOICHIOMETRY OF OXIDES OBTAINED FROM PLUTONIUU URANICYI ALLOYS OXIDIZED IN DRY $\mathrm{CO}_{2}$

From M. J. F. Notley, E. N. Hodkin, and J. A. C. Davidson, AERE-R-4070, 1962

\begin{tabular}{|c|c|c|c|c|}
\hline Composition & $\begin{array}{c}\text { Temper- } \\
\text { ature }\end{array}$ & $\begin{array}{c}\text { Rate, } \mathrm{ms} / \\
\mathrm{cm}^{2}-\mathrm{hr}^{\prime}\end{array}$ & $\begin{array}{l}\text { Lattice Parameter } \\
\text { of Oxide, A }\end{array}$ & $\begin{array}{c}\text { Stoichiometry } \\
\left(\mathrm{X} \text { in } \mathrm{CO}_{\mathrm{X}}\right) \\
\end{array}$ \\
\hline \multirow{4}{*}{$5 a t . \quad \mathrm{Ju}-\mathrm{U}$} & 500 & 1.0 & $5 .+567$ & \\
\hline & 600 & 4.7 & - & \\
\hline & 700 & 15.6 & 5.4617 & 2,105 \\
\hline & 800 & 43.0 & 5.4382 & \\
\hline \multirow[t]{4}{*}{10 at. ' $\mathrm{Pu}-\mathrm{U}$} & 500 & 1.05 & - & \\
\hline & 600 & $10.2-21.6$ & 5.4425 & 2.049 \\
\hline & 700 & 52.5 & 5,4346 & 2.053 \\
\hline & 800 & 61.3 & 5,4598 & \\
\hline
\end{tabular}
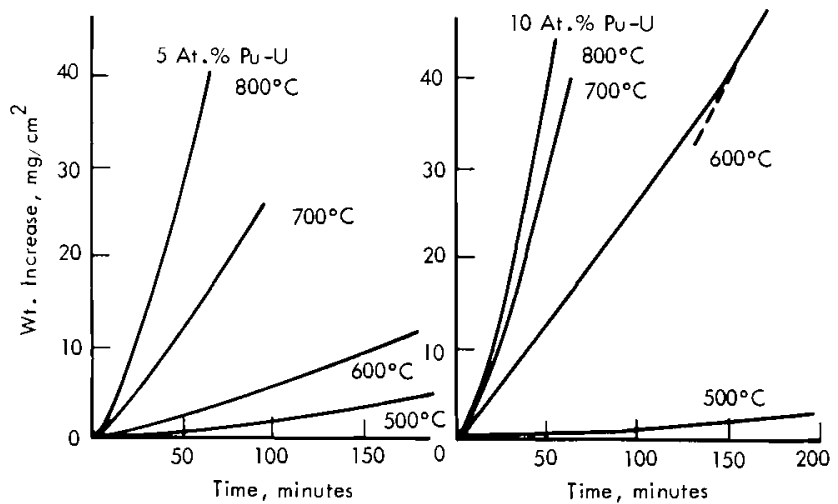

Oxidation of L:-Pu Alloys in Dry $\mathrm{CO}_{2}$.

From D. .I. Poole et al. in Plutonium 1960, p. 634

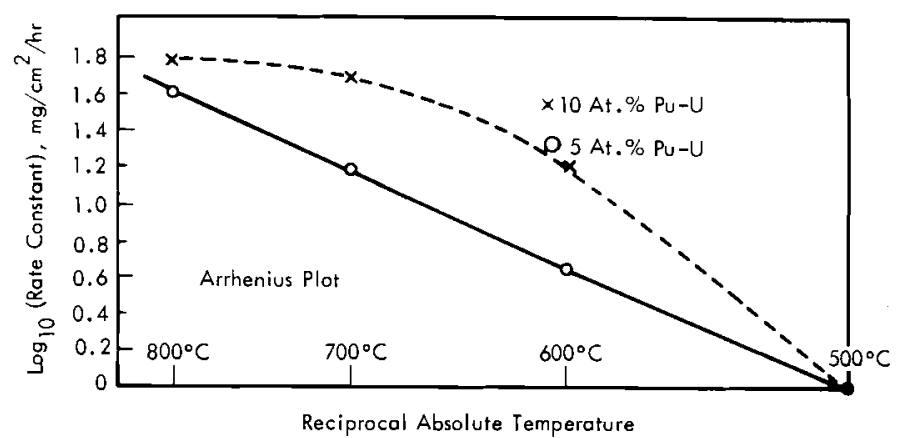

Arrhenius Plot of Oxidation of U-Pu Alloys in $\mathrm{CO}_{2}$ Dried Over Magnesium

From D. M. Poole et al. in Plutonium 1960, p. 634.

\section{U 600}

\section{Radiation Effects}

CAST PLUTONIUM-URANIUM ALLOYS

PREIRRADLATION AND POSTIRRADLATION DATA

From A. S. Coffinberry et al. in The Reactor Handbook, 2nd ed., vol. 1, p. 282, 284.1960.

\begin{tabular}{|c|c|c|c|c|c|c|}
\hline Specimen & $\begin{array}{l}\text { Plutonium } \\
\text { Nominal }\end{array}$ & $\begin{array}{l}\text { Content, Wt. } \\
\text { Analyzed }\end{array}$ & $\begin{array}{l}\text { Density } \\
\text { g/cm }\end{array}$ & $\begin{array}{l}\text { Indicated } \\
\text { Burnupa } \\
\text { Per Cent }\end{array}$ & $\begin{array}{l}\text { Calculated } \\
\text { Maximum } \\
\text { Temp, }{ }^{\circ} \mathrm{C}\end{array}$ & $\begin{array}{l}\text { Wt. Change, } \\
\text { mg }\end{array}$ \\
\hline $2 \mathrm{~A}$ & 0 & & 18.89 & 0.11 & 88 & -4.1 \\
\hline $2 \mathrm{~B}$ & 0 & & 18.89 & 0.12 & 91 & -2.3 \\
\hline $2 \mathrm{C}$ & 0 & & 18.86 & 0.10 & 83 & -2.6 \\
\hline $4 \mathrm{~A}$ &. & (Top 3.6: & 18.84 & 0.75 & 340 & 0.3 \\
\hline $4 \mathrm{~B}$ & $\bar{z}$ & bottom 3.4 & 18.81 & 1.1 & 480 & 3.1 \\
\hline $4 \mathrm{C}$ & ; & & 18.88 & 0.60 & 270 & 3.8 \\
\hline $6 \mathrm{~A}$ & 10 & (Top 7. & 18.92 & 1.6 & 720 & -- \\
\hline $6 \mathrm{~B}$ & 10 & S bottom $\pi$. & 17.94 & 1.9 & 820 & 532.3 \\
\hline $6 \mathrm{C}$ & 10 & & 18.70 & 0.81 & 360 & -242.9 \\
\hline $7 \mathrm{~A}$ & 1.5 & 10.6 & 18.75 & 1.5 & 740 & -15.1 \\
\hline$i \mathrm{~B}$ & 1.j & $15 .-1$ & 18.72 & 1.9 & 920 & -588.9 \\
\hline $7 \mathrm{C}$ & 1.5 & & 18.78 & 0.7 .5 & 370 & 3.1 \\
\hline
\end{tabular}

\begin{tabular}{|c|c|c|c|}
\hline Fis & on Gas & & \\
\hline A mount & $\because$ of & Length & \\
\hline $\begin{array}{l}\text { Released, } \\
\mathrm{cm}^{3}\end{array}$ & $\begin{array}{c}\text { Theoretical } \\
\text { Amt. }\end{array}$ & $\underset{7}{\text { Increase, }}$ & $\begin{array}{c}\text { Growth Rate, } \\
\text { in. /in. /ppm Burnup }\end{array}$ \\
\hline 0 & 0 & 0 & 0 \\
\hline-- & -- & 0 & 0 \\
\hline 0.037 & 57 & 4.9 & 48 \\
\hline-- & -- & 4.9 & 6.4 \\
\hline 0 & 0 & 6.1 & 5.4 \\
\hline-- & - & 5.4 & 8.8 \\
\hline 0.60 & 50 & - & - \\
\hline 1.3 & 105 & 18 & $8.7^{\mathrm{c}}$ \\
\hline 0.34 & 65 & 7.7 & $9.2^{\mathrm{d}}$ \\
\hline 0.86 & 88 & 11 & 6.9 \\
\hline 0.41 & 35 & 26 & $12^{\mathrm{c}}$ \\
\hline $0.07 . j$ & 24 & 17 & 21 \\
\hline
\end{tabular}

From results previously obtained on similarly sized enriched specimens, the following flux depressions were assumed respectively for 5,10 , and 15 per cent alloys: 5,15 , and 25 per cent.

1) At beginning of irradiation, using the above flux depressions and thermal conductivities 95,80 , and 75 per eent that of unalloved uranium.

c Probably too low a value because part of specimen was missing.

d Found in 1wo pieces. 


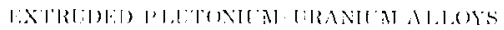

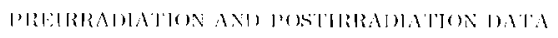

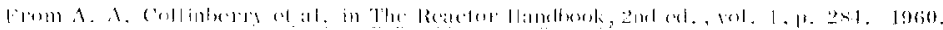

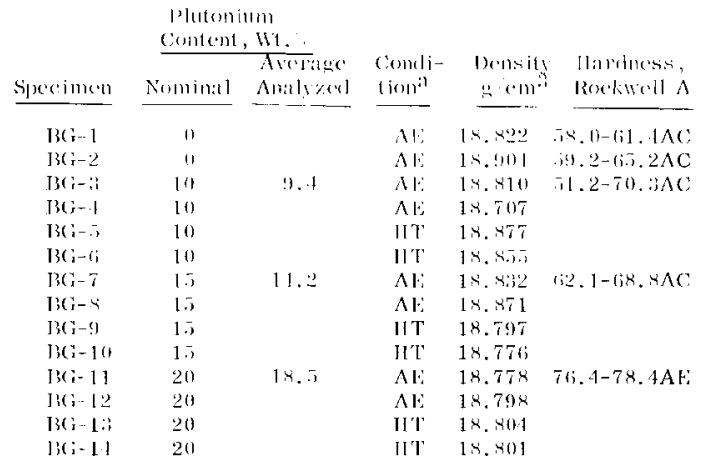

\begin{tabular}{|c|c|c|}
\hline \multirow{2}{*}{ 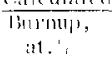 } & \multicolumn{2}{|c|}{ (: } \\
\hline & (9ונlor & Surfiler \\
\hline 0.0 .1 .5 & ४.) & s0 \\
\hline 0.0 .18 & 1૯ & 8.j \\
\hline 0.70 & $7 \times 0$ & 590 \\
\hline 0.3 .5 & 110 & 310 \\
\hline 0.45 & 520 & 400 \\
\hline 0.25 & $\because 00$ & 230 \\
\hline 0.32 & $\because 30$ & 290 \\
\hline 0.80 & 890 & (i) 0 \\
\hline 0.70 & $7 \times 0$ & .590 \\
\hline 0.14 & 190 & 150 \\
\hline 0.60 & 630 & 510 \\
\hline 0.40 & 460 & 350 \\
\hline $0 . .32$ & 890 & 450 \\
\hline 0.9 .5 & 500 & 230 \\
\hline
\end{tabular}

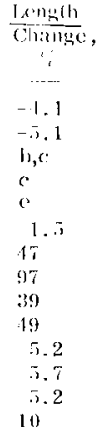

$\frac{\text { Giowdin laat }}{\text { in. }}$

Dia. [mom Turnu|,

chinge:

Wt.

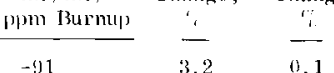

$\begin{array}{ll}-110 & 3.2 \\ 3.2\end{array}$

$\begin{array}{ll}0.2 & 0.1 \\ 3.2 & 0.1\end{array}$

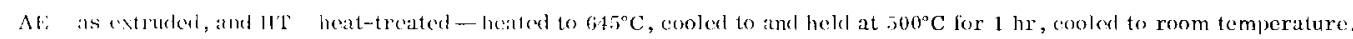

1) Pour exolition of specimen did not permit measurements.

c. Stuck in irratiation causule.

a lonited and burned bedore measurements eould be taken. e Could not be measured because balanee was out of order.

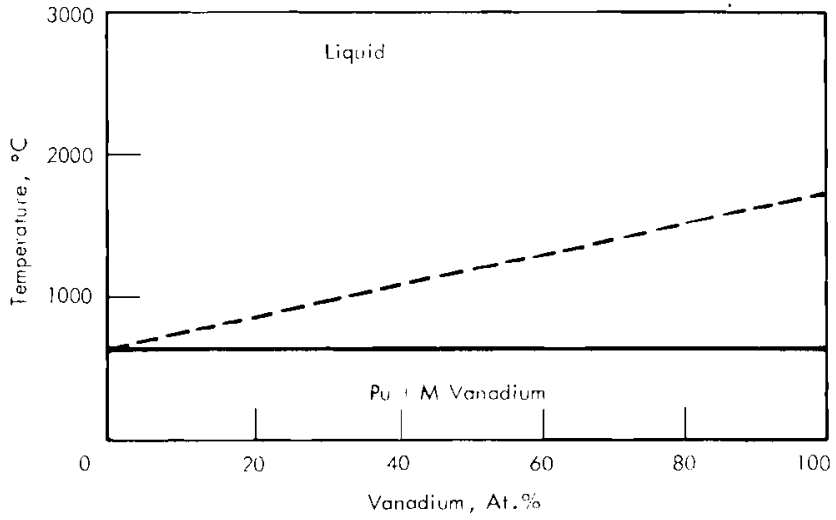

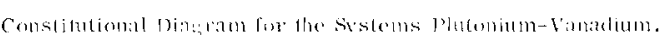

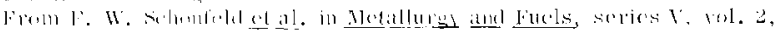

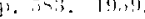

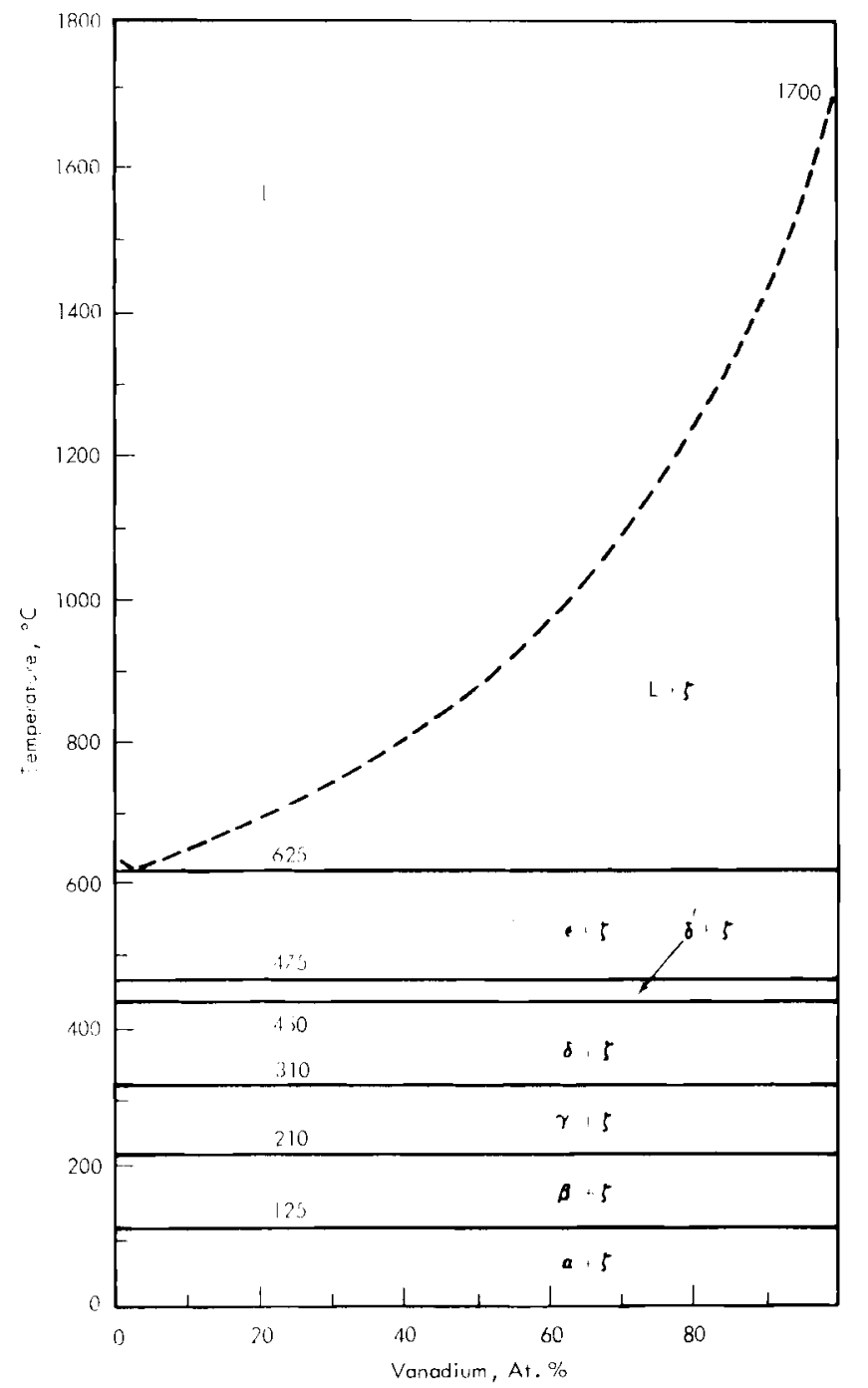

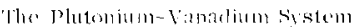

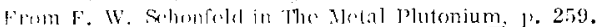



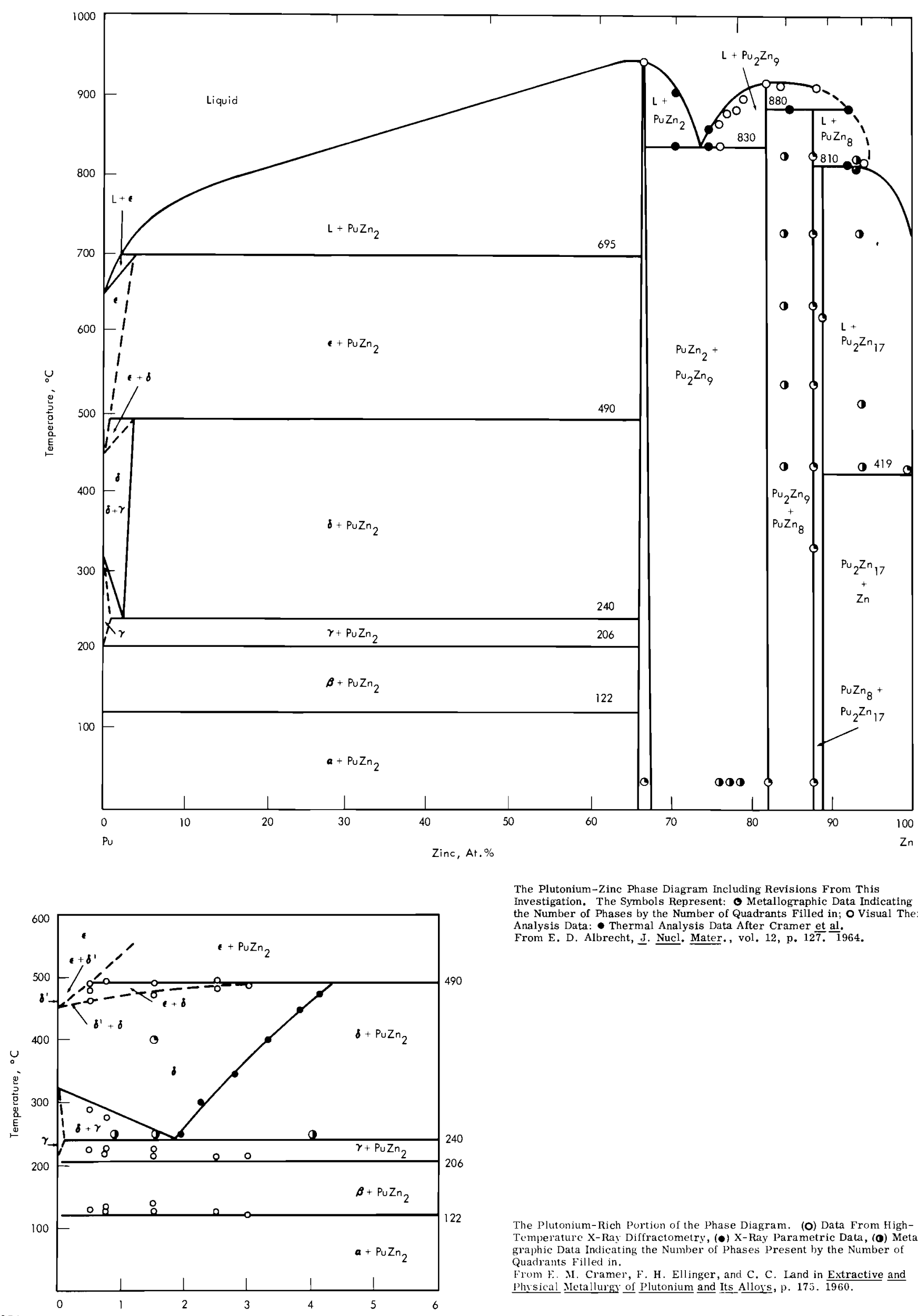

The Plutonium-Zine Phase Diagram Including Revisions From This

Investigation. The Symbols Represent: O Metallographic Data Indicating

the Number of Phases by the Number of Quadrants Filled in; O Visual Thermal

Analysis Data: - Thermal Analysis Data After Cramer et al.
From E. D. Albrecht, I. Nucl. Mater., vol. 12, p. 127. 1964 . 


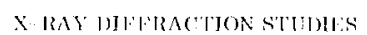

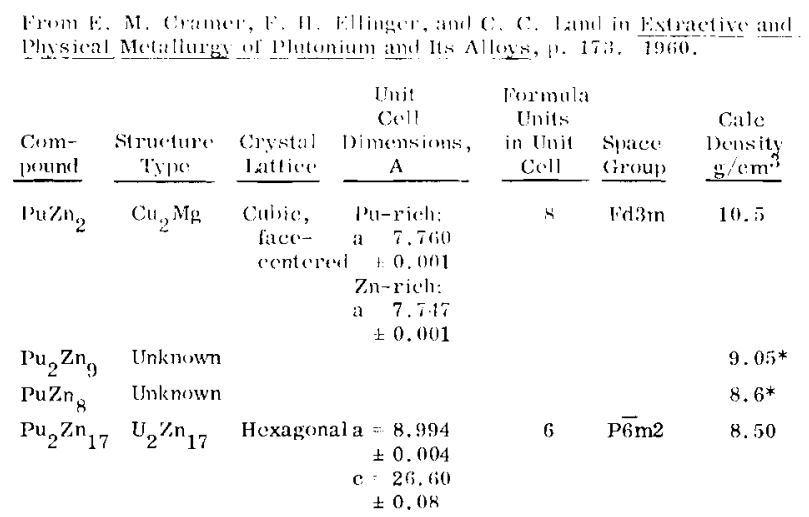

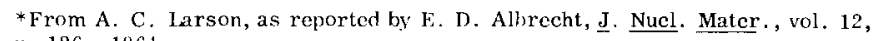
p. 126.1906 .

Density

J)ENSITY OF PU-7,॥ AJ,J,OYS AT $21^{n} \mathrm{C}$

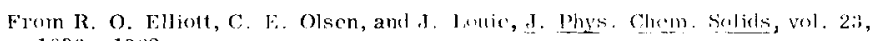
p. 1036 . 1942 .

\begin{tabular}{l}
$\begin{array}{c}\text { Composition } \\
\text { at. } 711\end{array}$ \\
\hline 1.51 \\
1.79 \\
2.15 \\
2.92 \\
3.35 \\
3.89
\end{tabular}

1. Heat treated at $450^{\circ} \mathrm{C}$ for $500 \mathrm{hr}$.

2. Heat treated a $180^{\circ} \mathrm{C}$ for $500 \mathrm{hr}$
DENSTTIES OF INTERMETALLIC COMPOUNDS $\left(\mathrm{g} / \mathrm{cm}^{3}\right)$ From E. I). Allrecht, J. Nucl. Mater., vol. 12, p. 12f. 1964.

\begin{tabular}{|c|c|c|}
\hline Compround & $\begin{array}{l}\text { X-Ray } \\
\text { Density }\end{array}$ & $\begin{array}{c}\text { Measured } \\
\text { Density }\end{array}$ \\
\hline$\mu^{\prime 1} Z_{n}$ & 10. i * & $10.8+$ \\
\hline נים & $9.0 ; 5^{* *}$ & 9.05 \\
\hline $\mathrm{l}^{\prime} \mathrm{uZn} \mathrm{n}_{8}$ & $8.0^{* * *}$ & 3.43 \\
\hline $\mathrm{Pu}_{2} \mathrm{Zn}_{17}$ & $8, \pi^{*}$ & $7.79+t$ \\
\hline
\end{tabular}

- Cramer et al.

* L'rivate Communication A. C. Larson, August 10, 1960. + Sample contained a small amount of alpha plutonium. + Sample containol a considerable proportion of voids.

\begin{tabular}{|c|c|c|c|c|}
\hline \multirow{2}{*}{$\begin{array}{l}\text { Composition } \\
\text { Zn, At, } \\
\end{array}$} & \multicolumn{3}{|c|}{$\begin{array}{c}\text { Specilie Rosistivity } \\
\text { n-cm }\end{array}$} & \multirow[b]{2}{*}{$300^{\circ} \mathrm{K}$} \\
\hline & $\underline{1} \underline{\underline{H}}$ & $20^{\circ} \mathrm{K}$ & $100^{\circ} \mathrm{K}$ & \\
\hline 3.35 & 4.1 .4 & 99.6 & 113.6 & 113.0 \\
\hline 3.89 & $\Delta x .1$ & 100.8 & 111.7 & 110.3 \\
\hline
\end{tabular}




\section{Zn 307 Hardness}

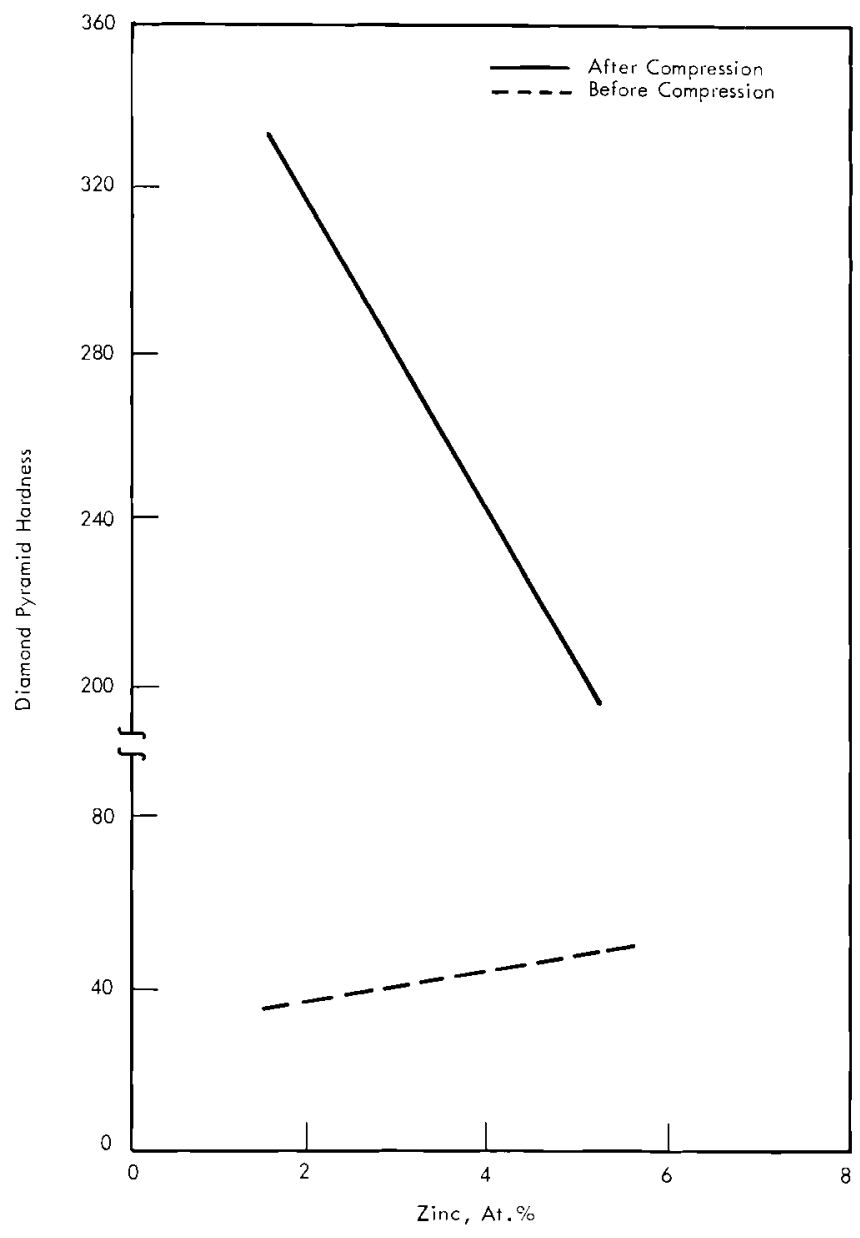

From R. O. Eilioat ancl K. A, Gschneidner, Jr., in Extractive and [hysical Iotallurey of plutonium and Its Alloys, p, 2.59, 1960

\section{Zn 401.1 Reactions with Gases - Air}

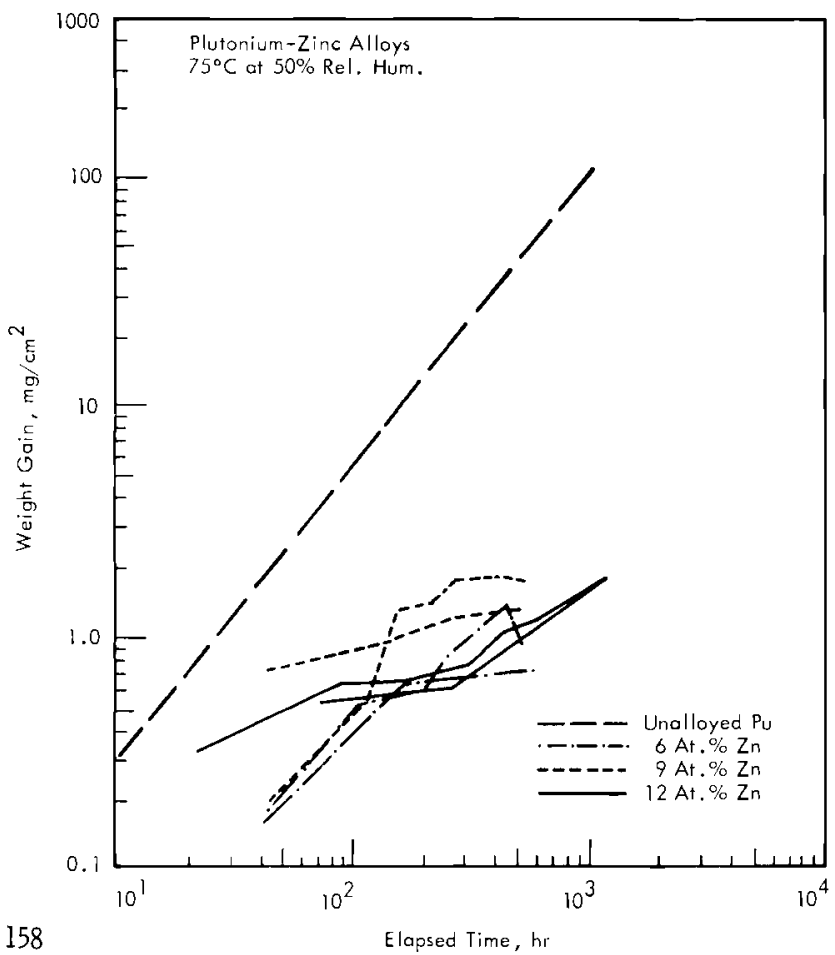

Comparison of the Corrosion Rehavions of Plutonium-Zinc Alloys and

Unalloyed Plutonimm Bxposed to $A \mathrm{i} 1^{\circ}$ at $\bar{\gamma}^{\circ} \mathrm{C}$ and $50^{\prime \prime}$. Relative Humidity.

From J. T. Waber et al. J. Nucl. Matce. vol, 3. p. 205. 1961
MICROHARDNESSES OF INTERMETALLIC COMPOUNDS

From E, D. Albrecht, J. Nucl. Mater, vol. 12, p. 126. 1964.

\begin{tabular}{lcc} 
Compound & $\begin{array}{c}\text { D.P.H.No. } \\
\text { (Range) }\end{array}$ & $\begin{array}{c}\text { D. P. H. No. } \\
\text { (Average) }\end{array}$ \\
\cline { 2 - 2 } $\mathrm{PuZn}_{2}$ & $340-370$ & 350 \\
$\mathrm{Pul}_{2} \mathrm{Zn}_{9}$ & $360-480$ & 420 \\
$\mathrm{PuZn}_{8}$ & $340-470$ & 390 \\
$\mathrm{Pu}_{2} \mathrm{Zn}_{17}$ & $200-320$ & 280
\end{tabular}

* Diamond Pyramid Hardness Numbers obtained at a load of $25 \mathrm{~g}$.
MERIT RATIOS FOR PLUTONIUM-ZINC ALLOYS EXPOSED TO AIR CONTAINING $50 \%$ RELATIVE HUMIDITY $75^{\circ} \mathrm{C}$

From J. T. Waber et al, J. Nucl, Mater., vol. 3, p. 206. 1961 .

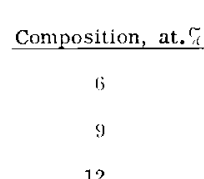

\begin{tabular}{|c|c|c|}
\hline \multicolumn{3}{|c|}{ Merit Ratio* } \\
\hline & & At $1000 \mathrm{hr}$ \\
\hline 34.0 & 44.1 & 122 \\
\hline 19.7 & 26.1 & 80 \\
\hline 31.1 & 36.8 & $62.5 \mathrm{t}$ \\
\hline
\end{tabular}

* Merit Ratio $=\frac{\text { Weight gain of unalloyed Pu }}{\text { Weight }}$ Weight gain of alloy 


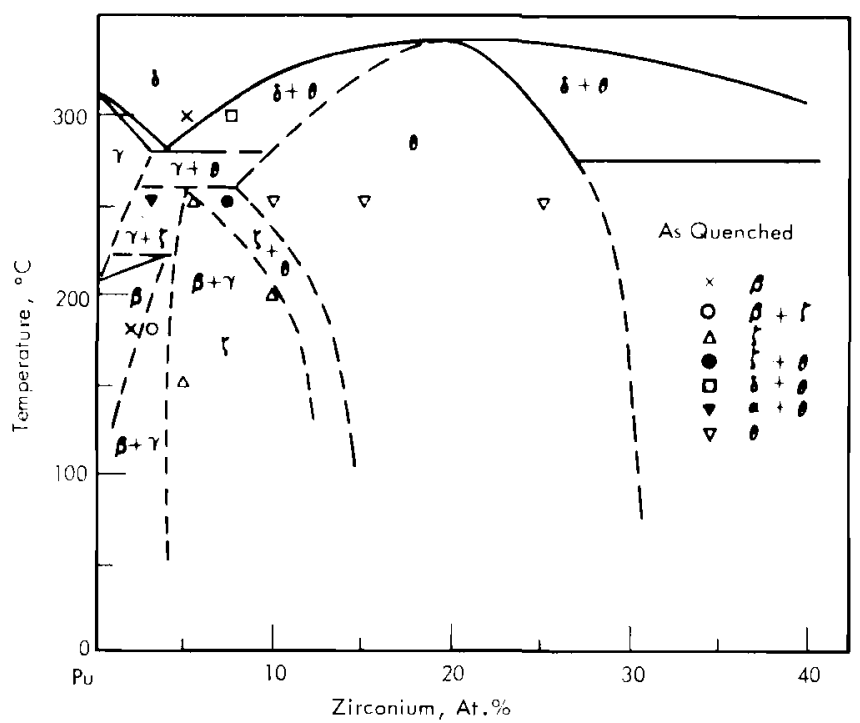

Partial Prase Diagram For Pu-Zr sustem. From F. H. Bllinger in Plutonium 1960, 1). 319 .

Solid lines trom D. M. Peole en al, in 1'LUTONIUM 196i0, 11. 21:9.

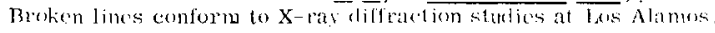




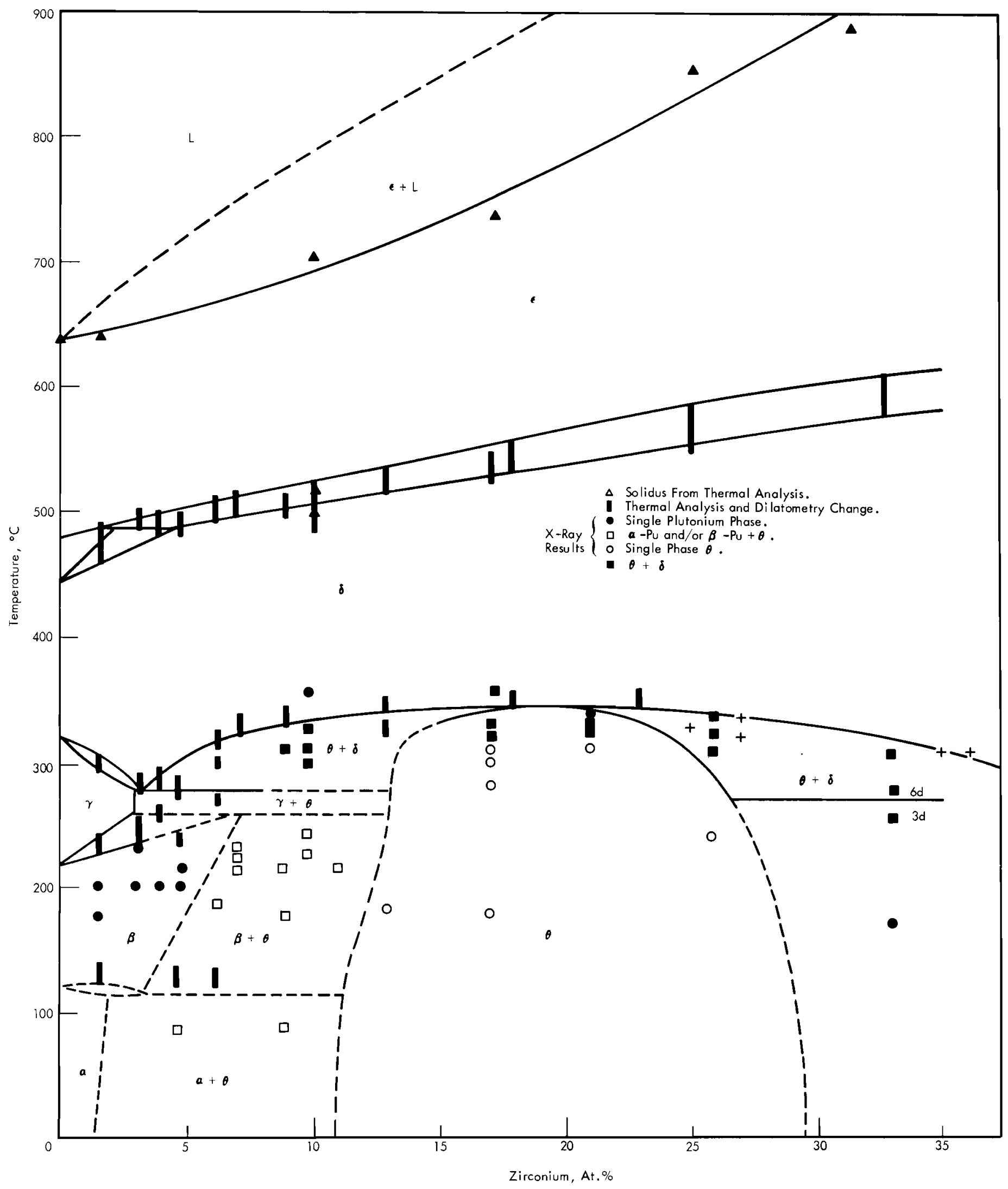

Plutonium-Zirconium Equilibrium Diagram 0-35 at. Zirconium. From J. A. C. 


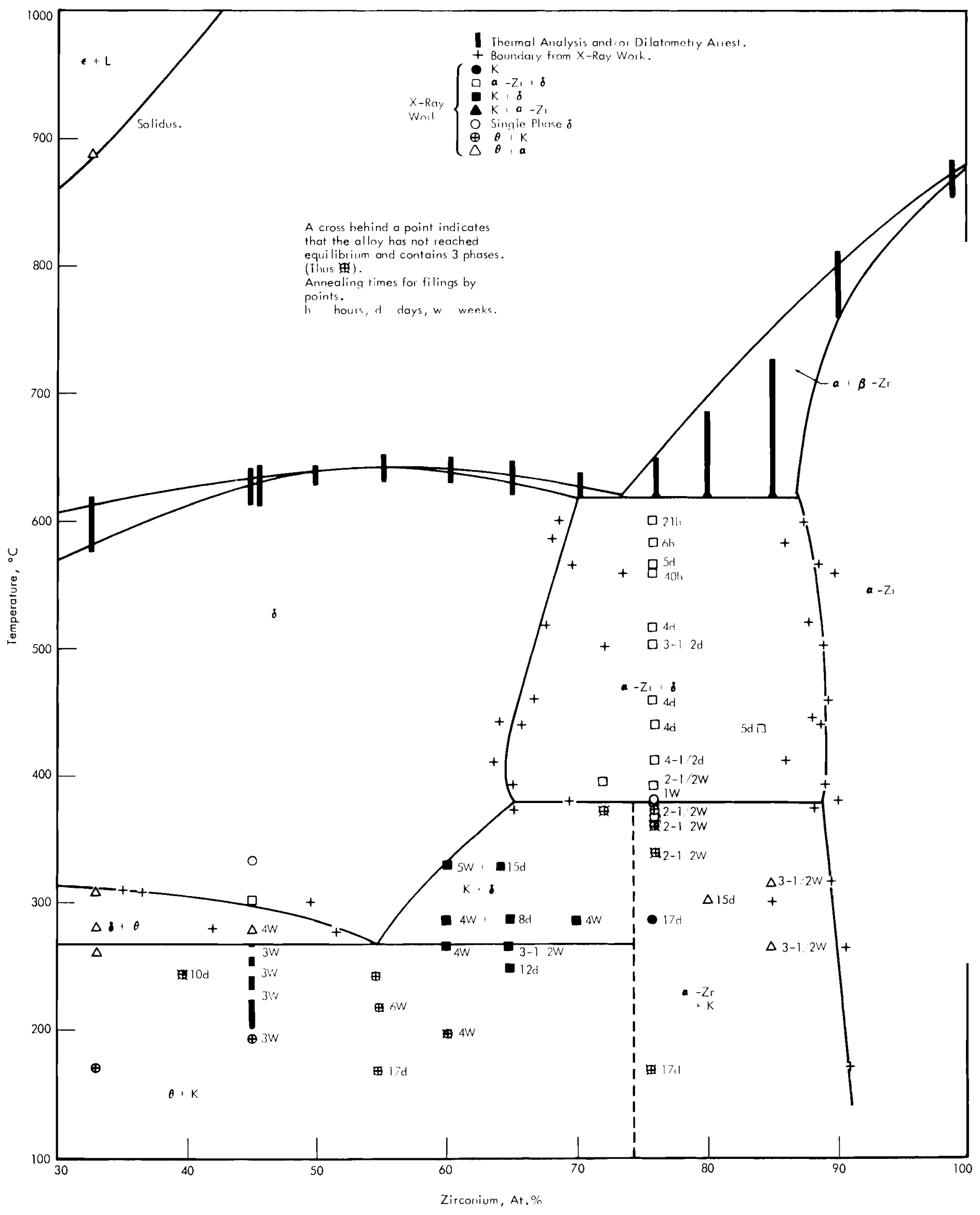

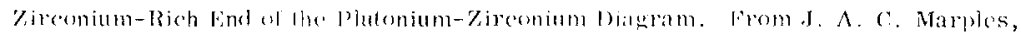




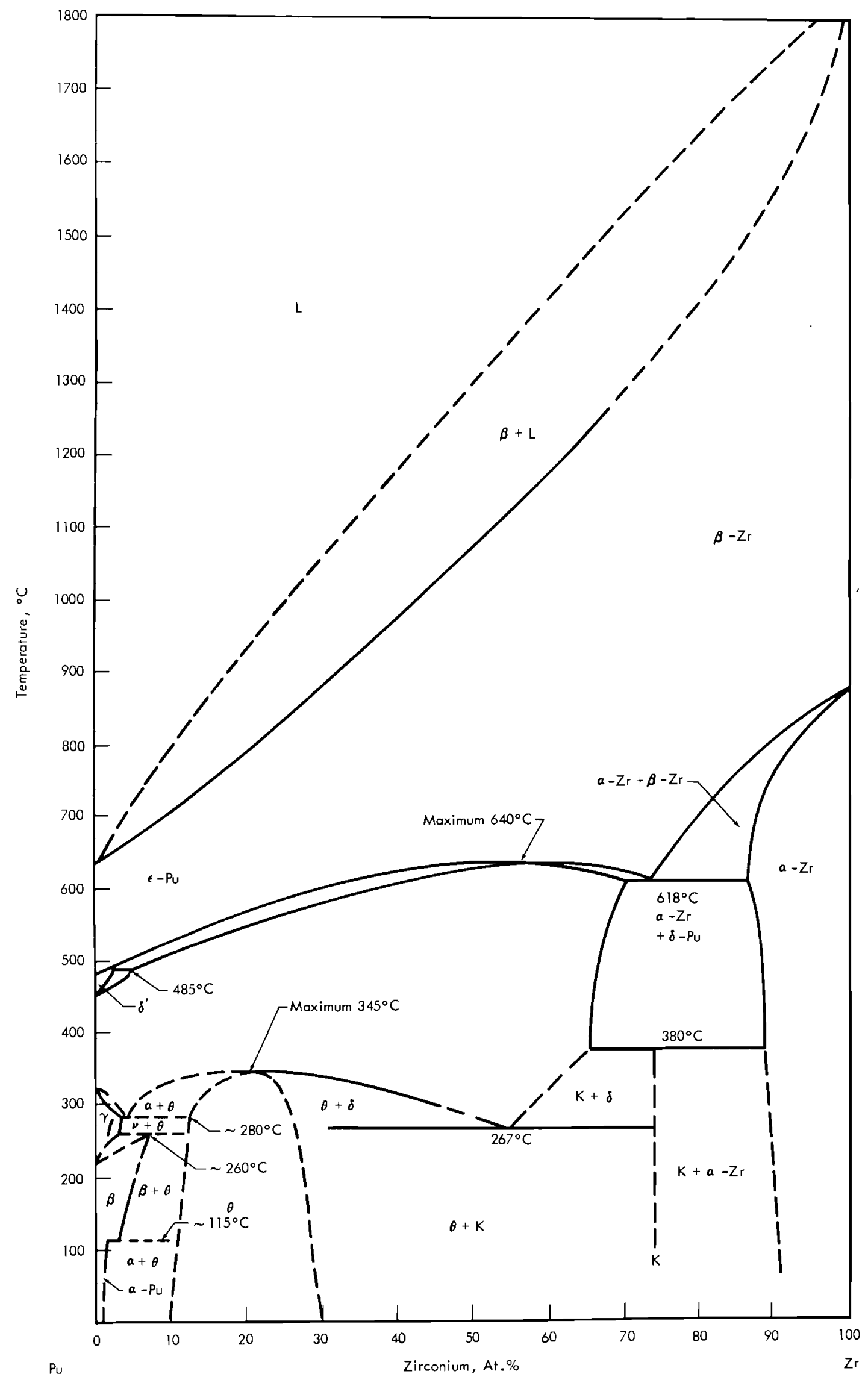




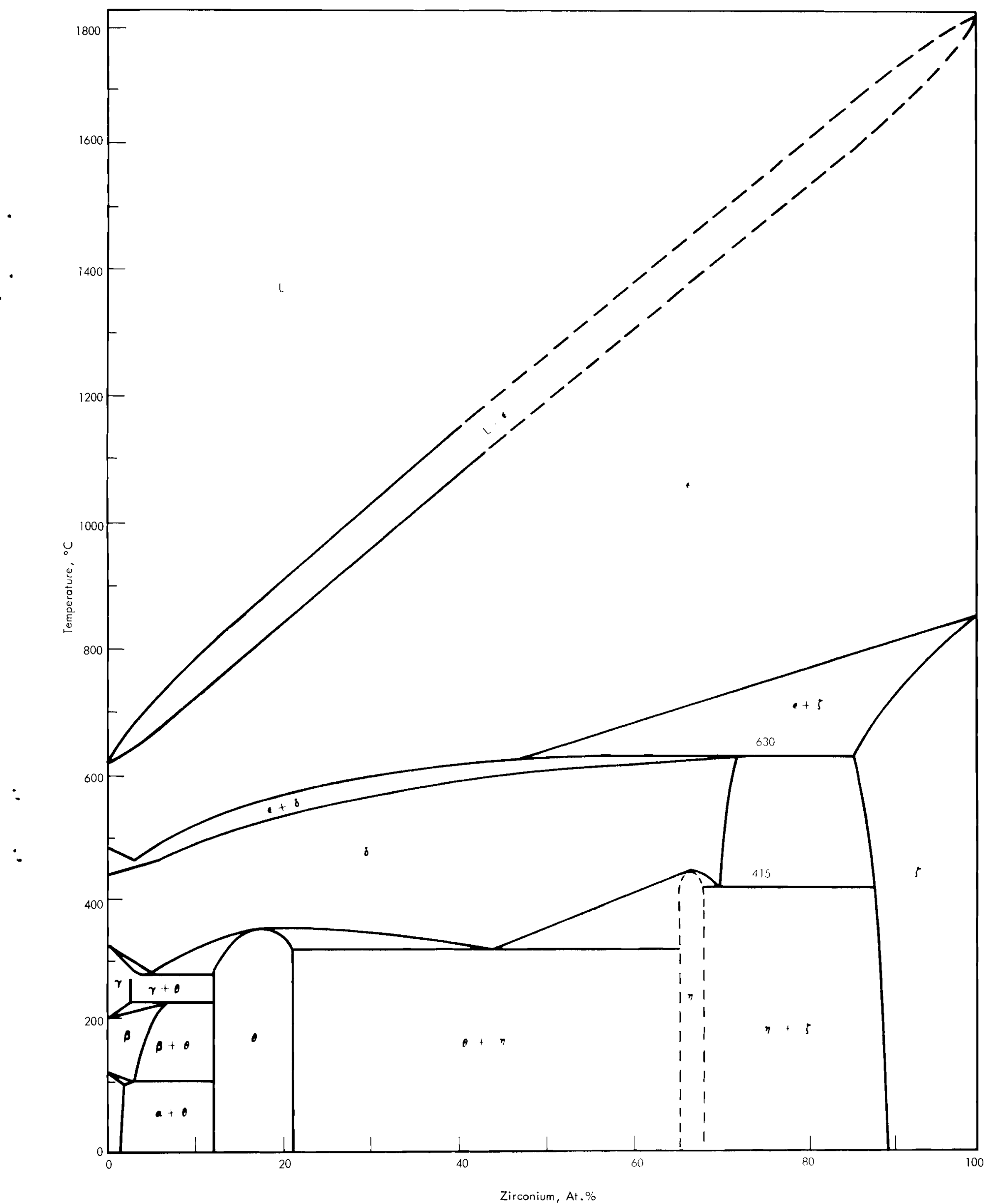

The Plutonium-\%irconim sistem. From l. W. Schonfeld in The Metal plutonium, 


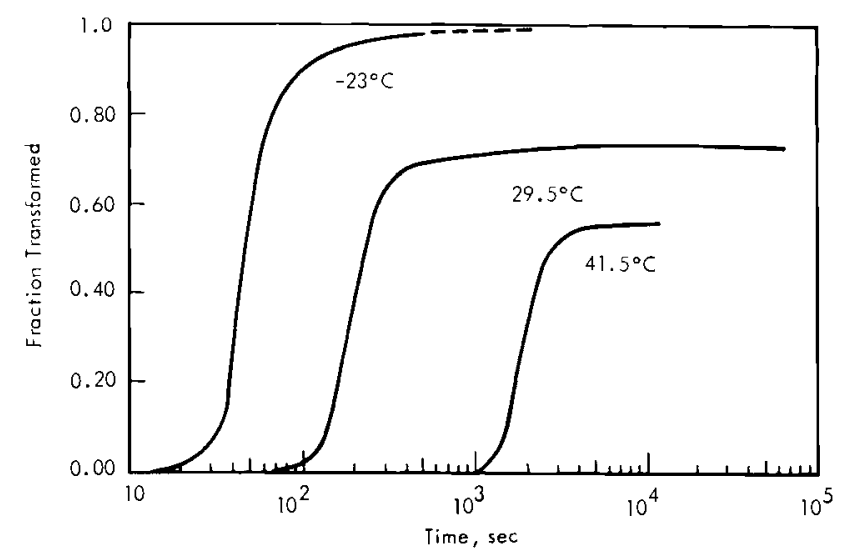

Isothernal Reaction Cur'ves For Pu-0.65 at.' $\mathrm{Z} \mathrm{l}^{\prime}$ Alloy. From J. M. Taylor, HW-SA-2.)

\section{Zr 103 crystal strucurue}

Two intermetallit phases have been observed in plutonium-zireonium alloys. Ios Alamos repolts that the plutonium-lieh phase is believed to eorrespond stojchiometrically to l'u $\mathrm{H}_{10} \mathrm{Zr}$. * Harwell reports that all observed lines on diffiation patterns are explicable in terms of P'u ${ }_{7} \mathrm{zr}$ and retained phases of pure plutenium. Russia ${ }^{\mp}$ rejorts a phase in the composition range 12.1 to

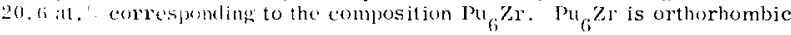
with a $10.39,1$, 10, 41 , and $\mathrm{c} 11,1 \times \mathrm{A}$. It has 8 formula units per unit

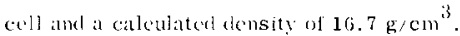

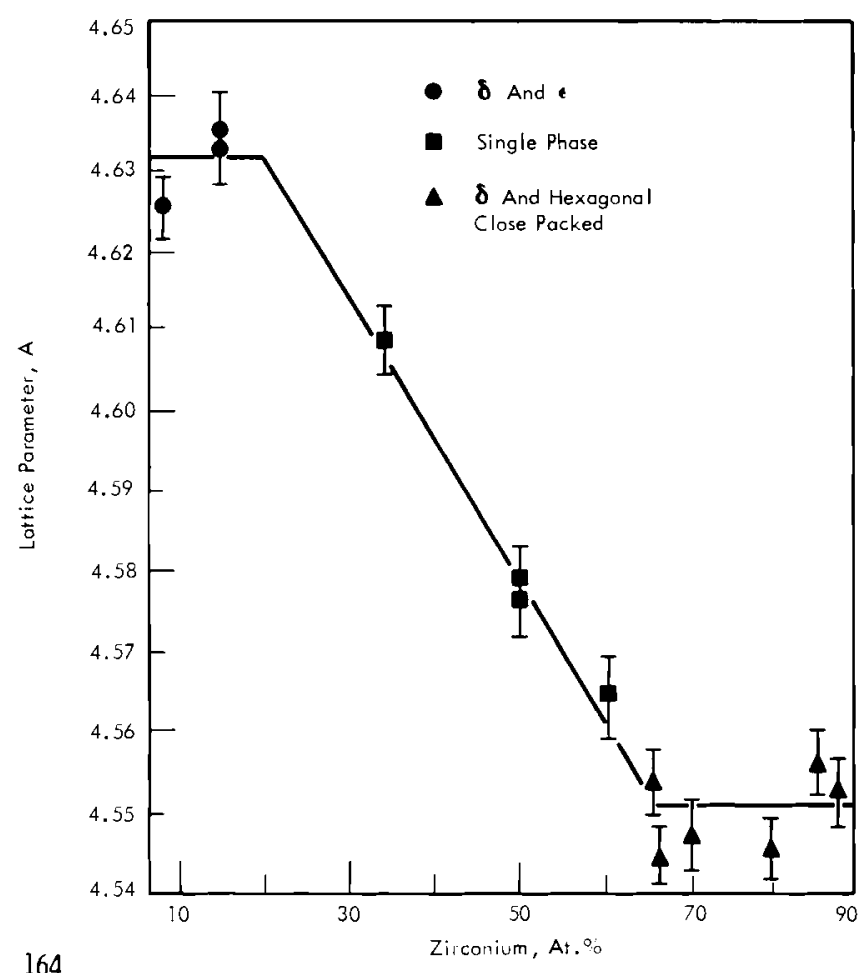

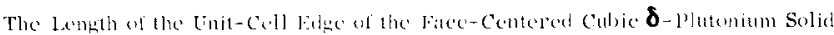
Solutions in the p'lutentum-Ziremium system.

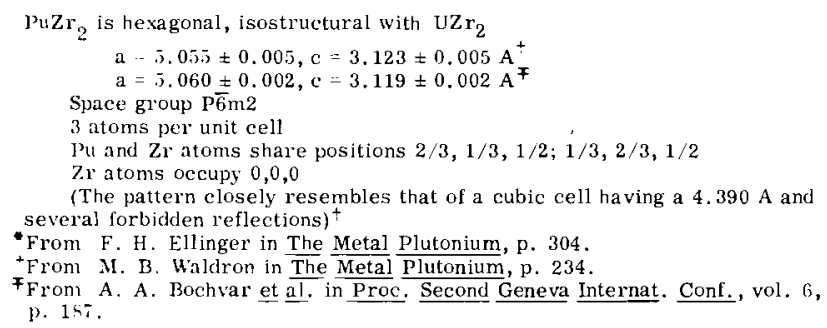

(The pattern closely resembles that of a cubic cell having a $4.390 \mathrm{~A}$ and several forbidden reflections) ${ }^{+}$

- From F. H. Ellinger in The Metal Plutonium, p. 304.

From M. B. Waldron in The Metal Plutonium, p. 234

From A. A. Bochvar et al, in Proc. Second Geneva Internat. Conf., vol. 6,

p. 1 isi.

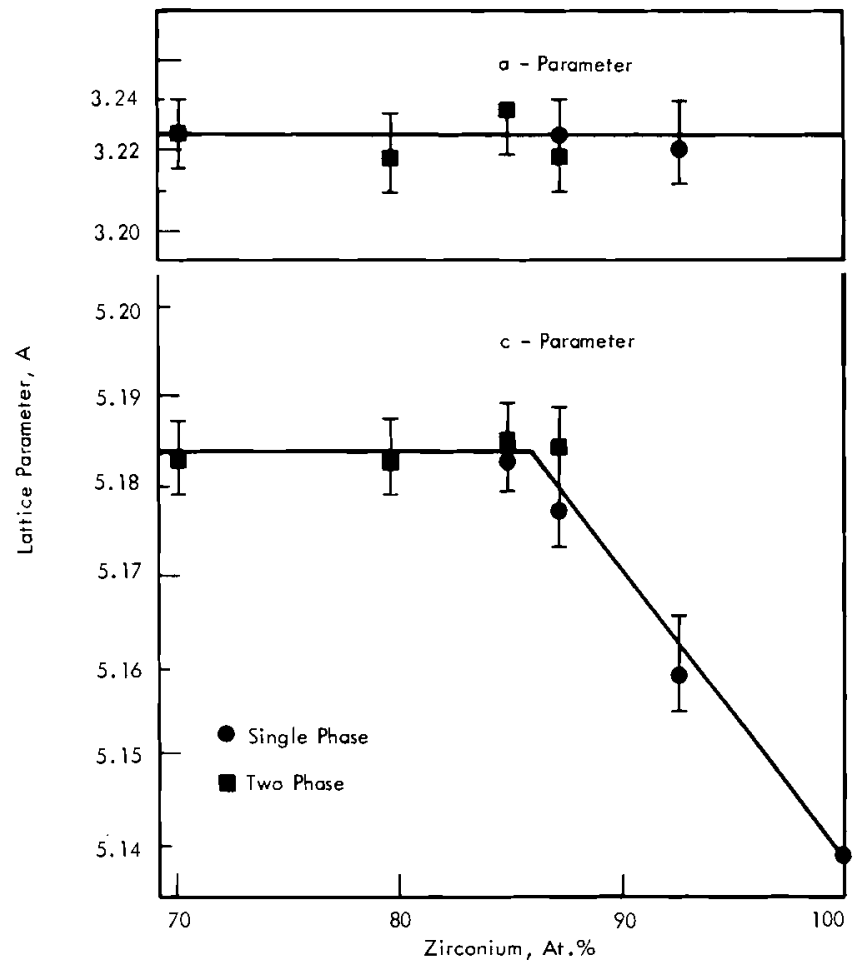

The bependente on Composition of $\mathrm{a}_{0}$ and $\mathrm{c}_{\mathrm{f}}$ of the Hexagonal a-Zirconium Solid Solutions in the Plutonium-Zirconium System. From M. B. Waldron in The 


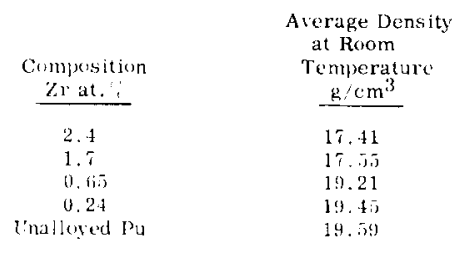

From J. M. Taylor, HW-SA-2560

\section{Thermal Expansion}

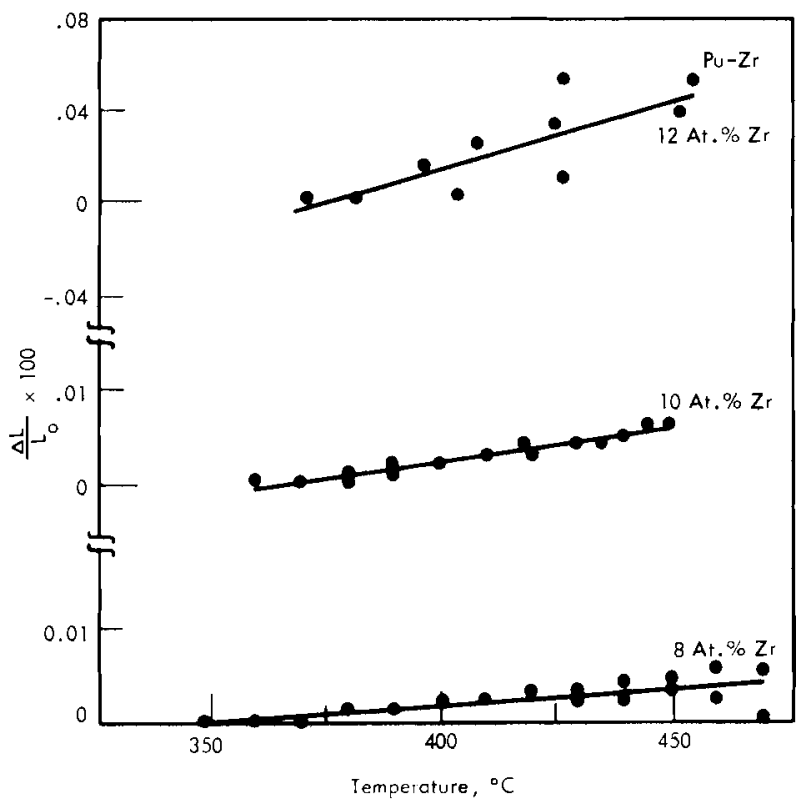

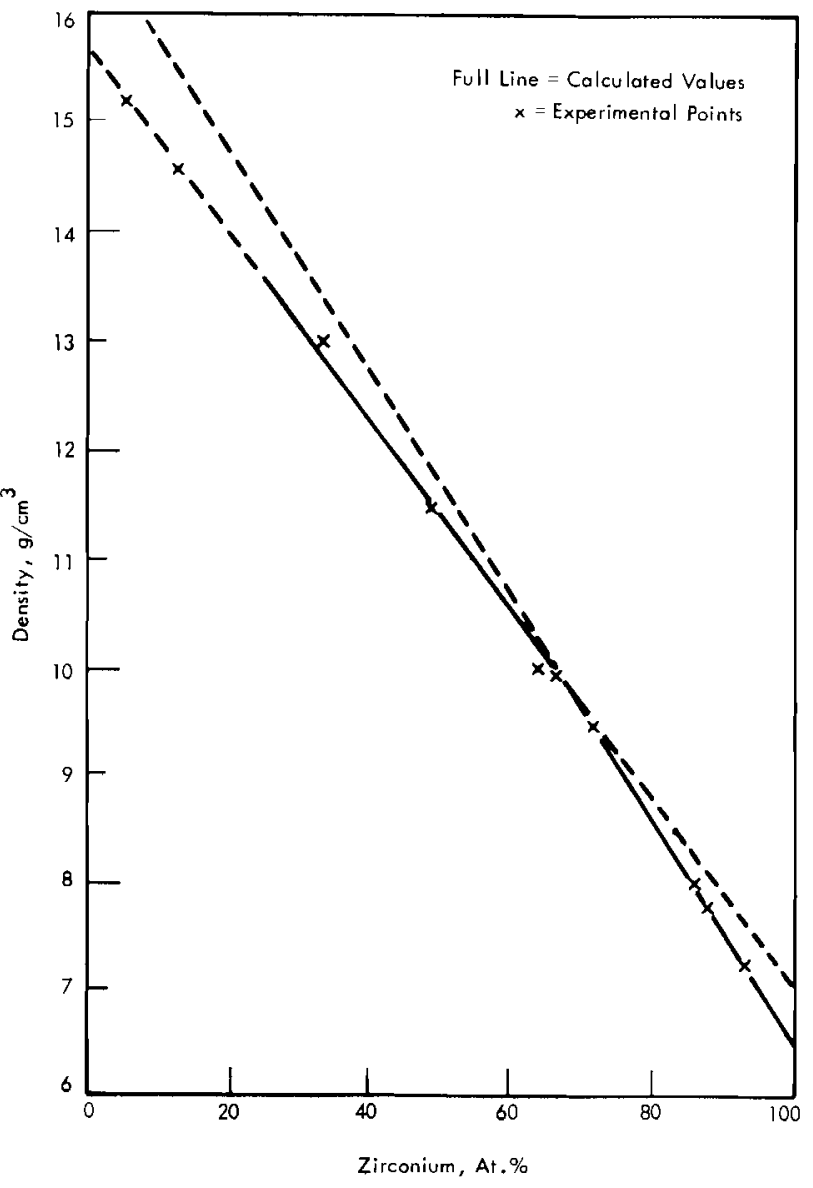

The Densities of Plutonium-Zirconium Alloys as a Function of Their Composition. From M. B. Waldron in The Metal Plutonium, 1). 236

\section{Zr 202.1}

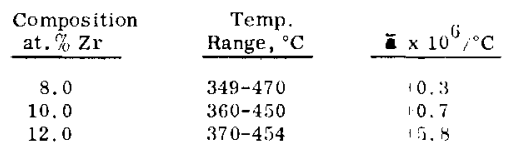

From R. O. Elliott et al. in Plutonium 1960, p. 151. 


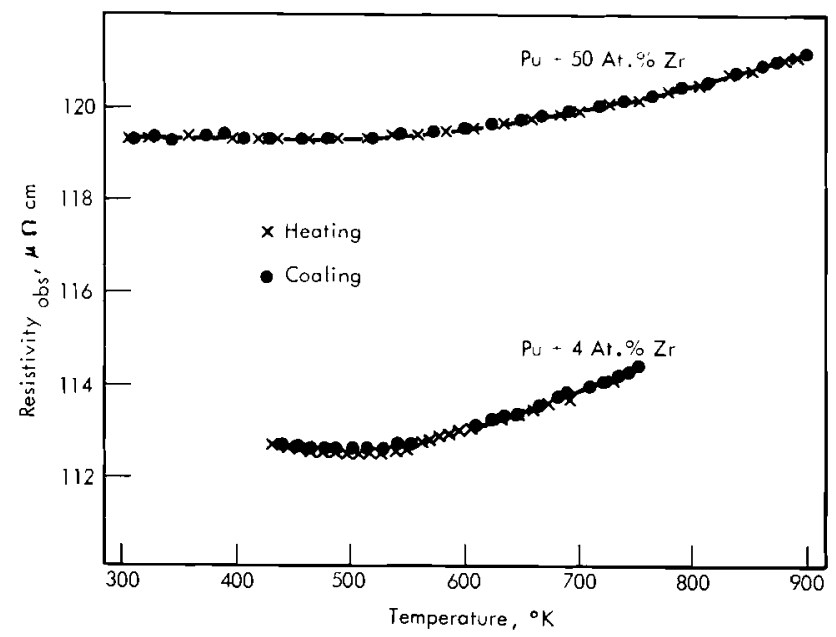

\section{$\operatorname{Zr} 204.1 \quad$ Magnetic Susceptibility}

Resistivilies of Pu-Zr $\delta$ - Phase Solid Solutions. From J. A. Lee et al. in Plutunium 1960, 1.4 .5 .

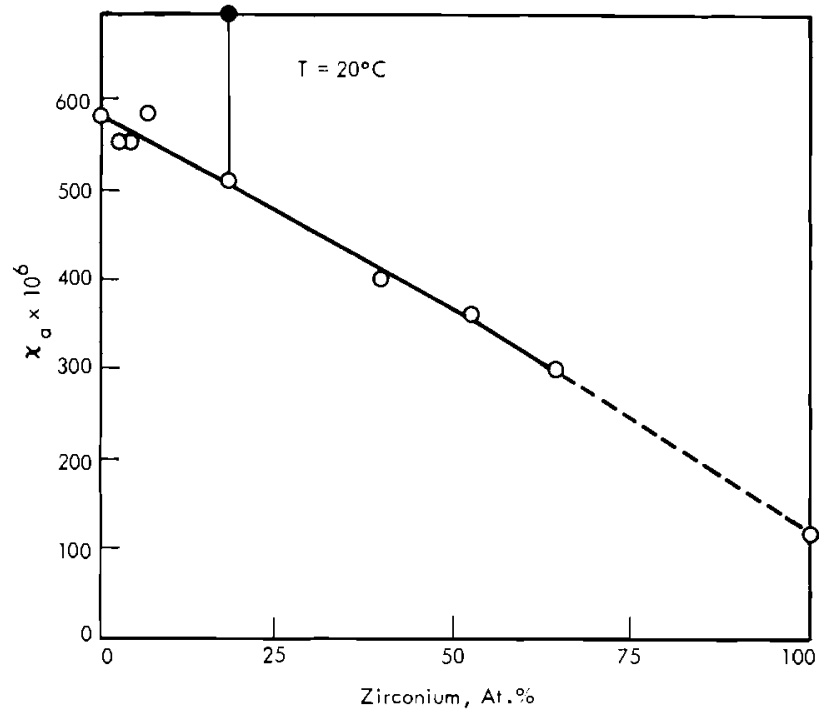

Paramagnetic Susceptibility of Plutonium-Zirconium Alloss.

O-Alloys of the $\delta$-plutonium Structure:

- Compound $\mathrm{Pu}_{6} \mathrm{Zr}$

From S. T. Komobcersky et al, in proc. Scoml Gonera Internat. Conf., vol. 6,

\section{Zr 301 Tensile Properties}

COMHPRISON OF MECHANICAL IPROPERTIES* OF VARIOUS COMPOSITIONS OF PLUTONIUM-ZIRCONIUM ALLOY ANI UNALLOYF,D PLUTONIUM AT ELEVATED TEMPERATURES

From J. M. Taylor, HW-SA-2560.

\begin{tabular}{|c|c|c|c|c|c|c|}
\hline $\begin{array}{l}\text { Composition } \\
\text { at. } \quad Z \text { Z }\end{array}$ & $\begin{array}{c}\text { Test } \\
\text { Temperature, } \\
{ }^{\circ} \mathrm{C} \\
\end{array}$ & $\begin{array}{l}\text { lltimate } \\
\text { Strength, } \\
\text { psi }\end{array}$ & Elongation, & $\begin{array}{l}\text { Rextuction } \\
\text { In Arou, }\end{array}$ & $\begin{array}{c}\text { Arerage Density } \\
\text { at Room } \\
\text { Temperature, } \mathrm{g} / \mathrm{cm}^{3} \\
\end{array}$ & Heat Treatment \\
\hline 2.4 & 180 & $1 \times, 000$ & -- &.- & 17.41 & 1 \\
\hline 2.4 & $1: 30$ & $-24,940$ & $\ldots$ & $\ldots$ & & 5 \\
\hline $1 . i$ & 1.40 & $16 i, 10.3$ & $\ldots$ & --- & $17 . . \overline{3}$ & $\vdots$ \\
\hline 1.7 & 130 & 26,0100 & --- & --- & & $\frac{5}{4}$ \\
\hline 0.15 .3 & $1 \times 0$ & 12,1000 & 100 & 1110 & 19.21 & $t$ \\
\hline 0.65 & $1: 31$ & $2 \cdot 1,22 \cdot 4$ & -- & -- & & $\S$ \\
\hline 11. 2.1 & 1.91 & 1,114 & +50 & $\therefore$ & $19.4 \pi$ & $t$ \\
\hline 11.21 & $1: 30$ & $1,1,300$ & 160 & $\because 11$ & & $\S$ \\
\hline 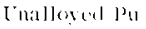 & 1.511 & $\therefore,-3>0$ & 5013.2 & 160 & $1\{1, .59$ & Beta Phase Plut \\
\hline 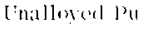 & $1: 30$ & 12,1110 & $2 ! 4$ & 1001 & & Beta Phase Plut \\
\hline
\end{tabular}

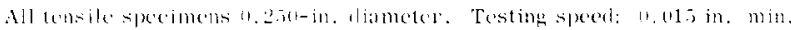

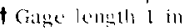

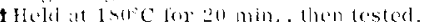




\begin{tabular}{|c|c|c|c|c|c|}
\hline $\begin{array}{l}\text { Test } \\
\text { Tem] } \\
{ }^{\circ} \mathrm{C} \\
\end{array}$ & $\begin{array}{c}\text { Litimate } \\
\text { Strengh, } \\
\text { psi } \\
\end{array}$ & $\begin{array}{c}\text { Yiclil } \\
\text { Strength, } \\
\text { psi } \\
\end{array}$ & Flongation, ${ }^{+}$ & $\begin{array}{l}\text { Recturtion } \\
\text { In Areu, }\end{array}$ & $\begin{array}{l}\text { Heat Treatment } \\
\text { And Remarks } \\
\end{array}$ \\
\hline 30 & 51,000 & $24,400^{\ddagger}$ & .076 & -- & $\begin{array}{l}\text { Specimel held at } 220^{\circ} \mathrm{C} \text { for } 24 \mathrm{hrs} \text {, quenched to room } \\
\text { temperature, tested } 7 \mathrm{hrs} \text { after quench. }\end{array}$ \\
\hline 70 & $4,, 346$ & $3 k, .50110$ & - & -- & $\begin{array}{l}\text { Held at } 220^{\circ} \mathrm{C} \text { for } 3 \text { hrs, Cooled to } 70^{\circ} \mathrm{C} \text {, and held for } \\
20 \mathrm{~min} \text {. and tested. }\end{array}$ \\
\hline 130 & 36,800 & 33,400 & -- & -- & $\begin{array}{l}\text { Held at } 220^{\circ} \mathrm{C} \text { for } 5 \text { hrs. Cooled to } 130^{\circ} \mathrm{C} \text {, and held for } \\
20 \mathrm{~min} \text {, and tested. }\end{array}$ \\
\hline 160 & 28,700 & 23,300 & -- & -- & $\begin{array}{l}\text { Held at } 220^{\circ} \mathrm{C} \text { for } \mathrm{s} \text { hrs. Cooled to } 160^{\circ} \mathrm{C} \text {, and held for } \\
20 \mathrm{~min} \text {. and tested. }\end{array}$ \\
\hline 180 & 23,835 & $20,0 \circ 0$ & -- & -- & $\begin{array}{l}\text { Held at } 220^{\circ} \mathrm{C} \text { for } 5 \text { hrs. Cooled to } 180^{\circ} \mathrm{C} \text {, and held for } \\
20 \text { min. and tested. }\end{array}$ \\
\hline 200 & 18,367 & 14,300 & -- & -- & $\begin{array}{l}\text { Held at } 220^{\circ} \mathrm{C} \text { for } 24 \mathrm{hrs} \text {, quenched to room temperature. } \\
\text { Heated to } 200^{\circ} \mathrm{C} \text {, and held for } 20 \mathrm{~min} \text {. then tested. }\end{array}$ \\
\hline 210 & 13,700 & 11,600 & $\cdots$ & -- & $\begin{array}{l}\text { Held at } 220^{\circ} \mathrm{C} \text { for } 24 \mathrm{hrs} \text {, quenched to room temperature. } \\
\text { Heated to } 210^{\circ} \mathrm{C} \text {, and heid for } 20 \mathrm{~min} \text {. then tested. }\end{array}$ \\
\hline 230 & 12,240 & 8,300 & $\ldots$ & 2 & $\begin{array}{l}\text { ficld at } 220^{\circ} \mathrm{C} \text { for } 24 \mathrm{hrs} \text {, quenched and heated to } 230^{b} \mathrm{C} \\
\text { and held } \text { (or } 20 \mathrm{~min} \text {. then tested. }\end{array}$ \\
\hline 250 & 10,290 & 7,300 & 5.0 & 2 & $\begin{array}{l}\text { Held at } 220^{\circ} \mathrm{C} \text { for } 24 \mathrm{hrs} \text {, quenched and heated to } 250^{\circ} \mathrm{C} \\
\text { for } 20 \mathrm{~min} \text {. then tested. }\end{array}$ \\
\hline 260 & 7,800 & 6,9001 & 6.3 & 40 & $\begin{array}{l}\text { Held at } 220^{\circ} \mathrm{C} \text { for } 24 \mathrm{hrs} \text {, quenched and heated to } 260^{\circ} \mathrm{C} \\
\text { for } 20 \mathrm{~min} \text {. then tested. }\end{array}$ \\
\hline
\end{tabular}

* Testing Speed: $0.015 \mathrm{in.} \mathrm{min.}{ }^{+}$Based on one in. gage length $\ddagger_{A} 0.01 \%$ offset yield strength.

STUDY OF THE FFEET OF AGING TTME ON THE TENSILE PROPERTIES* OF A BETA

STABILIZED Pu-2.4 at. Z Z ALLOY

From J. M. Tay'lor, HW-SA-2560.

\begin{tabular}{|c|c|c|c|c|c|c|}
\hline $\begin{array}{l}\text { Aging Time } \\
\text { After } \\
\text { Quench }\end{array}$ & $\begin{array}{l}\text { Ultimate } \\
\text { Strength, } \\
\text { psi }\end{array}$ & $\begin{array}{l}0.01 \% \\
\text { Yield Strength, } \\
\text { Is j }\end{array}$ & Elongation, & $\begin{array}{l}\text { Reduction } \\
\text { In Area, }\end{array}$ & $\begin{array}{l}\text { Modulus of } \\
\text { Elasticity } \\
\times 10^{-6}\end{array}$ & $\begin{array}{l}\text { Temperature } \\
\text { Of Test, } \\
{ }^{\circ} \mathrm{C}\end{array}$ \\
\hline $40 \mathrm{mi}$ & 40, & 22,100 & $0.07 \mathrm{i}$ & -- & 6.43 & 30 \\
\hline $7 \mathrm{hr}$ & \$1, 1100 & 28,400 & 0.28 & -- & 5.73 & 30 \\
\hline $24 \mathrm{hr}$ & 42,700 & 24,800 & 0.081 & $\cdots$ & 6.09 & 30 \\
\hline $48 \mathrm{hr}$ & 43,000 & 16,200 & 0.102 & -. & 6.59 & 30 \\
\hline
\end{tabular}

*Testing speerl: $0.015 \mathrm{in.} / \mathrm{min}$.

${ }^{+}$All tensile specimens were heat treated at $220^{\circ} \mathrm{C}$ for $24 \mathrm{hr}$ and then quenched in oil at room temperature.

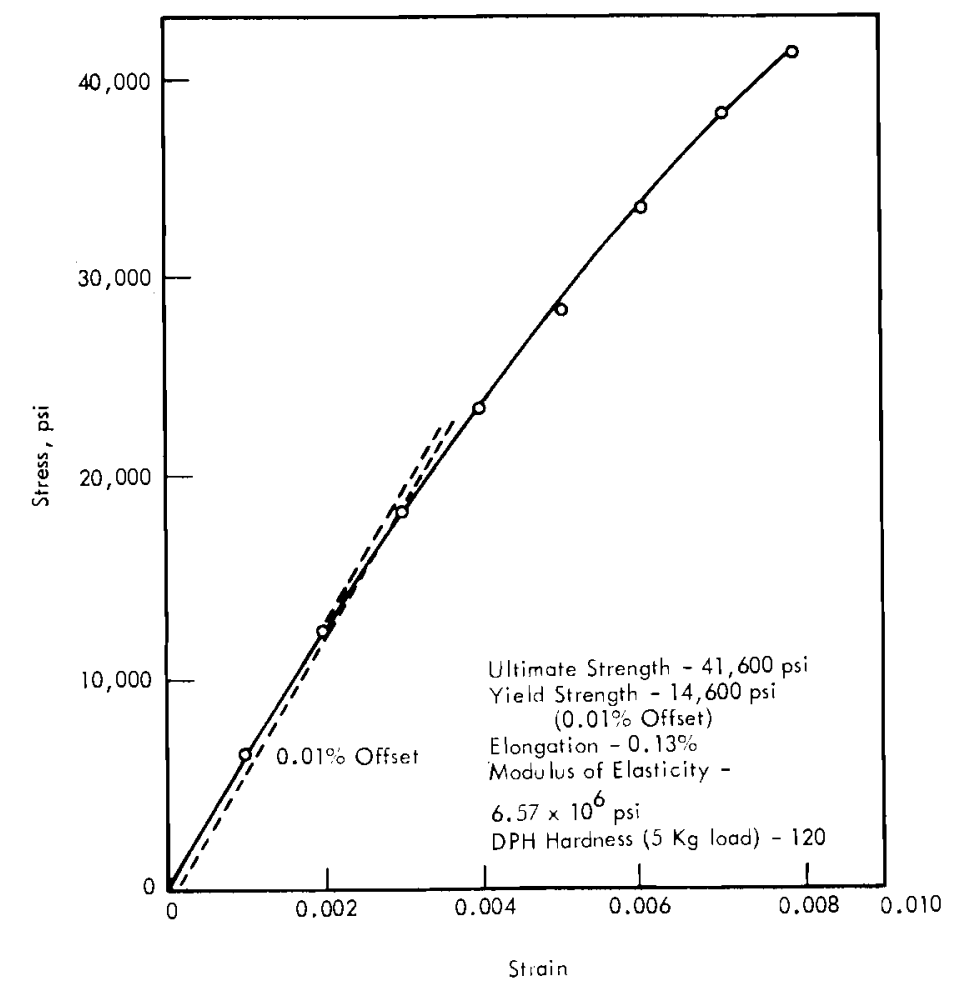



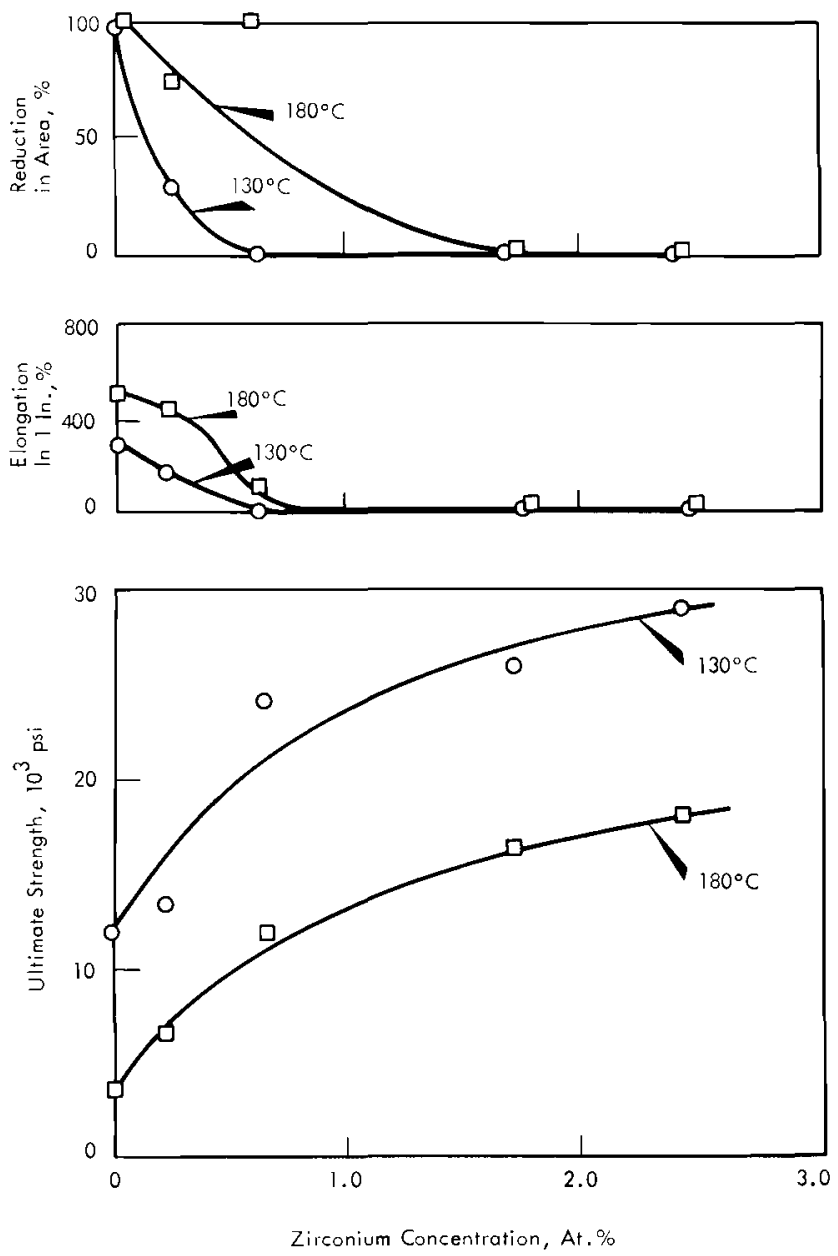

Lifect of Zireonium Concentration and Test Temperatures on the Tensile Preperties of IPlutomium-Zirconium Alloss. From J. M. Taylor, as

reported by 11. R. Galelner in Chapter 4, The plutonium Handbook, 1966.
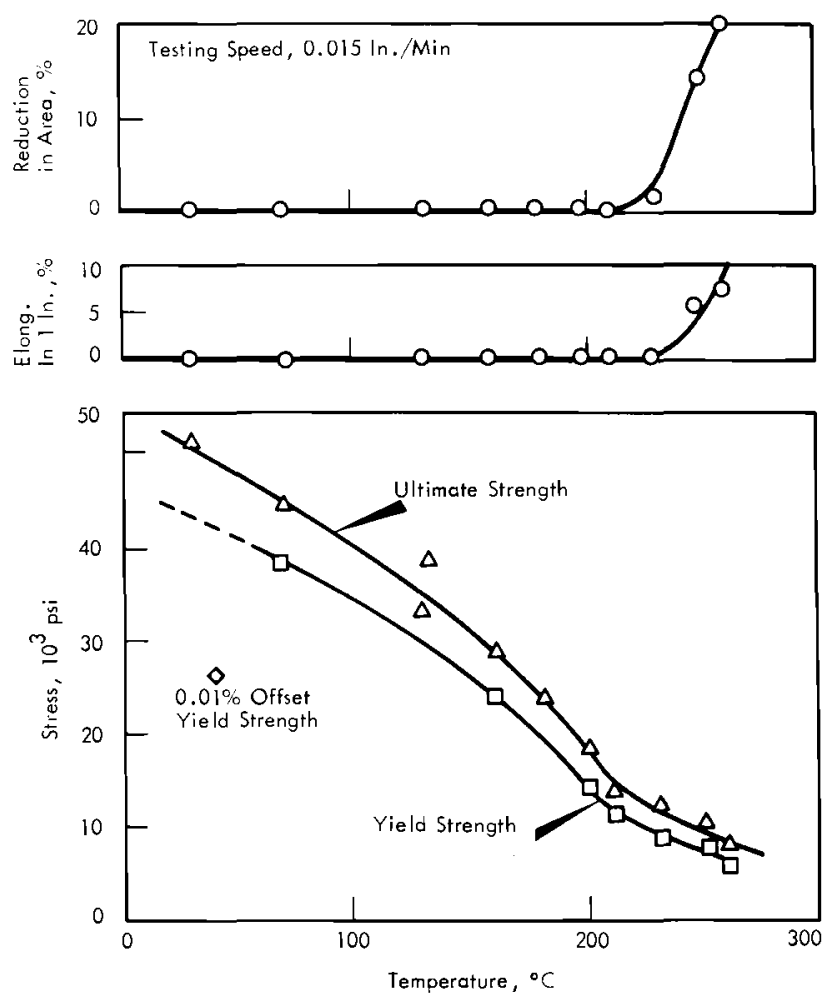

Effect of Temperature on Tensile Properties of Beta-Stabilized Plutonium-2,4 at."' Zirconiun Alloy. From J. M. Taylor, as reported by H. R. Gardner in Chapter 4, The Plutonium Handbook, 1966

\section{$\operatorname{Zr} 302$ conpressive roroerties}

EFFECT OF TEMPERATURE ON THE COMI'RESSION PROPERTIES* OF

BETA STABILIZED Pu-2.4 at. "' Zr ALLOY

From J. M. Taylor, HW-SA-2560.

\begin{tabular}{|c|c|c|c|c|}
\hline $\begin{array}{l}\text { Test } \\
\text { Termperature, } \\
{ }^{\circ} \mathrm{C}\end{array}$ & $\begin{array}{l}\text { Ultimate } \\
\text { Strength, } \\
\text { j)si }\end{array}$ & $\begin{array}{l}\text { Yield } \\
\text { Strength, } \\
\text { psi }\end{array}$ & $\begin{array}{l}\text { Plastic Decrease } \\
\text { In Length, }\end{array}$ & $\begin{array}{l}\text { Heat Treatment } \\
30 \mathrm{~min}{ }^{\circ} \mathrm{C}\end{array}$ \\
\hline rio & 07,0001 & $\pi 9, \times 00$ & 4.6 & 60 \\
\hline$\therefore 0$ & 64,600 & 37,900 & 5.3 & 50 \\
\hline 130 & 33,300 & 48,700 & 5.3 & 130 \\
\hline 160 & 45,600 & 41,000 & 6.01 & 160 \\
\hline 180 & 46,800 & 43,000 & 5.0 & 180 \\
\hline 200 & $32, \times 100$ & 27,700 & $\pi .0$ & 200 \\
\hline 220 & 28,500 & 26,900 & .7 .4 & 220 \\
\hline
\end{tabular}

Testing speed 0.115 in. min

+Total plastic decrease was determined from load-platen displiacement curve al point of fitilure. 


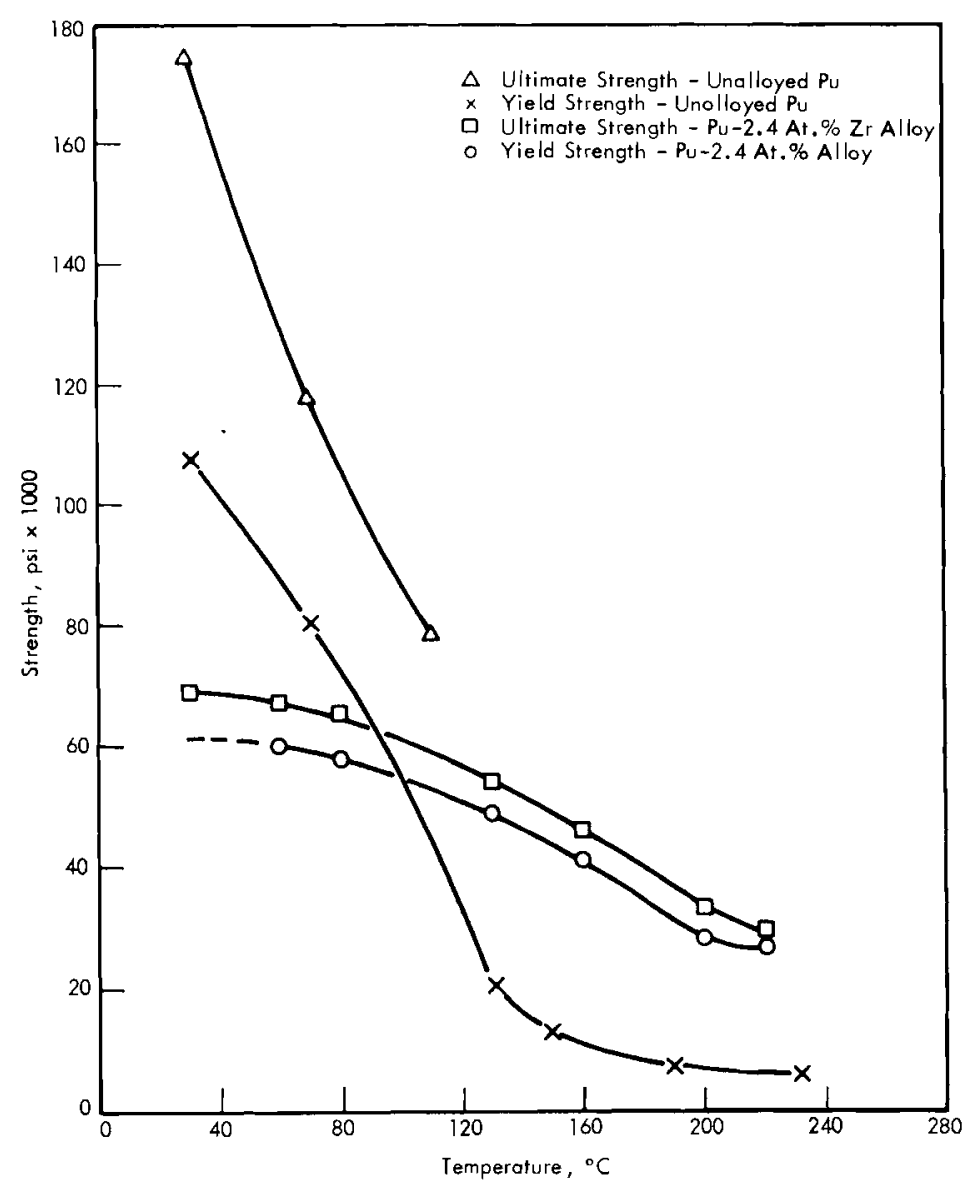

Comparison of Compressive Strengths of Pu-2.4 at. Zr Alloy and Unalloyed Plutonium. From J, M. Taylor, HW-SA-2560.

HARDNESS OF PU-Zr ALLOYS

From J. M. Taylor, as reported by H. R. Gardner in Chapter 4, The Plutonium Handbook, 1966.

\begin{tabular}{ccl}
$\begin{array}{c}\text { Composition, } \\
\text { at. \% } \mathrm{Zr}\end{array}$ & $\begin{array}{c}\text { Diamond } \\
\text { Pyramid } \\
\text { Hardness, } \\
5 \mathrm{~kg} \text { Load }\end{array}$ & \multicolumn{1}{c}{ Treatment } \\
\cline { 1 - 1 } 0.24 & 300 & As-Cast \\
0.6 .5 & 269 & As-Cast \\
1.7 & 107 & As-Cast \\
2.4 & 117 & $\begin{array}{l}\text { Annealed } 24 \mathrm{hr} \text { at } 220^{\circ} \mathrm{C} \text { and oil } \\
\text { quenched. }\end{array}$
\end{tabular}

ROOM TEMPERATURE HARDNESS FIGURES From D. M. Poole et al. in Plutonium 1960, p. 640.

$\frac{\text { Alloy Composition }}{\mathrm{Zr}^{-5} \text { at. } \mathrm{Pu}}$

$$
\begin{aligned}
& \text { Treatment } \\
& \hline \text { Rolled } \\
& \text { Rolled } \\
& \text { Rolled } \\
& \text { Rolled } \\
& \text { Rolled }
\end{aligned}
$$

Vickers Hardness Number, VHN

\begin{tabular}{r} 
VHN \\
\hline 207 \\
209 \\
189 \\
108 \\
97
\end{tabular}




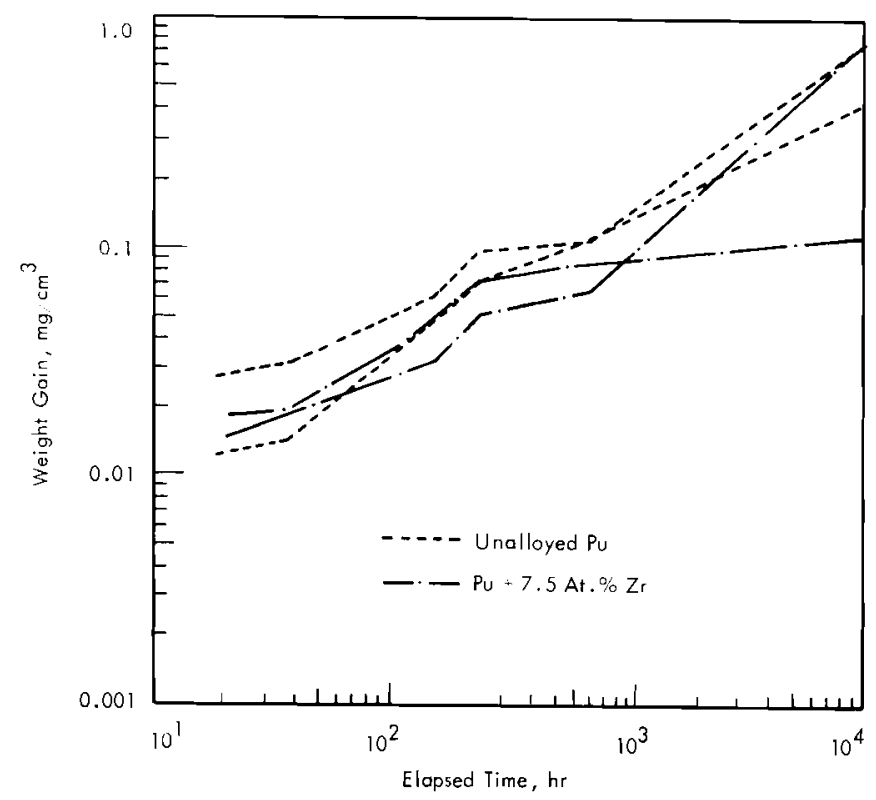

Corrosion Data for Pu and for Pu-Zr Alloy; Exposed to Air Dried With $\mathrm{MgClO}_{4}$ at $75^{\circ} \mathrm{C}$. From J. T. Waber in Proc. Second Geneva Internat. Conf, vol. 6,1$) .212$.

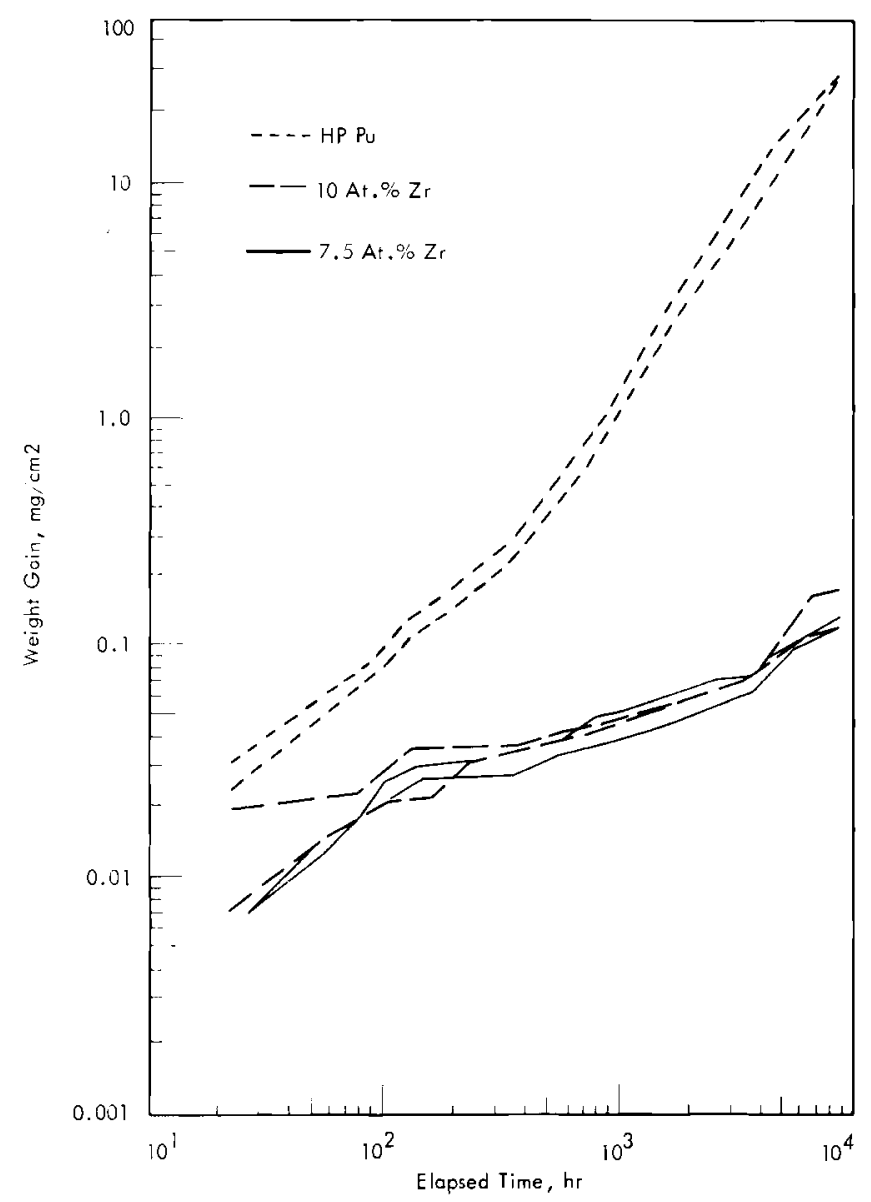

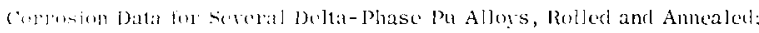

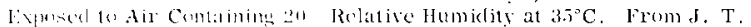

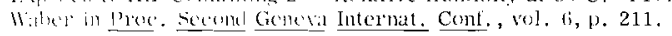

MLRIT RATIOS FOR ["U-Z1' $A$ I.LOYS EXI'OSED TO AIR AT $20^{\prime}$ RELATIVE H(MII)ITY AND $: 3^{\circ} \mathrm{C}$

lrom J. T. Walee in Proe. Sceond Geneva Internat. Conf., vol, 6, p, 211.

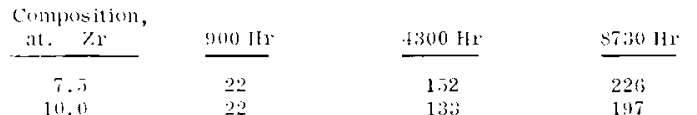

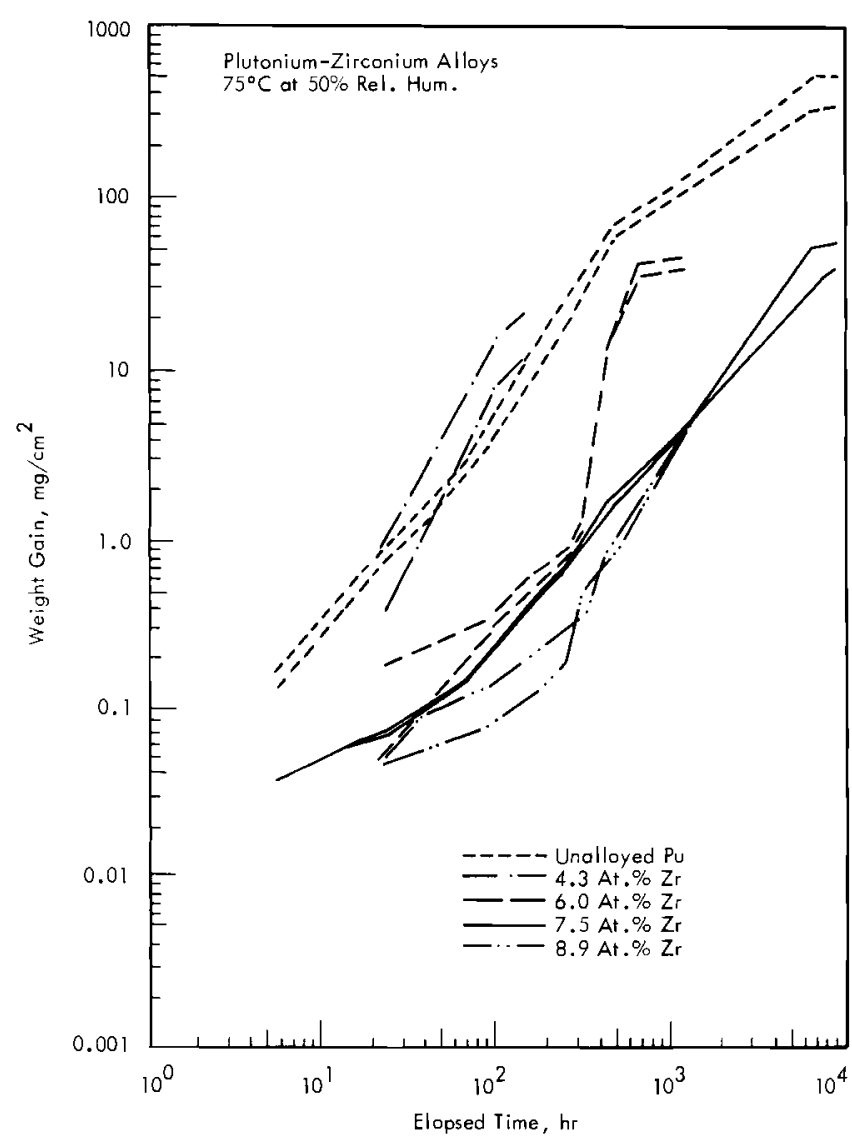

Comprarison of the Corrosion Behaviors of Plutonium-Zirconium Alloys and ['nalleged l'lutonium Exposed $10 \mathrm{Air}$ at $75^{\circ} \mathrm{C}$ and 50 ; Retative Humidity. From J. T. Maber et al., J. Nucl. Mater, vol. 3, p. 206.1961.

MH:RIT RITIOS FOR DE.T.T-PHASE Pu-Zr ALLOYS EXPOSED TO AIR AT .) RELATIVE HUMIDITY AND $75^{\circ} \mathrm{C}$

J. T. Waber el at. J. Nuel. Mater, vol. a, p. 206.1961.

\begin{tabular}{|c|c|c|}
\hline \multirow[b]{2}{*}{$Z r$, at.} & \multicolumn{2}{|c|}{ Merit Ratio Olserved } \\
\hline & at $300 \mathrm{~h}$ & at $1000 \mathrm{~h}$ \\
\hline 4.3 & 1.22 & 0.61 \\
\hline fi, 11 & 1. 1 & - \\
\hline 9.9 & 17.1 & 7.42 \\
\hline
\end{tabular}




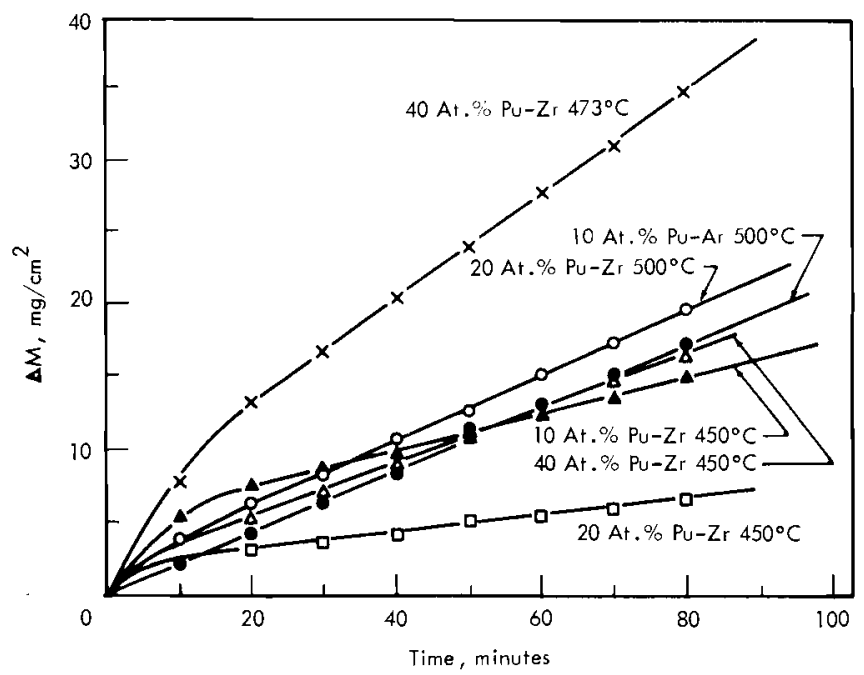

Oxidation of Pu-Zr Alloys Air Dried Over Magnesium Perchlorate.

From D. M. Poole et al. in Plutonium 1960, p. 630.

Reactions with Gases - Inert Gases

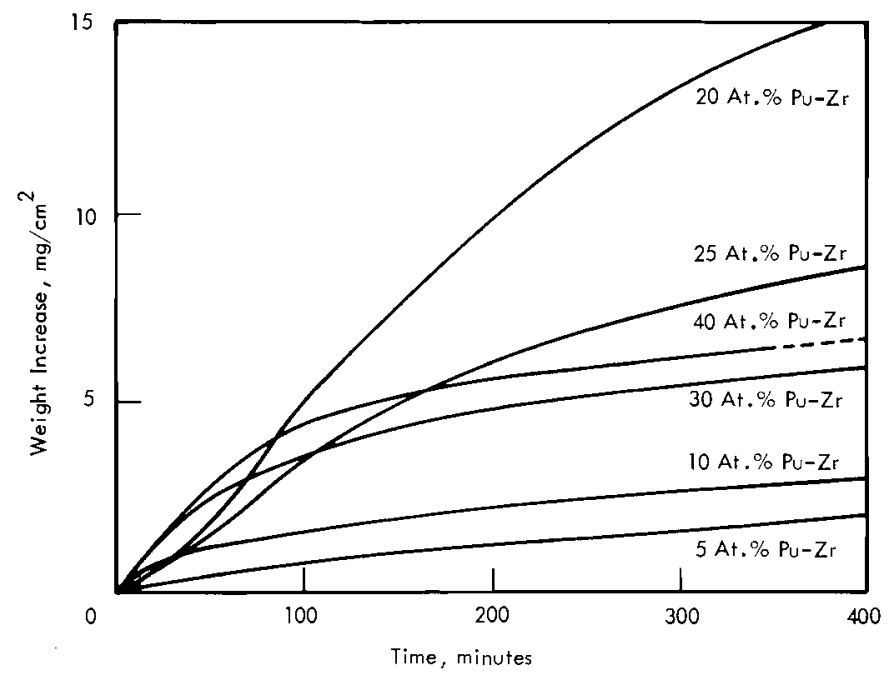

Oxidation of Rolled Pu-Zr Alloys in Dry $\mathrm{CO}_{2}$ at $700^{\circ} \mathrm{C}$

From D. M. Poole et al. in Plutonium 1960, p. 633.

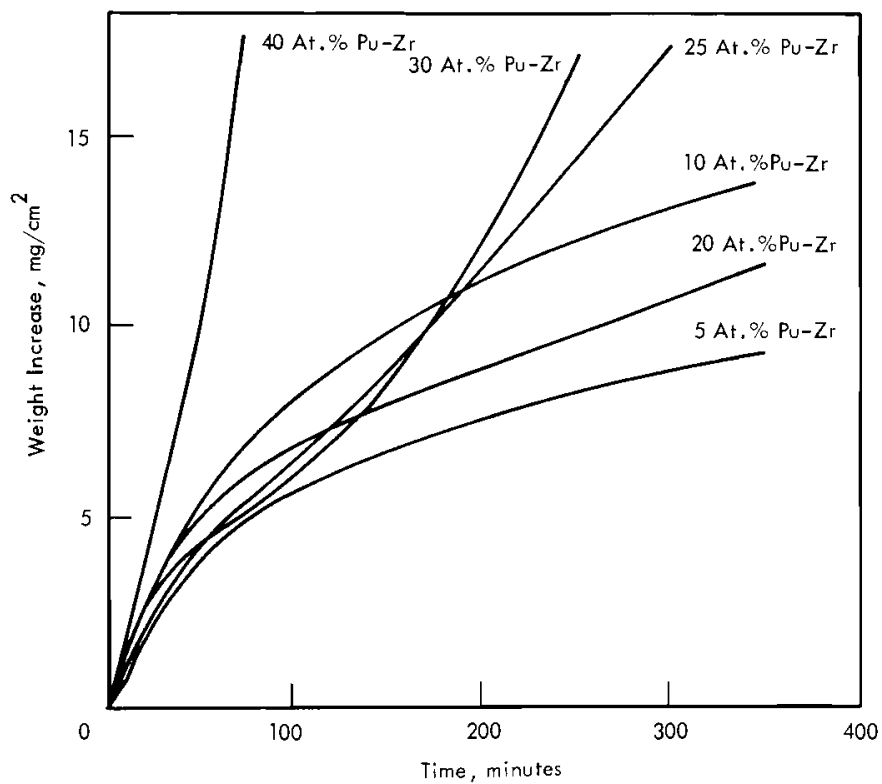

Oxidation of Rolled Pu-Zr Alloys in $\mathrm{CO}_{2}$ Dried Over Mapnesium Perchlorate at $800^{\circ} \mathrm{C}$. From D. M. Poole et al. in Plutonium 1960 p. 633. 


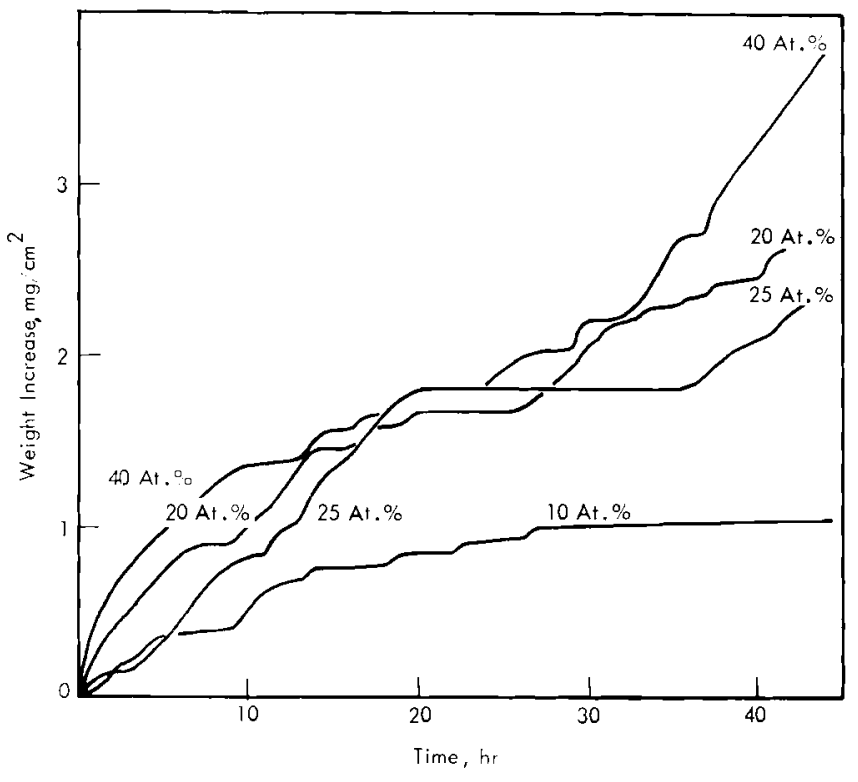

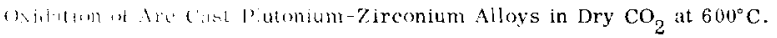

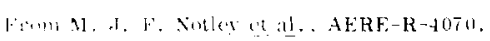

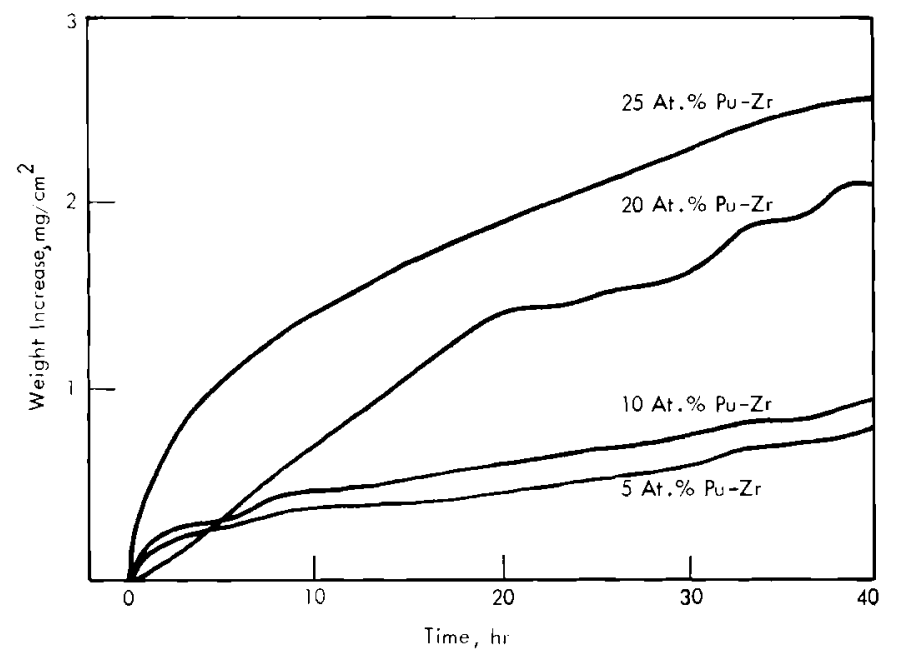

ONitatim of Rolled Mutonium-7ireonium $\mathrm{Allows}$ in Dry $\mathrm{CO}_{2}$ at $600^{\circ} \mathrm{C}$

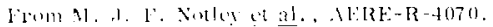

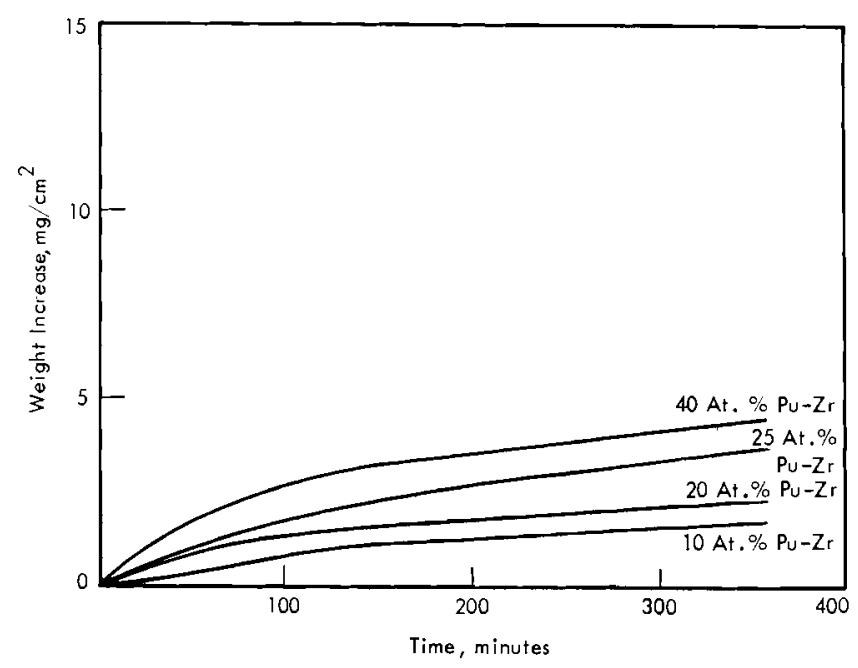

Oxidation of Are Cast Plutonium-Zirconium Alloys in Dry $\mathrm{CO}_{2}$ at $700^{\circ} \mathrm{C}$. From M. J, F, Notley et al., AERE-R-4070,

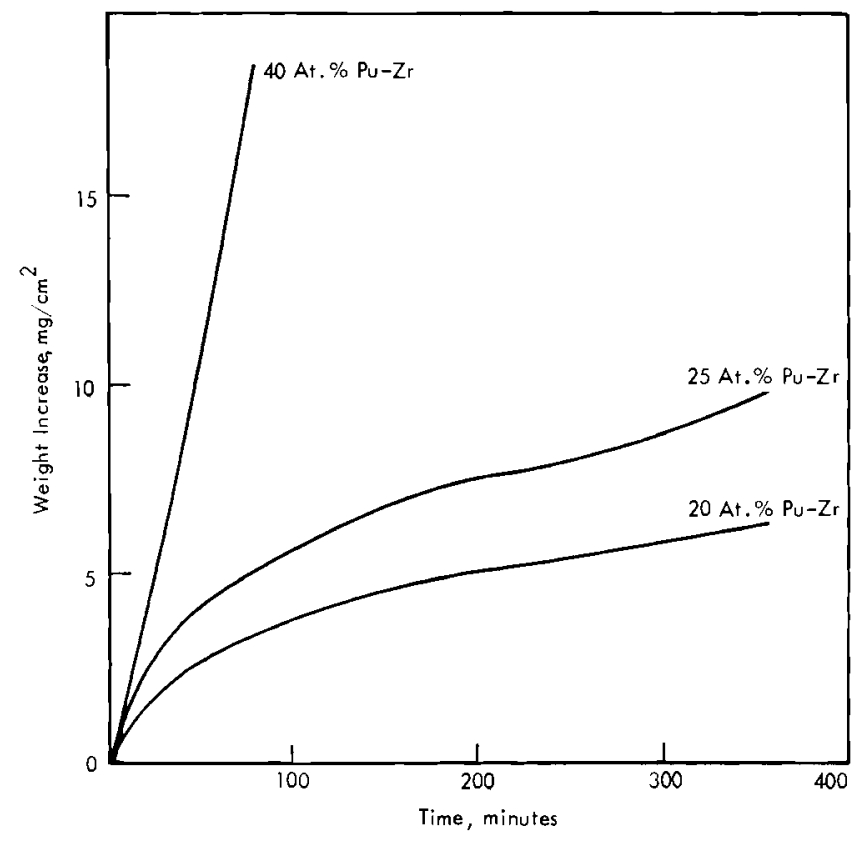

Oxidation of Are Cast Plutonium-Zirconium Alloys in Dry $\mathrm{CO}_{2}$ at $800^{\circ} \mathrm{C}$.

From M. J. F. Notley et al., AFrR:-R-4070. 


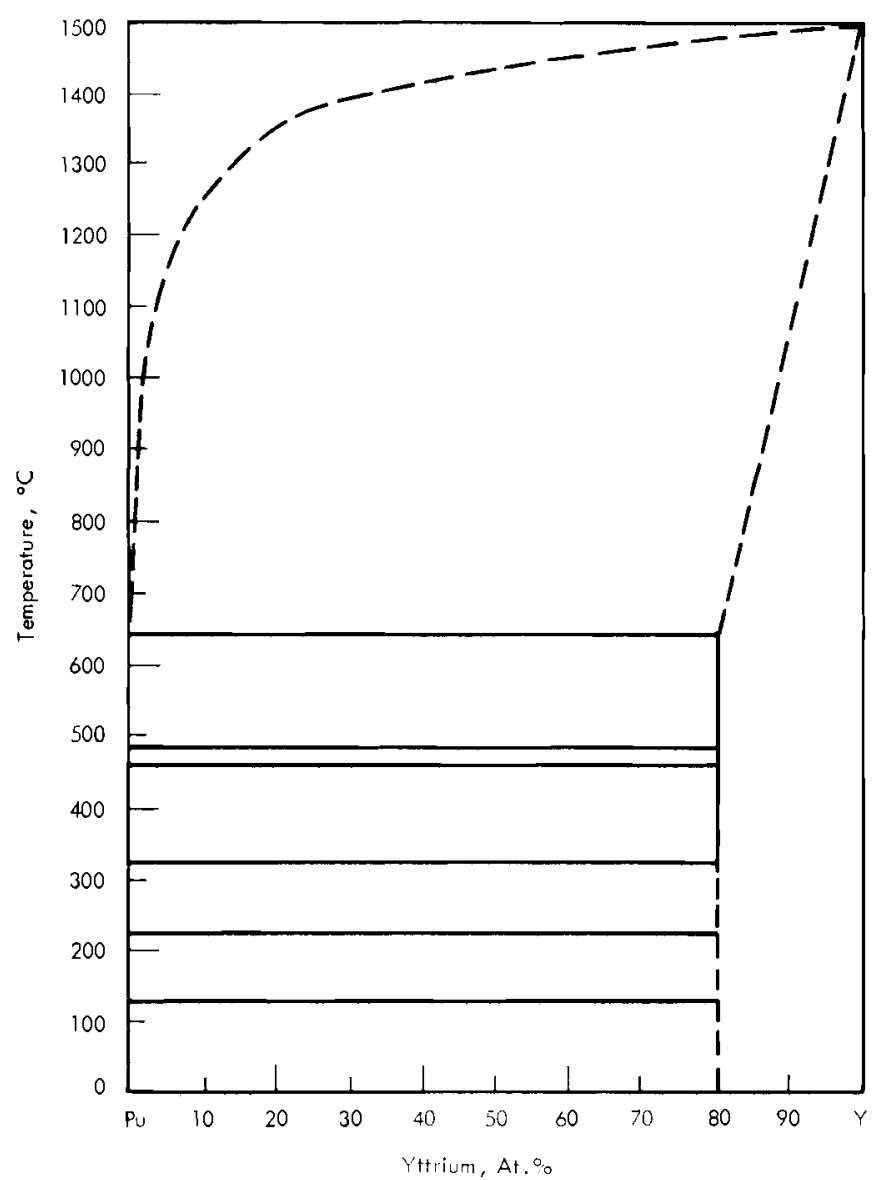

Plutonium-yttrium Phase Diagram. Jirom L., I. Wittenterg and G, R. Grove, M LM-1] 4 . . 


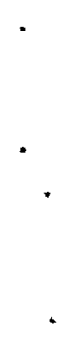

$\therefore$

, 


$$
\begin{aligned}
\text { PART } & \text { III } \\
\text { TERNARY } & \text { ALLOYS }
\end{aligned}
$$




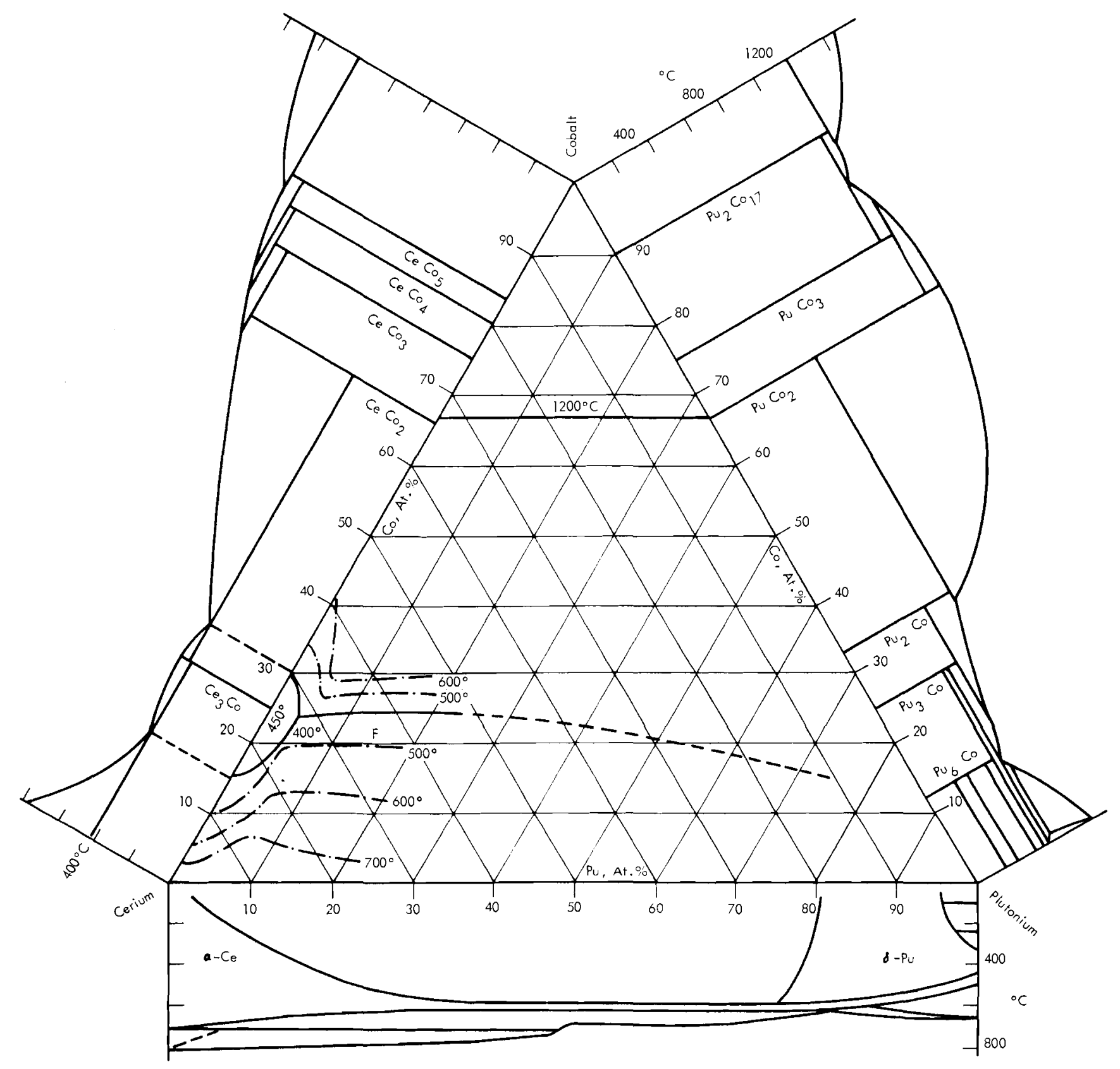

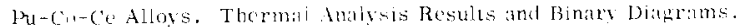

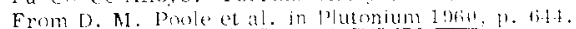




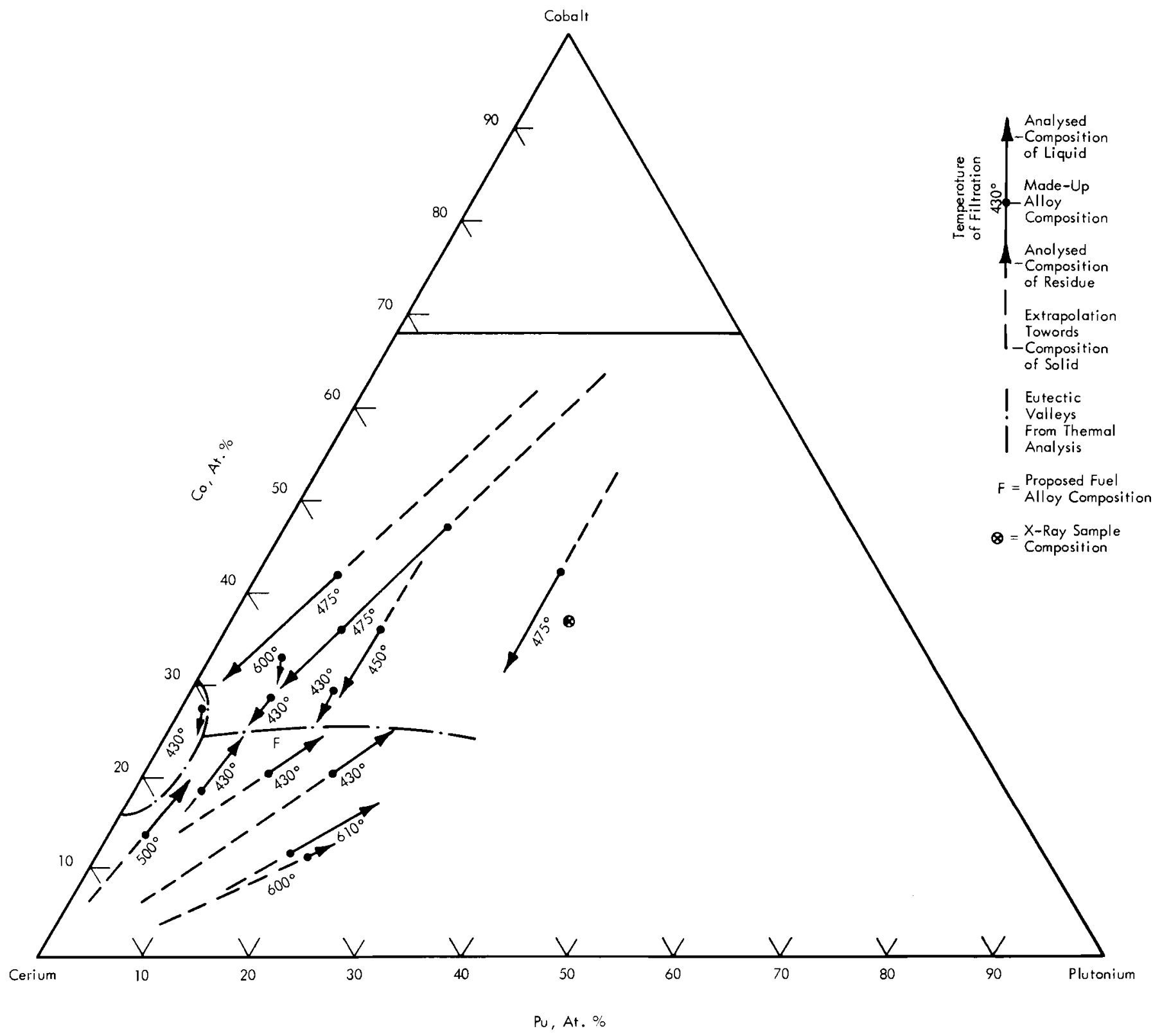

Pu-Co-Ce Allogis Fitration Results. From D, M. Poole et al. in Plutonium 1960, 1). tits. 

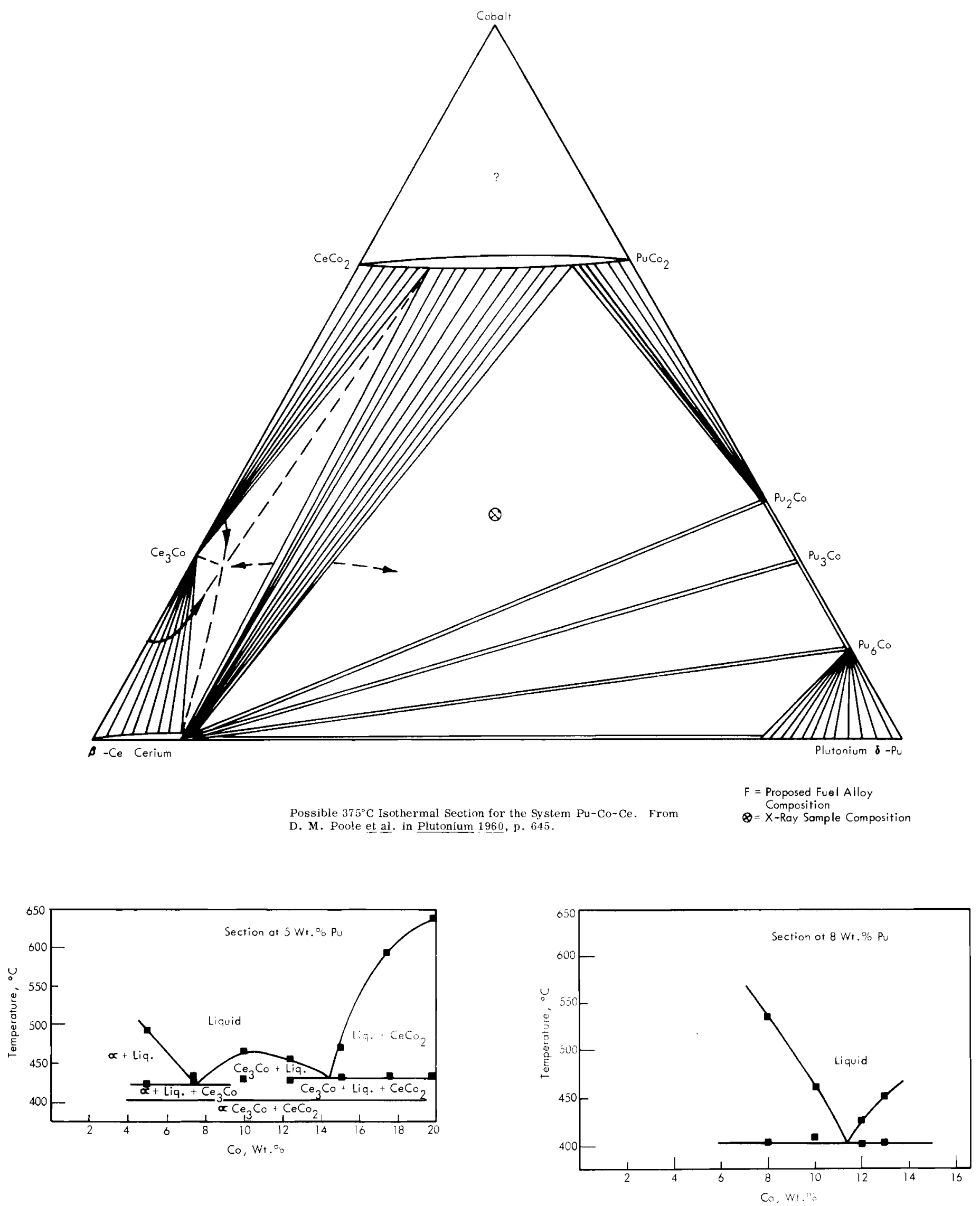

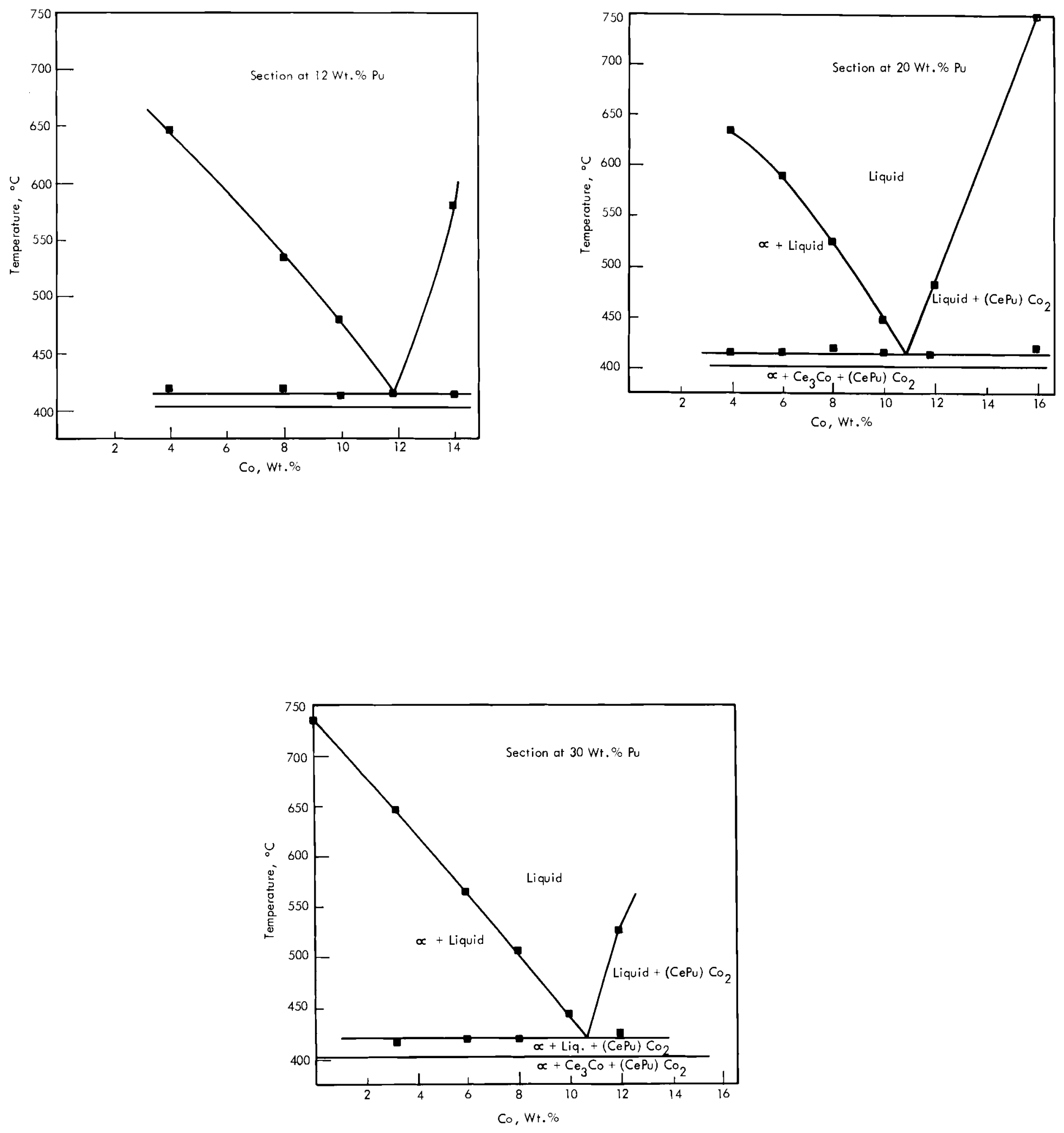

Cerium-Cobalt-Plutonium Alloys. From J. K. Critchley, AERE-M-488. 


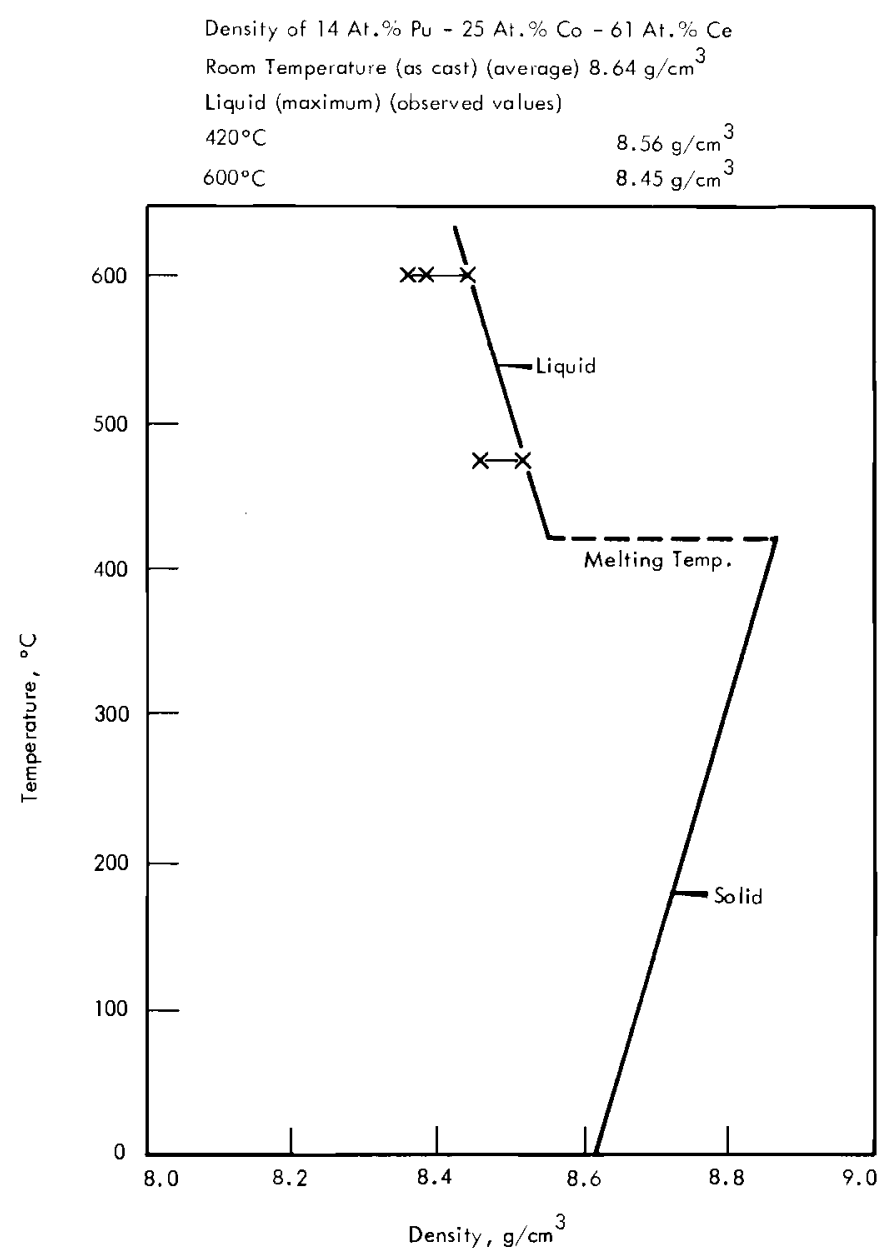

Density Variation in Liquid and Solid 14 at. \% Pu-25 at. \% Co-61 at. \% Ce Alloy. From D. M. Poole and J. L. Nichols, AERE-R-3122.

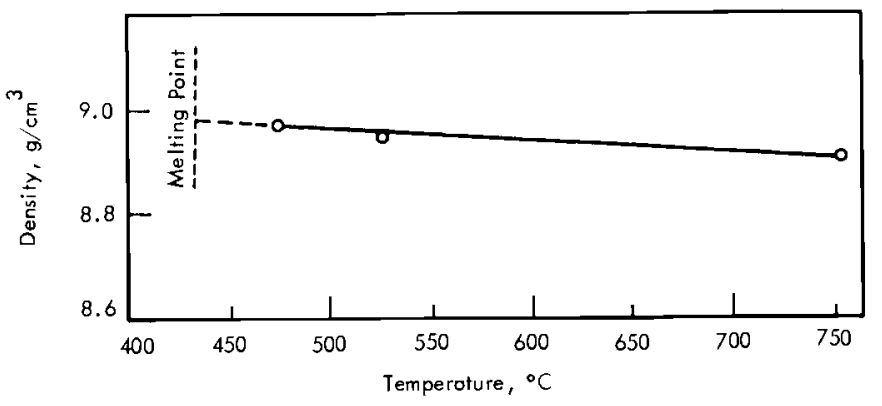

Density of Pu-Ce-Co Eutectic Alloy as a Function of Temperature.

From L. V. Jones et al., Am. Soc. Metals, Trans. Quart., vol. 55,

p. 824.1962 .

$$
\text { D }=9.082-2.31 \times 10^{-4} \mathrm{~T}
$$

where:

$$
\begin{aligned}
& \boldsymbol{D}=\text { density }, \mathrm{g} / \mathrm{cm}^{3} \\
& \mathrm{~T}={ }^{\circ} \mathrm{C}
\end{aligned}
$$

19.6 at. Pu, 56.2 at. Ce, 24.2 at. $\%$ Co

From L. V. Jones et al., Am. Soc. Metals, Trans. Quart., vol, 5ī, 


\section{Ce-Co 202.1 Thereral byonasison}

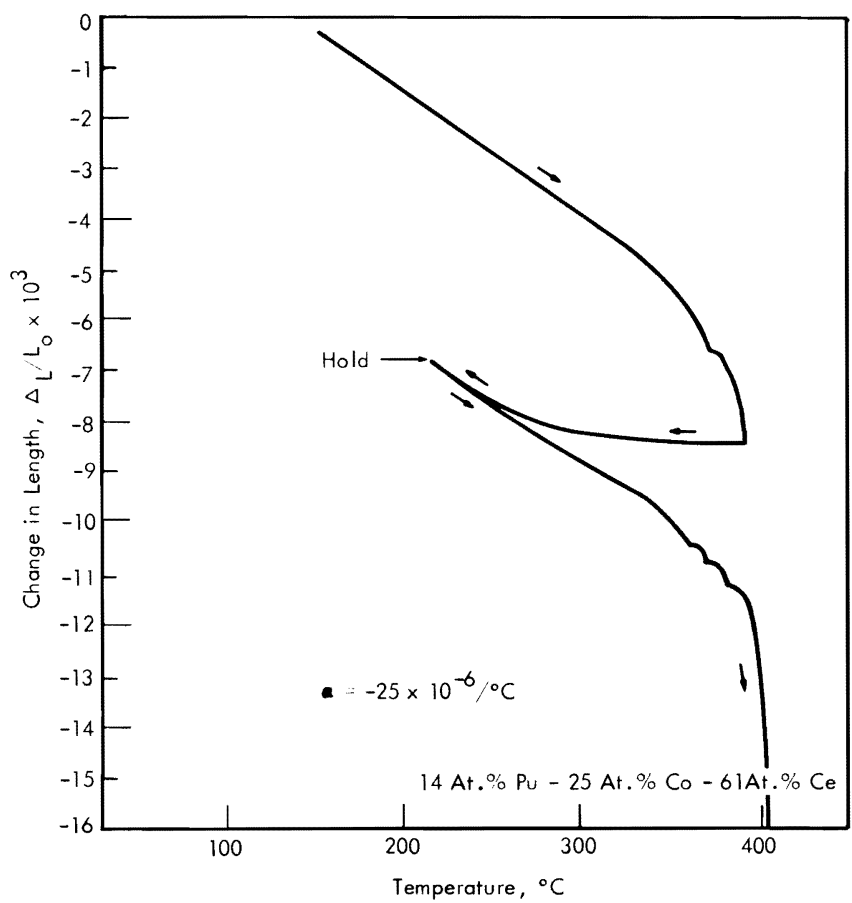

The value of the coefficient of expansion over the linear portion of the curve is equal to $-25 \times 10^{-6} /{ }^{\circ} \mathrm{C}$.

The volume coefficient of expansion of the liquid over the range $420-600^{\circ} \mathrm{C}$ is $72 \times 10^{-6} /{ }^{\circ} \mathrm{C}$.

Extrapolation of the solid and liquid density curves indicate a volume change on melting of $+4 \%$. From D. M. Poole et al. in Plutonium 1960 , p. 646 .

Dilatometer Record of Stlected Pu-Co-Ce Alloy. From D. M. Poole et al. in Plutonium 1960, 1. 646.

\section{Ce-Co 202.2 Thermal conductivity}

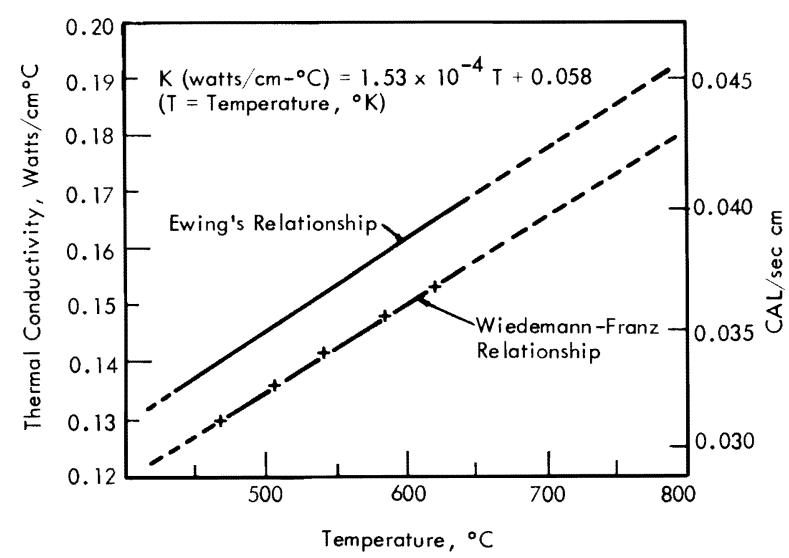

Thermal Conductivity of 14 at. Pu-25 at. Co-61 at. Ce Calculated From Observed Resistivity. From D. M. Poole et al. in Plutonium 1960, p. 647 . 


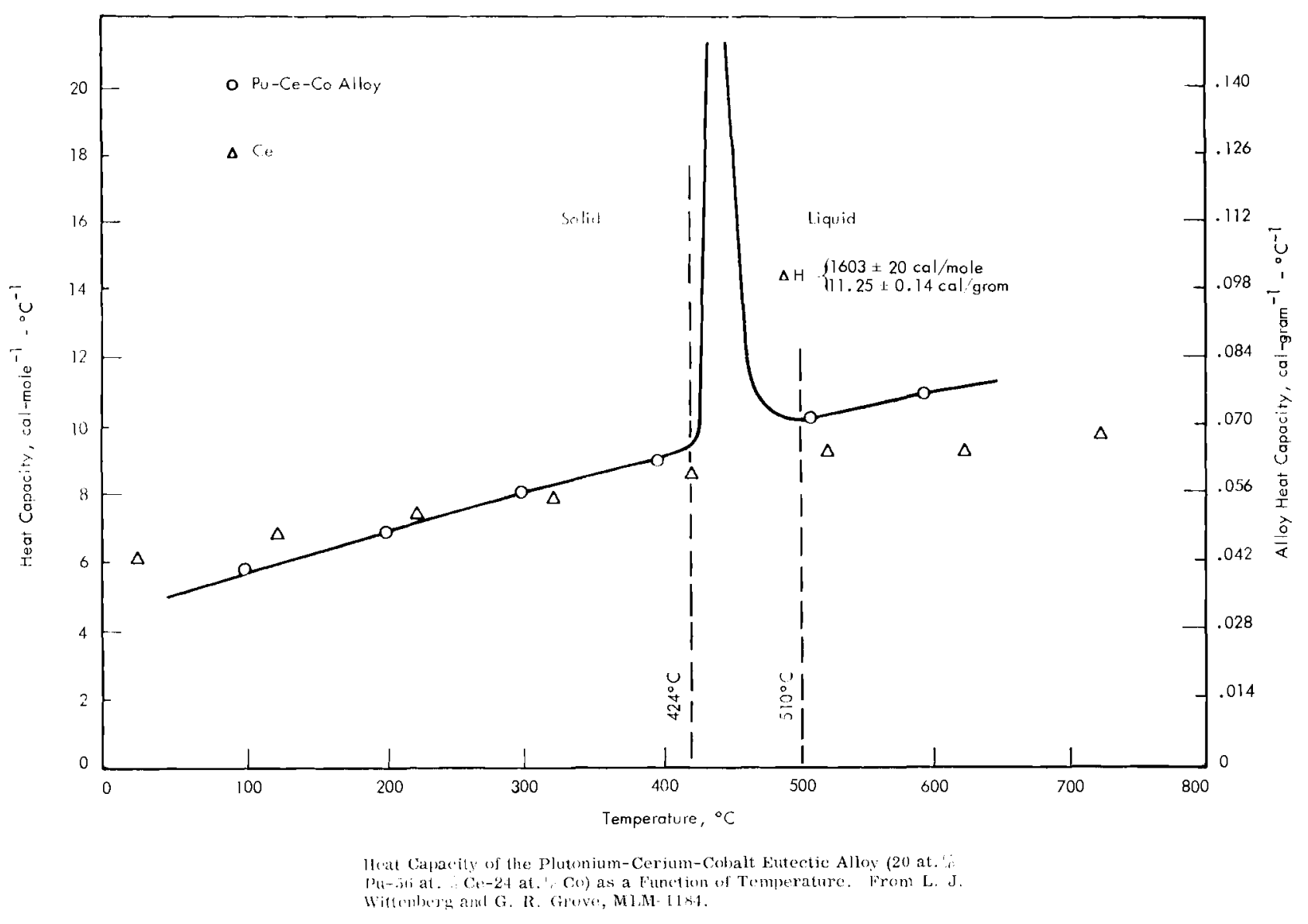

Temperature of and Volume Change during Transition

Heats of Transition
Ce-Co 202.5

See end of section Ce-Co 202.3

See end if section Ce-Co 202.3 . 


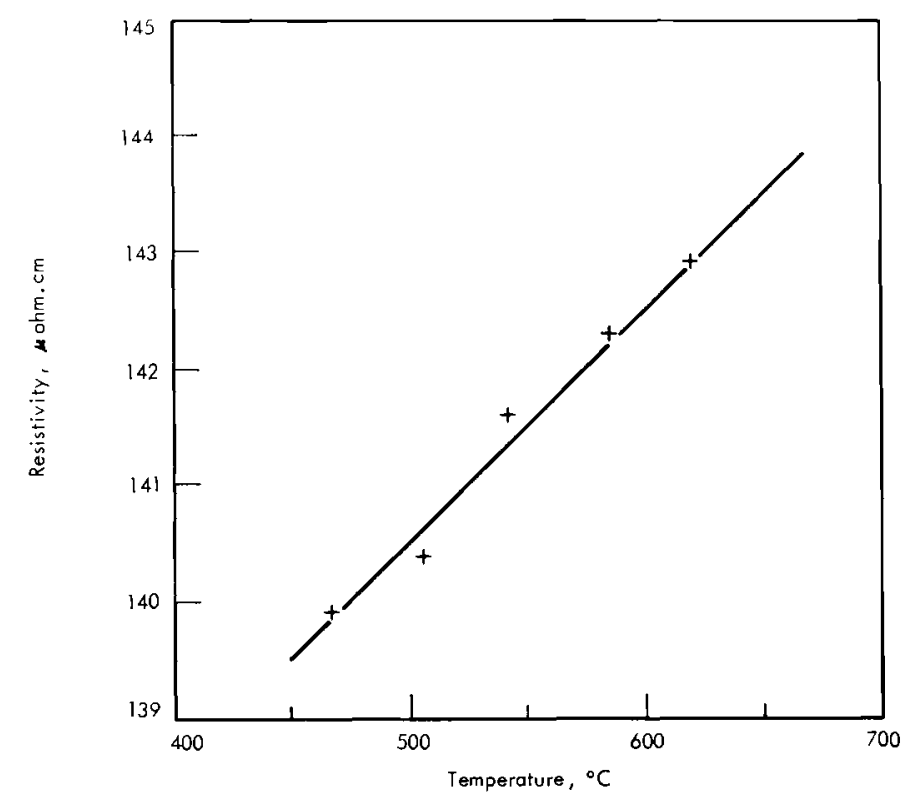

Measured Resistivity of Selected 14 at. Pu-25 at. Co-61 at. Ce Alloy. From D. M. Poole and J. L. Nichols, AERE-R-3122.

\section{Ce-Co 206 visosity}

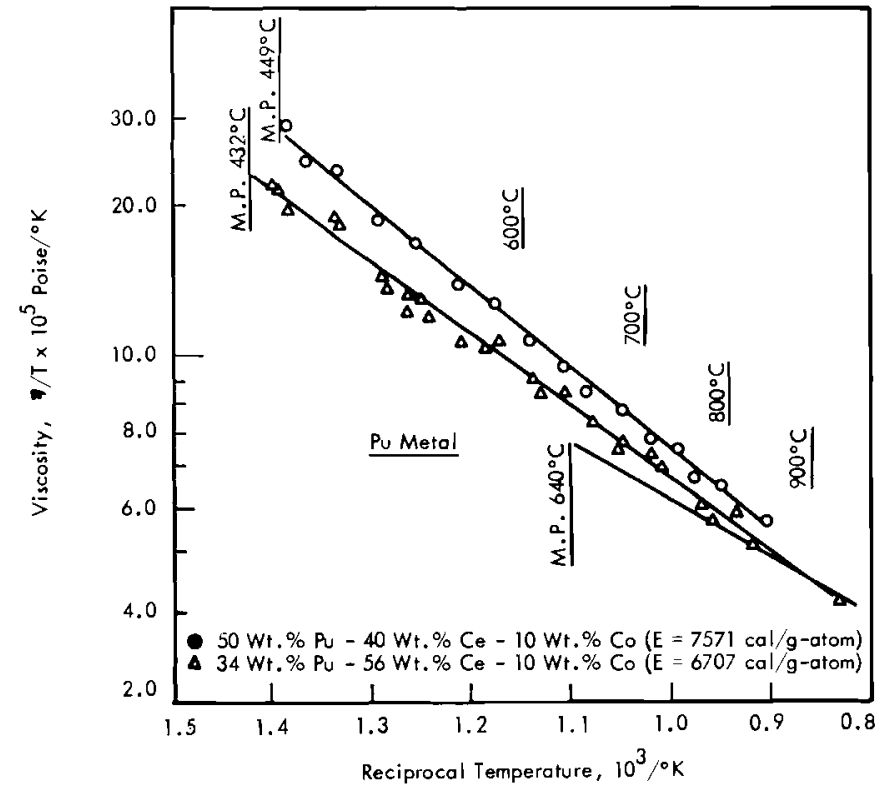

Viscosities of Plutonium-Cerium-Cobalt Molten Alloys as a Function of Temperature. From L. J. Wittenberg and G. R. Grove, MLM-1184.

\section{EQUATIONS FOR VISCOSITY DATA*}

From L. J. Wittenberg and G. R. Grove, MLM-1184.

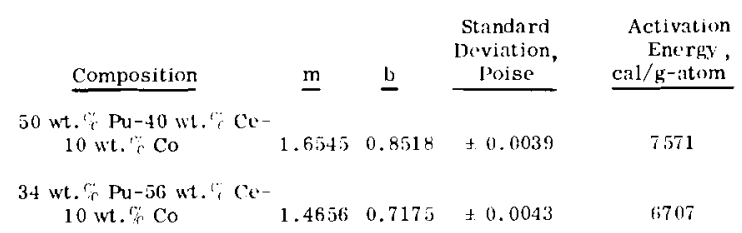

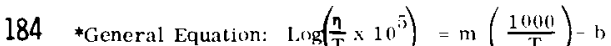

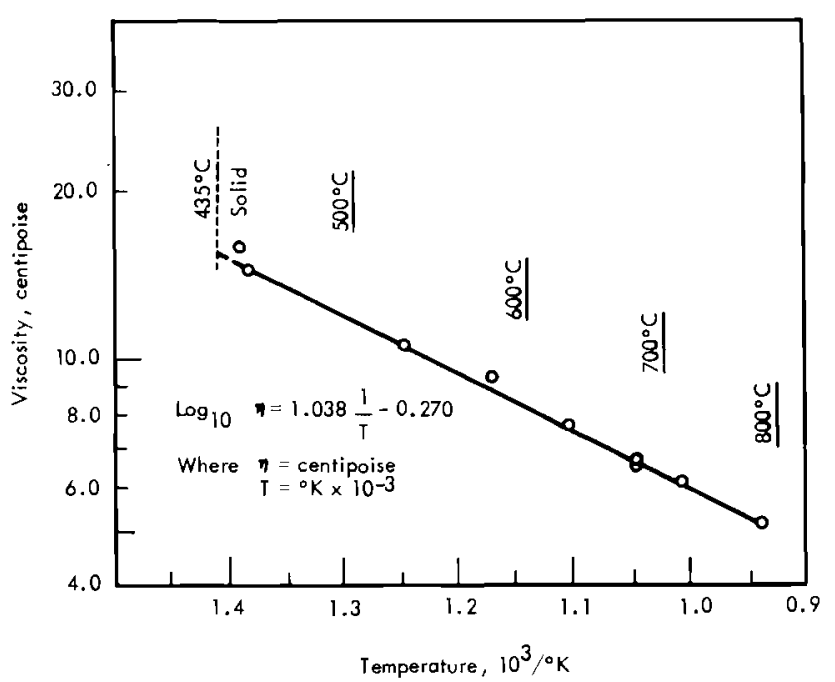

Viscosity of Pu-Ce-Co Eutectic Alloy as a Function of Temperature 19.6 at.\% Pu, 56.2 at. Ce, 24.2 at. $\%$ Co. From L. V. Jones et al., Am. Soc. Metals, Trans. Quart., vol. 55, p. 823. 1962.

\section{CALCU LATED VISCOSITIES OF Pu-Ce-Co}

From L. V. Jones et al., Am. Soc. Metals, Trans. Quart., vol. 55, p. 823. 1962

\begin{tabular}{|c|c|c|c|c|}
\hline $\begin{array}{l}\text { Temp, } \\
{ }^{\circ} \mathrm{C} \\
\end{array}$ & $\begin{array}{l}\text { Period, } \\
\text { Sec. }\end{array}$ & $\begin{array}{l}\text { Logarithmic } \\
\text { Decrement }\end{array}$ & $\begin{array}{l}\text { Density, } \\
\mathrm{g} / \mathrm{cm}^{3} \\
\end{array}$ & $\begin{array}{r}\text { Viscosity } \\
\text { Centipoise }\end{array}$ \\
\hline & & $\mathrm{Pu}-\mathrm{Ce}^{2}-\mathrm{Co}$ & & \\
\hline 801 & $2.66 \bar{i}$ & 0.0243603 & 8.893 & 5.08 \\
\hline 720 & 2.668 & 0.0259293 & 8.913 & 5.93 \\
\hline $6 \times 3$ & 2.666 & 0.0267754 & 8.923 & 6.44 \\
\hline 681 & 2.1600 & 0.0264774 & 8.924 & 6.26 \\
\hline 632 & 2.669 & 0.0284750 & 8.937 & 7.54 \\
\hline $5 \pi 9$ & 2.670 & $0.0306,03$ & 8.949 & 9.16 \\
\hline $5 \geq \mathrm{x}$ & 2.670 & 0.0321430 & 8.962 & 10.42 \\
\hline 4.71 & 2.674 & 0.0359024 & 8.978 & 14.28 \\
\hline$+41 ;$ & $2.6 i t$ & 0.0365210 & 8.980 & 15.54 \\
\hline
\end{tabular}


THE INTERGILANULAR PENETRATION (IGP) AND DISSOLUTION OF CERAMICS BY Pu-Cu-Ce ALLOYS (Conditions: $200 \mathrm{~h}$ at $7.50^{\circ} \mathrm{C}$, static, isothermal)

From B. J. Thimer in Corrosion of Reactor Materials, Vol. II, p. 310 .

\begin{tabular}{|c|c|c|}
\hline Ceramic & Test Fuel & Observations \\
\hline $\mathrm{TiC}_{0.93}+2.6 \mathrm{wt} . \mathrm{C}$ & & No IGP or dissolution \\
\hline 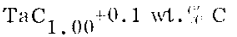 & 19 at. $\mathrm{Pu}$ & No IGP or dissolution \\
\hline $\mathrm{ZrC}_{0,13}+1.9 \mathrm{wt} ., \mathrm{C}$ & 55.6 at. $\mathrm{Ce}$ & Some IGP, no dissolution \\
\hline $\mathrm{HCC}_{0.91}+0.1 \mathrm{wt} . \% \mathrm{C}$ & 24.7 at. Co & No IGP or dissolution \\
\hline $\mathrm{BCO}$ & & $\begin{array}{l}\text { Not visibly attacked, } 120 \mathrm{ppm} \\
\text { Be produced in fuel }\end{array}$ \\
\hline $\mathrm{MgO}$ & & $\begin{array}{l}\text { Not visibly attacked, } 600 \mathrm{ppm} \\
\text { Mg produced in fuel }\end{array}$ \\
\hline $\mathrm{Al}_{2} \mathrm{O}_{3}$ & & $\begin{array}{l}\text { Not visibly attacked, less than } \\
200 \mathrm{ppm} \text { Al produced in fuel }\end{array}$ \\
\hline $\mathrm{TiB}_{1.77}$ & & $\begin{array}{l}\text { No IGP, less than } 200 \mathrm{ppm} \\
\text { Ti produced in fuel }\end{array}$ \\
\hline $\mathrm{ZrB}_{1.8}$ & & $\begin{array}{l}\text { No IGP, } 20 \mathrm{ppm} \mathrm{Zr} \text { produced } \\
\text { in fuel }\end{array}$ \\
\hline $\mathrm{CaO}$ & $\begin{array}{l}25.7 \text { at. } \mathrm{Pu} \\
49.2 \text { at. } \mathrm{Ce}\end{array}$ & $\begin{array}{l}\text { Extensive IGP and transgranu- } \\
\text { Iar penetration, } 150 \mathrm{ppm} \mathrm{Ca} \\
\text { produced in fuel }\end{array}$ \\
\hline $\mathrm{ThO}_{2}$ & 25.1 at. $\mathrm{CO}$ & $\begin{array}{l}\text { No IGP, } 90-195 \mathrm{ppm} \text { Th } \\
\text { produced in fuel }\end{array}$ \\
\hline $\mathrm{ZrO}_{2}$ & & $\begin{array}{l}\text { Extensive IGP, } 10-25 \mathrm{ppm} \mathrm{Zr} \\
\text { produced in fuel }\end{array}$ \\
\hline
\end{tabular}

ATTACK OF NIOBIUM, MOLYBDENUM AND 'IANTALUM CONTAINERS BY LOW MEITING POINT PLUTONIUM ALLOYS AFTER 7 DAYS AT $760^{\circ} \mathrm{C}$

From D. M. Poole et al. in Plutonium 1960, p. 648.

\begin{tabular}{|c|c|c|c|c|c|}
\hline Alloy & Container & $\begin{array}{c}\text { Approximate } \\
\text { Angle Of } \\
\text { Contact With } \\
\text { Wall } \\
\text { (Measured } \\
\text { Through } \\
\text { Liquid) } \\
\end{array}$ & $\begin{array}{l}\text { Appearance of } \\
\text { Interface } \\
\end{array}$ & $\begin{array}{l}\text { Depth } \\
\text { Of Visible } \\
\text { Inter- } \\
\text { action, } \\
\text { in. } \\
\end{array}$ & $\begin{array}{l}\text { Analysis } \\
\text { Of Contents, } \\
\text { wt. } \% \\
\end{array}$ \\
\hline \multirow[t]{3}{*}{$\mathrm{Pu}_{\mathrm{C}} \mathrm{Co}-\mathrm{Ce}$} & $\mathrm{Nb}$ & $0^{\circ}$ & $\begin{array}{l}\text { Irregular attack, white } \\
\text { angular compound } \\
\text { Iarticles formed. }\end{array}$ & $\sim 0.0005$ & $0.09 \mathrm{Nb}$ \\
\hline & Mo & $25^{\circ}$ & $\begin{array}{l}\text { Extensive intergranular } \\
\text { attack after } 6 \text { weeks at } \\
800^{\circ} \mathrm{C} \text {. }\end{array}$ & $(0.01)$ & (10 Mo) \\
\hline & Tia & $35^{\circ}$ & $\begin{array}{l}\text { Localized attack, non- } \\
\text { adherent film and de- } \\
\text { tached needle particles. }\end{array}$ & $\sim 0.0005$ & $\sim 0.5 \mathrm{Ta}$ \\
\hline
\end{tabular}


$\mathrm{Ce}-\mathrm{Cu} 101$

Phase Diagrams

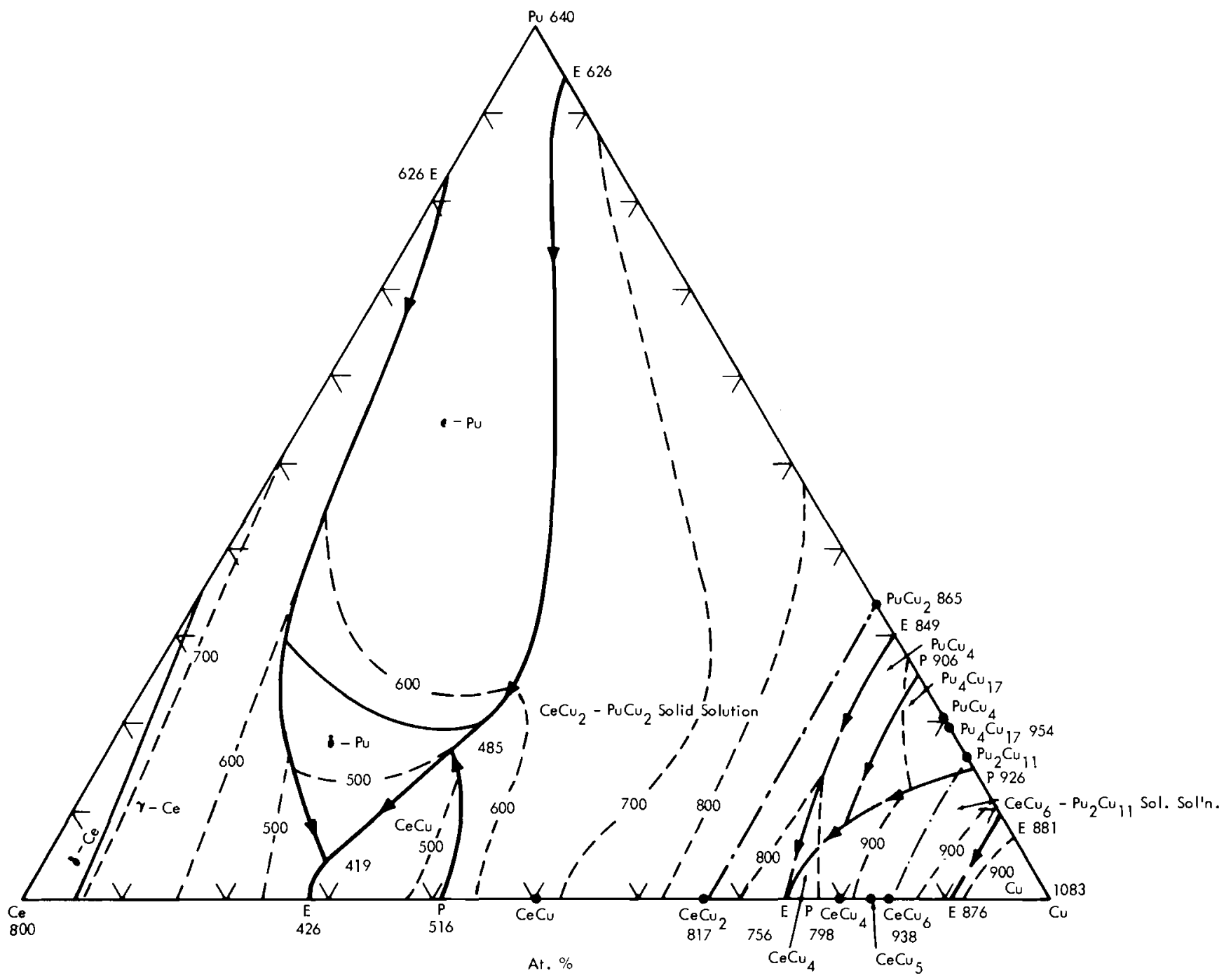

186

Plutonium-Cerium-Copper Phase Diagram. From L. J. Wittenberg and G. R. Grove, MLM-1184. 


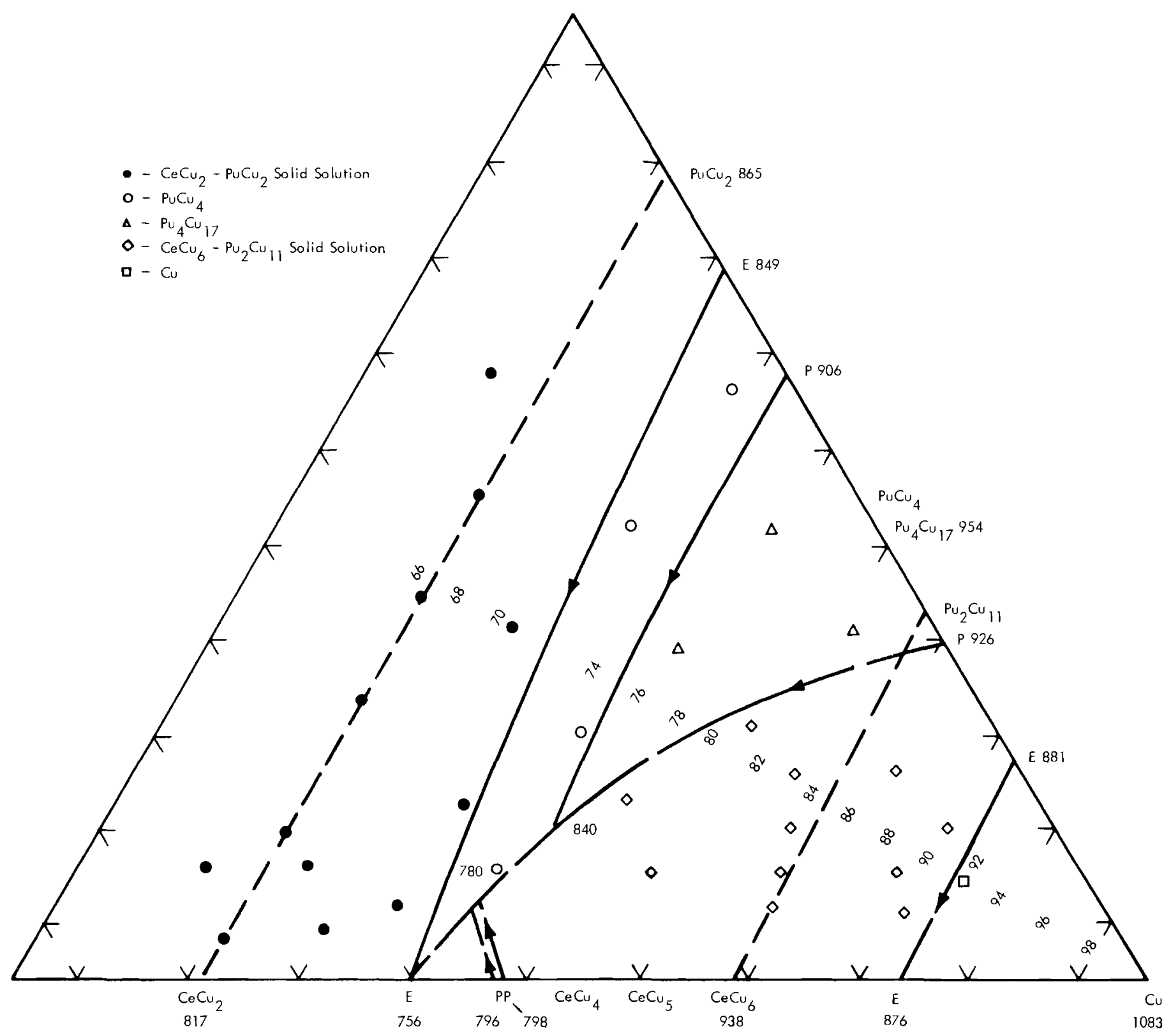

Copper-Rich Corner of the Plutonium-Cerium-Copper Phase Diagram. From L. J. Wittenberg and G. R. Grove, M LM-11 84 . 


\section{$\mathrm{Ce}-\mathrm{Cu} 600$ Raditiation thrects}

ATTACK OF NIOBIUM, MOLYBDENLN AND TANTALLM CONTAINERS BY LOW MELTING POINT PLUTONIUM ALLOYS AFTER $T$ DAYS AT $760^{\circ} \mathrm{C}$

From D. M. Poole et al. in Plutonium 1960, p. 648.

\begin{tabular}{|c|c|c|c|c|c|}
\hline Alloy & Container & $\begin{array}{c}\text { Approximate } \\
\text { Angle Of } \\
\text { Contact With } \\
\text { Wall } \\
\text { (Measured } \\
\text { Through } \\
\text { Liquid? }\end{array}$ & $\begin{array}{l}\text { Appearance of } \\
\text { Interface }\end{array}$ & $\begin{array}{l}\text { Depth } \\
\text { Of Visible } \\
\text { Inter- } \\
\text { action, } \\
\text { in. }\end{array}$ & $\begin{array}{l}\text { Analysis } \\
\text { Of Contents, } \\
\text { wt.? }\end{array}$ \\
\hline \multirow[t]{3}{*}{$\mathrm{Pu}-\mathrm{Cu}-\mathrm{Ce}$} & $\mathrm{Nb}$ & $0^{\circ}$ & $\begin{array}{l}\text { Rough reaction layer } \\
\text { plus occasional compound } \\
\text { needles. }\end{array}$ & $\sim 0.0001$ & $0.02 \mathrm{Nb}$ \\
\hline & Mo & $0^{\circ}$ & $\begin{array}{l}\text { Extensive intergranular } \\
\text { penetration. }\end{array}$ & $\sim 0.0025$ & $\sim 3.0 \mathrm{Mo}$ \\
\hline & $\mathrm{Ta}$ & $20-125^{\circ}$ & $\begin{array}{l}\text { Interrupted smooth re- } \\
\text { action layer below liquid } \\
\text { surface. Above liquid } \\
\text { level there were occasional } \\
\text { signs of extensive uneven } \\
\text { attack. }\end{array}$ & $\begin{array}{l}<0.0001 \\
1<\end{array}$ & $\sim 0.2 \mathrm{Ta}$ \\
\hline
\end{tabular}




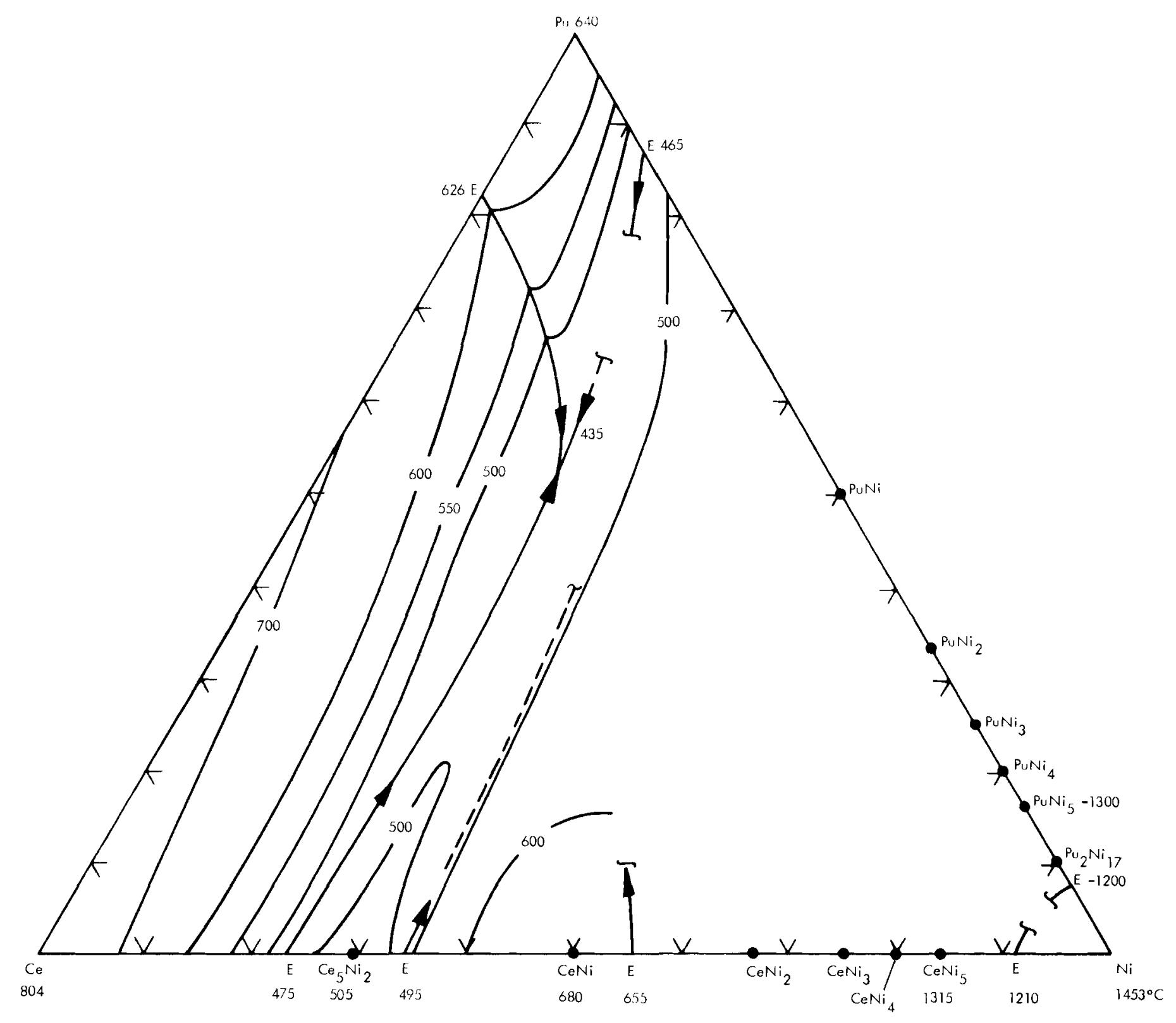




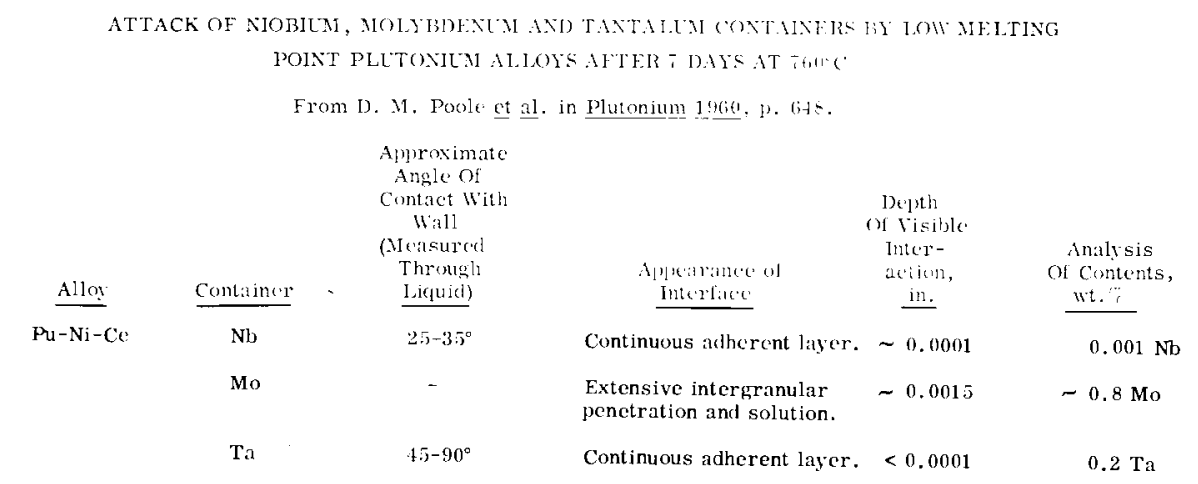

Mo.U 101 Phase iagrans

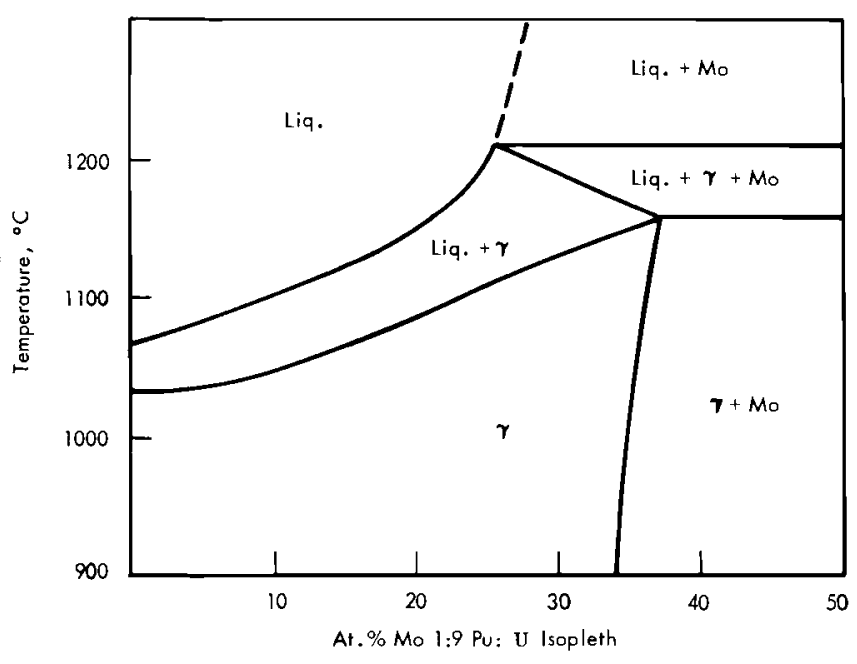

From P. G. Mardon et al. in Plutonium 1960, p. 343 .

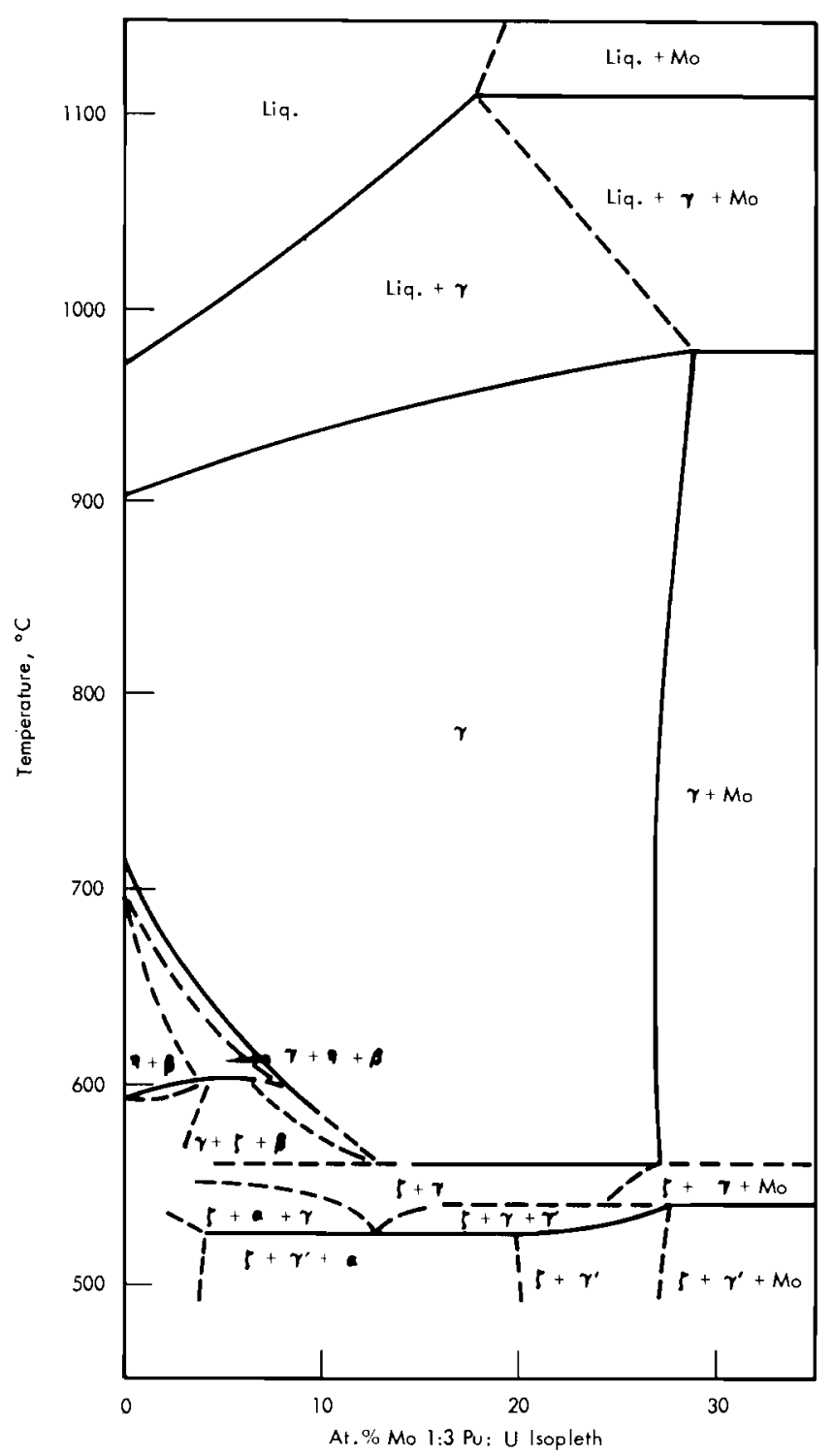



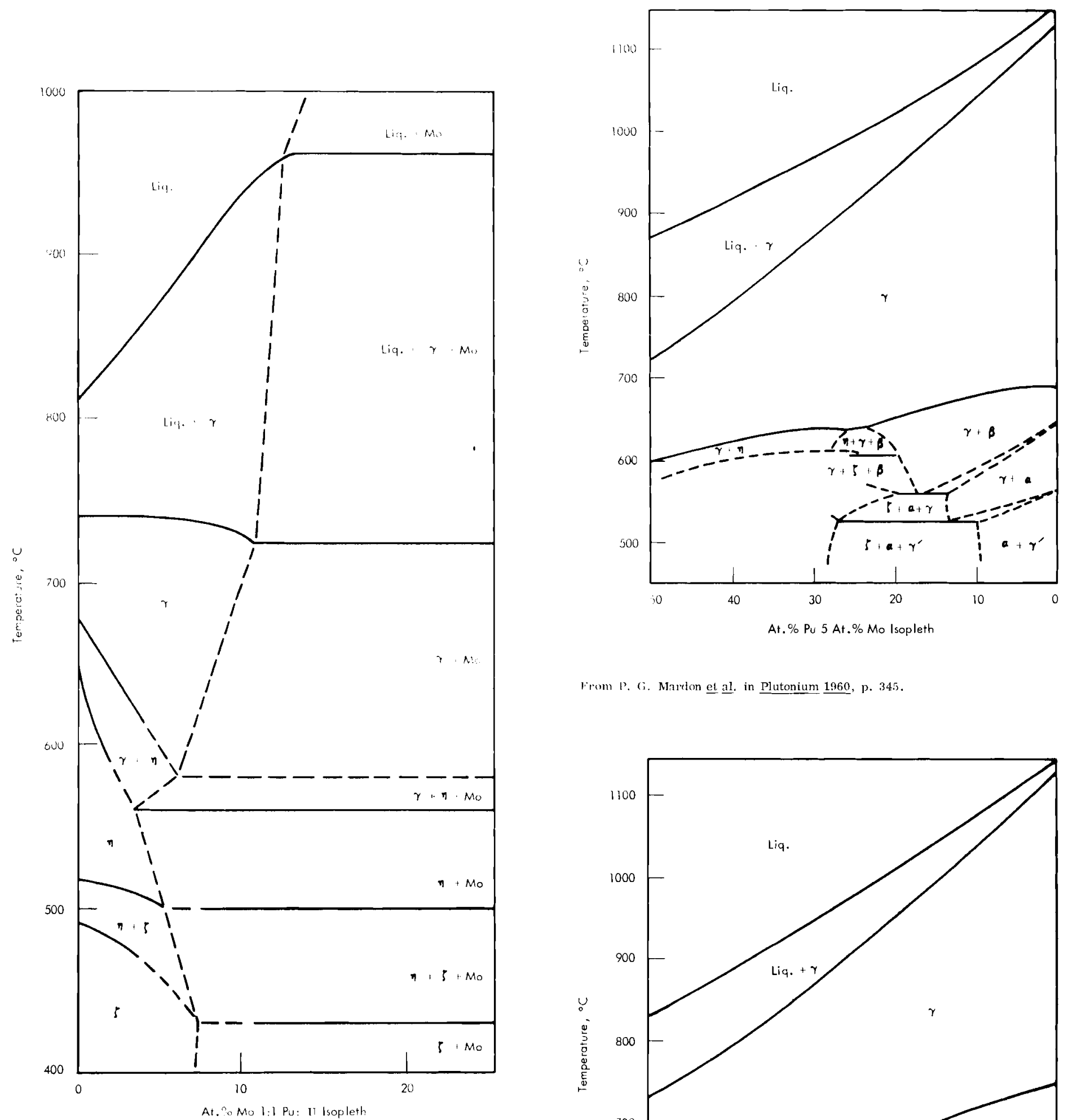

From l', G. Narion el al. in Plutonium 1960, p. 345.

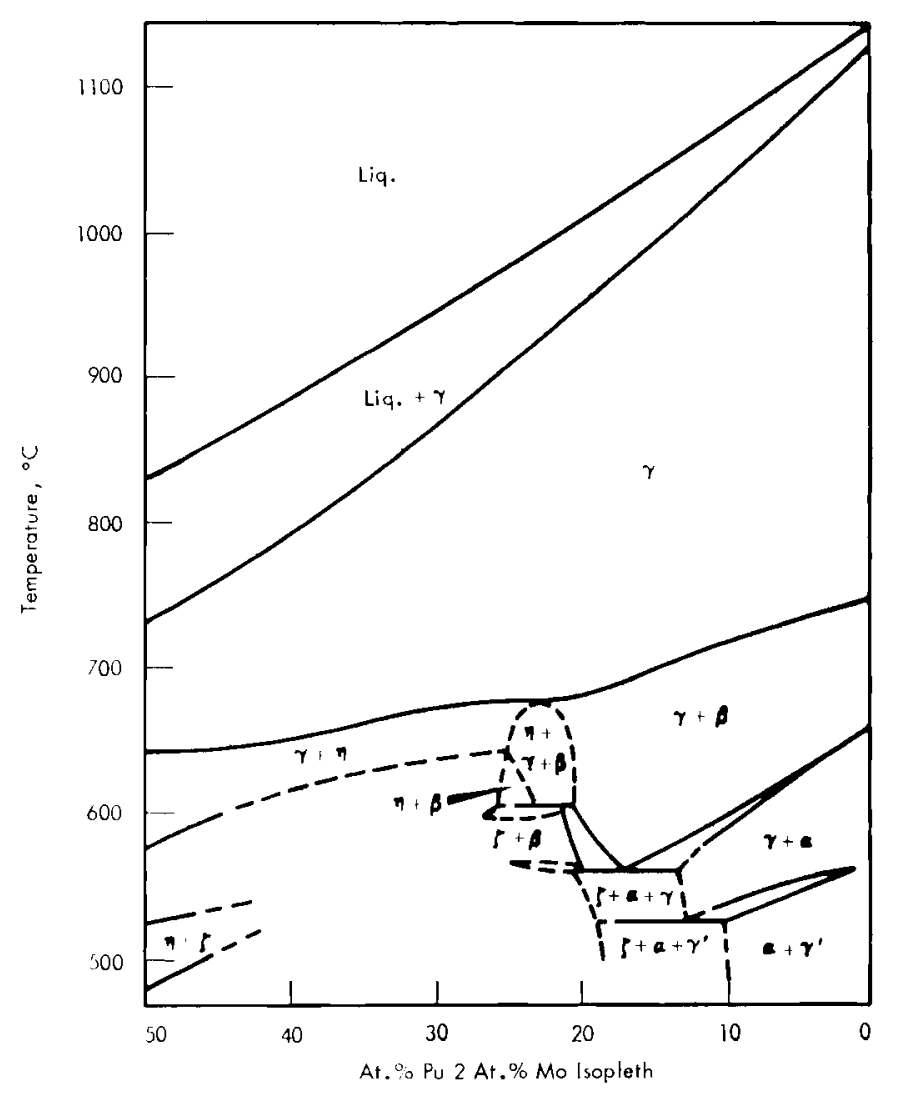




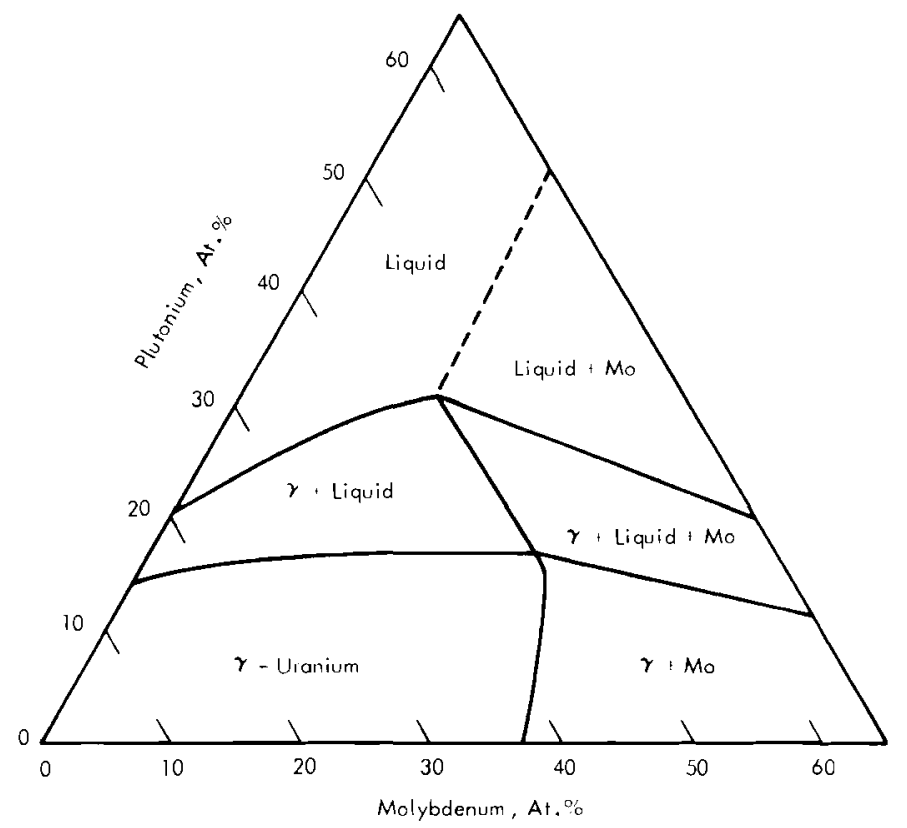

$1000^{\circ} \mathrm{C}$ Isotherm. From P. G. Mardon et al. in Plutonium 1960, p. 336.

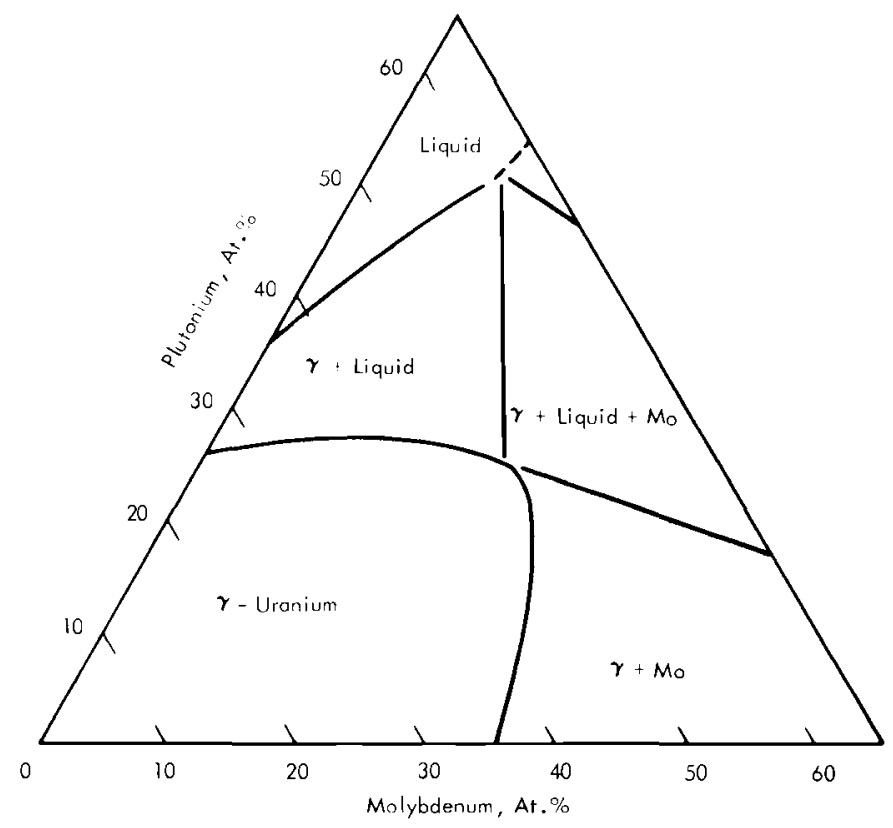

$900^{\circ} \mathrm{C}$ Isotherm. From P. G. Mardon et al. in Plutonium 1960, p. 336.

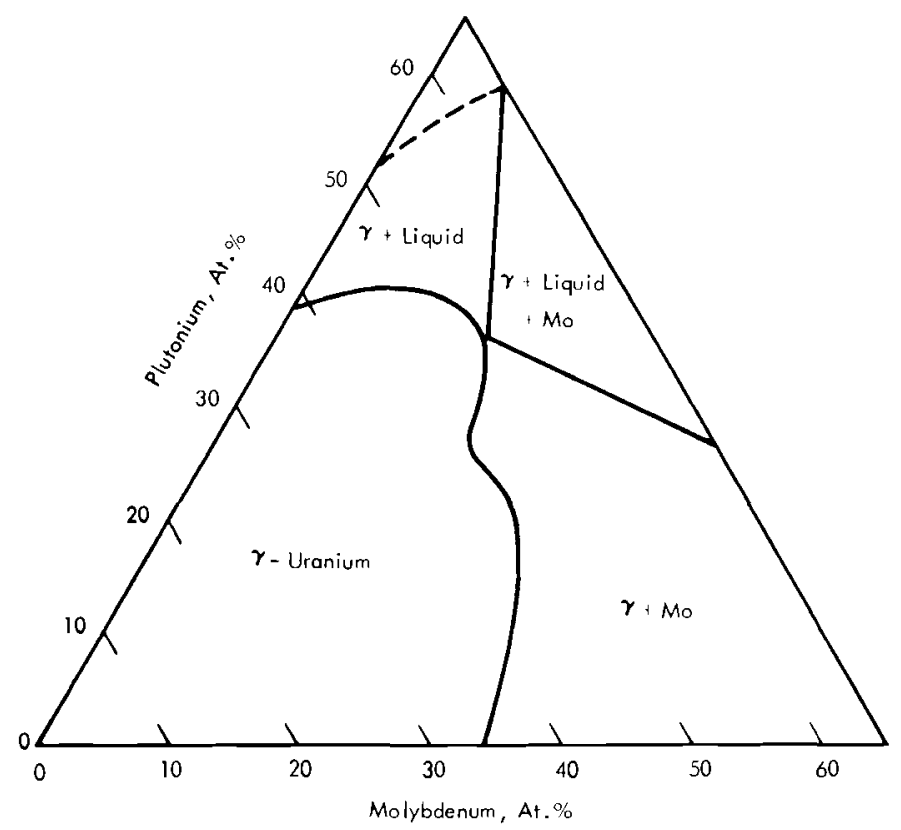

$800^{\circ} \mathrm{C}$ lsotherm. From P. G. Mardon et al. in Plutonium l960, 1. 3335. 192

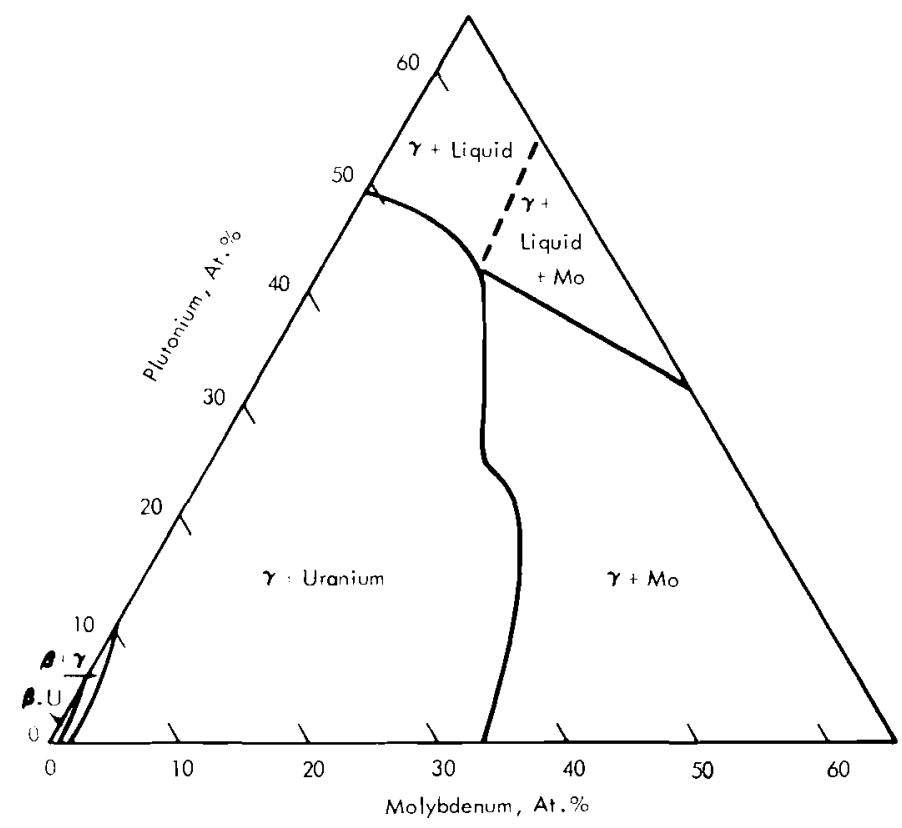

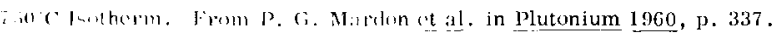




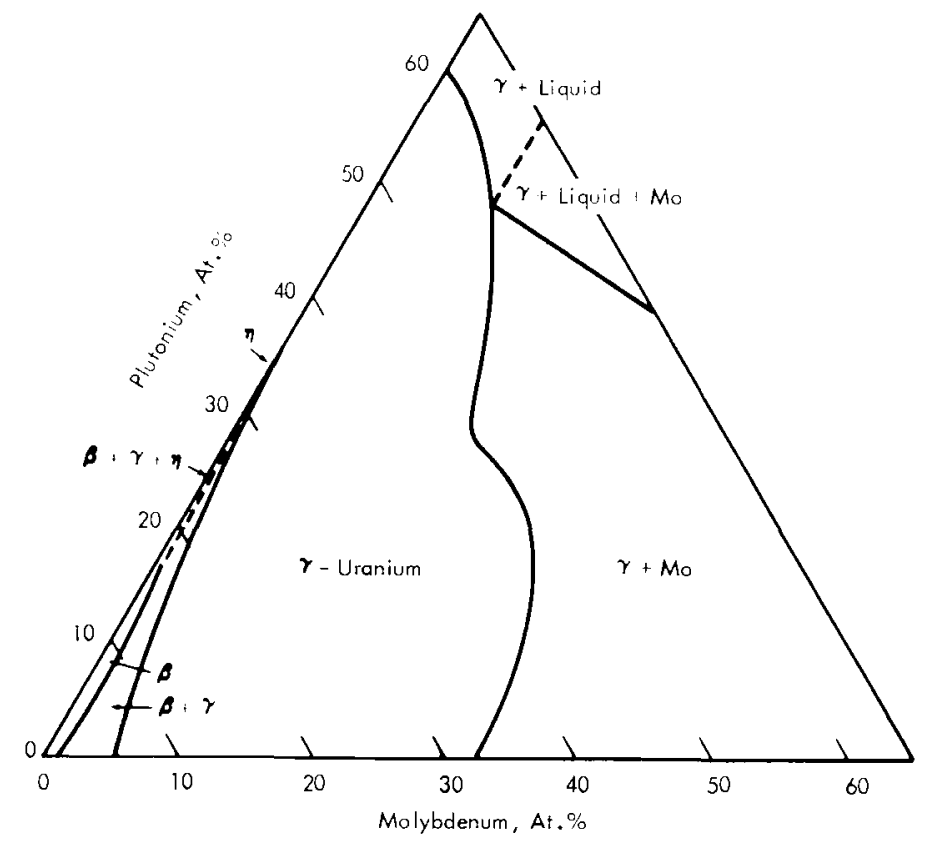

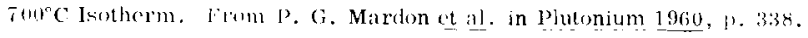

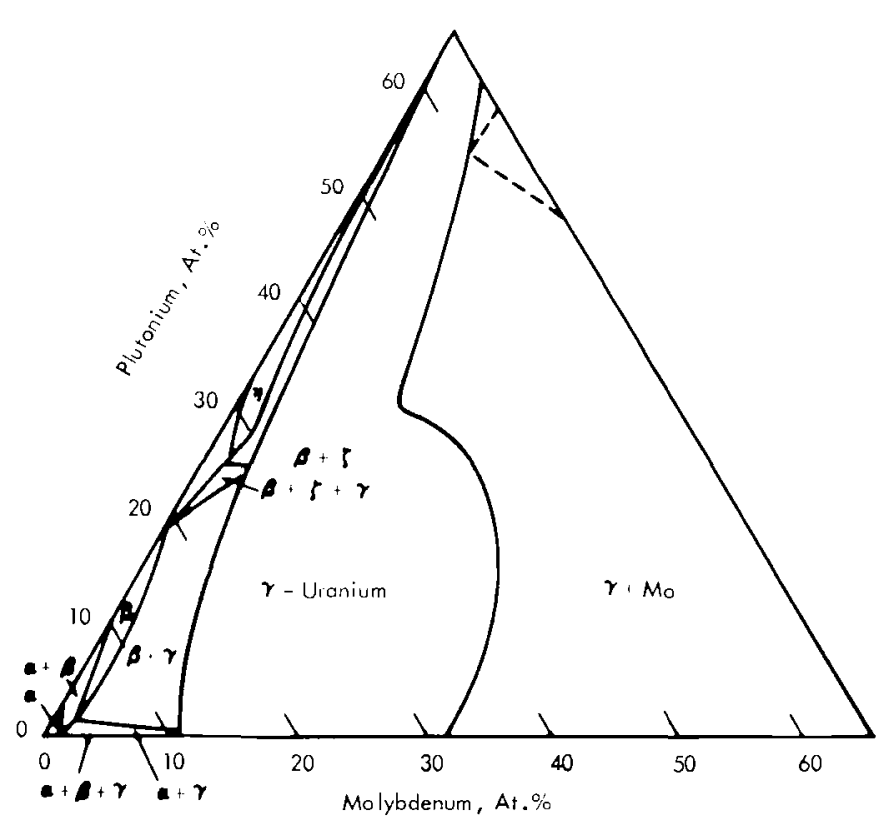

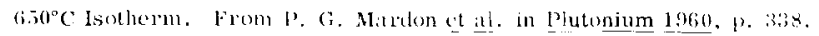

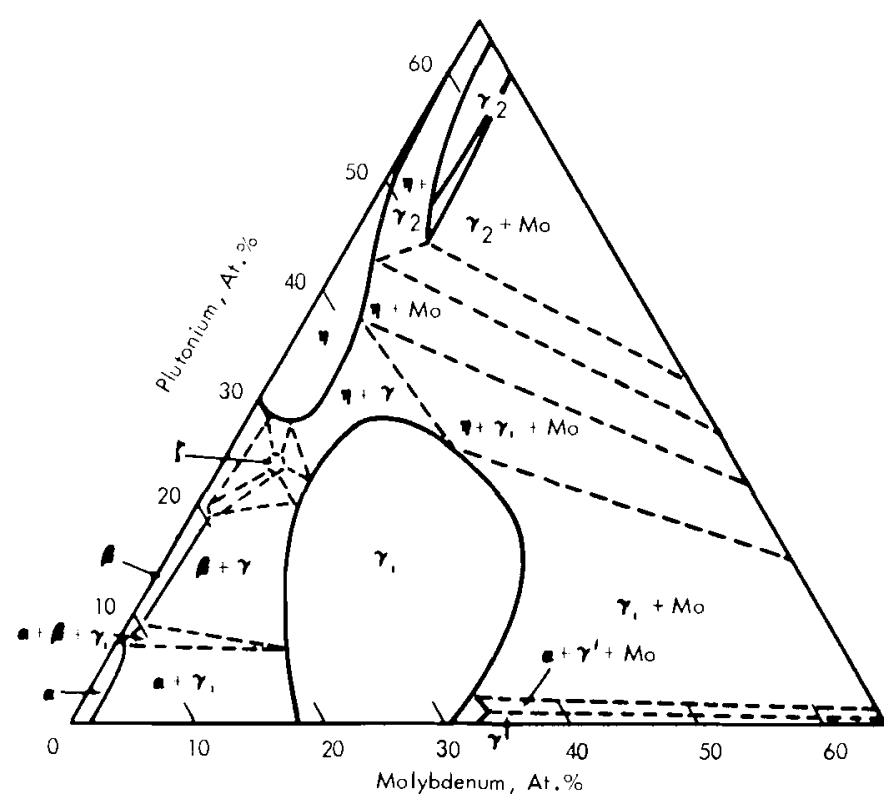

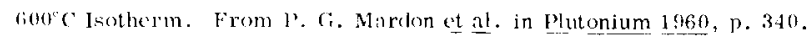

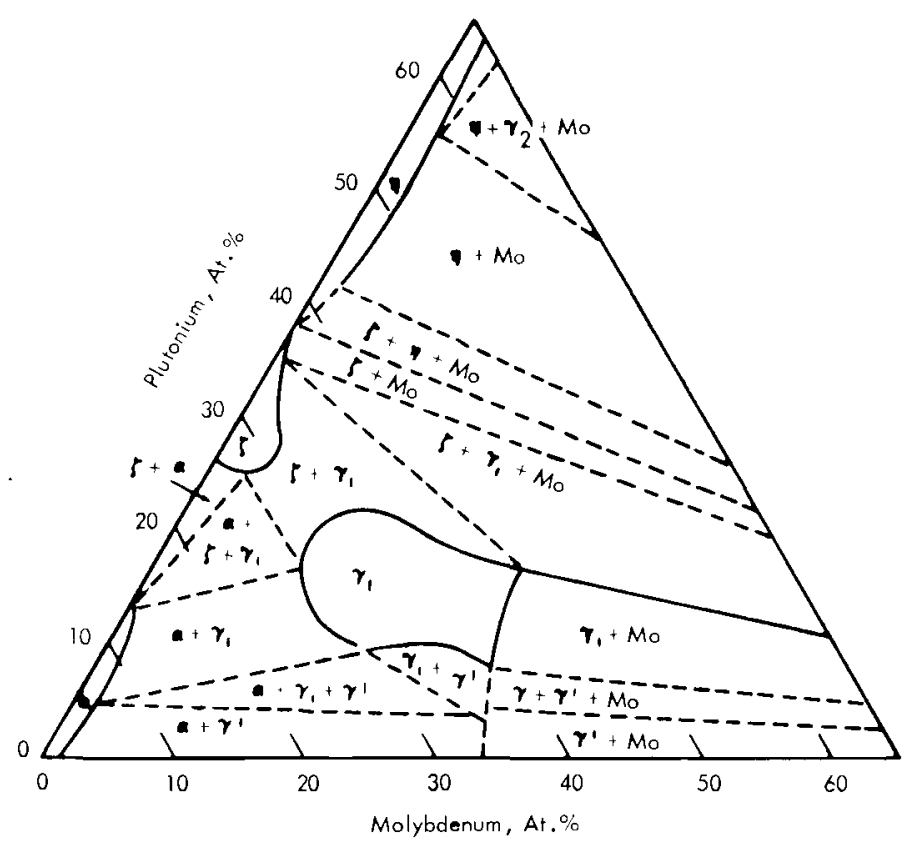

5.s C Lotherm. From P. G. Mardon et al. in l'lutonium 1960, p. 340. 


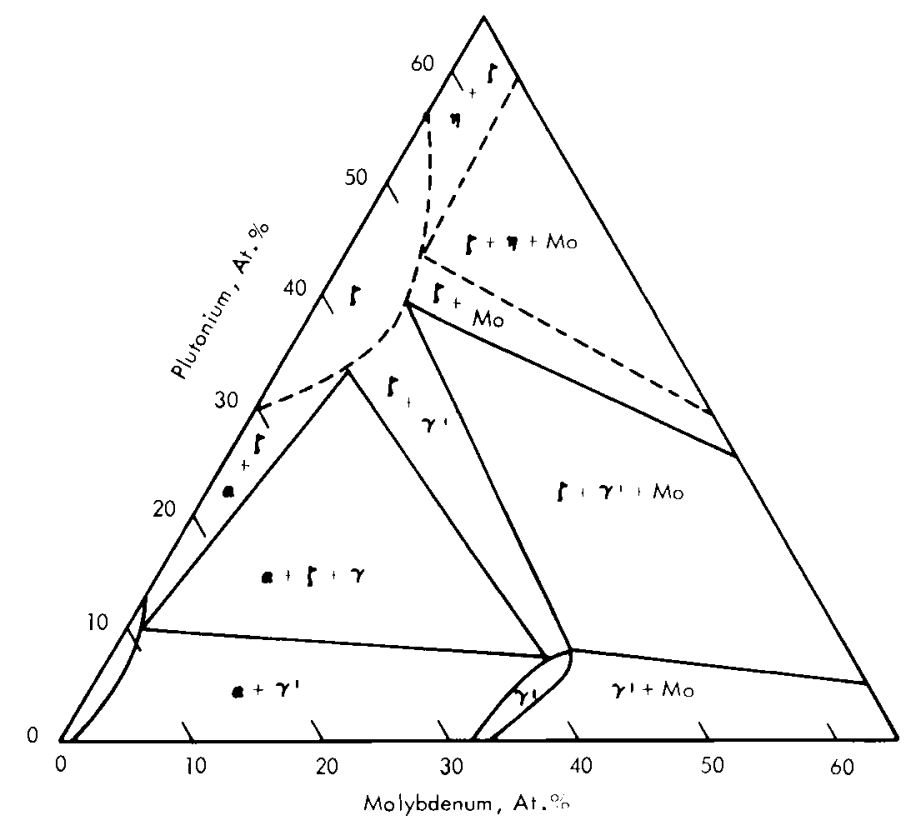

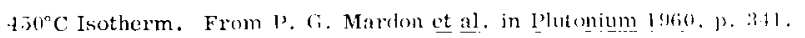
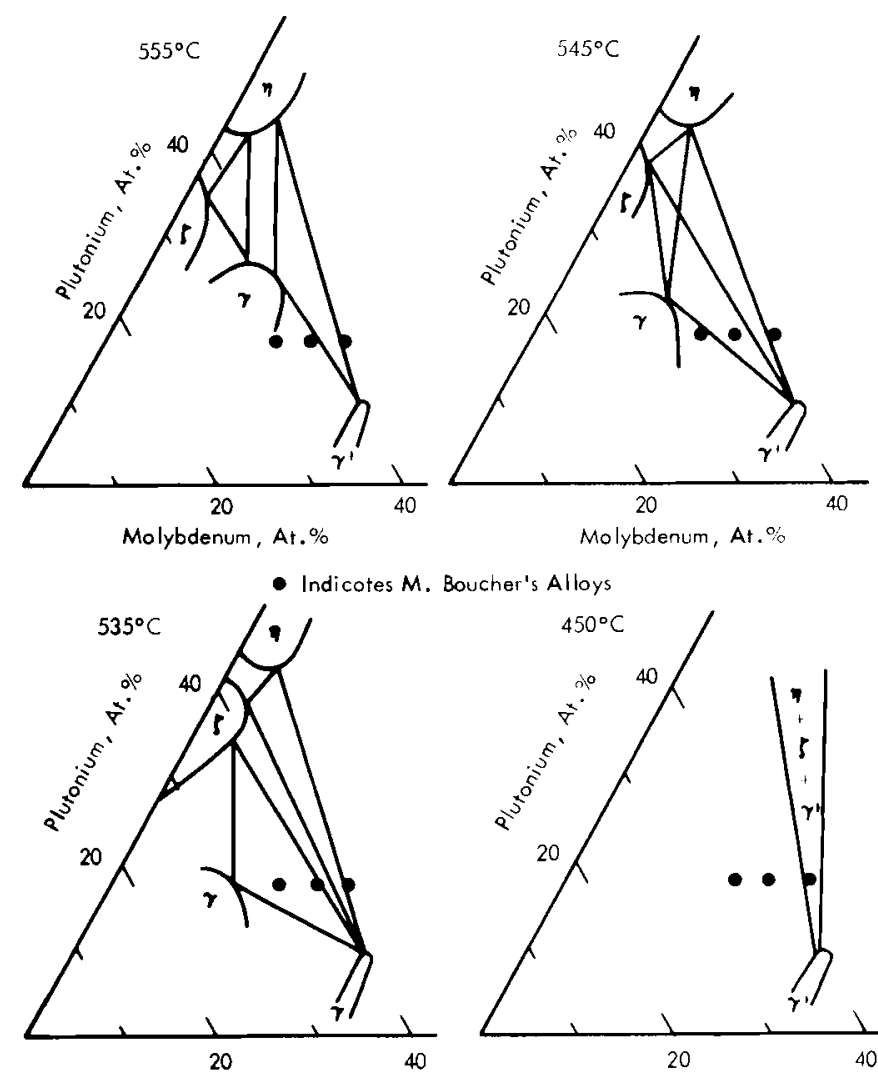

Molybdenum, At. \%

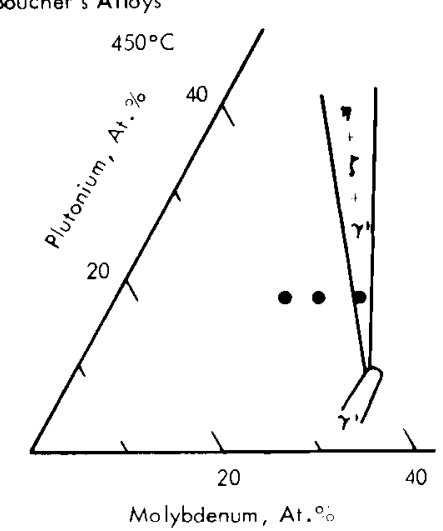

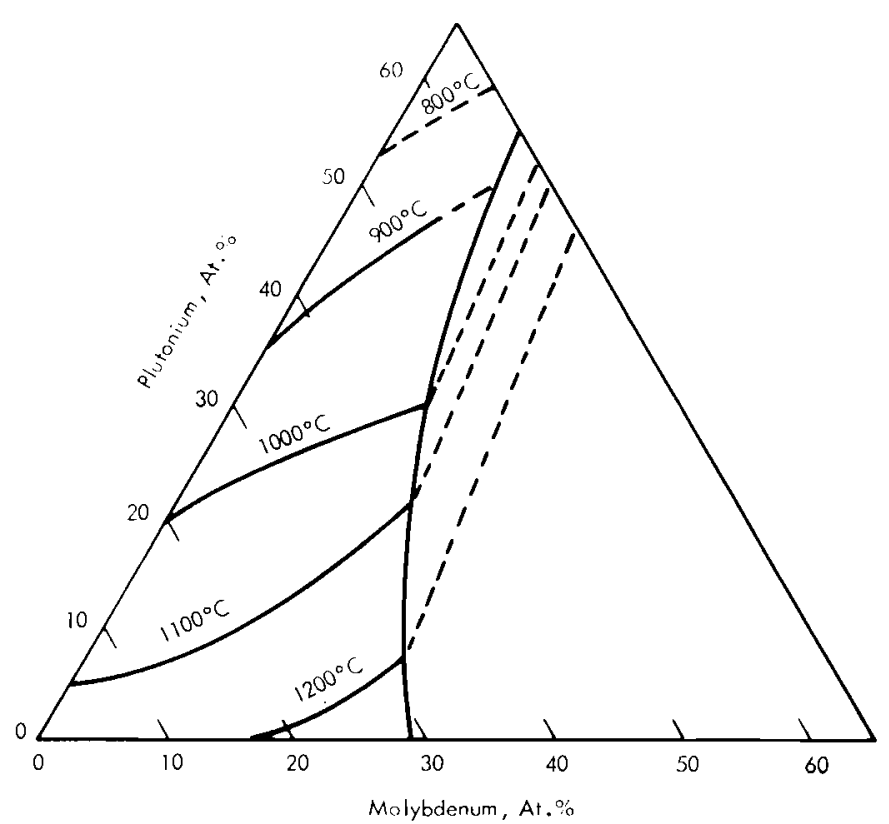

1.irpiiılus contours. From P. G. Mardon et aI. in Plutonium 1960, p. 334.

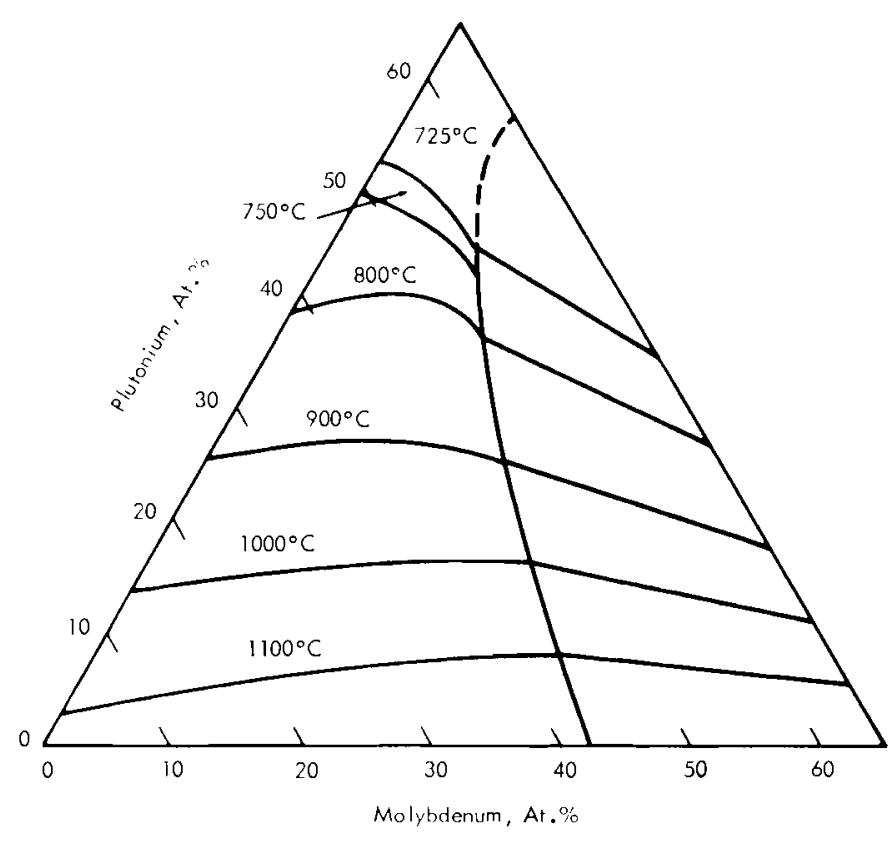

Solidus Contours. From P. G. Mardon et al. in Plutonium 1960 , p. 334. 

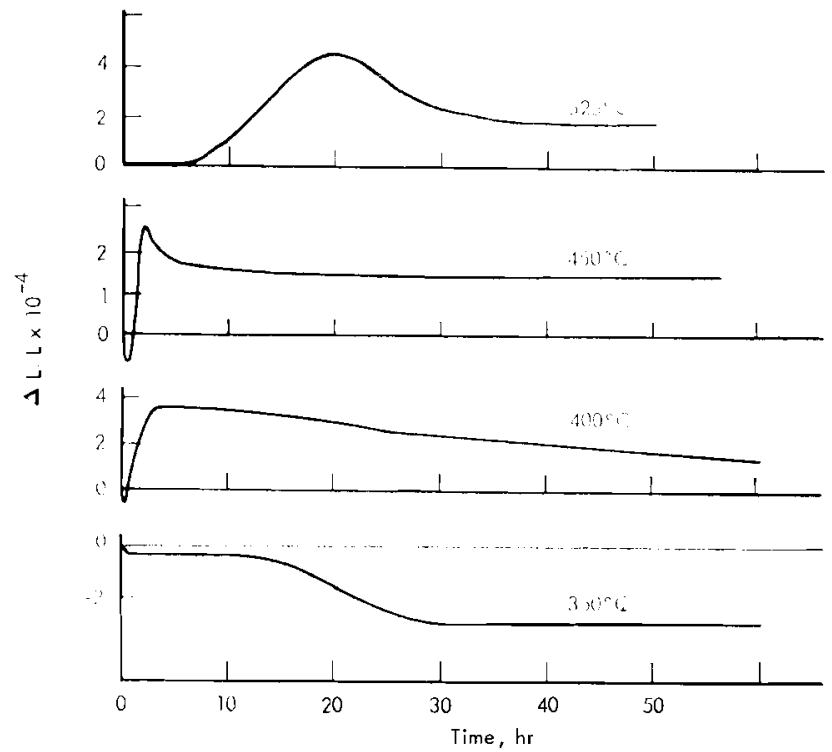

Change in Iength as a Function of Time for Specimens Held at Various Temperatues, fos', U-20\%, Pu-12', Mo, From R. Brucher in Plutonium $1960,1 \%$. 3ti
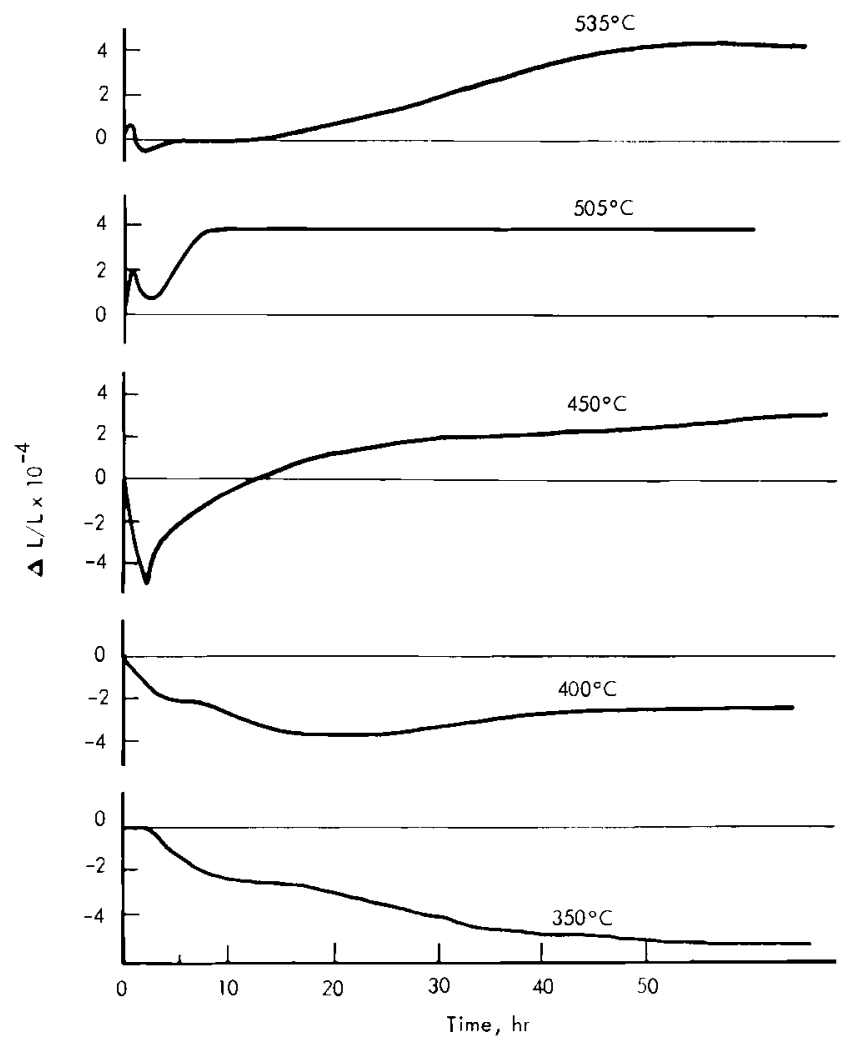

Change in Length as a Function of Time for Specimens Held at Various Temperatures, $70^{\circ} \mathrm{U}-20 \% \mathrm{Pu}-10 \% \mathrm{Mo}$. From R. Boucher in Plutonium 1960, p. 362 .

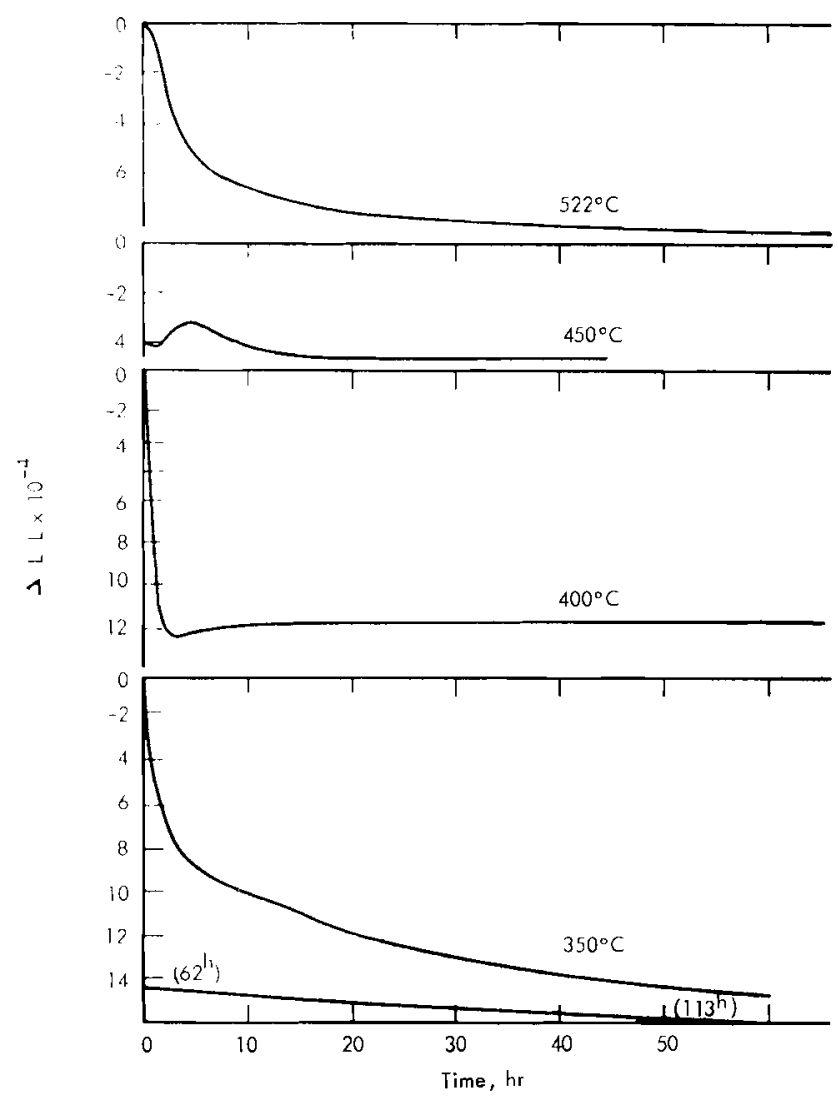

(hange in Length as a Function of Time for Specimens Held at Various Timperatures. $72 \% \mathrm{U}-20 \% \mathrm{Pu}-8 \%$ Mo. From R. Boucher in plutonium 1930, p. 363.

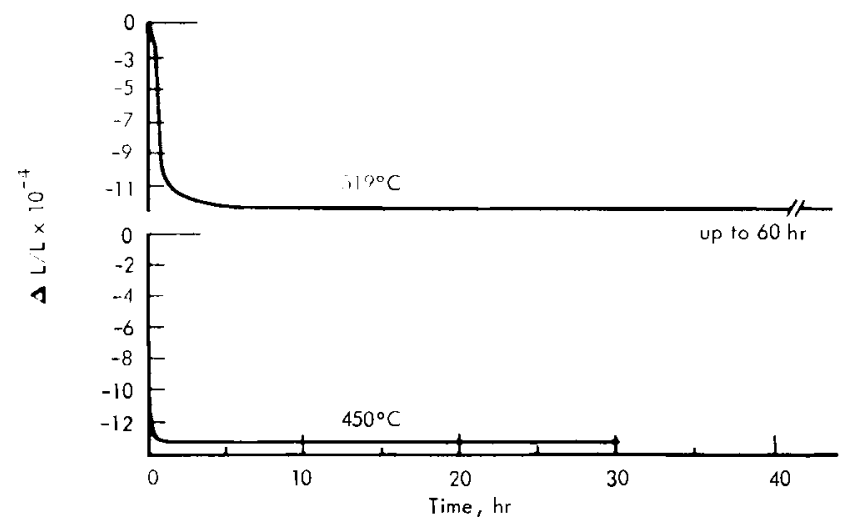

Curves of $\frac{\Delta \mathrm{L}}{\mathrm{L}}$ Versus Temperature. $74 \% \mathrm{U}-20 \% \mathrm{Pu}-6 \% \mathrm{Mo}$. From R. Boucher in plutonium 1960, p. 365.

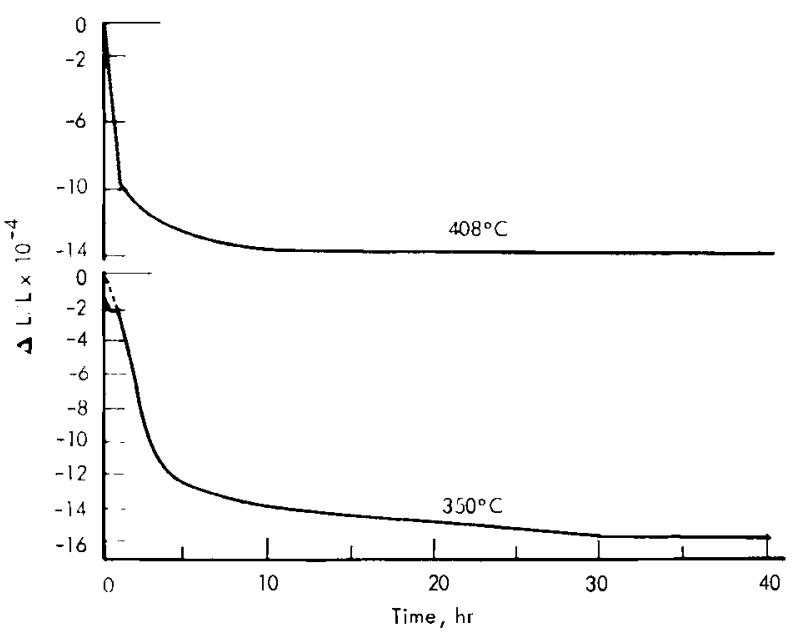




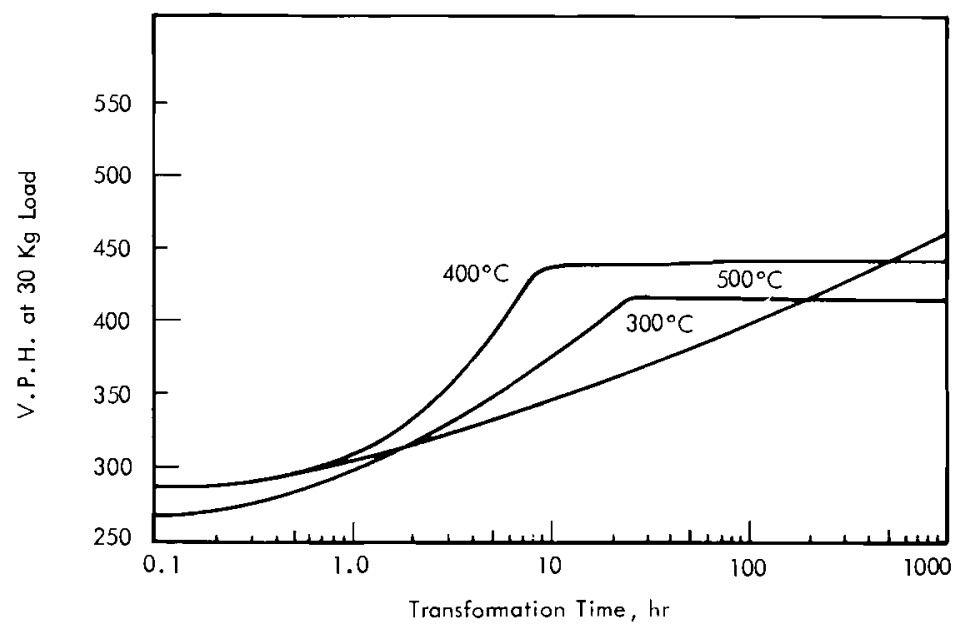

Transformation Behaviour of $\mathrm{U}-17$ at. $\mathrm{Pu}-17$ at. Mo Alloy Quenched From the $\gamma-\mathrm{U}$ Fjeld. From P'. G. Mardon in Plutonium 1960, p. 389.

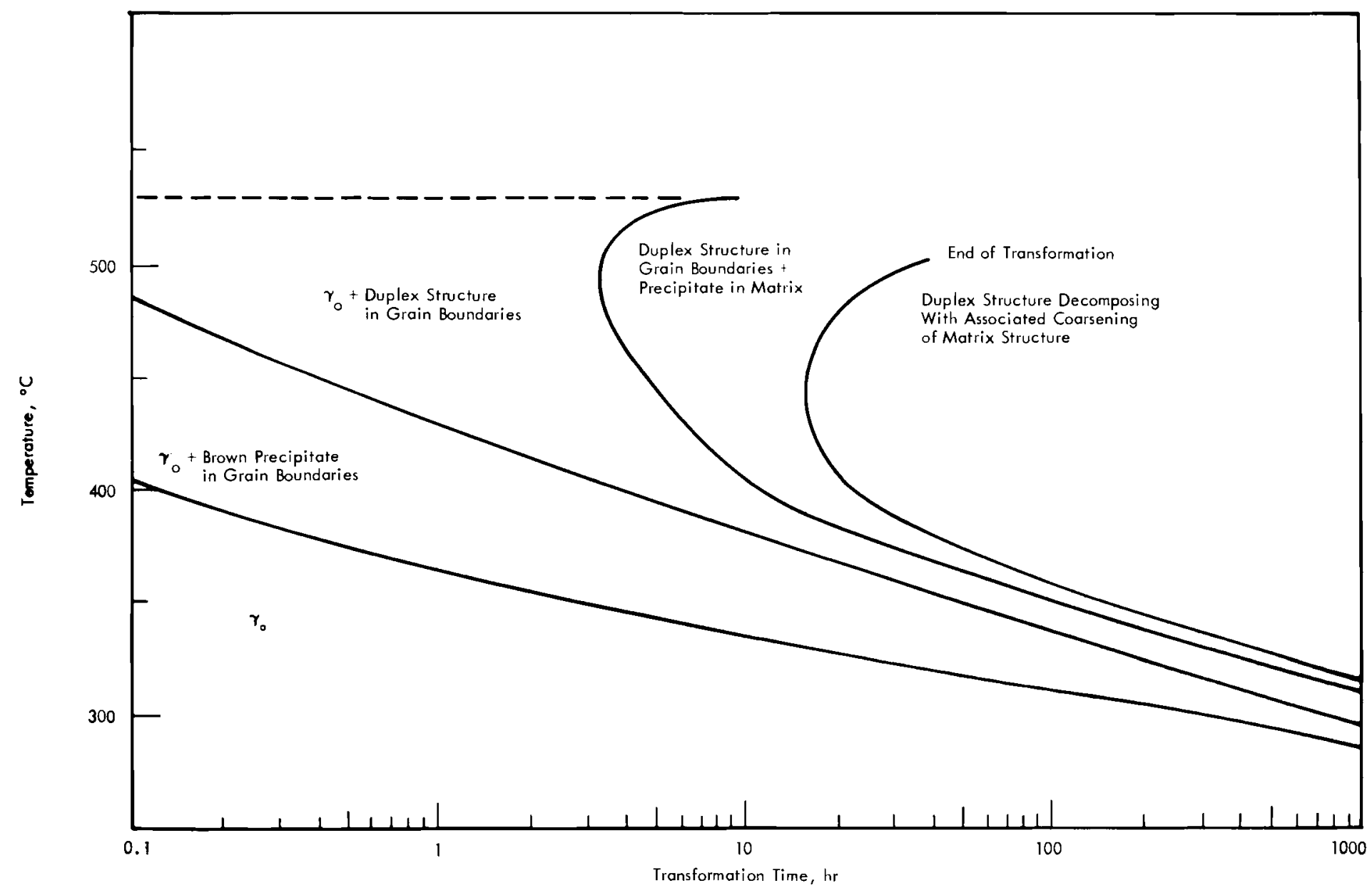

T-T-T Curves for $\mathrm{U}-17$ at. Pu-17 at. Mo Alloy Quenched From the $\gamma-\mathrm{U}$

Field. From P. G. Mardon in Plutonium 1960, p. 390 . 


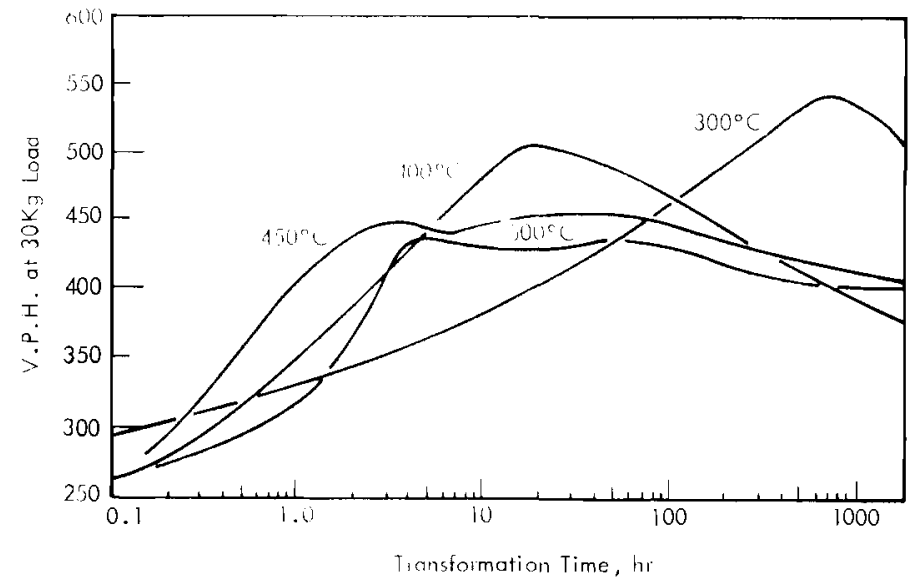

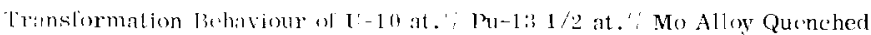

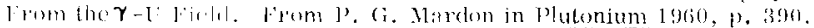

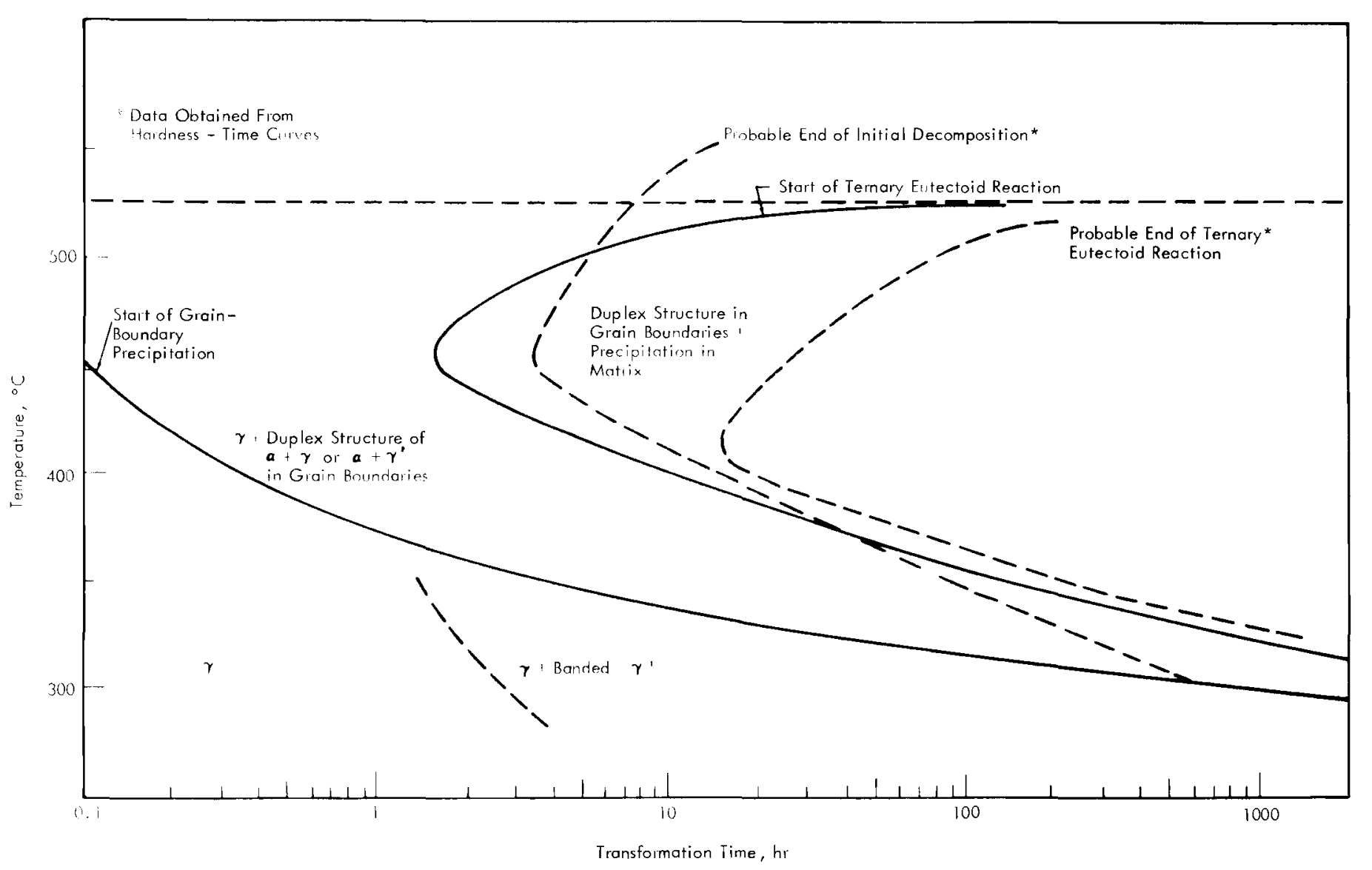

T-T-T Curves for U-10 at. Pu-13 $1 / 2$ at, Mo Alloy as Determined Modallographicall, From $\mathrm{P}$. G. Marton in Plutonium 1960, p. 391. 


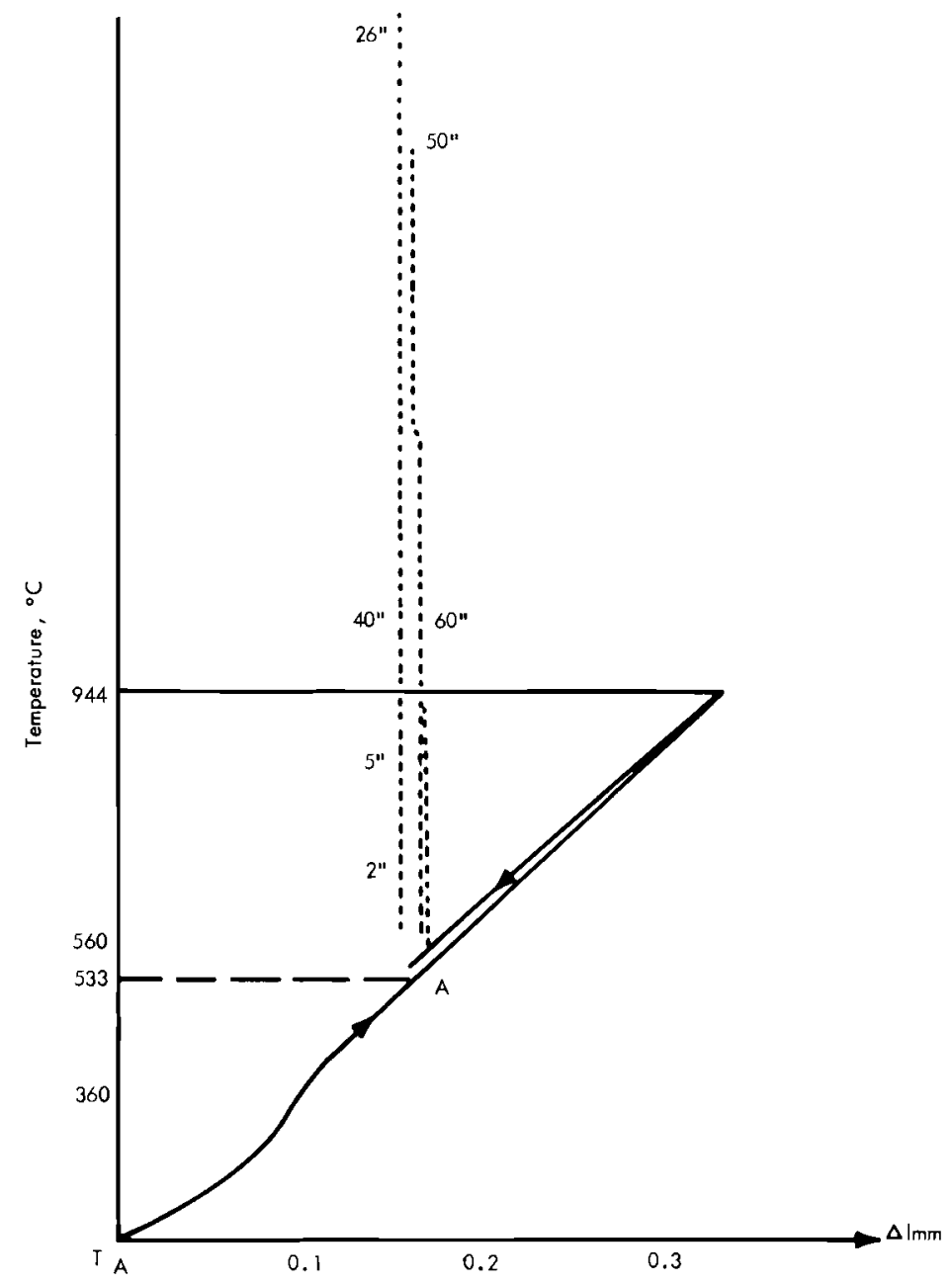

70 At. $\%$ U, 20 At. $\%$ Pu, 10 At. $\%$ Mo

$\left(\Delta\right.$, , time of $\left.533^{\circ} \mathrm{C}\right)$
Expansion vs temperature and expansion vs time curves.

\section{$\Delta l$, temperature curves are solid lines.}

$\Delta 1$, time curves are dotted lines, with a dot for every 10 minutes.

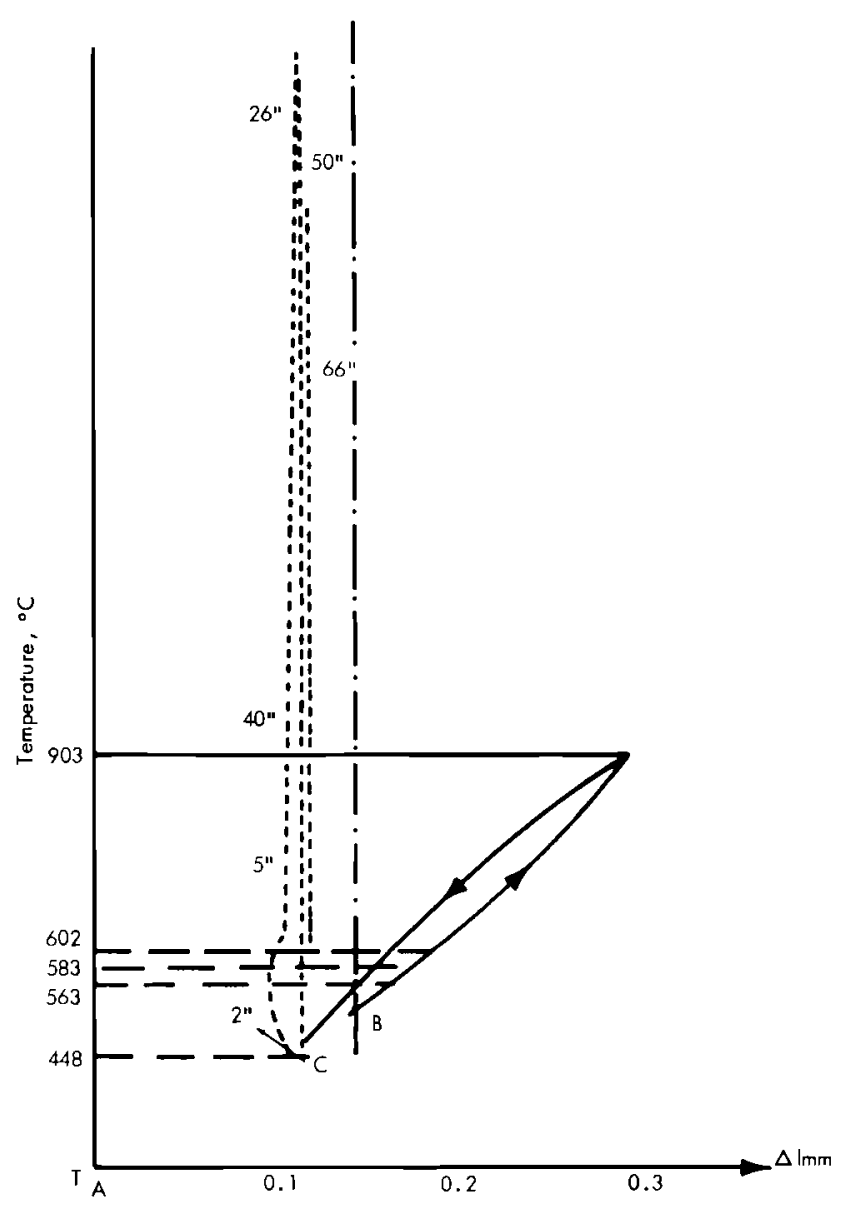

70 At. $\%$ U, 20 At. $\%$ Pu, 10 At. $\%$ Mo $\left(\Delta \mathrm{i}\right.$, time at $\left.448^{\circ} \mathrm{C}\right)$ 


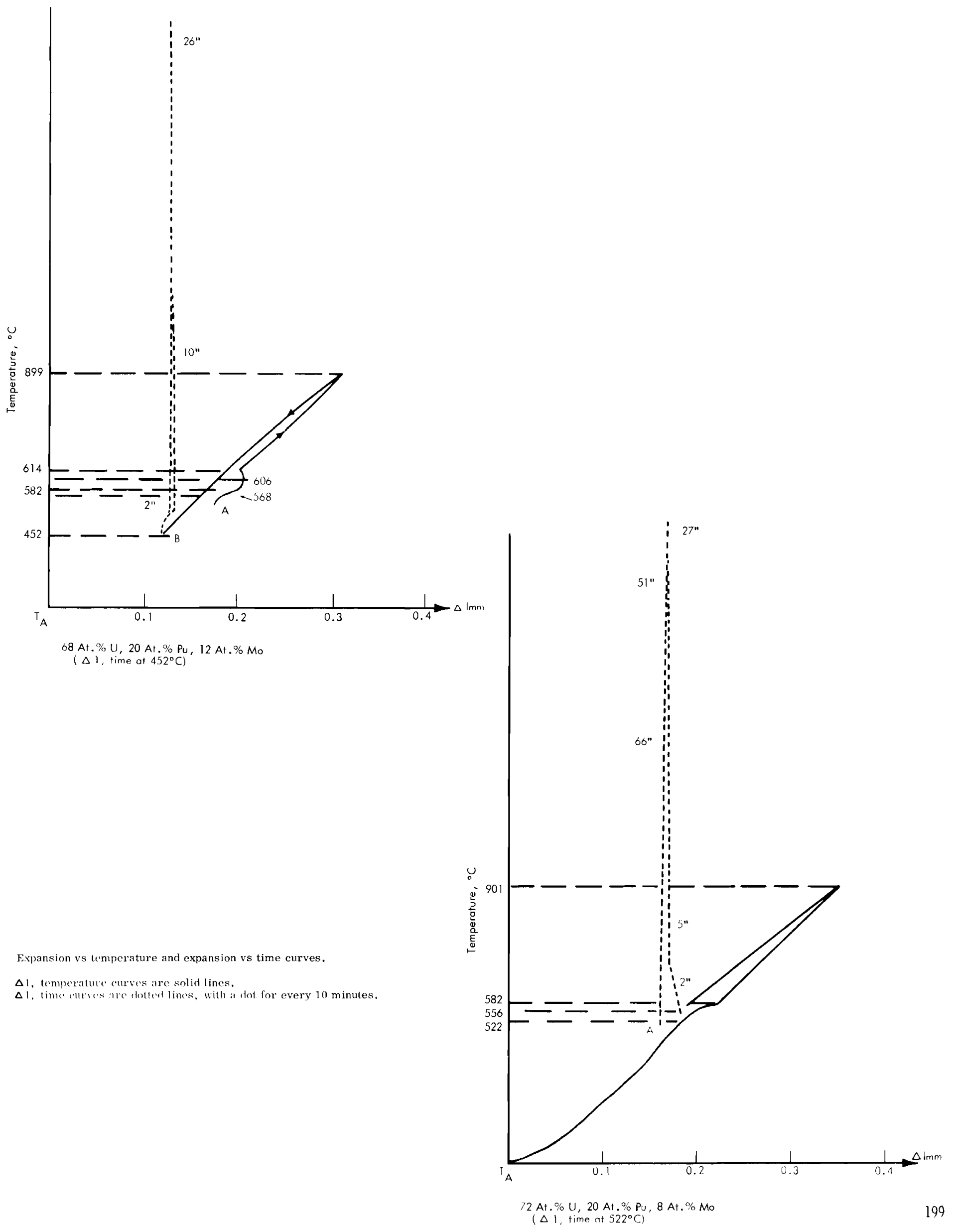




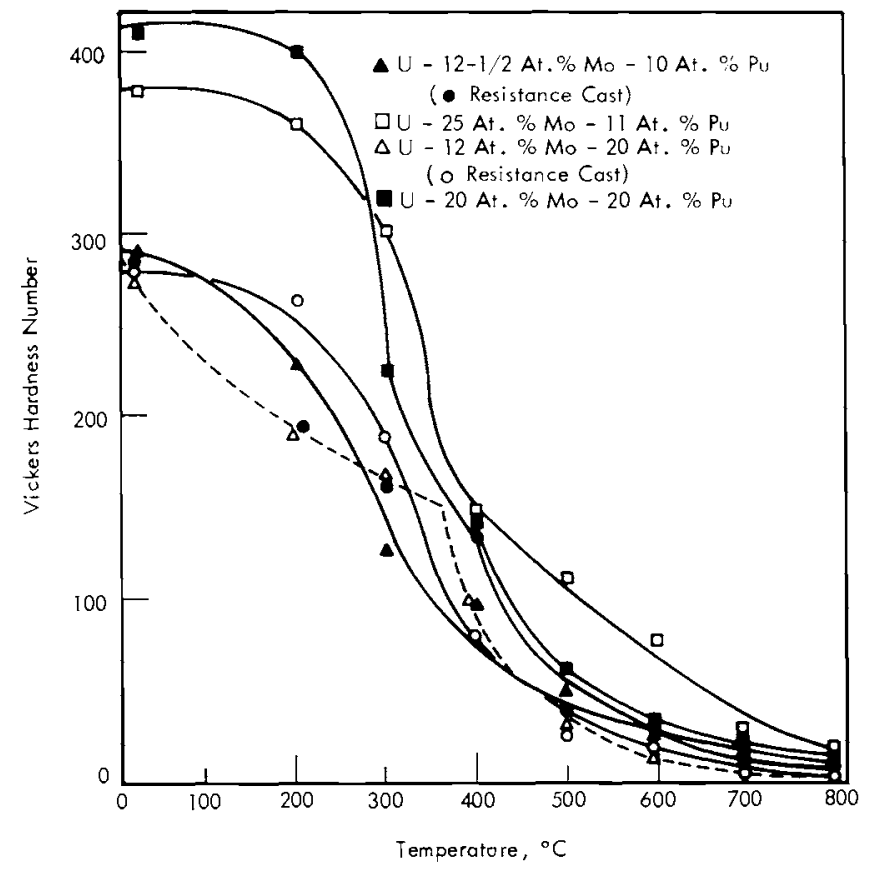

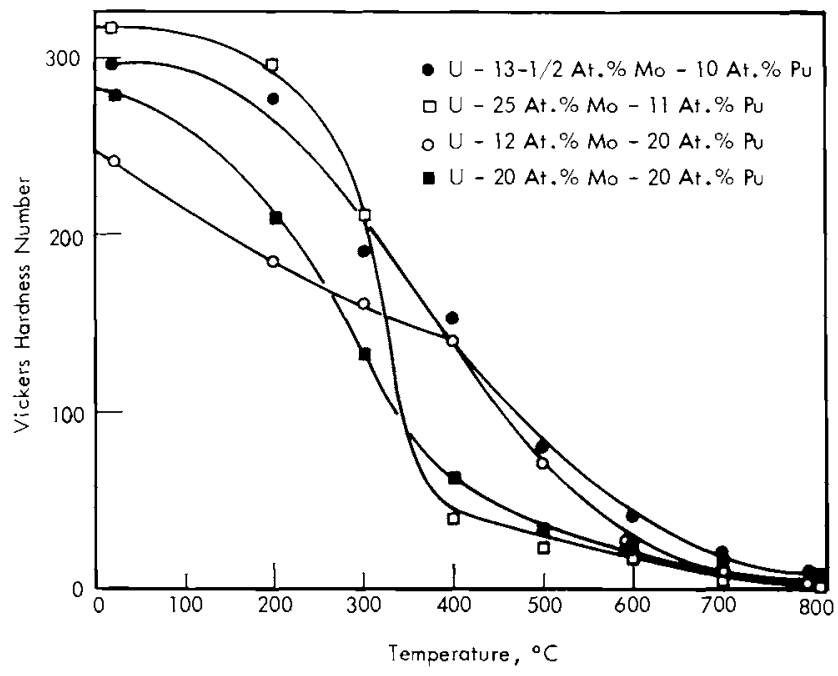

Effect of Temperature on Hariness of Homogenized and Forged UraniumMolybxlenum-Plulonium Alloys, Resistance Cast. From P. M. French,

as reported by H. R. Gardner in Chapter 4, The plutonium Handbook, 1966.

Effect of Temperature on Hariness of Homogenized and Rolled UraniumMolybicnum-Plutonium Alloys, Arc Cast Unless Otherwise Stated. From P. M. Frencl, as reported by H. R. Gardner in Chapter 4, The Plutonium Handloooli, 1066 .

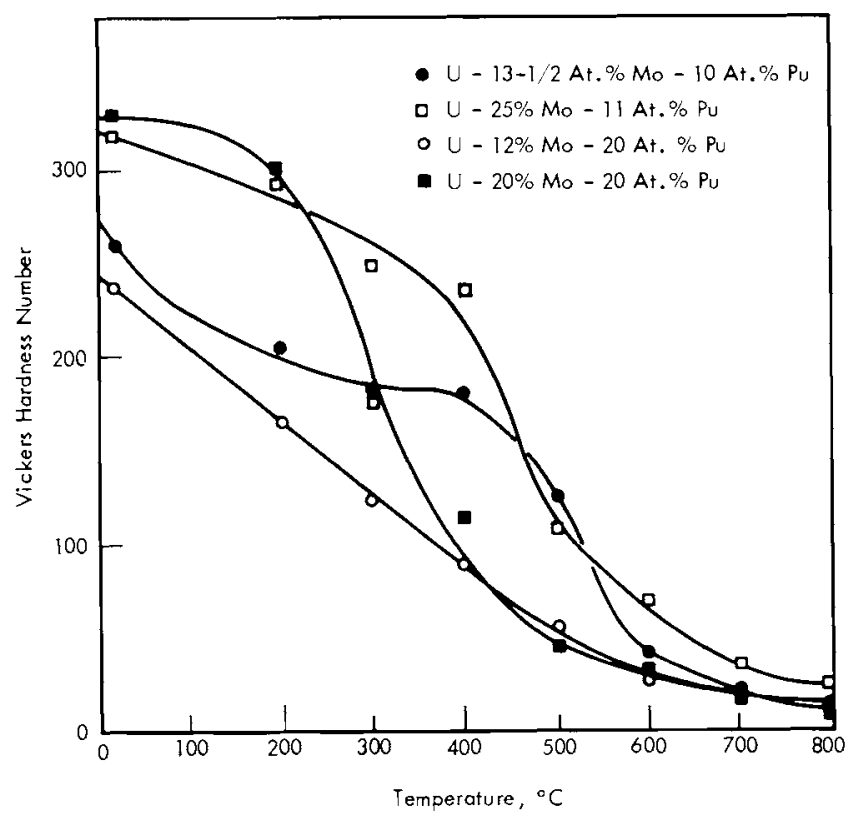




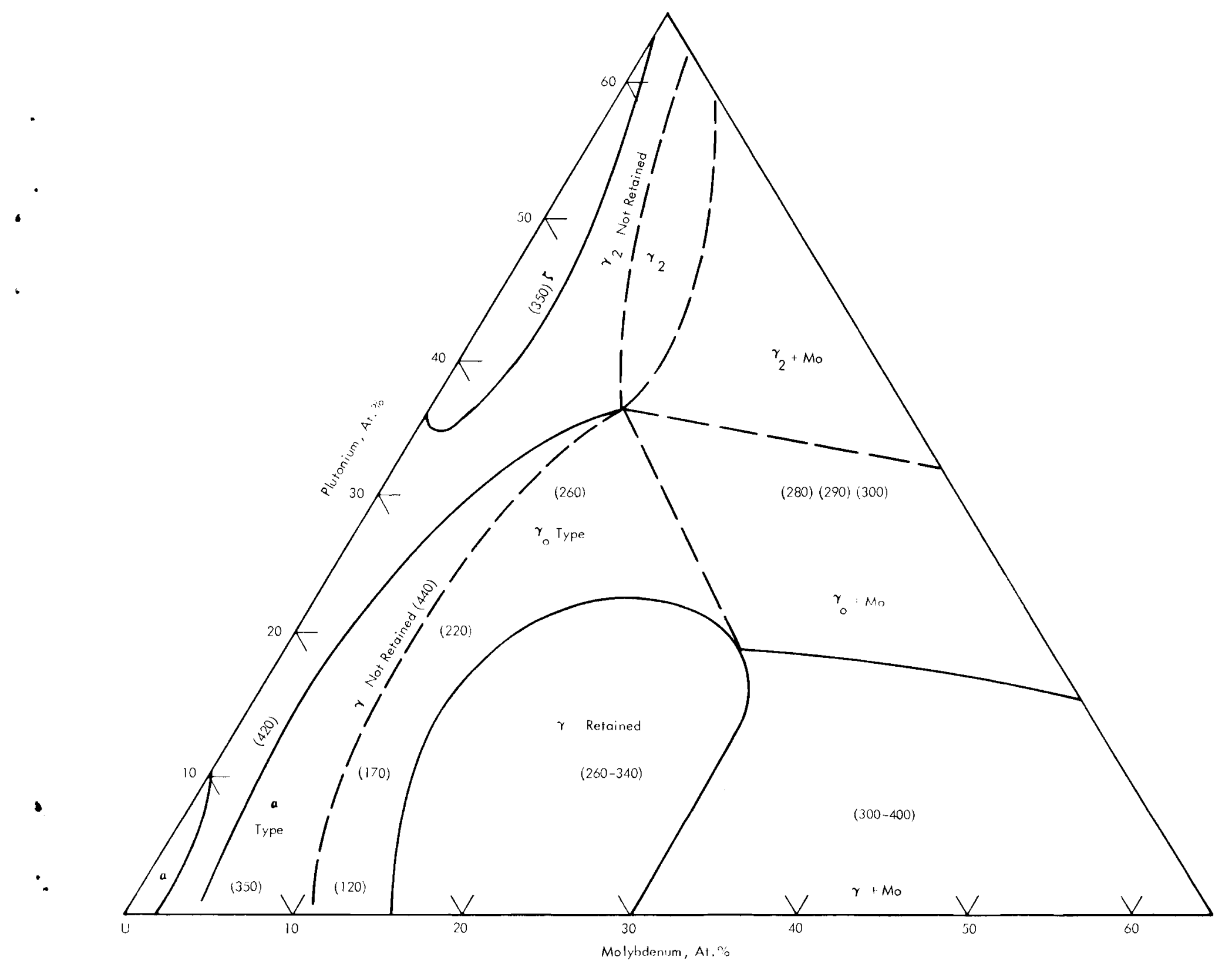

Room Temprobur Vickers Hardness of Quenehed Tranium-Molyblenum-

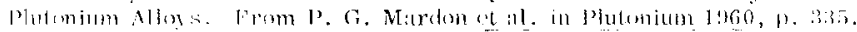




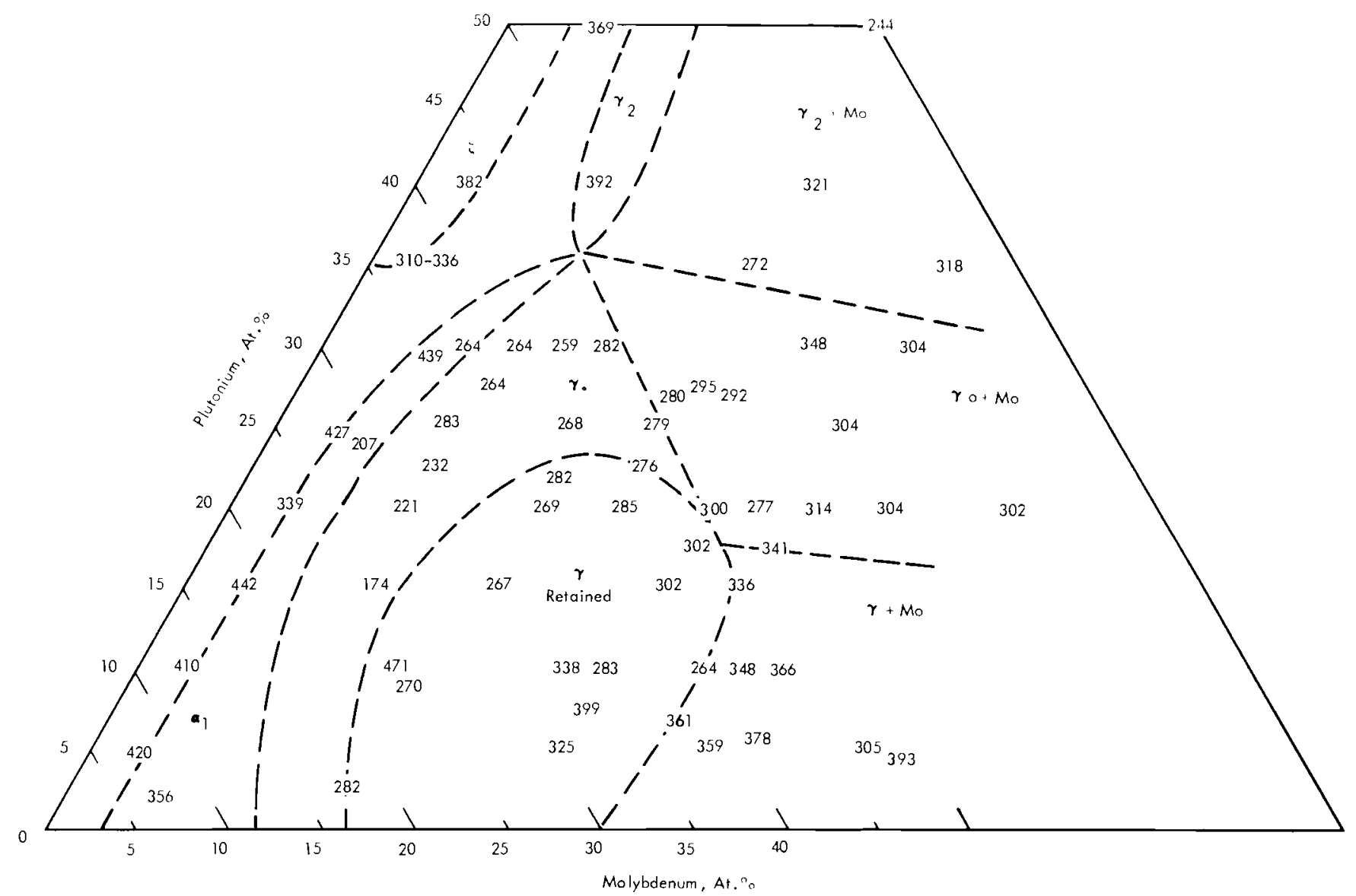

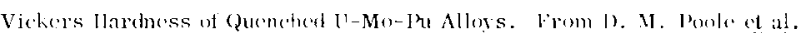
in Plutonium 1960, 1. (i.11).

ROOM TEMIERATURE IARIONESS FIGURES FOR URANIUM-MOLYBDENUMPl. TONIUM ALIOTS

From D. M. Fixole et a). in Plutonium $\frac{1960}{19}$ p. 642 .

\begin{tabular}{|c|c|c|c|}
\hline \multicolumn{2}{|c|}{ Alloy Composition } & \multirow[b]{2}{*}{ Ilout Treatment } & \multirow[b]{2}{*}{$\begin{array}{c}\text { Vickers Hinr(ness Numlu' } \\
\text { (V.H.N.) }\end{array}$} \\
\hline at. $M 0$ & a1. $P \mathrm{Pu}$ & & \\
\hline$\therefore$ & i) & Rolled & 492 \\
\hline 5 & 1.7 & Rolled & $41: 3$ \\
\hline 10 & 5 & Rolled & 160 \\
\hline 10 & 10 & Rolled & 252 \\
\hline 10 & 1.3 & Rolled & 3.1 \\
\hline 15 & $\bar{j}$ & Rolled & 266,400 \\
\hline 1.5 & $1 i$ & Are ciost & 303 \\
\hline 20 & $i$ & Rolled & $272,+123$ \\
\hline 20 & 10 & Rolled & 259,304 \\
\hline 20 & 15 & Are cist & 395 \\
\hline 25 & 2.73 & Rollou & 442 \\
\hline 25 & $\bar{j}$ & Rollerd & 426 \\
\hline 3.5 & $i$ & Mre tast & 327 \\
\hline 2.5 & $7 \ldots$ & Rolled & 408 \\
\hline 30 & $i$ & Ralled & $2 \times 2$ \\
\hline 30 & 10 & & $30 \mathrm{~K}$ \\
\hline
\end{tabular}

HOT IARIDNESS FIGITRES FOR IIRANIUM-MOLYBDENUM-PLUTONIUM 11.10YS

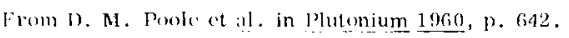

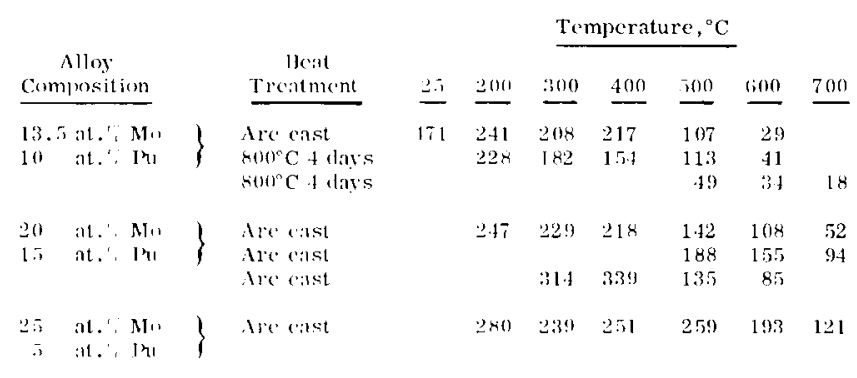

Vatrition in results ansed lo slow retion kineties below $600^{\circ} \mathrm{C}$.

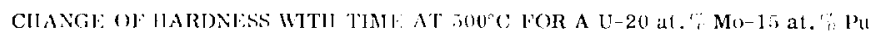
A.I. OS

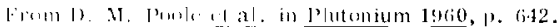




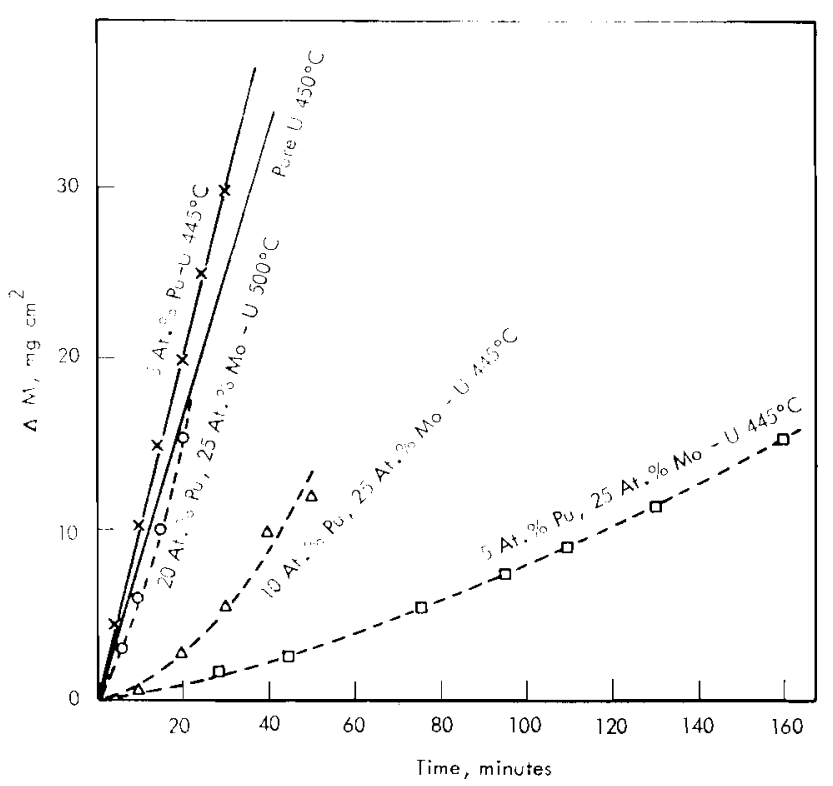

Oxidation of [ - 1'u and 1!-Mo-1Me Allows in Air Dried Over Magnesium

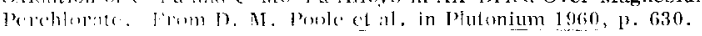

Inert Gases

Mo-U 401.4

(xillation in 1)ry $\mathrm{CO}_{2}$.

[:-Mu-Pu N/loys. A number of alloys of various compositions within the $\boldsymbol{\gamma}$ lick haw leen oxidizat and gave linear or increasing rites, but visua "Waminalion showed that all the attack was confined to a few regions and Lhit the remitinder of the specimen remained bright and apparently un"xidized: (Nehel or meehanieally-pulished specimens behaved in the same way. Metillogrilphic eximmination showed deep oxidation cavities with large "rickes radialting from them and oxidation appeared to have proceeded doun

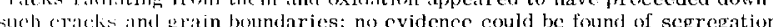
ste a

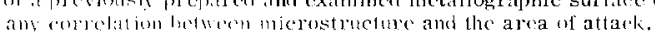

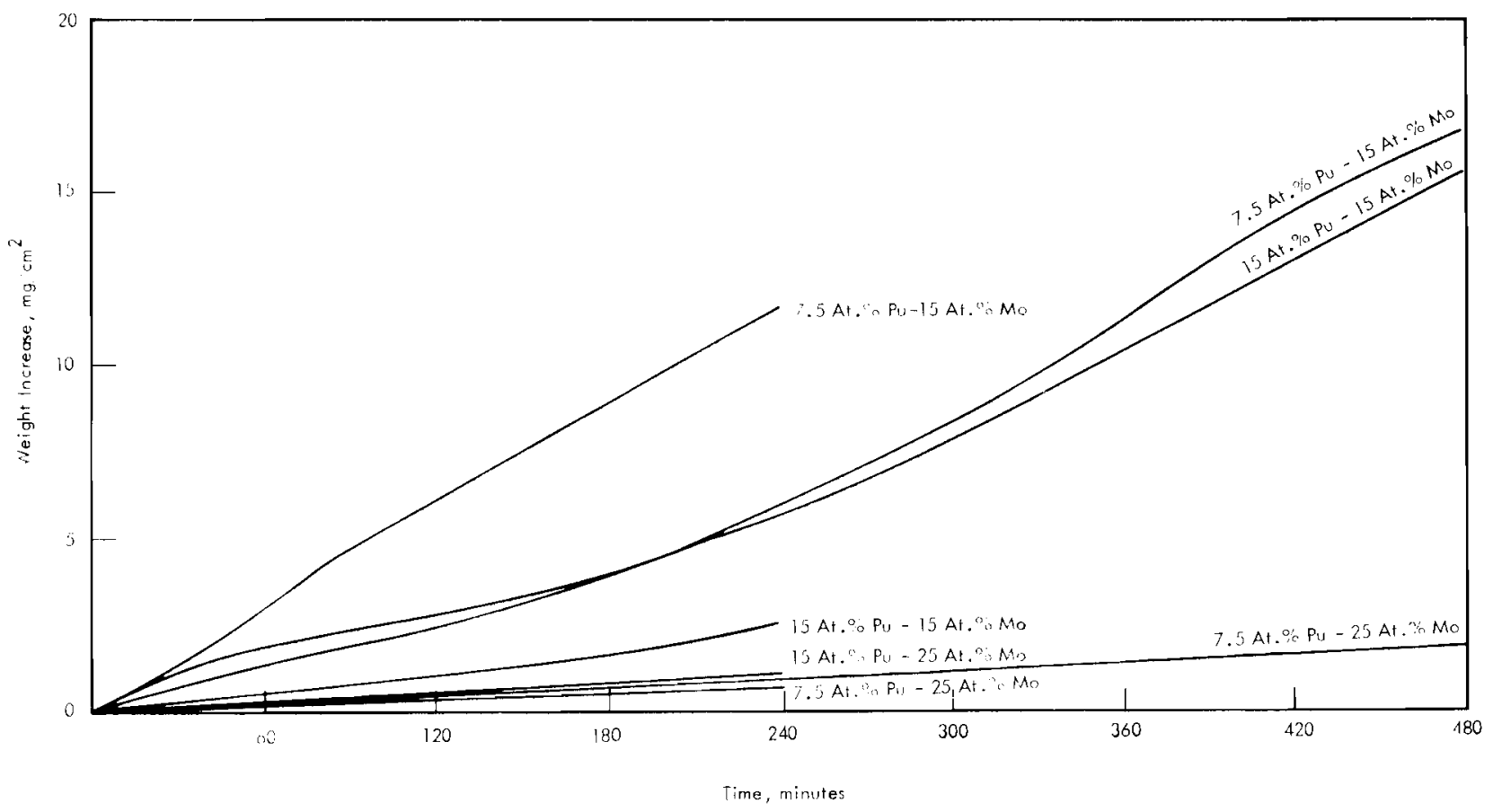

The overall rate of oxidition of the Lernary alloys is low and it would applar thit if the pitling could loc (aliminated the ir oxidation resistance would be

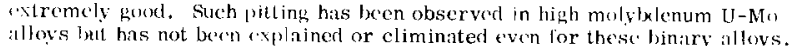

It appears that a surface liyer protects the specimen, for once attack starts it is latirly rapid. Attick is then propagated by cracks, mainly along grain boundaries, which open ender the stress caused by the oxide formation. It lias been sugpested that girains may become thermally insulated from the liulk of the material and that the temperature and rate of attack then rise rapitly. From D. M. Poole et al, in llutonium 1!160, 11). $63: 5,6336$ 
The interaction at temperatures between $(50)$ and $6.300^{\circ} \mathrm{C}$ between a range of U-Mo-Pu alloys and $18: 8: 1$ stainless steel has been studjerl. Allow sueci-

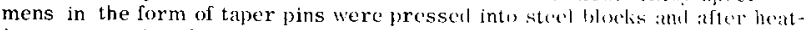
ing were sectioned, examincd metallographieally and the oxtent whe reaction zones estimated visually. From D. A. Joole (1) al. in Plutonium 1960, p. 637 .

Mechanically polished and lightly electronolished in 100 (mm $^{3}$ acetie acill, $25 \mathrm{~cm}^{3}$ water, $5 \mathrm{~g}$ chromic oxide at $1.75 \mathrm{~V}$ for $10 \mathrm{kec}$.

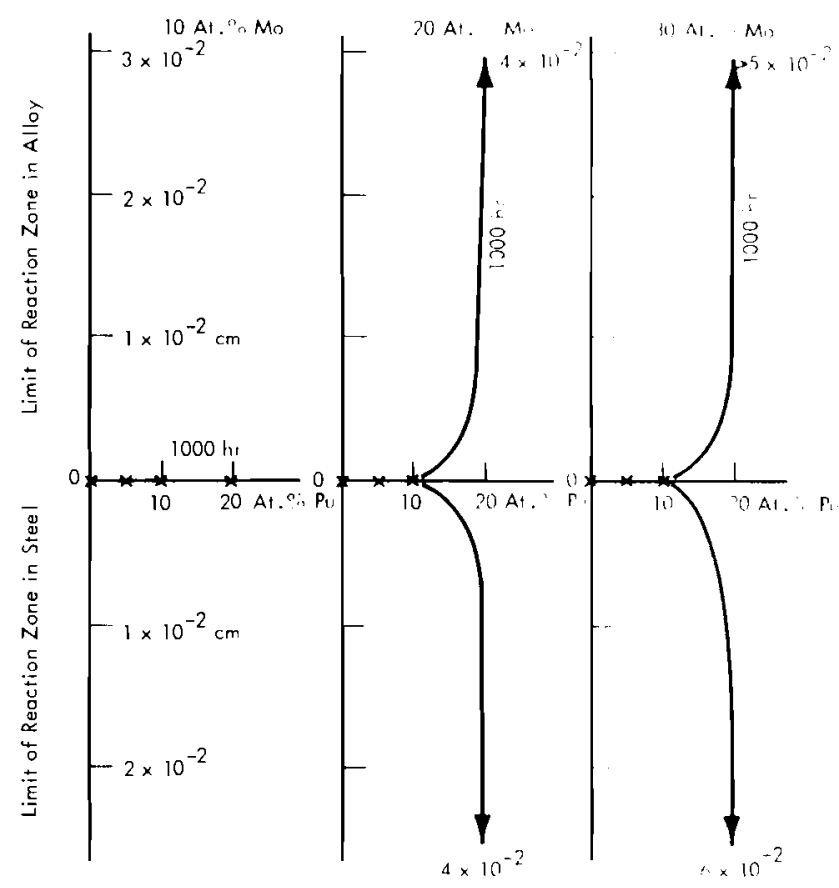

Compatability of U-Mo-Pu Alloys with 18:8:1 stainless Stecl $600^{\circ} \mathrm{C}$. From D. M. Poole et al. in Plutonium 1960, p. 033 .
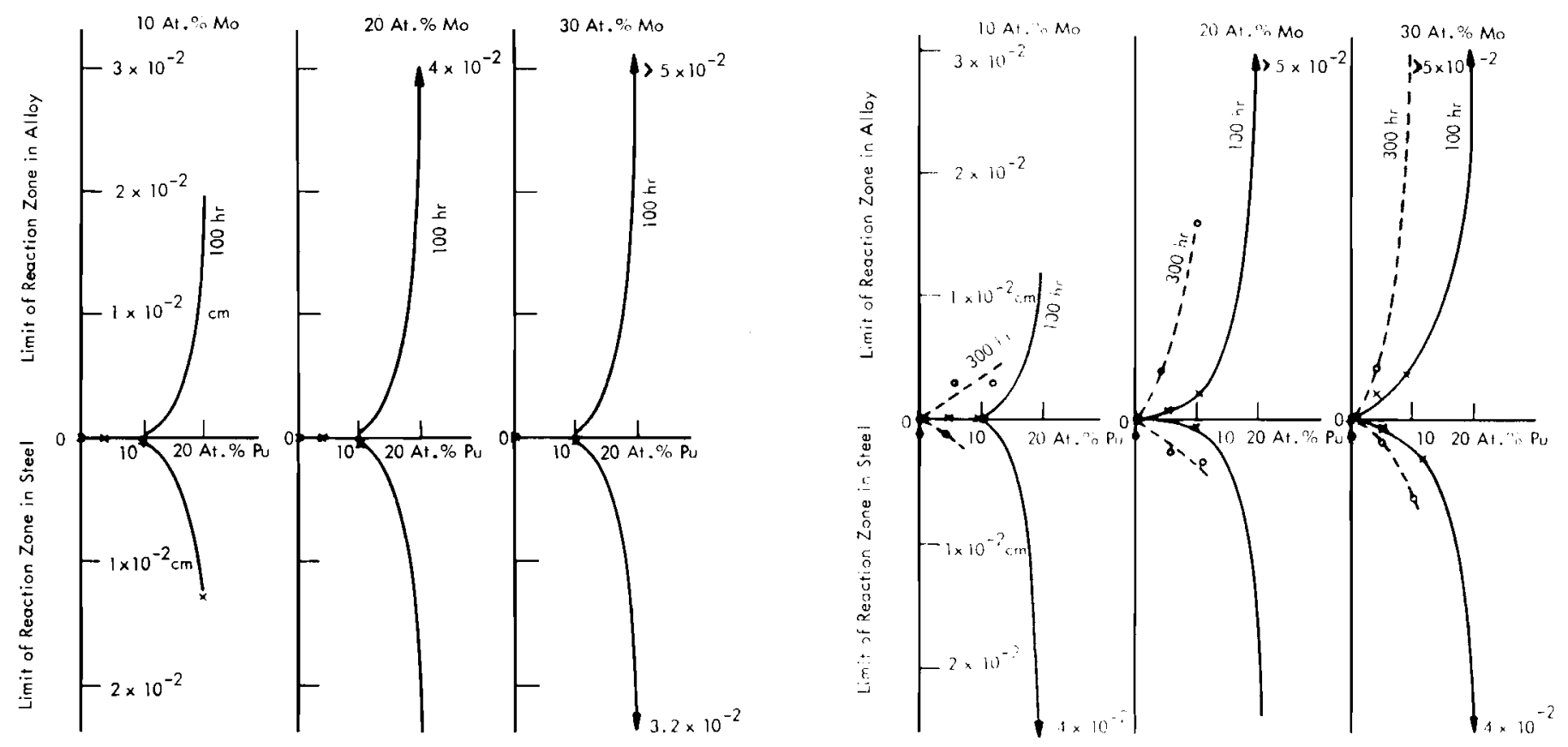

204 
R. Abramson et al. "sone l'roperties of Plutonium and AluminumPlutonium Alloy," $p .327$. Proeedings of the Second I Inited Nations: International conference on the Peacefu] iscs of Atomic linergy. vol. 6, pp. 17 -18:. Initei Nations, Geneva, 1958

E. 1). Albrecht. "The Plutenium-Zine Sistem," J. Nuel. Mlater.. vol. 12, p. 125.196.

P, J. Bacca, in W. J. Mariman. First Annual Plutonium Metallurgy Information Mleeting, LAB-CMB-1 1-3?04. Los Alamos Scientific Laboratory, March 2k, 1957. (Classified)

J. G. Ball and W. B. II. Iord, "A History of the Early British Work on I'lutonium Niletillurey," " . Inst. Metals, vol. 86, p. 376. $1957-58$.

A. A. Bituer and R. A. Frough. "Thorium Alloy Systems," Metallurgy ind Fuels, l'repless in Nucleal Finergy, edited by II. M. Finniston : and . P. Ilowe, Pergatmon I'ress, New York, [1]59. Series $V$, vol. 2, p. 383

A. F. Berndt. "Room Temperature Lattice Constant of Alloys of Plutonium in Alpha-l ranium," J. Nucl. Mater., vol. 9, p. 53. 19633 .

R. M. Bidwell et al. "The Development of IIigh Purity Tantalum and Alloys for Liquidl Plutonium Contained in Lampre I, "Nucl. Sci. Eng., vol. 14, 1, 109, 1962.

33. Blumenthal, Donsity Moisurements in Gloveboxes With Density Dittit on Monohromolongene, Cast Thorium. Thorium-Urani-

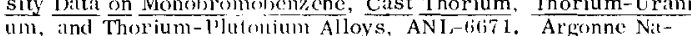
inn, ind Thorium-1'utultimt Alloys.

A. A. Bechurle et a1. "Interaction of Plutonium and other Metals

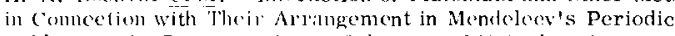
Table." 1.2 2197, l'bocenting of the Second Inited Nations Inter.

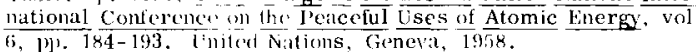

R. Boucher. "Cinetique les Transformations de l'hases d'Alliitges Uranium-Plutonium-Molybedne, "Plutonium 1960 , edited by E. Grison, W. B. II. Lard and R. I) Fowler cleaver-Hume press Ltcl. L Londull. 19\%il. [p]. 35,2-367.

H. C. Bowen. "High 'Trmperiture Aqueous corrosion of Aluminum-Plutunium :ind Aluminum-Silicon-Plutonium Allays, '

Corrosion. vol, 17. 160. $7,12,12,1961$

D. F. Bowersox. The solubility of Solected Floments in Liquid plutonium. Tant Plutonium,

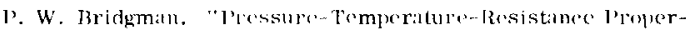
ties of Lanthanum, Bisinuth. Neptunium, J'lutonium, and Americium to $450^{\circ}$ ind $30 \mathrm{~K} 13, " \mathrm{~J}$. Phys, Chem. vol. $67, \mathrm{p}$ 2308. 1963

M. B. Brodsky. "Iall (ocfficient of Alphil I'lutonium, " P'hys. Rev., vol, 131, p. 1:37, 19633

S. E. Bronisz. "The Mechanical Propertics of Alphat Plutonium in Compression, "J. Nucl. Mater., vol. 9, j. 104. 1963.

S. E. Bronisz and A. F. Corum. "The Plastic Deformation of Monoclinic Alpha I'lutorium," $\perp$. Nucl. Mater., vol, 2, p. 353. 1960.

C. C. Burwell et al. "Corrosion Testing and Fuel Selection for LAMPRE I," Nucl. Sxi. Eng., vol, 14, pp. 12:3-134, 1962.

C. C. Burwell et al. "Corrosion Testing of LAMPRF-I Fucl,"

Trans. Am. Nucl. Soc., vol, 4, pp. 347, 348. 1961.

A. S. Coffinberry and F. H. Fllinger. "The Intermetillic Compounds of Plutonium, "proceedings of the International Conference on the Peaceful Lses of Atomic Energy, Genevit, 19.55, vol, 9. pp. $\frac{13}{13-146 .}$ Unitiol Nations. New York, 1956.

A. S. Coffinberry ind M. B. Waldron. "A Review of Physical Metallurgy of Plutunium, "Metzllurgy and Fuels, Progress in Nuclear Energy, ediled $\mathrm{I}, \mathrm{I} . \mathrm{M}$. Finniston and J. P. Howe

McGraw-Hill Beok (o.. Inc.. New York, 1956. Series V, vol, 1, pp. $355-410$

A. S. Coffinberry till. "Jlutonium and Its Alloys," Reactor Handlook, edited the (C. R. Tipton, Jr., Interscience Problishers. Ine., New Yolk, 1966), 2nd ed., vol, 1, l. $266 i$

A. A. Comstock and R. B. Gibney. Measurement of Plutonium Liquid Density, IAA-13/k, Los Alamos Scientific Liboratory, Jan 21,1952

R. G. Cope. "The Jelta I'lutonium Transition Temperature," Nature, vol. $186.1 \% .540 .1960$.

R. G. Cope et al. "The Plutomium-Ruthenium and PlutoniumNeptunium Binary Ihase Diturams, " Plutonium 1960, edited by E. Grison, W. B. II. Inld, and R. D. Fowler, Clearer-Hume Press Ltd., London, 1941. Wp. 280-289.

E. M. Cramer et al. "Ihe Dilatometry and Thermal Anilysis o Plutonium Metal," The Metal Flutonium, effited hy t. Soffinberry and $W$. N. Miner. The Innersity of Cheino l'ress. Chicago, 1961. pp. 112-122.
E. X. Crames, la, JJ, Fillinger. and C. C, latnd. "PlutoniumZine Phase Diagh'tur," Extlitetive ind physieal Metallurgy of Plutonium and Its Alloys, edlited by W. D. Wilkinson, lnterseience Publishers, New York, 1960, PJ, 169-180.

J. K. Critchley. Low Melling Point Aloys of Cerium with Iron,

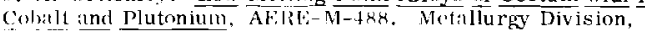
Atrimie: Energy IRescatech Fst:tlishment. Harwell, Berkshire. duls $19-19$.

D. T. Cromer and A. C. Larson. "The Crystal Structure of IniNi ${ }_{43}$ " Acta Cryst., vol. 12, p. 909. 1960.

1). T. Cromer anil C. E. OIsen, "The Crystal Structures of PriNi, :ure CeNi ${ }_{3}$, Acta Cryst., vol, 12, p. (i89. 1959.

D. T. Cromer and R. B. Rool, Jr. "The Crystal Structure of I’iNi." Actil Cryst., vol. 12, p. 942. 1959.

L. (;. T. Davy and J. S. White. "The Kinetics of the Transformation in Plutonium-Titanium Alloys," J. Nucl. Mater., vol. $12,1.221 .1964$.

J. K. Dawson. "Magnetochemistry of the Heaviest Elements, Jart VIII. Metallic Plutonium. " $\underline{J}$. Chem. Soc. (London), Part IIl, P. 33394 . 1954 .

3. d. Pacillenet in "Author's Replies to the Report and Comments on the Report and Papers, "Plutonium 1960, edited by E. Grison, W. B. 1f. Lord, and R. D. Fowler, Cleaver-Hume Press Ltd. London, 1961. pp. 105-10s.

E. Dempsey and A. E. Kay. "Some Investigations on Plutonium Metal," J. Inst. Metals, vol. 86, p. 379. 1957-1958.

J. E. Deveriall. The Thermal Conductivity of a Molten Pu-Fe rutectic $(9.5 \mathrm{a} / \mathrm{O}$ Fe). July 1958

F. H. Ellinger. "A Review of the Intermetallic Compounds of Plutonium," The Metal Plutonium, edited by A. S. Coffinberr and $W$. N. Miner, The University of Chicago Press, Chicago, 1901 , pr. 281-308.

F. II. F1linger. "Authors' Roplies to the Report and Comments on the Report and the Papers." "llutonium 1960, edited by $\mathrm{E}$. Grison. W. B. H. Lord, and iR, D. Fowler, Cleaver-Hume pross Ltd. . Iondon, 1961 . 13\%. $318-319$.

F. 11. Ellinger. "Crystal stricture of Delta-Prime I'lutonium and the Thermal Expansion (haricteristics of Delta, DeltaI'rimu, ind E.psilon Plutonium," J. Metals, vol. 8, p. 1256 19519. F. H. Ellinger et al. "Plutonium-Cerium Phase Diagram,"
Extrictive and Physical Metallurgy of Plutonium and Its Alloys, Extrictive and Physical Metallurgy of Plutonium and $\frac{\text { Its }}{\text { edited by } W \text {. D. Wilkinson, lnterscience Publishers, New York, }}$ 1960. pp. $149-167$

F. H. Ellinger et al. "The Plutonium-Gallium System," J. Nucl. Mater., vol. 12, p. 226. 19664.

F. H. Ellinger et al. "The Solubility Limits of Aluminum in Delta Plutonium and Some Revisions of the Plutonium-Aluminum Phase Diagram," J. Nucl. Mater., vol. 5, p. 165. 1962.

F. II. Ellingrer, R. O. Flliott, and E. M. Cramer. "The j) Jutonium-Uranium System," J. Nucl. Mater., voi. 1, p. 233.

R. O, Elliott and A, C. Larsion. "Delta-Prime Plutonium." The Mctal llutonium, edited by A. S. Coffinberry and W. N. Miner, The University of Chicago P'ress, Chicago, 1961. pl). $26: 5-280$.

R. (). Elliott and K. A. Gschneidner, Jr., "Behavior of Some Delta-Stabilized Plutonium Alloys at High Pressures," in Extractive and Physical Metallurgy of plutonium and lts Alloys editel by $W . D$. Wilkinson, Interscience Publishers, New York, 1960. pp. 243-2 12 .

R. O. Elliotl, C. E. Olsen, and S. E. Bronisz. "Effect of Prelerred Orientation on the Flectrical Resistivity of Alpha Plutonium at Low Trinporatures," Phys. Rev. Letters, vol. 12, 1. 272.1904.

R. (?. Filliott et al. "Flectrical Behavior Below $300^{\circ} \mathrm{K}$ of PlutoniumRich Delta-phase $\mathrm{S}$ ilid Solution Alloys Containing Cerium,

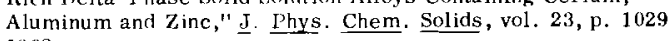
1962 .

R. O. Filliott et al. "Thermal Expansion of Some Delta Plutonium Solid Solution Alloys," Plutonium 1960, edited by E. Grison, W. B. H. Lord, and H. D. Fowler, Cleaver-Hume Press Lid., London, 1961 . pp. 142-155.

T $\mathbf{K}$. Engel et al. "A High Temperature Calorimeter for The rmal Property Measurements of I'lutonium Metal," The Third Conference on Nuclear Reactor Chemistry, Gatlinburg Tennessee, Oct. $9-11,1962$, TI1)-7641, pp. 266-272. 
P. M. French, Atomic Energy Iresearch Establishment, Jarwell, England, Personal Communieation, 196i:, as reporterl ls II. IR Gardner in Chapter 4, The Plutonium Handlook. 19risi.

H. 12. Gardner, Ifanford Atomic: Prolucts operation, Gencrub Electric Co., 19ti4

H. R. Gardner. "Fatigue Testing of Unalloyed Plutenium," J. Nucl. Mater, , vol, 10, p. 25, 1963,

H. R. Gardner. "Mechanion] I'roperties" (Chapter t), The

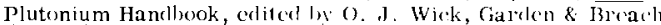
Science P'ublishers, Ine., New York, 19lifi.

II, IR. Garelnor. "Mechanical Proplerties ol' I'lutontum-Iron Alloys," in 0. J. Wick, Quartorly l'ogress Roperte, l'lutonium Metallurgy Operation, July-August-Scptemler, 1960, HW Hanforcl Atomic Products oneration, General Es lectric: $\mathrm{C}(0$. Oet. 15, 1960. (Classified)

H. R. Garelner, l'apers l'resented at the Fourtle I'lutonium Information Mecting at the Iawrence Radiation liboralory HW-71084. Hanford Atomic Prexluets Operation, Groneral libectric Co., Sertember 1!ibl. (Classified)

H. R. Gardner. Preliminary Ireport - A study of the Properties

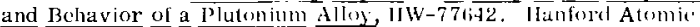

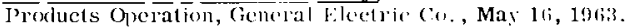
(Classified)

II. IR. Gardner. preliminary [repore on the Tensile propertics of a Plutonium Alloy, IIW-72stol. ILanforel Atomic Prociuets

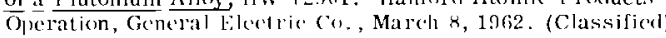

H. R. Gardner. "floe Imprat l'roperties of Unallored Plutonitum," Trans. Met. Soc, AlMli, rol, 221, 1, $201,1962$.

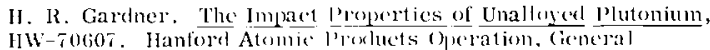
lilectric Co., Octoles 1961 .

H. R. Gardner. Torsion Thisting of plutonium, IHW-66909,

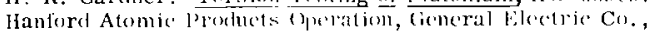
Oet. 21,1960 .

H. R. Garelner, in O. J. Hick. Quarterly J'ogress Ireport, Metallurgy Development Oueration, January-February-Mareh,

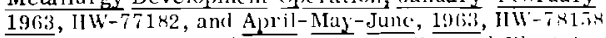
Hanford Atomic products (p)oration, ieneral Eilectrie Co. 19(3). (Classified)

H. R. Gardner. Cimpullisherl Data. IIanford Atomic: products Operation, General Electric (o. lonit.

H. R. Garelner and I. B. Mann. "Mcehanical Properties and Formability Studies on Thallored llutonium," Plutonium 1960, edited by E. Grison, W. I3. II. Lord, and R. D). l'owler, CleaverHume Press Led., Londonl. 196(61. p). 513-570.

H. R. Gardner and I. B. Mann. Mechanieal property and Formability Studies on lilutonium, JlW-65019. Hanford Atomic P'roducts Operation, Decemlece 1959.

H. IR. Gardner et al. "The Tensile Properties of Pure Plutonium and Aluminum-Plutonium Alluss," 1)/1081, Procecelings of the Second United Nations Interiational Conference on the peacefu Uses of Atomie Energy, wol.6, Bl). lisfi-6\%9. Unitci Nations, Geneva, $\frac{\text { Utom }}{1958}$

R. B. Gibney, Preliminary Report on Thermal and Electrical Conductivities of Some iPlutonium- Aluminum Alloys, IAMS-1080. Los Alomos Scientific Lab., March 15, 1950

E. R. Gilbert, Argonne National Laloratory, I'ersonal Connunication, February 1964, as reported by H. R. Gardiner in Chapter 4, The Plutonium Handlook. 1966.

A. E. Gorum, "Some Olsservations of the Flow and Fracture of Plutonium," in O. J. Wick and I. D. Thomas (Fds.), Proceeding of the Third Plutonium Metallurgy Conferenee, Ianuary 12, 13, 14 $\frac{\text { of the }}{1960} \frac{\text { Third }}{\mathrm{HW}-65} \frac{\text { Plutonium }}{439, \bar{V} \mathrm{l} .} \overline{1, \mathrm{pp} .52-65, \text { Inanford Atomic Products }}$ Operation, General Eleetric Co., Jan 15, 1960. (Classified)

A. V. Grosse. "The Viscusity of Liquid Metals and an Empirieal Relationship, Between Their Activation Energy of Viscosity and Their Melt inf Points," J. Inorg. Nuel. Chem., vol. 23, p. 3333 1961 .

A. V. Grosse. "The Viscosity of I.iquid Plutonium, l'rediced from a General Relationship Between the Activation Energy and Melting Points of Metals, and the Experimental | ata," J. Inorg. Nuel. Chem., vol. 25, 1\%, 13k. 194i3.

K. A. Gsehnejdner, Jr, et al. "lifects of Alloyiln Additions on the $\gamma$ Transformation of Cerium. Pari II. Elfects of Scandium, Thorium and Ilutonium Additions," I. Phys. Chem. Solids, vol. 23, p. 1191, 1962

J. D. Hill. "Allotropic Transformations in ['lutonium," Nature, vol. 186, p. 305. 1960 .

J. D. Hill. "Exploring the Transformation Behariour of the Allotropes of Plutonium," Less-Common Metals, (o) , 4, p. :341. 1962 .
C. T'. Hertom and li. I. Warel. "Rolling aud Mechanical Testing

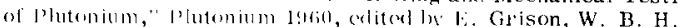

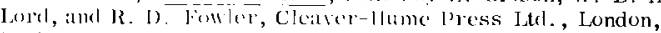
$1961.11) .1961-.12$.

H. H. His man ank J. J. Kut $\%$. Corrosion of Plutonium Alloys in

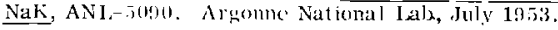

E. R. Jette. "I'lutonium Metal," Nuclear Metallurgy, IMD Special report Serios No. 1, p. 34. Amerian Institute of Mining and Metallurefica] Engineers, lne., New York, 195.

E. R. Jette. "Some physical Properties of Plutonium Metal," J. Chem. Phys, vol. 23, p. 365. 19.5.

E. R. Jette and A. S. Coffinberr. "Plutonium and lts Alloys," General Properties of Materials, The Reactor Handbook, vol. 3, sec. 1, USAEC Report AECD-3647, p. 237. 195.5.

L. V. Jones et al. "The Viscosity and Density of Molten Plutonium Metal and a Plutonium-Cerium-Cobalt Eutectic Alloy," Am. Soc. Metals, Trans. Quart., vol. 55, p. 819. 1962.

T. I. Jones. "An Initial Assessment of Aluminum-plutonium Alloys for Water-Cooled Power Reactors," Plutonium 1960 , edited by E. Grison, W. B. H, Lord, and R. D. Fowler, CleaverHume Press Ltd., London, 1961. pp. 720-744.

T. I. Jones. The Irradiation of Aluminum-Plutonium Alloys in Zircaloy-2 Sheathing. Part III: Post-irradiation Examination and Testing of Aluminum-Plutonium Alloys, CRRM-1097. Atomic Energy of Canada Limited, Chalk River, Ontario. July 1962.

A. E. Kay. "Replies and Comments," Plutonium 1960, edited JW E. Grison, W. B. H. Lord, and R. J). Fowler, Cleaver-Hume Press Lidd., Iondon, 1961. pp. 107-108.

A. E. Kay. "Some Physical and Physieochemical Properties of I'lutonium Metal," The Metal J'lutonium, edited by A. S. Coffinbery and $W$. N. Miner, The Univer'sity of Chicago p'ress, Chicago, 19)(1. |P). 194-204.

A. L. hay and P", l'. 'T. Iinford. "The klastice Constants of Plutonium," Plutonium 1960, orlited ly k. Grison, W. B. H. Lord,

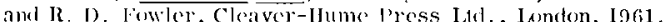
D). $51-i \pi$

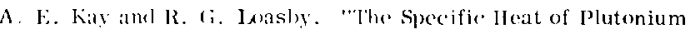
at lligh Tomperituses," phil. Mas., "righth series, vol. 9, no. 97 p. $12,196.4$.

J King et al, "Sill-Irradiation tilfeces in Staljilized plutonium,"Actal Metallurgicn, 161, 12, 1, 112. 1964.

E. King and J. A. Jee. "The Jom Temperature Resistivity of B-phase Plutonium," Cryogenies, wi. 3, 1. 177. 1963.

l. W. knight. "Culsic Expansion of I'lutonium and PlutoniumIron Eutertic Between loom I'emperature and 700 to $800^{\circ} \mathrm{C}$ Plutomium 1960, edited by E. Grison, W. R. II, Lord, and R. D. lowler, Cleaver-Hume Press Lld., Iondon, 1961. pp. 684-704.

S. T. Konolueersky. "Jhase Diagrams of Some Plutonium Systems," Conference of the Acarlemy of Sciences of the USSle on the PEACEFUL USES OF ATOMIC FNERGY

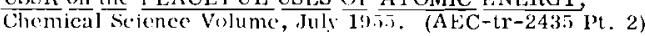
시. 207-21.4.

S. T. honobecrsky et al. "Some Physical properties of Uranium, plutonium, and their Alloys," 122230 , procedings of the Second Inited Nations Intornational Conference on the Peaceful Uses of Anited Nations International Conference on the Peaceful Uses of 1958

12. Lallement. "Mesure du Module d'Young du Plutonium Alpha a Basse Tomperature", phesies letters, vol . ., p. 182, 1963.

R. Iillement, "Resistivity of a I'utoniun Under Pressure at low Tenueratures," Physies letters, vol. 8, p. 166, 1964,

II. I. Jaquer (umpublished data, las Alamos, 1949-1953) in A. S, Coflinherry and M. B. Waldron. "A Review of Physical Metallurgy of Plutonium," Metallurgy and Fucls, Progress in Nuclear Energy, cdited by IL. M. Finniston and .T. P. Howe, MeGraw-Hill Book Co. Inc., New York, 1956. series V, vol. 1, 1) 383.

11. L. Jaquer. "Sound-Velocity Moasurements on Alpha-Phase Plutonium," The Metal Plutonium, celiterl hy A. S. Coffinberr

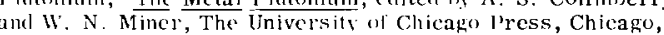
1!6il, (P). 15T-142

J. A. loe and J2. (). A. Hall. "Some Measurements on The Thermoclectric: Poller of Plutollium Motal," Iass-Common

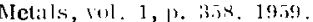

J. A, lee ct. al. "Aceumulation of sell-1)amage in I'lutonium,"

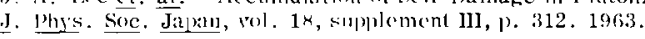

J. A. Ine et al. "Inw Temperature Resistivity of Self-Irradiated

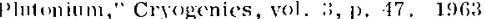

J. A. Ine et al. "Sonke Properties of Plutonium and PlutoniumRich Alless," Plutonium lofit, criter hy E. Grison, W. B. H. Lord, and R. I). Finler, Cleaver-1lume l'pess I.tht., London, 1961.

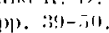




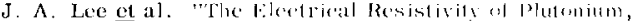

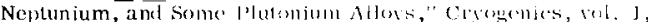
p. 52,1060 .

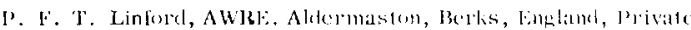

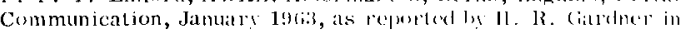
Chapter 1, The Plutonium liandbooli. Jasti

I'. F. T', Linford and A. L. Kar, Alomic Weapons Reseureh Establishment, lingland, l'ersonal Commoni ieation, November. 1961, as reported ly II. R. Ciatriner in Chapter 1, The Plutonium Hanclbonok. 19miti.

L. T. Lloyel and I. I. Nowidki. "Crystallographic Aubles for Al, hai plutonium," J. Nucl. Mater., vol, 10, p. 25. 19 (3i3.

R. G. Loaslyy. "Replies and comments," P'utonium 1960, cditer

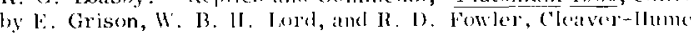

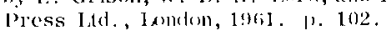

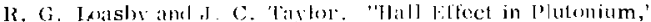

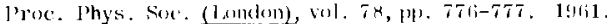

W. M. 1Amer. "Fhe $a-\delta$ Transformation in Plutunium," solis state Commun., sol. 1, p. wi. 19033

B. A. Ioomis. Suclling ol Aluminum-Clad Aluminum-[']lumium

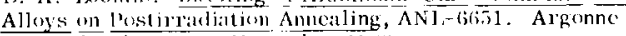
National Laboratory, Novomber 19tia.

1P. G. Mardon. "Authess' Roplies to the Report and Comments on the Report ancl Palpers," Plutonium 19tio, edited by F. Grisom,

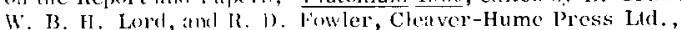

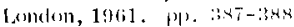

P. G. Mardon et al. "'The Comstitution and Falbrication of Uranium-Molyledenum-Plutonium Fucls, " Plutonium 1960, edited by E. Grison, W. B. H. Lard, and R, D. Fowler, cleaver-lume press I.td., l,omdon, 1961, pp. 329-352.

P. J. Mardon, J II. Pearee, and J. A. C. Marples. "Constitution Studies on the Neptuniun- Plutonium Alloy System," less-Common Metals, vol, :3, p. 2, 1 , 1 ! 1 .

J. A. C. Marples. "I'he Plutonium-Zineonium Plase Diagram," Less-Conmon Metals, vol, 2, p.:331. 1960.

A. E. Martin and G. D. Solmanoff. Properties of Plutonium Alloys, LA-301. Los Alamos Scientific laboratory, Aug. 26, 1947. (Classified)

D. MeWhan et al. "Compression and the $\boldsymbol{\alpha}-\boldsymbol{\beta}$ Phase Transition of J'lutonium," I. Ayzl. I'hys., vol. 30, p. 214. $19,39$.

G. T, Meaden. "Electronic Properties of the Actinide Metals at Low Temperatures," proe. Roy. Soc. (1.undon), Ser. A, vol. 276, p. 553.1963

G. T. Meaden and J. A. Lee. "The Thermoelectric Powers of the Actinide Metals at Low Temperatures," Cryogenics, vol. 2, p. 182.1962 .

K. Mendelssohn and D. A. Wigley. "Effect of Self-Irradiation on the Resistivity of llutonium," Physics Letters, vol. 1, p. 326. 1962 .

D. C. Miller and J. S. White, AWRE, Aldermaston, Berks, England, Private Communication, November 1962, reported by H. R. Gardner in Chapter 4, The Plutonium Handbook. 1966.

R. D. Nelson, as shown in J. I). Hill. The Thermodynamic Equilibrium Temperatures of the $\neq \&$ and $\neq$ Transformations

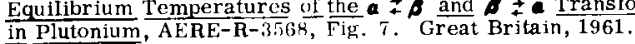

R. D. Nelson. Transformation Kineties of Beta Plutonium, HW-54016. Hanford Airnic Profiucts operation, General Electric Co., Dee. 4, 19:5i.

R. D. Nelson. "Transformation Kinetics of Beta Plutonium," Trans. Am. Soc. Motals, vol. 51, p. fi.9. 1959.

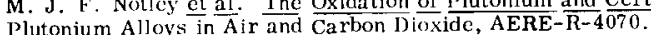

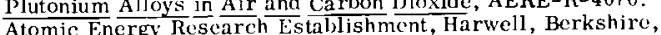
Atomic Energy
England, 1962 .

C. E. Olsen et al. "The Self-lrradiation of Plutonium and Its Delta Alloys," Radiation Daniage in Reactor Materials, International Atomic Energy Agency, Vienna, 1963. pp. 351-58.

C. E. Olsen, T. A. Sanderlaw, and C. C. Herrick. The Density of Liquid Plutonium Meta], IA-235. In. Alomos Scientific Laboratory, May 1969 .

D. M. Poole and J. L. Nichols. Properties of Some Plutonium Alloys of Potential Use as Iiquid Metal Fast Reaetor Fuels, $\overline{A E R E}-\bar{R}-3122$. Metallurgy Jivision, Atomic Encray Researel Establishment, Harwell, Berlishire. November 1959.

D. M, Poole et al. "phiss I)iagrams of Some plutonium Alloy Systems," plutonium 1960, editerl hy $R$. Grison, W. B. H. Iord, and R. D. Fowler, cleaver-Iume press Ldd, london, 1961. pp. 267-280.

D. M. Poole et al. "Properties al some l'lutonium Fucls,"

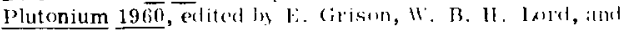

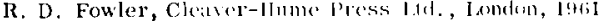
pp. $627-6,49$.
1) U. Pulle, (i. K. Milliamsem, ind .J. A. C. Marples. "A

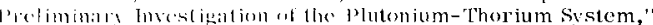

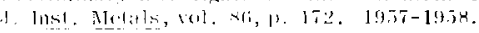

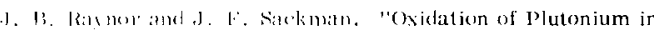
Henst Air ind Algon," Naluec, vol. 197, p. 588. 1963.

1. [3, Ithinclitmmer el al. "The Plutonium-Copper Phase

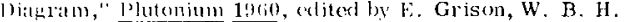

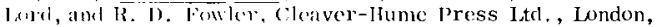

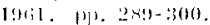

1. (1 Rolineon, Alomir. Heipons Researeh Establishment, Nkermaston, Berks, Linglancl, I'ersonal Communication, as reported by II. R. Cardere in Chaptel' 4, The Plutonium Itandiscoli. 19tits.

13. I3. $R(x)$, d $\mathrm{r}^{\circ} \Lambda$ Nentron Diffraction Examination of Alpha

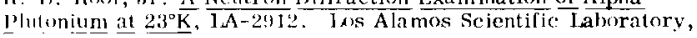

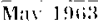

() I c I Iumnalls. "The Clystal structure of Some Intermetallic (amprumbis ol Pluturiun," Can. J. Chem., vol. 34, p. 133. 1956.

J. IF Sickman. "The Almospheric Oxirlation of Plutonium Metal," Piutonium 1960, edited hy l: Grison, W. B. H. Lord, and R. D. litile, Cleaver-llume le'ss Ltrl, London, 1961. pp. 222-229.

T. A. Sandenaw. "Determination of the Rate of Heat Generation "l I'lutonium Metal," J. Phys. Chem. Solids, vol. 23, p. 825. 19962.

'T. A. Sindenaw. "Effects of Self-]nduced Radiation Damage on the Low Temperature Heat Capacity of a Specimen of ElectroRolined High Purity Plutoninm," J. Phys. Chem. Solids, vol. 23 p. 1245.1962.

T. A. Sandenaw, "Heat Capacity, Thermal Expansion and Flectrical Resistivity of an 8 a/o Aluminım-Plutonium (DeltaI'hase Stabilized) Alloy Brow $300^{\circ} \mathrm{K}, " \mathrm{~J}$. Phys. Chem. Solids, vel. 16,1$) .329 .1960$

T. A. Sandenaw. "The Thermal tixpansion of Plutonium Metal Bolou $3900^{\circ} \mathrm{K}$ " Plutonium lofio, clited by $\mathrm{E}$ Grison, $\mathrm{W}$. B II

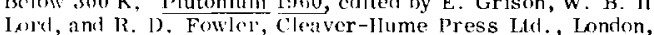

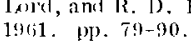

T. A. Sandenaw. "Results of Measurements of Physical Propertics of Ilutonium Metal," The Metal Plutonium, edited by A. S. colfinherry and $W$. N. Miner, The University of Chicago Press, Chicagn, 1961. pp. 152-1.5li

T. A. Sandenaw and C. F. Olsen. "Electrical Resistivity of P'utonium and Metal Jectween $1.73^{\circ} \mathrm{K}$ and $298^{\circ} \mathrm{K}$," Plutonium 19oro, edited by E. Grison, W. B. 1l. Lord, and R. D. Fowler, Claver-liume Press Ldd., Tondon, 1961. p. fi.

T. A. Sandenaw and R. B. Gibney. "The Electrical Resistivity and Thermal Conductivity of I'lutonium Metal," $\mathbf{J}$. Phys. Chem. Solids, vol. 6, p. 87. 19ik

T. A. Sandenaw, C. E. Olsen, and R. B. Gibney. "Heat Capaeity of Plutonium Metal Below $420^{\circ} \mathrm{K}$," Plutonium 1960, edited by E. Grison, W. B. H. Iord, and R. D. Fowler, Cleaver-Hume press Ltd., London, 1901. p. 72 .

II. E. Savage and E. R. Gilbert, Argonne National Laboratory, Jersonal Communication, February 1964 . As reported by H. R. Gardner in Chapter 4, The Plutonium Handbook.

F. W. Schonfeld, Los Alamos Scientific Lab., Unpublished Data, 10 \%1.

F. W. Schonfeld. "Plutonium Phase Diagrams Published by the Russians," The Metal Plutonium, edited by A. S. Coffinberry an W. N. Miner, The University of Chicago Press, Chicago, 1961. pp. $255-264$

F. W. Schonfeld. "Plutonium Phase Diagrams Studied at Los Alamos," The Metal Plutonium, edited by A. S. Coffinberry and W. N. Miner, The University of Chicago Press, Chicago, 1961. pp. $240-254$.

F. W. Schonfeld et al. "Plutonium Constitutional Diagrams," Metallurgy and Fuels, Progress in Nuclear Energy, edited by H. M. Finniston and J. P. Howe, Pergamon Press, New York, 1559. series $\mathrm{V}$, vol, 2, 19), 679-599.

J. E. Selle and D. E. Etter. "The Plutonium-Cerium System," Trans. Met. Soe. AIME. vol. 230, p. 1000. 1964

C. Z. Serpan, Jr, and l.. J. Wittenberg. "The Density of Liquid I'lutonium Melal," Trans. Met. Soce. AlME, vol. 221, p. 1019. 1961

1). R. Stephens. "The |'hase l)iagram of Plutonium," $\underline{\mathrm{J}}$. Phys. Chem. Solids, vol. 24, p. 1201. 1963.

1R. L. Tate and E. M. Cramer. "Self-Diffusion Studies of Delta plutonitum," Trans. Mot. Soc. AIME, vol. 230, p. 641. 1964.

12. F filte. "The lablecation of Billets Conta ining plutonium fir MTle luel Flements," The Metal plutonium, edjed by A. S collinmery and $W$. N. Mincer, The University of Chicago Press, Chicilyo, 1961. pp. 3.15-36. 


\section{ACKNOWLEDGMENTS}

Appreciation is expressed to the publishers or copyright assignees of the following publications for permission to use figures, tables, and direct quotations from their publications. All of the following are copyrighted except those marked by an asterisk $\left({ }^{*}\right)$.

Corrosion of Reactor Materials, Vol. II International Atomic Energy Agency.

Extractive and Physical Metallurgy of Plutonium and Its Alloys, American Institute of Mining, Metallurgical, and Petroleum Engineers.

The Metal Plutonium, The University of Chicago Press.

Metallurgy and Fucls, Progress in Nuclear Energy, Series V, Vols. 1 and 4, The Pergamon Press, Inc.

Nuclear Metallurgy - IMD Special Report Series No. 1, American Institute of Mining and Metallurgical Engineers, Inc.

Plutonium 1960, Cleaver-Hume Press Ltd.

Proceedings of the International Conference on the Poaceful Uses of Atomic Energy, 1955 , United Nations.*

Proceedings of the Second United Nations International Conference on the Peaceful Uses of Atomic Energy, United Nations.*

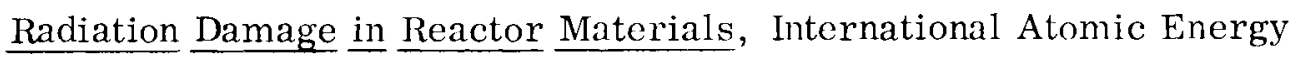
Agency.

Reactor Handbook, 2nd ed., Interscience Publishers, Inc.

Acta Crystallographica

Acta Metallurgica

American Society for Metals Transactions

Canadian Journal of Chemistry

Corrosion

Cryogenics

Journal of Applied Physics 
Journal of Chemical Physics

Journal of Inorganic and Nuclear Chemistry

Journal of Institute of Metals

Journal of Metals

Journal of Nuclear Materials

Journal of Physics and Chemistry of Solids

Journal of the Chemical Society (London)

Journal of the Less-Common Metals

Journal of Physical Chemistry

The Metallurgical Society of AIME Transactions

Nature

Nuclear Science and Engineering

The Philosophical Magazine

Physical Review

Physical Review Letters

Physics Letters

Proceedings of the Physical Society

Proceedings of the Royal Society (London), Series A

Solid State Communications

Transactions of the American Nuclear Society 
BNWL-37

\section{ONSITE DISTRIBUTION}

Copy Number

Pacific Northwest Laboratory

1
2
$3-12$
13
14
15
16
17
18
19
20
21
22
$23-26$
$27-200$

T. K. Bierlien

C. H. Bloomster

M. P. Burns

J. J. Cadwell

D. R. deHalas

H. R. Gardner

S. M. Gill

R. R. King, Jr.

E. D. McClanahan, Jr.

R. D. Nelson

T. C. Nelson, Jr.

R. W. Stewart

C. R. Tipton, Jr.

O. J. Wick

Technical Information Files

General Electric Company, Richland

201

202

A. E. Smith

Document Transfer \& Accountability

$\underline{\text { Richland Operations Office }}$

203

204

R. K. Sharp

Technical Information Library

\section{OFFSITE DISTRIBUTION (SPECIAL)}

Number of Copies

1

1
Argonne National Laboratory

Attn: F. G. Foote

Atomic Energy Research Establishment Weapons Group

Aldermaston, Berks, England

Attn: Dr. J. F. Sackman 
OFFSITE DISTRIBUTION (SPECIAL) (cont'd)

1

1

3

1

9

1

1
Battelle Memorial Institute

Attn: S. J. Paprocki

Dow Chemical Company, Rocky Flats

Attn: L. A. Matheson

Los Alamos Scientific Laboratory

Attn: R. D. Baker

W. J. Maraman

F. W. Schonfeld

Nuclear Materials Equipment Corporation Attn: I. D. Thomas

University of California, Livermore

Attn: M. E. Hasbrouck (6)

W. J. Ramsey (2)

D. A. Schauer

University of Leicester

Department of Chemistry

Leicester, England

Attn: Dr. J. B. Raynor

USAEC Division of Military Applications

Attn: Maj. I. L. Williams 